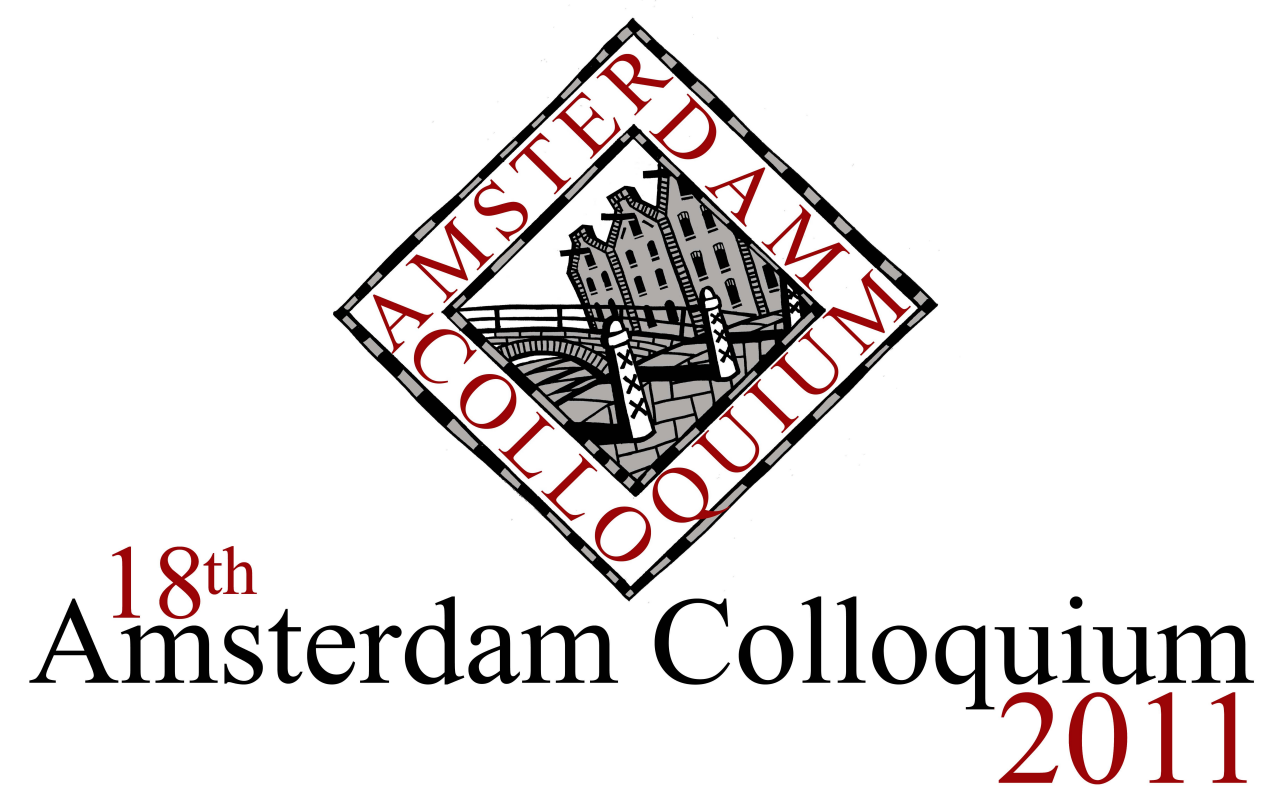

Pre-proceedings

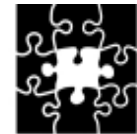

INSTITUTE FOR LOGIC, LANGUAGE AND COMPUTATION 


\section{Contents}

$\begin{array}{ll}\text { Workshop on Inquisitiveness } & 1\end{array}$

Martin Aher

Free Choice in Deontic Inquisitive Semantics (DIS) . . . . . 1

Robin Cooper \& Jonathan Ginzburg

Negative inquisitiveness and alternatives-based negation . . . 10

Edgar Onea \& Markus Steinbach

Where Question, Conditionals and Topics Converge . . . . . 20

Wataru Uegaki

Inquisitive knowledge attribution and the Gettier problem . . 30

Workshop on Formal Semantic Evidence

Ralf Naumann

Relating ERP-Effects to Theories of Belief Update \& Combin-

ing Systems . . . . . . . . . . . . . . . . 4 40

Janina Radó \& Oliver Bott

Underspecifed representations of scope ambiguity? . . . . . . . 51

Agata Maria Renans

Projective behaviour of Nur quantitative experimental research 61

Florian Schwarz \& Sonja Tiemann

Presupposition Processing - The Case of German wieder . . . 71

Poster SESSION . . . . . . . . . . . . . . . . 81

Márta Abrusán \& Kriszta Szendröi

Experimenting with the king of France . . . . . . . . . . 81

Gemma Boleda, Stefan Evert, Berit Gehrke \& Louise McNally

Adjectives as saturators vs. modifers: Statistical evidence . . . 91

Adrian. Brasoveanu \& Jakub Dotlačil

Licensing Sentence-internal Readings in English: An Experi-

mental Study . . . . . . . . . . . . . . . . . . 101

Lisa Bylinina \& S. Zadorozhny

Evaluative adjectives, scale structure, and ways of being polite 112

Emmanuel Chemla \& Lewis Bott

Processing: Free choice at no cost . . . . . . . . . . 126

Alex Djalali, Sven Lauer \& Christopher Potts

Corpus evidence for preference-driven interpretation . . . . . . 132

Francesca Panzeri \& Francesca Foppolo

Can children tell us something about the semantics of adjectives?142

Yasutada Sudo, Jacopo Romoli, Martin Hackl \& Danny Fox

Variation of Presupposition Projection in Quantifed Sentences 152 
Gemma Barberá

When wide scope is not enough: scope and topicality of discourse referents . . . . . . . . . . . . . . . . 162

Kathryn Davidson

When Disjunction looks like Conjunction: Pragmatic Consequences in ASL . . . . . . . . . . . . . . . . . . . 172 Josep Quer

Quantificational strategies across language modalities . . . . . 182 Ronnie Wilbur, Evie Malaia \& Robin Shay

Degree modification and intensification in ASL adjectives . . . 192

\section{General Program}

Márta Abrusán

Focus, Evidentiality and Soft triggers _. . . . . . . 201

Arno Bastenhof

Polarities in logic and semantics . . . . . . . . . . . . 211

Chris Blom, Philippe de Groote, Yoad Winter \& Joost Zwarts

Implicit Arguments: Event Modification or Option Type Cat-

egories? . . . . . . . . . . . . . . . . . . . . 221

Lucas Champollion

Each vs. jeweils: A cover-based view on distance-distributivity 231 Simon Charlow

Cross-categorial donkeys . . . . . . . . . . . . . . . . 241

Anna Chernilovskaya \& Rick Nouwen

On wh-exclamatives and noteworthiness . . . . . . . . 251

Ka-fat Chow

Generalizing Monotonicity Inferences to Opposition Inferences 261

Elizabeth Coppock \& David Beaver

Exclusive Updates! Brought to you by your local QUD . . . . 271

Tim Fernando

Steedman's Temporality Proposal and Finite Automata . . . . 282

Michael Franke

On Scales, Salience and Referential Language Use (A Revisit) 292

Jonathan Ginzburg, Raquel Fernández \& David Schlangen

On Semantics and Pragmatics of Dysfluency . . . . . . . . . . 302

Gianluca Giorgolo \& Stephanie Needham

Pragmatic Constraints on Gesture Use: The Effect of Down-

ward and Non-Entailing Contexts on Gesture Processing . . . 312

Daniel Hardt, Line Mikkelsen \& Bjarne Orsnes

Sameness, Ellipsis and Anaphora . . . . . . . . . . . . 322 
Vincent Homer

As Simple as It Seems . . . . . . . . . . . . . . . . . 332

I-Ta Chris Hsieh

On the Non-Licensing of NPIs in the Only-Focus . . . . . . 342

Julie Hunter

Now: A Discourse-Based Theory . . . . . . . . . . . . . . 352

Natalia Ivlieva

Obligatory implicatures and grammaticality . . . . . . . . . 362

Jacques Jayez \& Bob van Tiel

Only 'only'. An experimental window on exclusiveness . . . . 372

Udo Klein

Computing quantifer scope with witness sets . . . . . . . 382

Todor Koev

On the Grounding Status of Appositive Relative Clauses . . . 394

Sveta Krasikova

Definiteness in Superlatives . . . . . . . . . . . . 404

Noor van Leusen

The accommodation potential of implicative verbs . . . . . . 414

Louise McNally \& Henriette de Swart

Inflection and derivation: how adjectives and nouns refer to

abstract objects . . . . . . . . . . . . . . . . . 425

Friederike Moltmann

Tropes, Intensional Relative Clauses and the Notion of a Vari-

able Object . . . . . . . . . . . . . . . . . 4 . . . . . . . . . . . .

Reinhard Muskens

A Theory of Names and True Intensionality . . . . . . . . 446

Tohru Seraku

Multiple Foci in Japanese Clefts and the Growth of Semantic

Representation . . . . . . . . . . . . . 455

Ryan Waldie

Nuu-chah-nulth Evidentials and the Origo . . . . . . . . 465

Andreas Walker

Focus, Uniqueness and Soft Presupposition Triggers . . . . . . 475 Ting $\mathrm{Xu}$

You again: How is its ambiguity derived? . . . . . . . . 485 


\title{
Free Choice in Deontic Inquisitive Semantics (DIS)
}

\author{
Martin Aher
}

University of Osnabrueck, Institute of Cognitive Science

\begin{abstract}
We will propose a novel solution to the free choice puzzle that is driven by empirical data from legal discourse and does not suffer from the same problems as implicature-based accounts. We will argue against implicature-based accounts and provide an entailment-based solution. Following Anderson's violation-based deontic logic, we will demonstrate that a support-based radical inquisitive semantics will correctly model both the free choice effect and the boolean standard entailment relations in downward entailing contexts. An inquisitive semantics is especially suited to model sluicing effects where the continuation "but I do not know which" coerces an ignorance reading. It also demonstrates that the counterarguments to deontic reduction failed to take into account the different effects of inquisitive and informative utterances in con ferences invalid. Furthermore, we will argue that the problem of strengthening the antecedent that is used
as a counterargument against entailment-based accounts also fails under a refined notion of entailment.
\end{abstract}

\section{Introduction}

Example 1. A country may establish a research center or a laboratory

Example 2. A country may establish a research center.

Example 3. A country may establish a laboratory.

When (1) is international law, it gives permission to establish a research center and it gives permission to establish a laboratory. (Although it does not necessarily give permission to establish both.) The so called free choice reading reverses standard entailment relations between the disjuncts and disjunction such that (1) entails (2) and (3). It is now the same entailment relation as between a conjunction and its conjuncts. In a non-deontic setting disjuncts entail the disjunction.

Example 4. A country established a research center or a laboratory.

Example 5. A country established a research center.

Example 6. A country established a laboratory.

Either disjunct - (5), (6) - entails (4). Free choice disjunction has become one of the better documented puzzles in semantics since it was investigated by Hans Kamp [14].

The approach to free choice outlined here is based on an investigation of World Trade Organisation dispute texts, which documents discourse in a deontic setting between a complainant, respondent and a panel of judges. The results of previous work [1] suggest that judges organize legal discourse around central questions on whether specific laws have been violated. The complainant has the burden of proof to demonstrate that an act was performed and to specify the law that it violated. The respondent has to either deny the act or break the link between the act and the law.

A violation-based deontic logic gravitates around the question whether an act violates a specific law. A permission sentence in a law text provides information on what is not a violation. This should mostly be relevant in cases where there is also a general prohibition in force, so that the permission sentence effectively provides an exception. For example, in case pets are not permitted in malls, the following sentence explicitly tells you that you do not violate that prohibition by bringing a guide dog into the mall.

Example \%. Customers may bring guide dogs into the mall.

Legal discourse that gravitates around violations suggests an approach based on Anderson's reduction of a permission utterance $\diamond p$ to $p \rightarrow \bar{v}$ [4] which will be shown to be a successful approach to the free choice permission puzzle when implemented in the framework of inquisitive semantics. 


\section{Workshop on Inquisitiveness}

\section{Previous Accounts}

Zimmermann [21] provides a solution through positing a pragmatic mechanism that reinterprets disjunction as a conjunctive list of epistemic possibilities: $\diamond A \wedge \diamond B$. Unfortunately, such an approach does not explain why this reinterpretation arises in those contexts. Furthermore, Alonso-Ovalle [3], and Simons [20, p. 8] draws attention to the fact that disjunctive permission in a downward entailing context once again behaves standard - an effect Zimmermann fails to predict.

Many following accounts accepted that free choice is essentially a pragmatic effect and suggested that the phenomenon is an implicature. These accounts include Schultz [18], Eckardt [7], Fox [8] and the game theoretic implicature account by Franke [9]. As approaches to free choice have been extensively discussed in the literature, for example by Schultz [17] or more recently by Barker [5], we will concentrate on examining [7] to expatiate on general issues with implicature-based solutions.

Eckardt derives the free choice effect utilizing an implicature through the maxims of manner and quality. The free choice inference of disjunctive permission statements is derived through the following pragmatic steps.

1. May $S$ refers to a subset $\mathrm{W}$ of all deontic alternatives for a subject a in world $\mathrm{W}^{*}$

Presupposition: $\mathrm{W}$ needs to be a subset of a previously mentioned group $\mathrm{Y}$.

2. $\mathrm{A}(\mathrm{W})$ predicates over all objects in subset $\mathrm{W}$.

3. Disjunctive predication

(a) Speaker utters (A or B)(W) and has sufficient knowledge.

(b) Speaker violated the maxim of manner: be brief, as the speaker did not use either disjunct: $\mathrm{A}(\mathrm{W})$ or $\mathrm{B}(\mathrm{W})$.

(c) Inference from quality: Speaker believes $\mathrm{A}(\mathrm{W})$ is not true and $\mathrm{B}(\mathrm{W})$ is not true.

(d) Free choice effect: There are some worlds in $\mathrm{W}$ in which $\mathrm{A}$ is true and $\mathrm{B}$ is not, and other worlds in which $\mathrm{B}$ is true but $\mathrm{A}$ is not: $w_{1}, w_{2} \leq W: A\left(w_{1}\right) \wedge \neg B\left(w_{1}\right), \neg A\left(w_{2}\right) \wedge B\left(w_{2}\right)$

The weakness of this account lies in (3c) as the intuition behind the deontic free choice effect is that the speaker's information state supports only worlds in which $\mathrm{A}$ and $\mathrm{B}$ are permitted. The reason why $\mathrm{A}(\mathrm{W})$ and $\mathrm{B}(\mathrm{W})$ in (3d) are not true is that there exist some worlds in the speaker's information state in which one of them is not true. But this is contrary to the intuition outlined above.

$[20$, p. 14$]$ argues generally against implicature based accounts on the grounds that there does not seem to be a distinction between what is said and what is implicated in examples such as (1). Compare this to a classic example of generalized implicature from Grice [11, p. 32].

Example 8. $\mathrm{X}$ is meeting a woman this evening.

Grice states that such a statement generally implicates that the woman being met is not X's wife, mother, sister, etc. Thus, there exists a clear distinction between that which is said (X will meet a woman) and that which is implicated ( $\mathrm{X}$ will meet a potential romantic acquintance). The lack of such distinctions in free choice sentences poses a challenge to any implicature based account.

$[5$, p. 16] demonstrates that another marker of implicatures is visibly lacking, namely cancellability. Observe the following example.

Example 9. You may eat an apple or a pear, although in fact you may not eat an apple.

When an implicature in cancelled, the utterance only has the meaning of what is said. If (8) were cancelled by "... but it's only her mother." then the utterance would lose the implicature that the woman is a romantic acquintance. Yet, instead of reverting the phrase to that which is said as opposed to that which is implied, the added phrase in (9) appears to make the statement contradictory.

There appears to be another possible route for cancellation, which is to utter either of the following continuations.

Example 10. You may eat an apple or a pear, although in fact you may not eat both.

Example 11. You may eat an apple or a pear, although in fact I do not know which.

The consequence of uttering (10) does not cancel the free choice effect. Permission is given to eat an apple and permission is given to eat a pear. Yet, the continuation provides the additional information that eating both an apple and a pear is prohibited. This additional information does not conflict with free choice readings.

(11) intuitively suggests that the speaker is ignorant. The speaker has limited knowledge of the governing permissions and prohibitions, and utters the most helpful utterance available. It is well known in the literature that such utterances have standard disjuntive entailment relations, and it can easily be accounted for by inquisitive semantics.

[5] proposes a semantic approach similar to the one pursued here, by following [15] in positing a normative ideality $\delta$ such that if $\varphi$ is obligatory, then if $\varphi$ then $\delta$. This view is a contrapositive view of Anderson's reduction 
and, thus, is in no conflict with the current solution to the free choice puzzle at a foundational level. But in terms of details, the analysis of WTO examples in [1] suggests that legal reasoning does not concern idealities but rather violations. While this might be contingent on the deontic context, in terms of legal language, the violation-based solution remains preferable.

Furthermore, [5, p. 11] correctly notes that if obligation is rendered as "if $\varphi$ then $\delta$ " then doing $\varphi$ guarantees the ideal universe. In a standard model, this means that if you may eat an apple, then eating an apple and killing a postman will invariably lead to the ideal universe. Barker introduces a resource sensitive calculus to render such inferences invalid.

As will be demonstrated, Barker's approach seems intuitively to be on the right track, but it lacks certain aspects which will be included in the approach to follow. For example, a violation based system allows for inferences with different violations, such as when two different laws are relevant to judge a case. An ideality based model would require significant work to account for these cases.

Also, while Barker's account of the free choice effect is entailment based, he introduces pragmatics to attain the default reading of negated disjunctive permission sentences. It would be more aligned with his project to provide a fully semantic account. This observation, albeit not a counterargument in itself, also holds for the semantic account of Aloni [2].

\section{The Proposal}

Negation of disjunctive permission utterances is one of the fundamental problems for many accounts on free choice and this fact is often taken as support for the idea that the phenomenon should be resolved by an implicature. We shall thus base the inquisitive deontic model on an independently motivated prior version of inquisitive semantics that focuses on the effects of negation - Radical Inquisitive Semantics. An earlier version of the language used here was developed and explored by Sano [16]. Our proposal adds clauses for deontic permission and discusses entailment in the radical environment. Unlike the original [13], this version of Radical Inquisitive Semantics is restricted. Also note the definition for the negation of implication, the original motivation for such a formulation is discussed at length elsewhere [13, pp. 18-23, 28-30].

We shall only consider a propositional language of a finite set of propositional variables and the operators: $\bar{\varphi}, \wedge, \vee, \rightarrow, \neg$. Negation is defined as $\bar{\varphi}$ and $\neg$ is added as classical negation for comparison purposes and will only play a limited role in the deontic story.

We also need to define worlds as binary valuations for atomic sentences and states as non-empty sets of worlds. $\sigma$ and $\tau$ are variables that range over states, $w$ is the variable that ranges over worlds and $W$ is the set of all (classical) valuation functions. Propositions expressed by sentences are defined through support. When a state supports $\varphi$ then we write $\sigma \models^{+} \varphi$ and when a state rejects $\varphi$ then we write $\sigma \models^{-} \varphi$.

Definition 1. Radical inquisitive semantics.

1. $\sigma \models^{+} p$ iff $\forall w \in \sigma: w(p)=1$

$\sigma=^{-} p$ iff $\forall w \in \sigma: w(p)=0$

2. $\sigma \models^{+} \bar{\varphi}$ iff $\sigma \models^{-} \varphi$

$\sigma \models^{-} \bar{\varphi}$ iff $\sigma \models^{+} \varphi$

3. $\sigma=^{+} \neg \varphi$ iff $\forall \tau \subseteq \sigma . \tau \not \models^{+} \varphi$

$\sigma \models^{-} \neg \varphi$ iff $\sigma \models^{+} \varphi$

4. $\sigma \models^{+} \varphi \vee \psi$ iff $\sigma \models^{+} \varphi$ or $\sigma \models^{+} \psi$

$\sigma=^{-} \varphi \vee \psi$ iff $\sigma=^{-} \varphi$ and $\sigma \models^{-} \psi$

5. $\sigma \models^{+} \varphi \wedge \psi$ iff $\sigma \models^{+} \varphi$ and $\sigma \models^{+} \psi$

$\sigma \models^{-} \varphi \wedge \psi$ iff $\sigma \models^{-} \varphi$ or $\sigma \models^{-} \psi$

6. $\sigma \models^{+} \varphi \rightarrow \psi$ iff $\forall \tau \subseteq \sigma .\left(\tau \models^{+} \varphi\right.$ implies $\left.\tau \models^{+} \psi\right)$

$\sigma \models^{-} \varphi \rightarrow \psi$ iff $\exists \tau .\left(\tau \models^{+} \varphi\right.$ and $\forall \tau^{\prime} \supseteq \tau .\left(\tau^{\prime} \models^{+} \varphi\right.$ implies $\left.\left.\sigma \cap \tau^{\prime} \models^{-} \psi\right)\right)$

Definition 2. Propositions.

1. $\|\varphi\|^{+}:=\left\{\tau \subseteq W \mid \tau \models^{+} \varphi\right\}$

$\|\varphi\|^{-}:=\left\{\tau \subseteq W \mid \tau \models{ }^{-} \varphi\right\}$

The model is persistent, as $\|\varphi\|^{+}$and $\|\varphi\|^{-}$are closed under $\subseteq$ ie. when a state supports $\|\varphi\|^{+}$then so does each of its substates.

The clauses for possibilities and counter-possibilites differ from those in [13] with respect to the addition of a filter that ensures that possibilities and counter-possibilities are maximal states that support or reject a sentence. 


\section{Workshop on Inquisitiveness}

Definition 3. Maximality restriction.

Given any $\chi \subseteq \operatorname{Pow}(W), \chi_{M A X}$ is defined as all the $\subseteq$-maximal elements of $\chi$, ie. $\sigma \in \chi_{\text {MAX }}$ means that, for any $\tau \in \chi$ with $\sigma \subseteq \tau, \sigma=\tau$.

This allows us to define possibilities and counter-possibilities. We define for every sentence $\varphi$ in our language, the proposition $\lceil\varphi\rceil$ expressed by $\varphi$, and the counter-proposition $\lfloor\varphi\rfloor$ for $\varphi$. Both $\lceil\varphi\rceil$ and $\lfloor\varphi\rfloor$ will be sets of possibilities. We will refer to the elements of $\lceil\varphi\rceil$ as the possibilities for $\varphi$ and to the elements of $\lfloor\varphi\rfloor$ as the counter-possibilites for $\varphi$.

Definition 4. Possibilities and counter-possibilities.

1. $\lceil\varphi\rceil:=\|\varphi\|_{M A X}^{+}$

2. $\lfloor\varphi\rfloor:=\|\varphi\|_{M A X}^{-}$

To reason about deontic statements in the framework of inquisitive semantics, one requires an interpretation of permission statements with the modality "may". Following Anderson [4] and the way in which WTO judges reason, we take permission statements to provide information about what is not a violation. This can be captured via introducing the proposition $v$ that provides the information that a specific violation has occurred.

Generally, $v$ shall designate a specific law or regulation that is being violated. To account for different types of violations that can occur within a single legal framework, one can designate $v_{1}, v_{2}$, etc. for each specific violation. For example, $v_{1}$ may be taken as the proposition "Violation of law number 1 has occurred." As violation propositions are specific, violations can be reasoned about in the same manner as any other proposition. So the violation of one law does not lead to violations of other laws, nor does not violating one law save one from indictments due to other deeds. ${ }^{1}$

This will be defined in the semantics as follows.

Definition 5. Permissive "may".

1. $\sigma \models^{+} \diamond \varphi$ iff $\forall \tau \subseteq \sigma .\left(\tau \models^{+} \varphi\right.$ implies $\left.\tau \models^{+} \bar{v}\right)$

$\sigma \models{ }^{-} \diamond \varphi$ iff $\exists \tau .\left(\tau \models^{+} \varphi\right.$ and $\forall \tau^{\prime} \supseteq \tau .\left(\tau^{\prime} \models^{+} \varphi\right.$ implies $\left.\left.\sigma \cap \tau^{\prime} \models^{-} \bar{v}\right)\right)$

The effect of uttering $\lceil\diamond p\rceil$ is thus the same as uttering the following implication: $\lceil p \rightarrow \bar{v}\rceil$.

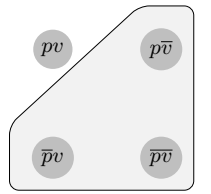

Fig. 1. $\lceil\diamond p\rceil$

For this account to provide a solution to the free choice puzzle, we must interpret "may" as taking scope over disjunction. And, indeed, this interpretation follows from general observations regarding disjunction and scope. Following Eckardt [7, pp. 9-10] we argue that in case of ambiguities, one chooses the strongest of the alternatives. In this paper, strongest is understood classically as most eliminative, while the standard measure for strength in inquisitive semantics is homogeneity [12, p. 23], which states that a sentence ought to be more eliminative and less inquisitive to be stronger. As can be seen by comparing figures (b) and (o), "may" taking scope over disjunction provides the stronger reading.

The solution to the free choice puzzle arises throught the following equivalence.

Example 12. $\lceil\diamond(p \vee q)\rceil \equiv\lceil(p \vee q) \rightarrow \neg v\rceil \equiv\lceil(p \rightarrow \bar{v}) \wedge(q \rightarrow \bar{v})\rceil$

In this formulation, a disjunctive permission sentence eliminates three worlds: $\langle p, \bar{q}, v\rangle,\langle\bar{p}, q, v\rangle$ and $\langle p, q, v\rangle$. The result is a single possibility that includes the remaining worlds.

${ }^{1}$ This formulation might yield interesting results with classic deontic logic puzzles, such as the Chisholm's paradox
and the gentle murder paradox. Yet, as these fall out of the scope of describing the natural language semantics of permissive disjunction sentences, it will not be discussed in this article. 


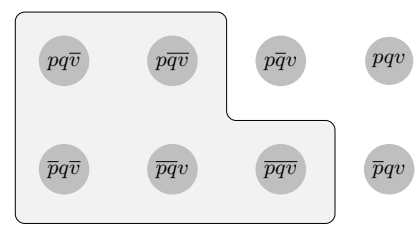

Fig. 2. A country may nominate a state funded research center or a private laboratory.

This appears to be in line with our intuitions regarding permission being granted for both disjuncts. Notice that the possibility for a disjunctive permission sentence does not prejudice whether $p$ and $q$ are in fact the case. The possibility is a set of worlds that includes $\langle\overline{p q v}\rangle$, which is a world in which neither $p$ nor $q$ is true. This accounts for the intuition that permission sentences do not require one to in fact perform the act that is permitted. Furthermore, the possibility includes the world $\langle\overline{p q} v\rangle$, where a violation does occur, but neither $p$ nor $q$ is true. This world allows for a more fine-grained analysis of interaction between different permissions and prohibitions, as a permission for one thing, in this case $p \vee q$ does not guarantee that a violation may not occur when another thing, for example $r$, is true.

\section{Radical Inquisitive Entailment}

The classic problem with violation based deontic logics is that from the assumption $p$ and the definition of obligation as $\bar{p} \rightarrow v$ one can derive the validity of $p \rightarrow \square p$ which is an obviously false prediction, known as the naturalistic fallacy. It isn't valid to derive from the fact that something is the case that it is also obligatory. The manner in which [4] derived this is the following.
1. $\square p:=\bar{p} \rightarrow v$
3. $\overline{\bar{p}}$
4. $\overline{\bar{p}} \vee v$
5. $\bar{p} \rightarrow v$
2. $p$
6. $p \rightarrow \square p$

Anderson's derivation poses a technical challenge, but it is not intuitively plausible that this counterargument will hold in natural language semantics. The steps that are most problematic are those from 3 to 4 and 4 to 5 . When one knows $p$ or $\overline{\bar{p}}$, it is dubious to assume that disjunctive addition does not create problems. It raises threefold issues. Firstly, one adds inquisitiveness, which previously wasn't present. Secondly, in a non-restricted system such as [6], one would draw attention to a new possibility, which needs to be justified. And, thirdly, one should be able to attest to the relevance of the added proposition. Unfortunately, challenging any of these three assumptions would require the addition of a great deal to this framework, so noting the limitations for this paper, we decided to concentrate on the move from step 4 to 5 . We can give a preliminary solution to this issue by investigating the notion of entailment with additional attention to negative responses. The proposed definition of entailment will also prove useful to deal with examples of strengthening the antecedent discussed earlier.

Inquisitive semantics provides the basic machinery for drawing the required distinctions that falsify the step from 4 to 5 . As these utterances constitute different possibilites, the effects of these utterances will similarly differ.

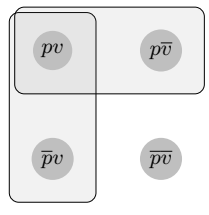

Fig. 3. $\lceil\overline{\bar{p}} \vee v\rceil$

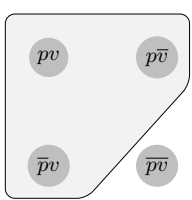

Fig. 4. $\lceil\bar{p} \rightarrow v\rceil$

While figure 3 represents a hybrid sentence that both eliminates one world and generates two possibilities, then $\lceil\bar{p} \rightarrow v\rceil$ depicted in figure 4 is a purely informative utterance that forms a single possibility that represents 


\section{Workshop on Inquisitiveness}

the connection between $\bar{p}$ and $v$. The fact that these utterances are informatively equivalent, but unavailable for use as substitutes in natural language was already noted by Grice [10, p. 67]. ${ }^{2}$

Intuitively, the utterances have a different effect on the conversation. While a disjunction has two possibilities and is thus inquisitive, the implication loses the inquisitive content. This is in itself a distinction that is dubious to ignore, but rather than block entailment at this stage, in a radical framework, one can capture the difference in conversational effect through investigating negative responses.

The idea behind drawing valid conclusions through inference is that the conclusion provides less information than the preceding step. This should mean that if one did not have a problem with the preceding step, then the following step cannot be problematic either. This is true in a classical setting and one can generalize that $\varphi \models \psi$ iff $\neg \psi \models \neg \varphi$. This should also hold for a disjunction to entail an implication, but the following provides reason to doubt this.

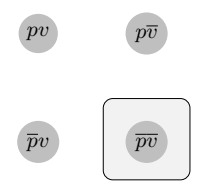

Fig. 5. $\lfloor\overline{\bar{p}} \vee v\rfloor$

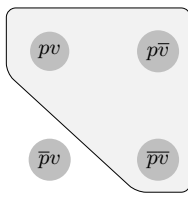

Fig. 6. $\lfloor\bar{p} \rightarrow v\rfloor$

As can be seen on figures 5 and 6 , to reject step 4 , one must eliminate two more worlds than to reject step 5. It follows that a person that did not have enough information to reject 4 might have enough information to reject step 5. For example, when a supposed interlocutor knows that $\bar{p} v$ is false, then this does not conflict with both possibilities of the disjunction, but it does conflict with the possibility for the implication. Thus, when responding to the disjunction, he can only reject one possibility and thus affirm the other one.

Looking at the implication, the world $\bar{p} v$ is also noteworthy as uttering an implication intuitively informs about whether the consequent follows from the antecedent. If the conversation weren't interested in whether the consequent follows the antecedent, it would be infelicitous to utter the implication in the first place. But if the combination of the antecedent and consequent is known to be false, the rule can only be proven to be false.

This suggests that standard inquisitive entailment [12, p. 10] does not capture the notion of drawing valid conclusions regarding disjunction and implication. In radical inquisitive semantics we require a more refined definition for entailment. We will follow the intuition that drawing conclusions is valid only when the preceding step is more difficult to negate than the following.

Definition 6. Radical inquisitive entailment.

1. $\varphi \models \psi$ iff $\forall \alpha \in\lceil\varphi\rceil: \exists \beta \in\lceil\psi\rceil: \alpha \subseteq \beta$ and $\bigcap\lceil\psi\rceil \subseteq \bigcup\lfloor\varphi\rfloor$

What radical entailment says is that when $\varphi$ entails $\psi$, then not only must every possibility in $\varphi$ be contained in a possibility for $\psi$, the intersection of counter-possibilities in $\psi$ must be contained in the union of counterpossibilities for $\varphi$.

With radical inquisitive entailment, step $4(\overline{\bar{p}} \vee q)$ in Anderson's counterargument no longer entails step $5(\bar{p} \rightarrow q)$ as the counter-possibility to step 5 includes the worlds $<p \bar{q}>,\langle\bar{p} q>$ and $<\overline{p q}>$ while the counter-possibility to step 4 consists of only one world $\langle p \bar{q}\rangle$.

Note that the clause for the entailment of counter-possibilities is restricted by taking the intersection and union of counter-possibilities, respectively. Unlike with the positive clause, one will probably find that drawing inferences from negative utterances can lead astray. This can be seen with $\lceil p \vee q \rightarrow \bar{v}\rceil \models\lceil p \rightarrow \bar{v} \wedge q \rightarrow \bar{v}\rceil$.

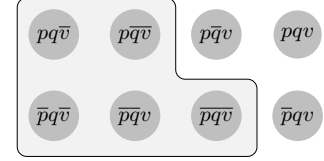

Fig. 7. $\lceil p \vee q \rightarrow \bar{v}$

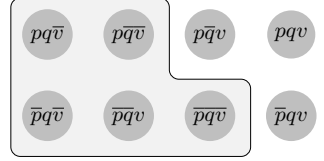

Fig. 8. $\lceil p \rightarrow \bar{v} \wedge q \rightarrow \bar{v}\rceil$

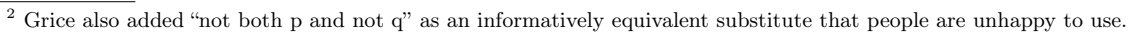




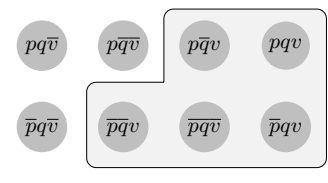

Fig. 9. $\lfloor\overline{p \vee q \rightarrow \bar{v}}\rfloor$

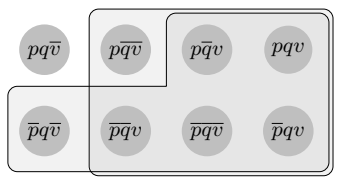

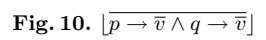

In radical inquisitive semantics, the negation of conjunction is inquisitive. This allows one to account for the fact that after a conjunction is rejected, one can ask: "Why?" and the other interlocutor can specify which conjunct was unacceptable. Yet, this fact creates a problem for drawing conclusions on the basis of counterpossibilities alone. The worlds $\langle p \overline{q v}>$ and $\langle\bar{p} q \bar{v}>$ are not eliminated, and instead remain in a counterpossibility, but inferring that either of these worlds is the case will not be warranted. These worlds might represent the world, but they also might not. Thus, to keep the additional functionality that having inquisitive rejection of conjunctive utterances provides, we have decided to tackle this issue of valid inferences at the level of entailment itself by taking intersection of the counter-possibilities for $\lfloor\psi\rfloor$ to limit inferences to only guaranteed worlds.

The radical definition of entailment also deals with the problem of strengthening the antecedent. If it is permitted to eat an apple, then intuitively it should not be the case that both eating an apple and killing a postman should still lead to a non-violation world. ${ }^{3}$ As one can see in the following figures, this inference is blocked by radical entailment.

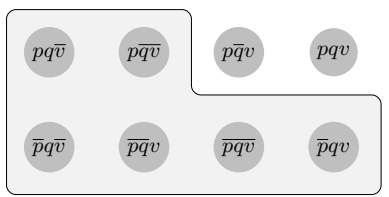

Fig. 11. $\lceil p \rightarrow \bar{v}\rceil$

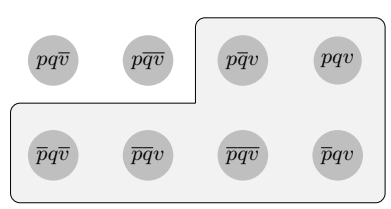

Fig. 12. $\lfloor p \rightarrow \bar{v}\rfloor$

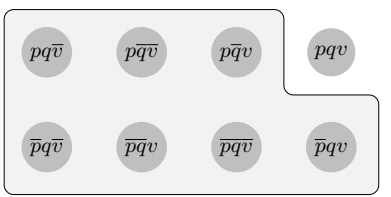

Fig. 13. $\lceil(p \wedge q) \rightarrow \bar{v}\rceil$

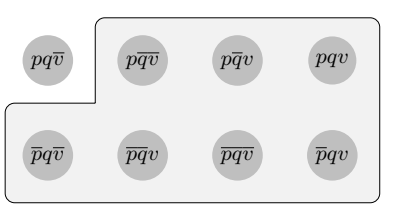

Fig. 14. $\lfloor(p \wedge q) \rightarrow \bar{v}\rfloor$

As the comparison of figures (12) and (14) demonstrates, the counter-possibility $\lfloor(p \wedge q) \rightarrow \bar{v}\rfloor$ is not contained in the counter-possibility $\lfloor(p \rightarrow \bar{v}\rfloor$, which means that the strengthened antecedent is not entailed by the original permission utterance.

Zimmermann [21] and Simons [19] draw our attention to the possibility of appending a disjunctive permission sentence with "... but I do not know which." such that the free choice effect gives way to an ignorance reading.

Example 13. A country may establish a research center or a laboratory, but I do not know which.

The entailment relations of these examples revert back to standard disjunction. We argue that this effect arises from the additional information blocking the modality from taking strongest scope - scoping over the disjunction. The result is a translation of (13) as a wide scope reading in which disjunction takes scope over "may" such that "may" distributes into the disjuncts.

Example 14. $\lceil\diamond p\rceil \vee\lceil\diamond q\rceil \equiv\lceil(p \rightarrow \bar{v})\rceil \vee\lceil(q \rightarrow \bar{v})\rceil$

${ }^{3}$ In the real world, eating an apple and murder would not be governed by the same violation, but to strengthen the argument, let us assume it is the same violation. 


\section{Workshop on Inquisitiveness}

As disjunction is a hybrid between inquisitive and informative utterances, a wide scope disjunctive permission utterance raises an issue for the speaker to solve, modeled as two possibilities as shown in the graph below.

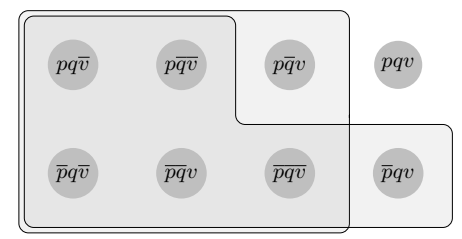

Fig. 15. A country may establish a research center or a laboratory but I don't know which.

The model still includes classical negation that takes the complement of a proposition. This allows us to model yet another effect. The following utterance is appended with "... and, also, I do not care."

Example 15. A country may establish a research center or a laboratory, but I do not know which, and, also, I do not care.

We model "I do not care." through double classical negation: $\neg \neg\lceil(\diamond \varphi \vee \diamond \psi)\rceil$ (known as non-inquisitive closure in $[12$, p. 4]), which results in the same possible worlds as (13), but it consists of only one possibility. This correctly does not guarantee that doing $p$ or $q$ does not result in a violation. Note, that the non-inquisitive closure only has an effect on inquisitive utterances, and "I do not care" is superfluous when added to the free choice examples.

\section{Conclusions}

This paper argued against the prevalent view that the solution to the free choice puzzle should be pragmatic, demonstrating that Anderson's reduction, when implemented in radical inquisitive semantics, provides an account that avoids many problematic cases known in the literature.

While negation has been especially problematic for previous solutions, specifying counter-possibilities and defining the negation of implication in the tradition of connexive logic provides a simple and principled account.

Counterarguments to Anderson's reduction were shown to rely on an overly simple notion of entailment and the refinement of standard inquisitive entailment into radical inquisitive entailment provided a solution to both of the largest issues concerning drawing implausible conclusions within a violation-based semantics.

Furthermore, a violation-based account aligns well with the way lawyers and judges use deontic language, which provides additional empirical support for an account where permission is defined as providing the information on what is not a violation. 


\section{References}

1. Aher, M.: Inquisitive semantics and legal language. Proceedings of the 15th student session of the european summer school for logic, language and information, Ed. Slavkovik, M. (2010) 124-131

2. Aloni, M.: Free Choice, Modals, and Imperatives. Natural Language Semantics 15 (2007) 65-94

3. Alonso-Ovalle, L.: Equal right for every disjunct! Quantification over alternatives or pointwise context change? Presentation at Sinn und Bedeutung 9 (2004)

4. Anderson, A.R.: Some Nasty Problems in the Formal Logic of Ethics. Nous 1 (1967) 345-360

5. Barker, C.: Free choice permission as resource-sensitive reasoning. Semantics and Pragmatics 3 (2010) 1-38

6. Ciardelli, I., Roelofsen, F., Groenendijk, J.A.G.: Attention! Might in Inquisitive Semantics. Semantics and Linguistic Theory (2009)

7. Eckardt, R.: Licencing 'or'. Presupposition and Implicature in Compositional Semantics. Palgrave MacMillan (2007) $34-70$

8. Fox, D.: Free choice disjunction and the theory of scalar implicature. Presupposition and implicature in compositional semantics. Palgrave MacMillan (2007) 71-120

9. Franke, M.: Free choice from iterated best response. Logic, Language and Meaning: 17th Amsterdam Colloquium, revised selected papers. Eds. Aloni, M; Bastiaanse, H; de Jager, T; Schulz, K (2009) 295-304

10. Grice, H. P.: Indicative conditionals. Studies in the Way of Words. Harvard University press (1989) chapter 4

11. Grice, H. P.: Logic and conversation. Studies in the Way of Words. Harvard University press (1989) chapter 2

12. Groenendijk, J.A.G., Roelofsen, F.: Inquisitive Semantics and Pragmatics. In Standford Workshop on Language, Communication and Rational Agency (2009)

13. Groenendijk, J.A.G., Roelofsen, F.: Radical Inquisitive Semantics. Preliminary version, presented at the Colloquium of the Institute for Cognitive Science, University of Osnabrueck (2010)

14. Kamp, H.: Free choice permission. Aristotelian Society Proceedings N.S 74 (1973) 57-74

15. Kanger, S.: New Foundations for ethical theory. Deontic Logic: Introductory and Systematic Readings. Ed. Hilpinen, R. Reidel Publishing Company (1971) chapter 12

16. Sano, K.: A Note on Support and Rejection for Radical Inquisitive Semantics. Unpublished. (2010)

17. Schultz, K.: You may read it now or later: A Case Study on the Paradox of Free Choice Permission. Master thesis, University of Amsterdam (2003)

18. Schultz, K.: A pragmatic solution for the paradox of free choice permission. Synthese: Knowledge, Rationality and Action 147 (2005) 343-377

19. Simons, M.: Dividing things up: the semantics of or and the modal/or interaction. Natural Language Semantics 13 (2005) $271-316$

20. Simons, M.: Semantics and Pragmatics in the Interpretation of or. Proceedings of SALT XV (2005) 205-222

21. Zimmermann, T. E.: Free Choice Disjunction and Epistemic Possibility. Natural Language Semantics 8 (2000) 255290 


\title{
Negative inquisitiveness and alternatives-based negation
}

\author{
Robin Cooper ${ }^{1}$ and Jonathan Ginzburg ${ }^{2}$ \\ 1 Department of Philosophy, Linguistics and Theory of Science, \\ University of Gothenburg, Box 200, 40530 Göteborg, Sweden \\ 2 Univ. Paris Diderot, Sorbonne Paris Cité \\ CLILLAC-ARP (EA 3967), 75004 Paris, France
}

\begin{abstract}
We propose some fundamental requirements for the treatment of negative particles, positive/negative polar questions, and negative propositions, as they occur in dialogue with questions. We offer a view of negation that combines aspects of alternative semantics, intuitionist negation, and situation semantics. We formalize the account in TTR (a version of type theory with records) [5, 7]. We sketch an account of the coherence of negative utterances in the dialogue framework KoS [10].
\end{abstract}

Keywords: interrogatives, negation, dialogue, type theory

\section{Introduction}

In the classical formal semantics treatments for questions the denotation of a positive polar interrogative (PPInt) $p$ ? is identical to that of the corresponding negative polar (NPInt) $\neg p$ ? $[15,14$, for example]. This is because the two interrogatives have identical exhaustive answerhood conditions. Indeed Groenendijk and Stokhof (1997), p. 1089 argue that this identification is fundamental. Recent work within Inquisitive Semantics [13, for example] seems to be equivocal about whether this identity should be preserved. ${ }^{3}$

However, other evidence calls the the identification of PPInt and NPInt denotations into question. (1a,b) based on examples due to [17] seems to describe distinct cognitive states. Hoepelmann, in arguing for this distinction, suggests that (1a) is appropriate for a person recently introduced to the odd/even distinction, whereas (1b) is appropriate in a context where, say, the opaque remarks of a mathematician sow doubt on the previously well-established belief that two is even. $(1 \mathrm{c}, \mathrm{d})$ seem to describe distinct investigations, the first by someone potentially even handed, whereas the second by someone tending towards DSK's innocence.

(1) a. The child wonders whether 2 is even.

b. The child wonders whether 2 isn't even.

c. Epstein is investigating whether DSK should be exonerated.

\footnotetext{
${ }^{3}$ Within Inquisitive Semantics $p$ ? is equivalent to $\neg p$ ? for $p$ a propositional variable, but this equivalence is not maintained for $\phi$ ? and $\neg \phi$ ?, where $\phi$ is a complex formula. We would like to thank Andzrej Wiśniewski for discussion of this issue.
} 
d. Epstein is investigating whether DSK shouldn't be exonerated.

That root PPInts and NPInts are appropriate in distinct contexts is well recognized in the literature since Hoepelmann and [19]. However, it is not merely the background that differs, it is also the responses triggered that are markedly and systematically different. A corpus study of the British National Corpus, whose results are displayed in Table 1, reveals that the two types of interrogatives exhibit almost a mirror image distribution: ${ }^{4}$ it suggests that PPInts $p$ ? are significantly biassed to eliciting $\mathrm{p}$, whereas NPInts $\neg p$ ? are almost identically biassed to eliciting $\neg p$ :

Table 1: Distribution of responses to Positive ('Did..?')/Negative ('Didn't..?') polar interrogatives in the British National Corpus

\begin{tabular}{|l|l|l|l|l|}
\hline Question type & Positive answer & Negative answer & No answer & Total \\
\hline Positive polar & $53 \%$ & $31 \%$ & $16 \%$ & $\mathrm{n}=106$ \\
\hline Negative polar & $23 \%$ & $54 \%$ & $22 \%$ & $\mathrm{n}=86$ \\
\hline
\end{tabular}

[12], who developed a view of questions as propositional abstracts, showed how such an account, combined with a theory of negative situation types developed in [4], can distinguish between PPInts and NPInts denotations and presuppositions while capturing the identity of resolving answerhood conditions. Their account relied on a complex ad hoc notion of simultaneous abstraction. In this paper we consider a number of phenomena relating negation and dialogue, on the basis of which we develop an account of propositional negation in the framework of Type Theory with Records (TTR) $[6,7]$. This account extends the earlier results in a type theoretic framework, based on standard notions of negation and abstraction. Along the way we also relate the view of negation to some recent psycholinguistic work. We conclude by sketching an account of the coherence of propositional negation in dialogue.

\section{Negation in simple dialogue}

(2) a. [[child B approaches socket with nail] A:(1) No. (2) Do you want to be electrocuted? (2') Don't you want to be electrocuted? B: (3) No. A: (4) No.

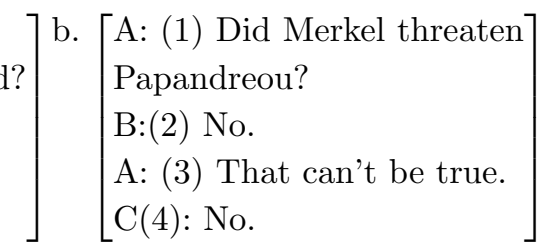

c. $[$ A: Marie est une bonne étudiante? B: Oui / \#Si. A: Marie n'est pas une bonne étudiante? B: \#Oui / Si.

\footnotetext{
4 Our pilot corpus study searched the BNC using SCoRE [21]. For the NPInts the sample reported below consists of all the NPInts of the form 'Didn't ...?' that we found. For the PPInts we found 1500 hits of the form 'Did ...?'. From these we selected a random sample of 106 . The 'no answer' category includes cases where either no response concerning the question was forthcoming or where it was difficult to understand how the information provided resolved the question.
} 
From $(2 \mathrm{a}, \mathrm{b}, \mathrm{c})$ one can extract some fundamental requirements for a theory of negation in dialogue. In (2a(1)) B's initial action provides the background for A's initial utterance of 'No', in which A ultimately expresses a wish for the negative situation type $\neg \operatorname{StickIn}(\mathrm{B}$,nail,socket). More generally, we argue that this type of use ('Neg(ative) Vol(itional) 'No') involves the specification of a negative situation/event type, thereby providing motivation for (3a). Additional motivation for this is provided by complements of naked infinitive clauses discussed below and the large body of work on the processing of negation, reviewed recently in [18]. Kaup offers experimental evidence that comprehending a negative sentence (e.g. Sam is not wearing a hat) involves simulating a scene consistent with the negated sentence. She suggests that indeed initially subjects simulate an "unnegated" scene (e.g. involving Sam wearing a hat). [23] offer additional evidence supporting the simulationist perspective. However, they argue against the "two step" view of negation (viz. unnegated and then negated), in favour of a view driven by dialogical coherence, based on QUD.

In the aftermath of $(2 \mathrm{a}(1)),(2 \mathrm{a}(2))$ would be a reasonable question to ask, whereas $\left(2 \mathrm{a}\left(2^{\prime}\right)\right)$ would be grounds for summoning the social services. This, together with our earlier discussion on PPInts and NPInts motivates (3b). Assuming $(2 \mathrm{a}(2))$ were uttered, B's response asserts the negation of the proposition $p_{\text {Want(B,electr(B)) }}$. A can now agree with B by uttering 'No'. That is, 'propositional' No always resolves to a negative proposition. This partly motivates (3c). Additional motivation for this is the existence in many languages, such as French and Georgian, of dialogue particles which presuppose respectively a positive (negative) polar question as the maximal element in QUD (MaxQUD), as in $(2 \mathrm{c})$.

In $(2 \mathrm{~b}(2)) \mathrm{B}$ retorts with $\neg p_{1}\left(p_{1}=\right.$ Threaten(Merkel,Papandreou)), whereas in $(2 \mathrm{~b}(3))$ A disagrees with B and affirms $\neg \neg p_{1}$. Clearly, we need $(2 \mathrm{~b}(3))$ to imply $p_{1}$, but this should not be identified with $p_{1}-\mathrm{C}$ 's utterance $(2 \mathrm{~b}(4))$ can be understood as agreement with $\mathrm{A}$, not with $\mathrm{B}$, hence motivating (3d).

(3) a. Constructive negation and negative situation types: $s: \neg T$ implies $s: T^{\prime}$, with T' a positive type that precludes $\mathrm{T}$

b. Positive/negative polar question distinction: $\mathrm{p}$ ? queries the truth of $p ; \neg p$ ? queries the truth of $\neg p$; these questions are distinct though have equivalent resolving answerhood conditions.

c. Negative propositions: negative propositions are recognizably distinct from positive propositions.

\section{d. Equivalence but non-identity of $p$ and $\neg \neg p$.}

\section{$3 \quad$ Negation and types}

The discussion here builds on the type theoretical dictum: "propositions as types". The idea is that we can consider propositions to be types of situations 
(possibly among other things). If a type has at least one witness it corresponds to a true proposition. A type with no witnesses corresponds to a false proposition.

In [8] we considered various options for treating negation in TTR considering negation as complement in possible worlds, intuitionistic negation, classical negation as a variant of intuitionistic negation, infonic negation, and negation in simulation semantics.

In our version of intuitionistic negation the negation of type $T$ is viewed as the type of functions $(T \rightarrow \perp)$ where $\perp$ is a necessarily empty type. In terms of TTR we say that $\{a \mid a: \perp\}=\emptyset$ no matter what is assigned to the basic types, thus giving $\perp$ a modal character: it is not only empty but necessarily empty. If $T$ is a type then $\neg T$ is the function type $(T \rightarrow \perp)$. This works as follows: if $T$ is a type corresponding to a proposition it is "true" just in case there is something of type $T$ (i.e. a witness or proof) and "false" just in case there is nothing of type $T$. Now suppose there is a function of type $\neg T$. If there is something $a$ of type $T$ then a function $f$ of type $\neg T$ would have to be such that $f(a): \perp$. But $\perp$, as we know, is empty. Therefore there cannot be any function of type $\neg T$. The only way there can be a function of type $\neg T$ is if $T$ itself is empty. Then there can be a function which returns an object of type $\perp$ for any object of type $T$, since, $T$ being empty, it will never be required to return anything.

This gives us a notion of negative type, that is a function type whose range type is $\perp$, which can be made distinct from positive types (which could be anything other than a negative type, though in practice we use record types as the basis for our propositions). In this way we fulfil (3c) by making negative types distinct from non-negative types. However, the proposals made in [8] did not yet give us a type of negative propositions. The problem is that for any type $T$ there are infinitely many corresponding negative types $(T \rightarrow \perp),((T \rightarrow \perp) \rightarrow \perp)$ and so on. All of these are types and therefore, if we allow a type Type of types ${ }^{5}$ they will all be of type Type. Things become a little more complicated when we want to talk of some particular collection of types closed under negation as we do below. If $\mathcal{T}$ is a type of types then we shall use $c l_{\neg}(\mathcal{T})$ to represent the type of types whose witnesses are the closure of the set of witnesses of $\mathcal{T}$ under $\neg$. We shall also use $\operatorname{map}_{\neg}(\mathcal{T})$ to represent the type $\mathfrak{T}$ such that $\neg T: \mathfrak{T}$ iff $T: \mathcal{T}$. This gets us a type whose witnesses involve one iteration of negation over the types belonging to $\mathcal{T}$, leaving out the types we started with, that is, a type of negative types.

Given this, and following [10], we introduce situation semantics style Austinian propositions into TTR [5,7]. These are objects of type (4a). An example of an Austinian proposition of this type would be (4b). Here RecType is the type of record types as defined in [5,7]. Denoting (3a) by AusProp, the type of Austinian propositions, we can say that NegAusProp, the type of negative Austinian propositions, is $(4 \mathrm{c})$.

(4) a. $\left[\begin{array}{ll}\text { sit } & : \operatorname{Rec} \\ \text { sit-type }: c l_{\neg}(\operatorname{Rec} T y p e)\end{array}\right]$

\footnotetext{
${ }^{5}$ We can do this if we are careful to avoid paradoxes, for example by stratifying the types as we do in $[5,7]$.
} 


$$
\begin{aligned}
& \text { b. }\left[\begin{array}{ll}
\text { sit } & =s \\
\text { sit-type }=\left[\mathrm{c}_{\text {run }}: \operatorname{run}(\operatorname{sam})\right]
\end{array}\right] \\
& \text { c. }\left[\begin{array}{ll}
\text { sit } & : \operatorname{Rec} \\
\text { sit-type }: \operatorname{cl}_{\neg}\left(\operatorname{map}_{\neg}(\operatorname{Rec} \text { Type })\right)
\end{array}\right]
\end{aligned}
$$

Truth for these Austinian propositions involves a notion of Austinian witness which in turn involves a notion of incompatible types. Two types $T_{1}$ and $T_{2}$ are incompatible just in case for any a not both $a: T_{1}$ and $a: T_{2}$, no matter what assignments are made to basic types. Incompatibility thus means that there is necessarily no overlap in the set of witnesses for the two types. In order to be fully viable incompatibility needs to be further restricted using a notion of alternativehood [3]. In some cases what the alternatives amount to is fairly straightforward and even lexicalized - classifying the table as not black requires evidence that it is green or brown or blue, say. But in general, figuring out the alternatives, as Cohen illustrates, is of course itself context dependent, relating, as we will exemplify below, to QUD.

Using the notion of "model" defined in [7], that is, an assignment of objects to basic types and to basic situation types constructed from a predicate and appropriate arguments, we can characterize the set of witnesses for a type $T$ with respect to "model" $M,\left[{ }^{\sim} T\right]^{M}$, to be $\left\{a \mid a::_{M} T\right\}$ where the notation $a:_{M} T$ means that $a$ is a witness for type $T$ according to assignment $M$. We can then say that two types $T_{1}$ and $T_{2}$ are incompatible if and only if for all $M$, $\left[{ }^{\sim} T_{1}\right]^{M} \cap\left[{ }^{\sim} T_{2}\right]^{M}=\emptyset .6$

We define a notion of Austinian witness for record types closed under negation:

(5) a. If $T$ is a record type, then $s$ is an Austinian witness for $T$ iff $s: T$

b. If $T$ is a record type, then $s$ is an Austinian witness for $\neg T$ iff $s: T^{\prime}$ for some $T^{\prime}$ incompatible with $T$

c. If $T$ is a type $\neg \neg T^{\prime}$ then $s$ is an Austinian witness for $T$ iff $s$ is an Austinian witness for $T^{\prime}$

The intuitions behind clauses (5b) and (5c) are based on the intuitive account of intuitionistic negation. (5b) is based on the fact that a way to show that $s$ being of type $T$ would lead to a contradiction is to show that $s$ belongs to a type that is incompatible with $T$. (5c) is based on the fact that if you want to show that a function of type $(T \rightarrow \perp)$ would lead to a contradiction requires finding a witness for $T$.

We say that an Austinian proposition $p$ is true iff $p$.sit is an Austinian witness for $p$.sit-type. Notice that if $p$ is true in this sense then $p$.sit-type will be nonempty, that is, "true" in the standard type-theoretical sense for propositions as types. If $p$ is an Austinian proposition as in (6a), then the negation of $p, \neg p$, is $(6 \mathrm{~b})$ :

(6) $\quad$ a. $\left[\begin{array}{ll}\text { sit } & =s \\ \text { sit-type }=T\end{array}\right]$ b. $\left[\begin{array}{l}\text { sit }=s \\ \text { sit-type }=\neg T\end{array}\right]$

\footnotetext{
${ }^{6}$ Notice that this definition of incompatibility is independent of our definition of negation below.
} 
We obtain the desideratum (3a) in virtue of the requirement involving an incompatible type in (4b). We obtain the desideratum (3c) because negative propositions are distinct from positive propositions. We obtain (3d) because double negations of propositions will be distinct from the original proposition but they will now (contrary to intuitionistic propositions) be truth-conditionally equivalent.

\section{Alternatives}

It is widely recognized that positive Naked Infinitive (NI) sentences describe an agent's perception of a situation/event, one which satisfies the descriptive conditions provided by the NI clause, as in $(7 \mathrm{a}, \mathrm{b})$. More tricky is the need to capture the 'constructive' nature of negation in negative NI sentences such as $(7 \mathrm{c}, \mathrm{d})$. These reports mean that $s$ actually possesses information which rules out the descriptive condition (e.g. for (7c) Mary avoiding contact with Bill), rather than simply lacking concrete evidence for this (e.g. Ralph shutting his eyes.). As [4] points out, Davidsonian accounts (e.g. [16]), are limited to the far weaker (7f):

(7) a. Ralph saw Mary serve Bill. b. Saw $(R, s) \wedge s$ : Serve(m,b).

c. Ralph saw Mary not serve Bill. d. Ralph saw Mary not pay her bill.

e. $\operatorname{Saw}(\mathrm{R}, \mathrm{s}) \wedge \mathrm{s}: \neg \operatorname{Serve}(\mathrm{m}, \mathrm{b})$. f. $\operatorname{Saw}(\mathrm{R}, \mathrm{s}) \wedge s: \operatorname{Serve}(m, b)$

[4] provides axioms on negative SOAs (infons) in situation semantics that attempt to capture this, as in (8a,b). (8a) states that if a situation $s$ supports the dual of $\sigma$, then $s$ also supports positive information that precludes $\sigma$ being the case. (8b) tells us that if a situation $s$ supports the dual of $\sigma$, then $s$ also supports information that defeasibly entails that $\sigma$ is the case.

(8) a. $\forall s, \sigma[s: \bar{\sigma}$ implies

$$
\begin{aligned}
\exists(P o s) \psi[s: \psi \text { and } \psi \Rightarrow \bar{\sigma}]] \\
\text { b. } \forall s, \sigma[s: \bar{\sigma} \text { implies } \\
\exists(\operatorname{Pos}) \psi[s: \psi \text { and } \psi>\sigma]]
\end{aligned}
$$

(5) accounts for (8a). In order to cover (8b) we could refine (5) as in (9).

(9) If $T$ is a record type, then $s$ is an Austinian witness for $\neg T$ iff $s: T^{\prime}$ for some $T^{\prime}$ incompatible with $T$ and there is some $T^{\prime \prime}$ such that $s: T^{\prime \prime}$ and $T^{\prime \prime}>T$

$T^{\prime \prime}>T$ should intuitively mean that the judgement $s: T^{\prime \prime}$ creates an expectation for a judgement $s: T$. This should mean that $T^{\prime}$ (the type that is incompatible with $T$ ) is regarded as an alternative for $T$ given $T^{\prime \prime}$. One way of handling these defeasible inferences is in terms of enthymemes as discussed in [2]. The idea is that among the resources available to an agent in a given situation there is a collection of enthymemes of the form $\lambda r: T_{1}\left(T_{2}\right)$, that is functions 
which map an object $r$ (e.g a situation) belonging to type $T_{1}$ to another type $T_{2}$ (which may depend on the object $r$ ). Such an enthymeme reflects an association between the type $T_{1}$ and $T_{2}$. Such resources may be part of the general knowledge that the agent has acquired. For example, Fillmore's [9] examples (10), uttered out of context, depend on such general knowledge. ${ }^{7}$

(10) a. Her father doesn't have any teeth.

b. \# Her husband doesn't have any walnut shells.

c. Your drawing of the teacher has no nose/\#noses.

d. The statue's left foot has no \#toe/toes.

We generally assume that people have teeth but not walnut shells and that humans have one nose but many toes. Such resources may also be local to a restricted domain or even a single dialogue or even part of a dialogue. So, for example, a previous turn in a dialogue is sufficient to create an association between husbands and walnut shells, thus making (10b) acceptable.

(11) A: My husband keeps walnut shells in the bedroom.

B: Millie's lucky in that respect. Her husband doesn't have any walnut shells.

This particular resource is quite likely not going to be used beyond this particular dialogue.

\section{$5 \quad$ Polar Interrogatives}

We are left with the desideratum (3b). We follow [12] in analyzing polar questions as 0-ary propositional abstracts. We rely on a standard type theoretic notion of abstraction, couched in terms of functional types. For instance, $(2 \mathrm{a}(2))$ and $\left(2 \mathrm{a}\left(2^{\prime}\right)\right)$ would be assigned the 0 -ary abstracts in (12a) and (12b) respectively. These are distinct functions from records of type [] (in other words from all records) into the corresponding Austinian propositions. This accords with the need to distinguish the distribution of their expected responses and the information states of questioners asking or agents investigating the corresponding issues:

$$
\begin{aligned}
& \text { a. } \lambda r: \operatorname{Rec}\left(\left[\begin{array}{l}
\text { sit }=\mathrm{s} \\
\text { sit-type }=[\mathrm{c}: \operatorname{want}(\mathrm{B}(\operatorname{electrocute}(\mathrm{B})))]
\end{array}\right]\right) \\
& \text { b. } \lambda r: \operatorname{Rec}\left(\left[\begin{array}{l}
\text { sit }=\mathrm{s} \\
\text { sit-type }=[\mathrm{c}: \neg \operatorname{want}(\mathrm{B}(\operatorname{electrocute}(\mathrm{B})))]
\end{array}\right]\right)
\end{aligned}
$$

As we mentioned in section 4 , the witnessing conditions associated with negative situation types could be strengthened as in (9) so that witnessing $\neg T$

\footnotetext{
7 (i) from the BNC illustrates an actual context for (10a):

(i) Marjorie: You just get in, have your tea, and Alexandro'll come round, come for a beer. Clive: There's Tony, look. He ain't got no teeth now, look. (BNC, KC2)
} 
involves the existence of $T^{\prime \prime}$ such that $s: T^{\prime \prime}$ and $T^{\prime \prime}>T$. Hence, wondering about $\left.\lambda r: \operatorname{Rec}\left(\begin{array}{l}\text { sit }=\mathrm{s} \\ \text { sit-type }=\neg \mathrm{T}\end{array}\right]\right)$ involves wondering about whether $s$ has the characteristics that typically involve $T$ being the case, but which-nonetheless, in this case - fail to bring about $T$. The simple answerhood relation of [12] recast in TTR will ensure that the exhaustive answer to $\mathrm{p}$ ? are $\{p, \neg p\}$, whereas to $\neg p$ ? they are $\{\neg p, \neg \neg p\}$, so the exhaustive answers are equivalent, as needed. ${ }^{8}$

\section{Characterizing contexts for negation}

We have already discussed the contextual presuppositions of dialogue particles like 'No' (NegVol and propositional use), 'Si', and 'Oui'. NegVol 'no' merely presupposes an event/situation concerning which the speaker can express her disapproval. Whereas the propositional uses require the QUD-maximality of $\mathrm{p}$ ?, where $p$ is the proposition they affirm/negate. In KoS [11,10, for example], the felicity of these particles in a post-assertoric or post-polar query context is assured by the following update rule:

$$
\left[\begin{array}{c}
\text { pre : }\left[\begin{array}{l}
{\left[\begin{array}{l}
\text { spkr: Ind } \\
\text { addr: Ind } \\
\text { p : Prop } \\
\text { LatestMove.cont }= \\
\text { Ask(spkr,addr,p?) } \\
\vee \text { Assert(spkr,addr,p) : IllocProp }
\end{array}\right]} \\
\text { effects : }[\text { qud }=\langle\text { p?, pre.qud }\rangle: \operatorname{poset}(\text { Question })
\end{array}\right]
\end{array}\right.
$$

What of the VP adverb 'not', in other words sentential negation? The rule in (13) provides a class of contexts in which clauses of the form 'NP $\neg \mathrm{VP}^{\prime}$ ' are felicitous, namely ones in which $\mathrm{p}$ ? is MAX-QUD, where $\mathrm{p}=\operatorname{cont}($ 'NP VP'). However, this characterization is partial, as demonstrated by the corpus examples (14), drawn from ([20]), who collected them from the International Corpus of English (GB) ${ }^{9}$ (14a,b) do not explicitly raise the issues, respectively, Was there a chemical attack on Israel? and Is the studio open at that time? (14c) is an instance of 'metalinguistic negation' in that it does not dispute content, but form, whereas (14d) is an instance of intra-utterance self-correction:

\footnotetext{
${ }^{8}$ In an extended version of this paper, we address the issue of how to accommodate an additional understanding/reading NPInts manifest, one that has been known since [19] as the outside negation reading, in which there is actually a positive bias to the question. We find the arguments of [22] that such a reading has a metalinguistic nature convincing, though we do not adopt his proposal that such utterances are complex assertion/query speech acts.

${ }^{9}$ http://www.ucl.ac.uk/english-usage/projects/ice-gb/
} 
(14) a. The army will only confirm that missiles have fallen in Israel ... It was not a chemical attack ... [S2B-015\#106] (Pitts' [137] )

b. I haven't got enough hours in the day ... unless I start teaching at midnight. But the studio's not open then. [S1A-083\#170] (Pitts' [141] )

c. A: there's lots of deers and lots of rabbits. B: It's not deers - it's deer. [S1A-006\#261] (Pitts' [107])

d. I might have to do the after-dinner speech at our annual, well, not annual, our Christmas departmental dinner. (Pitts' [112] )

We propose a generalization of (13). The latter licensed expressing $\neg p$ if $p$ has been asserted or $p$ ? queried, whereas (15) licenses $\neg p$ if q can be posed in the current context and $p$ is about $q$. asking $q$ is a relevant move given the current dialogue gameboard:

(15) Given a dialogue gameboard dgb0, a negative proposition $\neg p$ is felicitous in dgb0 iff the move ' $\mathrm{A}$ ask q' is relevant in dgb0 where About $(\mathrm{p}, \mathrm{q})$ holds.

(15) presupposes substantive notions of relevance or question raising. For the former we appeal to the notion of relevance developed in KoS (see [10]). For the latter see e.g. the framework of Inferential Erotetic Logic (IEL) e.g. [24]. We exemplify an account of (14a) with the latter and (14c) with the former.

A key component of the analysis in IEL is the use of m(ultiple)-c(onclusion) entailment - the truth of a set $\mathrm{X}$ of premises guarantees the truth of at least one conclusion. Given this, the question evocation can be defined as in (16):

(16) $X$ evokes a question $Q$ iff $X$ mc-entails $d Q$, the set of atomic answers of $Q$, but for no $A \in d Q, X=A$

According to this definition (17a) evokes (17b) and (17c) is About (17b).

(17) a. Missiles have fallen in Israel.

b. What kind of missiles were fired?

c. It was not a chemical attack.

In KoS an utterance $u$ by $\mathrm{A}$ in which $u 1$ is a sub-utterance of $u$ permits $\mathrm{B}$ to accommodate in $u$ 's immediate aftermath the issue (18a). This is inter alia the basis for explaining why (18c) is a coherent follow up to (18b) and can get the resolution (18d).

(18) a. What form $\operatorname{did} A$ intend in $u 1$ ?

b. A: There's lots of deers there.

c. B: Deers?

d. Did $A$ intend the form 'deers' in $u 1$ ?

Acknowledgements This research was supported in part by VR project 20091569, Semantic analysis of interaction and coordination in dialogue (SAICD). Some portions of this paper were presented at SemDial 2011 in Los Angeles. We thank the audience there as well as the reviewers for Amsterdam Colloquium for their comments. 


\section{References}

1. Artstein, R., Core, M., DeVault, D., Georgila, K., Kaiser, E., Stent, A. (eds.): SemDial 2011 (Los Angelogue): Proceedings of the 15th Workshop on the Semantics and Pragmatics of Dialogue (2011)

2. Breitholtz, E., Cooper, R.: Enthymemes as rhetorical resources. In: Artstein et al. [1]

3. Cohen, A.: How are alternatives computed? Journal of Semantics 16(1), 43 (1999)

4. Cooper, R.: Austinian propositions, Davidsonian events and perception complements. In: Ginzburg, J., Khasidashvili, Z., Levy, J.J., Vogel, C., Vallduvi, E. (eds.) The Tbilisi Symposium on Logic, Language, and Computation: Selected Papers, pp. 19-34. Foundations of Logic, Language, and Information, CSLI Publications, Stanford (1998)

5. Cooper, R.: Austinian truth, attitudes and type theory. Research on Language and Computation 3, 333-362 (2005)

6. Cooper, R.: Records and record types in semantic theory. Journal of Logic and Computation 15(2), 99-112 (2005)

7. Cooper, R.: Type theory and semantics in flux. In: Kempson, R., Asher, N., Fernando, T. (eds.) Handbook of the Philosophy of Science, vol. 14: Philosophy of Linguistics. Elsevier, Amsterdam (fthc)

8. Cooper, R., Ginzburg, J.: Negation in dialogue. In: Artstein et al. [1]

9. Fillmore, C.J.: Frames and the semantics of understanding. Quaderni di Semantica 6(2), 222-254 (1985)

10. Ginzburg, J.: The Interactive Stance: Meaning for Conversation. Oxford University Press, Oxford (2012)

11. Ginzburg, J., Fernández, R.: Computational models of dialogue. In: Clark, A. Fox, C., Lappin, S. (eds.) Handbook of Computational Linguistics and Natural Language. Blackwell, Oxford (2010)

12. Ginzburg, J., Sag, I.A.: Interrogative Investigations: the form, meaning and use of English Interrogatives. No. 123 in CSLI Lecture Notes, CSLI Publications, Stanford: California (2000)

13. Groenendijk, J., Roelofsen, F.: Inquisitive Semantics and Pragmatics (2009), Presented at the Stanford workshop on Language, Communication and Rational Agency, May 30-31, 2009

14. Groenendijk, J., Stokhof, M.: Questions. In: van Benthem, J., ter Meulen, A. (eds.) Handbook of Logic and Linguistics. North Holland, Amsterdam (1997)

15. Hamblin, C.L.: Questions in montague english. In: Partee, B. (ed.) Montague Grammar. Academic Press, New York (1973)

16. Higginbotham, J.: The logic of perceptual reports: An extensional alternative to situation semantics. Journal of Philosophy 80(2), 100-127 (1983)

17. Hoepelmann, J.: On questions. In: Kiefer, F. (ed.) Questions and Answers. Reidel (1983)

18. Kaup, B.: What psycholinguistic negation research tells us about the nature of the working memory representations utilized in language comprehension. Trends in Linguistics Studies and Monographs 173, 313-350 (2006)

19. Ladd, R.: A first look at the semantics and pragmatics of negative questions and tag questions pp. 164-171 (1981)

20. Pitts, A.: Metamessages of denial: the pragmatics of English negation. Ph.D. thesis, Cambridge University (2009)

21. Purver, M.: Score: A tool for searching the bnc. Tech. Rep. TR-01-07, King's College, London (2001)

22. Reese, B.: The meaning and use of negative polar interrogatives. Empirical Issues in Syntax and Semantics 6, 331-354 (2006)

23. Tian, Y., Breheny, R., Ferguson, H.: Why we simulate negated information: A dynamic pragmatic account. The Quarterly Journal of Experimental Psychology 63(12), 2305-2312 (2010)

24. Wiśniewski, A.: Questions and inferences. Logique et Analyse 173, 5-43 (2001) 


\title{
Where Question, Conditionals and Topics converge
}

\author{
Edgar Onea and Markus Steinbach* \\ University of Göttingen, \\ Courant Research Centre "Text Structures" \\ Nikolausberger Weg 23, 37073 Göttingen, Germany \\ edgar.onea@zentr.uni-goettingen.de \\ Markus.Steinbach@phil.uni-goettingen.de
}

\begin{abstract}
One puzzling fact about German is that what verb-one structures that surface as a yes-no questions can be interpreted as conditionals in a topic position. In this paper we provide an analysis for this phenomenon using the basic idea of inquisitive semantics that questions and assertions can be treated on a par as denoting sets of possibilities and some insights about the discourse function of different topic constructions in German. The key assumption is that in topic positions, questions can be interpreted as conditionals if and only if they contain a highlighted alternative possibility. We show that the analysis also correctly predicts the distribution of wh-questions and the distribution of so called irrelevance-conditionals containing auch('too').
\end{abstract}

Keywords: Topic, Conditionals, Inquisitive Semantics, Questions

\section{Introduction}

The standard difference between assertions and yes-no questions in German is that the former requires a verb-second (V2) construction, as in (1-a), whereas the latter exhibits a verb-first (V1) syntactic structure, as in (1-b). As opposed to yes-no questions, wh-questions, like assertions, exhibit a V2 structure but contain an initial wh-word, as in (1-c).

(1) a. Er kommt nach Hause.

he comes to home

'He comes home.'

b. Kommt er nach Hause?

comes he to home

'Does he come home?'

c. Wer kommt nach Hause?

who comes to home

'Who comes home?'

\footnotetext{
* This research has been supported by the German Initiative for Excellence funded by the DFG (German Science Foundation), which we gratefully acknowledge.
} 
However, a V1-clause can also be interpreted as a conditional, whenever it appears in sentence initial position, embedded into a V2 clause, as in (2-a). Crucially, the same applies neither to plain V2 constructions nor to wh-questions, as shown in (2-b) and (2-c) respectively.

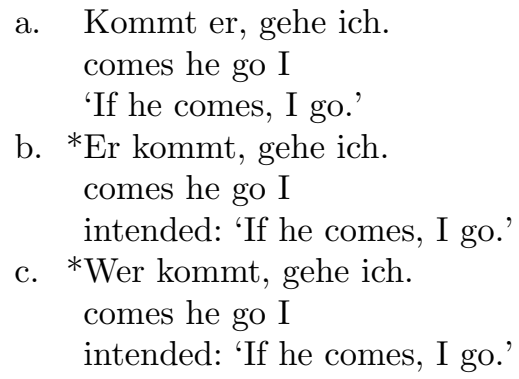

The main question this paper addresses, is accordingly: What is it about yes-no questions that makes a conditional interpretation possible in a topical position, as in (2-a). The answer to this question will be: yes-no questions can be analyzed as a set of alternatives containing one highlighted alternative. The rest of the ingredients of the analysis comes for free, if one makes the right assumptions about the function of two different sentence initial positions in German, namely frame setting and aboutness topics.

In the following section, we develop an analysis that correctly predicts the distributional facts in (1) vs. (2). For this we will use some existing theoretical tools, mainly the idea that yes-no questions can come with so-called highlighted alternatives, suggested in [Brasoveanu et al., 2011], and some general assumptions about the discourse function of two different topic constructions in German. In particular, we argue that yes-no questions can be aboutness but not framesetting topics. In section three, we extend the set of data under consideration and show how the addition of the discourse particle auch ('too') makes wh-questions in a higher topical position, which triggers a frame setting topic interpretation, acceptable. In addition, auch also successfully combines with yes-no questions, still preserving a conditional interpretation.

\section{Questions as conditionals}

In this section we present the basic analysis that predicts that, in German, yes-no questions can be interpreted as conditionals in a topical position. For this, we first briefly introduce the idea of inquisitive semantics including some relevant details. We then discuss the distribution of conditions in topic constructions in German. We show how a correct analysis of the discourse function of the aboutness topic together with the inquisitive analysis of V1-constructions correctly predict the distributional facts. 


\subsection{Inquisitiveness and highlighted alternatives}

The first main ingredient of our theory is the recently developed theory of inquisitive semantics [Groenendijk and Roelofsen, 2009]. One of the interesting features of inquisitive semantics relevant for our approach is that a unified treatment of questions and propositions becomes available. Traditionally, questions have been assumed to denote sets of sets of worlds, while propositions denoted sets of worlds. By contrast, in inquisitive semantics the notion of a proposition is shifted to sets of special sets of worlds supporting the proposition and not included into any other sets of worlds, called possibilities. A proposition is then inquisitive, if it contains more than one possibility, and informative, if it rules out at least some possibilities. This is exemplified in (3)
a. Peter smokes.
$\{\lambda w$. Peter smokes in $w\}$
Atomic assertion: informative but not inquisitive.
b. Peter smokes or Mary smokes
$\{\lambda w$. Peter smokes in $w, \lambda w$. Mary smokes in $w\}$
Disjunction: informative and inquisitive $=$ hybrid
c. Does Peter smoke?
$\{\lambda w$. Peter smokes in $w, \lambda w$. Peter does not smoke in $w\}$
Questions: non-informative, inquisitive.

In addition, we assume with [Brasoveanu et al., 2011] that the alternative possibilities may or may not be highlighted. Treat highlight as a technical notion. We signal 'highlighting' by underlining the corresponding alternative. We assume that in yes-no questions, the alternative explicitly mentioned is highlighted, hence, in positive yes-no questions the positive alternative is highlighted, and in negative ones, the negated one. We assume that we get this result in the process of composition, although we don't discuss the compositional details.
a. Does Peter smoke?
$\{\lambda w$. Peter smokes in $w, \lambda w$. Peter does not smoke in $w\}$
b. Doesn't Peter smoke?
$\{\lambda w$. Peter smokes in $w, \underline{\lambda w}$. Peter does not smoke in $w\}$

We define the operator Highlight as taking a set as an argument and returning the unique highlighted element in a set. Highlight and is undefined if either no such element exists or more than one element is highlighted:

$$
\text { Highlight }(\llbracket \text { Does Peter smoke? } \rrbracket)=\lambda w \cdot \text { Peter smokes in } w \text {. }
$$

Under the assumptions above, a V1 sentence in German denotes a set containing exactly two possibilities, as already suggested in [Lohnstein, 2000] and [Tuckenbrodt, 2006], however, we now take it that exactly one of them is highlighted. 


\subsection{The topic issue}

We assume with [Frey, 2004b], and [Ebert et al., 2008] that in German there are at least two types of left dislocated topic positions. The so called hanging topic position is available for frame setting topics, as in (6), whereas the position known as German left dislocation or fronting, shown in (7), is reserved for aboutness topics.

(6) Der/den Minister, den liebt nur seine Frau.

the.NOM/the.ACC Minister, the.ACC loves only his wife

'The minister, only his wife loves him.'

(7) Den Minister (den) liebt nur seine Frau. the.ACC Minister, (the.ACC) loves only his wife

'The minister, only his wife loves him.'

It has been observed already in [Schlenker, 2004], and also in [Ebert et al., 2008] that in German if-clauses can appear in both topic positions. [Ebert et al., 2008] argue that in the hanging topic position the interpretation of conditionals is the one known under the label of biscuit conditionals and exemplified in (8). Such conditionals are special because the truth of the consequence does not seem to depend on the truth of the antecedent. As opposed to this, conditionals in the left dislocation position behave as expected: the truth of the consequence typically depends on the the truth of the antecedent, as in (9). ${ }^{1}$

(8) Wenn du Hunger hast, es gibt Kekse im Kühlschrank.

If you hunger have there exist biscuit in-the fridge

'If you are hungry, there are biscuits in the fridge.'

(9) Wenn du welche gekauft hast, gibt es Kekse im Kühlschrank.

If you some bought have there exist biscuit in-the fridge

'If you bought some, there are biscuits in the fridge.'

[Ebert et al., 2008] analyse conditionals as definite descriptions over possible worlds as argued in [Schlenker, 2004]. Building on a old analogy between conditionals and definite descriptions, Schlenker claims that $p$, in if $p$ then $q$ interpreted relative to a world $w_{0}$, denotes the single world $w_{1}$, most similar to $w_{0}$ such that $w_{1} \in p$. The whole sentence is then analyzed as saying that $w_{1} \in q$. It is easy to see, that this captures the truth conditions of conditionals, since this predicts that if a world $w_{2}$ exists in which $p$ holds true but q does not,

\footnotetext{
${ }^{1}$ We use the position of the verb as a test. We assume that in German the verb always appears in the second position in assertions. Now, if the conditional counts in determining the second position of the verb, as in (9), we say that we have an instance of left dislocation or fronting, whereas if the conditional does not count, we have an instance of hanging topic, as in (8): practically, in a hanging topic construction, the verb appears in third place. Left dislocated or fronted constituents are syntactically more integrated into the matrix clause, however we do not discuss the exact syntactic details of the constructions in this paper. Note however, that intonational and further clues also help distinguishing, see [Ebert et al., 2008] and [Frey, 2004a] for details.
} 
this world will be less similar to $w_{0}$, and, hence, the intuition is captured that if the antecedent holds true but the consequence does not, independent reasons will apply that do not hold in the world of evaluation. An example clarifies this: (10-a) is true if in the most similar world to the world of evaluation $w_{0}$ in which the hearer puts the glass on the table, the hearer is happy. This does not predict that (10-a-ii) also comes out as true, as the world in which the hearer puts the glass on the table such that it breaks into pieces might be less similar to the world of evaluation, hence, (10-a) is silent about the truth of the consequence in it. Note that $\iota$ in Schlenker's system is interpreted as a choice function involving a parameter in subscript, and not as a classical Russelian $\iota$ operator.

a. If you put the glass on the table, I will be happy.

(i) happy (Speaker) $\left(\iota w_{w_{0}}\right.$. put (Speaker, Glas, Table $\left.)(w)\right)$

(ii) If you put the glass on the table such that it breaks into pieces, I will not be happy.

[Ebert et al., 2008] argue that while conditionals interpreted as aboutness topics are ultimately interpreted as the world argument of the consequence, in the hanging topic position, i.e. frame setting topics such as (8), are interpreted as independent referential acts, such that the world argument of the consequence is not the world depicted by the antecedent, but rather the world of evaluation, roughly as in (11).

$$
\begin{array}{ll}
\text { a. } & \exists \text { biscuit }\left(\iota w_{w_{0}} \cdot \operatorname{Buy}(\text { Speaker, biscuit })(w)\right) \\
\text { b. } & \operatorname{REF}\left(\iota w_{w_{0}} \cdot \text { Hungry }(\text { Speaker })(w)\right) \wedge \operatorname{ASSERT}\left(\exists \operatorname{Siscuit}\left(w_{0}\right)\right)
\end{array}
$$

\subsection{Our analysis}

We follow the main line of attack pursued in [Ebert et al., 2008], however, we take it that there is not enough evidence for the treatment of conditionals as definite descriptions over worlds.

For one thing, we see conceptual problems with the analysis in [Schlenker, 2004] already noticed in [Lewis, 1973] that the selection of the most similar world is problematic. In addition, we do not see how such an analysis could extend to V1-conditionals. Instead, we assume the more traditional analysis of conditionals as restricting the quantification of overt or covert modals. In particular, this means that a conditional interpretation is only possible if at LF at least a covert modal is available in the antecedent.

We assume that a proposition, interpreted in an aboutness topic position, will simply end up in its standard function, with the difference that we get some contrastive marking in the sence of [Büring, 2003]. If one explicitly signals that he is speaking about something, implicitly alternatives must have been available in the discourse. Otherwise, the additional marking seems unreasonable. We do not assume, however, that any exhaustiveness inferences must be associated with this notion of contrast. As opposed to this, a frame setting topic ends up as a referential act independent of the actual logical structure of the assertion, much like in [Ebert et al., 2008]. 
We also follow [Ebert et al., 2008] in assuming that both aboutness topics and framesetting topics must be, in a sense, referential. We note, however, that propositional arguments generally can appear in these positions. A few examples are given in (12). The referentiality of topics cannot imply that propositions must denote one single world in this case, otherwise one would need to postulate that every embedded clause should refer to one single world, which seems weird.

a. Weil Peter klug ist, (deshalbt) geht er nach Hause.

Because Peter clever is for-that goes he to home

'Peter goes home, because he is clever.'

b. Dass Peter klug ist, wissen wir.

That Peter clever is know we

'We know that Peter is clever'

c. Dass Peter klug ist, das wissen wir.

That Peter clever is that know we

'We know that Peter is clever'

More important seems, however the observation that inquisitive expressions such as disjunctions or non specific indefinites cannot appear in the topic position:
a. \#Peter oder Paul, die kommen spät.
Peter or Paul they come late
intended: 'Peter or Paul come late'
b. \#Peter oder Paul, der kommt spät.
Peter or Paul he comes late
intended: 'Peter or Paul come late'
c. \#Irgendein Mann, der kommt spät.
Some man he comes late
intended: 'Some man comes late'

We assume, therefore, instead of saying that propositions in topics denote one single world, that the topic operator, both for frame setting and for aboutness topic, can take propositional arguments only if the proposition, as a set of alternative possibilities, contains exactly one maximal possibility, i.e. the proposition is not inquisitive. This singleton requirement with respect to maximal alternatives is very similar to the referentiality requirement but is more general. In this case, the semantics of the aboutness topic operator is vacuous as far as the assertion is concerned, but there are discourse functional effects amounting to contrast, see [Buering, 1997]. The semantics of the frame setting topic amounts to asserting the matrix clause, drawing the attention of the hearer to the topical proposition beforehand. We do not discuss the formal implementation of this idea for the moment.

Crucially, V1-structures, interpreted as questions, are inquisitive and should not be allowed in any topic position. We assume, however, that applying the Highlight operator beforhand makes them suitable topical propositions. This leads to the interpretation of the V1 conditional as a proposition that somehow has to be combined with another full proposition. Assuming that the second 
proposition has some overt or covert modal, the V1-highlighted proposition will end up in the restrictor of that modal and hence yield a conditional interpretation.

Of course, this does not predict that also V2-sentences can simply appear in the aboutness topic-position, and receive a conditional interpretation. This is because we assume that V2 in German is associated with very direct rules of manipulation of the common ground, which exclude a more static interpretation as a topic.

In the case of the frame setting topic, again, we have exactly one highlighted alternative, and ultimately we expect a biscuit conditional interpretation, as argued by Ebert and colleagues. Indeed, this interpretation is available, in fact the only possible interpretation. Whence the contrast between (14-a) and (14-b). However, we observe that the function of frame setting topics yields oddity in (14-b): It is weird for the speaker to draw the attention of the hearer to a possibility that involves his own state of mind.

a. *Kommst du, ich gehe.

come you I go

intended: 'If you come I go.'

b. ?Hast du Hunger, es gibt was im Kühlschrank.

'If you are hungry, there is something in the fridge.'

For wh-clauses, we standardly assume that they denote multiple alternative possibilities. So, (15) can be modeled as a set of alternatives containing different instantiations of $\operatorname{wer}$ ('who')

Wer kommt nach Hause?

who comes to home

'Who comes home?'

a. $\quad\{\lambda w$. Peter comes in $w, \lambda w$. John comes in $w, \lambda w$. Max comes in $, \lambda w$. Dan comes in $w \ldots\}$

This multiple partition does not contain a highlighted alternative, hence the operator Highlight $(W H$ ?) is not applicable. Therefore, wh-clauses cannot be interpreted in a topic position. This prediction is correct, as can be seen in example (2-c) above. Hence, we explained why yes-no questions behave differently than both assertions and wh-questions with regard to their potential to be interpreted as conditionals.

\section{The presence of auch}

In this section we extend the range of data by including the discourse particle auch. We show that our analysis can cope with the new arising data as well. 


\subsection{The data}

In German, it is possible to generally add the particle auch ('too') to a conditional, hence yielding what has been called an irrelevance conditional in the literature, as shown in (16)

(16) Wenn Peter auch schläft, wir tanzen weiter.

If Peter too sleeps we dance on

'Even if Peter sleeps, we keep on dancing.' .

Once we add auch ('also') a V1 conditional can appear in a hanging topic position. This we can see, again since it is not 'counted' when checking the V2 constraint, as whithnessed in (17-a). Interestingly, leaving the V1-conditional with auch in the dislocation position we have analysed as an aboutness topic position, yields a marked or even unacceptable structure as in (17-b).

a. Endet es auch vor Gericht, wir zahlen die Miete (trotzdem) nicht. Ends it also in-front court we pay the rent (nevertheless) not 'Even if we end up in front of the court, we will not pay the rent.'

b. ??Endet es auch vor Gericht, zahlen wir die Miete (trotzdem) nicht. Ends it also in-front court pay we the rent (nevertheless) not

'Even if we end up in front of the court, we will not pay the rent.'

Moreover, adding auch not only 'saves' V1 structures in the hanging topic position, but actually makes even wh-structures acceptable, but, again, only in the hanging topic position, as can be observed in the contrast between (18) vs. (19).

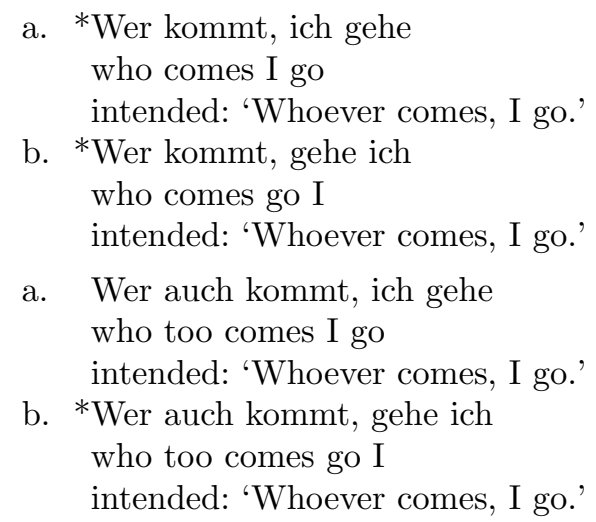

The arising puzzle is: Why does the presence of auch change the acceptability of $\mathrm{V} 1$ and wh-clauses in the different topic positions?

\subsection{Analysis}

We assume that the particle auch in German acts as an non-inquisitive closure operator in such constructions, i.e. it acts as a classical disjunction over the set of alternative possibilities denoted by the inquisitive propositions it occurs in. 
Using a version of inquisitive semantics, in which the non-inquisitive closure of a proposition, if generated compositionally, is added to its representation we end up with structures such as (20) vs. (21). We call such non-inquisitive propositions attentive propositions, and we assume with [Ciardelli et al., 2009] that their pragmatic function is to draw the attention of the hearer to the possibilities, which now are sub-possibilities of the "big" possibility, added by the non-inquisitive closure.

(20) $\llbracket$ Wer auch kommt $\rrbracket=\{\lambda w$. Peter comes in $w, \lambda w$. John comes in $w, \lambda w$. Max comes in , $\lambda w . \exists x . x$ comes in $w\}$

$\llbracket$ Kommst du auch $\rrbracket=\{\lambda w$. hearer comes in $w, \lambda w$. hearer doesn't come in $w, \mathbf{W}\}$

The assumptions above that frame setting topics have the discourse function to draw the attention of the hearer to a certain possibility, and that topics require a non-inquisitive proposition (or exactly one highlighted alternative) we end up with the prediction, that both wh- questions and yes-no questions can appear in the frame setting topic function in a hanging topic position, whenever auch is added, since auch turns both wh-questions and yes-no questions not only non-inquisitive but also attentive. This is exactly as required by the data.

Finally, we have to answer one additional question: Why can questions with auch not appear in an aboutness topic position, i.e. left dislocated. Why is it that e.g. (19-b) is bad in German.

Our answer to this question involves the pragmatics of aboutness-topics: In principle, an irrelevance conditional interpretation is possible in the aboutness topic position, however, typically, the additional marking has to be pragmatically justified. This happens, whenever an alternative aboutness topic lends itself. However, in these cases, the aboutness topic is always the entire set of worlds (excluding those in which noone comes for wh-questions), and it is very hard to imagine any alternative possibility to such an unlimited possibility. This correctly predicts the oddity of such examples. Because of this lack of alternatives, using auch constructions as aboutness-topics, appears marked or even unacceptable.

\section{Conclusion}

In this paper we have provided an analysis of V1-conditionals, i.e. yes-no questions with conditional interpretation in a topic position in German. Our analysis is based on the idea that topics can pick out the highlighted alternative of a polar question. We have shown that the analysis sketched above not only correctly predicts the possibility to use V1-questions as conditionals in German, but also correctly predicts the distributional facts regarding the presence and absence of the particle auch and the interaction with hanging topic position and the so called German left dislocation or fronting, which typically host frame setting topics and aboutness topics respectively. Adding up, the distributional picture that we correctly captured amounts to the one in Table 1: 


\begin{tabular}{|c|c|c|c|c|}
\hline & $\begin{array}{c}\text { Yes no } \\
\text { +auch }\end{array}$ & $\begin{array}{c}\text { Yes no } \\
\text {-auch }\end{array}$ & $\begin{array}{c}\text { Wh } \\
+ \text { auch }\end{array}$ & $\begin{array}{c}\text { Wh } \\
\text {-auch }\end{array}$ \\
\hline hanging topic & OK & $*$ & OK & $*$ \\
\hline left dislocation & $?$ & OK & $? ?$ & $*$ \\
\hline
\end{tabular}

Table 1. The distribution covered in this paper

The analysis leaves some questions open, however. For instance, we still have to examine the binding observations enumerated in [Ebert et al., 2008] which distinguish between hanging topics and left-dislocation, the presence or absence of the resumptive pronoun dann and its role in the interpretation, and finally, some of our more controversial data must be backed up with experimental research.

\section{References}

[Brasoveanu et al., 2011] Brasoveanu, A., Farkas, D., and Roelofsen, F. (2011). Polarity particles and the anatomy of n-words. In to appear in: Proceedings of SUB12, Utrecht.

[Buering, 1997] Buering, D. (1997). The meaning of topic and focus: the 59th Street Bridge accent. Routledge.

[Büring, 2003] Büring, D. (2003). On D-Trees, Beans, and B-Accents. Linguistics \& Philosophy, 26(5):511-545.

[Ciardelli et al., 2009] Ciardelli, I., Groenendijk, J., and Roelofsen, F. (2009). Information, issues, and attention. In Proceedings of SALT09.

[Ebert et al., 2008] Ebert, C., Endriss, C., and Hinterwimmer, S. (2008). Topics as speech acts: An analysis of conditionals. In Abner, N. and Bishop, J., editors, Proceedings of the 27th West Coast Conference on Formal Linguistics (WCCFL 27), pages 132-140, Sommerville, MA. Cascadilla Proceedings Project.

[Frey, 2004a] Frey, W. (2004a). A medial topic position for german. Linguistische Berichte, pages 153-190.

[Frey, 2004b] Frey, W. (2004b). Notes on the syntax and the pragmatics of german left dislocation. In Lohnstein, H. and Trissler, S., editors, The Syntax and Semantics of the Left Periphery, pages 203 - 233. Mouton de Gruyter.

[Groenendijk and Roelofsen, 2009] Groenendijk, J. and Roelofsen, F. (2009). Inquisitive semantics and pragmatics. MS University of Amsterdam.

[Lewis, 1973] Lewis, D. (1973). Counterfactuals. Harward.

[Lohnstein, 2000] Lohnstein, H. (2000). Satzmodus - kompositionell. Akadem, Berlin.

[Schlenker, 2004] Schlenker, P. (2004). Conditionals as definite descriptions (a referential analysis). Research on Language and Computation, 2(3):417-162.

[Tuckenbrodt, 2006] Tuckenbrodt, H. (2006). On the semantic motivation of syntactic verb movement to c in german. Theoretical Linguistics, 32(3):257-306. 


\title{
Inquisitive Knowledge Attribution and the Gettier Problem*
}

\author{
Wataru Uegaki \\ Department of Linguistics and Philosophy, MIT \\ wuegaki@mit.edu
}

\begin{abstract}
A disjunctive belief cannot be described as knowledge if the subject does not justifiably believe a true disjunct, even if the whole disjunctive belief is true and justified (Gettier 1963). This phenomenon is problematic if the verb know semantically operates on a (classical) proposition, as standardly assumed. In this paper, I offer a solution to this problem using Inquisitive Semantics, arguing that know operates on the set of alternative possibilities expressed by its complement. It will also be shown that the proposed semantics for know provides a novel account of its compatibility with both declarative and interrogative complements.
\end{abstract}

\section{Introduction}

The attitude verb know can embed either a declarative or an interrogative complement, in contrast to other attitude verbs, such as believe or ask/wonder, which take only one of the two complement types, as shown in (1).

(1) a. John knows \{that Sue came / who came\} to the party.

b. John believes $\{$ that Sue came /*who came $\}$ to the party.

c. John asked me/wonders $\left\{{ }^{*}\right.$ that Sue came / who came $\}$ to the party.

One of the basic issues in the semantics of question-embedding concerns this selection property of know and other verbs that behave similarly (e.g., forget, tell). Namely, how we can semantically account for the compatibility of know (and other verbs) with both a declarative and an interrogative complement.

The standard answer to this question states that the basic denotation of know selects for a proposition, which is the meaning of declarative clauses, and assumes some form of reduction from the meaning of embedded interrogatives to propositions (e.g. Karttunen 1977, Groenendijk and Stokhof 1984). However, such an account wrongly predicts that a believe-type verb should be able to embed an interrogative complement provided that the reduction is general. ${ }^{1}$

\footnotetext{
^ I thank Maria Aloni, Danny Fox, Irene Heim, Yasutada Sudo, Igor Yanovich, and especially Benjamin George for helpful discussion and criticism. Of course, they need not agree with the claims made in this paper, and all errors are my own.

1 An exception is Ginzburg (1995), who has a reduction in terms of coercion, but avoids this problem by positing an ontological distinction between the objects believe and know select for. Unfortunately, limited space prevents me from going into an extensive comparison between Ginzburg's and the current proposal, but it is important to note that the current proposal accounts for the difference in the selection restrictions in an ontology that is more conservative than Ginzburg's.
} 
In this paper, I propose an alternative approach to the issue that avoids this problem, focusing on a puzzling interpretation of a disjunction in a declarative complement of know, known as the GeTtier PROBLEM. Specifically, I will propose that know always operates on a set of alternative possibilities, which is typically the type of an interrogative meaning, even when know takes a declarative complement. I will argue that the solution to the Gettier problem crucially requires the proposed view of the meaning of know, and implement the analysis using the treatment of disjunction in Alternative Semantics and Inquisitive Semantics (Groenendijk 2009, Groenendijk and Roelofsen 2009).

\section{The Gettier Problem (Gettier 1963)}

Knowledge has been traditionally analyzed as a JUSTIFIED TRUE BELIEF (JTB) in epistemology. This traditional view is also underlying in the lexical entry for know in the standard semantic theory, as in (2), which basically treats the meaning of know as that of (justifiably) believe + factivity. ${ }^{2,3}$

(2) $\llbracket \mathrm{know} \rrbracket=\lambda p \in D_{\langle s, t\rangle} \lambda x \lambda w:[p(w)=1] . \operatorname{JDOX}_{x, w} \subseteq p$ where $\operatorname{JDOX}_{x, w}=\left\{w^{\prime} \mid w^{\prime}\right.$ is compatible with $x$ 's justified belief in $\left.w\right\}$

In his famous 1963 paper, Gettier presents counterexamples to this JTB analysis of knowledge. In the situation described in (3), the knowledge attribution in (4) is intuitively false even though the proposition 'Jones owns a Ford or a BMW' is a true and justified belief of Smith.

(3) Situation: Smith justifiably believes that Jones owns a Ford. (He saw Jones with the key of a Ford, driving a Ford etc.) He justifiably deduces from this belief that Jones owns a Ford or a BMW although he is unopinionated about whether Jones owns a BMW. However, it turns out that Jones in fact does not own a Ford, but he owns a BMW.

(4) Smith knows that [Jones owns a Ford or he owns a BMW].

A Gettier example need not involve a disjunction. The following is a case where the belief in question involves an existential quantification.

(5) Situation: "James, who is relaxing on a bench in a park, observes a dog that, about 8 yards away from him, is chewing on a bone. So he believes [that there is a dog in the park]. [However,] what he takes to be a dog is actually a robot dog so perfect that, by vision alone, it could not be distinguished from an actual dog. James does not know that such robot dogs exist. But in fact a Japanese toy manufacturer has recently developed them, and what

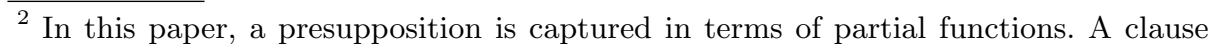
after a comma in a lambda term indicates a restriction on the domain of the function that the lambda term expresses.

3 The reference to a justified belief (JDOX) instead of a mere belief (DOX) might not be strictly standard in semantics. What I mean by 'standard' in the text here is the analysis of know as a straightforward extension of believe with additional conditions on its arguments and accessibility relation.
} 
James sees is a prototype that is used for testing the public's response. But, just a few feet away from the robot dog, there is a real dog. Sitting behind a bush, he is concealed from James's view." (Steup 2009, pp.7-8)

(6) James knows that there is a dog in the park.

Again, the knowledge attribution in (6) is intuitively false although the proposition 'there is a dog in the park' is a true and justified belief of James. This example is also important because it does not involve an inference from falsehood, which one might consider as the source of the problem in (3-4). The belief in (5) can be directly justified by James' visual perception.

This famous problem in epistemology is also a problem for the standard semantic analysis of know in (2), which incorrectly predicts sentences (4) and (6) to be true in the given contexts. ${ }^{4}$ In the next section, I will offer a solution to this puzzle using the treatment of disjunction in Inquisitive Semantics.

\section{Analysis in Inquisitive Semantics}

In this section, after briefly setting up the theoretical framework of Inquisitive Semantics in Section 3.1, I propose a solution to the Gettier problem by arguing that know operates on the set of possibilities denoted by its complement, unlike in the standard analysis where it operates on a classical proposition. In the last subsection, I show that the proposed meaning for know can be used with wh-complements with necessary modifications.

\subsection{Disjunction in Alternative/Inquisitive Semantics}

In Inquisitive Semantics (Groenendijk 2009, Groenendijk and Roelofsen 2009), the semantic value of a sentence is conceived of as possibly multiple ways of updating the common ground. Each update possibility (referred to simply as a POssibiLity) is modeled as a set of indices (of type $\langle s, t\rangle$ ) (Groenendijk and Roelofsen 2009). A sentence denotes a set (of type $\langle s t, t\rangle$ ) of such alternative possibilities qua index-sets. Also, for expository purposes, I model possibilities containing a presupposition as partial functions from indices to truth values in cases where the presupposition is relevant to the discussion.

In the context of this paper, particularly important is the treatment of disjunction. Along with the proposals in Alternative Semantics (Kratzer and Shimoyama 2002, Alonso-Ovalle 2006, a.o.), Inquisitive Semantics treats a sentential disjunction as a set union (Groenendijk and Roelofsen 2009).

\footnotetext{
${ }^{4}$ The puzzle might remind one of the free choice effect of a disjunction under a possibility modal or an imperative (Ross 1941, Kamp 1973, a.o.). However, the behavior of a disjunction under know is crucially different from free choice in licensing the inference pattern in (i), the failure of which is the hallmark of free choice, e.g., (ii).

(i) John knows that Sue came, but he does not know that Mary came. $\models$ John knows that Sue or Mary came.

(Disjunction under know)

(ii) You may take a pear, but you may not take an apple. $\not \models$ You may take a pear or an apple.

(Free choice permission)
} 
(7) $\llbracket \alpha$ or $\beta \rrbracket=\llbracket \alpha \rrbracket \cup \llbracket \beta \rrbracket$

That is, the meaning of a disjunction is the union of the sets of possibilities conveyed by each disjunct. In this way, Inquisitive Semantics captures the hybrid nature of disjunction: a disjunction is INQUISITIVE in proposing alternative possibilities just like a question, while it is also INFORMATIVE in eliminating a possibility (namely, one where neither disjunct is true) from the common ground just like a classical assertion. ${ }^{5}$

\subsection{First Attempt: Point-wise Function Application}

Having set up the theoretical background, let us return to the Gettier problem. One immediate question that arises is how an attitude verb semantically composes with its complement, given that the complement denote a set of alternative possibilities. The standard approach to subsentential composition in this kind of setup is to use the rule of Point-Wise Function ApplicAtion (PFA) in (8) following the literature of Alternative Semantics after Hamblin (1973).

(8) If $\llbracket \alpha \rrbracket \subseteq D_{\langle\sigma, \tau\rangle}$ and $\llbracket \beta \rrbracket \subseteq D_{\sigma}$, then

$$
\llbracket \alpha \beta \rrbracket:=\left\{d \in D_{\tau} \mid \exists a \in \llbracket \alpha \rrbracket \exists b \in \llbracket \beta \rrbracket[d=a(b)]\right\} \quad \text { (Point-wise FA; PFA) }
$$

A natural denotation for know one would expect in a compositional system like Alternative Semantics that utilizes PFA is simply the singleton set of its standard denotation, as given in (9).

(9) $\llbracket$ know $\rrbracket=\left\{\lambda p \in D_{\langle s, t\rangle} \lambda x \lambda w:[w \in p] . \operatorname{JDOX}_{x, w} \subseteq p\right\}$

If we apply the denotation of know in (9) to the set of possibilities in (10) via PFA, we get the set in (11) as the meaning of the Gettier example in (4).

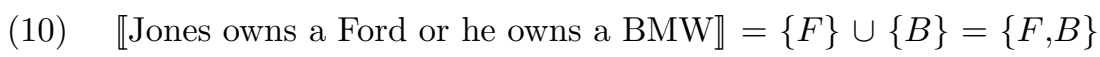

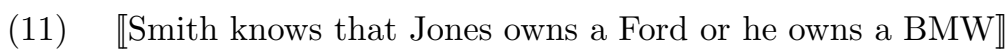
$=\left\{\lambda w:[w \in F] . \operatorname{JDOX}_{\mathbf{s}, w} \subseteq F, \lambda w:[w \in B] . \operatorname{JDOX}_{\mathbf{s}, w} \subseteq B\right\}$

Assuming that the truth of a sentence is determined as in (12) (along the lines of the notion SUPPORT in Groenendijk and Roelofsen 2009), we predict (11) to be false in the Gettier scenario in (3).

(12) $\varphi$ is true in $w$ iff there is some $p \in \llbracket \varphi \rrbracket$ such that $p(w)=1$

This is so because the first possibility in (11) is undefined for the situation, as the presupposition triggered by the factivity of know, i.e. $F$, is false. The latter possibility is false even though defined, as Smith does not believe $B$ in the situation. Thus, what we end up is the correct prediction that the Gettier example in (4) is false, somewhat surprisingly.

However, this analysis is clearly too strong as it predicts that a disjunction under know is always equivalent to a matrix disjunction of know. This means that we cannot capture a 'purely disjunctive' knowledge, which does not require a knowledge of any specific disjunct, as described in the following sentence.

\footnotetext{
${ }^{5}$ A sentence $\varphi$ is INQUISITIVE iff $\llbracket \varphi \rrbracket$ contains at least two possibilities (none of which contains another). A sentence $\varphi$ is InFOrmative iff $\bigcup \llbracket \varphi \rrbracket \neq \mathcal{W}$ (ie. for some index, all possibilities in $\llbracket \varphi \rrbracket$ are false.)
} 
(13) Smith knows that Jones owns a Ford or he owns a BMW, but he doesn't know specifically which he owns.

In order to capture the meaning of (13) in the current system, we seem to need an operation to 'collapse' multiple possibilities denoted by the complement into a single big possibility, along the lines of the NON-INQUISITIVE CLOSURE in (14).

$$
\llbracket ! \alpha \rrbracket:=\{\bigcup \llbracket \alpha \rrbracket\}
$$

(Non-inquisitive closure of $\llbracket \alpha \rrbracket)$

By applying this operation to the complement, we can capture the purely disjunctive knowledge in (13), as shown below.

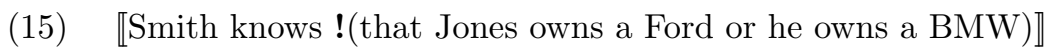
$=\left\{\lambda w:[w \in F \cup B] . \mathrm{JDOX}_{\mathbf{s}, w} \subseteq F \cup B\right\}$

The problem is how to constrain this closure operation. Allowing the operation freely would get us back to the original Gettier problem, as (15) is true also in the Gettier scenario. How can we allow attribution of a purely disjunctive knowledge in a sentence like (13) while block it in the Gettier case? The next section presents an answer to this question by analyzing knowledge as requiring a strongest true belief.

\subsection{Solution: Knowledge Requires a Strongest Justified Belief}

What distinguishes a Gettier case and a purely disjunctive knowledge as in (13) is that the subject believes a false disjunct in the former while she believes no specific disjunct in the latter. The descriptive generalization, therefore, is that purely disjunctive knowledge attribution, or 'collapsing' of alternative possibilities, is valid only if the subject believes no specific disjunct.

We can account for the generalization if a purely disjunctive knowledge entails that the subject has no justified belief about a specific disjunct. That is, the generalization is captured if we analyze $x$ knows $p$ or $q$ as ambiguous between the following two translations in classical logic.

(16) $x$ knows $p$ or $q$.

$$
\begin{aligned}
& \text { a. }\left(w \in p \wedge \operatorname{JDOX}_{x, w} \subseteq p\right) \vee\left(w \in q \wedge \operatorname{JDOX}_{x, w} \subseteq q\right) \\
& \text { b. }(w \in p \cup q) \wedge \operatorname{JDOX}_{x, w} \subseteq(p \cup q) \wedge \underline{\operatorname{JDOX}_{x, w} \not \subset p \wedge \operatorname{JDOX}_{x, w} \not \subset q}
\end{aligned}
$$

The reading in (16a) is equivalent to a matrix disjunction, in which the possibilities in the complement are not collapsed by the closure. On the other hand, the reading in $(16 \mathrm{~b})$ captures the purely disjunctive knowledge, in which the possibilities are collapsed by the closure. The crucial point here is the underlined condition in (16b), which requires the subject not to believe either disjunct specifically. This condition falsifies the Gettier case as the subject believes the false disjunct, i.e. that Jones owns a Ford in the Gettier scenario. As we saw above, the Gettier case is false in the reading in (16a), either. Thus, we succeed in predicting the Gettier example to be false under any reading. Generalizing the disjunction case, a Gettier case involving an existential quantification as in (5-6) above can be accounted for, as will be shown shortly in the formal analysis. 
Thus, the claim is that every standard Gettier case involves multiple possibilities induced by a disjunction/existential quantification over individuals, times or places. Knowledge attribution is invalid in these cases when the subject believes no true possibility while believing a false one. In Section 4, I discuss other putative Gettier cases that the analysis sketched here is not applicable to, and claim that they should be treated separately.

Now, let us move on the compositional implementation of this analysis. To derive the two readings in (16) in a unified way, I encode in the meaning of know that a knowledge requires a strongest justified belief among the relevant possibilities. Specifically, I propose the following denotation for know. Crucially, this denotation directly operates on the set of alternative possibilities in its complement, via ordinary Function Application but not via Point-wise FA.

$$
\begin{gathered}
\llbracket \mathrm{know} \rrbracket=\lambda Q \in D_{\langle s t, t\rangle}\left\{\lambda x \lambda w:\left[\exists p^{\prime} \in Q\left[w \in p^{\prime}\right]\right] .\right. \\
\left.\exists p \in u n i Q\left[w \in p \wedge \operatorname{JDOX}_{x, w} \subseteq p \wedge \operatorname{strong}\left(p, \operatorname{JDOX}_{x, w}, \text { uni } Q\right)\right]\right\} \\
-\quad \text { uni } Q:=\{\bigcup s \mid s \in \mathcal{P}(Q)\} \quad \text { (the closure of } Q \text { under union) } \\
\text { - strong }(p, q, S) \text { iff } \neg \exists p^{\prime} \in S\left[p^{\prime} \subset p \wedge q \subseteq p^{\prime}\right] \\
(p \text { is the smallest among the sets in } S \text { that are as big as } q)
\end{gathered}
$$

Below, I illustrate how this entry for know works in the specific examples. Let us consider example (4), repeated below. First, the closure-under-union (uni) of the possibility-set denoted by the complement is (18).

(4) Smith knows that Jones owns a Ford or he owns a BMW.

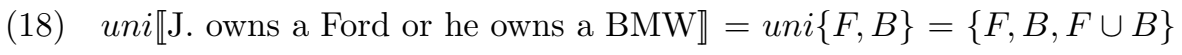

Applying (17) to this set, and supplying the subject via PFA, we get the meaning for the sentence in (19) below. The meaning in (19) contains a single possibility which presupposes that either $F$ or $B$ is true, and entails that there is a true proposition among (18) such that Smith justifiably believes it and that he believes no stronger possibility in (18).

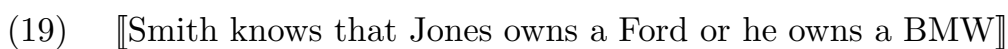

$$
\begin{aligned}
=\left\{\lambda w:\left[\exists p^{\prime}\right.\right. & \left.\in\{F, B\}\left[w \in p^{\prime}\right]\right] . \exists p \in\{F, B, F \cup B\} \\
& {\left.\left[w \in p \wedge \operatorname{JDOX}_{\mathbf{s}, w} \subseteq p \wedge \operatorname{strong}\left(p, \operatorname{JDOX}_{\mathbf{s}, w},\{F, B, F \cup B\}\right)\right]\right\} }
\end{aligned}
$$

In the following, I illustrate the evaluation of (19) under four possible belief states of the subject one by one, in all of which $F$ is actually false but $B$ is true. The four states are ones where Smith justifiably believes (i) $F$ but not $B$, (ii) $F \cup B$ but neither $F$ nor $B$, (iii) $B$ but not $F$, and (iv) both $F$ and $B$. The evaluation is summarized in the following list, where the (a)-clauses describe which possibility in (18) is Smith's strongest justified belief in each state, and (b)-clauses describe whether this possibility is true. If there is a proposition that meets these two conditions, (19) is evaluated true.

(i) $\operatorname{JDOX}_{\mathbf{s}, w} \subseteq F \cap \neg B$ (A Gettier state)

a. $F$ is Smith's strongest justified belief in $\{F, B, F \cup B\}$

b. $w \notin F$ (since $w \in \neg F \cap B$ ) $\Rightarrow$ (19) false. 
(ii) $\operatorname{JDOX}_{\mathbf{s}, w} \subseteq F \cup B \wedge \mathrm{JDOX}_{\mathbf{s}, w} \nsubseteq F \wedge \mathrm{JDOX}_{\mathbf{s}, w} \nsubseteq B$ (Purely disj. belief) a. $F \cup B$ is Smith's strongest justified belief in $\{F, B, F \cup B\}$

b. $w \in F \cup B$ (since $w \in \neg F \cap B) \quad \Rightarrow(19)$ true.

(iii) $\mathrm{JDOX}_{\mathbf{s}, w} \subseteq \neg F \cap B$

a. $B$ is Smith's strongest justified belief in $\{F, B, F \cup B\}$

b. $w \in B$ (since $w \in \neg F \cap B) \quad \Rightarrow$ (19) true.

(iv) $\operatorname{JDOX}_{\mathbf{s}, w} \subseteq F \cap B$

a. $B$ and $F$ are Smith's strongest justified beliefs in $\{F, B, F \cup B\}$

b. $w \notin F$, but $w \in B$ ( since $w \in \neg F \cap B) \quad \Rightarrow$ (19) true.

As illustrated above, we predict sentence (4) to be true in states (ii-iv) but false in state (i), namely the Gettier scenario where the subject justifiably believes a false disjunct but does not believe a true one. This is exactly the judgment pattern that we observe for a sentence with a disjunction under know.

In the reminder of this section, I illustrate the interpretation of an example involving an existential quantification, as in (6) repeated below.

(6) James knows that there is a dog in the park.

Although I have to gloss over the compositional details for the sake of space, the denotation of the complement in (6) will look like the following, generalizing the semantics for disjunction in Inquisitive Semantics.

(20) $\llbracket$ there is a $\operatorname{dog}$ in the park $\rrbracket=\left\{\{w \mid \operatorname{dog}(x)(w) \wedge \operatorname{inPark}(x)(w)\} \mid x \in D_{e}\right\}$

The meaning for the sentence in (6) will thus be the following, which contains the single possibility entailing that there is a true possibility in uni(20) such that James believes it and that there is no stronger possibility in uni(20).

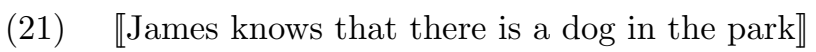

$$
\begin{aligned}
=\{\lambda w: & {\left[\exists p^{\prime} \in(20)\left[w \in p^{\prime}\right]\right] . } \\
\quad & \left.p \in \operatorname{uni}(20)\left[w \in p \wedge \operatorname{JDOX}_{\mathbf{j}, w} \subseteq p \wedge \operatorname{strong}\left(p, \operatorname{JDOX}_{\mathbf{s}, w}, \text { uni }(20)\right)\right]\right\}
\end{aligned}
$$

As the reader can check, (21) predicts that sentence (6) is false in the Gettier scenario described in (5), while it is true in other states parallel to those in (ii-iv) in the disjunction case above.

\subsection{Extension to Interrogative Complements ${ }^{6}$}

So far, I have been discussing only the embedding of a declarative complement by know. What about the embedding of an interrogative complement, as in (22)?

(22) Smith knows \{whether Jones or Lee/who\} came to the party.

If we assumed that the denotation of a wh-complement looks as in (23) (i.e. the Hamblin denotation), and directly applied the meaning of know in (17), we would face a problem: (22) is predicted to be true when Smith knows that either Jones or Lee came to the party, but does not know specifically who did.

\footnotetext{
${ }^{6}$ I thank Benjamin George for extensive discussion on the content of this section.
} 
(23) 【whether Jones or Lee came to the party $=\{J, L\}$

The problem is that a purely disjunctive knowledge, which we allowed in the case of declarative embedding, should somehow be blocked in the case of interrogative embedding. Remember that in the previous section, a purely disjunctive knowledge is made possible by the union operation by uni encoded in the meaning of know. To extend the analysis to interrogative embedding, however, I revise the analysis so that the uni operation is contributed not by the meaning of know, but by the declarative clause. ${ }^{7}$ Also, I propose that wh-complements contribute the partition operation (Groenendijk and Stokhof 1984) instead of uni. More specifically, I propose a revised meaning for know in (24) and the entries for the declarative and interrogative complementizers, as follows.

(24) $\llbracket$ know $\rrbracket=\lambda Q \in D_{\langle s t, t\rangle}\left\{\lambda x \lambda w:\left[\exists p^{\prime} \in Q\left[w \in p^{\prime}\right]\right]\right.$. $\left.\exists p \in Q\left[w \in p \wedge \operatorname{JDOX}_{x, w} \subseteq p \wedge \operatorname{strong}\left(p, \operatorname{JDOX}_{x, w}, Q\right)\right]\right\}$

$$
\begin{aligned}
& \text { a. } \llbracket \text { that } \rrbracket=\lambda Q \cdot \operatorname{uni}(Q) \quad \text { b. } \llbracket \text { wh(ether }) \rrbracket=\lambda Q \cdot \operatorname{part}(Q), \text { where } \\
& \operatorname{part}(Q):=\left\{\left\{w^{\prime} \sim_{Q} w\right\} \mid w \in \mathcal{W}\right\} \text { and } w^{\prime} \sim_{Q} w \text { iff } \forall p \in Q\left[p(w)=p\left(w^{\prime}\right)\right]
\end{aligned}
$$

Under this system, the meaning for the wh-complement in (23) will be the partition in (26), and the meaning for the whole sentence will be (27).

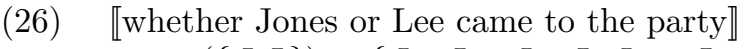

$$
=\operatorname{part}(\{J, L\})=\{J \cap L, \neg J \cap L, J \cap \neg L, \neg J \cap \neg L\}
$$

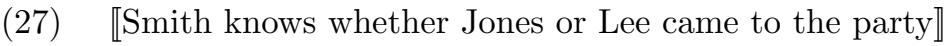

$$
=\left\{\lambda w:\left[\exists p^{\prime} \in(26)\left[w \in p^{\prime}\right]\right]\right. \text {. }
$$

$$
\left.\exists p \in(26)\left[w \in p \wedge \operatorname{JDOX}_{\mathbf{s}, w} \subseteq p \wedge \operatorname{strong}\left(p, \operatorname{JDOX}_{\mathbf{s}, w},(26)\right)\right]\right\}
$$

Thus, (27) predicts that (23) is true iff Smith has a justified true belief about the true cell in the partition in (26), just as in Groenendijk and Stokhof's (1984) partition semantics for wh-complements. Note that the condition on the strongest belief is here vacuous since cells in a partition do not contain each other. A purely disjunctive knowledge is not allowed since, if Smith believes that either $J$ or $L$ is true, but neither believes $J$ nor $L$ specifically, there is no proposition in (26) that Smith believes.

\section{Other Putative Gettier Cases}

In the literature, authors discuss other cases of putative Gettier problems which cannot be accounted for by the current analysis. One paradigm case is the fake barn case by Goldman (1976):

\footnotetext{
${ }^{7}$ It is interesting in this connection that Ciardelli et al. (2010) speculate that natural language declaratives in general involve their own version of non-inquisitive closure in (i), which is similar to my uni, in defining Inquisitive Semantics with Attentive Content.
}

(i) $! \varphi:=\varphi \vee \neg \neg \varphi$ 
(28) Situation: While Henry was driving through a certain country, he saw a building, and identified it as a barn. His sight is excellent and he is perfectly justified in identifying it as a barn. However, the country he was driving through was actually a strange country that has many fake barns. But, by accident, the building he saw was one of the few real barns. In fact, if he had seen a different one, he might have wrongly identified the fake barn as a real barn.

(29) Henry knows that what he saw was a barn.

The judgment that (29) is false in the situation in (28) cannot be accounted for by the current analysis since there is a true possibility in the denotation of the complement which is a strongest justified belief of Henry, namely that the particular building he saw was a barn.

Such cases are different from the standard cases discussed in the previous section in that they involve a skeptical hypothesis that is independent of the truth of the complement of know or the subject's belief state. In fact, as Kratzer (2002) notes, a case like (28-29) is judged less clearly as false if the the skepticism in the context is dubious or weaker. In contrast, the judgment of the falsity of the standard Gettier cases does not depend on the context independent both of the truth of the complement and of the subject's belief state. Thus, I claim that these other Gettier cases should be treated with a separate argument from the one accounting for the standard cases discussed in the previous section. More specifically, I argue that the skeptical hypotheses in the context of these cases raise the standard of belief justification, along the lines of Epistemic Contextualism (cf. e.g. DeRose 1992).

\section{Conclusions and Remarks on Other Attitude Verbs}

In this paper, I argued that the Gettier problem can be given a solution by analyzing know as operating on a set of alternative possibilities denoted by its declarative complement, using proposals in Alternative Semantics and Inquisitive Semantics. Also, we saw that this meaning for know can be easily extended to the case of interrogative complements. The difference between a declarative and an interrogative is that the former involves a closure under union, while the latter involves a partition. In sum, the Gettier problem provides support for the view that know always operates on a set of possibilities even when it combines with a declarative complement. Putting it differently, the problem motivates the view that 'knowing $p$ ' is to be able to resolve an issue raised by $p$.

Lastly, I below make a brief remark about how the selection restrictions of other attitude verbs can be accounted for. Under the current approach, the selection restrictions of attitude verbs such as believe and ask/wonder are accounted for in terms of the properties of the set of possibilities they select for. As for believe, I propose the following entry, which entails that the subject believes the union of the possibility set denoted by the complement.

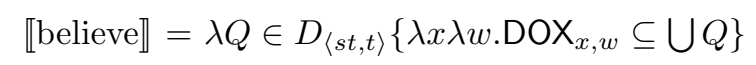


The entry in (30) is incompatible with a non-informative complement because it would derive a trivial entailment. This correctly accounts for the fact that believe does not take an interrogative complement since the union of a partition is equal to the universe. As for verbs such as ask or wonder, I claim that they require an inquisitive and non-informative complement. Therefore, these verbs cannot embed a declarative complement, which is either non-inquisitive or informative. I have to leave a further explanation of these restrictions for future research.

\section{References}

Alonso-Ovalle, Luis. 2006. Disjunction in Alternative Semantics. Ph.D. thesis, University of Massachusetts at Amherst.

Ciardelli, Ivano, Jeroen Groenendijk, and Floris Roelofsen. 2010. Information, issues, and attention. Ms., ILLC, University of Amsterdam.

DeRose, Keith. 1992. Contextualism and knowledge attributions. Philosophy and Phenomenological Research 52(4):913-929.

Gettier, Edmund. 1963. Is justified true belief knowledge? Analysis 23(6):121123.

Ginzburg, Jonathan. 1995. Resolving questions. Linguistics and Philosophy 18(5):459-527 (Part I) and 567-609 (Part II).

Goldman, Alvin. 1976. Discrimination and perceptual knowledge. Journal of Philosophy 73(20):771-791.

Groenendijk, Jeroen. 2009. Inquisitive semantics: Two possibilities for disjunction. In P. Bosch, D. Gabelaia, and J. Lang, eds., Seventh International Tbilisi Symposium on Language, Logic, and Computation. Springer.

Groenendijk, Jeroen and Floris Roelofsen. 2009. Inquisitive semantics and pragmatics. In J. M. Larrazabal and L. Zubeldia, eds., Meaning, Content, and Argument: Proceedings of the ILCLI International Workshop on Semantics, Pragmatics, and Rhetoric.

Groenendijk, Jeroen and Martin Stokhof. 1984. Studies on the Semantics of Questions and the Pragmatics of Answers. Ph.D. thesis, University of Amsterdam.

Hamblin, Charles L. 1973. Questions in Montague English. Foundations of Language 10(1):41-53.

Kamp, Hans. 1973. Free choice permission. Proceedings of the Aristotelian Society 74:57-74.

Karttunen, Lauri. 1977. Syntax and semantics of questions. Linguistics and Philosophy 1(1):3-44.

Kratzer, Angelika. 2002. Facts: Particulars or Information units? Linguistics and Philosophy 25(5-6):655-670.

Kratzer, Angelika and Junko Shimoyama. 2002. Indeterminate pronouns: The view from Japanese. In Y. Otsu, ed., The Proceedings of the Third Tokyo Conference on Psycholinguistics, 1-25. Tokyo: Hituji Shobo.

Ross, Alf. 1941. Imperatives and logic. Theoria 7(1):53-71.

Steup, Matthias. 2009. The analysis of knowledge. In E. N. Zalta, ed., Stanford Encyclopedia of Philosophy. The Metaphysics Research Lab and CSLI. 


\title{
Relating ERP-Effects to Theories of Belief Update and Combining Systems
}

\author{
Ralf Naumann \\ Institut für Sprache und Information \\ Universität Düsseldorf \\ Germany
}

\begin{abstract}
A major challenge for any attempt at combining linguistics with empirical neurophysiological data from brain research, like ERPs for example, is to give an answer to the question of how the gap between psycholinguistic and formal models of specific aspects of language on the one hand and the neural architecture underlying neurophysiological measures on the other can be bridged (Baggio et al. 2010). An interesting research program based on the extension of the event calculus in Van Lambalgen \& Hamm 2005 was launched in Baggio et al (2007, $2008,2010)$ where it was shown how a correlation between two known ERP-effects, the N400 and the LAN, and semantic phenomena like the progressive can be established. In this paper we will present an alternative formal theory which is based on the technique of combining systems and in which the dynamics of information change is separated from the more static aspects of knowledge representation. Using this multi-layered architecture, we hypothesize that the LAN is related to the process of updating a discourse model in the light of new information about changes in the world whereas the N400 concerns the more static aspects of situation models, e.g. the relation between events and persistent objects. ${ }^{1}$
\end{abstract}

Keywords: ERP, situation models, combining systems, aspect, belief revision

\section{Introduction}

A major obstacle for combining neuroscience and linguistics are the diverging ways in which the meaning and understanding of linguistic expressions (words) are defined. In theories of grounded cognition the meaning of a word is linked to (or even defined in terms of) past experiences and encounters with objects of the given kind. For example, the meaning of verbs like kick or lick, which refer to actions involving leg or mouth movements, is linked or identified with somatotopic activation patterns in corresponding motor cortices. Defining meaning in this way has the advantage of directly grounding the understanding and comprehension of language in the empirical evidence available to speakers.

\footnotetext{
${ }^{1}$ The research was supported by the German Science Foundation (DFG) funding the Collaborative Research Center 991 at the University of Düsseldorf.
} 
In addition to past experiences with objects of the given kind a second source of learning the meaning are distributional properties abstracted from the linguistic contexts in which the word occurs. This way of defining the meaning of words fits well into the conception of viewing the primary function of language as a set of processing instructions on how to arrive at a situation model for the state of affairs referred to by a sentence or a discourse (Zwaan \& Radvansky 1998).

This way of analyzing the meaning of verbs is almost orthogonal to the way they are analyzed in formal semantic theories. There verbs are classified e.g. as activities ( $r u n$ or lick), accomplishments (write) or semelfactives (kick). These classifications are based on properties of thematic relations like Theme and/or the notion of a nucleus structure. E.g. distinguishing incremental from constant Themes makes it possible to define accomplishment (write) and activity (push) verbs. The notion of a nucleus structure was introduced in Moens \& Steedman (1988). These structures are built in terms of three different parts: preparatory process, culmination and consequent state. Verbs differ with respect to the type of nucleus structure assigned to them. For example, accomplishments verbs like build have nuclei structures consisting of all three possible parts, whereas the nucleus structure of an activity verb like run consists only of a preparatory process.

One way of combining the two paradigms is the following. As emphasized in Zwaan \& Radvansky (1998:162), a situation model can be seen as a multidimensional system consisting of various dimensions like space, time, causation and objects. The various dimensions are linked to each other by particular relations guiding the flow of information between those dimensions. The different formal semantic theories can be taken as modeling a particular dimension of such a multidimensional system. For example, a nucleus structure defines the dynamic meaning component of a verb, i.e. the way an event of a given type (say a writing) evolves (or occurs) in space and time, and therefore the dimensions space and time in a situation model. The object dimension and its relation to the dynamic dimension is defined in terms of thematic relations like Actor or Theme. However, there are at least two missing links. First, the aspect of combining the different dimensions with each other (the flow of information) is missing in those semantic theories. Here I am going to propose using the framework of combining systems to fill this gap. Second, semantic theories must be related to neurophysiological and behavioural data from brain and cognitive science. In particular, the decompositions used in those theories (properties of thematic relations, the various constituents of a nucleus structure) don't seem to be directly related to the empirical evidence used by speakers to grasp the meaning of lexical items. A first step to bridge this gap has been undertaken in Van Lambalgen \& Hamm (2005) where the meaning of a sentence is seen as an algorithm (or a procedure) to construct a scenario (or, formally, a logic program) from the sentence. However, even in this approach it remains unclear how the proposed decomposition clauses are related to the empirical evidence about meanings available to comprehenders. The strategy proposed here to overcome this problem is based on the following empirical considerations. Events occur in time. This occurrence can be subdivided into 
different stages as described by a nucleus structure. Each stage of the development is characterized by (i) a subset of the objects involved in the event and (ii) particular activities and/or properties of those objects that are undertaken or affected during that stage. Given information about the type of event (verb), the stage the event is in (aspectual and temporal markers) and the role (thematic relation) of the object in the event, it is possible to infer (based on empirical knowledge of events of that type) the corresponding activities and/or properties and to built up the temporal dimension of the situation model in terms of them.

Two important questions that arise are: (i) How are situation models computed in the brain? and (ii) What is the relation between the various ERP components and the various dimensions of a situation model? Given that a situation model is multidimensional, certain effects may be related to particular dimensions and not to others. If this is true, Hagoort's (2003) thesis that those components are domain-specific must be extended to also include some form of dimension-specificity.

In the following section the experimental results of Baggio et al. (2008) are presented. In the third section an alternative framework is developed and compared to that of Baggio et al.

\section{The Progressive and left anterior negativity}

Using the formal semantic theory developed in Van Lambalgen \& Hamm (2005) and Hamm \& Van Lambalgen (2003) and based on ERP experiments, Baggio et al. $(2008,2010)$ present a unified account of the N400 and the LAN (left anterior negativity) by relating it to different semantic phenomena like the progressive and coercion. Similarly to other approaches, Baggio et al. link the N400 to the processing of semantic phenomena at the level of semantic (word) composition like priming (semantic relatedness) and the unification of variables. According to the analysis of the progressive in Hamm \& Van Lambalgen (2003), the meaning of a telic VP like write a letter is an algorithm (called a 'scenario'), implemented as a particular type of logic program from which the expression's reference, a minimal model of the scenario, can be derived via resolution (unification). For telic VPs such a scenario can be seen as a (simplified) plan to reach a particular goal. In the case of writing a letter this plan basically consists of the goal (the finished letter) and an activity to attain this goal, here writing (as opposed to say dictating). Since such a minimal model does not contain any information about obstacles (possibly) terminating the writing, it is possible to derive the obtaining of this goal at some later time using additional general constraints like the law of inertia. This conclusion concerning the existence of a goal state is defeasible. Now consider the sentences in (1).

(1) a. The girl was writing a letter when her friend spilled coffee on the paper.

b. The girl was writing a letter when her friend spilled coffee on the tablecloth. 
First, it was correctly predicted that a higher N400 effect occurred for tablecloth compared to paper. Baggio et al. explain this by the fact that in the context of a writing event (described in the main clause) the noun paper is semantically more expected as referring to the location where the letter comes into existence than the noun tablecloth. As a result, semantic unification is easier (proceeds more smoothly) for paper compared to tablecloth. Second, they (correctly) expected a higher LAN for the VP spill coffee on the paper compared to the VP with 'paper' replaced by tablecloth. This is explained by the fact that in the former case the minimal model derived for the main clause (MC) has to be recomputed due to the fact that the goal state (the completed letter) must be suppressed since the subordinate clause (SC) contains information about a terminating event. When this part of the sentence is processed by a speaker there is therefore a higher processing load which is neurophysiologically reflected in the higher sustained anterior negativity. By contrast, for tablecloth the minimal model is simply extended in a monotonic way. Finally, they correctly expected a correlation between higher LAN-amplitudes and speakers asserting that the letter was not finished (probe sentence for 1a).

\section{An alternative approach}

Following Zwaan \& Radvansky (1998), we assume that the successful understanding of a sentence consists in the ability to construct a situation model for the situation (or state of affairs) described by it. A situation model is a multidimensional system in which various dimensions are combined with each other. This multidimensional character will be modeled by using the technique of combining systems (Blackburn \& de Rijke 1997, Finger \& Gabbay 1992). A dimension is modeled as an ontology together with a family of $n$-ary relations. In the present context three such ontologies are distinguished: time points, eventualities and persistent objects, which are defined as follows: (i) Object structure $\underline{\mathbf{O}}=<O,\left\{A_{a}\right\}_{a \in A t t r}>$ with $O$ a (non-empty) set of persistent objects (like tables and chairs) and each $A_{a}$ is an $n$-ary relation on $O$; (ii) Eventuality structure $\underline{\mathbf{E}}=<E,\left\{P_{v}\right\}_{v \in \text { Verb }}>$ where $E$ is a (non-empty) set of event tokens (or event occurrences) and each $P_{v}$ is a unary relation on $E$, which is an event type and its elements are event occurrences or event tokens like runnings $(v=$ run) or writings $(v=$ write) and (iii) $\underline{\mathbf{T}}=<T,<_{T}>$ is a flow of time with $T$ a (non-empty) set of time points and $<_{T}$ a linear and atomic ordering.

The definition of the flow of information (i.e. the connections) between the various dimensions is based on the following considerations. Each event token $e$ occurs on a particular interval and is related to persistent objects from $\underline{O}$ that bear a particular relation to it like Actor or Theme. The first relation between $\underline{\mathbf{T}}$ and $\underline{\mathbf{E}}$ is defined by the function $\tau$ from $E$ to $\wp(T)$, which assigns to each $e \in E$ its run-time and which is required to be a convex set. In terms of $\tau$ the functions $P P, C u l$ and $C S$ are defined, mapping an event $e$ to the interval corresponding to the $\mathrm{PP}, \mathrm{Cul}$ (a singleton) and CS, respectively. This connection is called the 
temporal-causal dimension of a situation model. The relation between $\underline{\mathbf{E}}$ and $\underline{\mathbf{O}}$ is modeled by a set $\left\{R_{t r}\right\}_{t r \in T R}$ of (functional) thematic relations and is called the static dimension of a situation model. Events and actions bring about changes with respect to time-dependent properties of those persistent objects participating in them (i.e. which bear one of the thematic relations defined for the event/action). By way of illustration consider an event of a girl writing a letter. Such an event involves a goal (the completed letter) and an activity (writing) to bring about this goal. Since the writing involves an actor, it will be assumed that there is a state the writer is in when doing the writing. This state will be encoded by statewriting $(x)$, and be called a state-fluent. Formulas like completed(y), encoding the goal, are called goal formulas. During the occurrence of the event of writing the formulas are evaluated in a characteristic way. Whereas state $_{\text {writing }}$ (girl) is true during the whole event (except, possibly, at the beginning and the end point), completed(letter) is only true at the end point of the event and, in addition, after the event occurred. This relation is captured as follows. Let Attr $v$ be the restriction of Attr to those state-fluents and goal formulas related to event tokens from $P_{v}$. Then $Z$ is a function that assigns to each event $e$ and time point $t \in \tau(e)$ an object structure $\underline{O^{\prime}}=<O^{\prime},\left\{A_{a_{e}^{\prime}}^{\prime}\right\}_{a^{\prime} \in A t t r_{v}}>$ where $O^{\prime}$ is a subset of the objects involved in $e$ and $\left\{A_{a_{e}^{\prime}}^{\prime}\right\}_{a^{\prime} \in A_{t t r}}$ is the restriction of $\left\{A_{a^{\prime}}^{\prime}\right\}_{a^{\prime} \in A t t r v}$ to $O^{\prime}$. This structure is called the dynamic submodel of $e$ at $t$. In terms of $Z$ a function $Z^{*}$ is defined that assigns to an event $e$ a sequence of pairs $\left(t, M_{t}\right)$ that describes the evolution of the event $e$. How is the function $Z$ computed in the brain? Events evolve (develop) in time. This evolution can be subdivided into different stages as described, e.g. by a nucleus structure. Each stage of the development is characterized by (a) a subset of the objects involved in the event and (b) particular activities and/or properties of those objects that are undertaken or affected during that stage. So given information about (i) the type of the event (supplied by the verb), (ii) the stage the event is in (supplied by aspectual and temporal markers like progressive) and (iii) the role of the object (extracted from e.g. morphological information and/or word order), it is possible to retrieve, using empirically grounded knowledge about events and objects of those kinds in that particular role, appropriate activities and/or properties of the corresponding object for that stage or those stages that have already occurred up to the stage of the event described by the sentence. In our framework the above relationship is captured by axioms like those in (2) for write (see the end of the paper for reasons why the consequents are given in a very schematic way).

(2) $\quad$ a. Actor $(e)=x \wedge P P(e)=i \wedge P_{\text {write }}(e) \rightarrow$ state $_{\text {writing }}(x)(i)$

$$
\text { b. Theme }(e)=y \wedge P P(e)=i \wedge P_{\text {write }}(e) \rightarrow \text { state }_{\text {of_creation }}(y)(i)
$$$$
\text { c. Theme }(e)=y \wedge C u l(e)=\{t\} \wedge P_{\text {write }}(e) \rightarrow \operatorname{completed}(y)(\{t\})
$$$$
\text { d. Theme }(e)=y \wedge C S(e)=i^{\prime} \wedge P_{\text {write }}(e) \rightarrow \operatorname{completed}(y)\left(i^{\prime}\right)
$$

In these axioms information about the static dimension (thematic relation) and the temporal-causal dimension (second conjunct in the an- 
tecedent) together with type information about the event is used to infer information about the dynamic dimension. For a progressive sentence, only the first two axioms (2a) and (2b) are used, since the event is described as ongoing. By contrast, for a sentence in the simple past, the third axiom is used too and for a sentence in the (present) perfect, the antecedents of all four axioms are satisfied.

Empirical evidence for axioms of this kind comes from experiments that show that aspect and other grammatical devices influence not only the way an object is simulated but also which objects are focused (Madden \& Therriault 2008, Ferretti et al. 2009, Bergen \& Wheeler 2010). For example, in John is closing the drawer (ongoing event) the mental simulation focuses on the act of pushing the drawer undertaken by the Actor John whereas in John has closed the drawer the simulation is focused on the goal state and therefore on the Theme in that state, i.e. the closed drawer. The way an object is simulated is therefore closely related to its role in the event and to its properties affected by the event. On this view, decompositional predicates like those used in the consequents of the axioms in (2) are directly related to the way objects are simulated in the brain while computing a situation model whereas in the antecedents only information is used that can be directly extracted from the surface structure of the sentence.

An important aspect that is missing so far is non-determinism. If an event token occurs, it is in general not uniquely determined how the occurrence continues. For example, if a girl is writing a letter, she may finish it or not. Formally this non-determinism is modeled in the following way. Each pair $\left(t, M_{t}\right)$ is related to a set of (linearly) ordered sequences of pairs $\left(t^{\prime}, M_{t^{\prime}}\right)$ such that (i) each sequence is a possible way of how the event occurring at $t$ evolves after $\mathrm{t}$ and (ii) this set is ordered by a relation $\leq_{t}$, which is required to be at least reflexive and transitive. When taking both conditions together, one gets, for a given $t$, a computation tree (with root $t$ ) where each outgoing arc is assigned a weight, giving the degree of expectancy that this transition will occur (though these weights are not represented in the framework sketched above). This ordering reflects the expectancy or the priming a comprehender uses when processing a sentences with that verb. One basis of such an ordering is world knowledge about events of the given type, say writings. Another source is linguistic knowledge, in particular knowledge about the contexts in which the verb preferably occurs, e.g. whether the verb can be modified by temporal adverbials like in $x$ time or for $x$ time (' $>$ ' means that this context is more primed).

(3) a. John wrote the letter in an hour $>$ John wrote the letter for an hour.

b. John ran for ten minutes $>$ John ran in ten minutes.

Verbs which prime $i n$-adverbials compared to for-adverbials are normally related to goals and/or results. E.g. writing usually involves a particular object that is intended to be brought into existence, like a letter or an article for instance. By contrast, verbs priming for-adverbials are usually used to describe events that have no intended goal like running 
for example. From this it does not follow that write cannot be used without a goal, as examples like John wrote show, and run can be used as involving a goal: Bill ran a mile/to the station. However, these contexts are less primed. Posible orderings (primings) for write and run (or push) are: (i) write: $\mathrm{PP} \mathrm{Cul} \mathrm{CS}>\mathrm{PP}$ and (ii) run: $\mathrm{PP}>\mathrm{PP} \mathrm{Cul}(\mathrm{CS}$ ).

How is a situation model computed in the framework sketched above? It is assumed that this computation is based on the following two principles: processing (i) proceeds on a word-by-word basis and (ii) is done in accordance with Hagoort's Immediacy Hypothesis (IA): all sorts of information available to the comprehender is immediately used in parallel in order to arrive at a meaningful interpretation.

When a word is processed, information is added at different dimensions. This information will in general be underspecified. In particular, this will be the case if information is added to a dimension that is the combination of basic dimensions like the static or the dynamic dimension because then information from all dimensions involved is necessary to arrive at fully specified information. For instance, when processing wrote in The girl wrote a letter, one gets the following updates: (i) type information: the event is a writing (basic dimension $\underline{E}$ ); (ii) unification $\operatorname{Actor}(e)=\operatorname{girl}$ (static dimension) since the subject has already been processed. By contrast, information about the Theme is still underspecified: Theme $(e)=y$; (iii) 'triggering' the corresponding axioms for the nucleus structure (dynamic dimension). In the last section it will be shown how the data from Baggio et al. can be explained in our framework.

\subsection{The girl and the coffee: an alternative interpretation}

According to the Baggio et al. interpretation, the higher LAN is explained as follows. A formula that is true in the minimal model after processing the MC has to be retracted when this model is updated with the information contained in the (disabling) SC (revision of the model, non-monotonic update). This is formulated in the following hypothesis.

The Non-Monotonicity Hypothesis (NMH)

A higher LAN is expected when the current model has to be recomputed (revised) if new information is added that is incompatible with information in the current model. The amplitude of the LAN depends on the extent to which the model has to be recomputed.

One very simple method of calculating the measure of change to a model (or database) consists in counting the number of formulas that have to be revised (see Katsuno \& Mendelzon 1992). Consider again the disabling condition (1a). The spilling event causally interferes with the ongoing event of writing referred to in the $\mathrm{MC}$, bringing it (at least temporarily) to an end. Let this information be encoded by the formula $\operatorname{clip}\left(e_{\text {spill }}, e_{\text {write }}\right)$, which is added to the temporal-causal dimension of the current model. Integrating this information in the model does not effect the valuation of a formula in the dynamic submodel at $t_{\text {spill }}$. However, the girl is no longer simulated as writing a letter after $t_{\text {spill }}$ so that the formula state $_{\text {writing }}($ girl) will not be an element of the dynamic submodel 
at times $t^{\prime}>t_{\text {spill }}$. Similarly, the writing event is no longer described as occurring and the letter is no longer taken as coming into existence, yielding a total of three revisions. Of course, more sophisticated methods may be necessary.

The Non-Monotonicity Hypothesis not only applies to cases involving a goal state but to any non-monotonic change affecting the current model, in particular scenarios like those in (4) where a goal state is not part of the most expected nucleus structure, but the SC contains a disabling condition.

(4) The girl was pushing a cart towards the barn when she was hit by a car.

In (4) no goal state is involved because push a cart is atelic and the PP does not introduce a culmination. Therefore, no higher LAN is expected to occur according to the Baggio et al. analysis. By contrast, applying the $\mathrm{NMH}$, the hitting terminates the pushing, so that the girl is no longer simulated as being in a pushing state. The difference between (1a) and (4) consists in the fact that for the latter the number of revision is expected to be lower because no object is coming into existence so that there are only two revisions. Thus, the amplitude of the LAN for (1a) is expected to be higher compared to that for (4) using the simple counting criterion from above.

In our approach, the following, alternative explanation for the examples in (1) and (4) is possible. After processing the MC, the event is described as ongoing. For an accomplishment verb like write, this means that different ways of how the event continues are still open: the writing (PP) is continued until the culmination is reached (most primed); (ii) the PP is continued but the culmination is not reached due to an interruption, leaving the eventual attainment of the goal open and (iii) the writing is definitely terminated. Thus, no unique nucleus structure has been determined up to that point, in particular it is not determined whether the culmination or goal state (e.g. a completed letter) is eventually attained or not. When a disabling condition is encountered, the ordering on the possible continuations is changed. Several ways of how a new ordering is constructed are possible. At one extreme, the current most expected continuation is simply discarded. This will likely be the case when the $\mathrm{MC}$ is continued by when she got a heart attack or when she died (case (iii) above). An alternative reordering simply switches the current ordering between the different continuations so that the currently most expected ordering is still available but only demoted. This is likely to be the case for the example (1a) and case (ii) above because it is still possible that the girl (re)continues writing the letter on another sheet of paper. Changing the ordering of the possible continuations has the effect of changing the most expected scenario. This is formulated in the hypothesis below.

Reordering of Nuclei Structures Hypothesis (RH)

A higher LAN is expected whenever the ordering on the set of continuations at a point $t$ of the temporal-causal dimension is changed due to a disabling condition. 
On the RH, a reordering is also expected for (4). Recall that a verb like push is associated with a nucleus structure of type PP Cul CS, though this structure is less primed. Encountering the disabling condition in the SC of (4), this continuation has to be discarded. How is the cost of reordering the set of nuclei structures calculated? A very simple way of calculating the cost of a reordering operation consists in counting the number of reorderings. For example, completely discarding the most expected nucleus structure should be more costly than discarding a less primed nucleus structure. An answer to this question depends both on the exact way of how the ordering is defined and, of course, on experimental findings related to processing sentences in the brain. According to the $\mathrm{RH}$, the higher LAN in the Baggio et al. data is not due to a change in the valuation of (atomic) formulas. In the disabling condition the goal state is never part of the model because it is never asserted to have been attained. This state is, however, part of the most expected nucleus structure (scenario) and therefore expresses the generalization (expectation) that e.g. writing a letter normally leads to a completed letter. The higher LAN is rather an effect of a change wrt. the expectation of how the situation will dynamically evolve. A higher LAN can therefore be taken as the dynamic counterpart of the N400. Whereas the N400 is related to expectancy at the static dimension of a situation model, the LAN is related to its dynamic dimension.

What empirical evidence can be used to decide between the Non-Monotonicity Hypothesis and the Reordering Hypothesis (of course, they need not exclude each other)? In order to give a tentative answer to this question, we first have to look at the relation between formal models of the event calculus and neural networks in the framework used by Baggio et al. In the variant of the event calculus developed in Van Lambalgen \& Hamm (2005) the scenario associated with a clause (or sentence) has a minimal model. Minimal models can be regarded as the stable states of associated neural networks. Computing the minimal model for a clause, say the MC in the examples in (1), corresponds to the neural network settling into a stable state. Processing the next clause, say the SC in (1), the network settles into another stable state. The difference between (1a) and (1b), then, is that the transition from the stable state the network is in after processing the MC to the stable state after processing the SC is more costly in the disabling situation (1a) compared to the neutral situation (1b) because in the former but not in the latter case activation patterns across some units related to the goal state that were previously active have to be readjusted in the sense that the weights (or connection strengths) between these units are changed. This explanation of the relation between formal model and neural networks is based on two assumptions: (i) there is one global neural network that goes from one stable state to another during processing the different clauses of a sentence or discourse and (ii) the amplitude of the LAN is dependent on the extent to which connection strengths have to be readjusted.

In our multidimensional framework each nucleus structure associated with a verb has a minimal model for the dynamic dimension of the situation model. If the ordering on the nuclei structures is changed due to additional information (a different scenario becomes most expected), 
the connection strengths in the corresponding neural network have to be readjusted. It is this readjustment that triggers the higher LAN. On this view there is both a minimal model for the dynamic dimension of the SC and the MC (i.e. there are two separate minimal models, one for each dynamic dimension). At this point we apply a distinction introduced in Zwaan \& Radvansky (1998). They distinguish the integrated and the current model. Whereas the integrated model is the (global) model built up by the previous discourse, the current model is the model computed for the sentence that is being processed. After the current model has been computed, the integrated model is updated with this current model, resulting in a new integrated model. The important point is that if the minimal model is redefined, this has no effect on the valuation of formulas at the dynamic dimension in the current model because the valuation only concerns state-fluents and goal formulas. For example, if the MC describes an event as ongoing, only axioms like those in (2a) and (2b) are triggered. Their consequents remain true, independently of any disabling condition which is added while processing the SC. For the consequents of those axioms which are related to the $\mathrm{Cul}$ and the $\mathrm{CS}$, no decision is possible because no information about the corresponding state is available. As a consequence, neither the consequent nor its negation are part of the current model.

On this view the deactivation of units simulating the girl as being in a writing state is not the cause of a higher LAN. First, no formulas have to be retracted since the girl is not simulated as not being writing. More important, however, is the following argument. In a recent ERP study Willems et al. (2009) found a neural dissociation between action word understanding and mental imagery. Participants were presented with verbs related to hand actions (e.g. throw) and nonmanual actions (e.g. kneel). They either read these verbs (lexical decision task LD) or actively imagined performing the actions referred to by these verbs (imagery task IM). During the LD task effector-specific activation in premotor but not in primary cortex was elicited whereas in the IM task effector-specific activation in both types of cortices was found. The authors explain these findings by making a distinction between implicit mental simulation, which is related to prediction, and explicit mental imagery, which is reflective involving a covert enactment of the corresponding action.

Relating this distinction to our framework, one gets that computing (building up) the dynamic dimension of a siuation model corresponds to action verb understanding and therefore to implicit motor simulation in premotor (as opposed to primary motor) cortex. At this level (action) verbs are classified according to their associated ordered sets of nuclei structures. Such a classification is quite different from a classification in terms of explicit mental imagery. For example although write a letter and grasp a needle have identical (or at least very similar) nuclei structures, their corresponding mental imagery widely differ. Thus, although write a letter and grasp a needle differ with respect to their goal formulas (say, completed(letter) and be_grasped(needle)), these differences only show up when it comes to explicit mental imagery. And even for a verb like grasp, the mental imagery depends on the kind of object to be 
grasped: grasp a needle vs. grasp a barbell. Conversely, although stroke and write both (normally) involve hand movement, they are related to different ordered sets of nuclei structures. What is important for action verb understanding, therefore, is the set of ordered nuclei structures, i.e., the different ways an event can dynamically develop. On this view the consequents in dynamic axioms like those in (2) can be seen as amodal and highly abstract, symbolic descriptions of the concrete modal motor imagery that get replaced when implicit simulation ends and explicit imagery begins (see Willems et al. 2009:2397). In our framework those abstract descriptions are the consequents in dynamic axioms, which correspond to transitions in dynamic submodels. When simulation ends and imagery begins, an operation like transition refinement (see e.g. Blackburn \& de Rijke 1997) takes place which replaces these transitions with (or cues them to) a modal motor imagery in primary motor cortex.

\section{References}

1. Baggio, G. et al. (2008). Computing and recomputing discourse models, an ERP study, Journal of Memory and Language 59, 36-53.

2. Baggio, G. et al. (2010). Coercion and compositionality, Journal of Cognitive Neuroscience 22, 2131-2140.

3. Baggio, G. and M. van Lambalgen (2007). The processing consequences of the imperfective paradox, Journal of Semantics 24, 307330 .

4. Bergen, B. \& K. Wheeler (2010). Grammatical aspect and mental simulation. Brain \& Language 112, 150-158.

5. Blackburn, P. and M. de Rijke (1997). Zooming in, zooming out, Journal of Logic, Language, and Information, 6, 5-31.

6. Ferretti, T. R., H. Rohde, A. Kehler and M. Crutchley (2009). Verb aspect, event structure, and coreferential processing. Journal of Memory and Language, 61, 191-205.

7. Finger, M. and D. Gabbay (1992). Adding a temporal dimension to a logic system. J. of Logic Language and Information, 1, 203-233.

8. Hamm, F. and M. Van Lambalgen (2003). Event calculus, nominalisation, and the progressive, Linguistics and Philosophy 26, 381-458.

9. Katsuno, H. and A. Mendelzon (1991). On the difference between updating a knowledge database and revising it. KR-91, 387-394, Cambridge.

10. Madden, C. and D. Therriault (2009). Verb aspect and perceptual stimulations, The Quarterly Journal of Experimental Psychology, 62:7, 1294-1303.

11. Moens, M. and M. Steedman (1988). Temporal ontology and temporal reference, Computational Linguistics, 14:2, 15-28.

12. Van Lambalgen, M. and F. Hamm (2005). The proper treatment of events, Oxford: Blackwell.

13. Willems, R.M. et al. 2009. Neural dissociations between action verb understanding and motor imagery, Journal of Cognitive Neuroscience 22:10, 2387-2400.

14. Zwaan, R.A. and G.A. Radvansky (1998). Situation models in language comprehension and memory, Psychological Bulletin, 123:2, 162-185. 


\title{
Underspecified representations of scope ambiguity?
}

\author{
Janina Radó \& Oliver Bott \\ SFB 833, University of Tübingen
}

\section{Introduction}

Underspecified representations are commonly used to model semantic ambiguity, and scope ambiguity in particular. They offer an elegant way to capture the interpretation possibilities in a single compact representation, thus avoiding a combinatorial explosion of readings as the number of operators increases (see [3] for a critical discussion). This property has repeatedly been claimed to make them cognitively plausible: since only one representation is constructed, scopeambiguous sentences can be interpreted in a fast and efficient way.

There is indeed evidence that perceivers only specify the interpretation of the sentence as much as necessary for a particular task (e.g. pronouns are not automatically resolved, cf. [6], [11]). A similar claim has been made with respect to quantifier interpretation: scope is only resolved when necessary, e.g. when disambiguation is encountered, and may remain completely underspecified in some cases (see [11] for an example). Sanford and Sturt use the lack of scope preferences in some constructions to argue for underspecified scope representations.

It is important to note that the connection between underspecification and shallow processing is only indirect. Comprehenders often do not compute a representation that is detailed, complete, and accurate with respect to the input, but only one that is 'good enough' (see [4]). They process only as deeply as necessary under the circumstances and may end up with only a partial representation if that is sufficient for the task at hand rather than constructing a single connected representation (which may or may not be fully specified) for the complete sentence. Underspecified representations, on the other hand, are complete compact representations encoding all interpretation possibilities. They thus require deep processing but may not result in a specified representation (e.g. of scope), if there is no disambiguating information.

Intuitively, some scope readings are easier to get than others. This is also supported by psycholinguistic findings; for instance, [8] report a preference for the wide-scope interpretation of the second quantifier in the inverse linking construction in (1):

(1) George has a photograph of every admiral.

Such graded preferences have been captured in underspecification accounts by postulating weighted dominance constraints (e.g. [7]), which make some representations cheaper and easier to derive than others. The question now is whether 
the different readings are computed (and ranked) automatically, or only when the task e.g., a decision task requires it, as underspecification theory would have it. As it turns out, existing evidence does not allow us to answer this question (cf. [2]). For instance, [5] examined eye movements while perceivers read sentences like (2) (see also [10]) in order to find evidence for early disambiguation of quantifier scope.

(2) a. The celebrity gave a reporter from the newspaper every in-depth interview, but the reporter(s) was/were not very interested.

b. The celebrity gave every reporter from the newspaper an in-depth interview, but the interview(s) was/were not very interesting.

They report longer total reading times at the second quantifier in (2-a) than in (2-b), and interpret it as evidence for a scope conflict arising at the second quantifier in (2-a): linear order and grammatical role favor the wide-scope existential reading, whereas inherent properties of the quantifier support the wide-scope universal interpretation. In (2-b) all factors point to the wide-scope existential reading, thus processing is easier. This is reflected in off-line ratings as well: the $\exists \forall$ reading is accepted less in (2-a) than in (2-b). Crucially, however, both the reading time effects and the scope preferences are late effects; the scope conflict in (2-a) does not have any measurable influence on first-pass reading times. The results thus do not exclude the possibility that perceivers only computed a fully specified scope interpretation when they read the disambiguating information in the second clause.

To test the cognitive plausibility of scope underspecification we need to use a task that guarantees deep processing and look for evidence for scope interaction before disambiguating information is present. We designed a combined self-paced reading/verification experiment that we think fits the bill. Participants first read doubly quantified sentences and then had to decide whether the sentence matched a scope disambiguating card display. The sentences in the reading task exhibited a scope conflict between the inherent properties of the quantifiers and the construction they occurred in. Crucially, disambiguation was not available during the reading phase, so any reading time effects could be attributed to on-line resolution of scope relations. Moreover, participants were instructed to be accommodating and accept the picture if the corresponding reading was available at all. This way we hoped to encourage them to maintain an underspecified interpretation as long as possible.

\section{Methods}

Materials: We tested German inverse linking constructions and manipulated the quantifier in the embedded position. One type of complex quantifiers was built of the determiners genau ein (exactly one) and alle (all) as illustrated in the sample item in (3-a), whereas the other type embedded distributive jeder (each) as in $(3-b)$. The asterisks indicate segmentation in self-paced reading. 
a. Genau* ein Tier* auf* allen* Karten* ist* ein Affe.

Exactly one animal on all cards is a monkey.

b. Genau* ein Tier* auf* jeder* Karte* ist* ein Affe.

Exactly one animal on each card is a monkey.

Condition (3-a) exemplifies a case of scope conflict between quantifiers in the same DP: the inverse linking construction strongly biases towards inverse scope, but since the non-distributive alle (all) prefers not to outscope the first quantifier we expected competition between readings in the all conditions. This is different in (3-b) with jeder (each). Here, both factors point in the same direction therefore each should be interpreted with wide scope without any difficulty. As for the final interpretation, scope conflict should lead to fewer inverse interpretations in the all than in the each conditions.

In addition, we also tested doubly quantified sentences in which the quantifiers appeared in a non-embedded configuration.

$$
\begin{aligned}
& \text { a. Genau* ein Affe* ist* auf* allen* Karten* } \mathrm{zu}^{*} \text { finden. } \\
& \text { Exactly one monkey is on all cards to find. } \\
& \text { b. Genau* ein Affe* ist* auf* jeder* Karte* } \text { zu}^{*} \text { finden. } \\
& \text { Exactly one monkey is on each card to find. }
\end{aligned}
$$

Intuitively, the conditions (4) show the same contrast as (3), thus we expect the same pattern of interpretations in both construction types. In addition, comparing the DP- and the sentence conditions we can test when scope computation takes place if the initial interpretation is not underspecified. We will elaborate this point in the Predictions.

Each of the doubly quantified conditions (3) and (4) was paired with two disambiguating card displays yielding eight doubly quantified conditions in a 2 $\mathrm{x} 2 \mathrm{x} 2$ (construction $\mathrm{x}$ quantifier $\mathrm{x}$ picture) factorial design. The linear $\exists ! \forall$ card displays had the same object on all three cards, but the second card contained an additional object of the same category. Figure $1 \mathrm{a}$ is an example. The inverse $\forall \exists$ ! card displays had exactly one object of the relevant kind on each card, but different ones (cf. Figure 1b). Again, the second card provided the disambiguating information.

To control for potential differences in lexical processing between all and each we included the controls in (5). The controls were always paired with a card display that had the same object on all three cards (cf. Figure 1c).

$$
\begin{aligned}
& \text { a. Auf* allen* Karten* ist* ein Schimpanse. } \\
& \text { On all cards is a chimpanzee. } \\
& \text { b. Auf* jeder* Karte* ist* ein Schimpanse. } \\
& \text { On each card is a chimpanzee. }
\end{aligned}
$$

In total, this yielded ten conditions and we constructed 60 experimental items (ie. sentence/picture sets) in each of them. Additionally, we created 80 fillers which served several purposes. First, we made sure that all sorts of quantifiers would appear in the experiment. Second, the fillers varied in structure and in 


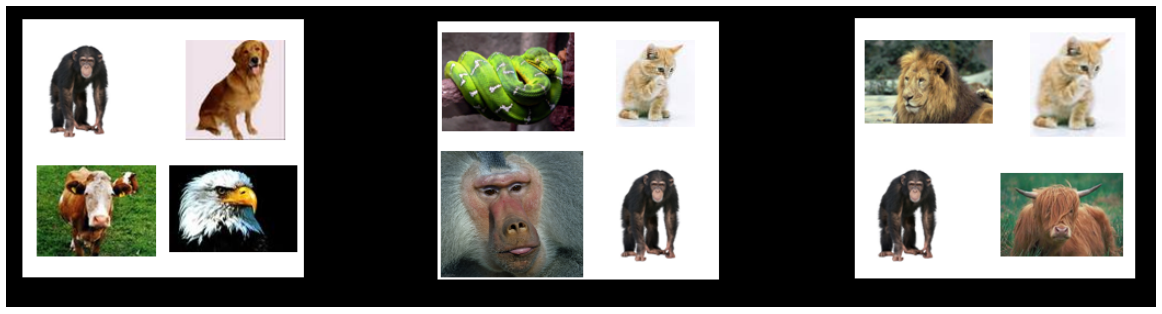

(a) $\exists ! \forall$ card display

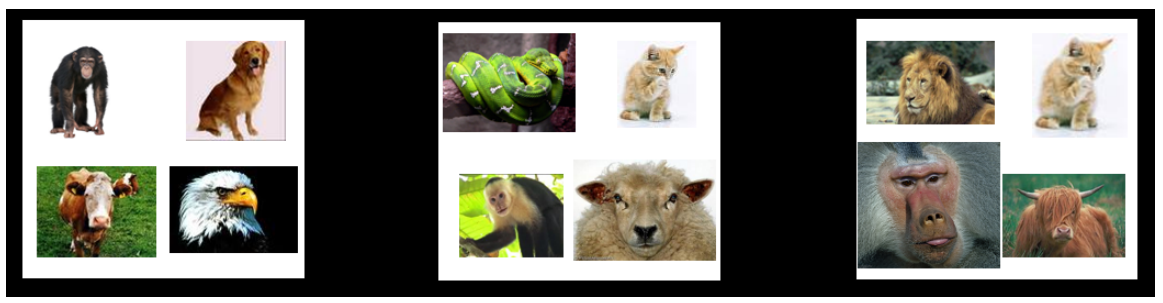

(b) $\forall \exists$ ! card display

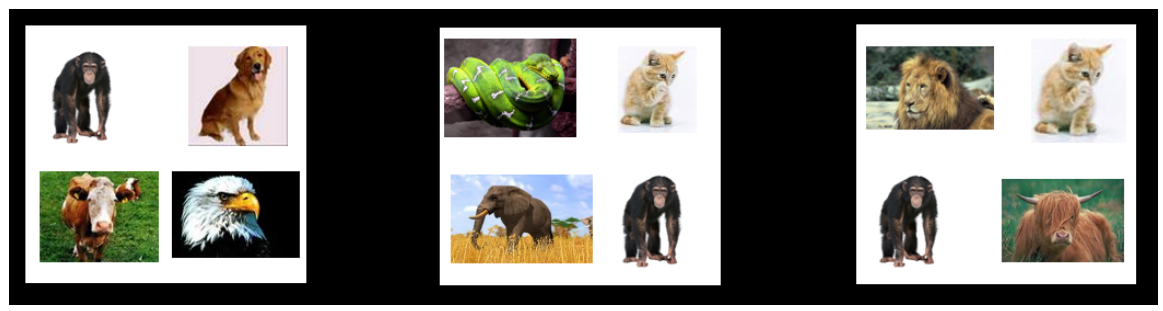

(c) control conditions

Fig. 1. Fully uncovered card displays for the sample item. Figure 1a is only compatible with wide scope of exactly one ( $\exists$ !). Figure $1 \mathrm{~b}$ disambiguates towards wide scope of the universal quantifier. Figure 1c is the card display presented with the control conditions. In the experiment, card displays were uncovered card by card from left to right.

a number of cases presented crucial information at the sentence final segment (eg. A drill can be found on all cards in their upper half). Third, a number of fillers had pictures that required accommodating interpretations, ie. a total of three objects for einige (a few). Finally, 40 of the fillers were clearly false to ensure that on a reasonable proportion of trials participants had to reject the card display. We took the experimental items and the fillers and constructed 10 lists according to a latin square.

Predictions: Assuming underspecification with weighted constraints, the quantifier manipulation should have no effect on the reading times. We do expect differences at the disambiguating card (card 2), however, since disambiguation should force scope resolution. 


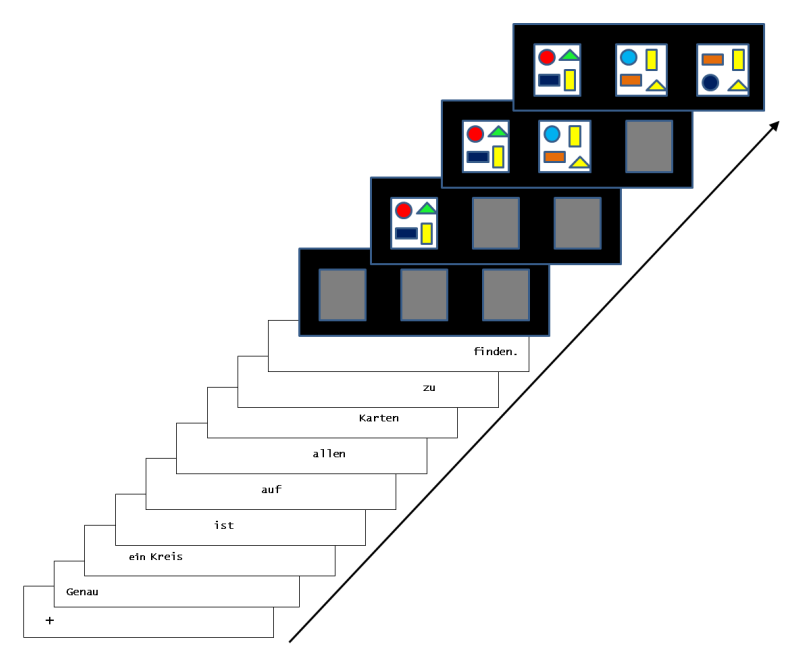

Fig. 2. Sample trial ('Exactly one circle can be found on all cards')

By contrast if scope is interpreted automatically, i.e. even without disambiguation, then the scope conflict should lead to processing difficulty already before the second card. In fact, we may find processing effects at the earliest point where scope computation may take place, i.e. at the second quantifier. We will dub this immediate full interpretation.

Finally, it is conceivable that semantic interpretation lags behind syntactic processing. At least some semantic interpretation processes require domains larger than individual words [1]. The same may hold for quantifier scope as well, especially as thematic roles seem to be one factor in determining the scope needs of a quantifier [9]. Then full scope interpretation would only be expected to take place once the verb and all of its arguments have been received. We will call this the minimal domain account. Under this view scope interpretation should be possible at the second quantifier in the sentence conditions in (4), but scope would remain underspecified until the lexical verb in the DP conditions in (3). Note that this still assumes the automatic computation of scope relations as soon as all relevant information is available.

To sum up, all three approaches predict a quantifier effect at the disambiguating card. In addition, under immediate full interpretation and the minimal domain approach we also expect reading time differences either at the second quantifier, or at least when the verb and all its arguments have been received. Thus the pattern of data in the inverse linking conditions will be crucial to distinguish between these two accounts. 
Procedure and Participants: Participants' task was to first read a sentence phrase by phrase employing non-cumulative self-paced reading with a moving window display. Segments outside the presentation window were covered by replacing all characters including punctuation marks with empty spaces. This was done to ensure that participants would not know how much material was yet to come. After reading the final segment the sentence disappeared from the screen and a layout of cards was presented. Participants had to uncover the cards one by one and for each card decide whether the sentence is already true or false or whether more information is needed and in that case move on to the next card. A sample trial is illustrated in Figure 2.

An experimental session started with written instructions followed by a practice session of 10 trials. In the instructions, we emphasized that whenever a picture was compatible with a sentence (even in case the interpretation is hard to get) participants should judge "yes, true". Then followed the experiment in a single block with an individually randomized presentation order for each participant. An experimental session took approximately 30 minutes. 40 participants (mean age 25.9; 31 female) from Tübingen University were paid 5 Euro for their participation.

\section{Results and Discussion}

Judgments: Table 1 presents the mean judgments in the ten conditions. Across the board, the linear reading was only accepted $17.2 \%$ of the time whereas the inverse conditions were on average accepted $85.1 \%$ of the time. However, acceptance in the linear conditions was $9.8 \%$ higher than in the false fillers which were only accepted $7.4 \%$ of the time indicating that linear scope, even though highly dispreferred, was still possible. Besides this very strong general preference for inverse scope, the distribution of readings showed a clear contrast between all and each. The inverse conditions were accepted $77.5 \%$ in the all sentence condition, $90.4 \%$ in the each sentence condition, $81.2 \%$ in the all DP condition and $91.3 \%$ in the each DP condition. We analyzed the judgments in the inverse linking and sentence constructions in a logit mixed effects model analysis including the fixed effects of construction, quantifier and reading and the random intercepts of participants and items. The analysis revealed significant fixed effects of reading $(z=-13.48 ; p<.01)$ and of quantifier $(z=3.15 ; p<.01)$. Besides a marginally reliable interaction between quantifier and reading $(z=-1.67 ; p=.09)$ no other effects reached significance (all $p \geq .29$ ). To further break down this interaction we computed separate logit mixed effects model analyses for the linear and the inverse scope conditions. While the linear scope conditions didn't differ significantly from each other (all $p \geq .23$ ), the analysis of the inverse conditions revealed a significant effect of quantifier $(z=3.44 ; p<.01)$. Taken together, the judgments provide evidence for a clear all/each contrast. Whereas each very strongly biases towards inverse scope, the bias is weaker with all. On the other hand, the complete lack of effects involving the factor construction suggests that the two constructions had comparable scope distributions. 


\begin{tabular}{lcc}
\hline \hline & \multicolumn{2}{c}{ Universal Quantifier } \\
\cline { 2 - 3 } & all & each \\
\hline Inverse Linking $\exists ! \forall$ & $15.4(2.3)$ & $19.2(2.5)$ \\
Inverse Linking $\forall \exists !$ & $81.2(2.5)$ & $91.3(1.8)$ \\
Sentence $\exists ! \forall$ & $16.3(2.4)$ & $17.5(2.5)$ \\
Sentence $\forall \exists !$ & $77.5(2.7)$ & $90.4(1.9)$ \\
Control & $95.8(1.3)$ & $97.1(1.1)$ \\
\hline \hline
\end{tabular}

Table 1. Mean proportions of "yes, true" judgments in percent (+ SE of the mean).

Verification Stage: Besides the judgments we analyzed two further dependent measures in the verification stage: the decision point, i.e. the particular card at which participants made their decision and the RTs of "yes, go on" button presses. The analysis of decision points revealed that participants decided at the earliest possible point. Most of the rejections in the linear card displays were launched directly from the second, disambiguating card without ever inspecting the last card. In the linear sentence all condition 130 out of a total of 201 rejections $(64.7 \%)$ came from the disambiguating card, the corresponding each condition had 116 out of 198 rejections (58.6\%), the inverse linking all condition had 121 out of $203(59.6 \%)$ and the inverse linking each condition 129 out of $194(66.5 \%)$. In the inverse card displays the situation was different. Here in $92.6 \%$ of the trials participants uncovered all three cards before they provided their judgment which in the majority of all cases was "yes, true". Thus, after processing the second card a verification strategy was clearly in place.

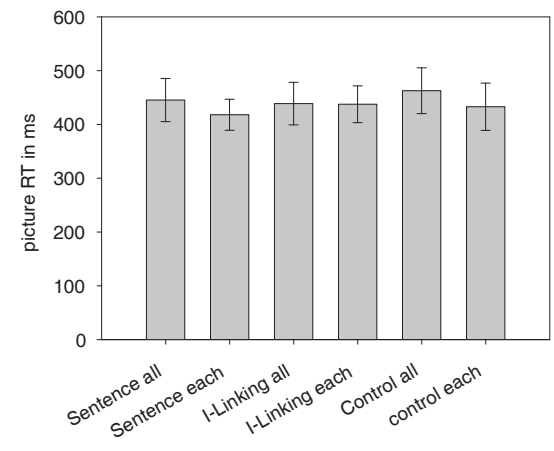

(a) fully covered cards

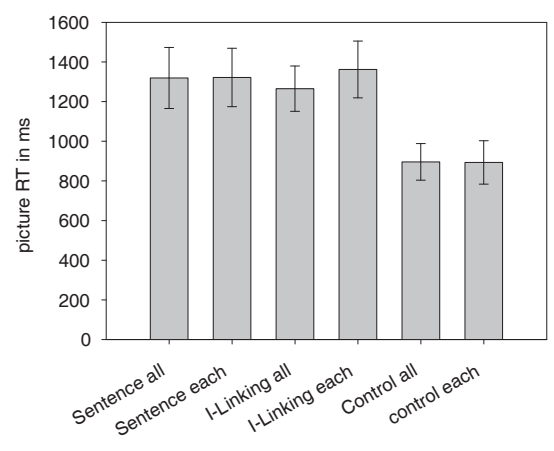

(b) first card

Fig. 3. Mean RTs of "yes, go on" button presses in ms (+ $95 \%$ confidence intervals; computed by participants) in the six sentence conditions. Figure 3a shows the RTs of the fully covered card layout. Figure $3 \mathrm{~b}$ shows the RTs of the first card. 
The analysis of RTs of "yes, go on" button presses provides us with a measure of how difficult a particular card was to evaluate. We corrected RTs for outliers by excluding values above 5s. RTs up to the second card were analyzed with mixed effects models including the fixed effects of construction (three levels: sentence vs. inverse linking vs. control) and determiner (two levels: all vs. each) and the random intercepts of participants and items. The picture RTs of the fully covered card display and the first card are depicted in Figure $3^{1}$. While there were no reliable differences between the conditions at the fully covered card display (LME: all $t<1$ ), the picture RTs of the (identical) first card clearly differed between conditions. Participants spent a mean RT of $1305 \mathrm{~ms}$ on the first card in the doubly quantified conditions compared to only $890 \mathrm{~ms}$ in the control conditions. Whereas the former conditions required evaluation of exactly one, i.e. categorization of all four objects and counting, the latter only required detection of the object in question. This clear-cut difference led to a significant contrast between the controls and the doubly quantified constructions (LME: estimate $=-387.00, t=-7.70, p<.01$ ). This was the only effect that turned out to be reliable. The contrast between the control condition and the doubly quantified conditions indicates that participants had already chosen the correct verification strategy before they uncovered the first card. The results of the verification stage are fully compatible with immediate full interpretation and the minimal domain account, but are unexpected in underspecification accounts where comprehenders delay interpretation until they encounter a disambiguation.

Reading Stage: We analyzed the reading times in the following six conditions: inverse linking constructions with each vs. all, sentence constructions with each vs. all and control conditions with each vs. all. To correct for outliers, we trimmed reading times by replacing values below $200 \mathrm{~ms}$ by a value of $200 \mathrm{~ms}$ and values above $2000 \mathrm{~ms}$ by a value of 2000 . This correction affected $3.65 \%$ of the data.

Figure 4 shows the mean reading times in the three construction types. In the sentence conditions there was a clear contrast between all and each: the determiner all was read on average $23.5 \mathrm{~ms}$ more slowly than each. In contrast, neither the inverse linking nor the control conditions revealed similar differences between determiners (mean difference jeder minus alle in inverse linking: $-7.6 \mathrm{~ms}$; control: $0.7 \mathrm{~ms}$ ). We analyzed the RTs of the determiner all vs. each in the inverse linking and the sentence constructions in repeated measures ANOVAs. The observed differences led to a significant main effect of construction $\left(F_{1}(1,39)=10.25\right.$, $\left.p<.01 ; F_{2}(1,59)=8.14, p<.01\right)$ and a significant interaction between construction and determiner $\left(F_{1}(1,39)=6.17, p<.05 ; F_{2}(1,59)=6.27, p<.05\right)$. To further break down the interaction we computed pairwise comparisons in each construction type. In the sentence conditions all took reliably longer to read than each $\left(t_{1}(39)=2.45, p<.05 ; t_{2}(59)=2.93, p<.01\right)$. Except for the

\footnotetext{
${ }^{1}$ Note that RTs of the second and third cards could not be properly analyzed because of almost no data in the linear conditions and systematic differences between cards in the different conditions.
} 
highly predictable following segment cards $\left(t_{1 / 2}<1\right)$ this effect persisted until the end of the sentence $\left(\right.$ to: $t_{1}(39)=3.36, p<.01 ; t_{2}(59)=2.81, p<.01$; find: $\left.t_{1}(39)=2.20, p<.05 ; t_{2}(59)=2.22, p<.05\right)$. This was different in the inverse linking conditions where all and each didn't differ at any region from the second quantifier until the end of the sentence (all $\left.t_{1 / 2}<1.5\right)^{2}$. The same held for the control conditions where all and each didn't differ anywhere in the sentence (all $\left.t_{1 / 2}<1.2\right)$. The lack of difference between all and each in the control conditions shows that the difficulty in the sentence conditions cannot be attributed to lexical factors. Instead, it must be due to interference between quantifiers.

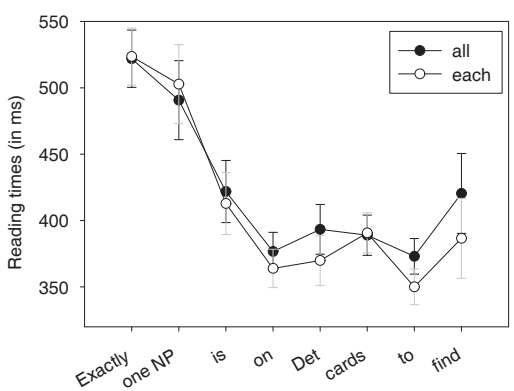

(a) sentence constructions

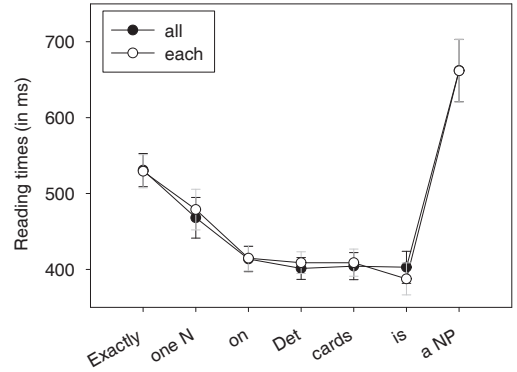

(b) inverse linking constructions

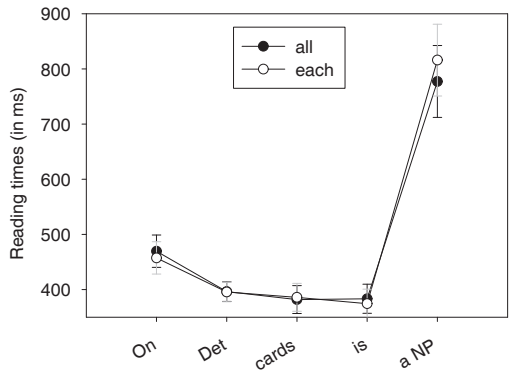

(c) control conditions

Fig. 4. Mean reading times in ms $(+95 \%$ confidence intervals; computed by participants) as a function of determiner.

\footnotetext{
${ }^{2}$ The minimal domain account would predict scope conflict at the point when the predication is complete. Finding no indication of scope conflict at the end of the sentence is therefore somewhat unexpected under this account. We suspect, however, that a delayed effect may have been covered by sentence wrap-up.
} 


\section{Conclusions}

To sum up, the experiment provides evidence for quantifier interpretation before disambiguation is encountered. Even though the participants were instructed to delay scope interpretation as long as possible, they computed scope relations already during reading. Our findings are thus clearly inconsistent with semantic underspecification accounts. The observed scope conflict might indicate that readers selected one interpretation right away. However, it is also possible that they computed both interpretations and ranked them according to the scope factors we have discussed. The results do not allow us to decide between these alternatives. We have shown, however, that a complete minimal domain is required for scope resolution. Our data thus call for introducing the notion of processing domain into cognitively realistic models of semantic interpretation. The results suggest that interpretation processes within a processing domain differ from those that take place at the domain boundary, and that the former may not be fully specified yet.

\section{References}

1. O. Bott (to appear). The processing domain of aspectual interpretation. In B. Arsenijevic, B. Gehrke \& R. Marín (eds.): Subatomic semantics of event predicates. Springer.

2. O. Bott, S. Featherston, J. Radó \& B. Stolterfoht (2011). The application of experimental methods in semantics. In C. Maienborn, K. von Heusinger \& P. Portner (eds.): Semantics: An international handbook. De Gruyter.

3. C. Ebert (2005). Formal investigations of underspecified representations. PhD thesis. King's College: London.

4. F. Ferreira and N.D. Patson (2007). The 'Good Enough' approach to language comprehension. Language and Linguistics Compass, 1 (1-2).

5. R. Filik, K.B. Paterson \& S.P. Liversedge (2004). Processing doubly quantified sentences: Evidence from eye movements. Psychonomic Bulletin \& Review. 11 (5).

6. S.B. Greene, G. McKoon \& R. Ratcliff (1992). Pronoun resolution and discourse models. JEP: $L M C, 18$.

7. A. Koller, M. Regneri \& S. Thater (2008). Regular tree grammars as formalism for scope underspecification. Proceedings of ACL-08.

8. H.S. Kurtzman \& M.C. MacDonald (1993). Resolution of quantifier scope ambiguities. Cognition, 48.

9. J. Pafel (2005). Quantifier scope in German. John Benjamins.

10. K.B. Paterson, R. Filik \& S.P. Liversedge (2008). Competition during the processing of quantifier scope ambiguities: Evidence from eye movements during reading. QJEP, 61(3).

11. A.J. Sanford \& P. Sturt (2002). Depth of processing in language comprehension: Not noticing the evidence. TCS, 6 (9). 


\title{
Projective Behaviour of Nur - Quantitative Experimental Research
}

\author{
Agata Maria Renans \\ Universität Potsdam, Institut für Linguistik \\ SFB 632 - Informationsstruktur \\ Karl-Liebknecht-Str. 24-25, \\ D-14476 Potsdam \\ renans@uni-potsdam.de
}

\begin{abstract}
The paper presents the results of the experiment on the projective meaning of nur (German: only). The data from German shows that the prejacent of nur projects easily out of counterfactual if-clauses, whereas its projective behaviour changes when it is embedded in indicative if-clauses. The obtained results classify projection out of counterfactuals as a reliable test for projective meanings in the cross-linguistic perspective, on the one hand, while shedding more light on the semantics of nur and conditionals, on the other.
\end{abstract}

\section{Introduction}

The meaning of a sentence with only can be fully described by two meaning components: (1) the prejacent (the meaning of the sentence without an exclusive particle), and (2) the universal (the negative, exclusive meaning of the sentence with only), e.g.:

(1) Only Mike ate ice-cream.

a. Prejacent $\rightarrow$ Mike ate ice-cream.

b. Universal $\rightarrow$ Nobody else but Mike ate ice-cream.

In the case of sentence (1), the prejacent is the proposition that Mike ate icecream, and the universal is the proposition that Nobody else but Mike ate icecream. Whereas it is commonly assumed that the universal is asserted, there are many competing theories regarding the semantic status of the prejacent of only, e.g., claiming that the prejacent is presupposed (Horn 1969), conversationally implicated (McCawley 1981, van Rooij \& Schulz 2005), entailed (Atlas 1993), or a non-speaker-oriented implicature (Roberts 2006).

The question is which diagnostics we can use to detect the projective meaning components and how we can distinguish different kinds of them. The bench of tests for identifying projective meaning are gathered and described by Beaver, Roberts, Simons, and Tonhauser (2009). One of the most popular tests for detecting a projective meaning is the so-called family of sentences test. It is claimed 
that the projective meaning should be interpreted outside the scope of (1) negation, (2) interrogation, (3) modals, and (4) the antecedent of a conditional.

In the experiments, I evaluate the projection out of the antecedent of a conditional test. The results show that the choice of the if-clause (counterfactual or indicative) influences the results of the experiment/elicitation on the projective meaning which is conducted with the help of this test. Projective meaning components (including the prejacent of nur) behave differently depending on whether they are embedded in counterfactual or indicative if-clauses.

\section{Projective Behaviour of Nur - Experiments}

\subsection{Experimental Set-Up}

To check whether the projective behaviour of the prejacent of the sentences with nur changes when the given sentence is embedded in the antecedent of the counterfactual and indicative conditionals, four experiments were conducted. Since in German indicative conditionals can be expressed either with the use of the particle wenn $(\approx i f)$ or falls $(\approx$ if, in case $)$, in order to eliminate the possibility that the results are modulated by the semantics of the given particle, two experiments with wenn and two experiments with falls were conducted. Moreover, experiments differed in the associated elements of nur: nur was associated either with Subject or with Direct Object. All conditionals in all four experiments were past-oriented and in each experiment the same lexical material was used in the antecedents of the conditonals ${ }^{1}$. The summary of the properties of all four experiments is presented in Table 1.

Table 1. Experiments

\begin{tabular}{lcc}
\hline & Indicative introduced by: & Element associated with nur \\
\hline Experiment 1 & Wenn & Subject \\
Experiment 2 & Wenn & Direct Object \\
Experiment 3 & Falls & Subject \\
Experiment 4 & Falls & Direct Object \\
\hline
\end{tabular}

17-26 first and second-year-students of linguistics took part in each of 4 experiments (84 participants together, 75 women, 9 men, average age: 21,91, all German native speakers). Each experiment comprised 6 items per condition (24 target sentences in total) and 26 fillers (50 tasks together). All the experiments were balanced: each participant saw the same amount of conditions and no participant saw the same item twice.

\footnotetext{
${ }^{1}$ The consequents differed due to different association patterns with nur. Keeping the same lexical material in the consequent of the conditionals in all four experiments would make the conditional sentences pragmatically infelicitous.
} 


\subsection{Methodology}

The design of the experiments is based on the methodology presented in Renans et al. (2011). Each task in each experiment consisted of a short context description, a target conditional sentence with nur (either (i) counterfactual or (ii) indicative) and a question (a) about the prejacent or (b) about the universal together with three possible answers ja (yes), nein (no), and nicht erkennbar (It's not known). The experiments were in the standard $2 \times 2$ conditions design, which is presented in Table 2. The informant's task was to answer the given questions.

Table 2. $2 \times 2$ conditions design in experiments (1)-(4)

\begin{tabular}{lcc}
\hline & Type of conditional: & Question about \\
\hline Condition a & counterfactual & prejacent \\
Condition b & counterfactual & universal \\
Condition c & Indicative & prejacent \\
Condition d & Indicative & universal \\
\hline
\end{tabular}

The tasks looked like as the following example ${ }^{2}$. Here is the exemplification of the counterfactual if-clause and nur associated with the Direct Object:

(2) Am Montag sollte jedes Kind seine Lieblingsspielzeuge mit in den On Monday should each child his favourite.toy with in ART Kindergarten bringen. Wenn Franz nur ein Bärchen kindergarten brin. If Franz only ART teddy-bear mitgebracht hätte, wären die Erzieherinnen sehr erstaunt bring.PAST have.KONJ, be.KONJ ART teacher very surprise gewesen.

be.PART

'On Monday every child was to bring his favourite toys to the kindergarten. If Franz had brought only a teddy-bear, the teachers would have been surprised.'

a. Hat Franz ein Bärchen mitgebracht?

have.PAST Franz ART teddy-bear bring.PART

'Did Franz bring a teddy bear?'

-ja (yes) -nein (no) -nicht erkennabar (It is not known)

b. Hat Franz noch etwas außer einem Bärchen

have.PAST Franz PART something beside ART teddy-bear

mitgebracht?

bring.PART

Did Franz bring anything else than a teddy-bear?

-ja (yes) -nein (no) -nicht erkennbar (It is not known)

$\overline{2}$ Notice that in the experiment, informants saw either question (a) or question (b). 
If the participant answers a question about the prejacent (condition $a$ and $c$ ) with yes, this means that the prejacent is interpreted outside the scope of the if-clause and hence projects out of the respective if-clause type. In the case of the universal, the interpretation of the results depends on the type of the conditional. In the case of (i) counterfactuals (condition $b$ ), answer yes means that the universal does not project out of the if-clause and answer no means that the universal projects out of the if-clause. In the case of (ii) indicatives (condition $d$ ), answer It is not known means that the universal does not project and answer no means that the prejacent projects. The interpretation of the results are shown in Table 3 (expected answers, assuming that the prejacent of nur projects and the universal is asserted, are written in boldface).

Table 3. Interpretation of the results

\begin{tabular}{llc}
\hline & No & Yes \\
\hline Counterfactual Prejacent $(a)$ & $\neg$ projection + projection & $?$ \\
& Universal $(b)+$ projection $\neg$ projection & $?$ \\
\hline Indicative & Prejacent $(c) \neg$ projection + projection & $\neg$ projection \\
& Universal $(d)+$ projection & $\neg$ projection $\neg$ projection \\
\hline
\end{tabular}

\subsection{Results}

The results of the experiments are shown in Tables $4,5,6$, and 7 . In all four experiments the answer patterns are similar. The results concerning the projective behaviour of the universal are as expected. They suggest that the universal does not project out of the if-clauses and its projective behaviour does not change with the change of the used conditionals. In all four experiments there are significantly more yes answers than no and It's not known answers in the case of the counterfactuals and there are significantly more It's not known answers than $n o$ and yes answers in the case of the indicatives, which are the expected answer patterns for the non-projective meaning components. Results concerning the projective behaviour of the prejacent are more surprising. We can observe the differences in its behaviour when it is embedded in the counterfactual and indicative if-clauses: prejacent of nur projects easily out of the counterfactual if-clauses, whereas it does not project so easily out of the indicative if-clauses.

The chi-square test showed that there was a significant interaction between conditions and the given answers, which means that the conditions influenced the answer patterns (for all experiments $p<0.001$ ).

To check whether the different answers were influenced by the type of the used conditional linear mixed-effects models (LME) were calculated. These models correspond to (logistic) regression models that take into account the variation due to participants and items. Since no answers were rare, $L M E$ models were 
Table 4. Experiment1: Nur associated with Subject, indicatives with wenn

\begin{tabular}{lrrrr}
\hline & No & Yes & $?$ \\
\hline Counterfactual Prejacent $(a)$ & $8(8 \%)$ & $\mathbf{8 6 ( 8 0 \% )}$ & $\mathbf{1 2}(12 \%)$ \\
& Universal $(b)$ & 5 & $90(88 \%)$ & $7(7 \%)$ \\
\hline Indicative & Prejacent $(c)$ & $2(2 \%)$ & $\mathbf{4 1 ( 4 0 \% )}$ & $\mathbf{5 9 ( 5 8 \% )}$ \\
& Universal $(d)$ & $16(16 \%)$ & $5(5 \%)$ & $81(79 \%)$ \\
\hline
\end{tabular}

Table 5. Experiment 2: Nur associated with Object, indicatives with wenn

\begin{tabular}{lrrrr}
\hline & No & Yes & $?$ \\
\hline Counterfactual Prejacent $(a)$ & $7(6 \%)$ & $\mathbf{8 6 ( \mathbf { 7 1 \% } )}$ & $\mathbf{2 7 ( 2 3 \% )}$ \\
& Universal $(b)$ & 0 & $104(87 \%)$ & $16(13 \%)$ \\
\hline \multirow{2}{*}{ Indicative } & Prejacent $(c)$ & $1(1 \%)$ & $\mathbf{4 1 ( 3 4 \% )}$ & $\mathbf{7 8 ( 6 5 \% )}$ \\
& Universal $(d)$ & $11(9 \%)$ & $7(6 \%)$ & $102(85 \%)$ \\
\hline
\end{tabular}

Table 6. Experiment 3: Nur associated with Subject, indicatives with falls

\begin{tabular}{lrrrr}
\hline & No & Yes & $?$ \\
\hline \multicolumn{2}{c}{ Counterfactual Prejacent $(a)$} & $7(6 \%)$ & $\mathbf{1 1 1 ( \mathbf { 8 8 \% } )}$ & $\mathbf{8 ( 6 \% )}$ \\
& Universal $(b)$ & $5(4 \%)$ & $113(90 \%)$ & $8(6 \%)$ \\
\hline Indicative & Prejacent $(c)$ & $5(4 \%)$ & $\mathbf{4 8 ( 3 8 \% )}$ & $\mathbf{7 3 ( 5 8 \% )}$ \\
& Universal $(d)$ & $6(5 \%)$ & $21(17 \%)$ & $99(78 \%)$ \\
\hline
\end{tabular}

Table 7. Experiment 4: Nur associated with Object, indicatives with falls

\begin{tabular}{|c|c|c|c|c|}
\hline & & No & Yes & $?$ \\
\hline Counterfactual & $\begin{array}{l}\text { Prejacent }(a) \\
\text { Universal }(b)\end{array}$ & $\begin{array}{l}9(6 \%) \\
5(3 \%) \\
\end{array}$ & $\begin{array}{r}123(\mathbf{7 9 \%}) \\
133(85 \%) \\
\end{array}$ & $\begin{array}{r}24(\mathbf{1 5 \%}) \\
18(12 \%) \\
\end{array}$ \\
\hline Ind & $\begin{array}{l}\text { Prejacent }(c) \\
\text { Universal }(d)\end{array}$ & $\begin{array}{r}5(3 \%) \\
4(2,4 \%) \\
\end{array}$ & $\begin{array}{r}49(\mathbf{3 1}, \mathbf{5 \%}) \\
11(7 \%)\end{array}$ & $\begin{array}{l}102(\mathbf{6 4}, \mathbf{5 \%}) \\
140(90 \%) 1(0,6 \%)\end{array}$ \\
\hline
\end{tabular}


calculated for yes and It's not known answers ${ }^{3}$. LME models showed that in all four experiments the type of the used conditional influenced the probability of yes and It's not known answers for the questions about the prejacent (Yes answers: Exp. 1: $z=-6.095, p<0.001$, Exp 2: $z=-6.092, p<0.001$, Exp. 3: $z=-7.815, p<0.001$, Exp. $4: z=-8.786, p<0.001$; It's not known answers: Exp. 1: $z=6.687, p<0.001$, Exp. 2: $z=6.798, p<0.001$, Exp. 3: $z=-7.781$, $p<0.001, \operatorname{Exp} 4: z=9.069, p<0.001)$. It means that Yes and It's not known answers for the question about the prejacent are modulated by the type of the conditional.

To assess the difference between the probability of Yes and It's not known answers in the case of the question about the prejacent in the indicative if-clauses (condition $c$ ), LME models were calculated on the proportions of each answer for each participant. There were significantly higher proportions ${ }^{4}$ of Yes than $I^{\prime}$ 's not known answers for Exp. $2(t=-4.933)$ and $4(t=-4.046)$, indicating that the prejacent of nur does not project out of the indicative if-clauses when it is associated with the Direct Object. For Exp. 3, the effect was marginal but with a trend in the same direction. However for Exp. 1 the effect was not significant. Nevertheless, the tendency towards non-projecting is also visible (40\% of Yes answers vs. $58 \%$ of It's not known answers).

Summarizing, the results of the experiments show that the prejacent of nur behaves differently when it is embedded in counterfactual and indicative ifclauses: the prejacent does not project or tends not to project out of the scope of the indicative if-clauses. In order to check whether the obtained results are due to the semantics of nur specifically or due to the properties of the projective meaning components in general, an experiment on projective behaviour of auch (German: too, also) was conducted.

\subsection{Projective Behaviour of Auch - Experiment}

The experimental set-up, the methodology, and the lexical material of the auchexperiment was the same as in Exp. (1). The tasks looked as follows:

(3) Am Montag sollte jedes Kind seine Lieblingsspielzeuge mit in den Kindergarten bringen. Wenn auch Franz ein Bärchen mitgebracht hätte, wären die Erzieherinnen nicht erstaunt gewesen.

'On Monday every child was to bring his favourite toys to the kindergarten. If also Franz had brought a teddy-bear, the teachers would not have been surprised.'

a. Hat Franz noch etwas außer einem Bärchen mitgebracht?

'Did Franz bring anything else than a teddy-bear?'

b. Hat Franz ein Bärchen mitgebracht?

'Did Franz bring a teddy bear?'

\footnotetext{
${ }^{3}$ Because of the lack of space, only the most important calcualtions are refered.

${ }^{4}$ For these post-hoc comparisons, $|t| \geq 2.4$ was taken to be significant
} 
In the case of (i) counterfactuals for both questions, answer Yes suggests that the respective meaning component projects, whereas answer No suggests that the respective meaning component does not project. In the case of (ii) indicatives for both questions, answer It's not known suggests that the respective meaning component does not project, whereas answer Yes suggests that it projects. The interpretation of the results of auch-experiment is presented in Table 8 (expected answers are written in boldface).

Table 8. Interpretation of the results: auch-experiment

\begin{tabular}{|c|c|c|c|c|}
\hline & & No & Yes & $?$ \\
\hline Counterfactual & $\begin{array}{l}\text { Additive meaning }(a) \\
\text { Core meaning } \quad(b)\end{array}$ & $\begin{array}{r}\neg \text { projection } \\
\neg \text { projection }\end{array}$ & $\begin{array}{l}+ \text { projection } \\
+ \text { projection }\end{array}$ & $\begin{array}{l}? \\
?\end{array}$ \\
\hline Indicative & $\begin{array}{l}\text { Additive meaning }(c) \\
\text { Core meaning }(d)\end{array}$ & $\begin{array}{l}\neg \text { projection } \\
\neg \text { projection }\end{array}$ & $\begin{array}{l}+ \text { projection } \\
+ \text { projection }\end{array}$ & $\begin{array}{l}\neg \text { projection } \\
\text { projection }\end{array}$ \\
\hline
\end{tabular}

13 second-year students of linguistics (11 women, 2 men, average age: 23,23, all German native-speakers) took part in the experiment. The results are presented in Table 9 .

Table 9. Experiment 5: Auch associated with Subject, indicatives with wenn

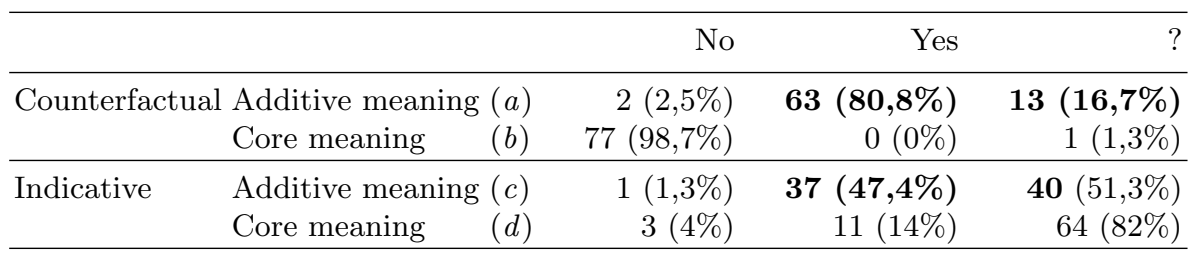

As in the case of Exp. (1)-(4), results for counterfactuals are as expected. They show that the additive meaning component projects easily out of the counterfactual if-clauses. The results for the indicative if-clauses are more surprising. Similar amount of Yes and It's not known answers for the question about the additivity in the case of the indicative if-clauses suggests that the additive meaning component can be interpreted both in and out of the scope of the if-clause. Moreover, as in the case of the prejacent of nur, we can observe the difference in the projective behaviour of the additive meaning component of auch depending on the type of the used if-clause.

The chi-square test showed that an interaction between the conditions and the given answers was significant $(p<0.001)$. In order to see whether the answers were modulated by the type of the conditional $L M E$ were calculated. They showed that the probability of Yes and It's not known answers were influenced by the type of the used conditional (Yes answer: $z=4.636, p<0.001$, It's not 
known answer: $z=-4.782, p<0.001)$. The results from Exp. (1) - (5) suggest that the described asymmetry is more systematical and is not caused by the specific semantics of nur. Notice, however that the observed effects are stronger in the case of the sentences with nur than with the sentences with auch.

\section{Analysis}

To analyse the results of Exp. (1)-(5) I adopt the restrictor analysis of conditionals in Kratzer (2011). She claims that in both indicative and counterfactual conditionals there is overt or covert modality. To understand conditional modality, we must start from Kratzer's approach to modals in general (Kratzer 1981). She captures the semantics of modals with the use of three notions: conversational background (CB), modal base, and ordering source. CB (a function from possible worlds to premise sets, which are sets of propositions) indicates the premises from which one can resolve the truth-value of the sentence with modals. There are many types of $\mathrm{CB}$, however here we are interested in the realistic $\mathrm{CB}$ and stereotypical CB.

Definition 1. Realistic $C B$ is a function $f$ such that for any world $w, w \in$ $\cap f(w)$. That is, $f$ assigns to every possible world a set of propositions that are true in it. (Kratzer 2011a)

A realisitc CB indicates a modal base (a set of accessible worlds) by transferring the premises to the closely related worlds. Note that the truth-values of modal sentences are resolved only regarding the modal base, not all the possible worlds. I hypothesize that the presuppositions (and other projective meaning components) should be present in a modal base.

Definition 2. Stereotypical $C B$ is a function $f$ such that for any world $w, f(w)$ represents what is normal in $w$ according to some suitable normalcy standard for w. (Kratzer 2011a)

A stereotypical CG which orders the worlds according to how closely they are related to the evaluation world taking into consideration the normal course of events in the evaluation world is an ordering source.

Given the above definitions, Kratzer (2011a) defines conditional modality. She claims that an if-clause additionally restricts the modal base of the consequent in reference to which the conditional sentence is interpreted.

Definition 3. For any conversational backgrounds f and $g$ : $[[\text { if } \alpha \beta]]^{f, g}=[[\beta]]^{f+, g}$, where for all $w \in W, f^{+}(w)=f(w) \cup[[\alpha]]^{f, g}$. (Kratzer 2011a)

Different kinds of conditionals are defined by different settings of the modal base and ordering source. Counterfactuals (in contrast to indicatives) are interpreted with the use of an empty modal base (Kratzer 2011a).

The modal base restricts the set of the possible worlds over which the covert modal in the consequent of the conditonal quantifies. Since presuppositions are 
in a modal base $f$, they also restrict the quantification domain of the covert modal in the consequent of a conditional. The crucial thing is that in the case of counterfactual if-clauses an initial modal base $f$ (from Def. 3 ) is empty. A modal base in reference to which the consequent is interpreted $\left(f^{+}\right)$is updated with the asserted proposition carried by the antecedent. However, since an initial modal base $f=\emptyset$, there are no presuppositions in $f^{+}$. Hence, the presuppositions do not take part in the restriction of the quantification domain of the consequent of the conditional. It follows that they are interpreted outside of the scope of the if-clause. Let us consider the following example:

(4) Also Mira came to the party.

a. core-meaning $\rightarrow$ Mira came to the party.

b. additive meaning $\rightarrow$ Somebody else than Mira came to the party.

c. If also Mira had come to the party, Anne would have been happy.

From Def. 3 follows that the consequent in (4-c) is interpreted in reference to the modal base $f^{+}$, which is the union of the empty set and the proposition expressed by the antecedent: $f^{+}(w)=\emptyset \cup[[\text { Mira came to the party }]]^{\emptyset, g}$. We can see that the presupposition (here: proposition that somebody else than Mira came to the party) does not restrict the possible worlds regarding to which the consequent is evaluated. Hence, the additive meaning component of auch is interpreted outside of the scope of the if-clause.

Indicative conditionals are interpreted in reference to a non-empty modal base. It means that the presuppositions (which are present in the initial modal base $f$ ) can restrict the modal base in reference to which the consequent is evaluated. Let us consider (4) and let us embed it in the indicative if-clause:

If also Mira came to the party, Anne was happy.

Sentence (5) is interpreted with respect to a non-empty modal base $f$ which includes the presupposition carried by the antecedent, i.e, the proposition that somebody else than Mira came to the party. The consequent is evaluated in reference to the modal base $f^{+}$which includes both the presupposition and the core-meaning of the antecedent: $f^{+}(w)=f(w) \cup\left[[\text { Mira came to the party] }]^{f, g}\right.$, where the presupposition [[Somebody else came to the party]] is included in $f(w)$. The presupposition (a proposition that somebody else came to the party) restricts the worlds over which the consequent quantifies.

Note that in the case of the indicatives, the presuppositions can be interpreted twice: in and out of the scope of the if-clause. The presupposition is present in a modal base, but it is still a projective meaning component, that is why there is still possible to interpret it out of the scope of the if-clause, what is indicated by the results of Exp. (5).

The difference between counterfactuals and indicatives is that the counterfactuals do not allow for the interpretation of the presuppositions within the scope of the if-clause, whereas indicatives provide such a possibility. The empty modal base prevents presuppositions to be interpreted in the scope of the counterfactual 
if-clause. A non-empty modal base which characterizes indicative conditionals allows us to interpret the presuppositions within the scope of the if-clause.

The mechanism of interpreting the conditionals with nur is the same as in the case of these with auch. The stronger effect of not projecting out of the scope of the indicative if-clauses is caused by the universal, which is an asserted part of the proposition in the antecedent and therefore obligatorily restricts the modal base. In sentence If only Mira came to the party, Anne was sad, the universal is a proposition that nobody else than Mira came to the party. In order to indicate all the possible worlds where nobody else than Mira came to the party, it is possible (but not obligatorily) to select the worlds where Mira came to the party. That is why the results of Exp. (1)-(4) showed a tendency to interpret the prejacent of nur in the scope of the indicative if-clause.

\section{Conclusion}

The paper reports the results of the experiments based on the methodology presented in Renans et al. (2011). Exp. (1)-(5) showed that there is a significant difference in the behaviour of the projective meaning components when they are embedded in indicative and counterfacutal if-clauses. The analysis of the results explained where this difference comes from. Therefore, the Projection out of the indicative if-clause test is evaluated as being inappropriate for the semantic fieldwork. The results of Exp. (1)-(5) showed that the quantitative experimental research can shed a new light on old (formal) semantic problems.

\section{References}

Atlas, J. D.: The Importance of Being 'Only': Testing the Neo-Gricean Versus NeoEntailment Paradigms. Journal of Semantics 10 (1993) 301-308

Beaver, D., Roberts, C., Simons, N., Tonhauser, J.: Addendeum: Investigating Properties of Projective Meaning. Ms., available at: http://ling.osu.edu/pragviki/file/bb2/Beaver-etal2009a.pdf

Beaver, D., Roberts, C., Simons, M., Tonhauser, J.: Not Just Projecting. Workshop on Projective Meaning (2009)

Beaver, D., Roberts, C., Simons, M., Tonhauser, J.: What Projects and Why. Proceedings of SALT XX (2010)

Horn, L.: A Presuppositional Analysis of 'Only' and 'Even'. Proceedings of the Annual Meeting of Chicago Linguistics Society (1969)

Kratzer, A.: The Notional Category of Modality, [in:] Modals and Conditionals, Oxford University Press (2011a)

Kratzer, A: Modals and Conditionals, Oxford University Press (2011), to be published

McCawley J.,: Everything that Linguists Have Always Wanted to Know about Logic but Were Ashamed to Ask. University of Chicago Press (1981)

Renans, A., Zimmermann, M., Greif, M.: Questionnaire on Focus Semantics. Potsdam Universität Verlag (2011)

Roberts,C.: 'Only', Presupposition and Implicature. Journal of Semantics, accepted with revisions (2006)

van Rooij, R., Schulz, K.: Only: Meaning and Implicatures. Maier, E., Bary, C., Huitink, J. Proceedings of Sinn und Bedeutung 9 (2005) 


\title{
Presupposition Processing - The Case of German wieder ${ }^{\star}$
}

\author{
Florian Schwarz ${ }^{1}$ and Sonja Tiemann ${ }^{2}$ \\ 1 Department of Linguistics and IRCS, University of Pennsylvania \\ florians@ling. upenn. edu \\ 2 Eberhard-Karls Universität Tübingen \\ sonja.tiemann@uni-tuebingen.de
}

\begin{abstract}
Presuppositions are vital for language comprehension, but little remains known about how they are processed. Using eye tracking in reading, we investigated two issues based on German wieder ('again'). First, we looked at the time course of presupposition processing by testing for processing costs of unsupported presuppositions. Secondly, we tested whether embedding wieder under negation affected a potential mismatch effect. Presupposition-induced effects showed up immediately after wieder, but only in the unembedded context, suggesting that embedding interferes with the detection of the mismatch. However, judgments in a follow-up rating study indicate that a mismatch is perceived in both the embedded and unembedded conditions when the PSP is not supported by the context. Taken together, these results suggest that detection of the mismatch under embedding is delayed in processing.
\end{abstract}

Keywords: Presuppositions, Psycholinguistics, Presupposition Processing, Presupposition Projection, Eye Tracking

\section{Introduction}

While the recent literature has seen a renewed peak in theoretical discussion of presuppositions, together with consideration of ever more intricate data (see Schlenker 2010 and Beaver and Geurts, to appear, for recent surveys), experimental approaches to presuppositional phenomena are still in their beginnings. Based on the general notion that presuppositions require some form of contextual support, previous experimental studies have found that lack of such support is reflected in various processing effects, e.g. regarding the choice of interpretation of a syntactically ambiguous structure and increase in reading times (Schwarz 2007) or the need for accommodation (Tiemann et al. 2011; see also Chemla 2009, and Chemla and Bott 2010 for other recent experimental studies). In a reading study using eye tracking, we investigated two issues concerning the processing of presuppositions. First, we test what form effects of presupposition failure, which have been found previously using Self-Paced Reading, have in

\footnotetext{
* This work was supported by EURO-XPRAG (ESF) and SFB 833 (DFG).
} 
this methodology. Eye tracking is more naturalistic and faster than Self-Paced Reading, and thus provides a more precise perspective on the time course of cognitive processes during reading. Secondly, our design compares these effects for unembedded occurrences with cases where a presupposition is introduced in the (syntactic) scope of negation (but standardly interpreted globally).

\section{Background}

Since different expressions that introduce presuppositions seem to vary in terms of ease of accommodation and other general properties (see, for example, the distinction between soft and hard triggers in Abusch 2009), it seems most prudent to focus experimental investigations on one presupposition trigger at a time. The present experiment focuses on the German trigger wieder ("again").

In the theories of Stalnaker 1973 and Heim 1990, presuppositions are restrictions on appropriate contexts. This means that a sentence like (1) is only felicitous if the context entails that Sue had danced before.

(1) Sue danced again.

An unmet presupposition results in presupposition failure and thus in uninterpretability of the sentence (cf. Heim and Kratzer 1998). Within this tradition, it is generally assumed that presuppositions are lexically encoded in the meaning of the presupposition trigger. The lexical entry for wieder then looks as in (2):

$$
\llbracket \text { wieder } \rrbracket=\lambda \mathrm{P} \cdot \lambda \mathrm{x} \cdot \lambda \mathrm{t} \cdot \lambda \mathrm{w}: \exists \mathrm{t}^{\prime}\left[\mathrm{t}^{\prime}<\mathrm{t} \& \mathrm{P}(\mathrm{x})\left(\mathrm{t}^{\prime}\right)(\mathrm{w})\right] \cdot \mathrm{P}(\mathrm{x})(\mathrm{t})(\mathrm{w})
$$

(3) captures formally that a sentence like (1) can only update a context if the context entails that there is a time $t^{\prime}$ before $t$ at which Sue has danced ( $c$ is Stalnaker's context set).

$$
\lambda \mathrm{c}: \mathrm{c} \subseteq\left\{\mathrm{w}: \exists \mathrm{t}^{\prime}<\mathrm{t} \& \text { Sue danced at t' in } \mathrm{w}\right\} . \mathrm{c} \cap\{\mathrm{w}: \text { Sue dances at } \mathrm{t} \text { in } \mathrm{w}\}
$$

In contrast with semantic theories of presupposition along these or similar lines, e.g. in the frameworks of dynamic semantics (Heim 1982) and Discourse Representation Theory (DRT, Kamp 1981, van der Sandt and Geurts 1991), several issues have recently given rise to a revived debate that includes various proposals for deriving presuppostions (of at least some presupposition triggers) pragmatically (Schlenker 2009, Simons 2001). In addition to the theoretical arguments that have been brought fourth in order to distinguish between these theories, experimental investigations can contribute to the debate in that the two types of accounts suggest different time courses for computing presuppositional content in online processing. The reasoning here is very much parallel to that presented in the experimental literature on scalar implicature processing. Increases in processing time have been taken to argue in favor of accounts of implicature generation where Gricean reasoning is carried out after the core literal content is computed (Bott and Noveck 2004, and much subsequent work). To the extent that pragmatic accounts of presuppositions also appeal to Gricean reasoning, 
we then might expect similar delays in effects related to the interpretation of presuppositions. If there is no delay in processing presupposed content, on the other hand, that would seem to fit more squarely with a view where presuppositions are encoded conventionally as part of the lexical content of the triggers (though it may not necessarily rule out certain versions of pragmatic accounts). Our experiments vary whether the context that again appears in supports its presupposition or not, which allows us to evaluate the time course of presupposition processing using the high temporal resolution of eye tracking during reading and thus contributes new empirical evidence to this debate.

A second experimental manipulation relates to one of the key properties of presupposed content, namely the fact that it is not affected by various embedding operators (including negation and various attitude verbs). For example, in (4),

(4) Sue [did NOT [dance again]].

even when assuming the syntactic structure indicated by the bracketing, the presupposition escapes the scope of negation, as it were, so that the entire sentence still presupposes that Sue danced before (rather than that she didn't dance before), just like the original version without negation. From the perspective of processing, keeping apart asserted and presupposed content and taking care to interpret these distinct aspects of meaning appropriately with respect to operators like negation constitutes a fairly delicate and complex task. Again appears syntactically in the scope of negation and has to combine with the verb in order to derive the appropriate presupposition, but the result of this then has to be interpreted globally, rather than in the scope of negation (unlike the asserted content contributed by, e.g., the verb). Investigating the online processing of presupposition triggers in the scope of operators like negation thus has the potential to provide important insights to our understanding of the underlying processes by which the global interpretation of presupposed content is derived. Our experiment is a first attempt to shed light on this issue by directly comparing processing effects based on presuppositional content both in configurations where it appears in the scope of negation as well as in global ones.

\section{Experiment}

\subsection{Methods and Material}

Design \& Stimuli. Our design makes use of a feature of German syntax, where wieder (again) and nicht (not) can appear in adjacent positions in either order. This makes it possible to construct target sentences which are minimally different with respect to whether again appears inside or outside the scope of negation. We presented such sentences in two different contexts, each of which supported the presupposition of one of the orders of wieder and nicht and contradicted the other. In the sample item from our materials below, the context sentence (5) supports the presupposition of (7a) (that Tina went ice-skating before), while (6) contradicts it (if not strictly speaking logically, then at least pragmatically). 
Conversely, (6) supports the presupposition of (7b) (that there was a preceding occasion where Tina did not go ice-skating), while (5) is inconsistent with it. ${ }^{1}$

(5) Tina went ice skating for the first time last week with Karl. The weather was beautiful, and they had a great time.

(6) Tina wanted to go ice skating for the first time with Karl last week. But the weather was miserable and they gave up on their plan.

(7) Dieses Wochenende war Tina $\{$ (a) nicht wieder / (b) wieder nicht $\}$ This weekend, was Tina $\{(\mathrm{a})$ not again / (b) again not $\}$ Schlittschuhlaufen, weil das Wetter so schlecht war. ice skating because the weather so bad was

The pairing of sentences and contexts yielded a fully counterbalanced $2 \times 2$ interaction design with two factors: Firstword (whether wieder or nicht appeared first) and Felicity (whether the context supports the presupposition or not).

Procedure \& Participants. 24 sentences with versions for each of the four conditions were created. In addition to the experimental items, there were 48 unrelated filler items. Subjects read the sentences on a computer screen while there eyes were being tracked by an EyeLink 1000 eye tracker from SR Research. For half of the items (of both the fillers and experimental sets), participants had to answer yes/no questions, which followed directly after the sentence, to ensure full comprehension of the materials. 32 native speakers of German from the University of Tübingen community participated in the experiment. Subjects were split into 4 groups, where each subject saw 6 of the sentences per condition.

\subsection{Results}

The primary focus in our analysis were the reading times on the verb following the $\{$ wieder nicht $\}$ sequence. Since the presupposition of wieder crucially relies on the verb of its clause, it is only at the point of the verb that it becomes recoverable from explicitly given materials. Reading times were also examined for $\{$ wieder nicht $\}$ itself. Standard reading measures were calculated for purposes of analysis. Based on prior self-paced reading experiments using the same general approach (Schwarz 2007, Tiemann et al. 2011), we expect increases in reading time when sentences are presented in contexts that are inconsistent with the presupposition. The time point at which such increases arise is indicative of the relevant presupposition having been computed at this point.

Data Analysis. All analyses used mixed-effect models with subjects and items as random effects, using the lmer function of the lme4 package in $R$ (Bates 2005), together with MCMC estimates for significance (Baayen et al. 2008). All effects significant at the $p<.05$ threshold are reported. Five reading time measures were

\footnotetext{
${ }^{1}$ At least on a global interpretation. See below for local interpretations.
} 
computed (Rayner 1998): first fixation duration, which measures the length of the very first fixation on the region of interest (here the verb); go-past time, which here is taken to measure the sum of all fixations on the region of interest prior to any fixations to the right of this region (but not including the time of regressive fixations); first pass time, which includes all fixations on the region when it is looked at the first time, up until leaving the region (to either the left or right); total duration, which sums all the fixations on the region of interest, no matter when they occur; regression path duration, which measures all fixations from first entering the region to first leaving it to the right (including all potential regressive fixations; this is sometimes also referred to as go past time); first pass regression proportion, which is the proportion of regressive eye movements following the first time of entering the region.

Means for the reading time measures on the verb are presented in table 1 . The primary result is an interaction betweeen Firstword and Felicity: when wieder was first (i.e., not embedded under negation), reading times on the verb were significantly higher in the infelicitous condition. When nicht was first, on the other hand (resulting in wieder being embedded under negation), there was no such slow-down (and except in total reading time, no significant difference between the felicitous and infelicitous context conditions).

Table 1. Reading time measures (in ms) and First Pass Regression Proportion (in \%)

\begin{tabular}{lcccc}
\hline & \multicolumn{2}{c}{ wieder nicht } & \multicolumn{2}{c}{ nicht wieder } \\
Reading Measure & felicitous & infelicitous & felicitous & infelicitous \\
\hline First Fixation & 173 & 185 & 171 & 171 \\
Go-Past & 253 & 327 & 282 & 258 \\
First Pass & 272 & 303 & 276 & 271 \\
Total & 296 & 382 & 352 & 298 \\
Reg. Duration & 296 & 382 & 352 & 298 \\
Reg. Proportion & $15.2 \%$ & $31.3 \%$ & $15.2 \%$ & $16.1 \%$ \\
\hline
\end{tabular}

There was a significant interaction for go-past $(p<.01)$, and total $(p<.001)$ reading times and for first pass regression proportion $(p<.05)$, as well as a marginal interaction for first fixation duration $(p=.071)$ and regression path duration $(p=0.065)$. There was a main effect of Firstword on all measures $(p<.05)$ with faster reading times (and lower regression proportions) on the verb in the nicht wieder conditions, which is not generally interpretable on its own given the cross-over interaction. The interaction was driven by a significant simple effect of Felicity for the wieder nicht conditions, with increases in reading measures for the infelicitous context (go-past time: $p<.01$, total time: $p<.01$, first fixation: $p<.01$, regression path: $p<.01$, regression proportion: $p<.001$ ). For the nicht wieder conditions, the only simple effect of Felicity appeared in the total reading time $(p<.05)$, and it was in the opposite direction, with a decreased reading time in the infelicitous condition. Regarding simple effects of 
Firstword, the only effect for the felicitous conditions was for total reading time $(p<.05)$, where reading times were faster in the wieder nicht condition than in the nicht wieder condition. In the infelicitous conditions, the wieder nicht conditions displayed faster reading times on the verb than the nicht wieder conditions ( total: $p<.01$, first fixation: $p<.05$, first pass: $p<.05$, go-past: $p<.0 .5$, regression path duration: $p=.11$, regression proportion: $p<.01$ ).

With respect to reading times on $\{$ wieder nicht $\}$ (taken as a unit, in either order), a parallel interaction effect showed up in the total reading times, with corresponding simple effects of Felicity for the wieder nicht condition and of Firstword for the infelicitous condition. There were no other significant interactions in this region, though there was a significant simple effect for first pass duration and a marginally significant effect for first fixation duration.

Given the lack of an effect of Felicity in the nicht wieder condition on the verb, follow-up analyses on later and larger regions were carried out. No increases in reading times were found for regions consisting of the verb plus 2 following words, the 3 words following the verb, the entire section of the sentence from nicht wieder to the end, or, for that matter, for the entire trial duration (i.e., total reading time for the entire paragraph).

\subsection{Discussion}

There are two main points to discuss with respect to the experimental results. The interaction shows that the effect of encountering a presupposition in a context that is inconsistent with it differs based on whether we are dealing with an embedded or an unembedded trigger. Furthermore, the presupposition of unembedded wieder gives rise to fairly immediate effects of inconsistency that are reflected throughout a variety of reading time measures. Of particular interest with regards to this latter point are the simple effects for first fixation duration and first pass regression proportion. Already during the first fixation of the verb (which last less than $200 \mathrm{~ms}$ ), the beginning of which arguably is the logically earliest point possible to fully compute the presupposition of wieder based on what has been explicitly provided, a $12 \mathrm{~ms}$ effect emerges. Based on the experimental design, the delay can be attributed to the inconsistency between the expressed presupposition and the provided context. But for such an inconsistency to arise, the relevant presupposition must of course have been computed. Similarly, the increase in first pass regression proportions indicates that upon first looking at the verb, there is an increased likelihood of returning to look at the preceding context, which is presumably triggered by noticing the same inconsistency. The experiment thus provides evidence that the presupposition of again is computed rapidly online. As mentioned above, this seems most consistent with theoretical proposals that assume it to be conventionally encoded, rather than derived by some type of pragmatic reasoning, which - based on what we know about scalar implicature processing - would seem to require some extra processing time. ${ }^{2}$

\footnotetext{
2 The extent to which this generalizes to other presupposition triggers remains to be explored. It is perfectly possible, and perhaps even likely, that triggers vary precisely in this respect (cf. Simons 2001).
} 
Returning to the first point, the picture is rather different for cases where wieder is embedded under negation. Given the standard global interpretation, the two contexts vary in precisely the same way as was the case for the unembedded occurrence of wieder (albeit their roles are reversed), with one context supporting the presupposition, while the other is inconsistent with it. If the global interpretation of the presupposition were available while reading the verb, we would expect to see an effect on reading times similar to the unembedded condition. However, on none of the reading measures was there a significant increase for the infelicitous condition. In fact, the only significant simple effect (for total reading times) went in the opposite direction (which is something that we do not yet have a clear explanation for). The lack of such an increase thus can be taken as an indication that the global interpretation is not available while the verb is being read.

In principle, there are two possible explanations for why this might be the case. First, it could be that more is involved in deriving a global interpretation in the context of an embedding operator like negation, compared to simply recognizing the presupposition of an unembedded trigger. Thus, the lack of an effect might be due to a lag in generating the appropriate presupposition in this more complex sentential context. An alternative exists, however, based on the possibility (ignored in our discussion so far) of local interpretations of presuppositions in the scope of negation. Perhaps the most well-known case of this concerns the existence presupposition of the definite article, as in (8), where a global interpretation of the presuppostion is inconsistent with the continuation.

(8) The King of France is not bald - because there is no King of France!

Similarly, in (9) it seems possible to negate the presupposition that Tina had been ice-skating before, rather than the asserted content.

(9) Tina didn't go ice-skating again last weekend - this was the first time!

A simple way of modeling this local interpretation is to simply assume that both the presupposed and the asserted content remain in the scope of negation, so that the overall interpretation of the sentence can be paraphrased as follows:

(10) NOT [Tina went ice-skating before AND went ice-skating this weekend]

While in principle, the falsity of either conjunct in the scope of negation would suffice to make this true, the fact that one could express the negation of the second conjunct more straightforwardly (by simply leaving out the presupposition trigger altogether) might bias this towards an interpretation where it is indeed the falsity of the conjunct contributed by the presuppostion trigger that is conveyed by an utterance of this sentence.

In any case, given a paraphrase along the lines of (10), if a local interpretation were available for the target sentence in the experimental materials, the nicht wieder sentences have interpretations that are perfectly consistent with either context. If the context states that Tina had been ice-skating some time recently, 
then the regular global presupposition of course remains consistent with that (and the local interpretation is not strictly speaking inconsistent with this either, if the paraphrase above is correct). And if the context states that she did not go ice-skating (and had never done it before, either), then the local interpretation (which is generally taken to convey that she had not been ice-skating before) is perfectly consistent with that. Thus, if both global and local interpretations for the presupposition of wieder in the scope of negation are available, we would not expect to see any reflexes of inconsistency in the reading times, since at least one of the readings always is consistent with the given contexts. In order to test whether local interpretations are indeed available for the experimental materials, a follow-up rating experiment was carried out.

\subsection{Follow-up Rating Experiment}

If local interpretations are indeed available for the presupposition of wieder when it appears in the scope of negation, we would expect this to affect speakers' acceptability judgments of these sentences in the two contexts. In particular, the type of interaction that we saw in the reading times should also be present in the judgments. If the local interpretation is not available (or only to a very limited extent), on the other hand, the nicht wieder sentences in what we have labeled as the infelicitous context above should be judged to be less acceptable than in the felicitous context. A rating questionnaire was conducted via the web using the WebExp2 software (http://www.webexp.info). The materials were exactly the same as those used in the eye tracking experiment, including all the fillers. Subjects were asked to rate the appropriateness of a given discourse on a scale from 1 (least appropriate) to 5 (most appropriate). The results are summarized in table 2.

Table 2. Results of the rating experiment

\begin{tabular}{lcccc}
\hline & \multicolumn{2}{c}{ wieder nicht } & \multicolumn{2}{c}{ nicht wieder } \\
& felicitous & infelicitous & felicitous & infelicitous \\
\hline Mean Rating & 3.94 & 2.63 & 3.23 & 2.34 \\
\hline
\end{tabular}

While there was a marginally significant interaction between Firstword and Felicity $(p=.059)$, as well as a marginally significant main effect of Firstword $(p=.059)$, more importantly there was a clearly significant main effect of Felicity $(p<0.001)$, with items containing felicitous contexts getting higher $(=$ better) ratings than those containing infelicitous contexts. While this effect was slightly more pronounced in the wieder nicht items (giving rise to the marginal interaction), there nonetheless is a significant simple effect for nicht wieder in the same direction $(p<.001)$, just as there is for wieder nicht $(p<.001)$. Thus the rating results clearly show that for both embedded and unembedded wieder, the 
when the context sentence was inconsistent with the (global) presupposition of wieder. This would be unexpected if the local interpretation of wieder under negation were readily available. The explanation of the reading time results in terms of the availability of such an interpretation thus is undermined by the rating results.

\section{Conclusion}

The results from the eye tracking experiment showed that reading times on the verb following $\{$ wieder nicht $\}$ were affected differently based on the order (and corresponding scope) of negation and wieder, with clear effects of infelicity in the unembedded wieder condition and no (or opposite) effects in the embedded wieder condition. The immediate presence of presupposition-based effects arguably is more consistent with semantic accounts of presupposition, which assume that the presupposed content is conventionally encoded in the lexical entries for the triggers.

With respect to the absence of reading time effects of Felicity in the embedded wieder condition, the results from the rating study show that this cannot be attributed to the general availability of a local interpretation of the presupposition of wieder, as this would predict the same interaction to show up in the ratings. An alternative explanation for the lack of reading time effects in this condition is that computing the global interpretation in the syntactic context of negation is more costly in terms of processing, and that this interpretation thereforre is not immediately available. What remains somewhat mysterious at this point is that no slow-downs in reading are to be found on any of the subsequent and larger regions that we analyzed. Characterizing the result as involving a delayed computation of the presupposition might lead us to expect to find the same type of increase in reading times, but on a later region. Nonetheless, the rating study clearly shows that the target sentences are perceived to be infelicitous in the infelicitous context, and it's hard to explain the absence of a reading time effect for the nicht wieder conditions if we assume that this infelicity becomes apparent immediately. Furthermore, there was a suggestive numerical increase in response times for the ratings in the infelicitous nicht wieder condition. While this did not give rise to a statistically significant interaction, there was a potential hint of a marginal simple effect of Felicity for this order $(p=.12)$. If it were possible to substantiate such an increase in a study that is more directly targeted at capturing the time course of the acceptability judgment, that could lend further and even more direct support to the hypothesis that computing global presupposition interpretations in the context of negation is more costly.

While limitations of space as well as the experimental focus of the present research have kept us from evaluating the impact on theoretical discussions of the interaction in detail, it would be of high theoretical significance if embedded presuppositions indeed involve more processing effort. In particular, this would seem very much consistent with theories that posit explicit and complex operations on levels of representation in the computation of global interpretations, such 
as the DRT analysis by van der Sandt and Geurts 1991 and van der Sandt 1992 (though this is certainly not the only possible account consistent with the data). But a more thorough exploration of such theoretical implications must await future occasion.

\section{References}

Abusch, D.: Presupposition Triggering from Alternatives. Journal of Semantics 27, 37-80 (2009)

Bates, D. M. and Sarkar, D.: lme4: Linear mixed-effects models using S4 classes, R package version $0.99875-6$. (2007)

Bates, D. M.: Fitting linear mixed models in R. R News 5, 27-30 (2005)

Baayen, R. H., Davidson, D. J., and Bates, D. M.: Mixed-effects modeling with crossed random effects for subjects and items. Journal of Memory and Language 59, 390$412(2008)$

Beaver, D. and Geurts, B.: Presupposition. The Stanford Encyclopedia of Philosophy (to appear)

Bott, L. and Noveck, I. A. Some utterances are underinformative: The onset and time course of scalar inferences. Journal of Memory and Language 51, 437-457 (2004)

Chemla, E., Bott, L.: Processing Presuppositions: Dynamic Semantics vs. Pragmatic Enrichment.Manuscript, Cardiff University, IJN, LSCP (2010)

Chemla, E.: Presuppositions of Quantified Sentences: Experimental Data. Natural Language Semantics 17, 299-34 (2009)

Heim, I. and Kratzer, A.: Semantics in Generative Grammar (1998)

Heim, I.: Presupposition Projection. Reader for the Nijmegen Workshop on Presupposition, Lexical Meaning, and Discourse Processes (1990)

Heim, I.: The Semantics of Definite and Indefinite Noun Phrases, Doctoral Dissertation, University of Massachusetts at Amherst (1982)

Kamp, H.: A Theory of Truth and Semantic Representation. Formal Methods in the Study of Language, 277-322 (1981)

Rayner, K.: Eye Movements in Reading and Information Processing: 20 Years of Research. Psychological 124, 372-422 (1998)

van der Sandt, R.: Presupposition Projection as Anaphora Resolution. Journal of Semantics 9, 333-377 (1992)

van der Sandt and R., Geurts, B.: Presupposition, Anaphora, and Lexical Content. Text Understanding in LILOG, 259-296 (1991)

Schwarz, F.: Processing Presupposed Content. Journal of Semantics 24, 373-416 (2007)

Schlenker, P.: Presuppositions and Local Contexts. Mind, 119, 474: 377-391 (2010)

Schlenker, P.: Local Contexts. Semantics \& Pragmatics 2, 1-78 (2009)

Simons, M.: On the Conversational Basis of Some Presuppositions. Proceedings of Semantics and Linguistics Theory 11 (2001)

Stalnaker, R.: Presuppositions. Journal of Phillosophical Logic 2, 447-457 (1973)

Tiemann, S., Schmid, M., Bade, N., Rolke, B., Hertrich, I., Ackermann, H., Knapp, J. and Beck, S.: Psycholinguistic Evidence for Presuppositions: On-line and Offline Data. Sinn und Bedeutung 15. Proceedings of the Annual Conference of the Gesellschaft für Semantik, 581-597 (2011) 


\title{
Experimenting with the King of France
}

\author{
Márta Abrusán \& Kriszta Szendröi \\ Lichtenberg Kolleg, Göttingen, Germany \& University College London, UK \\ abrusanealum.mit.edu \& k.szendroi@ucl.ac.uk
}

\begin{abstract}
Definite descriptions with reference failure have been argued to give rise to different truth-value intuitions depending on the local linguistic context in which they appear. We conducted an experiment to investigate these alleged differences. We have found that pragmatic strategies dependent on verification and topicalization, suggested in the context of trivalent theories, indeed play a role in people's subjective judgments. Overall however we think that our findings are best explained by combining these pragmatic strategies with an approach that assumes that definite descriptions have a bivalent semantics, as well as a pragmatic presupposition attached to them. We also suggest that the verification of a sentence -where possible - proceeds through a pivot constituent, and that this concept is relevant for the proper description of how speakers understand semantic meaning.
\end{abstract}

Keywords: definite descriptions, presupposition, topics, verifiability, experimental pragmatics

\section{Introduction}

According to Russell (1905, 1957), definite descriptions assert the existence of a unique individual that satisfies the description. When such an individual does not exist, as in (1), the sentence makes a false assertion. Famously, Strawson (1950, 1964) argued that Russell's theory cannot predict why speakers (like him) feel "squeamish" about assigning the truth-value 'false' to sentences such as (1). His proposal (which can be traced back to Frege 1892) was that definite descriptions instead of asserting, presuppose the existence of a unique individual that satisfies the description: when this presupposition is not met, the question of truth or falsity does not even arise and so the sentence does not have a truth-value. A third possibility (cf. Stalnaker 1974,1978 ) is that definite descriptions both assert and presuppose the existence of a unique individual satisfying the description.

(1) The king of France is bald.

Strawson (1964) has noted however that truth-value intuitions change when the same definite description is put in different contexts. He conceded that according to his intuition examples such as (2) do appear to be straightforwardly false. This in turn

adfa, p. 1, 2011.

(C) Springer-Verlag Berlin Heidelberg 2011 
requires some explanation from the view according to which (2) should not have a truth-value.

(2) The exhibition was visited yesterday by the king of France.

Since Strawson's observation, various factors have been identified in the literature that might influence our truth-value intuitions about sentences with reference failure noun phrases. Strawson (1964) himself (cf. also Reinhart 1981 and many others) have identified topic-comment structure as a factor. Others (cf. Fodor 1979, Lasersohn 1993, von Fintel 2004) stressed the importance of background knowledge based on which the sentence could be verified (or not), independently of the existence of the problematic referent. Sometimes, conflicting judgments have been asserted, which makes it hard to judge the relative import of the theories.

We have conducted a behavioral experiment that was designed to capture people's intuitions about sentences like (1)-(2). Our overall results argue against Russell's nonpresuppositional semantics for definite descriptions and also against Strawson's truthvalue gap approach, and suggest that the pragmatic presupposition approach of Stalnaker is more likely to be correct. But we have also found that the above-mentioned Strawsonian theories all identify relevant pragmatic factors that influence truth-value intuitions, even though none of them can fully predict the behavioral pattern we found: The sentence's topic-comment structure (as suggested by Strawson 1964, Reinhart 1981, etc) and the sentence's verifiability (as suggested by Lasersohn 1993, von Fintel 2004) are both important factors influencing truth-value judgments. At the end of this paper, we offer a brief discussion of how to reconcile these seemingly divergent factors.

\section{Possible Factors Behind Wavering Truth-Value Judgments}

Strawson (1969) has proposed that one factor behind difference in (1) and (2) is the topic-comment structure of the sentence. Topics are understood to be the constituents that the sentence is pragmatically about. Strawson proposed that when the definite description is not in a topic position, it is "absorbed" into the meaning of the predicate, and since it is not a referring expression anymore its presupposition is turned into an existential statement. This predicts the difference between (1) and (2): The noun phrase the king of France is in topic position in (1) hence in a context where it is known that France has no king, it leads to a presupposition failure, associated with "squeamishness". In (2) the same noun phrase is not in a topic position and so the sentence is simply false. The topic approach has been adopted and developed further by many researchers, most importantly Reinhart (1981, 1995). (See also Hajicova 1984, Gundel 1977, Lambrecht 1994, Erteschik-Shir 1997, Atlas 2004, Geurts 2007, Shoubye 2009, among others).

However, the importance of topichood has been questioned (cf. von Fintel 2004). One reason to doubt that the existential presupposition is absorbed into the predicate (or is just non-existent) when the definite description is not in topic is that 
the existential presupposition of definites seems to project out of embedded contexts, such as the antecedents of conditionals, whether or not the definite is in topic position. Thus (3) still seems to imply the existence of a French king, which suggests that the definite is still presuppositional, despite not being in topic.

(3) If the exhibition was visited by the king of France, the organizers must be happy.

This latter observation highlights a very important point, identified most clearly by von Fintel. Namely, that our intuitions about accepting or rejecting a sentence as true or false, and the sentence's actual semantic truth-value (and hence its presuppositionality) are two separate things. Speakers might feel that a sentence is false or true even when semantically it has no truth-value, as long as they can find some reason based on which they can reject (or accept) the sentence. The feeling of "squeamishness" arises only when all pragmatic repair strategies for dealing with a truth-valueless sentence fail.

The first proposal in this spirit was due to Lasersohn (1993). His main focus were examples such as (4) which are said to be judged false. Lasersohn's observation was that in a situation where the chair in front of the speaker is empty, or when it is occupied by somebody other than the king of France, speakers have enough grounds to reject the sentence as false: They can look at the chair and see that the king of France (whether or not he exists) is not in it.

(4) The king of France is sitting in this chair.

In the case of (1), in the absence of background knowledge about the hairstyle of French royals, speakers do not have enough grounds to reject or accept the sentence, and are left with the feeling of squeamishness. Lasersohn's theory rests on the following tenets: (a) Assume two kinds of truth-values: (i) semantically assigned values 1,0 , and a third value \#, which corresponds to 'neither 0 or 1' (ii) pragmatically assigned values TRUE, FALSE which represent the status of a sentence with respect to a given body of information, and correspond to acceptance and rejection. (b) Once we are faced with presupposition failure (neither 0 nor 1 ), there are fall-back strategies to fill in the gap and arrive at TRUE and FALSE. (c) Lasersohn's fall-back strategy: Step 1: revise the given body of information to remove the knowledge that there is no king of France. Step 2: See if the given body of information can be consistently extended to include the target proposition.

A consequence of Lasersohn's analysis is that only those propositions are predicted to have the truth-values TRUE or FALSE that are in direct conflict (or in accordance) with what can be concluded from the given body of information. This conclusion has been argued to be too weak by von Fintel (2004), based on examples such as (5), which he argues is felt to be FALSE, even in the absence of any information about who is on a state visit in Australia this week.

(5) The king of France is on a state visit to Australia this week. 
He proposes to add another fall-back strategy, besides (c) above: ${ }^{12}$ (d) Rejection/acceptance might (also) be based on the possibility of examining the intrinsic properties of a contextually salient independent entity (that everyone agrees exists).

This suggestion explains (5), even if the given body of information does not contain anything about who was visiting Australia. In principle, we could examine the properties of Australia and see whether the king of France is in it or not. Australia thus serves as a salient foothold for verification, based on which the truth of the sentence in (5) can be evaluated.

\section{The Experiment}

We tested 33 native speakers of English (mostly British English, all of them familiar with basic elements of British culture), aged 20-55, most of whom participated for a small fee. We investigated how participants judge different types of sentences with reference failure noun phrases. The participants first read instructions given to them on the computer screen, reproduced in (6).

(6) In this experiment, statements will appear on your screen. If you think a statement is true, you should click on the 'TRUE' button. If you think a statement is false, you should click on the 'FALSE' button. Sometimes, it may happen that you cannot decide. In those cases, you should click on the 'CAN'T SAY' button. Please do not dwell on your decision for too long. There is no right or wrong answer!

After a short practice session, participants were left alone with a program which presented the test items one-by-one on the screen. Each item contained one sentence, as shown in (7). Participants could use the mouse to click on the buttons. After they chose an answer, the next item appeared automatically.

(7) Example of an experimental trial:

\begin{tabular}{|l|}
\hline The king of France is bald. \\
FALSE CAN'T SAY TRUE \\
\hline
\end{tabular}

There were eleven test conditions, with eight test items in each condition. The test items were obtained by placing 8 definite descriptions that lack referents (listed in (8))

\footnotetext{
${ }^{1}$ That both (c) and (d) are needed is suggested by examples that fall under (c) but not (d): The king of France can jump 100 feet into the air unaided. (Example from intro by Bezuidenhout and Reimer to von Fintel 2004). However, if independent footholds for rejection/verification can also be general laws, then Lasersohn's account can be subsumed under von Fintel's, and thus (c) is a subcase of (d). It seems to us however that this would make it very hard to track what predictions von Fintel's proposal actually makes.
} 
in eleven types of sentential contexts, (the test conditions), illustrated in (9). More on the test conditions below.

(8) the king of France; the emperor of Canada; the Pope's wife; Princess Diana's daughter; the beaches of Birmingham; the Belgian rainforest; the coral reefs of Brighton; the volcanoes of Kent.

(9) Examples of test conditions, illustrated here with the king of France

0 The king of France is bald.

1 France has a king and he is bald.

2 The king of France is on a state visit to Australia this week.

3 The king of France is married to Carla Bruni.

4 The king of France, he was invited to have dinner with Sarkozy.

5 Sarkozy, he was invited to have dinner with the king of France

6 The king of France isn't bald.

7 The king of France is not on a state visit to Australia this week.

8 The king of France is not married to Carla Bruni.

9 The king of France, he wasn't invited to have dinner with Sarkozy.

10 Sarkozy, he wasn't invited to have dinner with the king of France.

The 88 test items were supplemented by almost twice as many filler items containing true and false controls. Altogether, there were 253 items presented in three blocks. The items were pseudo-randomised: there were no items from the same condition, or with the same NP, closer than 4 trials. The statements were presented in three separate blocks.

All three theories agree that sentences like The king of France is bald, i.e. Condition 0, should lead to truth-value gaps and predict FALSE judgments for the non presuppositional assertions in Condition 1. The theory of von Fintel predicts a higher number of FALSE judgments in Condition 2, 3, 4, 5 than in Condition 0 because the former contain an independent NP alongside the referentially challenged NP. It also does not predict any difference among Conditions 2, 3, 4, 5. Lasersohn predicts a higher percentage of FALSE answers for Condition 3: The items in this condition were individually paired with 8 true control items, e.g. the control item for (12-3) and (12-8) was that Sarkozy is married to Carla Bruni. It was predicted that participants who judge control items TRUE would have the necessary knowledge to reject the corresponding items in Condition 3. Strawson and Reinhart predicted that items in Condition 4 lead to truth-value gaps because in these the referentially challenged NP is tropicalized, while their variants in Condition 5 in which the same NP is not in topic are judged FALSE.

Following pilot studies, we have discovered that a large proportion of our participants judged items in our base condition, Condition 0, as FALSE. For this reason, we included a further five conditions, Conditions 6-10, which corresponded to Conditions $0,2-5$, respectively, but which involved sentential negation. Our assumption was that a FALSE judgment that reflects a rejection of a particular statement based on it being semantically false should turn into a TRUE judgment once the statement in question 
is negated. If a negated sentence such as The king of France is not bald is not judged to be TRUE, then likely the corresponding positive sentence is judged to be FALSE for some other reason than having the semantic value 0. Further, if The king of France is not bald is not judged to be TRUE, the non-Russellians are still in business and we can test the validity of the theories presented above. In particular, we make the following predictions. We would get a higher number of TRUE judgments in Condition 7 , the negated version of Condition 2, compared to Condition 6 , the negated version of Condition 0, to support von Fintel's theory. Lasersohn predicted a higher number of TRUE judgments in Condition 8, the negated version of Condition 3, than in Condition 6. While Reinhart/Strawson predicted higher number of TRUE judgments in Condition 10 than in Condition 9, which are the negated equivalents of Conditions 6 and 5 respectively.

\section{$4 \quad$ Results}

\subsection{Data}

All but one of our participants successfully finished the task. This participant was excluded. We compared the proportion (\%) at which subjects replied FALSE to the test items in Conditions 0-5 with ANOVA, following checks for normal distribution and homogeneity of variance. We found only weak or nearly significant differences between any of the conditions 0-5 ( $>>0.05$, Bonferroni post-hoc test), i.e. the positive conditions. Our subjects said FALSE to most of these most of the time. This was counter to our predictions, according to which we expected a low number of FALSE judgments in Condition 0, and a high number of FALSE judgments in Condition 1. Neither of the conditions 0-5 differed significantly from our FALSE-controls either.

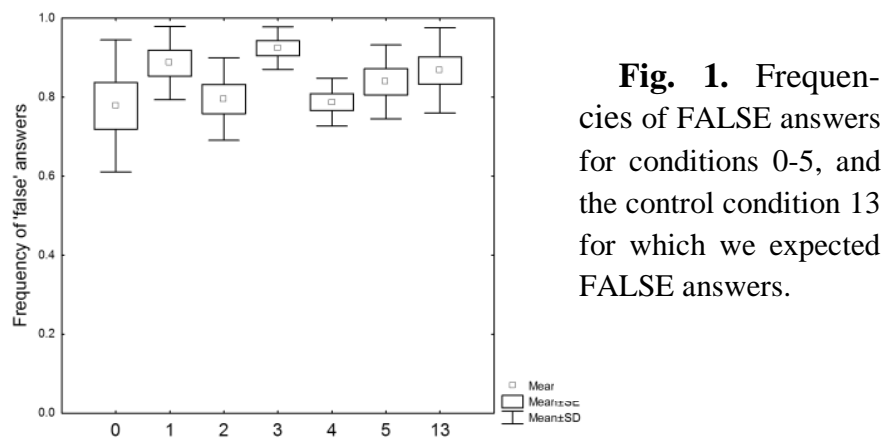

Since there was no significant difference in the percentage of FALSE judgments between any of the conditions $0-5$, we could not verify either a von Fintel effect ( 0 vs. 2), nor a Lasersohn effect ( 0 vs. 3 ) nor a topic-effect (4 vs. 5) by looking at positive sentences.

The negated versions of Conditions 0-5 (except Condition 1), namely Conditions 6-10 turned out to be more informative. We compared the proportion (\%) of TRUE responses in these conditions with ANOVA, (Bonferroni post-hoc test). As 
Figure 2 illustrates, we found that speakers responded TRUE at a significantly higher proportion to the items in Conditions 7, 8, 9, 10 than to the items in Condition 6 ( $\mathrm{p}<0.003$ in all cases), our base-line. The significant difference between Conditions 6 and 7 (and also 6 vs. 8, 9, 10) indicates that there is a von Fintel-effect. The significant difference between Conditions 6 and 8 supports Lasersohn's theory. Condition 8 also differed significantly from Condition 7 ( $\mathrm{p}=0.0012)$. In addition, we also found a significant difference $(\mathrm{p}=0.037)$ between Conditions 9 vs. 10: speakers responded with TRUE at a significantly higher proportion to Condition 10 than to Condition 9, which is in accordance with Strawson/ Reinhart's predictions, and indicates that topichood also plays a part in subjective evaluations of the truth of sentences with reference failure definite descriptions. The difference between conditions 7 vs. 9 and 8 vs. 10 was not significant $(\mathrm{p}>0.05)$.

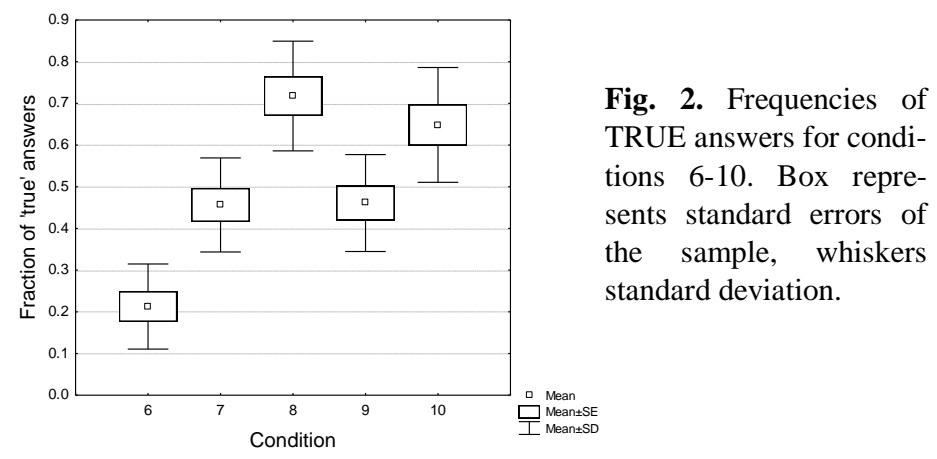

When comparing Conditions 6-10 with true controls, we found that all the conditions 6-10 (except condition 8) differed significantly from true controls as well, even if weakly $(\mathrm{p}<0.045)$. It is hard however to interpret this result, as Conditions 6-10 contained negation, but our control sentences did not.

\subsection{Interpreting the Results}

Positive vs. negative conditions. We found that the responses to Condition 0 did not differ significantly from Condition 1 , or from our control condition involving nonproblematic false statements. At a first glance, this result might seem to support the Russellian view. However, in the light of the answers we got for Conditions 6-10, the negative conditions, the Russellian position is hard to maintain as this view has no room to predict the systematic variation in conditions 6 to 10 .

The truth-value gap account of Strawson (also assumed by Lasersohn 1993 and von Fintel 2001) at first sight seems to have more room for maneuvers that might explain the overall pattern of data we found. On this account, all the sentences in conditions $0,2-10$ are truth-valueless: nevertheless it is possible to predict the results we got by assuming that our subjects interpreted the response option FALSE 'I do not think it is true' or 'I am rejecting this sentence' rather than 'I think this sentence is false'. Sentences in the positive Conditions 2-5 are judged to be FALSE either for the 
same reason, or because of Lasersohn's/von Fintel's idea according to which speakers opt for FALSE in order to avoid the conversational impasse created by a presupposition failure, but only if they have independent reasons to reject the sentence. The same reasoning also predicts a difference between conditions 6 vs. 7-10, which was also confirmed by our results. The problem is when we compare our answers in Condition 0 and Condition 6: To the extent that the above approaches would go along with our explanation for the high number of FALSE judgments in Condition 0 as instances of overapplication of FALSE to truth-valueless sentences, the same reasoning should apply to Condition 6. However, a post hoc comparison of the number of FALSE results for Conditions 0 and 6 revealed a significant difference (t-test $\mathrm{p}=0.0002$ ), our subjects were much more likely to reply FALSE to the examples in Condition 0 than to the examples in Condition 6. We do not see how to reconcile this finding with the truth-value gap approach.

The theory of pragmatic presuppositions, according to which definite descriptions both assert and presuppose the existence of a unique individual that satisfies the description, such as Stalnaker's (1974) theory can explain the full pattern of results (cf. also Karttunen and Peters 1979; Abbott 2000; Simons 2001; Schlenker 2008, 2009; (among others)). As for the positive sentences (Condition 0), speakers say these are false, because they are indeed semantically false, and the fact that these sentences at the same time exhibit a presupposition failure is not in itself a reason to change this judgment, at least as long as false sentences do not have to be added to the context set. As for the sentences in Condition 6 (the negative baseline sentences), although these are semantically true, speakers are reluctant to mark these sentences as such because they also manifest a presupposition failure, and therefore one should avoid adding them to the context set. Suppose TRUE implies a commitment to add the sentence to the context set: this suffices to prevent people from saying that such sentences are TRUE. But when there is a good pragmatic strategy based on which the presupposition failure can be ignored, as in Conditions 7-10, the sentences with negation (that are semantically true) will be felt as pragmatically TRUE as well. This predicts the difference we found between Condition 6 vs. conditions 7, 8, 910.

The von Fintel vs the Lasersohn-effect. We found a von Fintel effect: Condition 7 (and also Conditions 8, 9 and 10) received a significantly higher proportion of TRUE responses than Condition 6 . At the same time, we found that there was a significant difference between the proportion of TRUE responses to Condition 7, the 'pure' von Fintel condition, and Condition 8, the one that aimed to test Lasersohn's theory. We take this result to indicate that there is indeed a separate Lasersohn and von Finteleffect, and both effects measurably influenced truth-value judgments. For the von Fintel effect it is enough that an NP is present on the basis of which it would be possible to verify the sentence (given some appropriate knowledge dataset, say Wikipedia). For the Lasersohn effect, the properties of the NP provide a basis for verification given the speaker's actual dataset. This explains the finding that the Lasersohn effect is stronger: Our sentences that satisfied Lasersohn's criteria also satisfied von Fintel's criteria, but not the other way around. 
Topic effect. We also found that there was a significantly higher number of TRUE responses in Condition 10, where an existentially sound NP was topicalised, than in Condition 9, where the NP with referential failure was topicalised. Our results are thus consistent with Strawson's and Reinhart's prediction.

\section{$5 \quad$ Topics and Verifiability}

We think that overall our findings support the position that all the three proposals discussed above were right to some degree, in that they all identified relevant factors for truth value intuitions. One question that arises is whether the above are independent factors, or whether there is some connection among them.

We believe that verification and topichood are not unrelated notions. However, there is also a fair amount of confusion around the notion of topic: sometimes it is understood as a discourse based notion (cf. Kuno 1972, Gundel 1974, Givon 1983, von Fintel 1996, Roberts to appear, among others) but sometimes it is (also) couched in verificationalist terms (cf. Strawson 1964, Reinhart 1981, Lambrecht 1994, Erteschik-Shir 2007, among others). However, even the latter employ tests for topichood that are discourse based. We suggest to distinguish two related but different concepts from each other: a discourse based notion of topic, which is what the sentence is pragmatically about, and what we call pivot, which is the constituent in the sentence based on which the sentence is verified, in other words, the contextually salient entity that provides the foothold for verification in von Fintel's sense. Thus pivothood is a semantic notion that is concerned with the process of understanding a sentence. This invoves - at least in some cases - the possibility of verifying the sentence. The pivot is what the sentence is semantically about, if such an entity can be found, in this verificational sense of aboutness. It is not an obligatory element of understanding a sentence: In some cases it might not be possible to identify a unique pivot or indeed any pivot. Further, pivots do not have to be constituents of the sentence. This might be the case in examples with focus marking or clefting, where the set of alternatives provided by focus (or the background question) can serve as the pivot.

We believe speakers arrive at the truth-value intuitions TRUE and FALSE that we found as follows. For the reasons described above, we believe that the facts we found can be explained best on pragmatic presupposition theory such as Stalnaker's. Here is how the reasoning might go: (i) Sentences with reference failure NP's are semantically false (or true, if negated), but infelicitous because of presupposition failure. (ii) The presupposition failure can be ignored (and so the predicted infelicity can disappear) if a potential pivot that is independent from the reference failure NP is present in the sentence ( $\approx$ von Fintel). (iii) The above effect is even stronger, if the independent, referentially sound NP is topicalised $(\approx$ Strawson/Reinhart). This latter fact is because topics are default pivots, and hearers tend to verify sentences based on the sentence's topic. (iv) The effect is also stronger if the speaker has direct knowledge about the properties of the potential pivot, which contradicts the proposition irrespective of whether the entity denoted by the reference failure NP exists or not ( $\approx$ Lasersohn). 
Acknowledgements. Thanks to György Abrusán for his invaluable help with statistics. We are grateful to Nathan Klinedinst and Daniel Rothschild for insightful comments on an earlier draft, as well as Klaus Abels, Bob Borsley, Judit Gervain, Vanessa Harrar, Ad Neeleman and the audiences at the UCL LingLunch and the Departmental Colloquium at Essex University for their helpful questions and suggestions. The first author acknowledges financial support by the Mellon Foundation and the Lichtenberg Kolleg, Georg-August-Universität Göttingen. All remaining errors are our own.

\section{Selected References}

1. Erteschik-Shir, Nomi (2007). Information structure. Oxford University

2. von Fintel, Kai (2004). Would you believe it? The King of France is back! (Presuppositions and truth-value intuitions.) In Anne Bezuidenhout and Marga Reimer, (eds.). Descriptions and beyond: an interdisciplinary collection of essays on definite and indefinite descriptions and other related phenomena, Oxford University Press, 315-341.

3. Givon, Talmy (1983). Topic continuity in discourse: An introduction. In: Givon, T. (ed). Topic continuity in discourse: A quantitative cross-language study. John Benjamins: Amsterdam/Philadelphia.

4. Karttunen, Lauri and Peters, Stanley (1979). Conventional Implicatures in Montague Grammar, in Oh, C.-K. and Dineen, D. (eds.), Syntax and Semantics 11: Presupposition, New York: Academic Press, 1-56.

5. Kuno, Susumu (1972). Functional sentence perspective: A case study from Japanese and English. Linguistic Inquiry 3, 269-320.

6. Lasersohn, Peter (1993). Existence presuppositions and background knowledge. Journal of Semantics 10 (2), 113-122.

7. Lambrecht, Knud (1994). Information structure and sentence form: A theory of topic, focus and the mental representations of discourse referents. Cambridge University Press.

8. Reinhart, Tanya (1981). Pragmatics and linguistics: An analysis of sentence topics. Philosophica 27, 53-94.

9. Roberts, Craige (to appear) Topics. In Claudia Maienborn, Klaus von Heusinger \& Paul Portner (eds.) Semantics: An International Handbook of Natural Language Meaning. Mouton de Gruyter.

10. Russell, Bertrand (1905). On Denoting. Mind 14, 479-493.

11. Russell, Bertrand (1957). Mr. Strawson on Referring. Mind 66, 385-389.

12. Schlenker, Philippe (2008) Be articulate: A pragmatic theory of presupposition, Theoretical Linguistics, 34: 157-212.

13. Schoubye, Anders (2009). Descriptions, Truth-value Intuitions, and Questions. Linguistics and Philosophy 32 (6), 583-617.

14. Simons, Mandy (2001) On the conversational basis of some presuppositions, in Hastings, R. and Jackson, B. and Zvolensky, Z. (eds.), Proceedings of Semantics and Linguistics Theory 11, Ithaca, NY: CLC Publications, 431-448.

15. Stalnaker, Robert C. (1974). Pragmatic Presuppositions. In Munitz, Milton and Unger, Peter (eds.) Semantics and Philosophy: Essays. New York University Press.

16. Stalnaker, Robert C. (1978). Assertion. In Cole, P. (ed.) Syntax and Semantics 9. New York: New York Academic Press, pp. 315-32.

17. Strawson, Peter F. (1950). On Referring. Mind 59, 320-344.

18. Strawson, Peter F. (1964). Identifying reference and truth-values. Theoria 30 (2), 96-118. 


\title{
Adjectives as saturators vs. modifiers: Statistical evidence
}

\author{
Gemma Boleda $^{1}$, Stefan Evert ${ }^{2}$, Berit Gehrke $^{1}$, and Louise McNally ${ }^{1}$ \\ 1 Universitat Pompeu Fabra, Roc Boronat 138, \\ 08018 Barcelona, Spain \\ \{gemma.boleda, berit.gehrke, louise.mcnally\}@upf .edu, \\ http://www.upf.edu/ \\ 2 Technische Universität Darmstadt, Hochschulstrasse 1, \\ 64289 Darmstadt, Germany \\ evert@linglit.tu-darmstadt.de, \\ http://www.tu-darmstadt.de/
}

\begin{abstract}
This paper reports on a large-scale, statistical analysis of corpus data to support the null hypothesis that ethnic adjectives (EAs, e.g. French) are ordinary adjectives, rather than argument-saturating "nouns in disguise" (in, e.g., French agreement). In particular, EAs are argued to simply modify the noun they combine with, so that their special properties in inducing argument-like behavior arises from the interaction between the semantics of event nominals and that of the adjective.

Keywords: adjective, argument structure, kind modifier, modification, nominalization, lexical semantics, logistic regression model, statistical analysis
\end{abstract}

\section{Introduction}

Though surprising given standard assumptions about the formal semantics of adjectives, denominal relational adjectives, such as molecular and the so-called ethnic adjective (EA) subclass of these, e.g. French, have been repeatedly claimed in the syntactic literature to be able to saturate the presumably e-type arguments of the nouns they combine with $[16,6,10,1]$. On such a view, the compositional contribution of e.g. French in (1-a) would be identical to that of the PP in (1-b), saturating the agent argument of agreement, though specific semantic analyses for how this is done are not offered in this literature.

(1) a. French agreement to participate in the negotiations

b. agreement by France to participate in the negotiations

[3], in contrast, focusing specifically on ethnic adjectives, defend the null hypothesis that EAs are ordinary adjectives that simply modify the noun they combine with, such that the argument-like interpretation arises from the interaction between the semantics of the nominalization and that of the adjective. The goal of this paper is to show that large-scale, statistical corpus data analysis supports the latter analysis. 


\section{The two competing analyses}

We begin by sketching the main features of the argument-saturating analysis and the modifier analyses. For reasons of space, we only discuss [1] to exemplify the former and [3] for the latter, but our comments should generalize to other analyses that preserve the key features of each type of analysis. ${ }^{3}$

\subsection{The argument-saturating analysis}

[1], working in the framework of Distributed Morphology, treat thematicallyused EAs such as those in (1-a $)^{4}$ as covert nominals whose nominal source is visible at the level of interpretation. They bear the agent thematic role assigned to them by the (deverbal) noun they modify but lack case. Since every noun needs case, these EAs are deficient and become adjectives in the course of the syntactic derivation. The analysis of a (Greek) example of theirs is shown in (2).

$$
\begin{aligned}
\text { a. } & \text { germaniki epithesi } \\
& \text { German attack } \\
\text { b. } & {\left[\mathrm { DP } [ \ldots ] \left[\mathrm{FP} / \mathrm{AGRP}\left[\mathrm{a}(\mathrm{sp}) \mathrm{P}\left[\mathrm{a}(\mathrm{sp})^{\prime}\left[\mathrm{a}(\mathrm{sp})^{0} \operatorname{german}_{1}\left[\mathrm{a}(\mathrm{sp})^{0-\mathrm{ik}}\right]\right]\right]\right]\right.\right.} \\
& {\left[\mathrm{F}^{\prime} \mathrm{F}\left[{ }_{n P}\left[\mathrm{DP} t_{1}\left[\mathrm{n}^{\prime} \mathrm{n}[v P \mathrm{~V} \sqrt{\mathrm{EPITH}}]\right]\right]\right]\right] }
\end{aligned}
$$

German- starts out as a DP in the specifier of the noun phrase epithesi 'attack', represented in (2-b) via the root $\sqrt{ }$ EPITH. ${ }^{5}$ In this position, germanis necessarily assigned the agent theta role by the underlying verb, on analogy to genitive DPs, which are also generated in this position. Since german- is not valued for case, it is forced to move up and to adjoin as a head to a(sp), the head of an adjectival projection that generally occupies the specifier position of a functional category between $\mathrm{D}$ and $\mathrm{N}$, where it is spelled out as an adjective. On this account, both thematically-used EAs and genitive DPs (e.g. the Germans' (attack)) or PPs (e.g. (the attack) of the Germans) are base-generated in the same position, hence their relation to the nominal they combine with is the same, namely they saturate the agent argument of the nominal.

One argument put forward for nominal approaches to EAs is the fact that they do not behave like typical adjectives in some respects. For example, EAs cannot be used predicatively $((3-\mathrm{a}))$, are not gradable $((3-\mathrm{b}))$ and cannot be coordinated with 'normal' adjectives $((3-c))$ (examples from [1]).

(3) a. *The intervention in Cyprus was American.

b. *the very / more American invasion

c. *the immediate / quick / possible and American intervention

\footnotetext{
3 This section draws heavily on [3]; see that work for further details and discussion.

${ }^{4}$ EAs have been argued to have a second, classificatory use (e.g. French classifies wine in French wine); see references cited for discussion. [1] treat such adjectives as merely homophonous to thematically-used EAs, whereas e.g. [10] and [3] provide (contrasting) uniform accounts.

${ }^{5}$ Presumably, the nominalizing suffix -esi sits in $n$; these details are left out in [1].
} 
However, a serious problem for the nominal account is that the EA does not behave like a typical noun, either. Already [18] noted that EAs are 'anaphoric islands': the alleged nominal underlying the EA does not license anaphora ((4-a)). This fact is also acknowledged by [1], and they provide further examples that show the failure of EAs to bind reflexives ((4-b)), antecede personal pronouns, or control a relative pronoun (see [1] for examples).

(4) a. *The American ${ }_{i}$ proposal to the UN reveals its ${ }_{i} /$ her $_{i}$ rigid position. America ${ }_{i}$ 's proposal to the UN reveals its $_{i} /$ her $_{i}$ rigid position.

b. The Albanian destruction (*of itself) grieved the expatriot community.

[1] argue that the status of EAs as anaphoric islands follows from the fact that the underlying noun is morphologically deficient, which results in it becoming an adjective in the course of the derivation. They stipulate that the resulting 'adjective' is deprived of typical nominal anaphoric properties, and that anaphoric rules are sensitive to surface structure configurations only, even though for argumentsaturation purposes the nominal nature of EAs is still visible.

We consider this an inelegant solution at best. The facts in (3) do not force the abandonment of an adjectival analysis of EAs in favor of a nominal one, given that many indisputable adjectives (e.g. alleged, main, other) display similar properties (see also [11]); and since there is ample evidence that non-quantificational saturaters of e-type arguments quite systematically license discourse anaphora to token individuals, [1]'s analysis effectively renders EAs an anomaly. There are therefore good reasons to explore alternative analyses.

\subsection{The modifier analysis}

Building on [17] and others, [3] posit that nouns denote descriptions of kinds of individuals. EAs modify these descriptions, introducing a contextually-valued relation $(R$ in $(5-b))$ between the kind described by the noun and the country, ethnicity, etc. ${ }^{6}$ associated with the EA ( $\mathbf{R}$ is $[8]$ 's realization relation). Number turns the resulting property of kinds into a property of token entities $((5-\mathrm{d}, \mathrm{e}))$.

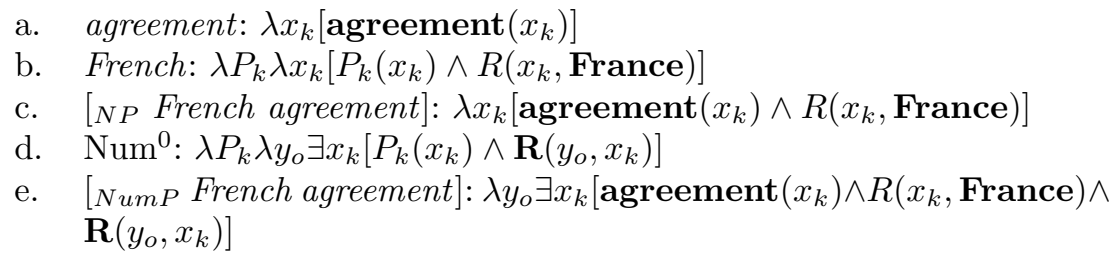

The relation $R$ is the source of the argument-saturating effect, as nothing in principle prevents it from corresponding to a thematic relation when the modified nominal describes a kind of eventuality. Since EAs do not denote entities, the

\footnotetext{
${ }^{6}$ For brevity, hereafter we refer to this entity simply as the country, since our study involved only names of countries.
} 
analysis predicts their general failure to introduce discourse referents, and thus their failure to license discourse anaphora.

Essential to understanding the relative distribution of EAs vs. PPs is the observation that the value of $R$ is restricted, as seen in the oddness of (6-a) out of context to describe a visit to Canada; a PP is strongly preferred, as in (6-b).

(6) a. Yeltsin met the prospective Democratic presidential candidate Bill Clinton on June 18. His itinerary also included ??an official Canadian visit.

b. Yeltsin met the prospective Democratic presidential candidate Bill Clinton on June 18. His itinerary also included an official visit to Canada. (BNC)

To account for this and similar restrictions on the classificatory use of EAs, [3] argue that in the default case, $R$ is the Origin relation defined in (7).

(7) $\operatorname{Origin}(x, y)$ iff $x$ comes into existence within the spatial domain of $y$.

Crucially, they posit that not only kinds of concrete objects (e.g. French bread) but also kinds of eventualities participate in this relation, by suggesting that the agent(-like) participant in a kind of eventuality can be considered its origin. Origin is a default value for $R$ because other interpretations are possible when prior discourse makes it clear what that specific relation is between the country and the referent of the head noun (see [3] for examples). Conceiving of the agent relation as a subcase of a default Origin value for $R$ allows [3] to provide a unified account of both the basic semantics for the thematic and classificatory uses of EAs and the restrictions on the specific interpretations under these uses.

In our empirical study, we looked at three contrasting predictions of the two analyses that could be translated into features that could be automatically extracted from a corpus containing only morphosyntactic annotation.

\subsection{Predictions}

Prediction 1. The argument-saturating analysis predicts the distribution of EAs vs. PPs to be roughly the same with event nominals, all other things being equal, given that both are treated as nominals that saturate an argument of the noun they modify. In contrast, the modifier analysis predicts event nominals to combine less often with EAs because when the event nominal has argument structure that must be saturated (i.e. is complex in the sense of [12]), the EA will not be able to do the job, and thus a $\mathrm{PP}$ will be required.

Prediction 2. Since, in nondefault cases, the modifier analysis relies on context to supply the identity of the relation between the referent of the head nominal and the country, this analysis predicts the distribution of EAs to be more restricted than that of PPs, as the latter make the relation explicit via the preposition and thus are not context-dependent in the same way. Specifically, EAs should occur only when the relevant relation $(R$ in $(5-b))$ is default or entailed by prior context. In contrast, the argument-saturating analysis predicts, 
all things being equal, no sensitivity to context in the distribution of EAs vs. PPs.

Prediction 3. A third difference in prediction is specific to analyses such as [3]'s that treat the EA as a modifier of kind descriptions as opposed to token descriptions. A modifier of kind descriptions produces a description of subkinds of the modified kind description. For example, French bread and Italian bread describe subkinds of bread. We assume that there must be nontrivial criteria that motivate the use of a subkind: a certain number of recognizable instances, an "act of baptism", a recognizable property that characterizes the subkind in contrast to other subkinds, etc. We therefore expect the use of EAs to be concentrated in a comparatively smaller number of nouns than the PPs, i.e. those that meet these criteria, rather than being thinly and evenly distributed across all nouns, as would be expected if the EA could denote a modifier of any token individual description. Conversely, though we see no reason in principle for PPs whose DP complements denote token individuals to be prohibited from serving as a modifier of kind descriptions, we also see no reason for them not to be used as complement or modifiers to descriptions of token entities. The argument-saturating analysis, again all things being equal, does not predict this asymmetric distribution, as it does not provide any basis to distinguish EAs and PPs in terms of the sorts of descriptions they can combine with.

In a previous study on the British National Corpus that did not employ a statistical model [5], we found that this prediction was in fact borne out and that the effect was even more pronounced with EAs with a low frequency and with event nominals. From this we concluded that use of the EA positively correlates with concept stability, i.e. the degree to which the full noun phrase describes a well-established subkind of (abstract or concrete) entities. We posited 1) that stable subkinds describable with EAs are unlikely to be formed for events (e.g. we do not classify e.g. agreements according to who makes them) and 2) that fewer stable concepts are formed for those countries we talk about less. However, as will be discussed in Section 4, with the statistical model we obtain different results, so this is a parameter that needs further exploration.

These different predictions grounded our decisions about which features in the corpus to include in the statistical analysis. For Prediction 1, the feature was whether the noun was an event nominal or not. For Prediction 2, since our corpus lacks any semantic annotation, we approximated prior contextual entailment of the value for $R$ with features that correlate with prior mention of the relation: the definiteness of the DP containing the target EA/PP and prior mention of the EA, the head noun, and the corresponding country noun. We also could not test Prediction 3 directly because it is sensitive to the number of types of lemmata, and our model operates on the token level. To approximate type frequency, we chose the frequency of the head noun as a factor, on the hypothesis that wellestablished concept descriptions will tend to be formed with nouns of a higher frequency, and thus that EAs will occur more often with these nouns than with low frequency nouns, once possible collocational relations between the EA and head noun as well as effects due to variation in the overall token distribution 
of the EA vs. the corresponding country noun are controlled for. We will see, however, that this hypothesis turned out to be incorrect.

\section{Method}

We conducted a study on the British National Corpus (BNC) in order to determine the factors influencing the choice between an EA and a PP, for a sample of 44 different countries whose adjective (e.g., French) and proper noun (France) forms occur between 1,000 and 30,000 times in the BNC. We tested the predictions outlined in the previous section by defining features that could be automatically extracted from a corpus (by running computer programs on the information contained in the BNC) and tested as factors in a statistical model. For instance, to test for definiteness of the NP containing the EA or PP (for Prediction 2), we searched for the words the, this, that, these, and those, followed by at most 4 (for EAs) or 5 (for PPs) arbitrary words excluding verbs, nouns, prepositions, pronouns, determiners, subordinating conjunctions, and punctuation (but optionally allowing for a comma after each adjective) preceding the relevant EA or PP. This type of approach is noisy, that is, the information thus gathered is just an approximation of the real syntactico-semantic information that we want to model. However, it has the advantadge that it can be applied on a large scale and that it can be refined and extended with very little effort, so that very different types of information can be explicitly coded and tested.

Our model contains information for the 74,094 occurrences of the relevant adjectives and prepositional phrases (target expressions from now on) found in the BNC. These data were analyzed with a logistic regression model [13], which predicts the probability of an adjective realization based on the specified factors and their interactions. Logistic regression has recently become a popular approach for the analysis of similar binary choice problems in quantitative linguistic studies, such as the English dative alternation [7]. ${ }^{7}$ For model fitting and analysis, we use the $\mathrm{R}$ package $\mathrm{rms}$ [14].

Our best model used 9 factors, which are listed below and grouped according to the theoretical predictions they are connected to. The factor labels shown here will also be used in the presentation and discussion of the results in Section 4 .

Prediction 1: tco1: the semantic sort of the head noun, according to the Top Concept Ontology resource (TCO, [2]). This resource restructures the noun hierarchy in WordNet $1.6^{8}$ into a coarse-grained ontology. We only use the highest level of the TCO concept hierarchy, which divides the nominal domain into, roughly, object, event, and abstract nouns.

Prediction 2: definite: the definiteness of the NP containing the target expression, defined as explained above; recent-mod: distance, in number of words, to the last mention of the target expression in the same discourse,

\footnotetext{
${ }^{7}$ See [13] for a detailed introduction to logistic regression, or [4, Sec. 6.3.1] for practical examples of its application in linguistics.

8 A lexical semantic resource for English, see http://wordnet.princeton.edu/.
} 
rescaled as a "decaying activation" $10 /(9+$ distance $)$, or 0 if no previous mention is found; last-mod-equiv: whether the previous mention is of the same form (adjective or noun) as the target expression; recent-head: same as recent-mod, but for the head noun and rescaled as $1 /$ distance. $^{9}$

Prediction 3: collocAN: the collocational strength between head noun and ethnic adjective, measured by a conservative estimate of pointwise mutual information ([15] and [9, p. 86ff.]); nhead: total frequency of the head noun (log-transformed); ntotal: total frequency of the target expression (both adjective and noun form, log-transformed); log-odds-ea-country: ratio between the frequencies of adjective and noun forms of the target expression in the corpus (log-transformed).

\section{Results}

From a logistic regression analysis, two basic insights can be gained: (i) which factors or combinations of factors ("interactions") play a significant role in the choice between EA and PP; and (ii) for each significant factor, to what extent and in which manner it increases or decreases the likelihood of an adjective realization (the "partial effect" of the factor).

Following [13, Ch. 10], we test the significance of factors and interactions by analysis of variance based on the asymptotic standard errors of coefficient estimates (so-called Wald statistics). Table 1 shows highly significant effects for all 9 predictive factors included in the model, lending initial support to the modifier analysis. There is no clear evidence for an interaction between the factors: most interaction terms are not significant or only weakly significant (not shown in the table). Considering the large sample size, we feel that inclusion of such interactions in the model is not justified at this point.

Figure 1 displays a graphical representation of the partial effect of each factor on the likelihood of an adjective realization. In the log odds scale, a value of 0 corresponds to equal likelihood of adjective and noun; positive values indicate that an EA is more probable than a PP. The baseline adjective likelihood of $70 \%$ corresponds to a log odds slightly below 1. For example, the middle left panel shows that speakers are more likely to use an adjective with low-frequency head nouns. The same holds if there was a prior mention of the target expression in the same discourse (top center panel). Prior mention of the head noun has an opposite effect (top left panel), but the shaded confidence band around the line indicates considerable uncertainty.

Prediction 1 is borne out, as event-denoting nouns strongly disprefer EAs: In the top right panel of Figure 1, adjective likelihood is considerably lower for 2nd Order Entities (the TCO equivalent of event-related nouns) than for 1st Order Entities (object-denoting nouns in the TCO). This is also the most significant effect in Table 1. The results also support Prediction 2, as (a) NPs with definite determiners (bottom center panel in Figure 1) have a slight preference for PPs,

\footnotetext{
${ }^{9}$ The different scaling formulae for recent-mod and recent-head were found by manual experimentation and resulted in a better fit of the logistic regression model.
} 


\begin{tabular}{|c|c|c|c|}
\hline \multicolumn{2}{|c|}{$\begin{array}{l}\text { Prediction Factor } \\
\end{array}$} & \multicolumn{2}{|c|}{ Chi-Square d.f. } \\
\hline 1 & tco1 & 2507.40 & $2<.0001$ \\
\hline \multirow{4}{*}{2} & definite & 42.19 & $1<.0001$ \\
\hline & recent-mod & 658.74 & $1<.0001$ \\
\hline & last-mod-equiv & 46.39 & $1<.0001$ \\
\hline & recent-head & 20.76 & $1<.0001$ \\
\hline \multirow{5}{*}{3} & collocAN & 14.30 & 10.0002 \\
\hline & nhead & 648.24 & $1<.0001$ \\
\hline & ntotal & 8.37 & 10.0038 \\
\hline & log-odds-ea-country & 1230.93 & $1<.0001$ \\
\hline & TOTAL & 5598.08 & $10<.0001$ \\
\hline
\end{tabular}

Table 1. Logistic Regression Model: Results of an ANOVA test on the model (Wald Statistics, response: ethnic adjective). Data distribution: Adjectives (EAs): 51,946 datapoints (70\%), nouns (PPs): 22,148 datapoints. See Section 3 for the interpretation of each factor.

(b) a recent mention of the target expression (modifier) favors the use of an EA (top center panel), and (c) a prior mention of the head noun is associated with a PP rather than EA realization (top left panel). ${ }^{10}$

However, the results do not support Prediction 3, as (a) the collocational strength between the EA and head noun has only a very small effect (bottom left panel), (b) frequent head nouns typically prefer the PP realization rather than the EA (contrary to what we expect based on a modifier analysis; middle left panel), and (c) the overall frequency of the target expression has virtually no effect (whereas we expected more familiar countries to combine more readily with EAs than infrequent countries; middle center panel). However, manual inspection of the corpus data revealed that many of the apparent counterexamples to Prediction 3 involve descriptions of unique individuals (e.g., Gulf of Mexico), which have been predicted to resist EAs [3]. Further examination of these data may thus lead to better results. Finally, lexical effects also seem to play an important role, as the overall corpus ratio of adjective/noun expressions has a very large effect on the EA likelihood (middle right panel).

The goodness-of-fit of the logistic regression model is not quite satisfactory yet, with a Nagelkerke $R^{2}$ of $11.5 \%$ and $c$ index of $67.5 \%$ indicating low discriminative power $[13$, p. 247$] .{ }^{11}$ As explained in the previous section, all the data used in this study were automatically obtained, a method that, for semantic analysis in particular, adds noise to the data set insofar as the factors are

${ }^{10}$ It has to be noted that (a) and (c) are relatively small effects despite their high significance, so they do not provide strong evidence either for or against the modifier analysis.

${ }^{11}$ The $c$ index shows how well the model can discriminate between an example of an EA realization and an example of a PP realization, with a value of 0.5 corresponding to random guesses. It has been shown that $c$ is identical to another popular evaluation measure, the area under ROC curve. 


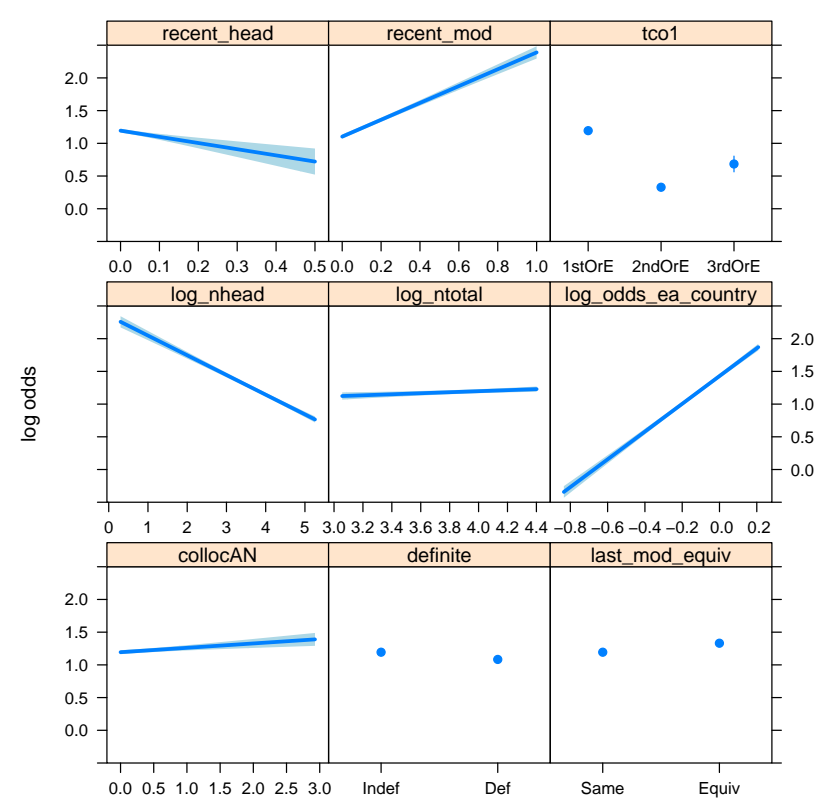

Fig. 1. Logistic Regression Model: graphical representation of the effect of each factor.

sometimes imperfectly correlated with meaning. Moreover, we are testing the effect of only 9 factors for more than 74,000 tokens. As the choice between EA and $\mathrm{PP}$ is a multi-faceted and intricate problem, we expect that adding more factors will improve the predictive power of the model. In particular, we plan to add factors such as posterior mention of the EA/country noun (as discourse topichood of the country may be a factor) and the presence of other modifiers in the target NP (since syntax allows only one EA per DP).

\section{Conclusions}

We presented statistical support for an account of ethnic adjectives as modifiers, as opposed to saturators. We tested three predictions of the modifier analysis on automatically extracted data for over 70,000 phrases and showed support for two of them; however, the statistical model is still not predictive enough. We have discussed ways in which the model can be enhanced in the future. Given that automatically extracted information is noisy, another enhancement we are planning is to build a supplementary statistical model on a much smaller, manually annotated set of data points. The annotation will include information that cannot be automatically extracted and that has been found to be relevant in the analysis of the results, e.g., whether the NP denotes a unique entity. 
Acknowledgments. This work was supported by grants from the Spanish Ministry of Science and Innovation (FFI2010-15006, TIN2009-14715-C04-04 (KNOWII), JCI-2010-08581), as well as by the European Union via the EU PASCAL2 Network of Excellence (FP7-ICT-216886) and by the Fundació ICREA.

\section{References}

1. Alexiadou, A., Stavrou, M.: Ethnic adjectivs as pseudo-adjectives: A case study in syntax-morphology interaction and the structure of DP. Studia Linguistica 65, $1-30(2011)$

2. Álvez, J., Atserias, J., Carrera, J., Climent, S., Laparra, E., Oliver, A., Rigau, G.: Complete and consistent annotation of wordnet using the top concept ontology. In: 6th Language Resources and Evaluation Conference (LREC 2008). Marrakech, Morocco (2008)

3. Arsenijević, B., Boleda, G., Gehrke, B., McNally, L.: Ethnic adjectives are proper adjectives. In: Proceedings of CLS 46 (to appear)

4. Baayen, R.H.: Analyzing Linguistic Data. A Practical Introduction to Statistics Using R. Cambridge University Press, Cambridge (2008)

5. Berndt, D., Boleda, G., Gehrke, B., McNally, L.: Semantic factors in the choice between ethnic adjectives and PP counterparts: Quantitative evidence (2011), paper presented at QITL-4, Berlin, March 2011

6. Bosque, I., Picallo, C.: Postnominal adjectives in Spanish DPs. Journal of Linguistics 32, 349-386 (1996)

7. Bresnan, J., Cueni, A., Nikitina, T., Baayen, R.H.: Predicting the dative alternation. In: Bouma, G., Krämer, I., Zwarts, J. (eds.) Cognitive Foundations of Interpretation, pp. 69-94. Royal Netherlands Academy of Science, Amsterdam, Netherlands (2007)

8. Carlson, G.N.: Reference to Kinds in English. Ph.D. thesis, University of Massachusetts at Amherst (1977)

9. Evert, S.: The Statistics of Word Cooccurrences: Word Pairs and Collocations. Dissertation, Institut für maschinelle Sprachverarbeitung, University of Stuttgart (2004), published in 2005, URN urn:nbn:de:bsz:93-opus-23714.

10. Fábregas, A.: The internal syntactic structure of relational adjectives. Probus 19.1, 135-170 (2007)

11. Gehrke, B., McNally, L.: Frequency adjectives and assertions about event types. In: Proceedings of SALT 19. pp. 180-197. CLC Publications, Ithaca, NY (2011)

12. Grimshaw, J.: Argument Structure. MIT Press, Cambridge, MA (1990)

13. Harrell, F.: Regression Modeling Strategies. Springer, Berlin (2001)

14. Harrell, F.: rms: Regression Modeling Strategies (2011), http://CRAN .R-project. org/package $=$ rms, $\mathrm{r}$ package version 3.3-2

15. Johnson, M.: Trading recall for precision with confidence sets (2001), unpublished technical report

16. Kayne, R.: On certain differences between French and English. Linguistic Inquiry 12, 349-371 (1981)

17. McNally, L., Boleda, G.: Relational adjectives as properties of kinds. In: Bonami, O., Hofherr, P.C. (eds.) Empirical Issues in Syntax and Semantics, vol. 5, pp. 179-196. http://www.cssp.cnrs.fr/eiss5 (2004)

18. Postal, P.: Anaphoric islands. In: Binnick, R.I. (ed.) Proceedings of the Fifth Regional Meeting of the Chicago Linguistics Society, pp. 205-239. University of Chicago, Department of Linguistics, Chicago (1969) 


\title{
Licensing Sentence-internal Readings in English An Experimental Study
}

\author{
Adrian Brasoveanu \& Jakub Dotlačil \\ Linguistics, UCSC, 1156 High St., Santa Cruz, CA 95064 \\ abrsvn,j.dotlacil@gmail.com
}

\begin{abstract}
Adjectives of comparison (AOCs) like same, different and similar can compare two elements sentence-internally, i.e., without referring to any previously introduced element. This reading can only be licensed if a semantically plural NP is present. In contrast to almost all previous literature, we argue in this paper that it is incorrect to describe a particular NP as either licensing or not licensing the sentence-internal reading of a specific AOC: licensing is more fine-grained. We use experimental methods to establish which NPs license which AOCs and to what extent. We show how the results can be interpreted against the background of a formal semantics analysis of AOCs and we argue that using Bayesian methods to analyze this kind of data has several advantages over the more traditional, frequentist approach.
\end{abstract}

\section{The phenomena}

Most, if not all, languages have lexical means to compare two elements and express identity / difference / similarity between them. English uses adjectives of comparison (henceforth AOCs) like same, different and similar for this purpose. Often, the comparison is between an element in the current sentence, e.g., the italicized NP the same movie in (1b) below, and a sentence-external element mentioned in the previous discourse, e.g., the underlined NP 'Waltz with Bashir' in (1a). AOCs can also compare sentence-internally, that is, without referring to any previously introduced element, as shown in (2). In this kind of cases, the sentence itself, as it were, provides the context for the comparison, hence the label of sentence-internal reading.

(1) a. Arnold saw 'Waltz with Bashir'.

b. Heloise saw the same movie / a different movie.

(2) Each of the students saw the same movie / a different movie.

The sentence-internal reading is available only if the sentence in which the AOC occurs also contains a semantically (but not necessarily morphologically) plural noun. Importantly, not all semantically plural NPs can license sentence-internal readings of AOCs. This has already been observed in previous literature on the topic (see [1], [2], [3], [4], [5], [8], among others). The previous literature also 
noted that many NPs license sentence-internal readings of only some AOCs (see [3] for a recent detailed discussion and summary of the previous literature).

However, it is much less known that the majority of semantically plural NPs cannot be described as either licensing or not licensing the sentence-internal reading of a specific AOC. Licensing is more fine-grained. The gradient nature of AOC licensing has not been acknowledged with the exception of [5] for different and has not been systematically studied. In this paper we report one experiment that begins to address this issue by establishing which NPs license which AOCs and to what extent. Furthermore, we argue that using Bayesian methods to analyze the resulting experimental data has several advantages over the more traditional, frequentist approach. The paper concludes with a brief discussion of the consequences of the experimental results for the semantic analysis of AOCs.

\section{Experiment}

\subsection{Method}

We used questionnaires to test people's intuitions about sentence-internal readings of three AOCs - same, different and similar, with four licensors - NPs headed by each, all, none and the (for a total of $3 \times 4=12$ conditions). Each condition was tested four times, twice in a scenario in which the condition was most likely judged as true and twice in a scenario in which the condition was most likely judged as false. There were 32 fillers.

An example of a scenario and three test items testing the sentence-internal reading of similar, same and different is given below. In the actual setup, each scenario was followed by five items, two of which were fillers. For each scenario, each of its corresponding test items had a different AOC and a different licensor.

(3) Gustav, Ryan and Bill are three bank managers who share a passion for Volvo, Rolls Royce and Porsche automobiles. Last year, each of them bought a new car. Gustav bought a Volvo PY30, Ryan bought a Volvo XRT2000 and Bill bought a Volvo H4.

a. Each of the bank managers chose a similar car.

b. All the bank managers chose the same car brand.

c. None of the bank managers chose a different car brand.

Each item was judged with respect to $(i)$ TRUTH: whether it is true, false or unknown given the accompanying scenario and (ii) ACCEPT(ABILITY): how acceptable it is on a 5 -point scale $(5=$ completely acceptable, $1=$ completely unacceptable). TRUTH was measured so that it could be distinguished from ACCEPT.

A total of 42 subjects in two undergraduate classes at UCSC completed the questionnaire for extra-credit. For each subject, we randomized both the order of the scenarios in the questionnaire and the order of the items for each scenario. We excluded two subjects because of their incorrect responses to fillers and one because only TRUTH was completed; one of the remaining 39 subjects filled in only three fourths of the questionnaire. Final number of observations: $n=1856$. 
Barplots of ACCEPT for the 12 conditions are shown in Figure 1, from the least acceptable, i.e., sentence-internal different when the licensor NP is headed by none, to the most acceptable, i.e., sentence-internal same when the licensor NP is headed by all.

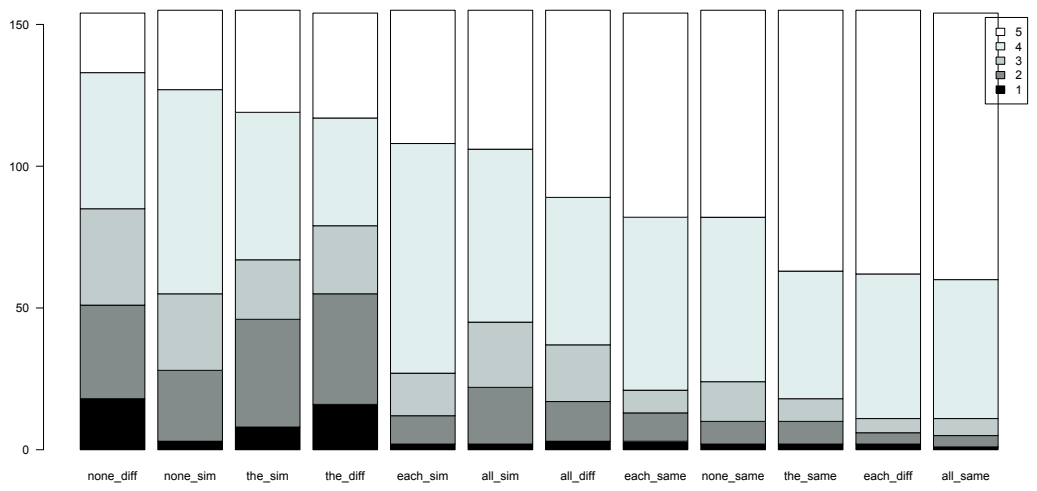

Fig. 1. Barplots of responses by quant-AOC combination

\subsection{Statistical modeling and resulting generalizations}

The response variable ACCEPT is ordinal, so we use ordered probit regression models to analyze the data. These models are similar to linear regression models in that the predictors are linearly combined and the weights / coefficients for each predictor are estimated from the data. The linear combination of predictors provides the mean for a normal distribution with a fixed variance (set to $1^{2}$ for simplicity). To put it differently, the linear combination of predictors provides an 'offset' for the mean of the standard normal distribution. The area under the probability density function obtained in this way is partitioned into five regions by four thresholds (also estimated from the data) and each region corresponds to one value of the ordinal variable.

We have 2 fixed-effects predictors: $(i)$ QUANT-AOC-factor with 12 levels since we have 12 licensor-AOC combinations, reference level: the each-different combination; (ii) TRUTH-factor with 3 levels T(rue), F(alse), U(nknown), reference level: T. Our main interest is in how QUANT-AOC affects ACCEPT while controlling for / factoring out the influence of TRUTH on ACCEPT.

A frequentist analysis shows that adding either of the fixed effects to the null (intercept-only) model significantly decreases deviance, but the interaction of the fixed effects does not $(\mathrm{p}=0.31)$. That is, licensor-AOC combinations and truth-value judgments significantly and additively influence acceptability judgments. Adding intercept-only random effects for items accounts for practically no variance, but adding random effects for subjects does (std.dev=0.56). Thus, the final regression model $\mathcal{M}$ we henceforth focus on has 2 fixed effects, QUANT-AOC and TRUTH (no interaction), and intercept-only random effects for subjects.

Our primary interest is to establish which NPs license sentence-internal readings of which AOCs and to what extent. That is, we are interested in a wide 
range of pairwise comparisons between various licensor-AOC combinations. But doing this in the null-hypothesis significance testing framework would require an unfeasibly large amount of data to achieve significance given the necessary $\alpha$-level correction for running all pairwise comparisons between the 12 licensorAOC combinations (66 comparisons in total).

In contrast, any number of pairwise comparisons can be carried out in a Bayesian framework because we do not use p-values as a criterion for decision making. Instead, we simply study the multivariate posterior distribution of the parameters obtained given our prior beliefs, the data and our mixed-effects order probit regression model $\mathcal{M}$. Pairwise comparisons of various licensor-AOC combinations are just different perspectives on, i.e., different ways of marginalizing over, this posterior distribution (see [6], [7] and references therein for more discussion). To determine whether there is a credible difference between any two conditions, we check whether 0 (=no difference) is in the $95 \%$ highest posterior density interval (HDI; basically, a 95\% confidence interval) of the difference: if 0 is outside the HDI, the two conditions are credibly different.

The Bayesian model we estimate has the following structure: $(i)$ we assume low-information / vague priors for the non-reference levels of QUANT-AOC and TRUTH - independent normal distributions with mean 0 and variance $10^{2}$; $(i i)$ the subject random effects are assumed to come from a normal distribution with mean 0 and variance $\sigma^{2}$, with $\sigma$ taken from a uniform distribution $\operatorname{Unif}(0,10)$. As we indicated above, the function linking the linearly combined predictors and the response ordinal value is the standard normal cumulative distribution function $\Phi$. The range of $\Phi$ is partitioned into five intervals (since the acceptability scale was 1-5) by 4 cutoff points / thresholds; the low-information priors for the thresholds are also independent normal distributions with mean 0 and variance $10^{2}$. We estimate the posterior distributions of the predictors QUANT-AOC and TRUTH, the standard deviation $\sigma$ of the subject random effects and the 4 thresholds by sampling from them using Markov Chain Monte Carlo techniques (3 chains, 125, 000 iterations per chain, we discard the first 25, 000 iterations and record only every $50^{\text {th }}$ one).

The posterior histograms for the most relevant comparisons are shown in Figures 2-4 below, grouped by AOC. The resulting generalizations are summarized at the top of each set of plots, where $>$ means the licensor(s) on the left is / are preferred to the licensor(s) on the right.

Figure 2 below shows that each is a better licensor of sentence-internal different compared to all, which in turn is better than definite plurals and negative quantifiers. However, we cannot confidently distinguish between definite plurals and negative quantifiers since the HDI of the difference between them includes 0 . Nonetheless, it is likely that slightly more data would enable us to distinguish between the acceptability of these last two licensors - in contrast to the rightmost plot in Figure 3, for example, where the difference between two licensors is virtually non-existent.

Thus, examining the posterior probability distributions reveals not only whether two conditions are different or not, but also how confident we are in postulating 
a difference and the magnitude of this difference. The corresponding generalizations for the licensors of same and similar are provided in Figures 3 and 4 .

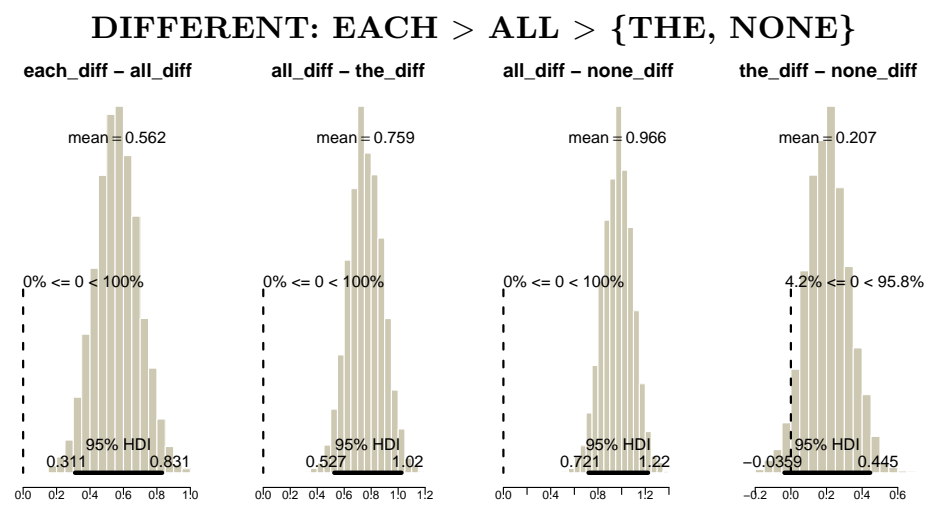

Fig. 2. Differences in acceptability between licensors of different

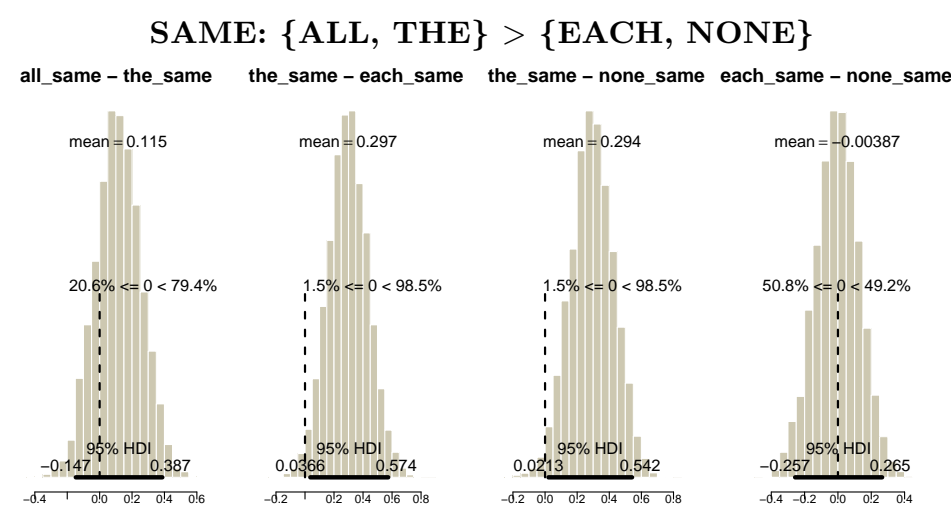

Fig. 3. Differences in acceptability between licensors of same

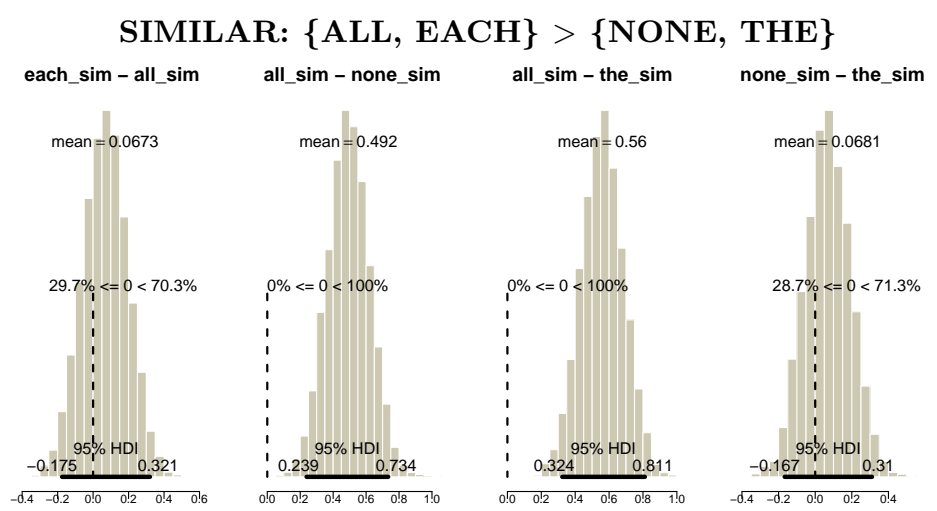

Fig. 4. Differences in acceptability between licensors of similar

Finally, Figure 5 shows the posterior distributions of the two non-reference levels of TRUTH and the four thresholds. False sentences (F) and sentences whose truth 
values are unknown (U) (due to their grammatically unclear status) lead to lower acceptability compared to true sentences. The rightmost plot shows the mean posterior thresholds plotted together with the standard normal probability density function. The fourth (rightmost) threshold, for example, is the cutoff point between values 4 and 5 of the ACCEPT response variable. We see that value 5 has the highest probability (the largest area under the probability density function).

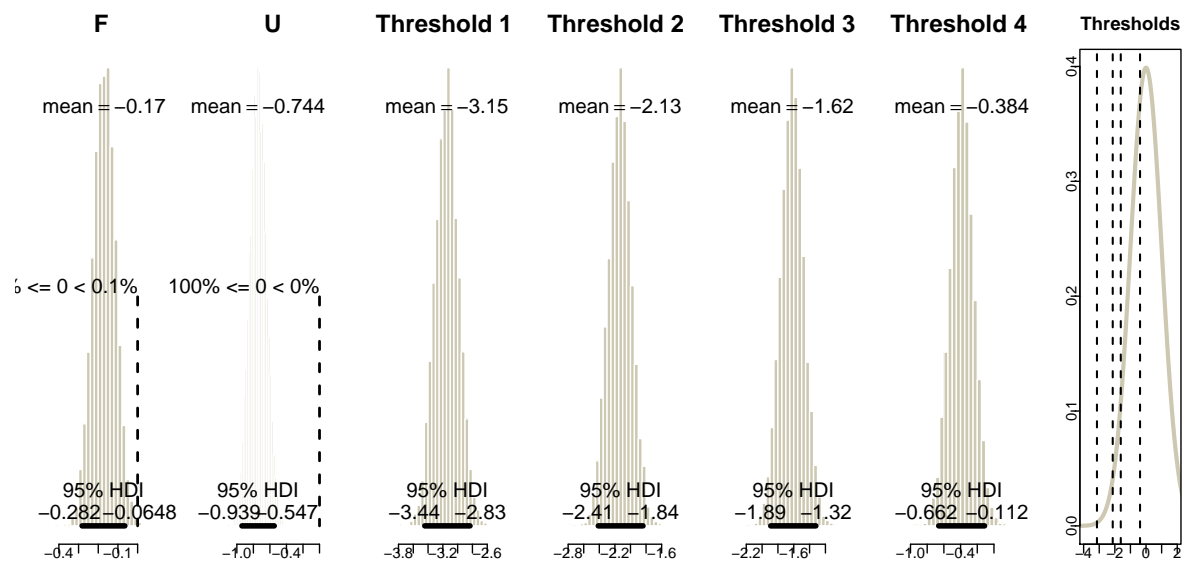

Fig. 5. Posterior distributions of TRUTH and thresholds

\section{Consequences for the semantic analysis of AOCs}

There is a long tradition of connecting the sentence-internal reading of at least some AOCs with distributivity. Here, we follow [3], who analyzes AOCs in a dynamic semantics that provides semantic values for natural language expressions in terms of sets of sequences of individuals and models these sequences as stacks.

Consider (4) below and the sequence of figures in (5a)-(5c) depicting the sequence of dynamic updates contributed by (4). The update contributed by each boy stores all the boys as the value of some variable, $u_{0}$ in our example. This is pictorially depicted by the one-column table following the leftmost arrow in (5a). The interpretation of the distributive operator dist contributed by each boy and of sentence-internal different are pictorially depicted in (5b): dist provides a temporary context inside of which the interpretation proceeds in three steps, namely $(i)$ pick two distinct boys, (ii) check that each of the two boys recited a poem and (iii) check that the two poems are different. In (5b-i), this sequence of steps is depicted for boy $y_{1}$ and boy $y_{2}$ and their corresponding poems. But dist requires these three steps to be repeated for any pair of boys in the set $u_{0}$, as shown in (5b-i)-(5b-v). For more details and the exact logical formulas, see [3].

(4) Each $^{u_{0}}$ boy recited $\mathrm{a}^{u_{1}}$ different $_{u_{1}}^{2}$ poem. 
(5)

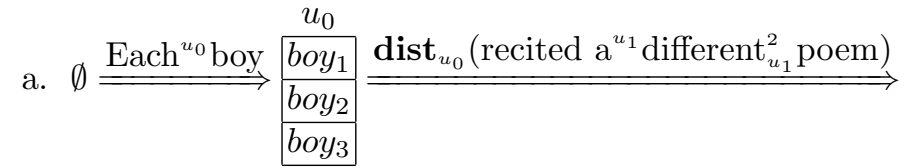

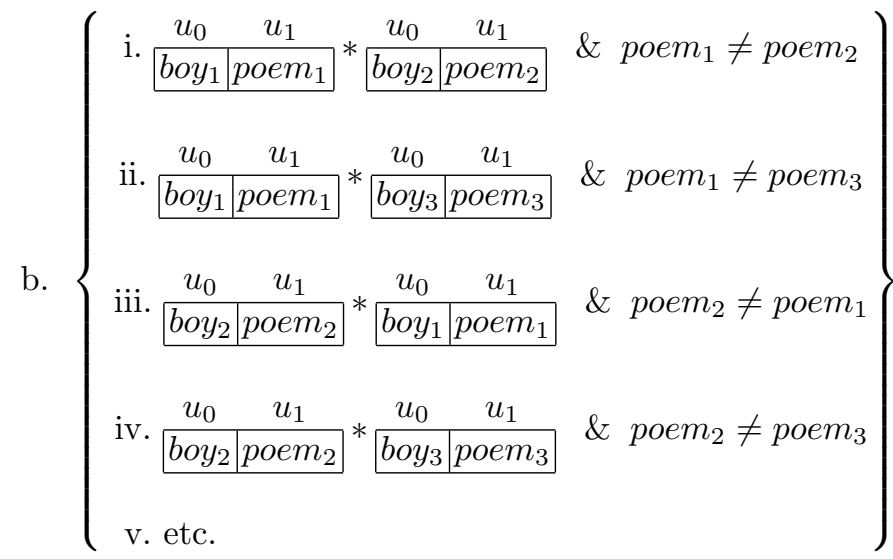
c. $\stackrel{\text { sum all updates }}{=}$\begin{tabular}{|c|c|}
$u_{0}$ & $u_{1}$ \\
\hline \begin{tabular}{ll} 
boy $_{1}$ & poem $_{1}$ \\
\hline boy $_{2}$ & poem $_{2}$ \\
\hline boy & poem \\
\hline
\end{tabular} \\
\hline
\end{tabular}
where $\begin{array}{ll}\text { boy }_{1} & \text { recited poem } \\ \text { boy }_{2} & \text { recited poem } \\ 2 \\ \text { boy }_{3} \text { recited poem }\end{array}$

Thus, in this account, the dist operator distributes over pairs of individuals and is necessary for sentence-internal readings of different. Besides the pairwise distributivity, [3] postulates another operator, dist-Comp, which creates a temporary context consisting of an individual and stacks that store all entities in the domain of quantification different from that individual. In (4), the dist-Comp operator would create a context comparing each boy and all the other boys.

Both the dist and the dist-Comp operator can capture distributive interpretations (hence their label dist), and both of them can account for sentenceinternal readings of different and same. However, sentence-internal readings of similar seem to be compatible only with dist-Comp: similarity is computed over the entire domain of quantification, which dist-Comp provides, and not simply over the individual pairs contributed by dist.

Consider the following example:

(6) Each manager bought a similar car.

Suppose there are three managers and two of them bought the same car brand, say, Volvo. The third manager bought a BMW, the color of which is similar to one Volvo and the design of which is similar to the other Volvo. In that case, it is true that for each pair of cars, the paired cars are similar (in some respect) - but (6) is intuitively false. We can capture this if sentence-internal similar is licensed by dist-Comp as opposed to dist.

Finally, same (and plural different, which we do not discuss in this paper) has another interpretation that gives rise to sentence-internal readings. If there 
is no distributivity in the clause, then same checks that only one entity (possibly plural) was introduced by its NP. The whole system of sentence-internal readings and their licensors is summarized in Table 1.

\begin{tabular}{|lccc|}
\hline & dist & dist-Comp & no distributivity \\
\hline \hline different & $\checkmark$ & $\checkmark$ & $*$ \\
same & $\checkmark$ & $\checkmark$ & $\checkmark$ \\
similar & $*$ & $\checkmark$ & $*$ \\
\hline
\end{tabular}

Table 1. Distributivity and sentence-internal readings in [3]

We are now going to discuss how this analysis, along with other accounts of sentence-internal readings, can account for the data from our experiment.

It has been observed in [5] that the distributive interpretation of predicates like build a snowman depends on the type of subject. In particular, the following cline in the availability of the interpretation was observed, where $>$ means the determiners / quantifiers on the left are preferred to the determiners / quantifiers on the right.

\section{(7) Distributive interpretation: EACH $>$ ALL $>$ THE}

The parallelism between the gradience of distributivity 'strength' associated with these determiners / quantifiers and the gradience of acceptability associated with sentence-internal readings of different provides support for accounts in which sentence-internal different requires distributivity to be licensed, as is the analysis of [3] discussed above, as well as [2], [4], [5], [8]. This is true regardless of the explanation for the gradient nature of distributivity 'strength' (but see [5] for one account).

At the same time, the results are problematic for accounts like [1], in which sentence-internal readings are incompatible with distributively interpreted licensors. From the perspective of [1], we would expect that all and the are better licensors than each, contrary to the facts.

Finally, none of the current accounts can explain why negative quantifiers are dispreferred licensors for different. These points have already been made in [5] with respect to the Dutch data. This paper extends them to English.

Regarding same, we have seen the following ordering of licensors:

\section{(8) Same: $\{$ ALL, THE $\}>\{$ EACH, NONE $\}$}

The difference between the two orderings of licensors supports the account of same in [1]. Under that analysis, same should not give rise to sentence-internal readings with distributive quantifiers, which squares well with the degraded status of each and none. The remaining question is why each and none are only slightly degraded, not uninterpretable, as the account in [1] would predict.

One possibility is that same is ambiguous, as discussed above and as assumed in [3] and [8]. One of the two meanings for same needs to appear in the scope of dist to have a sentence-internal reading, while the other meaning is compatible 
with a non-distributive plural licensor (see Table 1). Given the ordering in (8), the former meaning must be dispreferred / less accessible. Thus, our experiment seems to provide evidence for an ambiguity account of same, even though we still need to explain why one meaning of same should be preferred over the other.

One possibility is that the more complex meaning is dispreferred. Consider (9a) below: under the account in [3], dist creates temporary contexts storing pairs of non-identical boys and same needs to check that within each pair, the recited poems are identical. In contrast, the meaning of same in (9b) only needs to check that exactly one poem was introduced in discourse by the direct object. This second meaning of same is simpler in that we do not need to repeatedly examine pairs of poems (stored in two distinct discourse referents), we simply contribute a cardinality requirement on a set of witnesses. An investigation of the hypothesis that meaning (and / or processing) complexity can explain the licensing gradience in (8) is left for future research.

(9) a. Each boy recited the same poem.

b. All the boys/The boys recited the same poem.

Finally, sentence-internal similar is associated with the following scale of licensors:

\section{(10) Similar: $\{$ ALL, EACH $\}>\{$ NONE, THE $\}$}

The scale in (10) indicates that similar is close to different. The only difference between the two is that similar does not distinguish between all and each. These fine-grained parallelisms and contrasts between similar and different (or same) have not been previously noticed so far, as far as we know. As indicated above, the account in [3] generalizes to similar if we stipulate that NPs have another way of introducing distributivity, dist-Comp. But once again, the degraded status of negative quantifiers is surprising. While the account is stipulative, there are currently no better alternatives, as far as we know. A more careful specification and investigation of this account, including the contrast between dist and distComp on the theoretical side and the contrasts between similar, same and different on the empirical side, is also left for future research.

\section{Conclusion}

We have discussed experimental evidence showing that licensing sentence-internal readings of AOCs is gradient in nature. We have argued that this gradience supports an analysis of sentence-internal readings that connects them with distributivity. Furthermore, the particular ordering of licensors for same vs. different vs. similar provides evidence for an ambiguity account of same, as well as for two different distributivity operators. Some issues, like the particular status of negative quantifiers as licensors of different and similar, remain unclear and are left for future research. 


\section{Bibliography}

[1] Barker, C.: Parasitic scope. Linguistics and Philosophy 30, 407-444 (2007)

[2] Beck, S.: The semantics of Different: Comparison operator and relational adjective. Linguistics and Philosphy 23, 101-139 (2000)

[3] Brasoveanu, A.: Sentence-internal Different as quantifier-internal anaphora. Linguistics and Philosophy 34, 93-168 (2011)

[4] Carlson, G.: Same and Different: some consequences for syntax and semantics. Linguistics and Philosphy 10, 531-565 (1987)

[5] Dotlačil, J.: Anaphora and Distributivity. A study of same, different, reciprocals and others. Ph.D. thesis, Utrecht University, Utrecht (2010)

[6] Kruschke, J.K.: Bayesian data analysis. WIREs Cognitive Science 1, 658-676

[7] Kruschke, J.K.: Doing Bayesian Data Analysis: A Tutorial with R and BUGS. Academic Press/Elsevier, Oxford

[8] Moltmann, F.: Reciprocals and same/different: Towards a semantic analysis. Linguistics and Philosophy 15(4), 411-462 (1992) 


\title{
Evaluative adjectives, scale structure, and ways of being polite
}

\author{
Lisa Bylinina ${ }^{1}$ and Stas Zadorozhny ${ }^{2}$ \\ 1 Institute for Linguistics OTS, Utrecht \\ e.g.bylinina@uu.nl \\ 2 Yandex LLC \\ zador@yandex-team.ru
}

\section{Introduction}

This study is an attempt to add new facts (and new conclusions one could draw from them) to an old and settled topic. The evidence we will be using is of a quantitative sort, based on a corpus study. However, there will be nothing particularly quantitative or corpus-based in conclusions we draw from the data, as well as the semantic decisions we make given the results of the study.

At the same time the quantitative side of this work is of particular importance. This is an attempt to conduct a pilot corpus study from scratch - it includes collecting a corpus, basic pre-processing, calculating raw statistics, and formulating and testing certain types of linguistic hypotheses. This pipeline can be useful beyond the topic of this particular study. It does not require access to paid resources and allows to control various parameters to fit the needs of the study, and yet it is not very demanding technically. We hope to reuse and improve this set-up for other studies, the current paper being an example of the possible ways to use this pipeline. The overall goal is to make quantitative semantic tasks solved in more systematic way.

The topic we investigate here is the semantics of evaluative adjectives (EAs) - both positive (charming, industrious etc.) and negative (lazy, ugly etc.). (Bierwisch 1989) describes EAs as a subclass of gradable adjectives along with 'dimensional adjectives' (DAs) like tall, short, big, small etc.

EA vs. DA distinction is arguably not a clear-cut one, but there are several reasons for still making this distinction. We discuss them in further detail below, but they boil down to EAs and DAs referring to their underlying scales in different ways. (Bierwisch 1989) is still the only comprehensive study of how EAs and DAs are different, and no new substantial facts were added to Bierwisch's description since 1989.

We concentrate on further distinctions within the EA class - namely, how positive EAs are different from negative EAs in terms of scale structure. (Bierwisch 1989) treats them in exactly the same way, but observe the following contrast:

(1) a. Clyde is slightly stupid.

b. Clyde is slightly lazy. 
(2) a. ??Clyde is slightly smart.

b. ??Clyde is slightly industrious.

We will explore this and related contrasts further and see what they suggest. The study is limited to several degree modifiers and a pre-selected list of EA and DA adjectives.

Section 2 sets the theoretic stage for the study and formulates the problem; section 3 describes the corpus study. The results will be made sense of in section 4 , and section 5 concludes.

\section{Evaluative adjectives (Bierwisch 1989)}

\subsection{Gradable adjectives: background and conventions}

For our purposes we do not need to takes sides in the ongoing debates about nature of vagueness and gradability in natural language. We do not find it crucial for the case at hand to, say, choose between degree and delineation semantics for vagueness.

We will assume degree semantics of gradability for no particular reason. Here is an implementation of this view that analyzes gradable adjectives as measure functions: functions of type $\langle e, d\rangle$ from the domain of individuals to degrees on a certain scale (Bartsch and Vennemann 1973, Kennedy 1997, 2007):

(3) $\quad \|$ tall $\|=\lambda x$. tall $(x)$

where $\operatorname{adj}(x)$ is 'the degree on the appropriate scale that represents $x$ 's measure of adjective-ness'

Measure functions are converted into properties of individuals by degree morphology (comparative morphemes, intensifiers etc.). For the unmarked positive form (John is tall) a null POS morpheme is introduced, with a denotation along the lines of (4), where $\mathbf{d}_{s}$ is 'contextually appropriate standard of comparison, whatever that is' Kennedy (2007):

(4) $\quad \|_{D e g}$ POS $\|=\lambda g \lambda x \cdot g(x) \succeq \mathbf{d}_{s}$

\subsection{Scale structure and degree modifiers}

Under any view on vagueness/gradability, gradable adjectives are used for reasoning about domains that are or can be structured in a particular way, namely, (partially) ordered with respect to a certain dimension. For many purposes it would suffice to deal with the orderings on the domains of individuals and not involve a separate notion of scales as distinct objects, c.f. (Bale 2008). However, there are arguably facts that are not easy to capture if one does not assume a richer notion of scales. The obvious candidate is the absolute/relative distinction within the class of gradable adjectives (but see (van Rooij 2010)). This distinction manifests itself in a number of ways, for example, degree modifier distribution: 
(5) a. ??perfectly/??slightly \{tall, deep, expensive, likely\}

b. ??perfectly/??slightly \{short, shallow, inexpensive, unlikely\}

(6) a. ??perfectly/slightly \{bent, bumpy, dirty, worried

b. perfectly/??slightly \{straight, flat, clean, unworried\}

(7) a. perfectly/??slightly \{certain, safe, pure, accurate\}

b. ??perfectly/slightly \{uncertain, dangerous, impure, inaccurate\}

(8) a. perfectly/slightly \{full, open, opaque\}

b. perfectly/slightly \{empty, closed, transparent

These contrasts have been argued to signal the differences in structure of scales associated with different gradable adjectives, sketched below (Rothstein and Winter 2004, Kennedy and McNally 2005, Kennedy 2007):

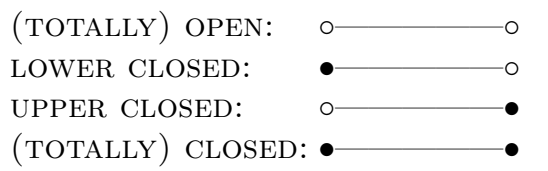

Adjectives that make use of a scale that is closed at least at one end are absolute adjectives, adjectives with totally open scales are relative ones. There have been several attempts to give an explanation of why distribution of degree modifiers needs to correlate with existence of a scale bound; some of them challenge the general picture presented above (Sassoon and Toledo 2011, Solt 2011, McNally 2011).

To give the simplest possible insight about why low degree modifiers like slightly are only possible with scales with a minimum, it would suffice to say that the positive form of relative adjectives requires of an entity to significantly exceed a contextual standard, i.e. stand out in a relevant respect; positive forms of absolute adjectives, on the other hand, do not include the significance component. We illustrate this by directly including significantly into semantics of $\mathrm{POS}_{r e l}$ combining with relative adjectives vs., say, $\mathrm{POS}_{\text {min }}$ combining with adjectives with lower bound scales (Kennedy 2007):

(9) $\quad$ a. $\left\|\operatorname{POS}_{r e l}\right\|=\lambda g \lambda c \in D_{\langle e, t\rangle} \lambda x . g(x) ! \succ \operatorname{norm}(c)(g)$ $\mathrm{c}=$ comparison class, $\mathrm{g}=$ gradable property, $! \succ=$ significantly exceed

b. $\left\|\operatorname{POS}_{m i n}\right\|=\lambda g \lambda x . g(x) \succ \min (\operatorname{SCALE}(g))$

Thus, relative standards cannot be addressed when reasoning about small differences, while absolute standards can. Whatever the right compositional analysis of degree modifiers is, this observation needs to hold for them as well as for POS.

In addition, very is usually taken to specifically target standards of relative adjectives: 'in normal usage, absolute adjectives reject modification by very' (Kennedy and McNally 2005, 370): 
(10) a. ??I always leave the door to my office very open.

b. ??That drug is currently very available.

\subsection{Dimensional vs. evaluative adjectives}

The distinction between evaluative (EAs) and dimensional adjectives (DAs) in (Bierwisch 1989) is motivated by two observations: 1) the antonymous pairs of EAs have a less obvious relation to each other than DA antonu spairs; sometimes it is hard to tell whether the two given EA items form a pair or not; 2) inference judgements for EAs vary a great deal inter- and intra-individually.

The intuition behind observation 1 is the following: 'Hans ist klein (Hans is short) assigns to Hans a certain degree of height, while Hans ist faul (Hans is lazy) does not mean that Hans has a certain degree of industriousness. Put somewhat differently, even a negative DA always specifies a positive value on the scale of its antonym, whereas this does not apply to a negative EA: even a short person has height, but a lazy person cannot be to any extent industrious' (Bierwisch 1989, 88). Here is a more theoretic way to formulate the same thought:

(11) (Bierwisch 1989, ix)

Antonymous DAs refer to the same scale of a given dimension and differ in the ordering on the scale, antonymous EAs refer to different scales or parts of scales.

Thus it is not obvious that every EA has a single antonym, at least in the sense that DAs do:

(12) DAs:
a. tall $\leftrightarrow$ short
b. heavy $\leftrightarrow$ light
c. hot $\leftrightarrow$ cold

(13) EAs:
a. brave, bold, courageous $\leftrightarrow$ cowardly, timid, fearful
b. clever, bright, shrewd, intelligent, brilliant $\leftrightarrow$ stupid, idiotic, foolish
c. pretty, beautiful, gorgeous, handsome $\leftrightarrow$ ugly, hideous, grotesque
d. lazy, indolent, unproductive $\leftrightarrow$ hard-working, industrious, workaholic

The second observation concerns the following kinds of inference patterns and the fact that speakers do not have a consensus on whether these inferences hold:

(14) a. How hard-working/lazy is Hans?

$\rightarrow$ Hans is hard-working/lazy.

b. Hans is as hard-working/lazy as Eva

$\rightarrow$ Hans is hard-working/lazy. 
c. Hans is more hard-working/lazier than Eva

$\rightarrow$ Hans is hard-working/lazy.

d. Hans is the most hard-working/laziest

$\rightarrow$ Hans is hard-working/lazy.

e. Hans is too hard-working/lazy for that

$\rightarrow$ Hans is hard-working/lazy.

f. Hans is hard working/lazy enough for that

$\rightarrow$ Hans is hard-working/lazy.

g. Hans is less hard-working/lazy than Eva

$\rightarrow$ Hans is hard-working/lazy.

This motivates a conclusion made in (Bierwisch 1989) that EAs are underlyingly not gradable at all, though they can used as gradable with the help of a type shift or coercion that results in a scale with a derived zero (=lowerbound). (Morzycki 2011) notes that this solution is 'immediately worrying, since non-dimensional adjectives straightforwardly form comparatives and occur with degree modifiers.' Our main goal is not to evaluate the idea of coercion from non-gradable to gradable per se, but to check whether the conclusion Bierwisch makes is a fully informed one.

The most important point in this solution for us is that positive and negative EAs are treated the same and are ultimately assigned the same scale structure. However, as we have seen in the introduction, there are contrasts between positive and negative EAs that look like differences in scale structure (1-2).

There is a clear need for a more wide-ranged study, first of all, to be able to make a claim about the data regarding low degree modification in EAs, and then see whether the data points in a direction of a particular analysis.

\section{Corpus study}

To collect the corpus we used BootCaT toolkit (Baroni and Bernardini 2004) software which allows to bootstrap corpora from web with a small initial set of seed words as its input. BootCat uses these seeds to query search engine (Google, Yahoo) and download urls from search results page to build initial corpora. These initial corpora are then used for expansion of seed words. The procedure repeats iteratively until the desired number of documents is reached.

Manipulating initial seed words is a way to control various parameters of the corpus, such as the language, genre or topic of downloaded documents. This is an extremely useful feature, though we did not make the best of it in the current study. In our work we used the general list of most frequent English words to obtain general corpora without bias towards specific genre or topic.

As result of applying the BootCaT procedure we have collected a corpus consisting of 156117 unique documents. These data were normalized (lowercasing, character trash removal etc.) and split into sentences using simple heuristics and set of standard UNIX tools. Then we collected basic statistics for bigrams (all pairs of consecutive words) in sentences - bigram frequency and marginal 
frequency (the frequency of first or second component of a bigram among all pairs).

We were interested in particular events - namely, co-occurrence of certain degree modifiers with certain adjectives. The modifiers we are interested in are low degree modifiers a bit, a little bit, slightly and somewhat (=LOW), and a standard-booster very (=VERY). The adjectives we are interested in are, first of all, positive EAs (=POS) and negative EAs (=NEG). Also we use data from relative adjectives (=REL) and lower-bound adjectives (=MIN). We draw the lists of adjectives from various sources - theoretic papers discussing gradability and scale structure (Kennedy and McNally 2005, Kennedy 1997, 2007, Rothstein and Winter 2004, Sassoon and Toledo 2011, McNally 2011) as well as works that include quantitative studies (Solt 2011, Sassoon 2011), and also various descriptive grammars of English that introduce semantic divisions within adjectives. We thank Stephanie Solt, Galit Sassoon and Chris Kennedy for sharing their lists and thoughts on possible ways to extend the lists we are using.

So we collect only events of the form $\langle x, y| x \in\{$ LOW, VERY $\}, y \in\{$ POS, NEG, MIN, REL $\}\rangle$. For each of the events we calculate the following frequencies: $f(x, y), f(x, *), f(*, y), f(*, *)$, where * stands for any word. Random sample of collected data:

\begin{tabular}{lllll}
$x$ & $y$ & $f(x, y)$ & $f(x, *)$ & $f(*, y)$ \\
\hline abit & responsible & 2 & 17582 & 25617 \\
abit & black & 1 & 17582 & 54527 \\
abit & bright & 2 & 17582 & 8687 \\
very & creepy & 12 & 194306 & 669 \\
very & brave & 58 & 194306 & 2058 \\
very & helpful & 1515 & 194306 & 18699 \\
very & angry & 230 & 194306 & 4980 \\
very & funny & 231 & 194306 & 7197 \\
somewhat & protective & 2 & 10091 & 4039 \\
very & inexpensive 131 & 194306 & 2749
\end{tabular}

Cross-tabulation of data:

\begin{tabular}{l|llll|l} 
class/mod & NEG & POS & MIN & REL & total \\
\hline a bit & 350 & 92 & 79 & 218 & 739 \\
a little bit & 66 & 12 & 8 & 19 & 105 \\
slightly & 114 & 42 & 202 & 57 & 415 \\
somewhat & 212 & 191 & 101 & 98 & 602 \\
\hline LOW & 742 & 337 & 390 & 392 & 1861 \\
VERY & 3032 & 21130 & 1299 & 15091 & 40522 \\
\hline \hline total & 3774 & 21467 & 1689 & 5483 &
\end{tabular}

We also filtered out events with frequency below 3 due to unreliability of probability estimates for low-frequency events, as discussed in (Evert 2005).

Our particular interest is how certain classes of modifiers are related to certain classes of gradable adjectives. The main goal of the paper is to know more 
about scale structure of positive and/vs. negative EAs, so the obvious pairs of distributions to compare are the following:

$$
\begin{gathered}
P(x=l o w, y=n e g) \text { vs. } P(x=l o w, y=p o s) \\
P(x=\text { very, } y=\text { pos }) \text { vs. } P(x=\text { ver } y, y=n e g)
\end{gathered}
$$

As we are using quite a lot of theoretic assumptions on how degree modifiers are sensitive to the scale structure, it would also be good to check these assumptions against our data before drawing any conclusions from results on (1) and (2). Contrasting the following pairs of distributions is aimed at proving these assumptions right or wrong on our data:

$$
\begin{gathered}
P(x=l o w, y=\min ) \text { vs. } P(x=l o w, y=r e l) \\
P(x=\text { very, } y=r e l) \text { vs. } P(x=\text { ver } y, y=\min )
\end{gathered}
$$

Finally, we try to see whether there are reasons to group positive or negative EAs with lower-bound or relative DAs based on co-occurrences with low degree modifiers:

$$
\begin{gathered}
P(x=l o w, y=n e g) \text { vs. } P(x=l o w, y=\text { min }) \\
P(x=l o w, y=\text { pos }) \text { vs. } P(x=l o w, y=r e l) \\
P(x=l o w, y=\text { neg }) \text { vs. } P(x=l o w, y=r e l) \\
P(x=l o w, y=\text { min }) \text { vs. } P(x=l o w, y=\text { pos })
\end{gathered}
$$

The same for very:

$$
\begin{aligned}
& P(x=\text { very, } y=n e g) \text { vs. } P(x=\text { ver } y, y=\min ) \\
& P(x=\text { very, } y=r e l) \text { vs. } P(x=\text { ver } y, y=\text { pos }) \\
& P(x=\text { very, } y=\text { rel }) \text { vs. } P(x=\text { very, } y=n e g) \\
& P(x=\text { very, } y=\min ) \text { vs. } P(x=\text { ver } y, y=\text { pos })
\end{aligned}
$$

First of all, we should establish a measure of relatedness between a modifier and an adjective and then compare the resulting distributions. We use pointwise mutual information (PMI) as a measure that was created precisely for these purposes (Fano 1961, Church and Hanks 1990): 


$$
P M I(x, y)=\log \frac{P(x, y)}{P(x) P(y)}
$$

In our case $P(x, y)$ is the probability of a bigram, $P(x)$ is the probability of a modifier preceding any word and $P(y)$ is the probability of an adjective following any word. Informally, mutual information compares the probability of observing $x$ and $y$ together (the joint probability) with the probabilities of observing $x$ and $y$ independently (chance). If there is a genuine association between $x$ and $y$, then the joint probability $P(x, y)$ will be much larger than chance $P(x) P(y)$. Thus, the large positive values of $P M I$ indicate stronger association between events; if it takes zero value, then $x$ and $y$ are independent; negative values indicate that $x$ and $y$ are in complementary distribution.

We can estimate the probabilities required for calculation of $P M I$ using maximum-likelihood estimation and previously collected bigram frequencies:

$$
\begin{gathered}
P(x, y)=\frac{f(x, y)}{f(*, *)} \\
P(x)=\frac{f(x, *)}{f(*, *)} \\
P(y)=\frac{f(*, y)}{f(*, *)}
\end{gathered}
$$

So maximum-likelihood estimation of PMI (13) will be:

$$
\overline{P M I}(x, y)=\log \frac{f(x, y) f(*, *)}{f(x, *) f(*, y)}
$$

To illustrate possible differences in distribution, we plot pairs of groups (112 ) by ordering them by $P M I$. Each color represents a class of adjectives and $P M I$ value for each adjective in two groups increased from left to right.

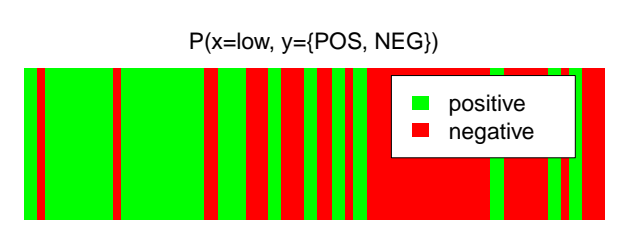

$P(x=v e r y, y=\{P O S, N E G\})$

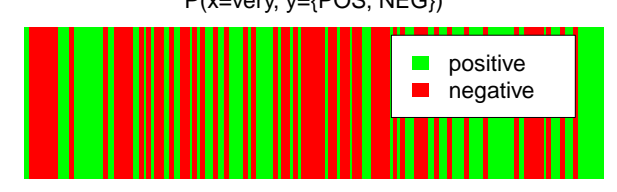

These two figures display distributions of positive and negative EAs with respect to their PMI with low degree modifiers (the first figure) and very (the second figure). Positive EAs (beautiful, smart etc.) are green, negative EAs (ugly, stupid etc.) are red. The figures visualize that negative EAs seem to group to the right of the low degree modifier figure (which speaks of more association), while the very picture looks free of any obvious tendency. 
This pair illustrates how well our data support the theoretic assumptions about scale structure sensitivity of degree modifiers. It is rather obvious from these figures that lower-bound adjectives (painted blue) have stronger association with low degree modifiers than relative adjectives do (painted yellow). However, the picture with very is not obvious and needs more attention. Theory predicts it to be a mirror image of the low degree modifier picture, but

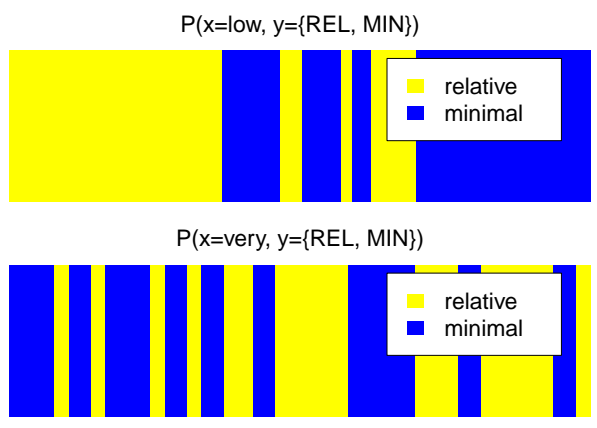
it is not.

Including the following four figures is motivated by the suggestion made in (Bierwisch 1989) that both positive (painted green) and negative EAs (painted red) are structurally similar to lower-bound adjectives (painted blue). It is not obvious from these pictures alone whether there is any tendency, but it can be seen rather well that these three classes are not strikingly different from each other:

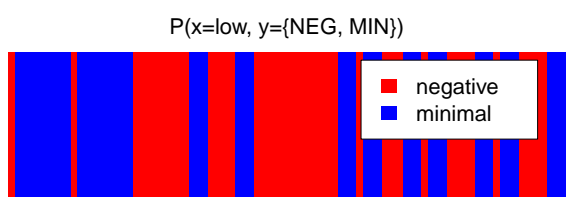

$P(x=$ very, $y=\{N E G, M I N\})$

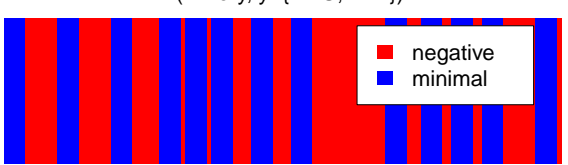

$P(x=$ low, $y=\{M I N, P O S\})$

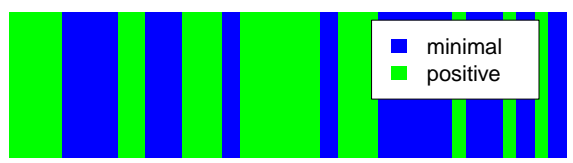

$P(x=$ very, $y=\{M I N, P O S\})$

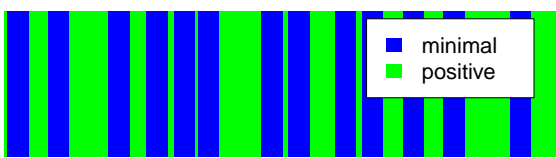

Comparing negative and positive EAs in the same way to relative adjectives (yellow) gives quite different results. Again, very is less informative than we would expect it to be, but there is a clear tendency for both positive and negative EAs to co-occur with low degree modifiers much more often than relative adjectives do:
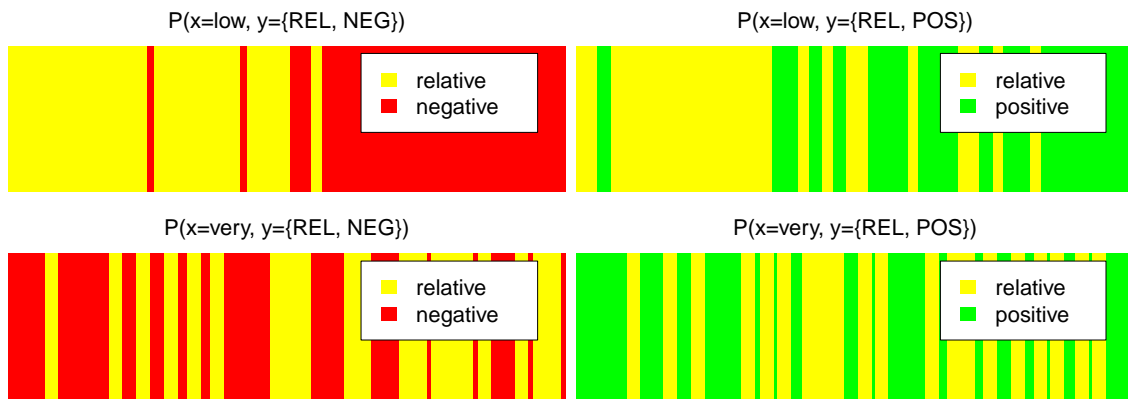

$P(x=$ very, $y=\{R E L, P O S\})$

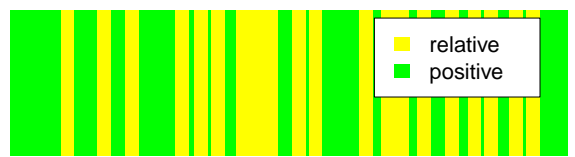


To qualitatively asses the observed differences, we performed series of statistical tests. We used Wilcoxon rank-sum test (MWW) (Mann and Whitney 1947, Hollander and Wolfe 1999), which allows assessing whether the two groups have a tendency to have different values (two-sided test) or whether one of the two groups tends to have greater values then the other group (one-sided test). The choice of this test was motivated by unknown distribution of samples (MWW is a non-parametric test).

For each pair of distributions (1-12) we formulate the following null hypothesis and an alternative hypothesis:

(15) a. $H_{0}$ : There is no difference in association score between two compared classes of adjectives with respect to a given modifier

b. $H_{1}$ : There is a significant difference in association score between two given classes of adjectives with respect to a given modifier

Based on our expectations from what theories of gradable adjectives predict, for some pairs we also formulate a different alternative hypothesis:

(16) $H_{1}$ : The association score tends to be larger for one class then for the other.

The following table represents results of MWW statistical test for distributions (1-12) with significance level 0.01 and frequency threshold 2 for low degree modifiers:

\begin{tabular}{l|lll}
$H_{1}$ & accept/decline & p-value & $\mathrm{W}$ \\
\hline$N E G \neq P O S$ & accept & $3.62 \mathrm{e}-05$ & 1924 \\
$N E G>P O S$ & accept & $1.81 \mathrm{e}-05$ & 1924 \\
$M I N \neq R E L$ & accept & $1.35 \mathrm{e}-06$ & 707 \\
$M I N>R E L$ & accept & $6.8 \mathrm{e}-07$ & 707 \\
$N E G \neq M I N$ & decline & 0.10503787 & 1194 \\
$N E G>M I N$ & decline & 0.05251893 & 1194 \\
$P O S \neq R E L$ & accept & $5.25 \mathrm{e}-06$ & 879 \\
$P O S>R E L$ & accept & $2.62 \mathrm{e}-06$ & 879 \\
$N E G \neq R E L$ & accept & 0 & 2863 \\
$N E G>R E L$ & accept & 0 & 2863 \\
$M I N \neq P O S$ & decline & 0.15485217 & 425 \\
$M I N>P O S$ & decline & 0.07742609 & 425
\end{tabular}

Results for very with the same settings: 


\begin{tabular}{l|lll}
$H_{1}$ & accept/decline & p-value & $\mathrm{W}$ \\
\hline$P O S \neq N E G$ & decline & 0.0534381 & 4634 \\
$P O S>N E G$ & decline & 0.02671905 & 4634 \\
$R E L \neq M I N$ & decline & 0.09397819 & 176 \\
$R E L>M I N$ & decline & 0.0469891 & 176 \\
$N E G \neq M I N$ & decline & 0.73924513 & 471 \\
$N E G>M I N$ & decline & 0.63470126 & 471 \\
$R E L \neq P O S$ & decline & 0.13602659 & 1248 \\
$R E L>P O S$ & decline & 0.0680133 & 1248 \\
$R E L \neq N E G$ & accept & 0.00339799 & 1099 \\
$R E L>N E G$ & accept & 0.00169899 & 1099 \\
$M I N \neq P O S$ & decline & 0.43602203 & 580 \\
$M I N>P O S$ & decline & 0.7845581 & 580
\end{tabular}

Results shows that we should reject statement about same distributions and may accept alternative hypothesis about significant difference for pairs $(1,2,4$, $5,11)$.

Described procedure has potential problems that are worth mentioning. The bigram set can contain bigrams that are not actually one syntactic unit but belong to different constituents, as we used raw text only, without any syntactic information.

\section{Interpreting the results}

This section evaluates the existing theories of gradable adjectives and their scale structure against the results of the corpus study presented in the previous section. We formulate expectations of the standard theories and sum up whether they are borne out by our study in the following list:

Expectation 1 Very goes with relative adjectives and not with absolute ones $\rightarrow$ Wrong

Expectation 2 Low degree modifiers go with lower-bound adjectives and not with relative ones $\rightarrow$ Right

Expectation 3 Neither POS nor NEG EAs go with very as well as relative adjectives do $\rightarrow$ Wrong

Expectation 4 Both POS and NEG EAs go with low degree modifiers, quite like lowerbound adjectives do $\rightarrow$ Right

Expectation 5 There is no difference between POS and NEG EAs in how well they go with low degree modifiers $\rightarrow$ Wrong

Expectation 6 There is no difference between POS and NEG EAs in how well they go with very $\rightarrow$ Right

Expectation 5 comes from (Bierwisch 1989) and is actually in a way expected to be wrong given examples (1-2) that were the initial motivation for our study. With only this fact in mind (now supported by statistics), one would be tempted to say that while negative EAs do have a scale with a minimum, positive EAs do not. When the correct Expectation 6 is added to the picture, the conclusion 
to draw from that would be something like the following (we did draw this conclusion in an earlier version of this paper):

Preliminary analysis Positive EAs have a totally open scale; negative EAs have a totally open scale as well, but they can also be interpreted in a different way that involves a scale with a minimum. Positive EAs lack this secondary interpretation.

This analysis crucially relies on what existing theories say about the way different classes of adjectives co-occur with different classes of degree modifiers. However, if we include relative adjectives and lower-bound adjectives in our study and compare them to each other and to positive and negative EAs pairwise, the picture changes. First, very as a test for relative adjectives does not seem to work at all. Second, when we look at low degree modifiers, we see that original hypothesis from (Bierwisch 1989) seems very close to what we observe. Both positive and negative EAs behave like lower-bound adjectives, and both positive and negative EAs behave differently from relative adjectives. This strongly suggests that all EAs have a scale with a minimum, quite as predicted. But this poses a problem for the observed differences between positive and relative EAs in how well they combine with low degree modifiers. We need to resolve this inconsistency.

Why the positive/negative EA asymmetry? Negative EAs generally express or implicate judge's negative feeling or attitude related to a certain object. As part of a general tendency to attenuate negative judgements, similar to what is described in (Baumeister et al. 2001, Rozin and Royzman 2001) a.m.o., judgements with negative EAs are quite often attenuated. The reason is that, basically, 'bad is stronger than good'. One could think of various ways to make sense of this intuition within a semantic theory of gradability. For instance, one could require of any mapping between positive and negative scales to be mediated by a certain coefficient, so that an interval on a positive scale would correspond to a bigger interval on a negative one. However this is implemented, the result should be that positive scales are in some sense more dense than negative ones, and this is a property of scales that is different from having or lacking a minimum or a maximum. We can now reformulate our analysis:

Analysis All EAs have lower-closed scales, but positive EAs have more dense scales than negative ones.

\section{Conclusion}

We conducted a corpus study of evaluative adjectives, both positive and negative. The particular goal of this study was to establish their scale structure and how positive and negative EAs are different from each other. As a result, we found that the original hypothesis of (Bierwisch 1989) is supported by our data, but there are still contrasts that are not captured by this hypothesis. We believe that pragmatic factors play a role, namely, that negative judgements have more 'strength' than positive ones, which explains massive low degree modification of negative but not positive EAs. 


\section{Bibliography}

Bale, A.: 2008, A universal scale of comparison, Linguistics and Philosophy 31(1), 1-55.

Baroni, M. and Bernardini, S.: 2004, Bootcat: Bootstrapping corpora and terms from the web, Proceedings of LREC 2004, pp. 1313-1316.

Bartsch, R. and Vennemann, T.: 1973, Semantic Structures: A Study in the Relation between Syntax and Semantics, Frankfurt.

Baumeister, R., Bratslavsky, E., Finkenauer, C. and Vohs, K.: 2001, Bad is stronger than good, Review of General Psychology 5, 323-370.

Bierwisch, M.: 1989, The semantics of gradation, in M. Bierwisch and E. Lang (eds), Dimensional Adjectives: Grammatical Structure and Conceptual Interpretation, Springer-Verlag, Berlin, pp. 71-261.

Church, K. W. and Hanks, P.: 1990, Word association norms, mutual information, and lexicography, Computational Linguistics 16, 22-29.

Evert, S.: 2005, The statistics of word cooccurrences : word pairs and collocations, PhD thesis, Universitt Stuttgart, Stuttgart.

Fano, R.: 1961, Transmission of Information: A Statistical Theory of Communications, The MIT Press, Cambridge, MA.

Hollander, M. and Wolfe, D.: 1999, Nonparametric statistical methods, Wiley series in probability and statistics: Texts and references section, Wiley.

Kennedy, C.: 1997, Projecting the adjective: the syntax and semantics of gradability and comparison, $\mathrm{PhD}$ thesis, UCSD.

Kennedy, C.: 2007, Vagueness and grammar: The semantics of relative and absolute gradable predicates, Linguistics and Philosophy 30, 1-45.

Kennedy, C. and McNally, L.: 2005, Scale structure, degree modification, and the semantics of gradable predicates, Language 81(2), 345-381.

Mann, H. and Whitney, D.: 1947, On a test of whether one of two random variables is stochastically larger than the other, Ann. Math. Stat. 18, 50-60.

McNally, L.: 2011, The relative role of property type and scale structure in explaining the behaviour of gradable adjectives, in R. e. a. Nouwen (ed.), Papers from the ESSLLI 2009 Workshop on Vagueness in Communication, Vol. 6517 of Lecture Notes in Artificial Intelligence, Springer, Berlin, pp. 151168.

Morzycki, M.: 2011, The several faces of adnominal degree modification, Proceedings of the 29th West Coast Conference on Formal Linguistics (WCCFL), Cascadilla Press, Somerville, Mass.

Rothstein, S. and Winter, Y.: 2004, Total adjectives vs. partial adjectives: Scale structure and higher-order modifiers, Natural Language Semantics 12, 259 288.

Rozin, P. and Royzman, E.: 2001, Negativity bias, negativity dominance, and contagion, Personality and Social Psychology Review 5, 296-320.

Sassoon, G.: 2011, A slightly modified economy principle: Stable properties have non-stable standards, Sinn und Bedeutung 16, Utrecht. 
Evaluative adjectives, scale structure, and being polite Lisa Bylinina \& S. Zadorozhny

Sassoon, G. and Toledo, A.: 2011, Absolute vs. relative adjectives: Variance within vs. between individuals, Proceedings of SALT21.

Solt, S.: 2011, Comparison to fuzzy standards, Sinn und Bedeutung 16, Utrecht. van Rooij, R.: 2010, Vagueness and linguistics, in G. Ronzitti (ed.), The Vagueness Handbook, Springer. 


\title{
Processing: free choice at no cost
}

\author{
Emmanuel Chemla ${ }^{1}$ and Lewis Bott ${ }^{2}$ \\ ${ }^{1}$ LSCP, CNRS/EHESS/ENS, Paris \\ 2 Cardiff University, UK
}

A disjunctive sentence such as (1) standardly carries the conjunctive inference that (2)a and (2)b are true.

(1) John is allowed to eat an apple or a banana.

(2) a. John is allowed to eat an apple.

b. John is allowed to eat a banana.

This phenomenon is known as Free Choice (FC) permission (Kamp, 1973). Current formal models tend to treat FC inferences as a special type of scalar implicature (mostly building on Kratzer and Shimoyama's 2002 insight, see, e.g., Schulz, 2003; Fox, 2006; Klinedinst, 2006; Alonso-Ovalle, 2008; Chemla, 2008, 2009; Franke, 2011). We present the first processing study of FC. Our results go against the expectations of recent formal analyses, and show that, unlike scalar implicatures, FC inferences come at no processing cost.

\section{Scalar implicatures}

A sentence such as (3) standardly conveys that its sister sentence (4) is false. Here is a derivation: The alternative sentence (4) is stronger than (3); (3) is thus not the best sentence to utter, unless (4) is false.

(3) Some elephants are mammals.

(4) All elephants are mammals.

\section{Processing}

Since Bott and Noveck (2004) (at least), a variety of experimental studies have investigated how this inference is derived in real time. They consistently found that the verification of a sentence is more demanding when its scalar implicature is taken into account (see Grodner, 2009 for discussion). To prove so, these studies relied on situations in which the target sentence would be (a) true without its scalar implicature, but (b) false with its scalar implicature. (3) is such an example: (a) it is true that there are elephants that are mammals, but (b) it is false that some but not all elephants are mammals. True/false judgments thus covary with the derivation of the scalar implicature. In a Truth Value Judgment Task, false answers, that correspond to the derivation of the scalar implicature, are found to be slower than true answers ("logical" answers, without the scalar implicature). 


\section{Free choice (FC)}

It has been shown that FC could be explained in a similar fashion. One implementation of this insight relies on the following hypotheses:

(H1) The sentences in (2) are alternatives to (1), in the same sense that (4) is an alternative to (3);

(H2) Sentences (2)a/b with their scalar implicatures become (5)a/b;

(H3) The enriched versions (5) are the sentences that end up negated when (1) is uttered.

In such a view, FC is analyzed as a second order kind of scalar implicature: the scalar implicatures of the alternatives are first derived, and then the alternatives, enriched with their own scalar implicatures, enter in the competition process to give rise to further scalar implicatures. The result is obtained because the conjunction of (1) and of the negations of each of the enriched alternatives in (5) entails FC, i.e. the conjunction of (2)a and (2)b.

(5) a. John is allowed to eat an apple, but not a banana.

b. John is allowed to eat a banana, but not an apple.

Such a view leads to the following prediction:

Processing prediction. According to the view sketched above, FC is a second order scalar implicature. Since first order scalar implicatures comes with a visible processing cost, we should be able to detect a similar or higher cost for FC inferences.

\section{Design}

We capitalized on the seminal idea that was used to test scalar implicatures: true/false answers were used as an indicator of whether FC inferences were derived. The target sentences were constructed with the help of a cover story in which the destruction of the planet was described as imminent, but that certain people were allowed to save certain types of objects. Specifically, zoologists were allowed to save living creatures, and engineers were allowed to save artificial objects. (One object per person at most, the rule says, to avoid issues about exclusive readings associated to disjunctions). We then tested sentences of the following type:

\begin{tabular}{|c|l|}
\hline TARGET & Mary-the-engineer $/ /$ is allowed to save // a monkey or a computer. \\
\hline Double-true & Mary-the-engineer // is allowed to save // a TV or a computer. \\
Double-false & Mary-the-engineer // is allowed to save // a monkey or a lion. \\
\hline Single -true & Mary-the-engineer // is allowed to save // a monkey. \\
Single -false & Mary-the-engineer // is allowed to save // a computer. \\
\hline
\end{tabular}

We presented the first bit of these sentences for $750 \mathrm{~ms}$, the next four words for $250 \mathrm{~ms}$ each, and the last bit remained until participants provided their true/false 
answers. The key example is the first one: "TARGET". According to the cover story, a FC interpretation would result in a false response (engineers are not allowed to save monkeys) whereas a logical interpretation would result in a true response (engineers are allowed to save computers). We compared response times of false responses (FC interpretations) to true responses (logical interpretations). The other conditions were included to control for various possible response biases, e.g., true responses may be faster than false responses, independently of any of the processes we are interested in.

\section{Experiment 1}

46 native speakers completed a verification task (two were excluded because they failed to answer appropriately to the control conditions). Control sentences were answered very accurately overall $(M=.93, S D=.039)$, illustrating that participants understood the task and the cover story. The proportion of free choice (false) responses to the experimental sentences was $M=.66, S D=.33$, and there was significantly greater variability in the experimental sentences than the control sentences. Overall then, the response choice data indicates that multiple interpretations were available for the experimental sentences.

\section{Response times}

Figure 1 shows the pattern of RTs for the correct responses to all five types of sentences. The TARGET sentences are broken down into FC responses (false) and logical responses (true). For both types of control sentences there is bias towards true sentences $\left(t_{1} \mathrm{~s}(43)>3.4, t_{2} \mathrm{~s}(19)>2.0\right.$, all $\left.p \mathrm{~s}<.05\right)$. For the TARGET sentences, however, FC responses (false) RTs are faster than logical (true) responses. While the simple comparison between FC and logical responses failed to reach significance, a repeated measures ANOVA with sentence type (single, double or target) and response (true or false) as factors revealed a significant interaction between sentence type and response type $\left(F_{1}(2,76)=3.1, F_{2}(2,38)=6.5, p \mathrm{~s}<.05\right)$. The difference between the $\mathrm{FC}$ and logical interpretations is therefore significantly smaller than the difference between the true and false responses for the different control sentences.

One explanation for our pattern of results is that FC interpretations are fast, but that a bias against false responding slowed down the FC responses. We therefore conducted an analysis in which the response bias was removed from latencies. For each participant, we computed the difference between the FC interpretations (false) and the false control sentences, and compared these scores against the difference between the logical responses (true) and the true control sentences. Any bias towards fast true responding in the TARGET condition should be removed by subtracting away the relevant control responses. This analysis revealed faster FC responses than logical responses (although only marginally so in the participants analysis, $p=.069)$. 


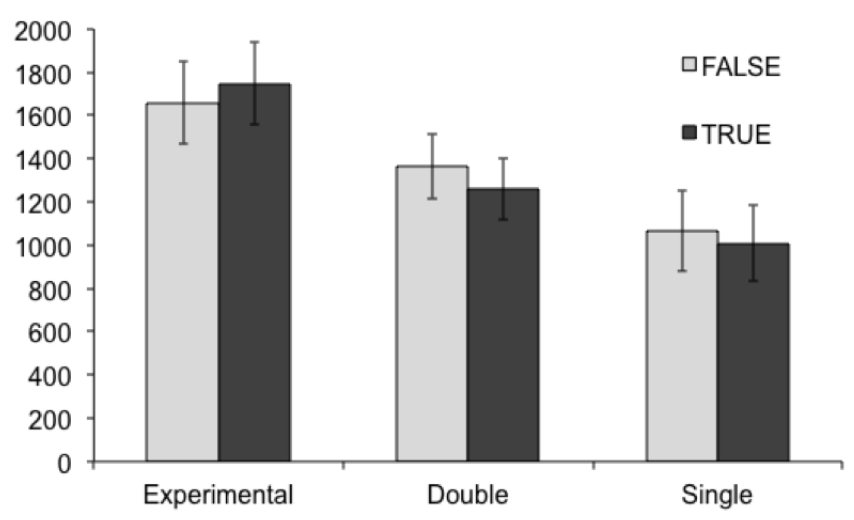

Fig. 1. Response times (ms) for experiment 1

\section{Experiment 2}

One of the difficulties of allowing participants to choose which interpretation they made to the experimental sentences is that is not possible to determine which responses are errors and which responses are correct interpretations. This means that the RT analysis contains noise and consequently has low power. Experiment 2 was similar to experiment 1, except that the 67 participants $(7$ excluded) were trained on sentences of the TARGET type with corrective feedback in a preliminary phase: half of them were trained to answer true (logical) and the other half to answer false (FC). Accuracy was high and approximately equal across conditions, both for the control sentences and for the TARGET sentences, in both groups $(M>.92, S D<.05)$.

\section{Response times}

Figure 2 shows the pattern of RTs. For control sentences, true responses were significantly faster than false responses $\left(t_{1} \mathrm{~s}(59)>4.0, t_{2} \mathrm{~s}(19)>2.8, p \mathrm{~s}<.01\right)$. But for the experimental sentences the reverse was true: FC responses (false) were derived marginally faster than logical (true) responses $\left(t_{1}(58)=1.7, p=\right.$ $\left..10, t_{2}(19)=5.0, p<.0005\right)$. This replicates the pattern observed in experiment 1 . As an extra test of the difference between FC and logical responses we used a mixed model regression analysis that combined participants and items. This analysis demonstrated significantly faster FC responses responses $(p<.05) .{ }^{3} \mathrm{We}$ also controlled for response bias, just as we did in experiment 1, by removing true control RTs from logical responses to the TARGET sentences, and false control

\footnotetext{
${ }^{3}$ We were unable to conduct this analysis for experiment 1 because of the large number of missing cells for many participants.
} 
RTs from FC responses. This revealed significantly faster FC responses when using single or double sentences as controls $\left(t_{\mathrm{s}}(58)>2.5, p \mathrm{~s}<.05\right)$.

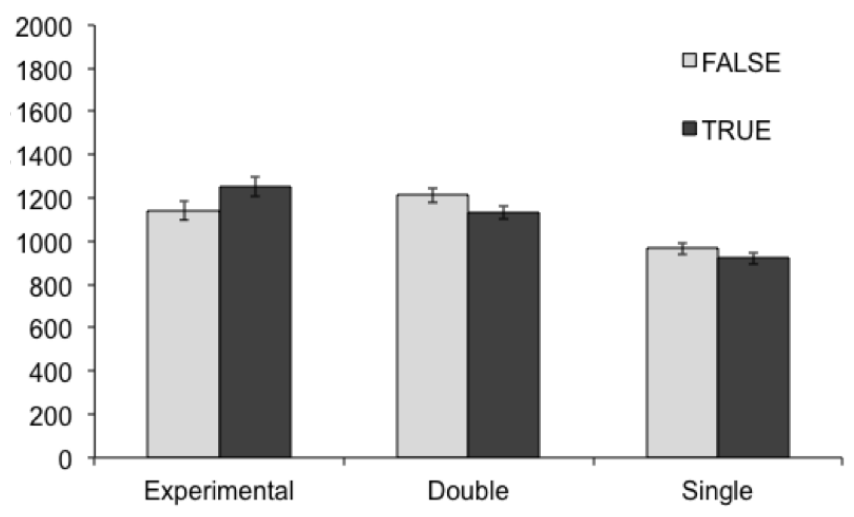

Fig. 2. Response times (ms) for experiment 2

\section{Conclusion}

We provide data from two studies modeled after the original experiments that detected a cost associated to the derivation of scalar implicatures. Our results show that, contrary to arguments coming from the theoretical literature, free choice inferences are different from scalar implicatures: they come with no processing cost, if not at a negative cost. In fact, this pattern of result is closer to the behavior of presuppositions (see Chemla and Bott, ress).

These results call for a greater differentiation between FC and scalar implicatures. They could lead to a reevaluation of the theoretical status of free choice. Alternatively, they could lead to a finer study of the origin of the cost found for scalar implicatures, which may tease apart scalar implicatures and free choice at a more superficial level. The alternatives involved in the two cases are of a different nature for scalar implicatures and for free choice (see Katzir, 2007). If processing cost for scalar implicature is mostly associated with alternatives (alternative derivation or comparison), then it could save the current, parsimonious scalar implicature accounts of free choice. 


\section{Bibliography}

Alonso-Ovalle, L. (2008). Innocent exclusion in an alternative-semantics. Natural Language Semantics 16(2), 115-128.

Bott, L. and I. A. Noveck (2004). Some utterances are underinformative: The onset and time course of scalar inferences. Journal of Memory and Language 51(3), 437-457.

Chemla, E. (2008). Présuppositions et implicatures scalaires: études formelles et expérimentales. Ph. D. thesis, EHESS.

Chemla, E. (2009). Similarity: towards a unified account of scalar implicatures, free choice permission and presupposition projection. Under revision for $\mathrm{Se}$ mantics and Pragmatics.

Chemla, E. and L. Bott (in press). Processing presuppositions: dynamic semantics vs pragmatic enrichment. Language and Cognitive Processes.

Fox, D. (2006). Free choice and the theory of scalar implicatures. unpublished manuscript.

Franke, M. (2011, June). Quantity implicatures, exhaustive interpretation, and rational conversation. Semantics and Pragmatics 4(1), 1-82.

Grodner, D. (2009). Speaker-specific knowledge in contrastive implicatures! In MayFest in Maryland.

Kamp, H. (1973). Free choice permission. Proceedings of the Aristotelian Society 74, 57-74.

Katzir, R. (2007). Structurally-defined alternatives. Linguistics and Philosophy 30(6), 669-690.

Klinedinst, N. (2006). Plurality and Possibility. Ph. D. thesis, UCLA.

Kratzer, A. and J. Shimoyama (2002). Indeterminate pronouns: The view from Japanese. The Proceedings of the Third Tokyo Conference on Psycholinguistics, $1-25$.

Schulz, K. (2003). You may read it now or later. A case study on the paradox of free choice permission. Master's thesis, University of Amsterdam. 


\title{
Corpus evidence for preference-driven interpretation ${ }^{\star}$
}

\author{
Alex Djalali, Sven Lauer, and Christopher Potts \\ Stanford University
}

\begin{abstract}
We present the Cards corpus of task-oriented dialogues and show how it can inform study of the ways in which discourse is goaland preference-driven. We report on three experimental studies involving underspecified referential expressions and quantifier domain restriction.
\end{abstract}

\section{Introduction}

There is growing interest in the notion that both production and interpretation are shaped by the goals and preferences of the discourse participants. This is a guiding idea behind the question-driven models of Ginzburg (1996), Roberts (1996), Groenendijk (1999), and Büring (2003), as well as the related broadly decision-theoretic approaches of van Rooy (2003), Malamud (2006), Dekker (2007), and Davis (2011). These models, which we henceforth refer to generically as goal-driven discourse models, are intuitively well-motivated, but they have so far been tested against only a limited number of mostly hand-crafted examples and highly specific phenomena (Schoubye 2009; Toosarvandani 2010), with relatively little quantitative or corpus evaluation that we know of (but see Cooper and Larsson 2001; Ginzburg and Fernandez 2010).

The central goal of this short paper is to introduce a new publicly-available resource, the Cards corpus, and show how it can be used to explore and evaluate goal-driven discourse models (see also Djalali et al. 2011). The Cards corpus is built from a two-person online video game in which players collaboratively refine a general task description and then complete that task together. The game engine records everything that happens during play, making it possible to study precise connections between the players' utterances, the context, and their general strategies. Because the corpus is large (744 transcripts, $\approx 23,500$ utterances) and its domain simple, it can be used to quantitatively evaluate specific pragmatic theories.

Here, we focus on the ways in which the players' conception of their task drives their understanding of referential and quantified noun phrases. After describing the corpus in more detail, we develop a simple goal-driven model that encodes and tracks certain important aspects of the players' preferences, and then we present three experiments designed to show how this model can be used to accurately resolve underspecified definites and quantifiers.

\footnotetext{
* We are indebted to Karl Schultz for designing the game engine underlying the Cards corpus. This research was supported in part by ONR grant No. N00014-10-1-0109 and ARO grant No. W911NF-07-1-0216.
} 


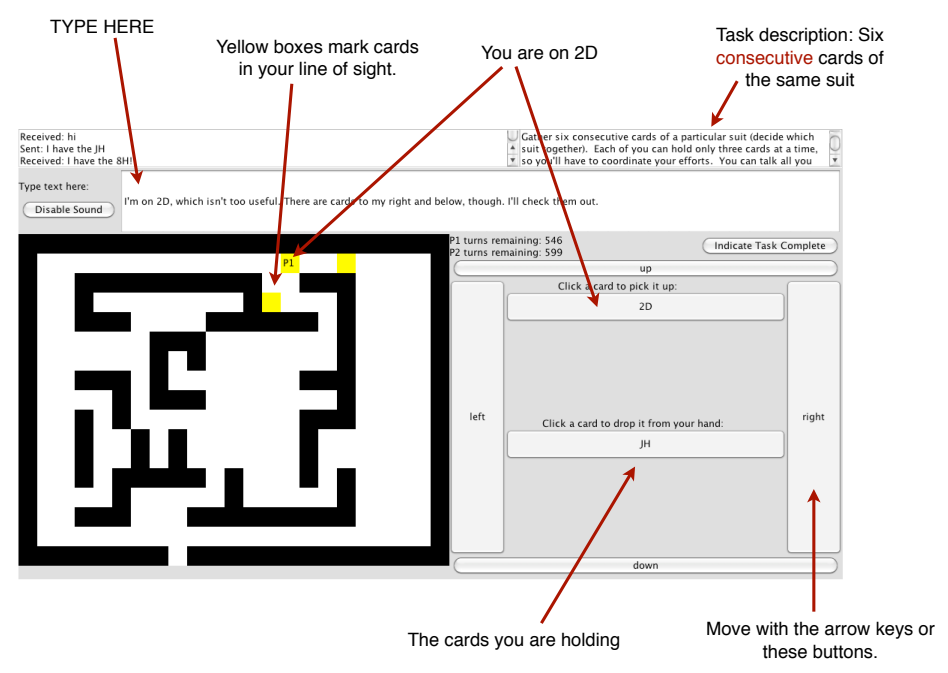

Fig. 1. An annotated version of the Cards gameboard.

\section{The Cards corpus}

The Cards corpus is built around a Web-based, two-person collaborative search task, partly inspired by similar efforts (Thompson et al. 1993; Allen et al. 1996; Stoia et al. 2008). We recruited players using Amazon's Mechanical Turk. The game-world consists of a maze-like environment in which a deck of cards has been randomly distributed. The players are placed in random initial positions and explore using keyboard input. A chat window allows them to exchange information and make decisions together. Each player can see his own location, but not the location of the other player. The visibility of locations of the cards are limited by distance and line-of-sight. Players can pick up and drop cards, but they can hold at most three cards at a time. In addition, while most of the walls are visible, some appear to a player only when within that player's line-of-sight.

When participants enter the game, they are presented with a description, some guidelines, and the annotated gameboard in figure 1. Before starting play, they are given the following task description (which remains visible in the upperright of the gameboard):

Gather six consecutive cards of a particular suit (decide which suit together), or determine that this is impossible. Each of you can hold only three cards at a time, so you'll have to coordinate your efforts. You can talk all you want, but you can make only a limited number of moves.

This task is intentionally underspecified. The players are thus forced to negotiate a specific goal and then achieve it together. In general, they begin by wandering around reporting on what they see, exchanging information as they go 


\begin{tabular}{|c|c|c|c|}
\hline Agent & Time & Action type & Contents \\
\hline Server & 0 & COLLECTION_SITE & Amazon Mechanical Turk \\
\hline Server & 0 & TASK_COMPLETED & 2010-06-17 10:10:53 EDT \\
\hline Server & 0 & PLAYER_1 & A00048 \\
\hline Server & 0 & PLAYER_2 & A00069 \\
\hline Server & 2 & MAX_LINEOFSIGHT & 3 \\
\hline Server & 2 & MAX_CARDS & 3 \\
\hline Server & 2 & GOAL_DESCRIPTION & Gather six consecutive cards ... \\
\hline Server & 2 & CREATE_ENVIRONMENT & [ASCII representation] \\
\hline Player 1 & 2092 & PLAYER_INITIAL_LOCATION & 16,15 \\
\hline Player 2 & 2732 & PLAYER_INITIAL_LOCATION & 9,10 \\
\hline
\end{tabular}

Table 1. Environment metadata in the corpus format.

until a viable strategy begins to emerge. Dialogue (1) is typical. (Between utterances, the players explore the environment and manipulate cards; this dialogue spans a total of 56 moves.)

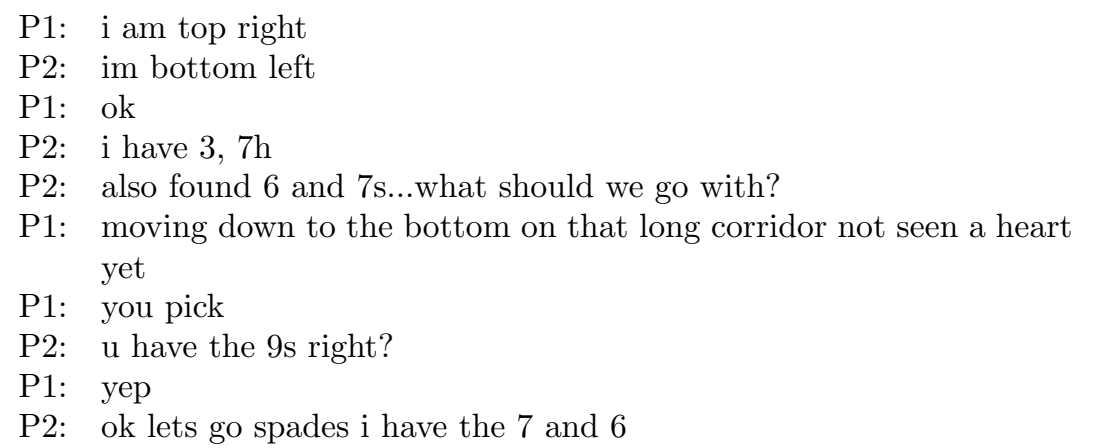

And then they pursue a specific solution. Because they can hold only three cards at a time, they are compelled to share information about the locations of cards, and their solutions are necessarily collaborative.

The current release (version 1) consists of 744 transcripts. Each transcript records not only the chat history, but also the initial state of the environment and all the players' actions (with timing information) throughout the game, which permits us to replay the games with perfect fidelity. In all, the corpus contains 23,532 utterances (mean length: 5.84 words), totaling 137,323 words, with a vocabulary size around 3,500. Most actions are not utterances, though: there are 255,734 movements, 11,027 card pick-ups, and 7,202 card drops. The median game-length is 414 actions, though this is extremely variable (standard deviation: 261 actions).

The transcripts are in CSV format. Table 1 is an example of the high-level environmental information included in the files, and table 2 is a snippet of gameplay. Computationally, one can update the initial state to reflect the players' actions, thereby deriving from each transcript a sequence of 〈context, event〉 pairs. This makes it easy to study players' movements, to make inferences about what 


\begin{tabular}{llll}
\hline Agent & Time & Action type & Contents \\
\hline Player 1 & 566650 & PLAYER_MOVE & 7,11 \\
Player 2 & 567771 & CHAT_MESSAGE_PREFIX & which c's do you have again? \\
Player 1 & 576500 & CHAT_MESSAGE_PREFIX & i have a 5c and an 8c \\
Player 2 & 577907 & CHAT_MESSAGE_PREFIX & i jsut found a 4 of clubs \\
Player 1 & 581474 & PLAYER_PICKUP_CARD & $7,11: 8 \mathrm{C}$ \\
Player 1 & 586098 & PLAYER_MOVE & 7,10 \\
\hline
\end{tabular}

Table 2. A snippet of gameplay in the corpus format.

guides their decision-making and, most importantly for pragmatics, to study their language in context.

The Cards corpus is available at http://cardscorpus.christopherpotts.net/. The distribution includes the transcripts, starter code for working with them in Python and $\mathrm{R}$, and a slideshow containing documentation. We think that the corpus fills an important niche; while there are a number of excellent taskoriented corpora available, the Cards corpus stands out for being large enough to support quantitative work and structured enough to permit researchers to isolate very specific phenomena and make confident inferences about the participants' intentions.

\section{Relevance and the evolving task}

The strategic aspects of interactions in the Cards corpus revolve around sequences of cards. In Djalali et al. 2011, we developed a hierarchy of abstract questions concerning the game, cards, and strategies, and showed that expert players and novice players negotiate this hierarchy differently: novices work systematically through it, whereas experts strategically presuppose resolutions of general issues so that they can immediately engage low-level task-oriented ones.

Here, we focus on how the relevance of particular cards changes as the players' strategies change. To this end, we define the value of a hand $H$, Value $(H)$, to be the minimum number of pick-up and drop moves from $H$ to a solution to the game. For example, Value $(\emptyset)=6, \operatorname{Value}(\{5 \mathrm{H}\})=5$, and Value $(\{5 \mathrm{H}, 8 \mathrm{H}\})=4$. Because dropping is costly, Value $(\{5 \mathrm{H}, 2 \mathrm{~S}, 3 \mathrm{D}\})=7$ : at least two cards have to be dropped before forward progress towards a solution.

The Value function gives rise to a measure of how relevant a card $c$ is given a hand $H$ :

$$
\text { Relevance }(c, H) \stackrel{\text { def }}{=} V(H)-V(H \cup\{c\})
$$

Where $c$ is intuitively relevant to $H$, this value is +1 , else it is -1 (or 0 if $c \in H$ ). Figure 2 illustrates the relevance sphere for a particular hand $H=\{2 \mathrm{H}, 4 \mathrm{H}, 5 \mathrm{H}\}$.

Our overarching hypothesis is that the players will seek cards that are relevant given their current holdings, and that this will be a driving force in how they resolve linguistic underspecification. The experiments in the next section seek 
to refine and support this basic idea. We should say, though, that the current notion of relevance is just an approximation of the players' underlying policy. We know, for example, that they often pick up irrelevant cards that might be relevant later - the cost of refinding cards is greater than the cost of having to drop those that turn out to be irrelevant. Our measure also does not take into account the costs of communication: the players have a slight bias for hearts, probably because this is the most iconic of the suits; they are reluctant to change suits once they have settled on one (even if this means extra exploration); and they favor solutions that don't span the Ace, since there is indeterminacy about whether such solutions are legitimate. We are confident that our results will only get stronger once these considerations are brought into the modeling. We mention them largely to emphasize that the corpus can support sophisticated investigations into decision making and pragmatics together.

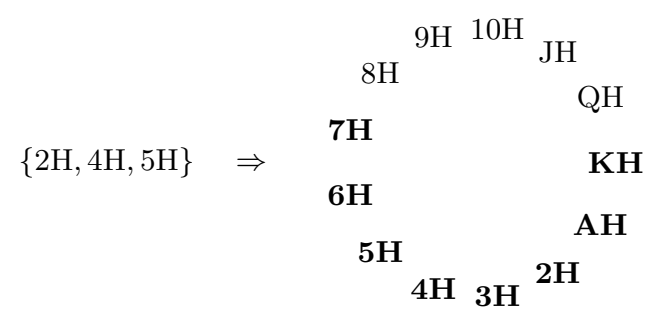

Fig. 2. Given the hand at left, the cards in bold are relevant in the sense that obtaining any one of them would move the players closer to a solution. The others would take the players farther from a solution, as measured by pick-up and drop moves, and are thus irrelevant given this hand.

\section{Experiments}

\subsection{Experiment 1: underspecified card references}

It is a testament to the importance of context that the majority of singular card references in the corpus (see table 3(a)) are like those in the following dialogue:

$$
\begin{aligned}
& \text { P2: Look for } 2 . \\
& \text { P1: }
\end{aligned}
$$

Absent knowledge of the context, we cannot know which cards these players are referring to. However, if we know that they are collectively holding $4 \mathrm{H}$ and $\mathrm{KH}$, then their intentions become clear: P2 refers to $2 \mathrm{H}$ and $\mathrm{P} 1$ to $3 \mathrm{H}$. Intuitively, such resolutions maximize relevance. More specifically, we hypothesized that, for any nominal referring expression $N$ and stage of play $t$, the intended referent will be in the set

$$
\operatorname{argmax}_{t, c \in \operatorname{Res}(N)} \operatorname{Value}\left(H_{t}\right)-\operatorname{Value}\left(H_{t} \cup\{c\}\right)
$$




\begin{aligned} & \hline Phrase type Count \\ & \hline Fully specified $103(37 \%) \\ &$ Underspecified $172(63 \%) \\ &$ Total 275 \\ & \hline\end{aligned}

(a) Singular definite card references

\begin{tabular}{rc}
\hline Inference & Count \\
\hline Correct & $164(95 \%)$ \\
Incorrect & $8(5 \%)$ \\
Total & 172 \\
\hline
\end{tabular}

(b) Results.

Table 3. Resolving underspecified card references via relevance.

where $H_{t}$ is the set of cards the players are currently holding at $t$, and $\operatorname{Res}(N)$ is the set of cards consistent with the descriptive content of $N$. (For example, $\operatorname{Res}(4)=\{4 \mathrm{H}, 4 \mathrm{D}, 4 \mathrm{~S}, 4 \mathrm{C}\}$ and $\operatorname{Res}(H)=$ the set of all hearts.)

To evaluate this hypothesis, we annotated the underspecified singular card references in 10 transcripts for what we took to be the intended referents and then wrote a computer program that chose the maximally relevant interpretation according to (4). (Where there was more than one such maximum, the program chose one at random.) We count a prediction as correct iff it matches the human annotation. The accuracy of this algorithm is extremely high (table 3(b)). What's more, we find that its mistakes tend to be clustered together near the start of transcripts, where even the interpreting player might have felt unsure about the speaker's intentions.

\subsection{Experiment 2: unrestricted quantification?}

From a strict logical perspective, quantifiers like everything, nothing, and anything carry universal force: the truth of a sentence involving them requires checking that every entity in the domain of discourse has (or does not have) the property they apply to. In this sense, P1 speaks falsely in (5) when he says, "I see nothing". We know this because the rich meta-data of the Cards corpus allows us to calculate exactly which cards P1 saw prior to this utterance. (He happened to have seen $5 \mathrm{C}$ and $10 \mathrm{~S}$.)

(5) P1: ok-i'll look at D and H, u look at C and S?

P2: ok

P1: i see nothing

It is clear why P1 is not perceived as speaking falsely, though: at the time of utterance, he had seen no cards that were relevant to his initial proposal, that is, no diamonds or hearts. In (6), the implicit restrictions are even more refined:

(6) P1: lets do spades

P1: I have the as, qs, and ks

[...]

P1: ok, i found js 


\begin{tabular}{llc}
\hline Quantifier & Literally true & Literally false \\
\hline anything & 2 & 6 \\
nothing & 0 & 6 \\
Total & $2(14 \%)$ & $12(86 \%)$ \\
\hline
\end{tabular}

Table 4. Experiment 2 results.

P2: Ok. I haven't found anything...lol

Here, P2's anything seems to range just over the cards that are relevant to the hand $\{\mathrm{AS}, \mathrm{QS}, \mathrm{KS}\}$, in the sense of (2). That is, P2 saw other cards, just not in this contextually privileged set.

The highly constrained nature of the Cards world means that we can precisely define the domain of discourse, which in turn permits us to identify exactly which contextual factors shape the domain in these cases. The first question we sought ask in this area is a seemingly simple one: what percentage of universally quantified claims are literally true, that is, true for an unrestricted interpretation of their quantifiers? To make our experiments manageable, we first extracted all utterances matching the regular expression in (7):

$$
\text { (find } \mid \text { found } \mid \text { see } \mid \text { saw) (any } \mid \text { no)thing }
$$

Such phrases include simple declaratives like I see nothing as well as interrogatives like Did you find anything? Although there are 35 matches for (7), we disregard ones like I didn't see anything around here, as they overtly restrict the domain of discourse and often involve indexical terms, whose semantics are more difficult to define.

We define one of these quantified phrases as literally true just in case it is true on an unrestricted interpretation of the quantifier, that is, a quantifier that ranges over the full deck of 52 cards. At any point $t$ in the game, each player $P$ will have walked over, and hence seen/found ('seeing' and 'finding' amount to the same thing in the Cards world, as players can identify only the cards they are currently standing on), a subset of the full deck of cards. Call this subset $S_{P, t}$. Suppose that, at time $t$, player $P$ says, "I found nothing". Then player $P$ 's claim is literally true just in case $S_{P, t}=\emptyset$ and literally false just in case $S_{P, t} \neq \emptyset$. If, at time $t$, a player $P$ asks "Did you find anything" and player $P^{\prime}$ responds "Yes", then $P^{\prime}$ 's response is literally true just in case $S_{P, t} \neq \emptyset$, and literally false just in case $S_{P, t}=\emptyset$. (Similarly if $P^{\prime}$ responds with 'No'.)

These definitions mean that we can quantitatively assess the percentage of universal claims that are literally true. The procedure is as follows: for each universally quantified claim made by a player $P$ at time $t$, build $S_{P, t}$ by following the full path taken by $P$ up to $t$ and adding to $S_{P, t}$ all and only those cards walked over by $P$ up to $t$. Table 4 summarizes the results of this experiment. As is evident, effectively no quantified phrases are literally true. Indeed, the only time players speak literally is during the initial stages of the game when they 
are trying to establish what sequence they should pursue, as in (8), in which P2 has found and is holding only the QC.

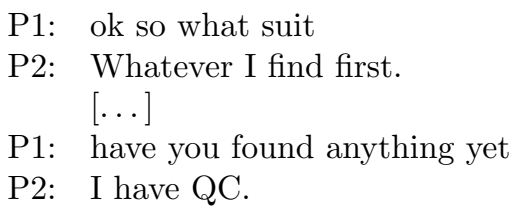

\subsection{Experiment 3: goal-based domain restriction}

The results of experiment 2 indicate that implicit quantifier restrictions are the norm. We turn now to the task of identifying which contextual factors the players use to provide these restrictions. It turns out the players' decisions about which suit to pursue are reliable indicators of how the domain of discourse is restricted. In the following dialogue, the players agree to pursue clubs:

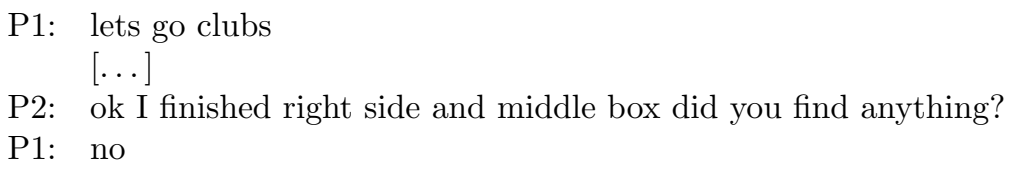

Literally speaking, P1 has spoken falsely, as (s)he found 11 cards prior to uttering "no". However, none of those findings include clubs. Thus, P1 speaks truthfully if we regard the earlier negotiation as restricting the domain for anything in P2's utterance.

In the terms of section 3, it looks like P1's proposal to limit to clubs makes all and only those cards relevant, which provides the basis for interpreting the quantifier. To test this idea, we hand-annotated all the transcripts involving the stimuli in experiment 2 for the players' mutually agreed-upon suit. The annotations mark the span of text in which the negotiation occurred with the suit they agreed upon. Phrases that indicated the start of such a negotiation included, but were not limited to, "let's go (for) $X$ ", "look for $X$ ", and simple suit mentions like "hearts?".

We now define one of the quantified phrases in (7) as restrictedly true just in case it is true on a restricted interpretation of the quantifier, that is to say, a quantifier that ranges only over cards with the agreed-upon suit. (A prominent edge case involved players who never overtly agreed upon any suit in particular, but rather deployed a strategy of stacking cards in a large pile and looking for any winning sequence. In such cases, we made the simplifying assumption that the domain of discourse included all and only the winning sequences.)

We were able to annotate the transcripts of 12 of the 14 quantified phrases considered in experiment 1 . We disregarded the two phrases that involved literally true uses of the quantifiers, as they were used when players are trying to establish what sequence they should agree upon, and reran the same experiment as above. The results are given in table 5. They are essentially the opposite of 


\begin{tabular}{lcl}
\hline Quantifier & Literally true & Literally false \\
\hline anything & 5 & 1 \\
nothing & 6 & 0 \\
Total & $11(92 \%)$ & $1(8 \%)$ \\
\hline
\end{tabular}

Table 5. Experiment 3 results.

those in table 4: when we restrict the domain of discourse to the players' agreed upon suit, utterances that were false become true. This is precisely the result one would expect on a model of discourse in which interpretation is governed by high-level factors relating to the discourse participants' understanding of the goals and issues at hand.

\section{Conclusion}

This paper introduced the Cards corpus, a highly-structured resource for doing corpus-driven computational pragmatics. In a series of experiments, we showed how the transcripts can be used to precisely define the domain, to ground denotations for quantified terms, and to pinpoint ways in which the context influences utterance understanding.

These experiments show that the Cards corpus can support quantitative evaluation of hypotheses in pragmatics. Going forward, we hope to expand our theoretical reach by pursuing the following inter-related goals:

- Increase the size and power of the corpus by collecting additional transcripts and altering the parameters of the game, e.g., number of moves, line of sight, and number of cards each player is able to hold.

- Extend our experimental techniques to a wider range of phenomena already present in the corpus. For example, there are many utterances in the corpus of the form " $4 \mathrm{H}$ " or even just " 4 ". In context, it is clear what the speaker intends: "Found the $4 \mathrm{H}$ ", "Can't find the $4 \mathrm{H}$ ", "Look for the $4 \mathrm{H}$ ", etc. We conjecture that, just as the context can be used effectively to resolve the underspecification of "4" (section 4.1), so too can it be used to resolve which predicate the speaker intended.

- Situate the above results in a fuller question-driven model of the sort employed by Djalali et al. 2011. Both implicit and explicit questions shape players' actions (where to move, what to pick up, when to speak, and so forth). For example, the players' negotiations about which suit to pursue (experiment 3) fit neatly into a goal-driven question hierarchy of the sort envisioned by Roberts (1996). Using a question model, we can study the players' linguistic behavior, and we can pursue questions in pragmatics and decision theory simultaneously, finding new ways in which language shapes, and is shaped by, the goals and preferences of the discourse participants. 


\section{Bibliography}

Allen, James F.; Bradford W. Miller; Eric K. Ringger; and Teresa Sikorski. 1996. A robust system for natural spoken dialogue. In Proceedings of ACL, 62-70.

Büring, Daniel. 2003. On D-trees, beans, and B-accents. Linguistics and Philosophy 26(5):511-545.

Cooper, Robin and Staffan Larsson. 2001. Accommodation and reaccommodation in dialogue. In Göteborg Papers in Computational Linguistics. Department of Linguistics, Göteborg University.

Davis, Christopher. 2011. Constraining Interpretation: Sentence Final Particles in Japanese. Ph.D. thesis, UMass Amherst.

Dekker, Paul. 2007. Optimal inquisitive discourse. In Maria Aloni; Alistair Butler; and Paul Dekker, eds., Questions in Dynamic Semantics, 83-101. Elsevier.

Djalali, Alex; David Clausen; Sven Lauer; Karl Schultz; and Christopher Potts. 2011. Modeling expert effects and common ground using Questions Under Discussion. In Proceedings of the AAAI Workshop on Building Representations of Common Ground with Intelligent Agents. AAAI.

Ginzburg, Jonathan. 1996. Dynamics and the semantics of dialogue. In Jerry Seligman, ed., Language, Logic, and Computation. CSLI.

Ginzburg, Jonathan and Raquel Fernandez. 2010. Computational models of dialogue. In Alex Clark; Chris Fox; and Shalom Lappin, eds., Handbook of Computational Linguistics and Natural Language Processing. Blackwell.

Groenendijk, Jeroen. 1999. The logic of interrogation. In Tanya Matthews and Devon Strolovitch, eds., Proceedings of SALT IX, 109-126. Cornell University.

Malamud, Sophia. 2006. Semantics and Pragmatics of Arbitrariness. Ph.D. thesis, Penn.

Roberts, Craige. 1996. Information structure: Towards an integrated formal theory of pragmatics. In Jae Hak Yoon and Andreas Kathol, eds., OSU Working Papers in Linguistics, 91-136. The Ohio State University Department of Linguistics. Revised 1998.

van Rooy, Robert. 2003. Questioning to resolve decision problems. Linguistics and Philosophy 26(6):727-763.

Schoubye, Anders. 2009. Descriptions, truth value intuitions, and questions. Linguistics and Philosophy 32(6):583-617.

Stoia, Laura; Darla Magdalene Shockley; Donna K. Byron; and Eric FoslerLussier. 2008. SCARE: A situated corpus with annotated referring expressions. In Proceedings of LREC.

Thompson, Henry S.; Anne Anderson; Ellen Gurman Bard; Gwyneth DohertySneddon; Alison Newlands; and Cathy Sotillo. 1993. The HCRC map task corpus: Natural dialogue for speech recognition. In HLT '93: Proceedings of the Workshop on Human Language Technology, 25-30. ACL.

Toosarvandani, Maziar. 2010. Association with Foci. Ph.D. thesis, UC Berkeley. 


\title{
Can Children Tell Us Something about the Semantics of Adjectives?
}

\author{
Francesca Panzeri and Francesca Foppolo \\ Università degli Studi di Milano-Bicocca, Milan, Italy \\ \{francesca.panzeri, francesca.foppolo\}@unimib.it
}

\begin{abstract}
We propose to investigate the process of the acquisition of relative gradable adjectives by children in order to gather new evidence to evaluate the main two theories that have been proposed to account for the meaning of gradable adjectives, i.e. the degree-based analysis and the partial function approach. We claim that younger children start by assigning a nominal like interpretation to relative gradable adjectives (tall means "with a vertical dimension"), and only at a later stage, for informativeness reasons, they access the comparative reading (tall means "taller than a standard"). We present and discuss the results of an experimental study in which we aimed at "turning adults into children". We show that, when informativeness is not at stake, even adults seem to access the nominal interpretation of relative adjectives. We eventually argue that the transition from the nominal to the comparative reading of relative adjectives is easily accounted for by a partial function approach.
\end{abstract}

Keywords. Semantics of adjectives, language acquisition, experimental semantics and pragmatics.

\section{The semantics of gradable adjectives}

The class of adjectives is heterogeneous, and different subclasses can be identified. Focusing on the distributional pattern, a distinction is drawn between gradable adjectives (GAs, those adjectives that can enter into a comparative construction and be modified by degree expressions, e.g., tall, intelligent, open), and not-gradable adjectives (e.g., Dutch, wooden, fourlegged). Ignoring so-called intensional adjectives (cf. [14]), not-gradable adjectives can be semantically viewed as referring to properties of individuals, that is, the meaning of the adjective Dutch can be identified with the set of individuals that are Dutch. The same meaning (function from individuals to truth-values) is not on the other hand straightforwardly attributable to gradable adjectives, ${ }^{1}$ given that GAs exhibit context depend-

1 Or, at least, to the subclass of relative gradable adjectives. Absolute gradable adjectives (full, open, straight, etc.) could in principle be analysed as denoting sets of individuals since they do not exhibit the same context dependency (cf. $[9,16])$. In this paper, however, for reasons 
ency (the same individual, for instance Bart, who is $188 \mathrm{~cm}$ tall, can be judged as tall for being an Italian man, and as not tall for being a basketball player) and borderline cases (if Bart is Dutch, we wouldn't be confident in judging the sentence neither true nor false, since the average height for men in Netherlands is $185 \mathrm{~cm}$ ).

To account for these facts, two main approaches have been proposed. The degree-based analysis (cf. the references in [8]) assumes that GAs differ from not-gradable ones in that they denote a relation between an individual and a degree, where degrees are conceived of as abstract entities ordered along a scale, associated with the dimension referred to by the adjective. Thus, the GA tall evokes a scale of degrees ordered with respect to the dimension of height, and a sentence containing tall is viewed as the assertion that an individual possesses the tallness property to certain degree. In fact, sentences involving bare uses of the adjective are analysed as concealed comparative sentences, thus "Bart is tall" gets interpreted as "Bart has a degree of height that exceeds a standard of comparison". For relative GAs, the standard of comparison has to be contextually retrieved, by making reference to the intended comparative class (e.g., Italian men or basketball players), or by taking into account other perceptually salient individuals (as in "tall compared to Leo").

Ewan Klein [10-12] on the other hand proposed a unified account for adjectives, viewing them as functions from individuals to truth values, with not-gradable (extensional) adjectives being total functions (thus corresponding to sets of individuals that share a property), and GAs being partial function. The idea is that, once we specify a particular domain of individuals that fall under the extension of a GA such as tall, the meaning of tall consists of a function that identifies the individuals who count as (definitely) tall, those who are (definitely) not tall, but for some individuals the function is undefined, i.e. it does not assign a value. Moreover, the restriction of the domain of application of the GA to particular subclasses (e.g., the set of Italian men or the set of basketball players) leads to different outputs of the value of the function (i.e., the same man can count as tall with respect to the class of Italian men, and as not-tall with respect to the class of basketball players). Thus, Klein accounts for the existence of borderline cases by means of the partiality of the GA function, and for the contextual variability of GAs by restricting their interpretation to different comparison classes.

of space, we will restrict our attention to relative gradable adjectives (and thus, in the final part, we will only report the results concerning the interpretation of relative adjectives, even if the experiments we ran aimed at investigating the analysis of absolute gradable adjectives as well). 
The degree-based account and the partial function approach have been compared on theoretical grounds (the partial function approach appears simpler and more in line with Frege's principle of compositionality, since it does not posit degrees as primitive entities, and it derives the meaning of the comparative construction from the meaning of the positive form, cf. [10]), and on empirical grounds (simplifying the question, adding particular constraints on the way individuals in the comparison classes are ordered, the two theories can be seen as nearly equivalent, cf. [15]). Our aim is to look for new evidence in favour or against these approaches by taking into account the process of language acquisition. Even if the proponents of these theories do not consider this question, ${ }^{2}$ and thus they do not make explicit prediction about the way children ought to interpret gradable adjectives, we think that children's linguistic behaviour could open a window into the semantics of adjectives.

\section{What children can tell us about the semantics of adjectives}

The process of language acquisition typically passes through different phases in which children may exhibit a linguistic behaviour that does not conform to adults' competence. For instance, in the first phase of the acquisition of the meaning of common nouns, children often make errors of over-extension, labelling $d a d d y$ any man, and this has been interpreted as evidence on the way children establish the mapping between words and objects. Our aim is to look at the way children understand and produce adjectives, focusing in particular on the errors they make, and then discuss how the two theories just discussed could account for these errors.

Even if adjectives are harder to acquire than nouns, children start using relative GAs as young as 2, and by the age of 3 they produce GAs such as big/small(little), tall/short, long/short, high/low, heavy/light. As for their interpretation, in experimental settings children appear to access the normative reading (judging an object to have the GA-property with respect to a normative class of comparison, e.g. judging a mitten to be big or small "as a mitten") and the perceptual reading (fixing the standard of comparison relative to another object physically present, cf. a.o. [4-5])). Nevertheless, it has been noticed that children make a consistent series of errors:

2 Except for Syrett's PhD thesis, which discusses children's interpretation of relative and absolute GAs. She adopts a degree-based account, and interprets children's performance under that perspective, even if she explicitly admits that some of children's unexpected behaviour would be straightforwardly explained assuming a kleinian-like semantics (cf. in particu$\operatorname{lar}[20]$, p. 41-42). 
they make substitution errors (e.g., they judge an object that is meant to be small as big, cf. [3], or they substitute tall with big, cf. [1]), and they exhibit "extreme labeling": when they are presented with a series of seven items that decrease along a relevant dimension (for instance, seven blocks decreasing in size), they tend to label as big (or small) only the extreme item of the series (cf. [18, 20]), whilst adults tend to put the cross-over point around the mid of the series. Since the experiments designed to test children's comprehension of relative GAs explicitly provided them with a comparative class, or perceptual cues, it is hard to tell what kind of interpretation children might access, and which framework better accounts for the facts. This is why we decided to design a new experiment.

\subsection{Experiment 1}

In a previous study (see [6]), we tested a group of 203 year-olds, 205 year-olds and adults as controls. In a first experiment, a truth-valuejudgment task we presented abstract objects in isolation, and asked subjects whether the description used by a puppet was correct, not correct, or whether they couldn't tell. Our experimental design aimed at not providing any contextual standard to judge relative GAs: the objects did not evoke any normative class, and were presented in isolation (without other perceptual cues) - for instance, we presented a $17,5 \mathrm{~cm}$ tall wooden rod describing it as "This is tall". Since the context did not provide any standard of comparison, we expected a prevalence of "I don't know" answers and/or chance distribution of "yes" and "no" answers. Our predictions were confirmed with adults: relative GAs received $60 \%$ of "I don't know", $30 \%$ of "yes", and $10 \%$ of "no"-answers. Quite surprisingly, 3 year-olds provided more than $88 \%$ of "yes"-answers (with "no" and "I don't know" at $6 \%$ each), accepting "This is rel-GA" (e.g. "This is big" or "This is small") applied to abstract objects in isolation. There seems to be a developmental trend, with 5 year-olds displaying 60\% of "yes", and 20\% each of "no" and "I don't know". Statistical comparisons by means of Fisher Exact Tests for count data revealed a significant general effect of Age in case of relative GAs $(\mathrm{p}<.001)$. A significant difference also emerged between 3 year-olds and 5 year-olds, between 3 year-olds and adults and between 5 year-olds and adults (all ps<.001). However, this effect might be partly due to the fact that children used the option "I don't know" much less than adults. Considering the proportion of "yes" answers over the total, the difference between the 3 and the 5 year-olds disappears $(p=.218)$, while it does not comparing the 5 year-olds and the adults $(\mathrm{p}<.01)$. In Table 1 we plot the distribution of participants' judgments by Age and Adjective with 
respect to relative GAs. Summing up, younger children tend to overwhelmingly accept descriptions of the form "This is rel-GA" applied to abstract objects in isolation (that is, when the context does not supply any standard of comparison), independently of the polarity of the adjective (i.e., they accept both antonyms).

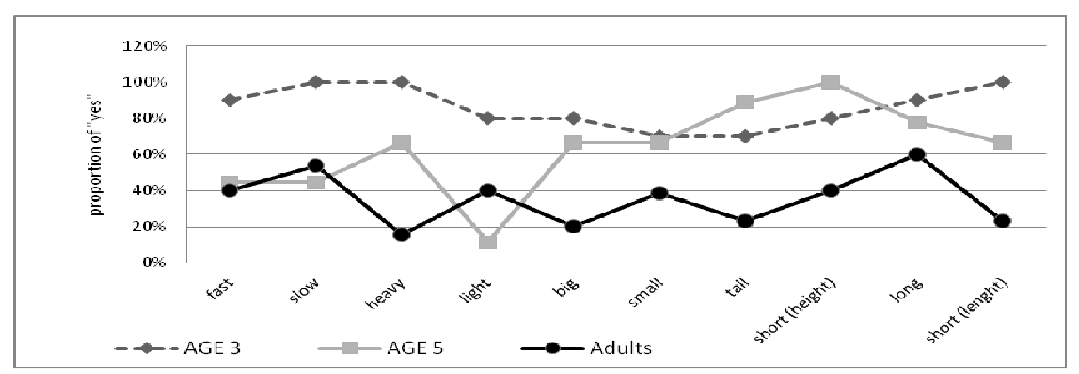

Table 1. Percentage of "yes" answers per Age of participants and Relative GA

We believe that this fact calls for an explanation, but we exclude the hypothesis that this high acceptance is simply due to the fact they do not know the meaning of the adjectives, and thus they opt for a "yes"-answer, because there's ample evidence in the literature that children know adjectives like the ones we tested from age 2 on (cf. the references in [20]), and moreover in a second experiment, a scalar judgment task, we presented a series of seven objects decreasing (or increasing) along a relevant dimension, and asked subject whether the description "This is rel-GA" could apply to each item (e.g., we presented a series of seven wooden rods ranging from $20 \mathrm{~cm}$ to $5 \mathrm{~cm}$ in height, and asked for each of them "Is this tall"?), and the group of 3 year-olds showed (the well-known phenomenon of extreme labelling, but also) a consistent behaviour - accepting the description for the first item of the series, and rejecting it for the last item, and thus we interpreted these results as evidence of the fact that children knew the meaning of the tested relative GAs. We also exclude the hypothesis that children are simply being extremely charitable, answering "yes" whenever they do not have strong reasons to reject the statement, because some of the fillers we tested were meant to prompt a "I don't know" response (e.g., a toy-zebra was described as "this is obedient", and, as expected, more that $90 \%$ of the adults reacted to these description using the option "I don't know"), and 3 year-old children split between a "yes" and a "no" answer: around 50\% accepted the description but half of them didn't, thus children do not choose the more charitable option whenever they can. 
Having excluded these simpler explanations, we propose to interpret the results assuming that there is a developmental trend in the interpretation of relative GAs: children start interpreting relative GAs as if they were referring to sets of individuals sharing a property (just like common nouns, and intersective adjectives), and only at a later stage they switch to the comparative-like reading. A similar hypothesis was proposed by H.H. Clark [2], who noticed that the positive relative GAs (tall, long, heavy...) are ambiguous between a nominal use and a comparative use. Thus, in (1) the relative GA tall is interpreted nominally, and the GA is neutral (that is, it does not imply that Lia is tall with respect to a standard of comparison), since it simply indicates that the dimension that is relevant for attributing a particular property to Lia is the vertical dimension; in (2), on the other hand, the relative GA is interpreted comparatively, and the reference to a contextual standard of comparison is necessary.

1. Lia is $156 \mathrm{~cm}$ tall.

2. Lia is tall.

If we assume that younger children firstly assign to relative GAs a nominal-like interpretation (and thus, tall would simply mean "that has a vertical dimension", fast "that has some speed", and so on) we could immediately account for the fact that younger children show a nearly at ceiling acceptance of the descriptions involving relative GAs, since, under this nominal interpretation, they would truthfully describe the objects presented. Only at a later stage, around 5 years of age, children would then realize that the nominal-like interpretation of relative GAs is highly underinformative in normal contexts, and would eventually realise that a comparative interpretation is needed.

\subsection{Experiment 2}

We are hypothesising that children start assigning to relative GAs a minimal interpretation (e.g. tall means "that has a vertical dimension"), that leads them to accept "This is rel-GA" referred to objects that do possess this minimal property even in the absence of a relevant standard of comparison that could trigger the comparative, stronger reading, and that they switch to the adult-like reading when they realise that in normal contexts such an interpretation would not add new information. This hypothesis assumes that there is contiguity between the nominal and the comparative interpretation, and that the latter is derived from the first one when informativeness is at stake. If this is correct, we should be able to detect the minimal, nominal-like, reading of relative GAs also in adults, once the 
informativeness requirement is not playing a role. ${ }^{3}$ In a novel experiment, our aim was to test adults' interpretation of relative GAs in a context that was manipulated in such a way to leave informativeness aside, in order to see whether in this new experimental setting adults would accept relative GAs the way younger children did in our previous study.

We tested 73 Italian adults, randomly assigned to one of two lists. Each list comprised a total of 29 items. Of these, fifteen were fillers: eight were clearly true, or clearly false, and the remaining seven were cases in which the adjective used was not applicable (NA) to the object, prompting a "no" response by the participants (for example, a toy plane was described as "This is vegetarian"). Differently from the first experiment, adults were given only two options of response: "yes" or "no", because children in the first study did not use the option "I don't know". In the second place, a training session was added, in which adults were asked to be as tolerant as they could with respect to the description provided by a puppet. The puppet was introduced as being an alien who had some hypotheses about the functioning of our language, and subjects were instructed to help him refining his hypotheses, correcting him when he was saying something clearly false, but accepting his sentences as long as they could apply in some way to the situation presented, even if his statements were not optimal. For instance, we presented a situation in which a toy-boy had four coins, and we recommended our subjects to reject clearly false sentences (e.g. the statement "The boy has seven coins"), but we also trained them to accept the alien's under-informative sentence "The boy has three coins", because even if the description was not optimal, correcting the alien could confound him because if he is told that it is false that the boy has three coins in a situation in which he actually has four, then the alien might think that the boy could not afford to buy an ice-cream that costs three coins. After this training session, we presented abstract objects in isolation, that were described by the alien as "This is adj", and asked the subjects to accept or reject these statements.

Compared to our previous study, on the basis of our hypotheses we predicted an increase of "yes" answers in case of Relative GAs, inasmuch as the adjectives could truthfully (even if not optimally) describe the objects under the minimal, nominal-like interpretation of the GAs. To make sure that adults were tolerant with critical cases only, we also predicted them

In a recent paper [7], Katsos and Bishop argue that children's performance with scalar implicatures (i.e., the fact they seem not to compute them, accepting underinformative sentences) is not imputable to the fact that they are not aware of under-informativeness, but to the fact that they more tolerant of pragmatic infelicity. Even if the question is interesting and worth pursuing, we will not explore this issue here. 
not to accept the descriptions in which the adjective used did not apply to the object, as it happened for the fillers classified as NA. For example, we expected adults to reject the description "This is vegetarian" referred to a toy-plane, on the basis that the property predicated by the adjective (i.e. "being vegetarian") cannot apply to a plane. Our results confirmed our predictions: adults' performance with fillers was at ceiling, and in particular, they did not hesitate in rejecting a description when the adjective used was not applicable to the object (cf. Filler-NA).

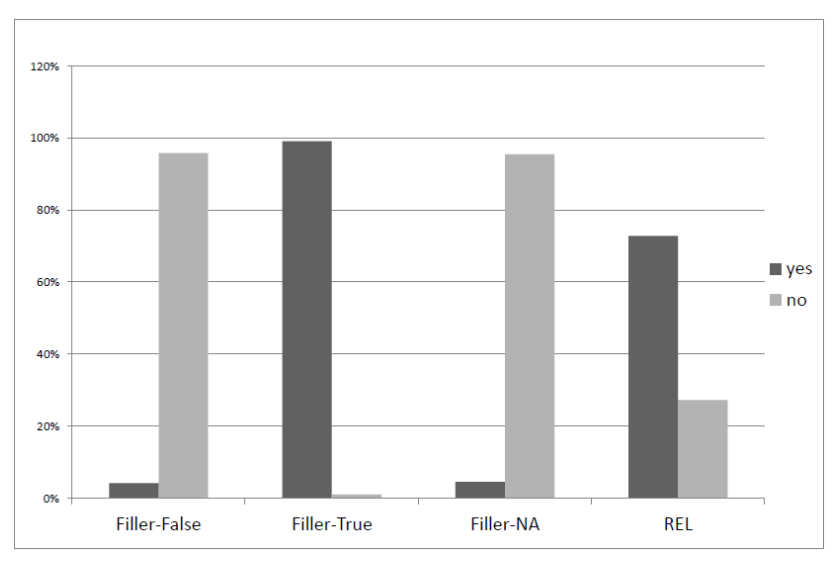

Table 2. Distribution of adult's responses in Experiment 2

Comparing adults' performance in the two studies in the interpretation of relative GAs, the increase of "yes" answer in this second experiment is significant, even taking into consideration the fact that in the second study the probability to answer "yes" was .50 , while it was .33 in the first experiment, where three options of response were given (X-squared $=85.35$, $\mathrm{df}=2, \mathrm{p}<.0001){ }^{4}$ Comparing adults' performance in the second study with children's performance in the first one (see table 3), adult's performance in case of relative GAs does not differ from the younger children. Statistical analysis confirms that: (i) adults do not differ from 3 year-old and 5 year-old children in case of relative GAs $(\mathrm{X}$-squared $=2.2709, \mathrm{df}=$ $1, \mathrm{p}$-value $=0.1318 ; \mathrm{X}$-squared $=1.7691, \mathrm{df}=1, \mathrm{p}$-value $=0.1835)$. The aim of turning adults into children did work in the end.

4 The proportion of "yes" answers in the second study is different from chance (X-squared = 46.2137, df $=1, \mathrm{p}<.0001)$. 


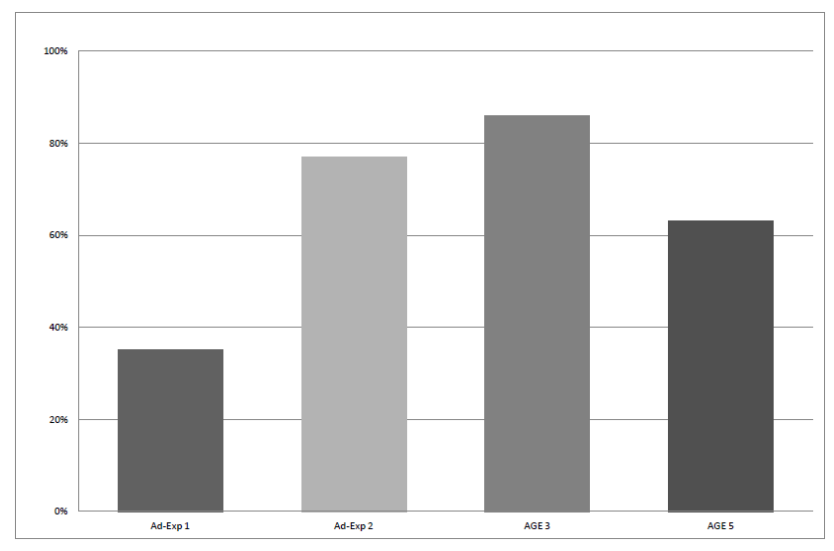

Table 3. Proportion of "yes" answers to rel-GAs: adults in Exp 1, Adults in Exp 2, 3 yo in Exp 1 and 5 yo in $\operatorname{Exp} 1$

\section{Conclusion}

Summing up, we suggest that children start interpreting relative GAs as common nouns, and other not-gradable adjectives, that is as denoting sets of individuals, and by identifying their denotation with the domain of application of the GA. At a later stage, for informativeness reasons, they realize that a stronger meaning - that involves a comparison - is needed, and thus they have an almost adult-like performance in tests in which they are asked to choose the "GA X", where X comes also with the intended normative class or perceptual cues. We believe that these facts can be easily accounted for by a partial function analysis of GAs - as partial functions from individuals to truth-values - assuming that children start treating GAs as total functions, and the evolutionary step consists in realising that the function denoted by the relative GA exhibit truth-value gaps and needs to be contextually restricted to particular domains of individuals (that is, comparison classes). On the contrary, we don't see any easy way to account for these results in degree-based semantics, since it requires as a first step to comprehend GAs the ability to construct abstract representation of degrees (scales), and moreover relative GAs are analysed as the assertion that an individual possesses the gradable property to a degree that exceeds a contextually given standard - and in out experimental settings even if the standard was not provided, younger children and adults in the second experiment did accept the relative GAs and judged them as applicable to the given objects. 


\section{References}

1. Brewer, W., Stone, J.B.: Acquisition of spatial antonym pairs. Journal of Experimental Child Psychology 19, 299-307 (1975)

2. Clark, H.H.: The primitive nature of children's relational concepts. In Hayes, J.R. (ed.) Cognition and the development of language, pp. 269-278. Wiley, New York (1970)

3. Donaldson M., Wales, R.J.: On the acquisition of some relational terms, In Hayes, J.R. (ed.) Cognition and the development of language. Wiley, New York (1970)

4. Ebeling, K.S., Gelman, S.A.: Coordination of size standards by young children. Child Development 59, 888-896 (1988)

5. Ebeling, K.S., Gelman, S.A.: Children's use of context in interpreting big and little. Child Development 65, 1178-1192 (1994)

6. Foppolo, F., Panzeri F.: Do children know when their room counts as clean? In: NELS 40: Proceedings of the 40th Annual Meeting of the North East Linguistic Society. GLSA Publications, University of Massachusetts, Amherst (to appear)

7. Katsos, N., Bishop, D.V.M.: Pragmatic tolerance: Implications for the acquisition of informativeness and implicature. Cognition 120.1, 67-81 (2011)

8. Kennedy, C.: Vagueness and Grammar: The Semantics of Relative and Absolute Gradable Adjectives. Linguistics and Philosophy 30, 1-45 (2007)

9. Kennedy, C. McNally, L.: Scale structure and the semantic typology of gradable predicates. Language 81.2, 345-381 (2005)

10. Klein, E.: A semantics for positive and comparative adjectives. Linguistics and Philosophy 4, 1-45 (1980)

11. Klein, E.: The interpretation of adjectival comparatives. Journal of Linguistics 18, 113-136 (1982)

12. Klein, E.: Comparatives. In von Stechow, A., Wunderlich, D. (eds.) Semantics: An International Handbook of Contemporary Research, pp. 673-691. de Gruyter, Berlin (1991)

13. Palermo, D.S.: More about less: A study of language comprehension. Journal of Verbal Learning and Verbal Behavior 12, 211-221 (1973)

14. Partee, B.H.: Lexical semantics and compositionality. In D. Osherson (series ed.), L. Gleitman \& M. Liberman (volume eds.) Invitation to cognitive science. Part I: Language, pp. 311-360. MIT Press, Cambridge. (1995)

15. van Rooij, R.: Vagueness and linguistics. In Ronzitti, G. (ed.) Vagueness: A Guide. Springer, Berlin (2010)

16. Rotstein, C., Winter, Y.: Total Adjectives Vs. Partial Adjectives: Scale Structure And Higher-Order Modifiers. Natural Language Semantics 12, 259-288 (2004)

17. Sera, M., Smith, L.B.: Big and Little: "Nominal" and Relative Uses. Cognitive Development 2, 89-111 (1987)

18. Smith, L.B., Cooney, N.J. \& McCord C.: What is "High"? The Development of Reference Points for "High" and "Low". Child Development 57.3, 583-602 (1986)

19. von Stechow, A.: Comparing Semantic Theories of Comparison. Journal of Semantics 3, 1-77 (1984)

20. Syrett, K.: Learning about the structure of scales: Adverbial modification and the acquisition of the semantics of gradable adjectives. Doctoral dissertation. Northwestern University, Department of Linguistics (2007) 


\title{
Presupposition Projection out of Quantified Sentences: Strengthening, Local Accommodation and Inter-speaker Variation ${ }^{\star}$
}

\author{
Yasutada Sudo ${ }^{1}$, Jacopo Romoli ${ }^{2}$, Martin Hackl ${ }^{1}$, and Danny Fox ${ }^{1,3}$ \\ ${ }^{1}$ Massachusetts Institute of Technology, ${ }^{2}$ Harvard University, \\ ${ }^{3}$ Hebrew University of Jerusalem \\ \{ysudo, fox, hackl\}@mit.edu, jromoli@fas .harvard.edu
}

\begin{abstract}
Presupposition projection in quantified sentences is at the center of debates in the presupposition literature. This paper reports on a survey revealing inter-speaker variation regarding which quantifier yields universal inferences - which $Q$ in $Q(B)\left(\lambda x . C(x)_{p(x)}\right)$ supports the inference $\forall x \in B: p(x)$. We observe an implication that if some yields a universal inference for a speaker, no, and any in a polar question do as well. We propose an account of this implication based on a trivalent theory of presupposition projection together with auxiliary assumptions suggested by [8].
\end{abstract}

Keywords: Presupposition Projection, Quantified Sentences, Trivalent Logic, Inter-speaker Variation

\section{Introduction}

The judgments regarding the presuppositions associated with quantificational sentences are often delicate, and different judgments are reported in the literature $([1,4,12])$. In this paper we focus on three particular types of quantificational sentences that are illustrated in (1).

(1) a. Some of the students drive their car to school

b. None of the students drive their car to school

c. Do any of the students drive their car to school?

We will refer to these three types of quantified sentence, an existential sentence, a negative existential sentence, and an existential polar question, respectively.

It is generally taken for granted that existential sentences like (1-a) do not have a universal inference, $\forall x: p(x)$ (but see $[3,5]$ ). According to some theories such as [16] (see also [12]), however, all quantificational determiner, including existential quantifiers, are predicted to carry a universal presupposition.

\footnotetext{
* We would like to thank Irene Heim and the audience at the Hackl lab meeting at MIT for the useful comments and suggestions. All the remaining errors belong to 
For negative existential sentences, [6] claims that it has a universal presupposition, while [1] contends that its presupposition is always existential, i.e. $\exists x: p(x)$. [1] goes one step further, and proposes a theory where the presupposition is existential for all quantificational determiners, not just none.

More recently [4] conducted an experiment whose results suggest that there are subtle differences among quantifiers. In particular, [4] provides evidence that existential sentences with modified numerals indeed lack a universal inference, while negative existential sentences tend to have a universal inference. However, [4]'s evidence is based on data pooled from all subjects and thus is not informative on possible variations in judgment among speakers. As we will show, such variation exists and it is an important aspect of the phenomenon that calls for an explanation.

This paper presents the results of an online survey that aims to investigate the possibility of inter-speaker variation in the distribution of universal inferences across the three types of quantified sentences mentioned above. In particular, our results indicate the following implication: if an existential sentence yields a universal inference for a speaker, a negative existential sentence and an existential polar question as well. We offer an account of this implication framed in a trivalent theory of presupposition projection ([2,7-10], among others). Trivalent theories predict a disjunctive presupposition for the three types of quantified sentences. Following [8], we assume that this presupposition is pragmatically marked and that two strategies can be used to mitigate this markedness: (i) pragmatic strengthening and (ii) insertion of the A-operator (defined below). We will see that the first strategy always yields a universal inference, whereas the second strategy never yields a universal inference for existential sentences, but could yield a universal inference for the other two types of quantificational sentences. We will propose that there are two types of speakers regarding the strategy they prefer to use, and demonstrate how this accounts for the implication found in the survey.

The organization of the paper is as follows. The survey is presented in Section 2. Our theoretical assumptions are introduced in Section 3, and our theory regarding inter-speaker variation is proposed in Section 4.

\section{Survey}

We conducted an on-line survey on Amazon Mechanical Turk (MTurk) ${ }^{1}$ whose main purpose was to investigate inter-speaker variation on which quantificational determiner yields a universal inference. We focus on the three types of sentences mentioned above, i.e. existential and negative existential sentences, and existential polar questions.

\footnotetext{
${ }^{1}$ https://www.mturk. com/mturk/welcome
} 


\subsection{Design}

We employed the 'covered box' method of [13]. The covered box method is a variant of picture selection method. In each trial of our survey, participants saw a sentence and a pair of pictures, and were asked to pick the picture that the sentence was about. One of the pictures was covered and invisible, while the other picture was overtly displayed. Participants were instructed to choose the covered picture only if the overt picture was not a possible match for the sentence.

The survey consists of 3 target trials, 3 control trials and 18 filler trials. The sentences used in the target trials are given below. They all contain both, which presupposes that there are exactly two entities satisfying the restriction.

(2) a. Some of these three triangles have the same color as both of the circles in their own cell

b. None of these three circles have the same color as both of the squares in their own cell

c. Do any of these three squares have the same color as both of the triangles in their own cell?

The overt picture in each of the target trials was designed in such a way that the universal inference is not satisfied in it. In addition for the trials with declarative sentences, the overt picture satisfies what is asserted (and implicated), e.g. some but not all of the three triangles have the same color as all of the circles in their own cell for (2-a). Therefore, the prediction is that the covered picture will be chosen if and only if the speaker gets a universal inference.

The overt pictures for the target trials are given in Figure 1. Each picture contains three cells, each of which in turn contains exactly one restrictor figure (e.g. a triangle for (2-a)). Crucially, only two of the cells have exactly two nuclear scope figures (e.g. circles for (2-a)), and the remaining one has only one. For trials with a polar question such as (2-c), the overt picture is colorless, and participants were instructed to imagine that somebody who is incapable of distinguishing colors is asking the question, and guess which picture they are asking about.

Fig. 1. Overt pictures in the target items

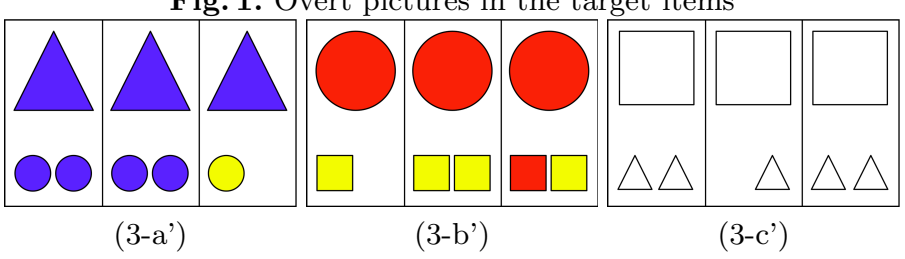

The three control items are identical in structure to the target items except for the following two points: the sentence mentions different restrictor and nuclear figures from the corresponding target item, and the overt picture satisfies 
the universal inference (i.e. all of the cells contain exactly two nuclear figures). Therefore all the participants are expected to choose the overt picture.

The eighteen filler items involve non-ambiguous quantificational sentences, eight of which are polar questions. None of them contain both taking scope below another quantifier. For half of the filler trials the overt picture matches the sentence, and for the other half, the overt picture does not satisfy the assertion and/or the presupposition of the sentence.

\subsection{Results}

193 participants were employed on MTurk, among which 10 non-native speaker participants and 78 other participants whose accuracy rate for the filler items was less than $80 \%$ (i.e. 4 or more mistakes) are excluded from the analysis. All participants were paid $\$ 0.20$ for their participation, and 36 of them are paid additional $\$ 0.25$ for answering all of the filler items correctly.

As there are two possible answers for each of the three target trials, there are eight possible answer patterns. The data from 115 native speakers of English is summarized in Table 1, where CP stands for the covered picture. ${ }^{2}$

Table 1. Results of the survey

\begin{tabular}{|c|c|c|c|c|c|c|}
\hline & $(2-b) \quad(2-c)$ & \# of $\mathrm{P}$ & & $(2-a)(2-b$ & (2-c) \# of $\mathrm{F}$ & \\
\hline 1 & Overt Overt & Overt & 33 & $\mathrm{CP}$ & Overt Overt & $\overline{2}$ \\
\hline 2 & Overt Overt & $\mathrm{CP}$ & 31 & $\mathrm{CP}$ & Overt CP & 1 \\
\hline 3 & Overt CP & Overt & 10 & $\mathrm{CP}$ & Overt & 0 \\
\hline 4 & Overt CP & $\mathrm{CP}$ & 27 & $\mathrm{CP}$ & $\mathrm{CP}$ & 11 \\
\hline
\end{tabular}

The distribution of participants across the answer patterns is clearly non-uniform. In particular, we observe the following implications:

(3) For a given speaker,

a. if the existential sentence (2-a) has a universal inference, then the negative existential sentence (2-b) and the existential polar question (2-c) do too;

b. if the existential sentence (2-a) does not have a universal inference, then the negative existential sentence (2-b) and the existential polar question (2-c) may or may not have a universal inference

\footnotetext{
${ }^{2}$ The error rate for the filler items is rather high, but inclusion of more subjects by lowering the cutoff accuracy rate does not undermine our results. Specifically, by lowering the cutoff accuracy to $75 \%$ (4 or less mistakes are allowed), 145 among the 183 native speaker participants, and by lowering it to $70 \%$ ( 5 or less mistakes are allowed), 168 subjects remain. In both cases, all patterns but 5-7 show an increase in number roughly proportionate to the number of the additional subjects, while 5-7 stay constant.
} 
It should also be remarked that 11 out of 115 participants chose the covered picture for the existential sentence, indicating that they obtained a universal inference for it. As remarked at the outset, it is generally considered that existential sentences have non-universal inferences, and our data shows a clear tendency in line with this intuition (see also [4]). Nonetheless, the existence of speakers preferring a universal reading is theoretically interesting, and as we will demonstrate, our theory accounts for both types of speakers.

The results of the control items are as follows. Recall that the overt picture satisfies the universal inference and hence is expected to be always chosen. In fact no participant chose the covered picture for the existential sentence, and only two subjects did so for the existential polar question. However, contrary to our expectation, the covered picture was chosen for the negative existential sentence by 23 out of 115 subjects. Although we cannot offer an explanation for this unexpectedly high figure for the negative existential sentence, it does not undermine the above observations, since excluding these subjects still indicates the same implicational patterns, as shown in the following table.

Table 2. Results from those participants who answered the control items correctly

\begin{tabular}{|c|c|c|c|c|c|}
\hline$(2-a)(2-b)$ & $(2-c)$ & \# of Participants & $\overline{(2-a)(2-b)}$ & $(2-c)$ & \# of Participants \\
\hline Overt Overt & Overt & 30 & CP Overt & Overt & $\overline{2}$ \\
\hline Overt Overt & $\mathrm{CP}$ & 27 & Overt & $\mathrm{CP}$ & 1 \\
\hline Overt CP & Overt & 6 & $\mathrm{CP}$ & Overt & 0 \\
\hline Overt CP & $\mathrm{CP}$ & 17 & $\mathrm{CP}$ & $\mathrm{CP}$ & 9 \\
\hline
\end{tabular}

\section{Trivalent Theory of Presupposition Projection}

\subsection{Three Truth Values and the Felicity Condition}

Trivalent theories $([2,7-10])$ postulate three, rather than two, truth values, denoted here by 0,1 and \#. The projection property of a given sentence is predicted via a pragmatic principle that requires a sentence to denote either 0 or 1 in each of the possible worlds in the current context set (in the sense of [19]). This pragmatic principle is stated as in (4).

\section{(4) Felicity Condition}

A (declarative) sentence $S$ can be felicitously used given a context set $C$ only if for all $w \in C, \llbracket S \rrbracket(w) \neq \#$

The Felicity Condition can be given a pragmatic motivation. It can be thought of as a consequence of a principle of conversation demanding that an utterance of a declarative sentence tell the conversational participants which worlds in the context to retain and which ones to discard. This demand will not be met if in 
any of the worlds in the context set the declarative sentence is neither true nor false (cf. [19]).

In this system, the presupposition of a declarative sentence is the proposition that needs to be true for the sentence to denote either 0 or 1 . As an illustration, consider the simple example below.

(5) John drives his car to school

Suppose that the denotation of this sentence is as in (6). To simplify the discussion, we only consider the presuppositions triggered by the possessive construction.

(6) $\lambda w \cdot\left\{\begin{array}{l}1 \text { if John has a car and drives it to school in } w \\ 0 \text { if John has a car and does not drive it to school in } w \\ \# \text { otherwise }\end{array}\right.$

According to the Felicity Condition in (4), for an utterance of (5) to be felicitous, it is required that John have a car in all of the possible worlds in the context set. Therefore (5) presupposes that John has a car.

\subsection{Extension to Polar Questions}

We now extend the above theory to polar questions. Following [14], we assume that questions denote sets of propositions (cf. [14]). Notice that the Felicity Condition in (4) does not apply to question denotations, and therefore we postulate a separate pragmatic condition for the use of questions. ${ }^{3}$ It is reasonable to assume that a question needs to be maximally relevant to the current conversation in the sense that it concerns all the possibilities that are currently open. We formulate this condition as follows.

\section{Felicity Condition for Questions}

A question $Q$ can be felicitously used given a context set $C$ only if for all $w \in C$, there is $q \in \llbracket Q \rrbracket$ such that $q(w) \neq \#$

This is evidently not meant to be the only condition on a felicitous use of a question. Other conditions include, for example, that the answer is not known yet, and that all propositions in the denotation are not known to be false, which amounts to requiring that for all $q \in \llbracket Q \rrbracket$, there is $w \in C$ such that $q(w)=1$. But as it turns out, only (7) matters for our purposes at hand.

In (8) we give a simple example for illustration.

(8) Does John drive his car to school?

Suppose that the denotation of this question is in (9).

\footnotetext{
${ }^{3}$ [8] suggests a bivalent reformulation of the theory using the notion of relevance with the aim of giving declarative sentences and questions a uniform treatment. We will not pursue this alternative in this paper.
} 
(9) $\quad\{\llbracket$ John drives his car to school $\rrbracket, \neg \llbracket$ John drives his car to school $\rrbracket\}$

$$
=\{\llbracket(5) \rrbracket, \neg \llbracket(5) \rrbracket\}
$$

The condition in (7) demands that in each world $w$ in the context set, either $\llbracket(5) \rrbracket(w)=1$ or $\llbracket(5) \rrbracket(w)=0$. Therefore, $(8)$ has the same presupposition as (5), namely that John owns a car.

\subsection{Disjunctive Presuppositions for Quantified Sentences}

As we will demonstrate in this subsection, trivalent theories assign a disjunctive presupposition to all of the three types of quantified sentences that we are interested in in the present paper. ${ }^{4}$ For ease of exposition, we schematically represent the meanings of quantificational sentences as in (10).

$$
\begin{aligned}
& Q(B)\left(\lambda x . C(x)_{p(x)}\right) \text { where } \\
& \text { a. } Q \text { is a determiner denotation } \\
& \text { b. } \quad B \text { is the restrictor of } Q \\
& \text { c. } \quad \lambda x . C(x)_{p(x)} \text { is the nuclear scope of } Q \text { with the presupposition that } \\
& \quad p(x)
\end{aligned}
$$

The predicted presupposition of the three types of quantificational sentences that we are after is $[\exists x \in B: p(x) \wedge C(x)] \vee[\forall x \in B: p(x)]$. Let us look at the three cases in turn.

Firstly, the truth conditions of an existential sentence are given in (11).

$$
\begin{aligned}
& \llbracket \text { some } \rrbracket(B)\left(\lambda x \cdot C(x)_{p(x)}\right) \\
& =\lambda w \cdot\left\{\begin{array}{l}
1 \text { if } \exists x \in B: p(x) \wedge C(x) \text { in } w \\
0 \text { if }[\forall x \in B: p(x)] \wedge[\neg \exists x \in B: p(x) \wedge C(x)] \text { in } w \\
\# \text { otherwise }
\end{array}\right.
\end{aligned}
$$

The Felicity Condition requires that for each possible world $w$ in the context set, either $\exists x \in B: p(x) \wedge C(x)$ or $[\forall x \in B: p(x)] \wedge[\neg \exists x \in B: p(x) \wedge C(x)]$. Thus the presupposition of (11) is the disjunction of these two propositions, i.e. $[\exists x \in B: p(x) \wedge C(x)] \vee[\forall x \in B: p(x)]$.

Given that a negative existential sentence is the negation of the corresponding existential sentence, i.e. (12), the exact same presupposition is predicted for negative existential sentences.

$$
\begin{aligned}
& \llbracket \text { none } \rrbracket(B)\left(\lambda x \cdot C(x)_{p(x)}\right) \\
& =\lambda w \cdot \begin{cases}1 & \text { if } \llbracket \text { some } \rrbracket(B)\left(\lambda x . C(x)_{p(x)}\right)(w)=0 \\
0 & \text { if } \llbracket \text { some } \rrbracket(B)\left(\lambda x . C(x)_{p(x)}\right)(w)=1 \\
\# \text { otherwise }\end{cases}
\end{aligned}
$$

Furthermore, an existential polar question has the same disjunctive presupposition, if we assume the denotation of $\llbracket ? \rrbracket \llbracket$ any $\rrbracket(B)\left(\lambda x . C(x)_{p(x)}\right)$, where '?' is the

\footnotetext{
${ }^{4}$ As demonstrated in $[2,7,9,10]$, a trivalent theory is applicable to various kinds of connectives, but as they are not our central concern, we will concentrate on the predictions for quantificational sentences here.
} 
question operator, to be the set $\left\{\llbracket\right.$ some $\rrbracket(B)\left(\lambda x . C(x)_{p(x)}\right), \llbracket$ none $\left.\rrbracket(B)\left(\lambda x . C(x)_{p(x)}\right)\right\}$. The Felicity Condition demands that for each possible world in the context set, either of these propositions is true, i.e. $[\exists x \in B: p(x) \wedge C(x)] \vee[\forall x \in B: p(x)]$ must be true in each world. Therefore the same disjunctive presupposition $[\exists x \in$ $B: p(x) \wedge C(x)] \vee[\forall x \in B: p(x)]$ is predicted.

\subsection{Two Strategies}

Following [8], we assume that the disjunctive presupposition $[\exists x \in B: p(x) \wedge$ $C(x)] \vee[\forall x \in B: p(x)]$ is pragmatically marked and triggers one of two repair strategies: (i) pragmatic strengthening or (ii) insertion of the operator that turns a trivalent proposition into a bivalent one, which we call the A-operator following [2].

Again following [8], we assume that when applied to the disjunctive presupposition, pragmatic strengthening yields the universal inference $\forall x \in B: p(x) .{ }^{5}$ Since three types of quantified sentences we are interested in here have the same disjunctive presupposition as shown in the previous subsection, pragmatic strengthening invariably yields the universal inference for all of them.

On the other hand the A-operator can result in a universal or weaker inference depending on its scope and the quantifier. The denotation of the operator is given in (13).

$$
\llbracket \mathrm{A} \rrbracket=\lambda p_{\langle s, t\rangle} \cdot \lambda w \cdot\left\{\begin{array}{l}
1 \text { if } p(w)=1 \\
0 \text { if } p(w)=0 \text { or } p(w)=\#
\end{array}\right.
$$

$\mathrm{W}$ assume that the A-operator is a phonologically null operator that may appear in any syntactic position where the sister constituent is of the propositional type $\langle s, t\rangle{ }^{6}$ Let us now examine what the A-operator yields for the three types of quantified sentences.

An existential sentence with the A-operator above the quantifier, i.e. $\llbracket \mathrm{A} \rrbracket\left(\llbracket\right.$ some $\left.\rrbracket(B)\left(\lambda x \cdot C(x)_{p(x)}\right)\right)$, denotes the following function.

$$
\lambda w \cdot\left\{\begin{array}{l}
1 \text { if } \llbracket \text { some } \rrbracket(B)\left(\lambda x . C(x)_{p(x)}\right)(w)=1 \\
0 \text { otherwise }
\end{array}\right.
$$

This does not have a universal inference, and only entails an existential inference $\exists x \in B: p(x)$. Also it is easy to see that the predicted denotation is the same, when the $\mathrm{A}$-operator is applied below the quantifier, i.e. $\llbracket$ some $\rrbracket(B)\left(\lambda x . \llbracket \mathrm{A} \rrbracket\left(C(x)_{p(x)}\right)\right)$. Therefore if the A-operator is present, the existential sentence only has an existential entailment.

\footnotetext{
${ }^{5}$ See [8] for discussion of the way issues pertaining to the proviso problem ([11, 17 , 18]) arise in this context and how they might be dealt with.

${ }^{6}$ Unlike [2], we do not impose any other condition on the use of the A-operator here, although ultimately some notion of preference among different scopes might be necessary.
} 
For a negative existential sentence, on the other hand, insertion of the Aoperator still can result in a universal inference. Consider $\llbracket \mathrm{A} \rrbracket\left(\llbracket\right.$ none $\left.\rrbracket(B)\left(\lambda x . C(x)_{p(x)}\right)\right)$ whose denotation is given in (15).

$$
\lambda w .\left\{\begin{array}{l}
1 \text { if } \llbracket \text { none } \rrbracket(B)\left(\lambda x . C(x)_{p(x)}\right)(w)=1 \\
0 \text { otherwise }
\end{array}\right.
$$

This entails the universal statement $\forall x \in B: p(x)$. In addition, there is a second use of the A-operator which gives rise to a weaker inference: when it applies below the quantifier, i.e. $\llbracket$ none $\rrbracket(B)\left(\lambda x . \llbracket \mathrm{A} \rrbracket\left(C(x)_{p(x)}\right)\right)$, the sentence is true in $w$ iff $\neg \exists x \in B:[p(x) \wedge C(x)]$ is true in $w$, from which neither an existential nor a universal inference is derived.

Finally the A-operator can yield a universal inference for an existential polar question too. When it is applied above the question operator, the predicted meaning is $\left\{\llbracket \mathrm{A} \rrbracket\left(\llbracket \operatorname{any} \rrbracket(B)\left(\lambda x . C(x)_{p(x)}\right)\right), \llbracket \mathrm{A} \rrbracket\left(\neg \llbracket \operatorname{any} \rrbracket(B)\left(\lambda x \cdot C(x)_{p(x)}\right)\right)\right\}$. Since the presupposition of a polar question is that either of the answers is true in each world in the context set, the presupposition is the disjunction $[\exists x \in B: p(x) \wedge$ $C(X)] \vee[\forall x \in B: p(x)]$. Thus the A-operator used in this way still results in the disjunctive presupposition. Because the disjunctive presupposition is marked and needs to be remedied, and pragmatic strengthening is still available, a universal inference ensues in this case. Alternatively, the A-operator may take lower scope, yielding $\left\{\llbracket \mathrm{A} \rrbracket\left(\llbracket \operatorname{any} \rrbracket(B)\left(\lambda x . C(x)_{p(x)}\right)\right), \neg \llbracket \mathrm{A} \rrbracket\left(\llbracket \operatorname{any} \rrbracket(B)\left(\lambda x . C(x)_{p(x)}\right)\right)\right\}$. Since this is a bipartition of any set of possible worlds, the presupposition is trivial. Similarly, when the A-operator takes scope below the quantifier, the resulting set, $\left\{\llbracket \operatorname{any} \rrbracket(B)\left(\lambda x \cdot \llbracket \mathrm{A} \rrbracket\left(C(x)_{p(x)}\right)\right), \neg \llbracket \operatorname{any} \rrbracket(B)\left(\lambda x . \llbracket \mathrm{A} \rrbracket\left(C(x)_{p(x)}\right)\right)\right\}$, is a bipartition of any set of possible worlds and only a trivial presupposition is predicted.

To sum up, pragmatic strengthening invariably yields a universal inference for all of the three types of quantified sentences. The A-operator, on the other hand, may give rise to weaker inferences depending on its scope and the quantifier. More specifically, existential sentences are never associated with a universal inference regardless of the scope of the A-operator, while negative existential sentences and existential polar questions can but do not have to have a universal inference.

\section{Account of the Implications}

The implications in (3) found in the survey are repeated here.

(3) For a given speaker,

a. if the existential sentence (2-a) has a universal inference, then the negative existential sentence $(2-b)$ and the existential polar question (2-c) do too;

b. if the existential sentence (2-a) does not have a universal inference, then the negative existential sentence $(2-b)$ and the existential polar question (2-c) may or may not have a universal inference 
In order to account for these implications, we propose that there are two types of speakers: (i) those who use the A-operator in one way or another for all three sentences, and (ii) those who never do. This explains the implication (3) as follows. Those who do not use the A-operator always resort to pragmatic strengthening, obtaining a universal inference for all of the three cases. This accounts for the implication in (3-a). On the other hand, those who use the A-operator never get a universal inference for existential sentences, but may get a universal inference for negative existential sentences and existential polar questions, depending on where it is inserted. This accounts for the implication in $(3-b)$.

\section{References}

1. Beaver, David (2001) Presupposition and Assertion in Dynamic Semantics. Stanford: CSLI.

2. Beaver, David and Emiel Krahmer (2001) A partial account of presupposition projection. Journal of Logic, Language and Information, 10: 147-182.

3. Charlow, Simon (2009) "Strong" predicative presuppositional objects. In Natahan Klinedinst and Daniel Rothschild (eds.), Proceedings of ESSLLI 2009 Workshop: New Directions in the Theory of Presupposition.

4. Chemla, Emmanuel (2009) Presuppositions of quantified sentences: experimental data. Natural Language Semantics, 17: 299-340.

5. Chemla, Emmanuel (ms) Similarity: towards a unified account of scalar implicatures, free choice permission and presupposition projection. Ms., ENS.

6. Cooper, Robin (1983) Quantification and Semantic Theory. Dordrecht: Reidel.

7. Fox, Danny (2008) Two short notes on Schlenker's theory of presupposition projection. Theoretical Linguistics, 34: 237-252.

8. Fox, Danny (2010) Presupposition projection, trivalent and relevance. Handout of the talk at University of Connecticut.

9. George, Benjamin (2008a) A new predictive theory of presupposition projection. In Proceedings of SALT 18. 358-375.

10. George, Benjamin (2008b) Presupposition Repairs: a Static, Trivalent Approach to Predicting Projection. MA thesis, University of California, Los Angeles.

11. Geurts, Bart (1999) Presuppositions and Pronouns. Amsterdam: Elsevier.

12. Heim, Irene (1983) On the projection problem of presuppositions. In Proceedings of WCCFL 2. 114-125.

13. Huang, Yi Ting, Elizabeth Spelke and Jesse Snedeker (ms.) What exactly do numbers mean? Ms., Harvard University.

14. Karttunen, Lauri (1977) Syntax and semantics of questions. Linguistics and Philosophy, 1: 3-44.

15. Peters, Stanley (1979) A truth-conditional formulation of Karttunen's account of presupposition. Synthese, 40: 301-316.

16. Schlenker, Philippe (2009) Local contexts. Semantics and Pragmatics, 3.

17. Schlenker, Philippe (to appear) The proviso problem: a note. To appear in Natural Language Semantics.

18. Singh, Raj (2008) Modularity and Locality in Interpretation. Ph.D. dissertation, Massachusetts Institute of Technology.

19. Stalnaker, Robert (1978) Assertion. In Peter Cole (ed.), Syntax and Semantics 9: Pragmatics. 315-332. NY: Academic Press. 


\title{
When Wide Scope Is not Enough: Scope and Topicality of Discourse Referents
}

\author{
Gemma Barberà \\ Universitat Pompeu Fabra \\ Roc Boronat, 138, Barcelona, Spain \\ gemma.barbera@upf.edu \\ http://parles.upf.edu/llocs/gbarbera/
}

\begin{abstract}
This paper analyses the semantic attributes discourse referents in Catalan Sign Language may have in order to have a corresponding location established in sign space. It is argued that a combination of scope and topicality is required when analysing the correlation between the introduction of entities into the discourse and assigning a spatial location.
\end{abstract}

Keywords: Catalan Sign Language (LSC), location, modal subordination, scope, sign space, specificity

\section{Introduction}

As natural languages in a visual-spatial modality, sign languages use the space in front of the signer's torso to articulate signs. Sign space is morphosyntactically relevant, since signs are spatially modulated for grammatical purposes to express number, person, and arguments of the verb. It is also relevant at the discourse level because entities introduced into the discourse model are identified with certain spatial locations localised on the horizontal plane [12], which is the plane that extends parallel to the floor $[18,2]$. However, the semantic attributes that discourse referents should have in order to be spatially localised have not been thoroughly analysed.

Catalan Sign Language (LSC) makes systematic use of signs directed to the horizontal plane, as commonly assumed for other sign languages (SLs) but also to the frontal plane, which is the plane that extends parallel to the signer's body. This paper focuses on the grammatical distinction denoted by the establishment of discourse referents within the two parts of the frontal plane, namely upper and lower. It is argued that this distinction is relevant for LSC grammar and it is explained in terms of scope behavior as well as topicality. My main claims are interrelated: (i) The expression of narrow scope quantifiers leads to a lack of spatial location establishment; however when focusing on specificity contexts, (ii) narrow scope related to specificity is overtly encoded in LSC grammar and, more particularly, it is expressed with marked spatial locations established on the upper part of the frontal plane; yet (iii) narrow scope variables can also 
establish a lower spatial location as long as they denote a prominent DR.

The rest of the paper is structured as follows. Section 2 analyses the relation between narrow scope marking and sign space in LSC. Section 3 presents contexts of modal subordination where variables attached to narrow scope quantifiers discursively behave as wide scope ones. Section 4 presents the interaction between scope and prominence, and section 5 concludes.

\section{Scope marking}

Discourse referents (DRs) are semantic objects which denote the object of thought or the thing the discourse is about. Once established in the discourse they can be referred back to by a pronoun or retrieved by a definite description [11]. A common assumption is that sign languages (SLs) localise DRs introduced into the discourse on the horizontal plane in sign space. However, not all the DRs introduced have a corresponding spatial location, nor they are only established on the horizontal plane. In LSC only DRs attached to wide scope quantifiers are spatially localised. The expression of narrow scope quantifiers leads to the lack of establishment of a spatial location, as contexts of dependent variables such as donkey sentences, genericity and quantified noun phrases (NPs) show. Nevertheless, narrow scope marking denoting specificity does not have a lack of spatial location establishment but rather a marked location established on the upper part of the frontal plane.

\section{$2.1 \quad$ Dependent variables}

Inspired in [6], dependent variables are variables introduced into the model the values assigned to which co-vary with those assigned to another variable. Here I consider contexts where a universal quantifier or an operator binds the variable. Classical Discourse Representation Theory [10] considers that donkey sentences include universal quantification which takes scope over the entire sentence, and unselectively binds all the free variables in it. In LSC donkey sentences ${ }^{1}$, nominals do not occur with a determiner index sign directed to space to establish a location, but rather are uttered as bare nouns and hardly ever localised. As shown by [15], verb agreement is realised in a neutral articulation (1). ${ }^{2}$

IF TOWN FARMER HORSE THERE-IS, SURE 1-TAKE-CARE- ${ }_{c}$. 'If a farmer owns a horse, he certainly takes care of it'.

\footnotetext{
${ }^{1}$ Cf. [19] for an analysis of donkey sentences in American SL and French SL, where it is argued in line with [13] that SL variables are overtly expressed.

${ }^{2}$ Glossing conventions: manual signs are represented by the capitalised word corresponding to the translation of the sign; IX3 (determiner index directed to the lateral parts); \#-VERB-\# (verb agreeing with subject and object: the numbers refer to the grammatical person); subindices mark direction towards sign space: l (low), u (up), ip (ipsilateral) cl (contralateral), ce (centre); +++ (reduplication of signs).
} 
Interestingly, in the DRT semantic representation of (1) the variable is represented under an embedded context.

$$
\begin{array}{|c|}
\hline \multicolumn{1}{|c|}{} \\
\hline x y \\
\hline \begin{array}{c}
\text { farmer(x) } \\
\text { horse }(\mathrm{y}) \\
\text { owns }(\mathrm{x}, \mathrm{y})
\end{array} \\
\hline
\end{array}
$$

Another context of narrow scope marking is that of genericity. Generic statements express general claims about kinds, rather than claims about particular entities. In LSC bare nouns assume a generic interpretation when they are not localised [14], as shown in (3). Any attempt to localise the DR in space is understood as referential, i.e. as denoting a specific man (4). Generic statements are represented according to the idea that a generic operator binds particular variables in its scope. As shown in (5) corresponding to (3), variables appear in the complex construction represented by a subordinate DRS bound by the generic operator.

\section{MAN PLAY LIKE} 'Men like to play'

(4) MAN IX3 ${ }_{i p}$ PLAY LIKE 'A/the/this man likes to play'
$(5)$

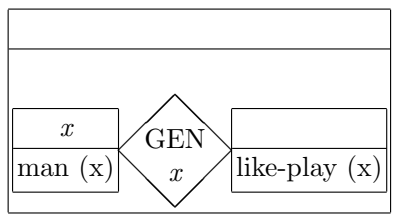

The third argument comes from quantified NPs. In American SL quantified expressions do not establish a spatial location, and sign space is only used to quantify over the domain [12]. In LSC the verbal morphology influences the quantificational interpretation of the bare noun STUDENT [14]. The movement of the verb into sign space denotes quantification, as well as distributivity.

(6) STUDENT EACH-ONE+++ TEACHER ASK +++

'Each pupil asked his/her teacher.'

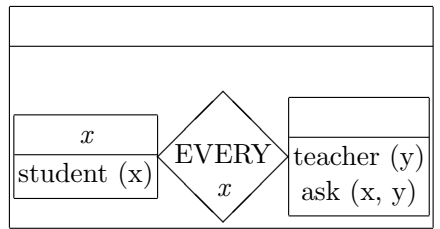

As seen in these examples, in LSC the binding of an operator correlates with lack of spatial location. Hence spatial locations can be defined as the overt manifestation of the DR attached to a quantifier that has wide scope. But this hypothesis can be slightly modified since in specificity contexts, narrow scope variables are overtly localised with a marked location established on the upper frontal plane. 


\section{$2.2 \quad$ Specificity marking}

Whether definiteness is grammatically encoded in SLs is still a matter of debate among SL linguists. While some works argue that an index sign directed to space is the formal marking of definiteness [1], other works have questioned the definiteness marking of index signs [5]. In contrast, in LSC there is no formal marking to distinguish (in)definiteness. As shown in (8), an NP co-occurring with an index sign directed to space is ambiguous between having a definite or an indefinite interpretation.

(8) TODAY IX1 INTERVIEW IX $3_{i p}$ WOMAN.

a. Today I have an interview with a woman.

b. Today I have an interview with the woman.

In LSC the location of indefinite NPs spatially differs according to the specificity interpretation. Signs can be localised on the lower and the upper part of the frontal plane and this distinction corresponds to the overt marking of specificity (9) and non-specificity (10), respectively.

(9) IX1 INTERVIEW IX $3_{l}$ WOMAN

I have an interview with a woman spec

IX1 INTERVIEW IX $3_{u}$ WOMAN

I have an interview with a woman ${ }_{\text {nonspec }}$

The properties specificity encompasses, namely scope and partitivity, can be distinguished in the two localisation processes. Scopal specificity is defined in terms of the interpretation of the indefinite NP outside the scope of an operator. According to this view specificity is equated with wide scope $[6,8,9]$. Hence indefinite NPs which are outside the scope of an operator are considered to have wide scope, and indefinite NPs under the scope of an operator are treated as narrow scope ones.

In LSC indefinite NPs are not ambiguous between having a specific or nonspecific reading. Specific NPs are established on the lower frontal plane, as shown in the LSC counterpart of (11), whereas non-specific NPs are established on the upper part (12).

$$
\begin{aligned}
& \text { I want to buy a cat. } \\
& \text { It is very obedient. }
\end{aligned}
$$

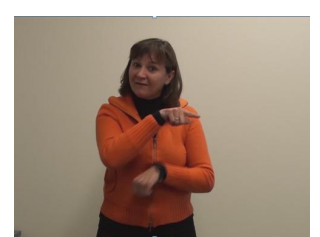

I want to buy a cat.

It must be obedient.

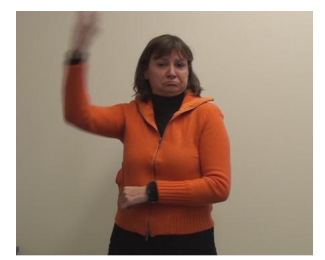

The implementation of specificity marking is formally represented with a variable appearing in the main DRS. This variable has wide scope over the other 
possible embedded variables in the subordinated DRS (13). Non-specificity is implemented with a subordinate variable embedded under the modality operator (14).

\begin{tabular}{|c|}
\hline$x y$ \\
\hline cat $(\mathrm{x})$ \\
$\frac{\text { buy }(1, \mathrm{x})}{\mathrm{it}(\mathrm{y})}$ \\
obedient $(\mathrm{y})$ \\
$\mathrm{y}=\mathrm{x}$ \\
\hline
\end{tabular}

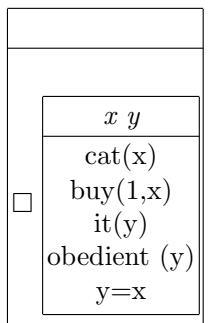

Partitive indefinite NPs receive a semantic partitive interpretation when the denotation of the NP is included within a given set and they have a restricted set as a possible value. The quantification ranges over some specific, non-empty, contextually fixed set. [4] views specificity as partitivity. She argues that in Turkish NPs ambiguity is resolved through case marking: NPs with overt case morphology are specific and they introduce into the domain of discourse entities from a previously given set; NPs without case morphology are non-specific.

In LSC there is a difference between NPs which have a restriction of the quantified NP and those which do not have such a restriction. This is marked in LSC with a difference on the two opposed directions on the frontal plane [15]. Under the restriction of the quantified NP, LSC locations are established on the lower frontal plane (15). When there is no such restriction, the upper frontal plane is used (16).

Some of the friends were hidden there for two years.

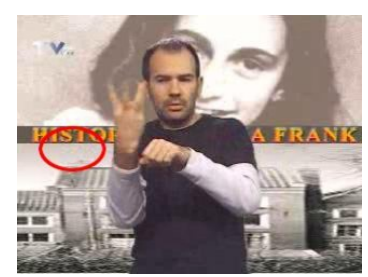

$(16)$

Someone denounced they
were there.

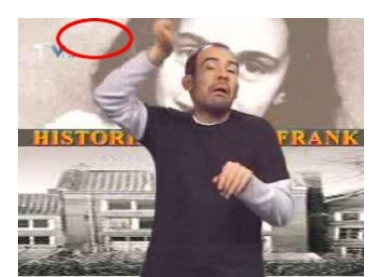

The quantifier in (15) is an element of the group denoted by the NP. This is shown in the corresponding DRS by the relation $\mathrm{x} \in \mathrm{X}$, where $\mathrm{X}$ corresponds to a non-atomic variable that is projected in the main universe. $\mathrm{x}$ is an atomic variable and it is a subset of X. Although $\mathrm{x}$ is not projected into the main DRS, it belongs to the set (17). In contrast, the sentence in (16) denotes a non-specific DR which does not belong to a contextually determined set. In the corresponding DRS, this is represented with an embedded variable which does not belong to any set from the main DRS (18). 

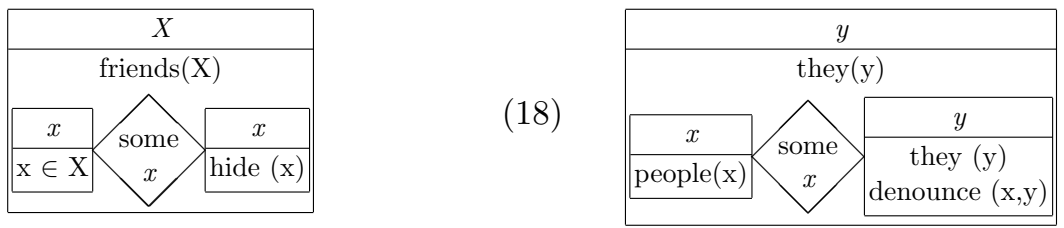

Previously, in 2.1 it has been shown that binding of an operator correlates with lack of establishment of spatial location. However, when considering specificity marking, binding of an operator can also correlate with the establishment of a marked location in the upper frontal plane. Upper locations denote that there is no restriction of the quantified NP and they occur with scopally non-specific DRs.

\section{Modal subordination}

This section is devoted to the analysis of narrow scope variables which behave as wide scope ones. Here I focus on modal subordination contexts which consist on noteworthy DRs introduced into the model the existence of which is not presupposed. Modal subordination are anaphoric contexts which are under the scope of a modal operator or a propositional attitude predicate, but display anaphoric relations that appear at first glance to violate generalisations about scope operators and anaphoric potential [17]. Under intensional contexts resumptive pronouns need to be bound by an operator in order to be a felicitous continuation.

In LSC modal subordination contexts, the variable is attached to a narrow scope quantifier but behaves as a wide scope variable. In (19) the DR is a nonspecific and non-identifiable one.

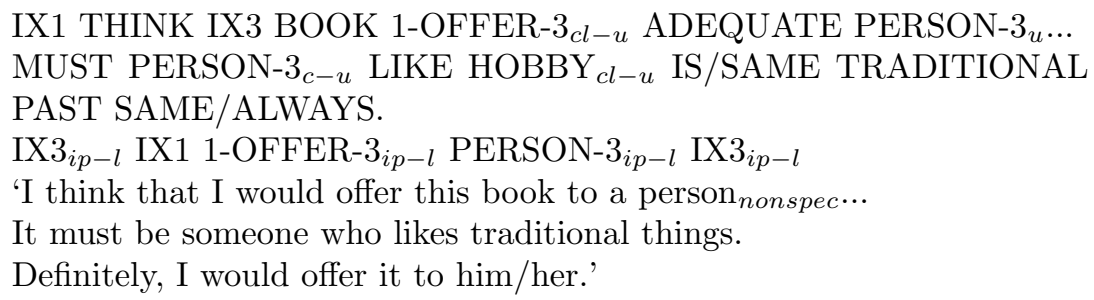

While introducing the antecedent ('someone who likes traditional things') the signer directs a darting eyegaze to an upper direction that goes from the ipsilateral, center and to the contralateral part (20). This eyegaze moves around along the upper frontal plane, without being directly fixed to an area. It functions as an overt operator denoting a de dicto mode. Once the intensional context is established by this de dicto mode, every subsequent sentence is anchored to this mode even across sentence boundaries and all the variables in the semantic representation are bound by the operator. However, as shown in the third utterance in (19) the signer directs pronominal signs and an agreement verb 
towards a lower spatial location. Thus a lower spatial location is established in the subsequent utterance.

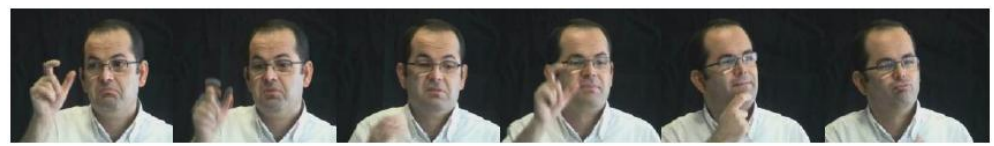

In LSC modal subordination contexts, a lower spatial location is established for a narrow scope variable once the intensional context has been set. Although modal subordination contexts seem to contradict the hypothesis presented in 2.2 where narrow scope variables have been analysed as being overtly expressed on the upper frontal plane, this apparent puzzle is resolved once we introduce the discursive notion of 'prominence'. Hence when studying the establishment of DRs in sign space, the analysis in terms of scope is not enough, but discourse structure and topicality of entities must also be incorporated.

\section{Prominent narrow scope variables}

Prominence is defined as the degree of relative salience of a unit of information, at a specific point in time, in comparison to the other units of information [3]. Centering Theory [7] represents probably the most influential account of entity-based prominence in discourse. Centering Theory is a processing model that relates the local utterance-by-utterance context and discourse anaphoric reference. It constitutes a basis to theorise about local coherence, prominence and choice of referring expressions. Centering has a set of basic notions, which are defined and adapted to the present account in what follows. A discourse model contains (i) a set of forward looking DRs, which is a set of $\operatorname{DRf}\left(\mathrm{U}_{k}\right)$ which appear in the DRS of $\mathrm{K}$ and that can be referred to in subsequent utterances; (ii) a backward looking $\mathrm{DR}$, which is a unique entity $\mathrm{DRb}\left(\mathrm{U}_{k}\right)$, defined for each utterance $\mathrm{U}_{k}$ (except for the intial segment) that refers back to a forward looking DR of the preceding utterance $\mathrm{U}_{k-1}$, and that, intuitively represents the DR which is the center of attention at utterance $\mathrm{U}_{k}$; and (iii) a preferred $\operatorname{DRp}\left(\mathrm{U}_{k}\right)$ which is the one that is on the top of the hierarchy of the set of DRs in the main DRS.

A DR is linked to the discourse topic of the fragment of discourse (i.e. it is the most prominent entity of that specific fragment) if it verifies the following formula:

$$
\operatorname{DRb}\left(\mathrm{K}_{n}\right)=\operatorname{DRb}\left(\mathrm{K}_{n-1}\right) \wedge \operatorname{DRb}\left(\mathrm{K}_{n}\right)=\operatorname{DRp}\left(\mathrm{K}_{n}\right)
$$

Although in most cases topics tend indeed to represent old information, this is neither a sufficient nor a necessary condition for topicality. Topics are better analysed in terms of their effect on the ongoing discourse and considering the effects of previous discourse on the given utterance, rather than as old information $[16,20]$. Thus in $(21)$ the corresponding variable verifies the previous utterance through the first argument of the formula $\left(\operatorname{DRb}\left(\mathrm{K}_{n}\right)=\operatorname{DRb}\left(\mathrm{K}_{n-1}\right)\right)$, 
but also the subsequent utterance through the second argument of the formula $\left(\operatorname{DRb}\left(\mathrm{K}_{n}\right)=\operatorname{DRp}\left(\mathrm{K}_{n}\right)\right)$.

The variable which verifies the formula in (21) will be connected to the discourse topic and will be thus the most prominent DR at a specific point in discourse. The set of forward looking variables $\operatorname{DRf}\left(\mathrm{U}_{k}\right)$ are not only restricted to the ones appearing on the main DRS of $\mathrm{U}_{k}$, but also to subordinated variables as long as they are embedded under the corresponding operator. In LSC, the $\operatorname{DRb}\left(\mathrm{U}_{k}\right)$ among the $\operatorname{DRf}\left(\mathrm{U}_{k}\right)$ will be correlated with a spatial location as long as it verifies (21) and independently of the scope of the quantifier attached to the variable. An example of a narrow scope variable which is prominent at a specific fragment of discourse is shown below.

I would offer the book to someone who likes traditional things. He would be very happy, and he would enjoy it a lot.

In (22) the variable that verifies (21) is z, as shown in (23). In the corresponding DRS a subindex $\mathrm{p}$ is assigned to the most prominent variable in the specific fragment of discourse.

$$
\left[\operatorname{DRb}\left(\mathrm{K}_{n}\right)=\operatorname{DRb}\left(\mathrm{K}_{n-1}\right) \wedge \operatorname{DRb}\left(\mathrm{K}_{n}\right)=\operatorname{DRp}\left(\mathrm{K}_{n}\right)\right] \equiv \mathrm{z}
$$

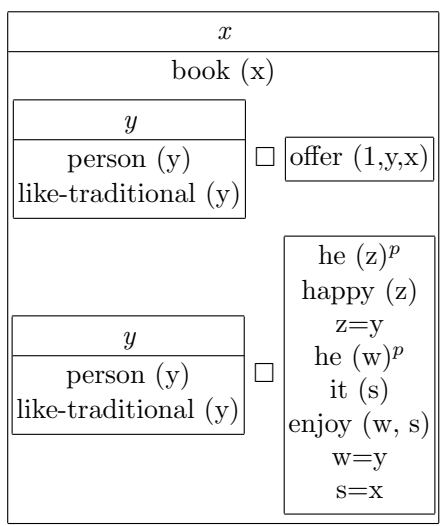

Both narrow and wide scope quantifiers attached to variables can be linked to the discourse topic and hence represent the most prominent DR. The assignment of the subindex into the corresponding variable allows to have the semantic representation for a fragment of discourse linked to its prominent structure. When the subindex is assigned, this correlates with the establishment of a lower spatial location in LSC sign space. 


\section{Conclusions}

The proposal offered has determined the properties that DRs may have, which lead to the establishment of a location in sign space. It has shown that the binding of an operator leads to a lack of spatial location establishment. However, when considering specificity contexts, it has been claimed that narrow scope can also be overtly expressed with locations established on the upper frontal plane. In fact, while lower spatial locations correlate with the expression of specificity, which can be explained by the properties specificity encompasses, namely wide scope and partitivity, upper spatial locations correlate with non-specificity, and this is explained through narrow scope and non-partitivity. But scope is not enough when studying the semantic attributes DRs need in order to have a corresponding spatial location in space, since the scope of the quantifier attached to the variable must be combined with the prominence of the variable at a point in the discourse. In cases of narrow scope marking the variable can establish a lower spatial location as long as it is connected to the prominent DR. Also a representational semantic level which integrates a theory of discourse structure with special focus on prominence has been offered. In the future cases of intermediate scope will be incorporated in the specificity analysis, as well as a refinement of the hierarchy motivations of prominence.

Acknowledgments. I am grateful to Josep Quer, Berit Gehrke and the audience at FEAST (Venice) for interesting comments on earlier versions of this work. Also my deaf colleagues Santiago Frigola and Delfina Aliaga deserve special credit for stimulating discussions. The research in this paper was partly made possible thanks to the Spanish Ministry of Science and Innovation (FFI200910492) and Generalitat de Catalunya - URLING (2009SGR00763). Of course, the remaining errors are all mine.

\section{References}

1. Bahan, B., Kegl, J., MacLaughlin, D., Neidle, C.: Convergent evidence for the structure of determiner phrases in American Sign Language. In: Gabriele, L., Hardison, D., Westmoreland, R. (eds.) FLSMVI. Proceedings of the Sixth Annual Meeting of the Formal Linguistics Society of Mid-America. Volume II: Syntax \& Semantics/Pragmatics. Indiana University Linguistics Club (1995)

2. Brentari, D.: A prosodic model of sign language phonology. The MIT Press, Cambridge, MA (1998)

3. Chiarcos, C., Claus, B., Grabski, M.: Salience in linguistisc and beyond. In: C. Chiarcos, B.C., Grabski, M. (eds.) Salience. Multidisciplinary perspectives on its function in discourse. Mouton de Gruyter, Berlin (2010)

4. Enç, M.: The semantics of specificity. Linguistic Inquiry 22(1), 1-25 (1991)

5. Engberg-Pedersen, E.: Space in Danish Sign Language: The semantics and morphosyntax of the use of space in a visual language. Signum Press, Hamburg (1993)

6. Farkas, D.F.: Dependent indefinites. In: F. Corblin, D.G., Marandin, J. (eds.) Empirical Issues in Formal Syntax and Semantics, pp. 243-267. Peter Lang Publishers (1997) 
7. Grosz, B., Joshi, A., Weinstein, S.: Centering: A framework for modelling the local coherence of discourse. Computational Linguistics 2(21), 203-225 (1995)

8. von Heusinger, K.: Specificity and definiteness in sentence and discourse structure. Journal of Semantics 19, 245-274 (2002)

9. Ionin, T.: This is definitely specific: Specificity and definiteness in article systems. Natural Language Semantics 14, 175-234 (2006)

10. Kamp, H., Reyle, U.: From discourse to logic. Introduction to modeltheoretic semantics of natural language, formal logic and discourse representation theory. Kluwer Academic Press, Dordrecht (1993)

11. Karttunen, L.: Discourse referents. In: McCawley, J. (ed.) Syntax and Semantics: Notes from the Linguistic Underground, pp. 363-386. Academic Press, New York (1976)

12. Klima, E., Bellugi, U.: The signs of language. Harvard University Press, Cambridge, MA (1979)

13. Lillo-Martin, D., Klima, E.: Pointing out differences: ASL pronouns in syntactic theory. In: Fischer, S., Siple, P. (eds.) Theoretical Issues in Sign Language Research, Vol. 1: Linguistics, pp. 191-210. University Chicago Press, Chicago (1990)

14. Quer, J.: Quantifying across language modalities: generalized tripartite structures in signed languages (2005), presentation at the I Workshop on Sign Languages, EHU Vitoria-Gasteiz

15. Quer, J.: Signed agreement: Putting some more arguments together (2010), presentation at TISLR10. Purdue University, Indiana

16. Reinhart, T.: Pragmatics and linguistics. an analysis of sentence topics. Philosophica 27, 53-94 (1981)

17. Roberts, C.: Modal subordination and pronominal anaphora in discourse. Linguistics and Philosophy 12, 683-721 (1989)

18. Sandler, W.: Phonological Representation of the Sign: Linearity and Nonlinearity in American Sign Language. Foris, Dordrecht (1989)

19. Schlenker, P.: Donkey anaphora: the view from sign language (ASL and LSF). Linguistics \& Philosophy (to appear)

20. Vallduví, E.: The Informational component. Garland Press, New York (1992) 


\title{
When Disjunction looks like Conjunction: Pragmatic Consequences in ASL
}

\author{
Kathryn Davidson \\ University of California, San Diego \\ 9500 Gilman Drive. \#0108 \\ La Jolla, CA 92093-0108 \\ kdavidson@ling.ucsd.edu
}

\begin{abstract}
In American Sign Language (ASL), conjunction and disjunction are often conveyed by the same coordinators (transcribed as "COORD"). So the sequence of signs WANT TEA COORD COFFEE can be interpreted as "I want tea or coffee" or "I want tea and coffee" depending on contextual or world knowledge or other linguistic information such as prosodic marking and the addition of disambiguating lexical material. In this paper I show that general use coordinators appearing in ASL can be a test case for understanding the role of the lexicalization of scalar items in the semantic/pragmatic phenomenon known as scalar implicature by collecting quantitative data from 10 adult native signers of ASL and 12 adult signers of English using a Felicity Judgment paradigm. Results show that there is a significant difference in interpretation of the general use coordination scale from other lexically-based scales in ASL and the lexically-based coordination scale in English, suggesting that lexical contrast between scalemates is important for scalar implicature calculation.
\end{abstract}

Keywords: sign languages, experimental pragmatics, scalar implicature, conjunction, disjunction

\section{Introduction}

In natural languages such as English, the disjunctive coordinator "or" can sometimes be interpreted as conjunction ("and"), as shown by the paraphrase in parentheses in (1).

(1) You can have coffee or tea.

(You can have coffee and you can have tea.)

This paper focuses on a different type of relationship between these operators that is found in American Sign Language (ASL), where two very common strategies of coordination work as general use coordinators which can be interpreted as either disjunction or as conjunction in the very same sentence (unlike (1), where the paraphrase is structurally different). 
Although the topic of this paper is general use coordination, it is important to note that there are multiple forms for different types of coordination in ASL, of which only a subset are general use coordination. For example, there is a sign AND which is typically used when translating titles into ASL directly from English, but is grammatical when used as a connective in its own right in ASL. There are also two ways to uniquely signal disjunction in ASL: (a) through fingerspelling $O R$ using the manual alphabet letters "O" and "R" consecutively clearly showing its relation to English, and through a mostly antiquated sign that is homophonous with the Whquestion sign WHICH. In addition to these ways of conveying conjunction and disjunction, ASL very frequently employs a general use coordination strategy, which are described in more detail in section 2 . In section 3 , I present new data from a felicity judgment experiment showing how this way of lexicalizing coordination is accompanied by decreased calculation of scalar implicatures. Section 4 discusses consequences for pragmatic theory and concludes. All grammaticality judgments in this paper were provided from a deaf signer whose parents and grandparents were all deaf, and all signed in ASL. Each judgment was confirmed by at least one other fluent signer of ASL, although these signers varied more in their language background.

\section{Two types of General Use Coordinators in ASL}

\subsection{COORD-shift}

There are two different types of general use coordination in ASL, which I label COORD-shift and COORD-L, respectively. The first, COORD-shift, involves moving the body (a combination of torso, head, and/or eyes) slightly for each coordinated element and signing each of the coordinated items in separate places in the signing space (Fig. 1a). For ease of reading I have transcribed it in the way that manual signs are transcribed with the convention of using capitalized English words to represent a sign in ASL that can be roughly trsanslated with that English word. For COORDshift, however, there is just a change in position from one side of the body to the other; the placement of the notation 'COORD-shift' marks the timing of this change in location.

As shown in (2)-(3), COORD-shift is a general use coordinator: it can convey either disjunction (2) or conjunction (3), depending on the context.

\section{MARY HAVE COFFEE COORD TEA, DON'T-KNOW WHICH.}

'Mary had coffee or tea, I'm not sure which.'

MARY HAVE COFFEE COORD TEA, SHE THIRSTY.

'Mary had coffee and tea, she was thirsty.'

In (2), the clause containing the coordinator is followed by an elided clause, specifically, a clause whose complement is an embedded sluiced constituent interrogative introduced by a D-linked wh-word. This clause is compatible only with a disjunctive interpretation of the preceding coordination phrase. On the other hand, 
in (3) the clause containing the coordinator is followed by a phrase biasing the interpretation of the coordinator towards a conjunctive interpretation.

As disjunction, COORD-shift can appear in alternative questions (4a) as well as both inclusive (4b) and exclusive (4c) disjunctive statements, although signers report that ideally its use in alternative questions would also co-occur with further clarifying linguistic information, such as a sentence-final wh-word WHICH (4a').
a. ?HER PARENTS WILL BUY HER CAR COORD-shift SHE WILL TRAVEL?
'Will her parents buy her a car, or will she [use the money to] travel?'
a'. HER PARENTS WILL BUY HER CAR COORD-shift SHE WILL TRAVEL, WHICH?
'Will her parents buy her a car, or will she [use the money to] travel?'
b. HER PARENTS WILL BUY HER CAR COORD-shift SHE WILL TRAVEL, (DON'T-KNOW WHICH)
'Her parents will buy her a car or she will travel, I'm not sure which.'
c. HER PARENTS WILL BUY HER CAR COORD-shift SHE WILL TRAVEL, (MAYBE BOTH)
'Her parents will buy her a car or she will travel, maybe both.'

Syntactically, COORD-shift can coordinate clauses (4), predicates (5) and noun phrases (6). When coordinating constituents of each of these types, COORD-shift can occur in alternative questions and inclusive statements as well, although in the case of alternative questions, these are usually also reported to be not as good as if WHICH were added at the end of the question, as in (4a').

MARY SWIM COORD-shift RUN, (DON'T-KNOW WHICH).

'Mary swims or runs, I'm not sure which.'

'Mary had coffee or tea, I'm not sure which.'

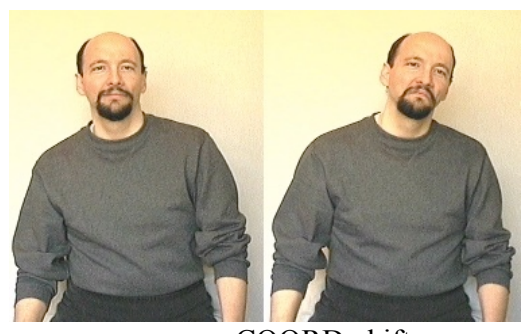

a. COORD-shift

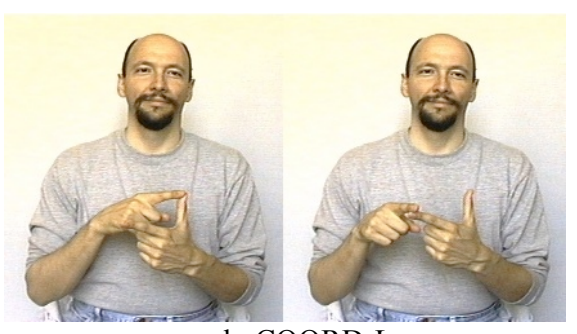

b. COORD-L

Fig. 1. COORD-L and COORD-shift, General Use Coordinators in ASL, from [1] 


\subsection{COORD-L}

The second form of general use coordination in ASL, COORD-L, consists of the signer's dominant hand pointing with a $G$ "extended index finger" (or sometimes a $B$ "mitten-shaped") handshape to successive fingers on the non-dominant hand, beginning with the thumb. Possible number of fingers on the non-dominant hand range from two (the thumb and forefinger) to all five fingers, which is also the range of coordinated items that this strategy allows. An illustration of COORD-L can be seen in Fig. 1b. Another name for this strategy of coordination is List Buoy [2].

Like COORD-shift, COORD-L can convey either conjunction or disjunction, and when used as disjunction can occur in alternative questions and inclusive and exclusive statements. Syntactically, COORD-L can connect clauses, predicates and nouns. For some but not all signers (notated as \%), the use of COORD-L is reported to feel too heavy prosodically to connect two small light nouns as shown in (8), contrasted with full clauses in (7).

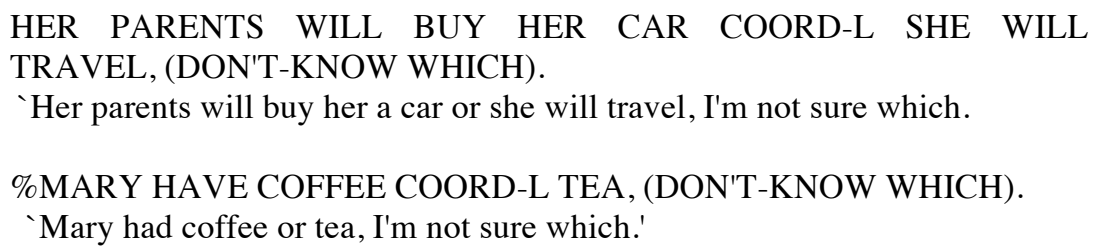

Overall, COORD-L seems to share the syntactic and semantic properties of English coordinators, but may sometimes prefer a more restricted set of prosodic environments. Because of the potential interference of this prosodic restriction, COORD-shift is used as the general use coordinator in the experimental design in section 3 , as the experiment involves coordination of small prosodically light nouns.

\subsection{General Use Coordination in Other Languages}

Both COORD-shift and COORD-L pattern semantically and syntactically much like English disjunction in coordinating all types of constituents and being used for various semantic purposes, and yet depending on the context they can also be interpreted as conjunction. Although this seems to be rare from the point of view of English, there are some reports of other languages in which conjunction and disjunction are similarly disambiguated by context. One is example is Maricopa, a Yunan language, which juxtaposes items for coordination ([3] as reported in [4]) similarly to COORD-shift in ASL. In (9) the verb is marked with the plain future tense, and it is believed with a higher certainly than the sentence in (10), in which the verb has the additional marking of a modal/evidential element. Consequently, (9) is interpreted conjunctively while (10) is interpreted disjunctively, even though in both cases the NP coordinates are simply juxtaposed. 
(9) John-s Bill-s vPaawuum.

John-NOM Bill-NOM 3.come.PL.FUT

"John and Bill will come."

(10) John-s Bill-s v Paawuumsaa.

John-NOM Bill-NOM 3.come.PL.FUT.INFER

"John or Bill will come."

In Japanese, NPs can also be coordinated by juxtaposition, and in this case meaning also depends on the surrounding context or by adding to ('and') or $k a$ ('or') to disambiguate [5]. Although [6] and [7] provide an extensive typological investigation of conjunction, very little attention is given to disjunction. The pattern in ASL presented here suggests that further investigation of disjunction in ASL and other languages like Maricopa and Japanese may lead to uncovering generalizations concerning the relationship between conjunction and disjunction, and especially the ways that these logical relationships can be conveyed by juxtaposing items in a list without the use of overt lexical items like English 'and' or 'or'.

\section{Quantitative Measures of Scalar Implicatures based on General Use Coordination}

This section presents data from an experiment involving native signers of ASL and native speakers of English using a Felicity Judgment Task. Included is a comparison of the disjunctive/conjunctive ("coordination") scale in ASL with the coordination scale in English, as well as a comparison of the coordination scale in ASL with a more prototypical scale in ASL (quantifiers). There are many examples in the literature testing scalar implicature calculation in languages other than English ([8][9][10], among others) and all find similar behavioral results among their participants with implicatures based on prototypical scales like quantifiers. So, we can expect that where ASL makes a similar lexical distinction to English, as in its quantifier scale (<ALL, SOME $>$ ), there should be a similar rate of scalar implicatures.

However, it appears that no previous work directly compares one language to another which makes a different lexical distinction of the same scalar items, and this is the first experiment testing a language that completely lacks a lexical distinction between potential scalar items. If calculation by deaf native signers on the coordination scale in ASL looks just like the quantifier scale in ASL, and like the coordination scale in English, then we can conclude that having contrasting lexical items is not an important feature of a semantic scale. On the other hand, if there is less scalar implicature calculation on the coordination scale in ASL than other scales in ASL, or than the coordination scale in English, this would suggest that lexical contrast is an important part of scalar implicature calculation. The experiment below tests these predictions, and finds that in fact there is less scalar implicature calculation based on coordination in ASL. 


\subsection{Methods}

Participants were 22 adults from the greater San Diego area. Ten were adults who self-identify as deaf and have been learning and using American Sign Language from birth because they had at least one deaf parent. All were unable to hear normal speech, and all used ASL in their home, at work, or both. These participants were recruited directly through email requests from a laboratory database of interested participants or indirectly through recommendations by friends. All received reimbursement in cash or gift cards. These ten participants will be referred to as "native signers of ASL". The twelve remaining participants were typically hearing undergraduate students at the University of California who are native speakers of English and have had no exposure to ASL. These participants received course credit for participating in the experiment. These participants will be referred to as "native speakers of English".

\subsection{Procedures and Stimuli}

Each testing session lasted 30-35 minutes. Both the instructions and the task itself were presented on the laptop in video form, by a native signer of ASL (ASL version) or a native speaker of English (English version). Participants were instructed that for each trial of the experiment, a picture will appear on the screen, and that after looking at the picture, they should press the Space Bar key and a video description will begin to play next to the picture. Participants were told to press the smile face if they were "satisfied that the description matches the picture." If they were "not satisfied, and think that the description does not match the picture", they were instructed to press the frown face. It was impossible in both the ASL version and in the English version to replay a video.

Participants saw three practice trials to acquaint them with the task. These practice trials were followed by further instructions, and a confirmation that the task was understood. Finally, 48 experimental trials were presented. Of these, 24 were fillers used as experimental conditions for other studies, and 24 were experimental conditions in the current study. The current experiments' 24 trials consisted of 12 trials of each sentence type: (a) Quantifiers, which are a prototypical scale in ASL and in English and (b) Coordination, which has a lexical contrast in ASL but not in English. Responses were recorded using Psyscope experimental software.

The quantifier scale was used as the baseline case for scalar implicature calculation in this experiment for ASL, both compared to the coordination scale in ASL and compared to the quantifier scale in English. ASL has multiple signs which can be translated into English as "some" or "all"; in this experiment, the version of the quantifiers SOME and ALL that are shown in Fig. 2 were used. These quantifiers can serve as a prototypical scale in ASL because they contrast lexically in the same way in ASL as they do in English. 


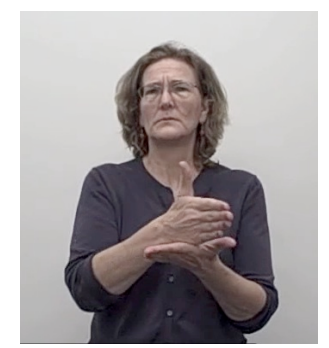

SOME

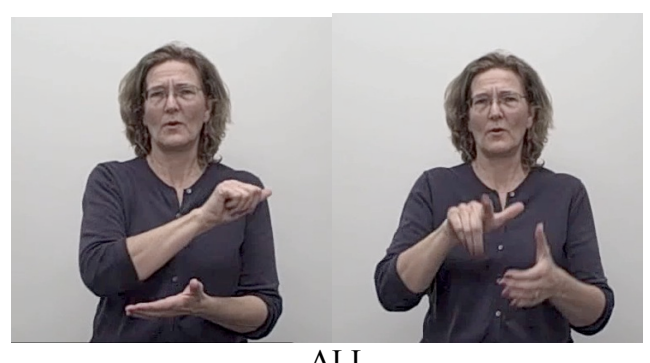

ALL

Fig. 2. SOME and ALL used in the Quantifier sentence type.

In Quantifier trials, each picture consisted of a set of three objects of which either some of the objects or all of them fulfilled a characterization about that object (e.g. red cans, lit candles, full glasses, etc.). Under the Match condition (a total of 4 trials), the characterization applied to all of the objects (e.g. three cans, all red), and the description was accurate (e.g. CANS, ALL RED "All of the cans are red."). Under the Mismatch condition (4 total trials), the characterization applied to only two of the objects (e.g. three cans, only two are red), and the description was not accurate (e.g. CANS, ALL RED "All of the cans are red."). Finally, under the Test condition (4 total trials), the characterization applied to all of the objects (e.g. three cans, all red), and the description was not maximally informative (e.g. CANS, SOME RED "Some of the cans are red."). In this way, the weak scalar term SOME was only evaluated by participants in the Test condition, so that they were never directly comparing this condition to use of the term when it was maximally informative. Trials for all sentence types were counterbalanced so that each sentence frame (e.g. red cans) appeared in only one trial type (Match, Test, Mismatch) for each participant, and each third of participants saw the sentence frame in a different trial type.

COORD-shift was used as the general use coordinator. When interpreted as disjunction, there was additional brow-raising nonmanual marking on the disjuncts (see Fig. 3) and I labeled this use of the coordinator COORD-shift(or). The other use, without brow-raising and conveying conjunctive meaning, was labeled COORDshift(and). In each of the Coordination trials, the picture consisted of two different objects (e.g. a mug and a bowl), and then either one or two of the same type of object (e.g. spoons) in relation to the first objects.

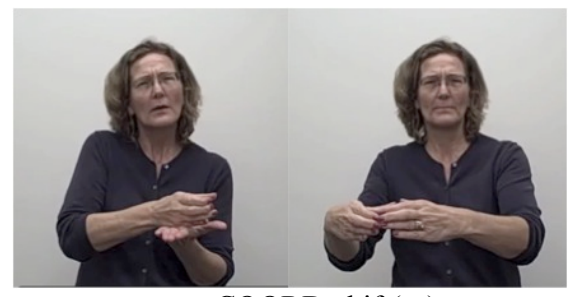

COORD-shift(or)

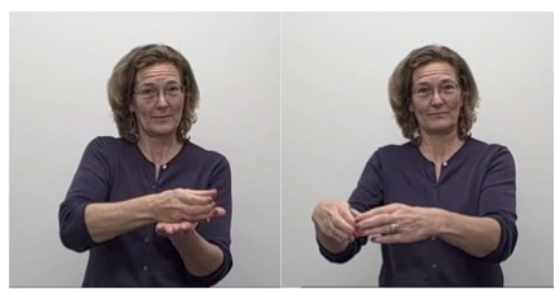

COORD-shift(and)

Fig. 3. COORD-shift(or) and COORD-shift(and) used in the Coordination sentence type. 
In the Match condition in these trials, each of the two different objects were related to one of the similar objects, and the description was accurate (e.g. HAVE SPOON IN CUP COORD-shift(and) SPOON IN BOWL. "A spoon is in the mug and a spoon is in the bowl."). Under the Mismatch condition, only one of the two different objects were related to one of the similar objects, but the description said that they both were equally related (e.g. HAVE SPOON IN CUP COORD-shift(and) SPOON IN BOWL. "A spoon is in the mug and a spoon is in the bowl."). Finally, under the Test condition, each of the two different objects were related to one of the similar objects, but the description was not maximally informative due to the disjunctive nonmanual marking on the general use coordinator (e.g. HAVE SPOON IN CUP COORDshift(or) SPOON IN BOWL "A spoon is in the mug or a spoon is in the bowl")

\subsection{Results}

Concerning prototypical scales based on lexical contrast, native speakers of English showed no significant difference in their acceptance of the Test conditions for the Quantifier scale and the Coordination scale (in fact, the mean accuracy rate (rejection) for each scale was each 0.77 , with a standard deviation of 0.36 for coordination and 0.38 for quantifiers). Both were also accepted (i.e. indicated with a smile face) significantly less often than the control Match sentence in each sentence type $(\mathrm{t}(11)=7.10, \mathrm{p}<0.0001$ for quantifiers; $\mathrm{t}(11)=6.76, \mathrm{p}<0.0001$ for coordination), indicating that scalar implicature calculation was occurring for both scales in English, at the same rate, which is what we would expect from previous research. Native signers of ASL also accepted the Test condition of the prototypical quantifier scale in ASL significantly less often than the Match condition of the quantifier scale $(t(9)=15.38, p<0.0001)$, indicating that scalar implicatures were being calculated for the Test condition, as expected because they were based on a prototypical scale. Moreover, they behaved just like their English counterparts: there was no significant difference on scalar implicature calculation in the quantifier scale between native signers of ASL and native speakers of English $(\mathrm{t}(20)=1.03, \mathrm{p}>0.1)$. This indicates a typical rate of scalar implicature calculation by this group of signers on a prototypical scale in ASL.

However, Coordination in ASL exhibited a different pattern: native signers show significantly less calculation of scalar implicatures based on the coordination scale in ASL compared to native speakers on the Coordination scale in English $(t(20)=25$, $\mathrm{p}<0.01)$ (Fig. 3). Since there was no difference between the acceptance of the Quantifier scales in both languages, we cannot attribute this to a general behavioral difference in the populations of native ASL signers and native English speakers. Furthermore, within ASL there was significantly less scalar implicature calculation on the Coordination scale than on the Quantifier scale $(\mathrm{t}(9)=7.57, \mathrm{p}<0.0001)$. These results are striking, considering that they are both responses to the same trial type (the underinformative Test conditions) by the same set of native signers; nonetheless, there were more rejections (i.e. pragmatic strengthening) in the Quantifier sentence type than the Coordination sentence type. Together with the difference seen between ASL and English on the coordination scale in the two languages, this supports the 
view that the instantiation of scale members as separate lexical items is important for a high rate of scalar implicature calculation.

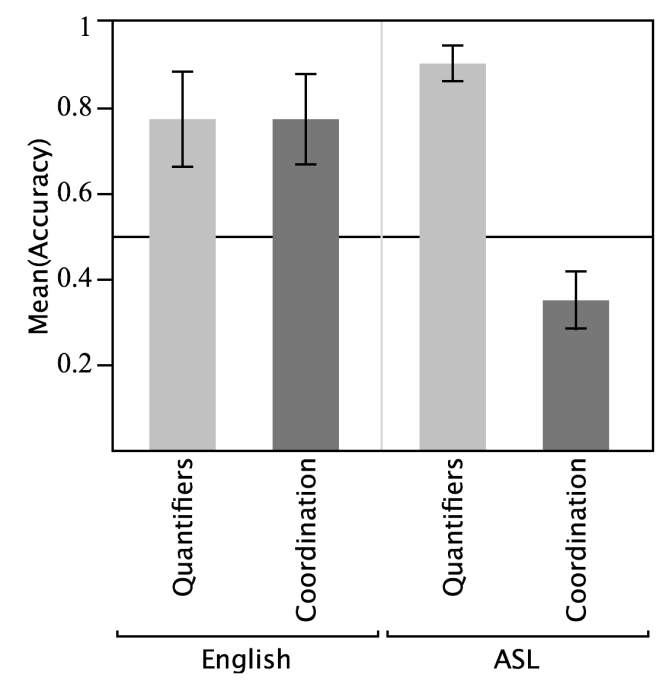

Fig. 3: Calculation of implicatures (i.e. rejection rates for underinformative descriptions) based on quantifier and coordination scales in ASL and English

Recall that the general use coordinator COORD-shift is ambiguous between disjunction or conjunction. So, one explanation of the data presented so far could be that signers chose to interpret the coordinator in the most charitable way: they were accepting the Match sentences, but also the Test sentences, which had disjunctive nonmanual marking but which under a coordination interpretation would be felicitous. For this we can turn to the Mismatch data, where under a charitable interpretation the participants would be expected to accept the descriptions. Instead, participants overwhelmingly reject the descriptions in the coordination Mismatch case $(\mathrm{M}=0.83$, $\mathrm{SD}=0.17$ ), which is not significantly different from the percent of accepted trials in the Match case $(\mathrm{M}=0.80, \mathrm{SD}=0.20)(\mathrm{t}(9)=0.29, \mathrm{p}>0.1)$, but is significantly different from rejections in the Test case $(\mathrm{M}=0.35, \mathrm{SD}=0.21)(\mathrm{t}(9)=5.02, \mathrm{t}<0.001)$. We can conclude that while participants are not likely to reject Test trial descriptions, this is specific to underinformative descriptions not triggering a scalar implicature, and not due to an overall charitable answering strategy for coordination in ASL.

\section{Conclusions}

General use coordination in ASL is similar to strategies reported in Maricopa and in Japanese in allowing both disjunctive and conjunctive interpretations, depending on the surrounding context, while ASL is unique among these language in having 
multiple forms of general use coordination, COORD-shift and COORD-L, and in being a signed language.

The existence of general use coordination allows for a test of the role of lexical contrast in the calculation of scalar implicatures. In many studies of scalar implicature, coordination is a frequently studied scale, due to its status a prototypical scale in English, allowing for a comparison of the calculation on this scale in ASL with the same scale in English, which showed less implicature calculation on the ASL scale. Furthermore, within ASL the coordination scale triggered less implicatures than the lexically-contrastive quantifier scale. Together, these suggest that the semantics relationship of the scalemates is not enough to order items on a scale. In addition to "in salient opposition: of the same form class, in the same dialect or register, and lexicalized to the same degree" [11], scalemates seem to also be required to be separate lexical items that are linked to each other via a scalar relationship. Further testing on similar structures in Japanese, or even Maricopa, as well as more complex structures within these languages and in ASL can indicate further the role that this property plays in scalar implicature calculation.

Acknowledgments. Much gratitude to Marla Hatrak, Ivano Caponigro, Rachel Mayberry, Carol Padden, Brandon Scates, Peggy Lott, Cami Miner, Corinne Brion, Philippe Schlenker, members of the UCSD Sign Language Reading Group, Multimodal Language Development lab, and Semantics Babble.

\section{References}

1. Lifeprint.com, http://www.lifeprint.com/dictionary.htm

2. Liddell, S.: Grammar, Gesture, and Meaning in American Sign Language. University Press, Cambridge (2003)

3. Gil, D.: Aristotle Goes to Arizona and Finds a Language Without "And". In: Zaefferer, D. (ed.) Semantics Universals and Universal Semantics. pp. 96-130. Walter de Gruyter (1991)

4. Haspelmath, M.: Coordinating Constructions. J. Benjamins, Philadelphia (2004)

5. Ohori, T.: Coordination in Mentalese. In: Haspelmath, M. (ed.) Coordinating Constructions. J. Benjamins, Philadelphia (2004)

6. Haspelmath, M.: Coordinating Constructions. J. Benjamins, Philadelphia (2004)

7. Haspelmath, M.: Coordination. In: Shopen, T. (ed.) Language Typology and Syntactic Description Vol. II: Complex Constructions. Cambridge University Press, Cambridge. (2007)

8. Noveck, I.: When Children are More Logical Than Adults: Experimental Investigations of Scalar Implicature. Cognition 78, 165--188 (2001)

9. Papafragou, A., Musolino, J.: Scalar Implicatures: experiments at the semantics-pragmatics interface. Cognition 86, 253--282 (2003)

10. Slabakova, R.: Scalar Implicatures in Second Language Acquisition. Lingua 120, 2444-$2462(2010)$

11. Levinson, S. Presumptive Meanings: the Theory of Generalized Conversational Implicature. MIT Press, Cambridge (2000) 


\title{
Quantificational strategies across language modalities
}

\author{
Josep Quer \\ ICREA-Universitat Pompeu Fabra, Barcelona \\ josep.quer@upf.edu
}

\begin{abstract}
The study of quantification traditionally focused on structures where quantificational meanings are encoded in determiners. Only as a later development attention was paid to quantificational strategies that rely on adverbs, or affixes. In this paper I discuss three varieties of quantificational strategies attested in two sign languages (ASL and LSC) and argue that even the apparent instances of determiner quantification in those languages make use of the more "constructional" way of encoding quantificational meanings that partially reflect the mapping onto tripartite structures overtly. Further, lexical quantification is addressed in the domain of distributivity.
\end{abstract}

Keywords. Quantification, sign languages, D-quantification, A-quantification.

\section{Introduction}

The study of quantification in natural language traditionally focused on structures where quantificational meanings are encoded in determiner like in the nominal domain. This bias results from circumscribing the empirical range of inquiry to IndoEuropean languages, and mostly to English. This tendency was only countered relatively recently by cross-linguistic reasearch by formal linguists on less studied or undescribed languages, the most prominent examples of it being Bach et al.'s (1995) and Matthewson (2008) volumes. The works presented there make a clear case for reconsidering the inherited research agenda on the basis of theoretical analysis of a broader and typologically and genetically more diverse set of languages, as descriptive grammars usually lack the level of detail and theoretical insight required for solid crosslinguistic semantic inquiry.

Sign languages (SLs) have only become the object of systematic linguistic analysis in the past few decades, with very irregular coverage of description and a rather limited language sample. American Sign Language (ASL) has been the most thoroughly studied one. However, semantics is the least well-known area of SL grammars, let alone from a formal semantics perspective. ${ }^{1}$ This applies to the grammar of quantification, as well, with the exception of Partee (1995) and Petronio (1995) on ASL. The present paper aligns with those two works in two senses:

1 For a sample of the few exceptions in the study of different topics in the semantics of sign langages, see the works by J. Quer, Ph. Schlenker, R. Wilbur, for instance, as well as those referenced in the text on quantification in SLs. 
- It explores the ways in which language modality (visual-gestural vs. aural-oral) might have an impact on the expression of quantificational meanings in natural languages. It resorts to the main divide proposed in Bach et al. (1995), namely quantification expressed in the nominal domain by the determiner system vs. quantification conveyed through other means like adverbials or affixes (D- vs. Aquantification, see below for details). Following Heim (1982) and Partee (1992, 1995), tripartite structures are taken as the unifying generalization across those two types of quantifying strategies in natural language.

- It brings SLs to the forefront of the discussion by providing new and unpublished data from Catalan SL (LSC) (Quer 2005) and by comparing it with parallel phenomena described and analyzed in ASL.

This study confirms that the two SLs under discussion display very similar ways of encoding quantification, both of the D- and A-types. It is suggested that the more constructional way of expressing quantified meanings in SLs could be related to their dourse-oriented character of their surface structures, which makes mapping into tripartite structures partially transparent.

Section 2 presents the basic ingredients of the D- vs. A-quantification divide and sets up the stage for the sections to follow. Section 3 adresses A-quantification structures in LSC that support the hypothesis that tripartite quantificational structures are a common means to encode quantificational meanings overtly. Section 4 tackles D-quantification in LSC and argues that the relevant part of the restriction gets overt marking. Section 5 discusses affixal quantification in the verb as pluriactional marking. The paper concludes with general considerations drawn from the empirical and theoretical discussion.

\section{D-Quantification vs. A-Quantification}

The classical analysis of quantification relied on the properties that quantified nominal expressions of the form 'every/most/some N' have in languages like English. The most prominent representative of this view is probably Generalized Quantifier Theory in Barwise \& Cooper (1981). However, a whole line of inquiry into quantification was initiated by Lewis and developed by Kamp (1981) and Heim (1982) on the basis of quantificational readings triggered by adverbs like always or usually, as in (1a), which can be paraphrased as (1b). What (1b) does is make the quantificational structure explicit. The quantificational relation is represented in a more abstract or general format in a tripartite structure as in (2).

(1) a. A quadratic equation usually has two different solutions.

b. Usually, $\mathrm{x}$ is a quadratic equation, $\mathrm{x}$ has two different solutions.

(2) Operator [Restrictor] [Nuclear scope] 
In a Kamp/Heim type of analysis, the indefinite NP a quadratic equation introduces an open variable without quantificational force. The open variable is unselectively bound by the quantificational adverb (Q-adverb) usually. This operator binds the unbound instances in the restrictor and in the nuclear scope. The virtual equivalence of (1a) to a sentence like Most quadratic equations have two different solutions, featuring a D-quantifier instead of a Q-adverb, has led to developing a very fruitful avenue of research on quantification from both a theoretical and a descriptive point of view, as natural languages turn out to vary significantly as to the ways they realize quantification, well beyond enconding it solely in the NP/DP domain.

Partee et al. (1987) coined the terms D-Quantification to refer to the "classical" nominal quantifed nominal phrases that resort to the determiner system, next to A-Quantification. The latter is used to denote a cluster of other quantificational coders, namely Adverbs, Auxiliaries, Affixes, and Argument-structure adjusters that "can be thought of as alternative ways of introducing quantification in a more "constructional' way" (Partee 1995: 544).

A further development in this approach has been the attempt to understand the intraction of information structure partitioning and quantificational structure, as in Partee (1992, 1995) and Bach et al. (1987). The main thrust behind this connection is to understand how topic/focus determines the projection of material onto a tripartite structure. Partee (1995: 545-546) expresses this generalized view of tripartite structure as in Figure 1, featuring "a number of hypothesized syntactic, semantic, and pragmatic structures that can be argued to be correlated with each other and with the basic tripartite scheme." The underlying motivation is of this analytical tool lies in the fact that a broad range of quantificational structures show focus-sensitivity. It should be kept in mind, though, that syntactic constraints override the generality of tripartite structures, thus leading to more complex mappings from syntax to semantics.

In what follows it will be argued that relevant evidence from SLs (LSC and ASL) provides further support for the proposed view. With this limited sample, it is shown that languages in the visual-gestural modality remain within the limits attested crosslinguistically in this specific domain of the syntax/semantics-pragmatics mapping.

\begin{tabular}{|c|c|c|}
\hline $\begin{array}{l}\text { Operator } \\
\forall \\
\text { must } \\
\text { not } \\
\text { almost every } \\
\text { always } \\
\text { mostly } \\
\text { Generic }\end{array}$ & $\begin{array}{l}\text { Restrictor } \\
\text { "cases" } \\
\text { if-clause } \\
\text { subordinate clauses } \\
\text { common noun phrase } \\
\text { topic } \\
\text { presuppositions } \\
\text { domain } \\
\text { antecedent } \\
\text { context }\end{array}$ & $\begin{array}{l}\text { Nuclear Scope } \\
\text { main clause } \\
\text { assertion } \\
\text { focus } \\
\text { consequent } \\
\text { main predication }\end{array}$ \\
\hline
\end{tabular}




\section{A-Quantification structures in SLs}

\subsection{With overt operators}

Conditional and generic statements with indefinite descriptions are typical instances where quantificational variablity is attested in the presence of overt Q-adverbs. LSC is no exception in this respect. Note that the language marks familiar or specific DPs by means of an accompanying index (cf. also Barberà 2011): nominal descriptions bound unselectively by a generic or habitual operator appear as bare nouns in the antecedent, as in (3). ${ }^{2}$ The arguments in the consequent of (3) are not realized by pronouns and the potentially inflecting verb TAKE-CARE displays a neutral form without overt marking for object agreement.

$$
\mathrm{br}^{3}
$$

\section{IF PEASANT HORSE THERE-BE, SURE TAKE-CARE}

'If a farmer has a horse, he certainly takes care of it.'

It seems quite uncontroversial to posit that antecedent and consequent instantiate here the restrictor and the nuclear scope of a tripartite structure. Following much work in the dynamic approaches to such cases, we take SURE in (3) to lexicalize a modal epistemic necessity operator that raises at the level of LF and unselectively binds the argument variables within its scope. The same kind of interpretation is triggered by Q-adverbs, as in (4):

br

IF PEASANT HORSE THERE-BE, ALWAYS TAKE-CARE

'If a farmer has a horse, he always takes care of it.'

Generic statements have been argued to be overtly marked in ASL by the manual sign TEND (Wilbur 1998, Wilbur \& Patschke 1999), as exemplified in (5). Parallel structures in LSC in (6) feature the sign ÉS that characterizes this type of statements. It can be argued that such specialized signs lexicalize the generic operator in these languages.

$$
\begin{aligned}
& \text { LION } \text { SELF:CL1 \#PREDATORY TEND } \\
& \text { 'The lion is a predatory cat.' } \\
& \text { br } \\
& \text { a. IX JAPAN EARTHQUAKE ÉS++ } \\
& \text { 'Japan is in a seismic area.' }
\end{aligned}
$$

[ASL]

2 For a recent detailed treatment of donkey-sentences of the 'bishop' variety in ASL and LSF (French SL), see Schlenker (2011).

3 Standard conventions for glossing SL data are followed here: manual signs are represented with a gloss in capital letters roghly corresponding to the sign; a tier above the manual signs indicates the scope in the coarticulation of non-manual signals like 'br' (brow raise), 'bf' (brow forrowing) or 'hs' (headshake). +++ indicates reduplication. 


\author{
b. $\quad$ LLEÓ DEPREDAR+++ ÉS \\ 'The lion is a predator.'
}

\title{
3.2 Without overt operators
}

As predicted in the framework of generalized tripartite structures, no overt operator is required for them to obtain if the relevant contextual and morphosyntactic conditions are met. LSC example (7) is an instance of a generic or habitual predication. The nominal description in the antecedent and the lack of any indication of episodicity in the main predication allow for it. Note that the antecedent has no overt manual introducer and is only flagged by brow raise (see Wilbur \& Patsche 1999 for extensive arguments in favor of analyzing brow raise as the marker of restrictions of non-Wh operators in ASL).

br

FRIEND PERSON COME, IX1 3-INVITE-1
'If/When a friend comes, I treat him/her.'

Non-conditional generic predications essentially rely on the same ingredients: nonepisodicity, lack of index marking of the generic subject and presence of brow raise on it, as in (8):

(8) WOMAN PLAY LIKE NOT
'Women don't like to play.'

In both types of examples it is legitimate to argue that the interpretation is the result of the licensing of a covert generic operator GEN that unselectively binds the open argument variables (cf. Krifka et al. 1995).

The structures reviewed in this section constitute an example of transparent mapping of quantificational sentences onto tripartite structures: the conditional antecedent and the brow-raise marked subjects correspond to the material in the restrictive clause, while the rest is trivially projected into the nuclear scope.

\section{D-Quantification and partial overt realization of tripartite structures}

The SLs under study display D-Quantifiers which can form regular quantificational phrases. A strong tendency to split the noun and the quantifier has been identified in both ASL and LSC, where the nominal restrictor occurs either in argument position or in a left-peripheral position marked with brow raise, as in ASL (9) (adapted from Boster 1996, Partee 1995, Petronio 1995): 

br
a. BOOK I WANT ALL/SOME/THREE
br
b. $\quad *$ ALL/SOME/THREE I WANT BOOK

The same kind of split is observed with restricted Wh-phrases, where rightward movement in LSC strands the restriction in argument position or else the restriction appears in the left periphery marked with brow raise again, as in (10a) and (10b) respectively:
a. IX 2 CAR $\overline{\text { BUY WHICH }}$
'Which car did you buy?'
b. BOOK IX3 READ HOW-MANY
'How many of the books did s/he read?'

However, the type of quantifiers exemplified in (9) and (10) are weak and thus symmetrical in their two arguments, so they require no tripartite structure for the computation of their truth contitions. The Q-N split observed here is arguably an effect of information structure considerations. At least in LSC, an SOV language, the sentence final position is the one naturally receiving focal stress. It is this information structure partition what determines the surface split of operator and restrictor, thus yielding a only surface resemblance to proper tripartite structured quantification.

Nevertheless, there is one especially interesting case involving negation. LSC has no proper negative determiners (see Matthewson 2008, and especially Zerbian \& Krifka 2008 on Bantu). ${ }^{4}$ Despite the appearance of a negative determiner in a fragment answer like (11B), it can be shown that the several existing negative markers are sentential operators.

$\begin{array}{llll}\text { (11) A: } & \text { THIS MORNING STUDENT } & \text { COME WHO } \\ \text { B: } & \begin{array}{l}\text { NO-RES2 } \\ \text { 'None.' }\end{array}\end{array}$

It can be shown that negative operators behave as adverbial unselective binders that sit in the Specifier of NegP (Quer 2003, Quer \& Boldú 2007). As negative operators,

4 One note of caution should be included here, as a sign NINGÚ 'no(ne)' is attested as a negative adnominal determiner in utterances that are perceived as influenced by Spanish or Catalan. Further research is need to ascertain whether this is a contact borrowing or it has become integral part of the quantificational system of LSC. 
they bind the unbound variables of the predicate, be it the event argument (12), the subject (13) or both subject and object arguments (14).

(12) IX1 $\stackrel{\text { hs }}{\text { SMOKE }}$

'I haven't smoked.'

hs $\quad$ hs

(13) YESTERDAY NIGHT $\overline{\text { COME }} \overline{\text { NO-RES2 }}$

'Noone came last night.'

(14) BRING NO-RES2

'Nobody brought anything.'

If an argument of the predicate does not contribute a variable to the structure, it cannot be unselectively bound by negation, as observed in (15) and (16), where only the object positions can be bound by the negative opetaror:

(15) PEOPLE+++ SOME

'Some people didn't bring anything.'

In this connection it is interesting to note cases like (16)-(17) in LSC, where the sign ALL appears in the subject description of a negative statement. The interpretation that obtains confirms the recurrent observation that 'all', as opposed to 'every', is not a proper operator, but rather a predicate indicating exhaustification of the intended set.
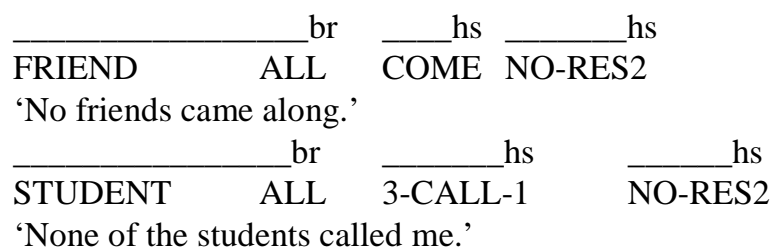

Still, we do find bona fide proportional quantifiers like MAJORITY, the equivalent of English most in LSC:

(18) STUDENT MAJORITY EXAM PASS

'Most students passed the exam.'

This case makes clear that proper cases of D-Quantification in SLs impose a less straighforward mapping from overt quantificational statements onto tripartite structures. For basic cases like (18), though, one simply needs to assume that the quantifier will raise at LF to take scope over restrictor (overtly encoded through brow raise above the nominal constituent in the left periphery) and the nuclear scope (the remaining material). 


\section{$5 \quad$ Lexical quantification}

Under the label A-Quantification, a rather heterogenous set of resources to encode quantificational meanings are included in Bach et al. (1995). Verbal affixes of quantificational nature are among them, next to Q-adverbs. Partee (1995) herself actually suggests that A-quantification might not form a natural class and it might need to be further split into true unselective quantifier structures, on the one hand, and lexical quantification applied directly to a verb or other predicate type, on the other. She argues that morphology as operator on the verb can be quite different from unselective binding, because the operator is directed to a specific argument or arguments of the verbs.

In their seminal work on ASL, Klima \& Bellugi (1979) analyze and classify a whole set of verbal inflections related to aspect, number and thistributivity. Among the last group we find the following:

- [dual]: action with respect to an argument of cardinality two;

- [multiple]: action to many, viewed as a single episode;

- [exhaustive]: distributed action to each individual in a set.

Mostly coinciding with the charaterization drawn for ASL by Petronio (1995), the following LSC paradigm illustrates the behaviour of quantificational inflection on the verb and the corresponding interpretations. All of them quantify over the internal argument of the verb. Such inflections are clearly related to what is known as pluriactionals in many spoken languages, but a detailed analysis along these lines is still pending.

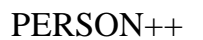

STUDENT

IX^TWO

IX1

1ASK3[dual]

'I asked the two students.'

PERSON++ STUDENT
'I asked the three students.'

IX^THREE

IX1 1ASK3[mult]

$$
\begin{aligned}
& \text { PERSON++ STUDENT IX INHREE IX1 1ASK3[exh] } \\
& \text { 'I asked each one of the three students.' }
\end{aligned}
$$

The exhaustive inflection is also known as distributive marking. A crucial fact in this connection is that in LSC the same morphological mechanism is at play in the expression of distributivity in the nominal domain. The short reduplication along an arc attested in (21), for instance, is the same morpheme that marks distributive-key and the distributive-share in the language (Gil 1995). Typically the reduplicated form of the numeral ONE appears with both functions, but it can also appear on other nominal elements like the possessive (22) or the kinship marker (23): 
'Each student hasked his/her teacher.'

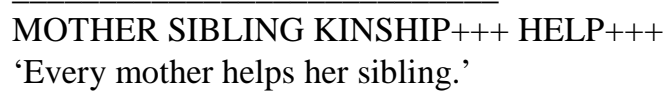

Such cases seem to suggest that what might be seen as verbal inflection encoding quantificational interpretations has a more widesprad use in the grammar of the language, as a homogeneous way of expressing distributivity.

\section{Conclusion}

The overview of the data on quantified structures in LSC and ASL tentatively allows to confirm that languages in the visual-gestural modality resort to essentially the same kinds of mechanisms attested for the expression of quantification in spoken languages. At the same time, it validates the generalization of tripartite structures as an important heuristic and analytical tool that helps capture the correlations and correspondences in the different strategies languages employ.

Acknowledgments. The original work reported in this paper was presented at conferences in 2004/2005 and their audiences are gratefully thanked for their feedback and comments, as well as two anonymous reviewers. Delfina Aliaga, Santiago Frigola, Josep Boronat and Joan Frigola have contributed as Deaf LSC experts with their intuitions in invaluable ways. This research has been partly made possible by the grants awarded to the author by the Spanish Ministry of Science and Innovation (FFI200910492) and to URLING by the Govern de la Generalitat de Catalunya (2009SGR00763), as well as by COST Action IS 1006 SignGram.

\section{References}

1. Bach, E. et al. (eds.): Quantification in natural languages. Kluwer, Dordrecht (1995)

2. Barberà, G. When wide scope is not enough: Wide scope and topicality in discourse referents. In Proceedings of Amsterdam Colloquium (2011)

3. Barwise, J., Cooper, R. Generalized Quantifiers and Natural Language. Linguistics and Philosophy. 4, 159-219 (1981)

4. Boster, C.T.: On the Quantfier-Noun Phrase Split in American Sign Language and the Structure of Quantified Noun Phrases. In International Review of Sign Linguistics, Volume 1, Emondson, W.H., Wilbur, R.B., (eds.) pp. 159-208. Lawrence Erlbaum, Mahwah, New Jersey (1996)

5. Gil, D.: Universal Quantifiers and Distributivity. In Quantification in natural languages, Bach, E. et al. (eds.) pp. 321-362. Kluwer, Dordrecht (1995)

6. Heim, I.: The Semantics of Definite and Indefinite Noun Phrases. Doctoral dissertation, University of Massachusetts/Amherst (1982) 
7. Kamp, H.: A Theory of Truth and Semantic Representation. In Truth, Representation and Information, Groenendijk J. et al. (eds.) pp. 1-41. Foris, Dordrecht (1981)

8. Klima, E.S., Bellugi, U.: The signs of language. Harvard UP, Harvard (1979)

9. Krifka, M. et al.: Genericity: An Introduction. In The Generic Book, Carlson, G.N., Pelletier, F.J. (eds.), pp. 1-124. The University of Chicago Press, Chicago (1995)

10. Matthewson, L.: Quantification: A Crosslinguistic Perspective. Emerald, Bingley (2008)

11. Partee, B. H.: Topic, Focus, and Quantification. In SALT I Proceedings, pp. 159-189. Cornell University: DMLL Publications (1992)

12. Partee, B. H.: Quantificational structures and compositionality. In Quantification in natural languages, Bach, E. et al. (eds.) pp. 487-540. Kluwer, Dordrecht (1995)

13. Petronio, K.: Bare noun phrases, verbs and quantification in ASL. In Quantification in natural languages Bach, E. et al. (eds.) pp. 603-618. Kluwer, Dordrecht (1995)

14. Quer, J.: Operadores negativos en Lengua de Signos Catalana. Ms. ICREA \& Universitat de Barcelona (2003)

15. Quer, J.: Quantifying across Language Modalities: Generalized Tripartite Structures in Signed Languages. Invited lecture at the $1^{\text {st }}$ Workshop on Sign Language. University of the Basque Country/EHU, Gasteiz-Vitoria (2005)

16. Quer, J., Boldú, R.M.: Lexical and morphological resources in the expression of sentential negation in Catalan Sign Language (LSC). In Actes del 7è Congrés de Lingüística General, Universitat de Barcelona. CD-ROM, Barcelona (2006)

17. Schlenker, P.: Quantifiers and Variables: Insights from Sign Language (ASL and LSF. In Formal Semantics and Pragmatics: Discourse, Context, and Model, Partee, B.H., Glanzberg, M., Skilters, J. (eds.) The Baltic International Yearbook of Cognition, Logic and Communication, Vol. 6, (2011)

18. Wilbur, R.B.: Generic and habitual structures in ASL: The role of brow raise. Talk at Theoretical Issues in Sign Language Research, Gallaudet University, Washington, D.C. (1998)

19. Wilbur, R.B., Patschke, C.: Syntactic Correlates of Brow Raise in ASL. Sign Language and Linguistics 2.1, 3-41. (1999)

20. Zerbian, S., Krifka, M.: Quantification across Bantu languages. In Quantification: A Crosslinguistic Perspective, Matthewson, L. (ed.) pp. 383-414. Emerald, Bingley (2008) 


\title{
Degree Modification and Intensification in American Sign Language Adjectives
}

\author{
Ronnie B. Wilbur ${ }^{1}$, Evie Malaia ${ }^{1,2}$, Robin A. Shay ${ }^{1}$ \\ ${ }^{1}$ Purdue University \\ $\{$ willbur, shayra\} @purdue.edu \\ ${ }^{2}$ University of Texas, Arlington \\ malaia@uta.edu
}

\begin{abstract}
Scalar adjectives lacking closed upper boundaries (like far) can be coerced to have a closed upper boundary reading when combined with a degree modification with too which provides the limit, e.g. too far to walk. Parallel to the mapping of event structure to scalar structure in adjectives [4], we observe that scalar adjectives are end-marked in ASL. These adjectives receive marking similar to telic verbs, indicating that, like the visibility of event structure in verbs, scalar structure, or at least the upper boundary, is also visible in ASL. The Event Visibility Hypothesis (EVH) was formulated for sign languages based on the observation that telic verb signs are distinguished from atelics by end-marking reflecting the final state of telic events. Here, the EVH is extended to a general Visibility Hypothesis for sign languages.
\end{abstract}

Keywords: gradable adjectives, American Sign Language, degree modification, intensification

\section{Introduction}

To date, adjectives in ASL have not received detailed investigation comparable to that of verbs and nouns. Two studies involving adjectives both focused on syntax [1-2]. MacLaughlin [1] explored the distinction between attributive and predicative adjectives and their related word order. Bernath [2] investigated their syntax and suggested that the different word orders result from movement of the noun. He further raised the question of whether adjectives like SICK ${ }^{1}$ should be considered as adjectives at all, given that they can be aspectually modified, and suggested that they should instead be treated as verbs, e.g. BE-SICK or AIL. The present project takes a different perspective by focusing on the semantics of the adjectives. In particular, it focuses on gradable adjectives and their interaction with degree modification, reporting new observations on how such modification is marked.

Section 2 introduces gradable adjectives, scales, and the degree modifications to be dealt with. Section 3 presents examples of ASL gradable adjectives and how they are

1921 The glosses for signs are traditionally written in capital letters. 
marked under degree modification. Section 4 considers the interaction of ASL gradable adjectives with the semantics of too used in the form too Adjective to Verb (e.g., too hot to eat, too far to walk). Section 5 ties the pieces together. We relate the marking of adjectives in too $A$ to $V$ to marking of end-state boundaries in ASL signs denoting telic events, previously discussed under the rubric of the Event Visibility Hypothesis $(\mathrm{EVH})$ [3]. We suggest that this similarity of marking is not coincidence but related to the existence of boundaries/limits in the scales in both cases. We extend the EVH, broadening it to a more general Visibility Hypothesis (VH).

\section{Gradable Adjectives, Scales, and Degree Modification}

Following Kennedy and McNally [4], we take a relational approach and assume that gradable adjectives denote a relation $(G)$ between individuals $(x)$ and degrees $(d)$ on a scale reflecting an appropriate dimension for that adjective. For example, the adjective expensive could be represented as a relation between objects and degrees of cost so that the cost of $x$ equals $d$.

$$
[[\text { expensive }]]=\lambda d \lambda x \text {.expensive }(x)=d
$$

However, this representation does not take into account the idea that an object could have a cost $d$ that would not be considered expensive but rather normal, fair, or even cheap. To decide that something should be called expensive, there needs to be a way of determining when a cost is big enough to be considered expensive.

The notion of a scale for a dimension such as cost requires that the degrees of cost can be ordered in such a way that it is always possible to tell whether one particular degree is above or below another one. The variation along this scale is what allows us to talk about an adjective being gradable. For simplicity, let us assume that there is a generally agreed upon standard of comparison value $(s)$ on the scale above which the cost of something is expensive. That is, the cost of $x$ must be greater than (>) the standard $d_{\mathrm{S}}$ on the scale of degrees of cost.

$$
[[\text { expensive }]]=\lambda d \lambda x \text {.expensive }(x)>d_{\mathrm{S}}
$$

However, what is expensive for a cup of coffee is different from what is expensive for a new car, that is, the standard of comparison may vary by context. Thus, the application of a gradable adjective to an object (deciding to call something expensive) always requires a comparison. Sometimes the comparison is contextually dependent (relative adjectives) and sometimes it is fixed (absolute adjectives) [5], even if it is not overtly mentioned.

Now assume that as the distance between the cost of $x$ and the standard of comparison $d_{\mathrm{S}}$ becomes greater and greater, we want to talk about larger degrees of expensive. In English, this can be done with degree intensifiers such as very and too. Kennedy and McNally [6] provide a semantic analysis of very as in (3). Very applies to a gradable adjective and has the effect of increasing (boosting) its value by a contextually-determined large amount. 


$$
[[\text { very }]]=\left\{\left\langle G,\left\langle d_{\mathrm{S}(G)}, x\right\rangle\right\rangle \mid \exists d\left[G(x) \geq d_{\mathrm{S}(G)}+d \wedge \operatorname{LARGE}(d)\right]\right\}
$$

\section{ASL Gradable Adjectives and Their Marking Under Degree Modification}

To begin, the signing of an ASL lexical adjective is similar to that of any other lexical sign, in that the sign components (handshapes, place of articulation, movement, etc.) are lexically specified (as outlined in [7]). There are prosodic contextual effects, so that the actual production depends on sign position in its phrase (there is Phrase Final Lengthening), relative degree of stress or emphasis, and current signing rate [8]. ${ }^{2}$

While there is a sign for very (Fig.1), it is considered 'English register' rather than daily ASL, and its use is extremely limited. Except for discussion of it, it does not occur anywhere in our extensive ASL corpus. Intensification is seen in alternate ways.
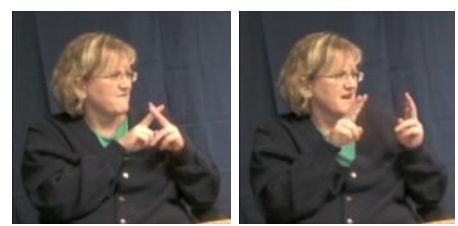

Fig. 1. VERY very; rejected as ASL degree intensifier

\subsection{Plain Adjectives}

The typical production of an adjective sign will be considered the baseline against which intensified productions must be compared. The baseline production is the one in which the standard for application of the adjective has been met, as in (2) above. Typically, the signs are accompanied by mouthing of the English word or by mouth positions that do not change during the movement of the sign, as seen in FAR (Fig. 2).
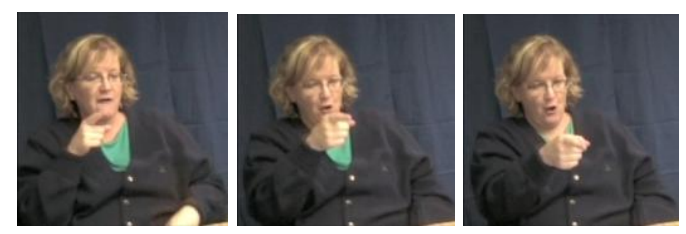

Fig. 2. The sign FAR

Among the adjectives we investigated for this paper are: BIG, CLOSE, FAR, HARD, HEAVY, LATE, LONG, MUCH, NICE, OBVIOUS, SMALL, SOON, SORRY. Data

2 We emphasize lexical here because there are also classifier-based constructions that are contextually-dependent. The form of the ASL translation of thick depends on whether one means thick liquid, thick horizontal object (book lying down), or thick vertical object (book standing up), and so on. That is, there is no lexical sign for thick. 
includes elicited and natural productions, the latter coming from our lab archives, online videologs, published and youtube videos.

\subsection{Phonological Marking of Intensification}

An intensified ASL adjective must meet two criteria. First, it must contain the semantic degree boosting effect given in (3). Second, a sign language specific criterion: the intensified adjective must be visually distinct from the baseline adjective form. This leads to an unusual topic in a semantics paper, namely phonological marking that represents degree morphology. But it is not unusual in sign language research to talk about the semantics-phonology interface and the visibility of event structure in formation of predicate signs formalized as the Event Visibility Hypothesis (EVH) [3] [9].

In our data, we observed the following modifications to adjective signs under intensification:

- Overall increase in tension of the hands and face;

- Movement modifications;

- Add or enlarge movement trajectory;

- [delayed release] of the start of the movement;

- Non-manual modifications (face, head, body);

- Frown on face;

- Head tilt away from neutral.

Many intensified adjectives with [delayed release] have a prefixal hold prior to the onset of hand movement and, if there is any mouth position change, it occurs with the onset of hand movement. ${ }^{3}$ To illustrate, we present a sequence of stills from the signing of FAR-intensified (Fig. 3). As can be seen, the production is distinct from plain FAR shown in Fig. 2. The mouth and hand position are held at the beginning (pictures 1 and 2), then hand and mouth movement begin, and the sign ends with the end of the hand movement and no further change in the mouth position (it stays open). The head is tilted for the entire sign.

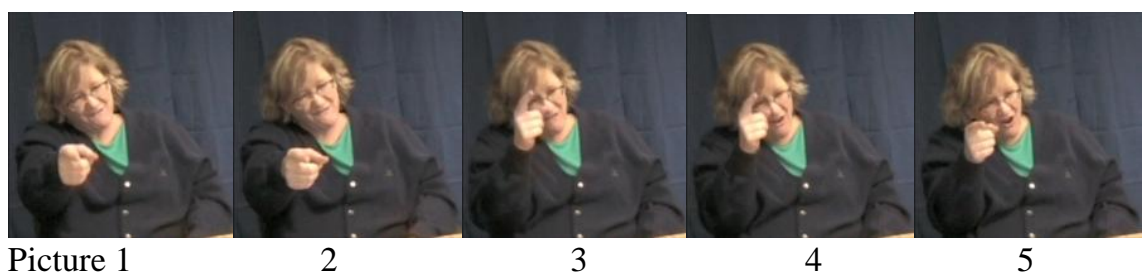

Fig. 3. FAR-intense with [delayed release], mouth opens with start of hand movement (3), mouth open at end (5); note head tilt, frown eyes and forehead.

3 Some forms, HEAVY, prefixed additional movement prior to lexical movement rather than the hold seen in FAR, CLOSE, LITTLE. Both types constitute a delay of movement onset. 


\subsection{The Intensification Adverb Y-OO}

Our investigation led to the realization that there is another sign in ASL that could be considered to have the meaning of so, very (Fig. 4). This sign has no known conventional gloss, and since we have not fully tested its semantics, we have dubbed it $\mathrm{Y}$ $\mathrm{OO}$, reflecting its use of $\mathrm{Y}$ handshape and circling movement. ${ }^{4} \mathrm{Y}-\mathrm{OO}$ can be made with one or both hands. One observation is that Y-OO cannot modify all gradable adjectives. For example, it cannot occur with the sign SORRY, which readily takes the intensification modifications described in 3.2. But it does occur with HARD, FAST, HEAVY, BRIGHT/CLEAR/OBVIOUS, AWESOME, among others.

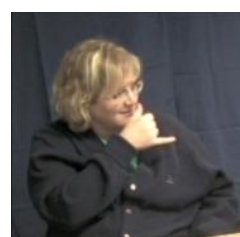

Fig. 4. The adverb sign Y-OO so, very

Furthermore, with the adjectives accompanied by Y-OO, we did not see [delayed release]. Another adjective structure we investigated was Adj like a $\mathrm{N}$, for example, hard like a brick. This translated as Y-OO HARD SAME-AS (fs)brick so/very hard like (a) brick. In multiple appearances, HARD did not show [delayed release]. It may be that intensification morphology and Y-OO are mutually exclusive, but further investigation is clearly needed.

\subsection{Direct Translations of English too}

There are several ways that the meaning too can be shown. We observe some adjective signs produced with the simultaneous mouthing of too. Other adjectives may be preceded by the loan sign \#TOO (derived from the fingerspelling of the English word too). So far, we are unable to predict which adjectives take which form, nor can we rule out the possibility of both markings occurring with the same adjective. The use of these two forms occurs in both elicited and corpus examples, but only in intensification contexts, not in the too $A$ to $V$ discussed below.

\section{4 'Too Adjective to Verb'}

In order to understand the interesting behavior of ASL adjectives in the context too A to $V$, we need to consider the meaning of too, and the role that is played by the infinitive to $V$. Too is often described as a form of degree morphology, along with English -er, more, so, and others. In one sense, like these other intensifiers, it implies a greater

4 This sign should not be confused with the sign glossed SILLY, which has diagonal linear movement or bending at the wrist. 
degree. But too differs from the others in containing the notion of excess, that is, beyond a limit, especially in the context too A to $V$. Meier [10] analyzes this construction by treating too as a comparative quantifier relating two values (treated as extent predicates but not as counterfactual conditionals [11-12]). The first extent is the maximal extent of the gradable adjective. The second extent comes from the maximal or minimal extent from a set of extents satisfying an 'incomplete conditional' related to the sentential complement to $V$. Consider the example of too A to $V$ (4).

The food is too good to throw (it) away.

Meier argues that the sentence in (4) has the form in (5), where two values are compared. One is that of the actual goodness of the food. The other is the maximal value of food-goodness to be allowed to throw it away (from the sentential complement represented as modalized proposition).

$$
x \text { is too adjective MODAL } p \text {. }
$$

The paraphrase she suggests for too good to throw away is "the value $v$ such that the food is $v$-good is greater than the maximum of all values $v^{*}$ such that if the food is $v$ good, we are allowed to throw it away". Note that this paraphrase has (a) a statement that the food is $v$-good, (b) a statement that this value is greater than the maximum of the conditional, (c) the conditional if $X$, then $Y$ in the form if food is $v$-good, we are allowed to throw it away, and (d) the modal be allowed to. Similarly, for negative adjectives, e.g. too young to date, the paraphrase would be the same but the comparison in (b) would require that the value be smaller than the minimum of the conditional. The paraphrases in (c) and (d) provide the contribution of the complement to $V$.

Let us turn now to the ASL structure of interest. What happens to an adjective like FAR when it is put into 'too far to walk' and why does it happen? Given that too is a degree intensifier, we should not be surprised to find that TOO-FAR exhibits the [delayed release] of the hand movement identified in section 3 (Fig. 5). ${ }^{5}$

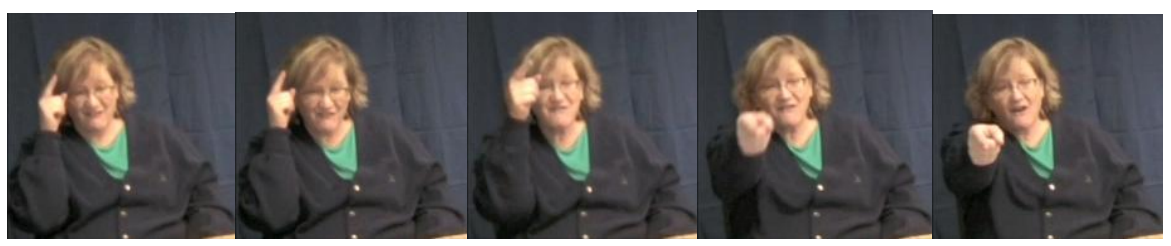

Fig. 5. Too far to walk: TOO-FAR with [delayed release] (pictures 1-2), mouth opens at end of hand movement (picture 5)

However, there are at least three differences in the too $A$ to $V$ productions compared to the A-intensified productions seen in Fig. 3. Note first that the head tilt seen in Fig. 3 is not used. Second, there is a striking difference in the mouth behavior, with the mouth opening at the start of the hand movement in Fig. 3 but not until the end of the

5 [5] refers to too as a sufficiency morpheme, while [10] separates it from sufficiency enough and refers to it as a morpheme of excess. 
hands movement in Fig. 5. Third, what cannot be seen in a sequence of still pictures is the sharp ending of the adjective sign movement in the too $A$ to $V$ contexts. The mouth position change at the end of the movement and the sharp ending of the movement itself caught our attention, because we have seen these two linguistic features together before, namely at the end of movements in verb signs that denote a telic event [9][13]. The mouth position change is referred to as a transition non-manual (TNM). The sharp ending of the movement is due to a rapid deceleration from the peak velocity to the end stop [14-15]. This end-marking is considered to reflect the end state of a telic event, and led to the formulation of the Event Visibility Hypothesis: In the predicate system, the semantics of event structure is visible in the phonological form of the predicate sign. Its presence in the too $A$ to $V$ context suggests that it may have a broader function.

Kennedy and McNally [4] discuss the parallelism between spoken language adjectives derived from verbs and the aspectual properties of those verbs with respect to their common scales, noting there is a general correlation between event structure and scale structure. Their suggestion is that the deverbal adjectives inherit their scale structure either from the event denoted by the source verb or from the entity to which the adjective applies. In particular, deverbal gradable adjectives derived from state and activity denoting atelic verbs tend to be associated with scales that are open on the upper end (have no maximum value) because atelic events have no natural endpoint. In contrast, deverbal adjectives derived from achievement and accomplishment (those with incremental themes) denoting telic verbs are associated with closed upper scales (do have a maximum value) because telic events have endpoints reflecting the end state of the event. ${ }^{6}$

What we have found is that our gradable adjectives display the same end marking that we observe on telic predicates in ASL. That is, they behave as though they had end states, or more appropriately for adjectives, closed upper boundaries. Yet only one of our current adjectives, HARD, passes the tests for closed upper boundary, and indeed it is totally closed [6] [16]. That means that the other adjectives, e.g., FAR, which themselves have open upper scales, are displaying the behavior of a closed upper scale in the too $A$ to $V$ context even though alone they do not have maximum values.

\section{$5 \quad$ Putting the Pieces Together}

To combine with too, a maximum value is required, which is what [10] provides through the hidden incomplete conditional analysis. Thus, scalar (non-deverbal) adjectives lacking closed upper boundaries (like far) can be coerced to have a closed upper boundary reading when combined with a type of measure phrase providing the limit in the construction too $A$ to $V$. How do we compose too $A$ to $V$ ?

6 The ASL adjectives that we investigated for this report are not deverbal, but it would be interesting to see how ASL adjectival predicates behave in this regard. 
Beginning with sentence (6), we use rule (2) and treat the distance of the restaurant as being greater than a distance that justifies the use of too.

The restaurant is too far to walk (to).

$$
\lambda d \lambda x \operatorname{far}(x)>d_{\text {too }}
$$

This needs to be combined with the value that is walkable, which we get from the modalized conditional suggested by [10].

$$
\text { If } x \text { is } d \text {-distance, we can walk to it. }
$$

The distance to the restaurant exceeds the maximum value that we can (are able to, or are allowed to) walk to. ${ }^{7}$

$\lambda d \lambda x$ too-far $(x)>$ MAX $d$ such that if $x$ is $d$-distance, we can walk to it.

That is, the distance is greater than the maximum value of the scale of walkable, and this maximum is where the closed upper boundary comes from in too far to walk. Thus, the restaurant is at a distance that is greater than the maximum distance that we can walk (to).

The ASL structure has the following pieces in it. The adjective FAR takes the prefix [delayed release] for intensification; this affects both the hand movement and the mouth change. It also takes end marking consisting of sharp movement to a stop and the T-NM consisting of [closed -> open]. [7] notes that [delayed release] in the delayed completive aspect attaches to the first timing slot of a telic verb; here we suggest that it attaches to the first timing slot of the adjective. ${ }^{8}$ The end marking attaches to the second timing slot of telic verbs, and here to the second slot of the adjective, and is aligned to the right edge. Thus, the mouth does not open until the end of the movement. The sharp movement is the result of later peak velocity and greater deceleration than plain signs. The end result is that the adjective has the same motion characteristics as a delayed completive telic verb (but without the tongue wagging that [7] notes). This same form is seen with other open scale adjectives, e.g. HEAVY, but interestingly, not with HARD, which is a closed scale adjective. Instead, HARD is modified by $\mathrm{Y}-\mathrm{OO}$, and there is no mouth change or noticeably different movement pattern compared to its plain form. A more systematic investigation is needed.

This parallel between end marked verb signs and end marked too-Adjectives suggests that the original formulation of the Event Visibility Hypothesis, based on verbs only, is too narrow. We propose an extended version, the Visibility Hypothesis, which reflects the idea that sign languages have grammaticalized resources from physics and geometry for perceptual and production purposes to convey the meanings that humans wish to express. However, this is not the 'iconicity' that many people think is there.

7 As [10] notes, this can be epistemic or deontic. Explicit modals (TOO-FAR WALK CAN'T, TOO-HARD EAT CAN'T) were rejected. One paraphrase was suggested: CAN'T LIFT WHY, BOX HEAVY-intens. I can't lift the box because it's too heavy.

8 The typical sign is monosyllabic, consisting of two timing slots which associate to the movement. 
The semantics-phonology interface goes well beyond the typical notion of 'iconicity' ('guessability') [9]. End-marking is an example of grammaticalization of physics (deceleration) for linguistic purposes. Thus, whereas [4], among others, noted the parallel between event predicates and scalar adjectives in spoken languages, in ASL and probably other sign languages, you can actually see it.

Acknowledgements. The work was partially supported by NIH grants DC00524 and DC011081 to R. B. Wilbur.

\section{References}

1. MacLaughlin, D.: The Structure of Determiner Phrases: Evidence from American Sign Language. Dissertation, Boston University (1997)

2. Bernath, J.: Adjectives in ASL. In 10th Theoretical Issues in Sign Language Research, pp. 162-163. Purdue University, West Lafayette, IN (2010)

3. Wilbur, R. B.: Complex Predicates Involving Events, Time and Aspect: Is This Why Sign Languages Look So Similar? In Quer, J. (ed.) Signs of the Time, pp. 217-250. Signum, Hamburg (2008)

4. Kennedy, C., McNally, L.: Scale Structure, Degree Modification, and the Semantics of Gradable Predicates. Lang. 81, 345-381 (2005)

5. Kennedy, C.: Vagueness and Grammar: Semantics of Relative and Absolute Gradable Adjectives. Ling \& Philo. 30,1-45 (2007)

6. Kennedy, C., McNally, L.: From Event Structure to Scale Structure: Degree Modification in Deverbal Adjectives. In: Mathews, T., D. Strolovitch (eds.) SALT IX, 163-180. CLC Publications, Ithaca (1999)

7. Brentari, D.: Prosodic Model of Sign Language Phonology. MIT Press (1998)

8. Wilbur, R. B.: Effects of Varying Rate of Signing on ASL Manual Signs and Nonmanual Markers. Language and Speech 52(2/3): 245-285 (2009)

9. Wilbur, R. B.: The Semantics-Phonology Interface. In D. Brentari (ed.), Cambridge Language Surveys: Sign Languages, pp. 357-382. Cambridge University Press (2010)

10. Meier, C.: The Meaning Of Too, Enough, And So . . That. Natural Language Semantics 11, 69-107 (2003)

11. von Stechow, A.: Comparing Semantic Theories of Comparison. Journal of Semantics 3: 177 (1984a)

12. von Stechow, A.: My Reaction to Cresswell's, Hellan's, Hoeksema's and Seuren's Comments. Journal of Semantics 3: 183-199 (1984b)

13. Schalber, K.: Phonological Visibility of Event Structure in Austrian Sign Language: A Comparison of ASL and OGS. MA Thesis, Purdue University (2004).

14. Malaia, E., Wilbur, R. B.: Kinematic Signatures of Telic and Atelic Events in ASL Predicates. Language and Speech (in press).

15. Malaia, E., Ranaweera, R., Wilbur, R. B., Talavage, T. M.: Neural Representation of Event Structure in American Sign Language: fMRI Comparison of Cortical Activations in Deaf Signers and Hearing Non-signers. Neuroimage (in press).

16. Demonte, V.: Adjectives. In Maienborn, C., von Heusinger, K., Portner, P. (eds.) Semantics: An International Handbook of Natural Language Meaning. Mouton de Gruyter (2011) 


\title{
Focus, Evidentiality and Soft triggers
}

\author{
Márta Abrusán \\ Lichtenberg Kolleg, Georg-August Universität Göttingen, Germany
}

\begin{abstract}
Soft triggers are fairly easily suspendable in context. Two main environments in which this happens have been identified: (a) The presupposition of soft triggers can be suspended by focus (cf. Beaver 2004) (b) Simons (2007) has observed that many soft triggers such as hear, see, believe, discover, know, etc. have semantically parenthetical uses which are not presuppositional. This paper offers a way of predicting these facts in the context of a theory applicable to soft triggers.
\end{abstract}

\section{Introduction}

Soft triggers are fairly easily suspendable in context, as was pointed out by Karttunen (1971), Stalnaker (1974), Chierchia and McConnell-Ginet (2000), Simons (2001), Beaver (2004), Abbott (2006), Klinedinst (2009), Romoli (2011), among others. Observe a classic example:

(1) As far as I know, everything I've said is correct. But if I realize later that I have not told the truth, I will confess it to everyone. (Karttunen 1971)

Such examples of suspendability have been taken to suggest by many of the above authors that - at least in the case of soft triggers - a pragmatic explanation of why the presupposition arises is desirable.

Yet how presuppositions could be predicted has been an elusive and rarely addressed question. While the few attempts in the literature to explain presuppositions of at least certain items provided valuable insights (cf. Sperber and Wilson 1979, Simons 2001, Abusch 2010, Simons et al. 2010), they either did not make correct predictions or failed to be sufficiently explanatory. Stalnaker (1974) and Schlenker (2010) laid out a blueprint for a triggering mechanism, but did not provide a theory themselves.

Abrusán (2011) proposed a mechanism for predicting the presuppositions of soft triggers according to which entailments of a sentence $\mathrm{S}$ that are independent from the main point of $S$ are presupposed. The main point of a sentence $S$ is defined by grammar, and is given by those entailments that are by nature about the event time of the matrix predicate of S. Entailments of S that describe events that are not necessarily about the event time of the matrix predicate of $\mathrm{S}$ are independent from the main point, hence presupposed. However this proposal was not context-sensitive and was therefore not able to handle cases of presupposition suspension.

This paper argues that besides the grammatically defined main point, a secondary, pragmatic main point can be derived as well by markers such as focus and 
evidential expressions. In these cases, sentences have two main points that are relevant for presupposition triggering: the default (grammatical) and a secondary (pragmatic) one. Entailments that are to be presupposed have to be independent from both of these. This predicts that in the examples of presupposition suspension no presupposition is triggered to begin with. A useful metaphor for the perspective of the present paper comes from vision science: Visual attention is thought to be guided both by automatic (bottom-up) processes and by context driven (top-down) processes that modulate the outcome of the first process. We might view the presuppositions of soft triggers as a result of how attention structures the informational content of a sentence: bottom-up processes derive the grammatically defined main point, while contextual (top-down) processes modulate what becomes the main point in a given context.

\section{Aboutness}

The notion of aboutness in this paper is that of being about an entity (object). This treatment is an extension of the notion of being about an argument worked out by Demolombe and Fariñas del Cerro (2000) for first order logic. The proposal has two parts: the definition of variants of a possible world with respect to an object and the definition of being about an object.

\subsection{Variants}

We define variants of possible worlds with respect to objects. Roughly speaking, two worlds $\mathrm{w}$ and w' are c-variants if they only differ by the properties of the object denoted by the constant symbol c. In this case we need to allow that cvariants differ in the truth assignment to atomic sentences where the expression $\mathrm{c}$ appears as an argument of the matrix clause. Let $\mathrm{M}$ be a model $\langle W, D, R, \llbracket \rrbracket\rangle$, where

- W is a set of possible worlds

- D is a domain of things ${ }^{1}$

$-\mathrm{R} \subseteq \mathrm{W} \times \mathrm{W}$ is an accessibility relation on $\mathrm{W}$

- for a non-logical atomic predicate $p^{n}, \llbracket p^{n} \rrbracket^{w} \subseteq D^{n}$

For the purpose of calculating variants, a sentence such as a knows that $p$ at $t$ will be treated as if it had the more simple syntax $K(a)(t)$, where $K$ stands for knows that $p$. The language is assumed not to contain the identity predicate. We can then define for every possible world $\mathrm{w} \in \mathrm{W}$, variants $\mathrm{w}$ ' of $\mathrm{w}$ as follows:

$-D_{\mathrm{w}},=D_{\mathrm{w}}$

- $\llbracket \mathrm{x} \rrbracket^{w^{\prime}}=\llbracket \mathrm{x} \rrbracket^{w}$, for each constant or variable symbol $\mathrm{x}$

\footnotetext{
$\overline{1}$ To keep the above discussion more simple, I assume that both individuals and time intervals belong to $\mathrm{D}$. Things in $\mathrm{D}$ are typed however, $\left(\mathrm{so} \mathrm{D}=\mathrm{D}_{\mathrm{e}} \cup \mathrm{D}_{\mathrm{I}}\right)$ and predicates have a type restriction on the their arguments.
} 
- if $p$ is a predicate symbol of arity $n$

- if $t$ is an $\mathrm{n}+1$-tuple the first $\mathrm{n}$ terms of $L_{\mathrm{c}}$ and the final element is from $\mathrm{W}$ and which contains no occurrence of the constant $c$, then $\left\langle\llbracket \mathbb{\llbracket} \rrbracket, w^{\prime}\right\rangle \in$ $\llbracket \mathrm{p} \rrbracket$ iff $\langle\llbracket \mathrm{t} \rrbracket, w\rangle \in \llbracket \mathrm{p} \rrbracket$.

- if an element $\left\langle d_{1}, \ldots, d_{n}\right\rangle$ of $D^{n}$ is such that for every $j$ in $[1, \mathrm{n}], d_{\mathrm{j}} \neq \llbracket c \rrbracket$, then $\left\langle d_{1}, \ldots, d_{n}\right\rangle \in \llbracket \mathrm{p} \rrbracket^{w^{\prime}}$ iff $\left\langle d_{1}, \ldots, d_{n}\right\rangle \in \llbracket \mathrm{p} \rrbracket^{w}$.

\subsection{Aboutness}

Given the above notion of variants, we might define aboutness as follows:

(2) Aboutness

A sentence $\mathrm{S}$ is about the object denoted by the constant symbol $\mathrm{c}$ iff there are two worlds $\mathrm{w}$ and $\mathrm{w}^{\prime}$ in $W$ which are c-variants and $\llbracket \mathrm{S} \rrbracket^{w}=1$ and $\llbracket \mathrm{S} \rrbracket^{w^{\prime}}=0$

Conversely, we can also give a definition of what it means for a sentence to not be about an object c:

(3) The property of not-being about

A sentence $\mathrm{S}$ is not about the object denoted by the constant symbol $\mathrm{c}$ iff for every w, w' in $W$ st. w and w' are c-variants $\llbracket \mathrm{S} \rrbracket^{w}=\llbracket \mathrm{S} \rrbracket^{w^{\prime}}$.

\subsection{Examples}

(4) $\quad$ a. $\xi=$ Fido is tired

b. $\varphi=$ Some individual is tired

c. $\quad \psi=$ Fido is tired or Fido is not tired

The sentence $\mathrm{S}$ above is about Fido iff there are two Fido-variants w, w', st. $\llbracket \xi \rrbracket^{w}=1$ and $\llbracket \xi \rrbracket^{w^{\prime}}=0$. Notice that the definition in (2) quantifies over all worlds, therefore $\varphi$ is also about Fido, because there are two worlds which differ only in the properties of Fido, st. and $\llbracket \varphi \rrbracket^{w}=1$ and $\llbracket \varphi \rrbracket^{w^{\prime}}=0$, e.g. if Fido is the only tired individual in w. $\psi$ however is not about Fido because $\psi$ is a tautology, and therefore it is true in every two worlds w, w' that are Fido-variants.

\section{Abrusán's (2011) Proposal}

Abrusán (2011) presented a mechanism to predict the presuppositions of soft triggers. The main intuition, inspired by Stalnaker (1974), is that entailments of a sentence that are in some sense independent from the main point of the sentence are presupposed. It was assumed that that the main point of a sentence is given by those entailments that are by nature about the event time of the matrix predicate. Propositions that describe events that are not (or do not have to be) about the event time of the matrix predicate of $\mathrm{S}$ are independent, and hence presupposed. 
Let's illustrate the idea with a simple example. Consider (5), in which $\mathrm{t}_{1}$ denotes the event time interval of the matrix predicate, and $t_{2}$ is some interval before $t_{1}$, given by the context. Let's look at the sentence $\mathrm{S}$ and two of its (many) entailments $\varphi$ and $\psi$ :

$$
\mathrm{S}=\text { John knows }\left(\text { at } \mathrm{t}_{1}\right) \text { that it was raining }\left(\text { at } \mathrm{t}_{2}\right) \text {. }
$$

a. $\varphi=$ John believes (at $\left.t_{1}\right)$ that it was raining (at $\left.t_{2}\right)$.

b. $\quad \psi=$ It was raining $\left(\right.$ at $\left.t_{2}\right)$.

In an intuitive sense, $\varphi$ is about the time denoted by $t_{1}$, but $\psi$ is not: changing the properties of the world at $t_{1}$ will not affect the truth value of $\psi$ but it might affect the truth value of $\varphi$. This is exactly what is captured by Demolombe and Fariñas del Cerro's (2000) definition of aboutness: There are no two t t-variants $_{1}$ (i.e. worlds that differ in nothing but the properties of $t_{1}$ ) which differ in the truth value of $\psi$. Therefore, $\psi$ is not about $t_{1}$. But there might be two worlds that are $t_{1}$ variants and differ in the truth of $\varphi$ in them, e.g. $w_{1}$ in which John believes $\left(\right.$ at $\left.t_{1}\right)$ that it was raining, and $w_{2}$ in which John does not believe (at $\left.t_{1}\right)$ that it was raining.

In effect the aim was to tell independent events apart: Select the main event described by the sentence, and decide what other information conveyed by the sentence describe independent events from the main one. But this is a very difficult task and cannot be easily accomplished just by looking at events themselves because of the very complex mereological structure of events. For example, is the event of raining part of the complex event of John's knowing it? If not, why not? The idea of looking at event times instead of events themselves serves the purpose of making independence more tractable: Events that happen at different times are clearly different events. Further, in some cases, e.g. mathematical truths (John knows that $2+2=4$ ), the possibility of invoking events is not obvious at all. However, since event times are more abstract than events, evoking event times is still possible even in these cases.

Of course one might wonder about sentences such as (6), where the embedded proposition and the matrix predicate are assumed to be true at the same time. The simple picture above predicts that the embedded proposition in (6) is not independent from the main assertion, and is therefore not presupposed, contrary to fact:

(6) $\mathrm{S}=$ John knows $\left(\right.$ at $\left.\mathrm{t}_{1}\right)$ that it is raining $\left(\right.$ at $\left.\mathrm{t}_{1}\right)$.

a. $\varphi=$ John believes (at $\left.t_{1}\right)$ that it is raining (at $\left.t_{1}\right)$.

b. $\psi=$ It is raining (at $\left.t_{1}\right)$.

Thus we need a way to distinguish accidental co-temporaneity from non-accidental ones. In the above example, though it so happens that the embedded proposition and the matrix proposition are true at the same time, this is only an accident. But the co-temporaneity of the matrix time of $\varphi$ with the matrix time of $\mathrm{S}$ is not an accident, but follows from the lexical interpretation of know. To distinguish these two cases, Abrusán (2011) and Abrusán (2011) proposes to compute 
the triggering mechanism on an abstraction of the sentence in which accidental temporal overlap can be ignored, as opposed to non-accidental ones. I refer the reader to the above papers for further detail.

\section{Contextual Effects}

The triggering mechanism outlined above was viewed as a function that takes as its input the semantic entailments of a sentence S, and flags one or more of these as presupposed. It has been argued however (cf. Stalnaker 1974, Chierchia and McConnell-Ginet 2000, Simons 2001, Beaver 2004, Abbott 2006, Abusch 2010, Schlenker 2010) that certain examples might neccessitate taking the context into account as well. This section looks at two such cases, and how they might be integrated into the above framework, without claiming to be exhaustive.

\subsection{Parenthetical Uses of Verbs}

Simons (2007) observes that certain clause embedding verbs such as hear, see, believe, discover, know, etc. have semantically parenthetical uses. In these cases the embedded clause carries the main point of the utterance, while the matrix clause serves an evidential function of identifying information source, emotional attitude, etc. An example is the conversation below:

(7) A: Why didn't Louise come to the meeting yesterday?

B: I heard that she's out of town.

Some of the verbs that admit such parenthetical uses are members of the class commonly thought of as factive verbs (e.g see, know, discover, etc). Simons notes that when used in this parenthetical manner, these verbs loose their presuppositionality: In the examples below the information that Luise is out of town is presented as new information, and not as information that is already entailed by the common ground or presupposed. Some illustrative examples from Simons' paper:

(8) A: Why isn't Louise coming to our meetings these days?

B: Henry discovered/realized/figured out/learned that she's left town.

(9) Yikes! I just remembered/realized that I didn't turn off the stove!

As regards projection facts, it also seems that the above verbs can act in a way as if their complement was not presupposed. Such facts have been long noted in the literature (cf. Stalnaker 1974, Chierchia and McConnell-Ginet 2000, Simons 2001, Beaver 2004, Abbott 2006, Abusch 2010, among others), and for this reason the terminology semi-factives has been sometimes used to describe the above verbs:

(10) a. If Henry discovers that Louise is in NY, he'll be furious.

b. Why is Henry in such a bad mood? Did he discover that Louise is in NY? 
c. Henry is in a terrible mood. Perhaps he's discovered that Louise is in NY.

Simons proposes that in the above cases the main verb is used in an evidential way. Evidentials are words or morphemes that express the source of information or the type of evidence that the speaker has for the information being conveyed. In many languages of the world these markers of information are highly grammaticalized, and might even be obligatory. (cf. Speas 2008, Aikhenvald 2006). The most common information types expressed are direct information (which might e.g. be visual, auditory, other sensory, etc.) and indirect information (e.g. reported, inferred, etc.), with variation among languages of how the evidence types are divided. Most often, evidentials are analyzed as either illocutionary operators (cf. Faller 2002, Davis et al. 2007, etc.) or as modal operators (cf. Garrett 2000, Izvorski 1997, etc.), with languages possibly differing in the semantic properties of their evidentials. Another source of theoretical (and perhaps empirical) variation is whether the content of evidential markers becomes part of the propositional content. Further, given that evidentials either serve an illocutionary or a modal function, the content modified by the evidential is (also) the main point of the utterance. This can also be seen with the evidential verbs surveyed above, as was pointed out by Simons, many of which allow a so-called slifting construction. In such cases the main verb is inserted as a parenthetical comment into the subordinated clause, as in (11):

John, I heard, is out of town.

Simons' (2007) idea has interesting consequences for the present proposal. Above it was assumed that there is a grammatically defined main point, the information that is about the tense of the matrix verb. But in cases where the matrix verb is used in an evidential way, there might also be a second (pragmatic) main point besides the grammatically defined default one, which is derived contextually. The second main point concerns the clause "modified" by the evidential, the syntactically embedded clause. Technically the secondary main point is the information that is about the time of the syntactically embedded verb. Since presupposed material has to be independent from the main point (i.e. not be about the event time of the main point), once the embedded clause becomes the main point as well, the content of the embedded clause is not predicted to be presupposed any more, despite being entailed. This is exactly what is happening in these examples.

Part-time triggers Certain expressions, as was noticed by Schlenker (2008, 2010), trigger a presupposition when they appear in certain contexts but not in others. Schlenker calls such expressions 'part-time triggers'. An example is the verb announce. In some contexts, it does not entail the truth of its complement and in these contexts it does not presuppose the truth of its complement either. In other contexts, it entails and presupposes the truth of its complement:

Mary has announced that she is pregnant 
a. Scenario 1: Mary is 30 years old and she is expected to be reliable. Therefore the context entails the truth of the embedded proposition. $\rightarrow$ (12) presupposes that Mary is pregnant

b. Scenario 2: Mary is 7 years old and is not expected to be reliable. Therefore the context does not entail the truth of the embedded proposition.

$\rightarrow$ (12) does not presuppose that Mary is pregnant

As Schlenker points out, the verb announce contrasts minimally with the verb inform, which seems to lexically entail and presuppose the truth of its complement in the above context. The mechanism proposed in this paper can be extended to handle the above facts by allowing contextual entailments to enter the pool of candidate entailments for presuppositions. Then if the embedded proposition is contextually entailed, it is also be predicted to be presupposed, as in (12a). Otherwise, as in (12b), it isn't.

Interestingly, there are further cases, discussed in Schlenker (2006), which show that inform itself is a part-time trigger. In contexts in which the truth of the complement is in question and the subject is assumed to be a very reliable source of information regarding the truth of the complement, the factive inference of both announce and inform disappears:

(13) George is the family butler. He is very reliable. If he says p, then we can infer that $\mathrm{p}$ is the case, and if he does not say $\mathrm{p}$, we can infer that $\mathrm{p}$ is not the case.

Has George announced/informed the guests that dinner is ready?

$\rightarrow$ there is no implication that dinner is ready.

This can also be accounted for in the present proposal. A plausible thing to say is that the butler being a completely reliable source for the information in question (whether dinner is ready), the embedded clause becomes the pragmatic main point. In effect, the question is interpreted as 'Is dinner ready (according to the most reliable source)?'. This makes the truth of the embedded clause not independent from the secondary main point (indeed it is equivalent to it) and therefore the truth of the embedded clause is not predicted to be presupposed, despite being entailed.

\subsection{Focus}

Presupposition suspension or canceling can also be brought about by focus (cf. Beaver 2004). As Beaver observes, (14b), but not (14a) suggests that the student has plagiarized his work, as well as a sense of complicity between speaker and student: ${ }^{2}$

\footnotetext{
${ }^{2}$ Example (14a) is slightly modified from the original, the focused part being the entire embedded clause in the present discussion, but only the verb plagiarized in the original version. These two cases are hard to tell apart phonetically in English, but Hungarian indicates that more likely the latter is the case.
} 
(14) a. If I discover that [your work is plagiarized $]_{F}$, I will be [forced to notify the Dean $]_{\mathrm{F}}$.

b. If the T.A. [discovers $]_{F}$ that your work is plagiarized, I will be [forced to notify the Dean $]_{F}$.

Focus is usually taken to be the part of a sentence that conveys the new or highlighted information, thus the information that directly answers a background question. In this sense, focus grammatically signals the presence of a background question. I will assume here that grammatically marked background questions can introduce a secondary (or pragmatic) main point, similarly to evidentials. Secondary main points concern the event time of the most direct proposition that answers the background question. The presupposition triggering mechanism looks both at the default (grammatical) and the secondary (pragmatic) main points and requires the presupposition to be independent from both of these. This derives the above data in the present framework.

In (14a), focusing the embedded clause indicates that the background question is What will I discover? The direct answer to this question is a proposition, namely the proposition denoted by the embedded clause. The pragmatic, secondary main point therefore concerns the information that is about the tense argument of this proposition, i.e. the tense argument of the embedded clause. For this reason, the information conveyed by the embedded clause is not independent from the secondary main point, and is not predicted to be presupposed. In (14b) focus on the matrix verb indicates that the background question is What will the TA do? The direct answer to this question is a proposition that restates the main clause of the antecedent of the conditional and therefore the pragmatic main point of the sentence concerns the main clause and the matrix tense. Since this is the same as the default (grammatical) main point, we derive the same presupposition as in the default, unfocused case: the truth of the embedded complement is independent from the main point (i.e. it is not about the matrix tense argument) and is presupposed.

In Hungarian the pattern identified by Beaver can be replicated more generally, even with factive verbs such as tud 'know'. The main verb tud 'know', just like most verbs that take propositional complements, can have a pronominal argument in the main clause (in this case azt 'that.acc') that is anaphoric to the embedded proposition. This pronoun can be focused: when it is, as in (15a), the truth of the complement is not presupposed any more. This is in contrast to $(15 \mathrm{~b})$, in which instead of the propositional anaphor the verb is focused: here factivity is preserved:

a. Kétlem, hogy Péter [azt $]_{F}$ tudja, hogy esik az eső. doubt.1sg that Peter that.acc knows that falls the rain 'I doubt that what Peter knows is that it is raining.'

b. Kétlem, hogy Péter [tudja $]_{F}$ (azt), hogy esik az eső. doubt.1sg that Peter knows (that.acc) that falls the rain 'I doubt that Peter $[\text { knows }]_{F}$ that it is raining.' 
The Hungarian facts can be predicted in the same way as the English data above. Thus the present account is able to derive why in certain contexts the presuppositions of soft triggers disappear: in these cases focus indicates the presence of a background question and a secondary main point that is different from the default main point. Since the presupposition has to be independent from this secondary main point as well, presuppositions are simply not generated. ${ }^{3}$

\subsection{Comparison with Simons et al. (2010)}

Recently, Simons et al. (2010) have proposed that entailments that do not address the question under discussion project. The present proposal is similar to this idea in that presuppositions will be suspended if a background question invokes a secondary main point. However, in contrast with Simons et al. in this paper this is only a secondary mechanism and is only licensed by markers that are relevant categories for grammar; simply arbitrarily changing the question under discussion has no effect on the presuppositions generated.

Acknowledgments Many thanks to the audience at SEP in Göttingen for their helpful questions about this material, all the people who have commented on previous, related papers and the Mellon Foundation and the DFG in the framework of the Lichtenberg-Kolleg of the Georg-August-Universität Göttingen for financial support. All remaining errors are my own.

\section{References}

Abbott, B. (2006). Where have some of the presuppositions gone? In B. J. Birner and G. Ward (Eds.), Drawing the boundaries of meaning: Neo-Gricean studies in pragmatics and semantics in honor of Laurence R. Horn, pp. 1-20. Philadelphia: John Benjamins.

Abrusán, M. (2011). Predicting the Presuppositions of Soft Triggers. Manuscript, Lichtenberg Kolleg.

Abrusán, M. (2011). Triggering verbal presuppositions. In N. Li and D. Lutz (Eds.), Semantics and Linguistic Theory (SALT) 20, pp. 684-701. eLanguage. Abusch, D. (2010). Presupposition triggering from alternatives. Journal of Semantics 27(1), 37-80.

\footnotetext{
${ }^{3}$ Another case of presupposition-suspension based on focus might be the example in (i). In Hungarian, the example only works if the adverb recently is present, and is in focus position.
}

(i) I notice you are chewing on your pencil. Have you recently stopped smoking? (Geurts 1994)

Possibly, the adverb makes the entire stretch of time signaled by recently the main point of the utterance. This allows a non-presuppositional understanding of the question, if the time of smoking falls within the stretch of time designated by the adverb. 
Aikhenvald, A. (2006). Evidentiality. Oxford University Press, USA.

Beaver, D. (2004). Have you noticed that your belly button lint colour is related to the colour of your clothing? In R. Bauerle, U. Reyle, and T. E. Zimmerman (Eds.), Presupposition: Papers in Honor of Hans Kamp.

Chierchia, G. and S. McConnell-Ginet (2000). Meaning and grammar: an introduction to semantics. MIT Press Cambridge, MA, USA.

Davis, C., C. Potts, and M. Speas (2007). The pragmatic values of evidential sentences. In M. Gibson and T. Friedman (Eds.), Proceedings of Semantics and Linguistic Theory 17, pp. 71-88. Ithaca, NY: CLC Publications.

Demolombe, R. and L. Fariñas del Cerro (2000). Towards a logical characterization of sentences of the kind "sentence p is about object c". In S. Holdobler (Ed.), Intellectics and computational logic: papers in honor of Wolfgang Bibel. Kluwer Academic Press.

Faller, M. (2002). Semantics and pragmatics of evidentials in Cuzco Quechua. Ph. D. thesis, Stanford University.

Garrett, E. J. (2000). Evidentiality and assertion in Tibetan. Ph. D. thesis, University of California Los Angeles.

Geurts, B. (1994). Presupposing. Ph. D. thesis, University of Osnabrück.

Izvorski, R. (1997). The present perfect as an epistemic modal. In Proceedings of SALT , Volume 7, pp. 222-239. Cornell: CLC Publications.

Karttunen, L. (1971). Some Observations on Factivity. Papers in Linguistics 5, $55-69$.

Klinedinst, N. (2009). Totally Hardcore Semantic Presuppositions. ms. UCL.

Romoli, J. (2011). The presuppositions of soft triggers are not presuppositions. Talk presented at SALT 21.

Schlenker, P. (2006). Presupposed Entailments: The Triggering Problem Revisited. Talk presented at Sinn und Bedeutung 11.

Schlenker, P. (2008). Be Articulate: A Pragmatic Theory of Presupposition Projection. Theoretical Linguistics 34(3), 157-212.

Schlenker, P. (2010). Local Contexts and Local Meanings. Philosophical Studies.

Simons, M. (2001). On the conversational basis of some presuppositions. In J. B. Hastings, R. and S. Zvolenszky (Eds.), Semantics and Linguistic Theory, 11.

Simons, M. (2007). Observations on embedding verbs, evidentiality, and presupposition. Lingua 117(6), 1034-1056.

Simons, M., J. Tonhauser, D. Beaver, and C. Roberts (2010). What projects and why. In N. Li and D. Lutz (Eds.), Proceedings of Semantics and Linguistic Theory 20, pp. 309-327. Cornell: Ithaca.

Speas, P. (2008). On the syntax and semantics of evidentials. Language and Linguistics Compass 2(5), 940-965.

Sperber, D. and D. Wilson (1979). Ordered entailments: An alternative to presuppositional theories. Syntax and Semantics XI: Presupposition, 299-323.

Stalnaker, R. C. (1974). Pragmatic presuppositions. Semantics and Philosophy, 197-214. 


\title{
Polarities in logic and semantics
}

\author{
Arno Bastenhof \\ Utrecht University
}

\section{Introduction}

Categorial type logics (CTL, [10]) attempt the reduction of natural language grammar to proof theory. Thus, syntactic categories are replaced with formulas, and a sentence's derivability is identified with the provability of its grammaticality. CTL may be considered a realization of Montague's Universal Grammar program $([9])$, in the sense of offering a mathematical framework for studying both the syntax and semantics of natural and formal languages, in accordance with the principle of compositionality. In particular, CTL benefits of the close correspondence between the logician's formulas and the computer scientist's types, as cemented in the Curry-Howard isomorphism.

In practice, CTL serves as an umbrella term covering a plethora of logical calculi, each more or less agreeing on a fixed context-free core, but diverging in their explorations into the expressivity realms beyond. Still, one typically observes a shared asymmetry inherited from Lambek's founding work [7]. Roughly, derivability is considered a relation between a possible multitude of hypotheses (the categories, or formulas, assigned to the individual words) and a unique conclusion (the formula categorizing the phrase made up from the hypotheses).

Proposals for restoring symmetry are of more recent times, inspired by Girard's classical linear logic. While gaining in mathematical elegance, little improvement has been claimed towards strengthening linguistic coverage. ${ }^{1}$ The current paper challenges this situation, treating scopal ambiguities. Typically, their categorial analyses involve non-context-free mechanisms, realized by controlled use of structural rules, or relax compositionality to a mapping of derivations into non-singleton sets of readings. In contrast, we show that once symmetry is embraced, already within the most structure-sensitive of logics (rejecting both commutativity and associativity) all combinatorially possible scopal readings may be accounted for, even when keeping to a unique reading for each derivation. We use a variation on Classical Non-associative Lambek calculus (CNL, [3]), dubbed 'polarized CNL', or simply $\mathbf{C N L}^{\text {pol }}$. While ensured equivalent provability-wise with CNL, CNL ${ }^{p o l}$ makes a finer distinction between proofs, mapped in turn to separate readings. The concept of polarity, our main ingredient, originates in Girard's constructivization of classical logic ([5]), recognizable by computer scientists as a continuation-passing style (CPS) translation.

We proceed as follows. After introducing $\mathbf{C N L}^{\text {pol }}$ in $\S 2$, we motivate its applicability to scopal ambiguities in $\S 3$ by example. To gain a more precise

\footnotetext{
${ }^{1}$ See [11] for an exception, and $\S 5$ for a comparison with the approach discussed here.
} 
understanding of the flexibility of our approach, we consider in $\S 4$ Hendriks' type-shifting rules ([6]), showing their derivability within $\mathbf{C N L}^{\text {pol }} . \S 5$ concludes with an evaluation, including discussion detailing the limitations of our work.

\section{Polarized classical non-associative Lambek calculus}

Classical non-associative Lambek calculus was introduced by de Groote and Lamarche ([3]) as a classical conservative extension of Lambek's non-associative syntactic calculus (NL, [8]). 'Classicality' here refers to the existence of a linear negation $A^{\perp}$ on formulas $A$ satisfying involutivity $\left(A^{\perp \perp}=A\right)$. Besides the usual multiplicative conjunctions, or tensors $(A \otimes B)$, their dual disjunctions, or pars $(A \oplus B)$ now arise through the De Morgan laws as $\left(B^{\perp} \otimes A^{\perp}\right)^{\perp}$. Consequently, direction-sensitive implications $(A / B)$ and $(B \backslash A)$ need no longer be considered primitive, but may rather be defined in terms of the par and negation by $\left(A \oplus B^{\perp}\right)$ and $\left(B^{\perp} \oplus A\right)$ respectively. It was shown in [3] that CNL faithfully embeds NL.

We adapt CNL according to Girard's constructive interpretation of (traditional) classical logic ([5]), dubbing the result polarized CNL. Roughly, formulas are partitioned into those considered positive(ly polar) and negative(ly polar), with two additional connectives, the shifts $\downarrow$ and $\uparrow$ mediating between the two. Polarity is interchanged through $\cdot^{\perp}$, lending proof-theoretic support. Intuitively, the shifts may be considered 'semantic annotations', recording the locations for inserting negations in the image of semantic interpretation. The following definition details the formula language, explicating the concept of polarity.

Definition 1. Positive formulas $(P, Q, \ldots)$ and their negative duals $(M, N, \ldots)$ are defined by mutual induction, starting from a countable set of atoms $p, q, \ldots$

$$
\begin{aligned}
& P, Q \quad:=p|(P \otimes Q)|(\downarrow N) \quad \text { (Positive(ly polar) formulas) } \\
& M, N \quad::=\bar{p}|(M \oplus N)|(\uparrow P) \quad \text { (Negative(ly polar) formulas) }
\end{aligned}
$$

Linear negation $\cdot^{\perp}$ acts as primitive on atoms only (written $\cdot \overline{)}$ ), while extending to complex formulas through De Morgan's laws: $p^{\perp}=\bar{p}, \bar{p}^{\perp}=p,(P \otimes Q)^{\perp}=$ $Q^{\perp} \oplus P^{\perp},(M \oplus N)^{\perp}=N^{\perp} \otimes M^{\perp},(\downarrow N)^{\perp}=\uparrow N^{\perp}$ and $(\uparrow P)^{\perp}=\downarrow P^{\perp}$.

In practice, we stick to atomic formulas $s$ (categorizing sentences), $n p$ (noun phrases) and $n$ (common nouns). For the target language of semantic interpretation, we take simply-typed $\lambda$-calculus with base types $e$ (for individuals) and $t$ (truth values). Besides the familiar function types $(\sigma \rightarrow \tau)$ (written $\langle\sigma, \tau\rangle$ in $[4])$, semantic types $\sigma, \tau$ may include products $(\sigma \times \tau)$. In mapping formulas to types, function types are restricted to $(\sigma \rightarrow t)$, abbreviated $\neg \sigma$.

Definition 2. We associate, by mutual induction, formulas $P, N$ with types $\llbracket P \rrbracket^{+}, \llbracket N \rrbracket^{-}$. At the level of atoms, we set $\llbracket s \rrbracket^{+}=\llbracket \bar{s} \rrbracket^{-}=t$ (sentences interpret by truth values), $\llbracket n p \rrbracket^{+}=\llbracket \overline{n p} \rrbracket^{-}=e$ (noun phrases by entities) and $\llbracket n \rrbracket^{+}=$ $\llbracket \bar{n} \rrbracket^{-}=\neg e$ (common nouns by first-order properties), while for complex types,

$$
\begin{array}{rlrl}
\llbracket P \otimes Q \rrbracket^{+} & =\llbracket P \rrbracket^{+} \times \llbracket Q \rrbracket^{+} \llbracket M \oplus N \rrbracket^{-}=\llbracket N \rrbracket^{-} \times \llbracket M \rrbracket^{-} \\
\llbracket \downarrow N \rrbracket^{+} & =\neg \llbracket N \rrbracket^{-} & \llbracket \uparrow P \rrbracket^{-} & =\neg \llbracket P \rrbracket^{+}
\end{array}
$$




$$
\begin{array}{cc}
\frac{\Delta \vdash s^{\prime}: P^{\perp} \quad \Gamma, x: P \vdash s}{\Gamma, \Delta \vdash s\left[s^{\prime} / x\right]} \text { Cut } & \frac{}{x: A \vdash x: A^{\perp}} A x \\
\frac{\Gamma, \Delta \vdash s}{\Delta, \Gamma \vdash s} d p^{1} & \frac{\Gamma \bullet \Delta, \Theta \vdash s}{\Gamma, \Delta \bullet \Theta \vdash s} d p^{2} \\
\frac{\Gamma \vdash s: N}{\Gamma, \downarrow N^{x} \vdash(x s)} \downarrow & \frac{\Gamma, P^{x} \vdash s}{\Gamma \vdash \lambda x^{\llbracket P \rrbracket^{+} s: \uparrow P} \uparrow} \\
\Gamma, P^{y} \bullet Q^{z} \vdash s & \frac{\Gamma \vdash s: M \quad \Delta \vdash s^{\prime}: N}{\Delta \bullet \Gamma \vdash\left\langle s^{\prime}, s\right\rangle: M \oplus N} \oplus
\end{array}
$$

Fig. 1. Polarized classical non-associative Lambek calculus.

Derivability establishes pairings of binary-branching trees $\Gamma, \Delta$ of formulas, referred to by structures. Their leaves carry positive formulas, negative $N$ appearing only when prefixed by $\downarrow$. Furthermore, in anticipation of the term assignment to derivations, formulas are labeled using $(\lambda$-)variables $x, y, \ldots$

Definition 3. Structures $\Gamma, \Delta$ collect pairings $P^{x}$ into binary-branching trees:

$$
\Gamma, \Delta::=P^{x} \mid(\Gamma \bullet \Delta)
$$

Proofs involve derivability judgements $\Gamma, \Delta \vdash s$ and $\Gamma \vdash s: N$, with $s$ a $\lambda$-term of type $t$ in the first case, and of type $\llbracket N \rrbracket^{-}$in the latter, while containing free variables $x$ of type $\llbracket P \rrbracket^{+}$for each $P^{x}$ in $\Gamma(, \Delta)$. Compared to [3], we have chosen an unconventional left-sided sequent notation, better reflecting the intuitionistic nature of the semantic target language.

Definition 4. Figure 1 details the mutually defined derivability judgements $\Gamma, \Delta \vdash s$ and $\Gamma \vdash s: N$. Double inference lines are used in $\left(d p^{2}\right)$ to indicate that derivability goes in both directions.

We make the following observations regarding the above definition.

1. The rules $\left(d p^{1}\right)$ and $\left(d p^{2}\right)$ bear resemblance to Belnap's display postulates $([2])$, in allowing one to isolate the main formula of a logical inference as the whole of one of the sequent's components by turning its context 'inside out'. They are not to be mistaken for associativity and commutativity postulates.

2. Compared to $\mathbf{C N L}, \mathbf{C N L}^{p o l}$ exploits both sides of the turnstile symbol $\vdash$, though treating them asymmetrically in order to restore the tight connection with $\lambda$-calculus. The newly added shifts serve solely to control traffic across $\vdash$. Provability-wise, these are harmless extensions over CNL, as argued for below in Theorems 6 and 7. When considering the actual proofs, however, shifts, together with the display postulates, offer a means of creating abstractions over any of the variables found in a sequent without compromising structure-sensitivity. In contrast, NL severely restricts the abstraction mechanism, accounting for its limited semantic expressivity. 
Theorem 5. Cut is admissible, as are non-atomic instantiations of axioms.

Theorem 6. Under the forgetful translation, removing shifts and writing all sequents one-sided, derivations in $\mathbf{C N L}^{\text {pol }}$ map into those of CNL.

Theorem 7. There exists a decoration of CNL's formulas by shifts relative to which derivations can be translated into $\mathbf{C N L}^{\text {pol }}$.

The previous theorems, proven in [1], ensure equivalence, provability-wise, of CNL and CNL $^{p o l}$. The proof of Theorem 7 in particular bears resemblance to a continuation-passing style (CPS) translation, as further elaborated upon in [1].

\section{Case analyses}

We illustrate the versatility of our approach through a survey of several classical examples. As further motivated in $\S 4$, the underlying generalization is that any combinatorially possible scopal reading may be accounted for through generous use of shifts inside the lexicon. Conversely, parsimonious use allows for certain readings to be blocked ( 33.4 ), although this approach has its limitations (cf. $\S 5$ ). Below, we first provide a sample lexicon ( $\$ 3.1)$, through which we can explain object-wide scope readings ( $\S 3.2$, as a warm-up), embedded scope (§3.3), scope sieves (§3.4), and, finally, intensionality and coordination (§3.5).

\section{$3.1 \quad$ Lexicon}

A lexicon associates words $w$ with formulas $P$ and semantic interpretation a term $s$ of type $\llbracket P \rrbracket^{+}$, and is usually written informally as a collection of sequents $w \vdash$ $s: P^{\perp}$ (noting $\llbracket P \rrbracket^{+}=\llbracket P^{\perp} \rrbracket^{-}$, by a routine induction). If this notation is taken seriously, allowing in particular words at the leaves of structures, lexical insertion into a derivation proceeds through Cut. In defining the lexical denotations $s$, we allow recourse to the usual predicate-logical connectives, considered constants $\wedge, \vee, \supset$ (of types $t \rightarrow(t \rightarrow t)), \forall$ and $\exists$ (of types $(e \rightarrow t) \rightarrow t$ ), with the binary operations written in the usual infix style. Here, $\supset$ refers to implication, its notation meant to prevent confusion with the type-forming operator $\rightarrow$.

F.2 presents a sample lexicon, using constants ALICE (of type $e$ ), PERSON, UNICORN, YAWN $(e \rightarrow t)$, FIND $(e \rightarrow(e \rightarrow t))$, SEEK $(((e \rightarrow t) \rightarrow t) \rightarrow(e \rightarrow t))$, THINK and HEAR $(t \rightarrow(e \rightarrow t))$. We have written $(N / P)$ and $(P \backslash N)$ for $\left(N \oplus P^{\perp}\right)$ and $\left(P^{\perp} \oplus N\right)$ respectively, to facilitate comparison with traditional CTL. Finally, we use paired abstractions $\lambda\langle x, y\rangle s$ as abbreviations for $\lambda z s\left[\pi^{1}(z) / x, \pi^{2}(z) / y\right]$.

\subsection{Object-wide scope}

As a warm-up, consider the familiar linear and inverse scope readings (1a), (1b)

1. Everyone found a unicorn.

1a. For every person $x$, there exists some unicorn $y$ s.t. $x$ found $y$.

1b. There exists some unicorn $y$ s.t. for every person $x, x$ found $y$. 


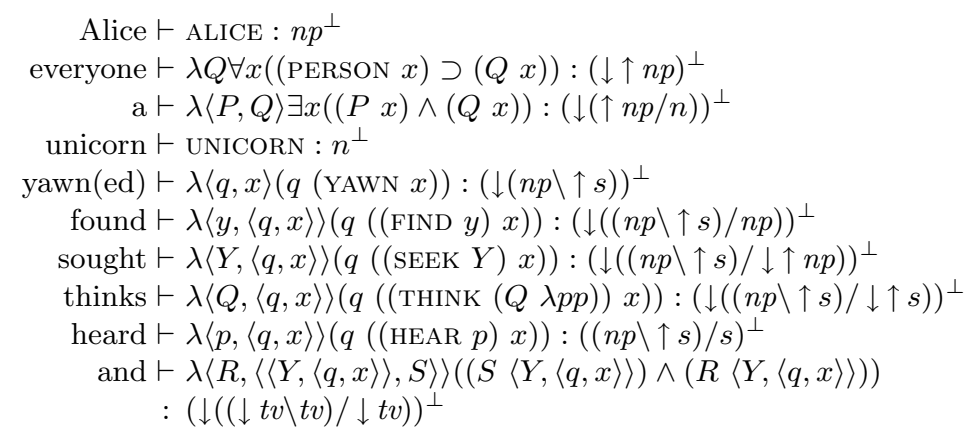

Fig. 2. Sample lexicon, where $t v$ abbreviates $(n p \backslash(\uparrow s / \downarrow \uparrow n p))$.

The formulas assigned to the quantified noun phrases involved reflect their interpretation as second-order properties through the use of double shifts, inserting double negations at the level of types (i.e., implications with result type $t$ ). We first construct the following derivations, using the formulas found in F.2 (cf. F.3)

$$
\begin{array}{r}
\downarrow \uparrow n p^{x} \bullet\left(\downarrow((n p \backslash \uparrow s) / n p)^{y} \bullet\left(\downarrow(\uparrow n p / n)^{u} \bullet n^{v}\right)\right), \downarrow \bar{s}^{z} \\
\vdash\left\{\begin{array}{l}
(x \lambda a(u\langle v, \lambda b(y\langle b,\langle z, a\rangle))\rangle)) \\
(u\langle v, \lambda b(x \lambda a(y\langle b,\langle z, a\rangle\rangle))\rangle)
\end{array}\right.
\end{array}
$$

with lexical insertion (through Cut) deriving, after $\beta$-conversion, the desired

$$
\vdash\left\{\begin{array}{r}
\text { everyone } \bullet \text { (found } \bullet \text { (a } \bullet x((\text { unicorn })), \downarrow \bar{s}^{z} \\
\exists y((\operatorname{UNICORN} y) \supset \exists y((\text { UNICORN } y) \wedge(z((\text { FIND } y) x)))(1 \mathrm{a})
\end{array}\right.
$$

noting the presence of a free variable $z$ for the resulting category. If desired, one may apply $(\uparrow)$ to create an abstraction, deriving $\uparrow \downarrow \bar{s}$.

\subsection{Embedded scope}

We illustrate non-local scope construal from inside a complement clause:

2. Alice thinks a unicorn yawned.

2a. Alice thinks there exists a unicorn $y$ s.t. $y$ yawned.

2b. For some unicorn $y$, Alice thinks $y$ yawned.

By having the verb select for a clausal complement of category $\downarrow \uparrow s$ instead of $s$, we provide the deductive machinery with enough freedom to construct both of the desired readings. Using the formulas found in F.2, we derive three terms,

$$
\begin{aligned}
& n p^{a} \bullet\left(\downarrow((n p \backslash \uparrow s) / \downarrow \uparrow s)^{t} \bullet\left(\left(\downarrow(\uparrow n p / n)^{u} \bullet n^{v}\right) \bullet \downarrow(n p \backslash \uparrow s)^{w}\right)\right), \downarrow \bar{s}^{z} \\
& \vdash\left\{\begin{array}{l}
(t\langle\lambda q(u\langle v, \lambda y(w\langle q, y\rangle)\rangle),\langle z, a\rangle\rangle) \\
(u\langle v, \lambda y(t\langle\lambda q(w\langle q, y\rangle),\langle z, a\rangle\rangle)\rangle) \\
(u\langle v, \lambda y(w\langle\lambda p(t\langle\lambda q(q p),\langle z, a\rangle)), y\rangle)\rangle)
\end{array}\right.
\end{aligned}
$$

collapsed into two readings after lexical insertion: 


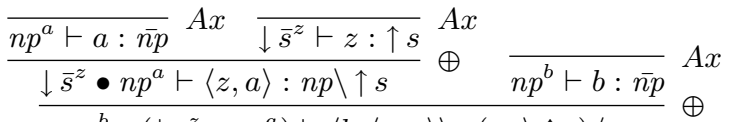

$$
\begin{aligned}
& n p^{b} \bullet\left(\downarrow \bar{s}^{z} \bullet n p^{a}\right) \vdash\langle b,\langle z, a\rangle\rangle:(n p \backslash \uparrow s) / n p \\
& \overline{n p^{b} \bullet\left(\downarrow \bar{s}^{z} \bullet n p^{a}\right), \downarrow((n p \backslash \uparrow s) / n p)^{y} \vdash(y\langle b,\langle z, a\rangle\rangle)} \downarrow \\
& \overline{\left(\downarrow \bar{s}^{z} \bullet n p^{a}\right) \bullet \downarrow((n p \backslash \uparrow s) / n p)^{y}, n p^{b} \vdash(y\langle b,\langle z, a\rangle\rangle)} D p \\
& \overline{\left(\downarrow \bar{s}^{z} \bullet n p^{a}\right) \bullet \downarrow((n p \backslash \uparrow s) / n p)^{y} \vdash \lambda b(y\langle b,\langle z, a\rangle\rangle): \uparrow n p} \uparrow \quad \overline{n^{v} \vdash v: \bar{n}} A x
\end{aligned}
$$

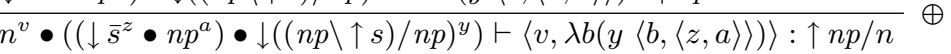

$$
\begin{aligned}
& \overline{n^{v} \bullet\left(\left(\downarrow \bar{s}^{z} \bullet n p^{a}\right) \bullet \downarrow((n p \backslash \uparrow s) / n p)^{y}\right), \downarrow(\uparrow n p / n)^{u} \vdash(u\langle v, \lambda b(y\langle b,\langle z, a\rangle\rangle)\rangle)} \downarrow \\
& \overline{\left(\downarrow((n p \backslash \uparrow s) / n p)^{y} \bullet\left(\downarrow(\uparrow n p / n)^{u} \bullet n^{v}\right)\right) \bullet \downarrow s^{z}, n p^{a} \vdash(u\langle v, \lambda b(y\langle b,\langle z, a\rangle\rangle)\rangle)} D p \\
& \frac{\left(\downarrow((n p \backslash \uparrow s) / n p)^{y} \bullet\left(\downarrow(\uparrow n p / n)^{u} \bullet n^{v}\right)\right) \bullet \downarrow \bar{s}^{z} \vdash \lambda a(u\langle v, \lambda b(y\langle b,\langle z, a\rangle\rangle)\rangle): \uparrow n p}{(\uparrow}
\end{aligned}
$$

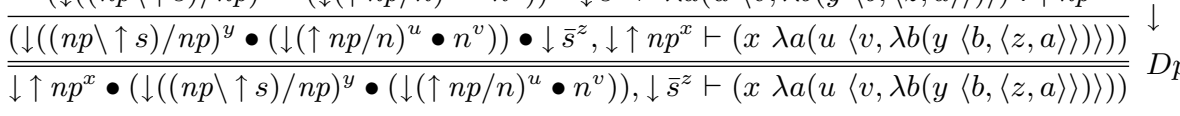

$$
\begin{aligned}
& \overline{n p^{a} \vdash a: \overline{n p}} A x \quad \overline{\downarrow \bar{s}^{z} \vdash z: \uparrow s} A x \\
& \downarrow \bar{s}^{z} \bullet n p^{a} \vdash\langle z, a\rangle: n p \backslash \uparrow s \quad \oplus \quad \overline{n p^{b} \vdash b: \overline{n p}} A x \\
& n p^{b} \bullet\left(\downarrow \bar{s}^{z} \bullet n p^{a}\right) \vdash\langle b,\langle z, a\rangle\rangle:(n p \backslash \uparrow s) / n p \\
& \overline{\overline{n p^{b} \bullet\left(\downarrow \bar{s}^{z} \bullet n p^{a}\right), \downarrow((n p \backslash \uparrow s) / n p)^{y} \vdash(y\langle b,\langle z, a\rangle\rangle)}} \downarrow \\
& \frac{\overline{\left(\downarrow((n p \backslash \uparrow s) / n p)^{y} \bullet n p^{b}\right) \bullet \downarrow \bar{s}^{z}, n p^{a} \vdash(y\langle b,\langle z, a\rangle\rangle)}}{\left(\downarrow((n p \backslash \uparrow s) / n p)^{y} \bullet n p^{b}\right) \bullet \downarrow \bar{s}^{z} \vdash \lambda a(y\langle b,\langle z, a\rangle\rangle): \uparrow n p} \uparrow \\
& \overline{\overline{\left(\downarrow((n p \backslash \uparrow s) / n p)^{y} \bullet n p^{b}\right) \bullet \downarrow \bar{s}^{z}, \downarrow \uparrow n p^{x} \vdash(x \lambda a(y\langle b,\langle z, a\rangle\rangle))}} \downarrow \\
& \frac{\overline{\left(\downarrow \bar{s}^{z} \bullet \downarrow \uparrow n p^{x}\right) \bullet \downarrow((n p \backslash \uparrow s) / n p)^{y}, n p^{b} \vdash(x \lambda a(y\langle b,\langle z, a\rangle\rangle))}}{\left(\downarrow \bar{s}^{z} \bullet \downarrow \uparrow n p^{x}\right) \bullet \downarrow((n p \backslash \uparrow s) / n p)^{y} \vdash \lambda b(x \lambda a(y\langle b,\langle z, a\rangle\rangle)): \uparrow n p} \uparrow \bar{~} \overline{n^{v} \vdash v: \bar{n}} A x
\end{aligned}
$$

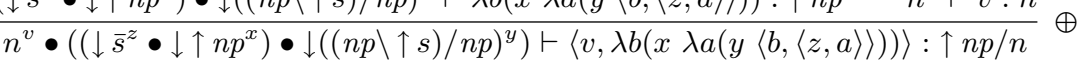

$$
\begin{aligned}
& \frac{\overline{n^{v} \bullet\left(\left(\downarrow \bar{s}^{z} \bullet \downarrow \uparrow n p^{x}\right) \bullet \downarrow((n p \backslash \uparrow s) / n p)^{y}\right), \downarrow(\uparrow n p / n)^{u} \vdash(u\langle v, \lambda b(x \lambda a(y\langle b,\langle z, a\rangle\rangle))\rangle)}}{\overline{\downarrow \uparrow n p^{x} \bullet\left(\downarrow((n p \backslash \uparrow s) / n p)^{y} \bullet\left(\downarrow(\uparrow n p / n)^{u} \bullet n^{v}\right)\right), \downarrow \bar{s}^{z} \vdash(u\langle v, \lambda b(x \lambda a(y\langle b,\langle z, a\rangle))))}} D p
\end{aligned}
$$

Fig. 3. Deriving everyone found a unicorn. (Dp) abbreviates multiple $\left(d p^{2}, d p^{1}\right)$-steps.

Alice $\bullet$ (thinks $\bullet(($ a $\bullet$ unicorn $) \bullet$ yawned $)), \downarrow \bar{s}^{z}$ $\vdash\left\{\begin{array}{l}(z((\text { THINK } \exists y((\text { UNICORN } y) \wedge(\text { YAWN } y))) \text { ALICE }))(2 a) \\ \exists y((\text { UNICORN } y) \wedge(z((\text { THINK }(\text { YAWN } y)) \text { ALICE })))(2 b)\end{array}\right.$

\subsection{Scope sieves}

Further insight into the categorization of verbs selecting for clausal complements is provided by the following example, illustrating scope sieves. Here, non-local scope is enforced, as typically observed with perception verbs (cf. [6], p.108):

3. Alice heard a unicorn yawn. 
The desired result obtains by having the matrix verb select for $s$ instead of $\downarrow \uparrow s$. For reasons of space, we only show the end result obtained after lexical insertion.

$$
\begin{aligned}
& \text { Alice } \bullet(\text { heard } \bullet((\text { a } \bullet \text { unicorn }) \bullet \text { yawn })), \downarrow \bar{s}^{z} \\
\vdash & \exists y((\text { UNICORN } y) \wedge(z((\text { HEAR } y) \text { ALICE })))
\end{aligned}
$$

On the other hand, it does not seem clear how to enforce the local reading (e.g., as needed for scope islands). See $\S 5$ for further discussion.

\subsection{Coordination and intensionality}

We conclude with intensionality and coordination, treated together to show their interplay. Our treatment of intensionality is simplified w.r.t. our choice of semantic type for interpreting sentences, although this can be easily remedied if the reader so desires. Furthermore, found is treated extensionally, in line with [4].

4. Alice sought and found a unicorn.

4a. Alice sought a unicorn, and there exists a unicorn $y$ s.t. Alice found $y$. 4b. There exists a unicorn $y$ s.t. Alice sought $y$ and Alice found $y$.

Again, showing only the end results after lexical insertion for reasons of space:

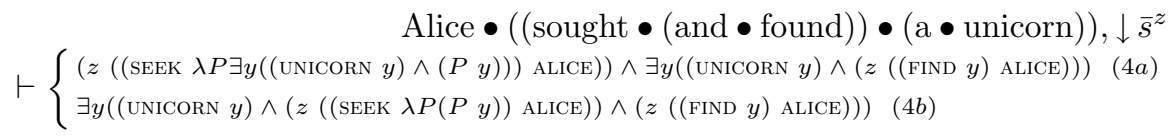

\section{Type-shifting}

The previous section attempted the description of $\mathbf{C N L}^{\mathrm{pol}}$ 's semantic expressivity by empirical illustration. To arrive at a more precise characterization, we now compare our treatment of scopal ambiguities with a more well-understood solution. Hendriks ([6]), generalizing [12], proposed a flexible correspondence between syntax and semantics by closing the readings associated with a given syntactic derivation under the following derived semantic inference rules, referred to collectively by type shifting:

1. Value Raising. Assuming right-associative bracketing, terms of type $\sigma_{1} \rightarrow$ $\ldots \rightarrow \sigma_{n} \rightarrow \sigma$ may be lifted to the type $\sigma_{1} \rightarrow \ldots \rightarrow\left(\sigma \rightarrow \tau^{\prime}\right) \rightarrow \tau^{\prime}$, for any $\tau^{\prime}$. E.g., a proper name 'Alice' with interpretation of type $e$ undergoes value raising to derive a term of the type $(e \rightarrow t) \rightarrow t$ for generalized quantifiers.

2. Argument Raising. Inhabitants of $\sigma_{1} \rightarrow \ldots \rightarrow \sigma_{n} \rightarrow \tau$ may transition into $\sigma_{1} \rightarrow \ldots \rightarrow\left(\left(\sigma_{i} \rightarrow \tau^{\prime}\right) \rightarrow \tau^{\prime}\right) \rightarrow \tau$. Typically applies to the subject and object positions of transitive verbs. For example, $e \rightarrow(e \rightarrow t)$ undergoes argument raising twice in order to derive the type of a binary relation on generalized quantifiers. Depending on which of the arguments is raised first, we obtain different scopal readings.

3. Argument Lowering. Inhabitants of $\sigma_{1} \rightarrow \ldots \rightarrow \sigma_{i} \rightarrow \tau$ may be derived from terms of type $\sigma_{1} \rightarrow \ldots \rightarrow\left(\left(\sigma_{i} \rightarrow \tau^{\prime}\right) \rightarrow \tau^{\prime}\right) \rightarrow \tau$. For example, lowering and subsequent raising (for the choice $\tau^{\prime}=t$ ) of the object position of an intensional verb allows for the derivation of de re readings. 
By applying the right combinations of these rules, one could derive all combinatorially available scopal readings for a given sentence, as proved by Hendriks. What is important to note is that all rules involved are derivable within $\lambda$-calculus. In contrast, analogous rules for the traditional incarnations of CTL necessitate full associativity and commutativity, leading to serious overgeneration. Consequently, type-shifting seemed exclusive to the realm of semantics, necessitating the relaxation of compositionality if it was to be made any use of.

We claim the existence of derivable rules of inference within CNL $^{\text {pol }}$ mapping to Hendriks' type-shifting schemas under semantic interpretation. Consequently, the strict correspondence between syntax and semantics can be restored, while preserving semantic expressivity. One restriction, however, applies: the types $\tau^{\prime}$ in the above explanation of type-shifting always are to be $t$. In practice, this still leaves us with sufficient generality to account for scoping within clausal domains.

Theorem 8. The following rules of Value Raising (VR), Argument Raising (AR) and Argument Lowering (AL) are derivable within CNL $^{\text {pol: }}$ :

$$
\begin{array}{rlrl}
\Gamma \vdash s: N & \Rightarrow \Gamma \vdash \lambda x(x s): \uparrow \downarrow N & (V R) \\
\Gamma \vdash s:(\downarrow(M / Q))^{\perp} & \Rightarrow \Gamma \vdash \lambda\langle x, v\rangle(x \lambda u(s\langle u, v\rangle)):(\downarrow(M / \downarrow \uparrow Q))^{\perp}\left(A R^{r}\right) \\
\Gamma \vdash s:(\downarrow(P \backslash N))^{\perp} & \Rightarrow \Gamma \vdash \lambda\langle v, x\rangle(x \lambda u(s\langle v, u\rangle)):(\downarrow(\downarrow \uparrow P \backslash N))^{\perp} & \left(A R^{l}\right) \\
\Gamma \vdash s:(\downarrow(M / \downarrow \uparrow Q))^{\perp} \Rightarrow \Gamma \vdash \lambda\langle u, v\rangle(s\langle\lambda x(x u), v\rangle): & (\downarrow(M / Q))^{\perp} & \left(A L^{r}\right) \\
\Gamma \vdash s:(\downarrow(\downarrow \uparrow P \backslash N))^{\perp} & \Rightarrow \Gamma \vdash \lambda\langle v, u\rangle(s\langle v, \lambda x(x u)\rangle): & (\downarrow(P \backslash N))^{\perp} & \left(A L^{l}\right)
\end{array}
$$

Example 9. We illustrate the above result with a lexical entry from Figure 2:

$$
\text { found } \vdash \lambda\langle y,\langle q, x\rangle\rangle(q((\text { FIND } y) x)):(\downarrow((n p \backslash \uparrow s) / n p))^{\perp}
$$

Note the term involved is of type $\neg(e \times(\neg t \times e))$, which by uncurrying is isomorphic to $e \rightarrow(e \rightarrow \neg \neg t))$. Applying Argument Raising (i.e., $\left.\left(A R^{r}\right)\right)$, we get

$$
\text { found } \vdash \lambda\langle Y,\langle q, x\rangle\rangle(Y \lambda y(q((\operatorname{FIND} y) x))):(\downarrow((\downarrow \uparrow n p \backslash \uparrow s) / n p))^{\perp}
$$

which is of type $\neg(\neg \neg e \times(\neg t \times e))$, i.e., $\neg \neg e \rightarrow(e \rightarrow \neg \neg t)$.

Still lacking is the means to target other positions besides the direct object for Argument Raising (or -Lowering), a possibility rendered available in Hendriks' presentation by allowing for any number of arguments preceding the one to which the operation was applied. In our case, the following result allows any intervening arguments to be stripped off one by one, and to be added back after the desired type-shifting rule has been applied.

Lemma 10. The following are derivable rules of inference:

$$
\begin{array}{cc}
\frac{\Gamma \bullet P^{x} \vdash s:(\downarrow N)^{\perp}}{\Gamma \vdash \lambda\langle x, y\rangle(s y):(\downarrow(N / P))^{\perp}}> & \frac{P^{x} \bullet \Gamma \vdash s:(\downarrow N)^{\perp}}{\Gamma \vdash \lambda\langle y, x\rangle(s y):(\downarrow(P \backslash N))^{\perp}}< \\
\frac{\Gamma \vdash s:(\downarrow(N / P))^{\perp}}{\Gamma \bullet P^{x} \vdash \lambda y(s\langle x, y\rangle):(\downarrow N)^{\perp}}>^{\prime} & \frac{\Gamma \vdash s:(\downarrow(P \backslash N))^{\perp}}{P^{x} \bullet \Gamma \vdash \lambda y(s\langle y, x\rangle):(\downarrow N)^{\perp}}<^{\prime}
\end{array}
$$


Example 11. Continuing where we left off in E.9, $\left(A R^{l}\right)$ combines with $(>)$ and $\left(>^{\prime}\right)$ to allow for Argument Raising of the subject position, resulting in

$$
\text { found } \vdash \lambda\langle Y,\langle q, X\rangle\rangle(X \lambda x(Y \lambda y(q((\text { FIND } y) x)))):(\downarrow((\downarrow \uparrow n p \backslash \uparrow s) / \downarrow \uparrow n p))^{\perp}
$$

being of type $\neg \neg e \rightarrow(\neg \neg e \rightarrow \neg \neg t)$ after uncurrying. Note we could also have applied $\left(A R^{l}\right)$ first, followed by $\left(A R^{r}\right)$ to derive

$$
\text { found } \vdash \lambda\langle Y,\langle q, X\rangle\rangle(Y \lambda y(X \lambda x(q((\text { FIND } y) x)))):(\downarrow((\downarrow \uparrow n p \backslash \uparrow s) / \downarrow \uparrow n p))^{\perp}
$$

The latter allows for the derivation of object-wide scope readings, whereas raising the subject after the object favors subject-wide scope.

\section{Evaluation}

We have presented polarized classical non-associative Lambek calculus, arguing for its applicability to the analysis of scopal ambiguities despite its resource sensitivity, and despite keeping to a strict correspondence between syntax and semantics. In contrast, earlier categorial accounts typically involved non-contextfree mechanisms by allowing for controlled associativity and commutativity, or relaxed compositionality to a relation. We have compared our approach to one such traditional account, to wit, Hendriks' use of type-shifting in associating syntactic derivations with sets of readings. Below, we briefly discuss, first, a point of critique concerning our capacity to block scopal readings that, while combinatorially possible, are unrealized by linguistic reality. Second, we draw attention to a curious discrepancy with Hendriks' account of verbs taking clausal complements, and, finally, make a brief comparison with a proposal for the categorial analysis of scope closely related to ours.

\subsection{Blocking scopal readings}

While we could ensure derivability of all combinatorially available scopal readings for the various examples we considered, this same property also constitutes the main limitation of our approach: situations where linguistic reality excludes certain readings are not so easily accounted for. While we had a small success with the analysis of scope sieves, scope islands fall outside our coverage. The reasons for this go deep: through the display postulates, any variable-labeled formula may be isolated as the whole of one of the sequents' components, which may subsequently be abstracted over through $(\uparrow)$. In other words, accounting for scope islands means to restrict the display postulates, which constitute an essential ingredient for the notion of structure adopted by CNL, as exemplified graphically by De Groote and Lamarche's proof nets ([3]).

\subsection{On verbs taking clausal complements}

Our analysis of verbs taking clausal complements is host to an interesting curiosity, setting it apart from Hendriks' account. One might first expect the category 
$\downarrow((n p \backslash \uparrow s) / s)$ assigned to heard in $\S 3.4$ to foresee at least in the local reading, with type-shifting required for non-local scope construal. Such a situation would parallel Hendriks' [6], which starts from a minimal semantic type assignment. Instead, we find that only the non-local reading is derived, whereas a local reading necessitates the prefixing of the $s$ argument by a double shift, cf. the category $\downarrow((n p \backslash \uparrow s) / \downarrow \uparrow s)$ assigned to thinks in $\S 3.3$.

\subsection{Comparison}

Another account of scopal ambiguities similar to ours was recently put forward by Moortgat ([11]), working within the closely related Lambek-Grishin calculus (LG). There, scopal ambiguities were accounted for by adding minimal (co)negations to the logical vocabulary (referred to by (co-)Galois connections), used similarly to our shifts. We claim polarization allows for a better understanding of this proposal. Like CNL, LG turns out amendable to a polarized reformulation, as demonstrated in [1]. Moortgat's compositional semantics may then be recast as a translation of $\mathbf{L G}$ into $\mathbf{L} \mathbf{G}^{\text {pol }}$, revealing that his minimal negations simply serve to enforce the appearance of shifts in the target.

\section{References}

1. A. Bastenhof. Polarized classical non-associative lambek calculus and formal semantics. In S. Pogodalla and J-P. Prost, editors, Logical Aspects of Computational Linguistics - 6th International Conference, LACL 2011. Proceedings, volume 6736 of Lecture Notes in Computer Science, pages 33-48. Springer, 2011.

2. N.D. Belnap. Display Logic. Journal of Philosophical Logic, 11(4):375-417, 1982.

3. P. De Groote and F. Lamarche. Classical non associative Lambek calculus. Studia Logica, 71:355-388, 2002.

4. L.T.F. Gamut. Logic, Language and Meaning, Volume 2: Intensional Logic and Logical Grammar. University of Chicago Press, 1991.

5. J-Y. Girard. A new constructive logic: classical logic. Mathematical Structures in Computer Science, 1(3):255-296, 1991.

6. H. Hendriks. Studied flexibility. Categories and types in syntax and semantics. PhD thesis, ILLC Amsterdam, 1993.

7. J. Lambek. The mathematics of sentence structure. American Mathematical Monthly, 65:154-169, 1958.

8. J. Lambek. On the calculus of syntactic types. In R. Jakobson, editor, Structure of Language and its Mathematical Aspects, Proceedings of the Twelfth Symposium in Applied Mathematics, 1961.

9. R. Montague. Universal grammar. Theoria, 36(3):373-398, 1970.

10. M. Moortgat. Categorial type logics. In Handbook of Logic and Language, pages 93-177. Elsevier, 1997.

11. M. Moortgat. Symmetric categorial grammar: residuation and Galois connections. CoRR, abs/1008.0170, 2010.

12. B. Partee and M. Rooth. Generalized conjunction and type ambiguity. In R. Bäuerle, C. Schwarze, and A. von Stechow, editors, Meaning, use, and interpretation of language, pages 361-383. Gruyter, Berlin, 1983. 


\title{
Implicit Arguments: Event Modification or Option Type Categories?
}

\author{
Chris Blom ${ }^{1}$, Philippe de Groote ${ }^{2}$, Yoad Winter ${ }^{3}$, and Joost Zwarts ${ }^{3}$ \\ 1 CAI Master Program, Utrecht University \\ 2 LORIA/INRIA \\ 3 UiL OTS, Utrecht University
}

\begin{abstract}
We propose a unified syntactic-semantic account of passive sentences and sentences with an unspecified object (John read). For both constructions, we employ option types for introducing implicit arguments into the syntactic-semantic categorial mechanism. We show the advantages of this approach over previous proposals in the domains of scope and unaccusatives. Unlike pure syntactic treatments, option types immediately derive the obligatory narrow scope of existential quantification over an implicit argument's slot. Unlike purely semantic, event-based treatments, our proposal naturally accounts for syntactic contrasts between passives and unaccusatives, as in the door *(was) opened by John. After the main system is introduced, some of its further predictions in the domain of optional arguments and event modification are analyzed.
\end{abstract}

\section{Introduction}

Many verbs allow adjacent constituents like the ones underlined in (1) to be optional, as illustrated by (2).

(1) a. John read the book.

b. John introduced Paul to Mary.

c. The vase was broken by John.

(2) a. John read.

b. John introduced Paul.

c. The vase was broken.

Unspecified objects (UOs, Levin [12]) as in (2a) are licensed with verbs like eat, drink, bake etc. In semantic terms, the optionality of object NPs with such verbs is similar to the optionality of subcategorized PPs as in (1b) and by phrases in passive sentences like (1c). When these constituents are missing, as in (2), the sentence still makes an existence claim with respect to the unfilled predicate slot [5]. This is illustrated by the equivalences in (3).

(3) John read $\Leftrightarrow$ John read something.

John introduced Paul $\Leftrightarrow$ John introduced Paul to someone.

The vase was broken $\Leftrightarrow$ The vase was broken by someone or something. 
Because of this 'semantic implicitness' of the verbal slot in (2), we refer to the underlined constituents in (1) as optional arguments. ${ }^{4}$

Our aim in this paper is to give a basic syntactic/semantic account of optional arguments in categorial grammar and explain their semantic properties. The main two problems we deal with are therefore:

P1 How can an argument be missing (2), while the sentence still makes an existential import involving the unfilled slot(3)?

P2 How can verbs with an optional argument compose with this argument when it is present (1), neutralizing the purely existential effect?

After reviewing some previous accounts and their limitations, we propose a new account of these two questions using option types in Abstract Categorial Gram$\operatorname{mar}(\mathrm{ACG}[6,13])$.

\section{Previous accounts: meaning postulates and event modification}

Existential implicit arguments must be interpreted with narrow scope (Fodor \& Fodor, [5]). Consider the following examples.

(4) a. John did not read yesterday.

b. John did not read something yesterday.

(5) a. The door was not opened yesterday.

b. The door was not opened by something/someone yesterday.

The overt indefinites in (4b) and (5b) are scopally ambiguous. By contrast, in (4a) and (5a) the only reading is existential narrow scope: "John did not read anything" and "the door was not opened by anything/anyone", respectively. As Fodor \& Fodor observe, obligatory narrow scope of quantifiers over implicit arguments challenges pure syntactic theories that introduce an existential argument at some covert syntactic level. F\&F account for contrasts as in (4) using a meaning postulate connecting a transitive entry of UO verbs with another, intransitive entry. As F\&F show, this immediately accounts for narrow scope effects in cases like (4a). A similar idea governs ambiguity-based derivations of passives, with

\footnotetext{
${ }^{4}$ We will not try to give here a full syntactic analysis of the distinction between optional arguments and adjuncts. First, the disability to iterate (optional and optionality) arguments (cf. * John ate/bought a sandwich a hamburger) extends to other constituents that syntacticians may consider as adjuncts: *John went to Paul to Mary, * John baked a cake for Mary for Sue, ${ }^{*}$ John cut the rope with a knife with an axe, etc. Another challenge for defining the distribution of optional arguments is that some typical adjuncts may also have an existence entailment when missing. For instance, John will sing entails John will sing sometime. Note however that many adjuncts are clearly distinguished from optional arguments in having no existential entailment: Mary worked does not entail Mary worked for someone, John baked a cake does not entail John baked a cake for someone, John went does not entail John went somewhere (he may have evaporated in space), and John cut the rope does not entail John cut the rope with something (he may have had super-natural powers).
} 
or without a by phrase (Bach [1], Dowty [4]). However, this 'classical' approach to passives is quite cumbersome (Landman [9]). For instance, to account for the optionality of the two underlined constituents in a passive sentence such as Paul was introduced by John to Mary, classical accounts would have to assign four different meanings to the passive form of the verb introduce, connected to each other using meaning postulates.

A more promising semantic approach to passives and UOs was suggested by Carlson [2], who treats both by phrases in passives and objects with UO verbs as neo-Davidsonian event modifiers. Carlson treats the object in John read 'Lolita' as an adjunct that modifies reading events. When the object is missing (John read), Carlson treats the existence entailment as an implied property of events: when there is a reading, something must be read (cf. Larson [10], Lasersohn [11]). Similarly, Carlson treats the understood agent in a passive sentence like Mary was praised as a property of 'praising events': when there is a praising there is a praiser. These intuitions can be implemented as a single meaning postulate on predicates over events, which nicely improves F\&F's approach. A similar event-based account of passives is proposed by Landman in [9].

However, Carlson's approach becomes less appealing when considering unaccusative verbs. A sentence like the door opened is not equivalent to someone/something opened the door. This shows that 'opening events' do not require an agent. Hence, the understood agent in passives like the door was opened is not explained. ${ }^{5}$ Furthermore, treating by phrases as adjuncts does not account for their ungrammaticality with unaccusatives: if by phrases simply modify events by specifying an agent, why are they systematically disallowed in cases like *the door opened by John? To block a meaning like "John opened the door" for such strings, Carlson (p.268) must resort to ad hoc syntactic assumptions.

\section{The Optional Argument Principle and option types in Abstract Categorial Grammar}

The considerations in section 2 support a semantic approach that is more consistent with current syntactic theories about implicit arguments (Landau [8]). We propose the following principle (cf. Dekker [3]). ${ }^{6}$

\footnotetext{
${ }^{5}$ This is no problem for Landman's proposal, which uses the passive morphology for deriving the semantic requirement that an agent exists in events described by passive sentences. However for this reason, Landman's approach does not naturally extend to UOs, where intransitive forms are homonymic to transitives. Hence, in such situations overt morphology alone cannot account for the requirement that a patient/theme exists in all events described by UO verbs.

${ }^{6}$ Dekker proposes treating some optionality phenomena using dynamic binding. Extending Dekker's approach to passives and UOs may have comparable advantages to the present account. It would require augmenting Dekker's proposal with a clear distinction between verb modifiers (e.g. many adverbials), which can iterate, and optional arguments that cannot iterate (e.g. optional objects and by phrases in passives). In Dekker's system the former must be treated as dynamic argument modifiers, whereas the latter are treated as static argument saturators. Once this distinc-
} 
Optional Argument Principle (OAP): Verbal forms may specify optional arguments in their syntactic-semantic type. The existential semantic interpretation of an implicit argument is lexically introduced.

To implement OAP-based optionality in categorial grammar, we mark optionality by adding the symbol 'o' on the optional argument's type. For instance:

Intransitive verbs (smile, unaccusative open):

$$
\mathrm{np} \rightarrow \mathrm{s}
$$

Transitive verbs with obligatory object (praise, unergative open):

$$
\mathrm{np} \rightarrow(\mathrm{np} \rightarrow \mathrm{s})
$$

Transitive verbs with optional object (read):

$$
\mathrm{np}^{\circ} \rightarrow(\mathrm{np} \rightarrow \mathrm{s})
$$

Passive forms of transitive verbs (was read, was praised, was opened): ${ }^{7}$

$$
\mathrm{np}_{\text {by }}^{\circ} \rightarrow(\mathrm{np} \rightarrow \mathrm{s})
$$

Option types are simple cases of sum types in functional programming [7]. There are two ways of filling in an optional slot of a function: by providing an argument of the appropriate type, or by using a universal filler, marked ' $*$ '. Let $F$ be a function of type $\mathrm{a} \rightarrow \mathrm{b}$, with an obligatory argument slot of abstract type a. To make this slot into an optional one, an option operator distinguishes the case where the argument is present from usages of the filler. For the second case, we use a default result. Formally, an object $x$ of type $a^{\circ}$ is either of type a or the $*$-filler (disjoint from a's domain). Let $d$ be a default result of type $\mathrm{b}$. The option operator of type $\left(\mathrm{a}^{\circ} \times(\mathrm{a} \rightarrow \mathrm{b}) \times \mathrm{b}\right) \rightarrow \mathrm{b}$ is defined by:

$\operatorname{option}(x, F, d)=d$ if $x=*$; otherwise option $(x, F, d)=F(x)$.

The function $\lambda x \cdot \operatorname{option}(x, F, d)$ is therefore of type $\mathrm{a}^{\circ} \rightarrow \mathrm{b}$.

In ACG, the connection between the morpho-syntactic level and the semantic level is handled by mapping each simple abstract type (np, s etc.) to a morphosyntactic type and a semantic type. We assume the simple morpho-syntactic type $f$ for strings and the simple semantic types $e$ and $t$ for entities and truth-values respectively. We assume the following mappings of the simple abstract type $\mathrm{np}$ and $\mathrm{s}$ to morpho-syntactic and semantic types:

$$
\begin{aligned}
& \mathrm{np} \mapsto\langle f, e\rangle: \text { a noun phrase surfaces as a string and denotes an entity } \\
& \mathrm{s} \mapsto\langle f, t\rangle \quad \text { : a sentence surfaces as a string and denotes a truth-value }
\end{aligned}
$$

Complex types like $\mathrm{np} \rightarrow \mathrm{s}$ (for intransitive verbs) or $\mathrm{np} \rightarrow(\mathrm{np} \rightarrow \mathrm{s}$ ) (for transitive verbs) are inductively mapped to the corresponding morpho-syntactic and semantic types according to the following rule.

Let a be an abstract type that is mapped to the morpho-syntactic type $a_{1}$ and the semantic type $a_{2}$. Let $\mathrm{b}$ be an abstract type that is mapped to the morpho-

tion is properly treated, we believe that Dekker's approach can be extended to an approach that is very similar to our account of optional arguments - see especially Dekker's remarks on this issue on [3, p.573].

7 The abstract type $\mathrm{np}_{\mathrm{by}}$ is used for by phrases in passive constructions, and is needed for morpho-syntactic reasons alone (see section 5). The semantics of this type for the purposes of this paper is treated as equivalent to the semantics of $n p$ types. 
syntactic type $b_{1}$ and the semantic type $b_{2}$. Then $\mathrm{a} \rightarrow \mathrm{b}$ is an abstract type that is mapped to the morpho-syntactic type $a_{1} \rightarrow b_{1}$ and the semantic type $a_{2} \rightarrow b_{2}$. Likewise, $\mathrm{a}^{\circ}$ is an abstract type that is mapped to the morpho-syntactic type $a_{1}^{o}$ and the semantic type $a_{2}^{o}$.

In short we denote:

$$
\begin{aligned}
& \mathrm{a} \mapsto\left\langle a_{1}, a_{2}\right\rangle \\
& \mathrm{b} \mapsto\left\langle b_{1}, b_{2}\right\rangle \\
& (\mathrm{a} \rightarrow \mathrm{b}) \mapsto\left\langle a_{1} b_{1}, a_{2} b_{2}\right\rangle \\
& \mathrm{a}^{\mathrm{o}} \mapsto\left\langle a_{1}^{o}, a_{2}^{o}\right\rangle
\end{aligned}
$$

For instance:

$$
(\mathrm{np} \rightarrow \mathrm{s}) \mapsto\langle f f, e t\rangle
$$

: an intransitive verb surfaces as a function from strings to strings, and denotes a function from entities to truth-values

$$
(\mathrm{np} \rightarrow(\mathrm{np} \rightarrow \mathrm{s})) \mapsto\langle f(f f), e(e t)\rangle
$$

: a transitive verb surfaces as a function from strings to functions from strings to strings, and denotes a function from entities to functions from entities to truth-values

Specifically, we assume the entry SMILE for the intransitive verb smile:

SMILE $=\lambda s_{f} . s \bullet$ smiled $_{f}:$ smile $_{e t}$

In words: the morpho-syntactic entry for the verb smile is the function sending every subject string $s$ to the corresponding sentence string composed of $s$ and the string smiled; the corresponding semantic function is the function smile from entities to truth-values.

Similarly, we assume the following ACG entry PRAISE for the transitive verb praise:

PRAISE $=\lambda o_{f} \cdot \lambda s_{f} . s \bullet$ praised $_{f} \bullet o:$ praise $_{e(e t)}$

In words: the morpho-syntactic entry for the verb praise is the function sending every object string $o$ to the function mapping every subject string $s$ to the corresponding sentence string composed of $o, s$ and the string praised; the corresponding semantic function is the function praise from entities to functions from entities to truth-values.

Simple sentences like John smiled and John praised Lolita are analyzed at the abstract level as SMILE(JOHN) and PRAISE(LOLITA)(JOHN), respectively. Using the verbal entries above and the treatment of names as entity-denoting strings, these sentences are associated with the following strings and truth-values:

$\operatorname{SMILE}(\mathrm{JOHN})=$ John $\bullet$ smiled $: \operatorname{smile}(\mathbf{j o h n})$

$\operatorname{PRAISE}(\operatorname{LOLITA})(\mathrm{JOHN})=$ John $\bullet$ praised $\bullet$ Lolita $:$ praise $($ lolita $)($ john $)$

Using option types in ACG we extend this simple treatment to transitive verbs with an optional object. For instance, we assume the following ACG entry READ for the transitive verb read, of abstract type $\mathrm{np}^{\circ} \rightarrow(\mathrm{np} \rightarrow \mathrm{s})$ : 


$$
\begin{aligned}
\operatorname{READ}= & \lambda o_{f} \cdot \lambda s_{f} \cdot \operatorname{option}\left(o, \lambda u_{f} . s \bullet \operatorname{read}_{f} \bullet u, s \bullet \operatorname{read}\right): \\
& \lambda o_{e} \cdot \lambda s_{e} \cdot \operatorname{option}\left(o, \lambda u_{e} \cdot \operatorname{read}_{e(e t)}(u)(s), \exists x \cdot \operatorname{read}(x)(s)\right)
\end{aligned}
$$

In words: the morpho-syntactic entry for the verb read uses the object string $o$ in the object position if it is given, and leaves the object position null if the object argument is not given; the corresponding semantic function uses the object entity $o$ as the first argument of the binary function read, and existentially saturates the position if the object argument is not given.

In this way, the sentences John read and John read Lolita are associated with the following strings and truth-values:

$\operatorname{READ}(\mathrm{JOHN})=\operatorname{John} \bullet \mathrm{read}: \exists x \cdot \operatorname{read}(x)(\mathbf{j o h n})$

$\operatorname{READ}(\mathrm{LOLITA})(\mathrm{JOHN})=$ John $\bullet$ read $\bullet$ Lolita $: \operatorname{read}($ lolita $)($ john $)$

The treatment of passives is analogous. For instance, the passive form was praised (by) is analyzed as follows.

$$
\begin{aligned}
\operatorname{PRAISE}_{\text {pass }}= & \lambda o_{f} \cdot \lambda s_{f} \cdot \operatorname{option}\left(o, \lambda u_{f} \cdot s \bullet \text { was-praised }_{f} \bullet u, s \bullet \text { was-praised }_{f}\right): \\
& \lambda o_{e} \cdot \lambda s_{e} \cdot \mathbf{o p t i o n}\left(o, \lambda u_{e} \cdot \text { praise }_{e(e t)}(s)(u), \exists x \cdot \text { praise }(s)(x)\right)
\end{aligned}
$$

The sentences Lolita was praised and Lolita was praised by John are associated with the following strings and truth-values (for the definition of BY see Table 1):

PRAISE $_{\text {pass }}($ LOLITA $)=$ Lolita $\bullet$ was-praised $: \exists x$.praise $($ lolita $)(x)$

$\operatorname{PRAISE}_{\text {pass }}(\mathrm{BY}(\mathrm{JOHN}))($ LOLITA $)=$ Lolita $\bullet$ was-praised $\bullet$ by $\bullet$ John $:$ praise $($ lolita $)($ john $)$

\section{Some exceptional cases}

There are some exceptional kinds of UO verbs and passive verbs that do not give rise to existential entailments when the object or by phrase is missing. Consider the following examples.

(6) a. John kicked $\stackrel{?}{\Rightarrow}$ John kicked something.

b. John bit $\stackrel{?}{\Rightarrow}$ John bit something.

(7) a. Mary was left alone $\stackrel{?}{\Rightarrow}$ Someone left Mary alone. [2].

b. The traps were avoided $\stackrel{?}{\Rightarrow}$ Someone avoided the traps. [11]

Similar cases were used by Carlson [2] to argue for event-oriented modification by objects and by phrases. Carlson assumes that since existence entailments do not appear with some such cases, this motivates an ontology-based account of the existence entailments that as a rule appear with these constructions. In addition to the reasons we gave in section 2 for doubting Carlson's general approach to UOs and passives, it should be remarked that lexical UOs like kick and bite are rather rare. ${ }^{8}$ Because of their rarity, the UO verb alternations

\footnotetext{
${ }^{8}$ In her extensive typology of verb alternations in English, Levin [12] characterizes verbs such as kick or bite as showing "characteristic property alternations". This is because of sentences like our horse kicks or our dog bites regularly, which indicate a tendency towards kicking or biting. Levin does not mention simple past tense sentences as in (6), where transitive verbs without objects give rise to episodic readings.
} 
that such transitive verbs show in English may be a result of an accidental lexical ambiguity/polysemy, rather than a general process, as Carlson assumes. With passives as in (7), it is even more unclear whether there is any need to assume a general non-existential strategy for passives. Note that both sentences in (7) involve a negative component for the verbal element (alone $=$ with no one, avoid $=$ not get close to). Arguably, the non-existential effect is due to an existential reading of the agent argument which is in the scope of these negative components. Thus, examples such as (7) do not provide evidence for "non-existential passives".

\section{A general strategy of 'optionalization'}

So far in this paper we have exemplified specific entries of various option types and the way they are interpreted. We would now like to define a general procedure of 'optionalization'. Thus, given an ACG lexicon $\mathcal{L}$ without option types, we would like to transform $\mathcal{L}$ into an lexicon $O P T(\mathcal{L})$ with option types. This is done using a specification of some of the arguments of entries in $\mathcal{L}$ as optional arguments and using this specification for systematically optionalizing $\mathcal{L}$.

For example, consider the optionality-free toy ACG verbal lexicon in Table 1. ${ }^{9}$ This lexicon contains the verbal forms discussed in section 3 , but without any treatment of optionality: all arguments are treated as obligatory, against common linguistic judgements about the verbal arguments that are underlined in Table 1. Our general method transforms such a description of verbal entries into a proper ACG lexicon with types for optional arguments and the option operator in entry values as required by their types. This general method guarantees an economical representation for the two features common to all the optional arguments treated: no effect of the presence or lack of an optional argument on the form of the verb; and narrow scope existential quantification over the semantic slot in the verb's meaning.

Our general procedure for mapping such an option-free lexicon $\mathcal{L}$ to the corresponding optionalized lexicon is based on mapping a option-free entry of abstract type $\underline{\mathrm{a}} \rightarrow \mathrm{b}$, where the a argument is specified as desirably optional, to a lexicon entry of abstract type $a^{\circ} \rightarrow b$. For instance, the entry READ in Table 1 does not respect the optionality of the verb's object argument. However, the procedure below will guarantee that the underlined argument in the type $\underline{\mathrm{np}} \rightarrow \mathrm{np} \rightarrow \mathrm{s}$ will be properly treated as optional in the resulting lexicon, with an entry for the same verb of type $\mathrm{np}^{\circ} \rightarrow \mathrm{np} \rightarrow \mathrm{s}$.

Let $\varphi=\varphi_{1}: \varphi_{2}$ be a lexical entry of abstract type $\mathrm{a} \rightarrow \mathrm{b}$, where the argument type $a$ is defined as desirably optional. Such entries are marked $\underline{a} \rightarrow b$ in Table 1. In order to define optionalization inductively, we assume that the abstract types $a$ and $b$ have no underlined sub-parts. To capture correctly the behavior of optional arguments, we assume further that these types satisfy the following restrictions:

\footnotetext{
9 To achieve a more conspicuous notation we avoid parentheses in this table, reading the type notation $\alpha \rightarrow \beta \rightarrow \gamma$ with right-association as in $\alpha \rightarrow(\beta \rightarrow \gamma)$.
} 


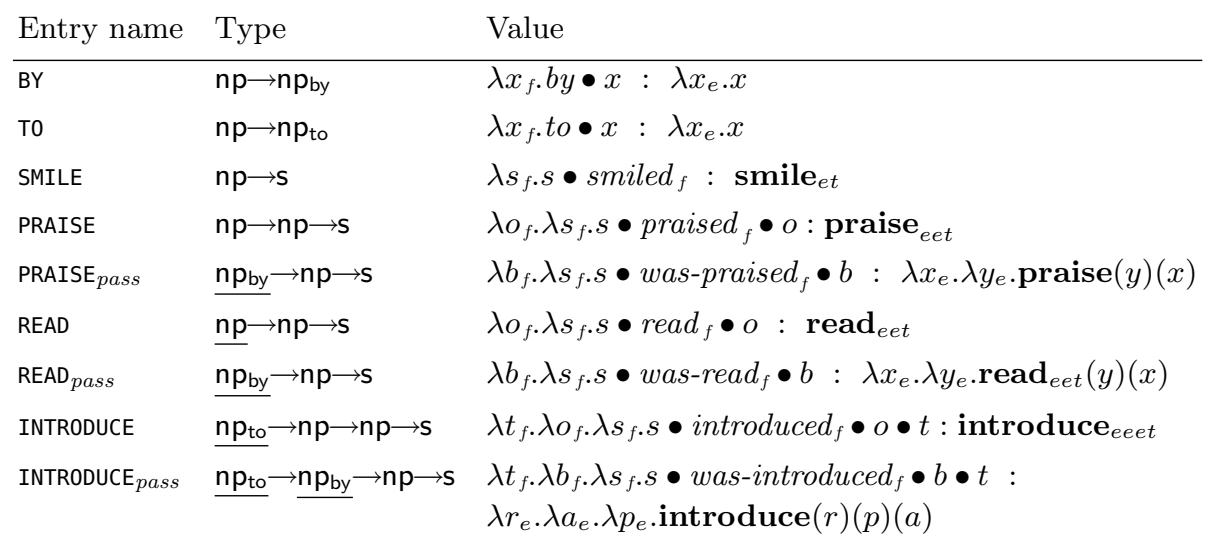

Table 1. Toy ACG lexicon without option types

- The concrete morpho-syntactic type of a is string: $\mathrm{a} \mapsto\left\langle f, \tau_{\mathrm{a}}\right\rangle$.

- The concrete semantic type of $b$ is boolean: $\mathrm{b} \mapsto\left\langle\sigma_{\mathrm{b}}, \tau_{\mathrm{b}}\right\rangle$, where $\tau_{\mathrm{b}}$ is a boolean type. ${ }^{10}$

The 'string' requirement is needed to allow filling in optional slots by the empty string; the 'boolean' requirement is needed to allow existential quantification over morpho-syntactically empty slots.

Given these assumptions on the type of the entry $\varphi$, we define the corresponding optionalized entry opt $(\varphi)=\varphi_{1}^{\prime}: \varphi_{2}^{\prime}$ of type $\mathrm{a}^{\circ} \rightarrow \mathrm{b}$ as follows:

$$
\begin{aligned}
& \varphi_{1}^{\prime}=\lambda x_{f^{\circ}} \text {.option }\left(x, \varphi_{1}, \varphi_{1}(\epsilon)\right) \\
& \varphi_{2}^{\prime}=\lambda x_{\tau_{\mathrm{a}} o} . \text { option }\left(x, \varphi_{2}, C L O S\left(\varphi_{2}\right)\right)
\end{aligned}
$$

The operator CLOS existentially saturates the first argument of $\varphi_{2}$, which is inductively defined as follows:

$$
\text { If } \tau_{\mathrm{b}}=t \text { then } \operatorname{CLOS}\left(\varphi_{2}\right)=\exists x_{\tau_{\mathrm{a}}} \cdot \varphi_{2}(x) \text {. }
$$

Otherwise, let $\tau_{\mathbf{b}}=\tau_{1} \tau_{2}$, where $\tau_{2}$ is boolean by induction. We define:

$$
\operatorname{CLOS}\left(\varphi_{2}\right)=\lambda y_{\tau_{1}} . \operatorname{CLOS}\left(\lambda x_{\tau_{\mathrm{a}}} \cdot \varphi_{2}(x)(y)\right) \text {. }
$$

For instance, for the constant read of type $e(e t)$, we have:

$$
C L O S(\text { read })=\lambda y_{e} \cdot C L O S\left(\lambda x_{e} \cdot \operatorname{read}(x)(y)\right)=\lambda y \cdot \exists x \cdot \operatorname{read}(x)(y)
$$

As a result, the optionalized version of the entry for READ in Table 1 is opt (READ) $=$ $\varphi_{1}^{\prime}: \varphi_{2}^{\prime}$ s.t.:

$$
\begin{aligned}
\varphi_{1}^{\prime} & =\lambda x f_{f^{o} .} . \operatorname{pption}\left(x, \lambda o_{f} \cdot \lambda s_{f} . s \bullet \operatorname{read}_{f} \bullet o, \lambda s_{f} . s \bullet \operatorname{read}_{f} \bullet \epsilon\right) \\
\varphi_{2}^{\prime} & =\lambda x_{e^{o}} . \operatorname{option}\left(x, \operatorname{read}_{e e t}, C L O S\left(\operatorname{read}_{e e t}\right)\right) \\
& =\lambda x_{e^{o}} . \operatorname{option}\left(x, \operatorname{read}_{e e t}, \lambda y \cdot \exists x^{\prime} \cdot \operatorname{read}\left(x^{\prime}\right)(y)\right)
\end{aligned}
$$

The definition of the opt operator is not yet sufficient in order to optionalize entries of abstract type $a \rightarrow b$ where $b$ has underlined types in it. This is necessary, as illustrated by the entry INTRODUCE pass in Table 1, representing the form and meaning of the verbal passive be introduced (by $X$ to $Y$ ), which has two optional

${ }^{10}$ Standardly, we define the set of 'boolean' semantic types as the smallest set of semantic types that contains $t$ and any type $\tau_{1} \tau_{2}$ where $\tau_{2}$ is boolean. 
arguments. To allow optionalization of such entries as well, let $\varphi=\varphi_{1}: \varphi_{2}$ be a lexical entry of a abstract type $X$. The type $X$ may be primitive. In case $\mathrm{X}=\mathrm{a} \rightarrow \mathrm{b}$, we adopt the same assumptions above on a (string morpho-syntactic type) and $b$ (boolean semantic type), but now $b$ possibly has underlined types in it. Given these assumptions on the type $\mathrm{X}$, we inductively define the optionalized entry $\operatorname{OPT}(\varphi)$ as follows:

If $\mathrm{X}$ is primitive, then the entry $\operatorname{OPT}(\varphi)=\varphi$.

If $X=\mathrm{a} \rightarrow \mathrm{b}$ (the argument a should not be optionalized), then the optionalized entry is inductively defined by $\operatorname{OPT}(\varphi)=\lambda x_{\mathrm{a}} \cdot \operatorname{OPT}(\varphi(x))$.

If $X=\underline{a} \rightarrow \mathrm{b}$ (the argument a should be optionalized), then the optionalized entry is inductively defined by $\operatorname{opt}\left(\lambda x_{\mathrm{a}} \cdot \operatorname{OPT}(\varphi(x))\right)$.

For instance, applying OPT to the option-free entry INTRODUCE ${ }_{\text {pass }}$ in Table 1 , of type $\underline{\mathrm{np}_{\mathrm{to}}} \rightarrow \mathrm{np}_{\text {by }} \rightarrow \mathrm{np} \rightarrow \mathrm{s}$ (first two arguments are underscored as desirably optional), results in:

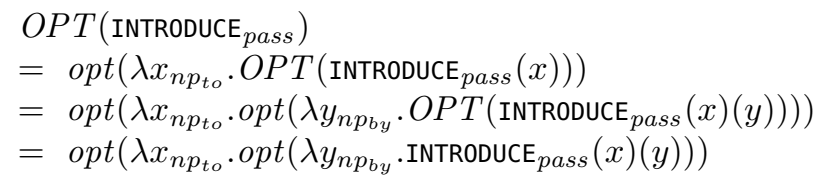

In the morpho-syntactic level, this amounts to:

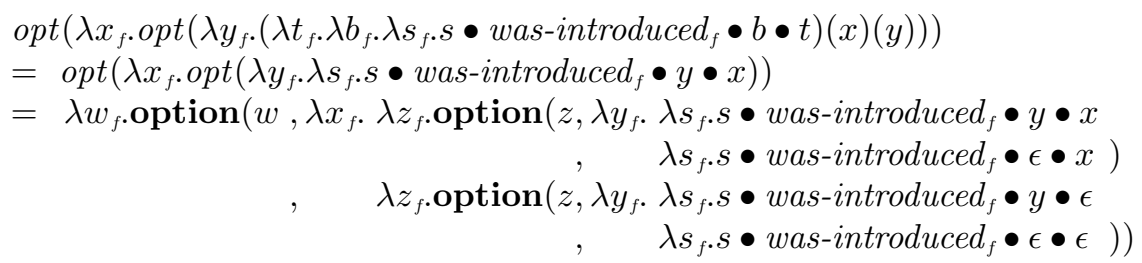

This covers the four combinations of the two present/missing morpho-syntactic arguments.

Similarly, in the semantic level we have:

$$
\begin{aligned}
\operatorname{opt}\left(\lambda x . \operatorname{opt}\left(\lambda y_{e} \cdot\left(\lambda r_{e} \cdot \lambda a_{e} \cdot \lambda p_{e} \cdot \text { introduce }_{e(e(e t))}(r)(p)(a)\right)(x)(y)\right)\right) \\
=\operatorname{opt}\left(\lambda x _ { e } \cdot \lambda z \cdot \operatorname { o p t i o n } \left(z, \lambda y_{e} \cdot \lambda p_{e} \cdot \text { introduce }_{e(e(e t))}(x)(p)(y)\right.\right. \\
\left.\left., C L O S\left(\lambda y_{e} \cdot \lambda p_{e} \cdot \text {.introduce }_{e(e(e t))}(x)(p)(y)\right)\right)\right)
\end{aligned}
$$

And applying $C L O S$ gives:

$$
\begin{aligned}
& \operatorname{opt}\left(\lambda x_{e} \cdot \lambda z . \operatorname{option}\left(z, \lambda y_{e} \cdot \lambda p_{e} . \text { introduce }_{e(e(e t))}(x)(p)(y)\right.\right. \\
& \left.\left., \lambda p_{e} \cdot \exists y_{e} . \text { introduce }_{e(e(e t))}(x)(p)(y)\right)\right)
\end{aligned}
$$

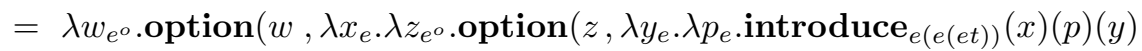

$$
\begin{aligned}
& \left., \lambda p_{e} \cdot \exists y_{e} . \text { introduce }_{e(e(e t))}(x)(p)(y)\right) \\
& , \lambda z_{e^{o}} . \exists x_{e} \cdot \operatorname{option}\left(z, \lambda y_{e} \cdot \lambda p_{e} \cdot \text { introduce }_{e(e(e t))}(x)(p)(y)\right. \\
& \left.\left., \lambda p_{e} \cdot \exists y_{e} . \text { introduce }_{e(e(e t))}(x)(p)(y)\right)\right)
\end{aligned}
$$

This covers the four combinations of the two present/existentially-closed semantic arguments. The same procedure also correctly derives the other specific entries discussed in section 3 . 


\section{Conclusions}

Optionality in the argument structure of verbs is a well-known phenomenon that is treated in one way or another by most syntactic theories. We started out by pointing out the narrow-scope behavior of existential quantifiers over empty argument slots, which, although familiar, is not treated systematically by syntactic frameworks we are aware of. The behavior of unaccusative verbs in contrast to their passive forms has led us to conclude that by phrases in passives should be treated similarly to optional arguments, and not using event-modifiers as in some versions of event semantics. The Optional Argument Principle aims to describe the relations between the syntactic optionality of arguments and their semantic properties. Within the framework of Abstract Categorial Grammar (ACG), we introduced a standard interpretation of option types, which enabled us to describe a general transformation of grammars without argument optionality to grammars that encode it in a uniform way.

Acknowledgements: We thank Marijana Marelj for helpful discussions. The work was partially supported by a Van Gogh grant to the 2nd and 3rd author by the FrenchDutch Academy. The work of the 3rd author was partially supported by a VICI grant number 277-80-002 by the Netherlands Organisation for Scientific Research (NWO). The work of the 4 th author was supported by grant number 360-70-340 by the Netherlands Organisation for Scientific Research (NWO).

\section{References}

1. Bach, E.: In defense of passive. Linguistics and Philosophy 3, 297-341 (1980)

2. Carlson, G.: Thematic roles and their role in semantic interpretation. Linguistics 22, 259-279 (1984)

3. Dekker, P.: Existential disclosure. Linguistics and Philosophy 16, 561-588 (1993)

4. Dowty, D.: Grammatical relations and Montague Grammar. In: Jacobson, P., Pullum, G. (eds.) The Nature of Syntactic Representation. Kluwer, Dordrecht (1982)

5. Fodor, J.A., Fodor, J.D.: Functional structure, quantifiers, and meaning postulates. Linguistic Inquiry 11, 759-770 (1980)

6. de Groote, P.: Towards abstract categorial grammars. In: Proceedings of the 39th annual meeting of the Association for Computational Linguistics (ACL) (2001)

7. Gunter, C.: Semantics of programming languages: structures and techniques. The MIT Press (1992)

8. Landau, I.: The explicit syntax of implicit arguments. Linguistic Inquiry 41, 357$388(2010)$

9. Landman, F.: Events and Plurality: the Jerusalem lectures. Kluwer, Dordrecht (2000)

10. Larson, R.K.: Events and modification in nominals. In: Proceedings of Semantics and Linguistic Theory, SALT8. pp. 145-168 (1998)

11. Lasersohn, P.: Lexical distributivity and implicit arguments. In: Proceedings of Semantics and Linguistic Theory, SALT3 (1993)

12. Levin, B.: English Verb Classes and Alternations. The University of Chicago Press, Chicago (1993)

13. Muskens, R.: Language, Lambdas, and Logic. In: Kruijff, G.J., Oehrle, R. (eds.) Resource Sensitivity in Binding and Anaphora, pp. 23-54. Studies in Linguistics and Philosophy, Kluwer (2003) 


\title{
Each vs. jeweils: A cover-based view on distance-distributivity
}

\author{
Lucas Champollion* \\ Eberhard Karls Universität Tübingen \\ Nauklerstr. 35 \\ 72074 Tübingen, Germany \\ champoll@gmail.com
}

\begin{abstract}
Zimmermann (2002) identifies two kinds of distance-distributive items. The first kind (e.g. each) is restricted to distribution over individuals; the second kind (e.g. German jeweils) can also be interpreted as distributing over salient occasions. I explain this behavior by formally relating this split to the two distributivity operators proposed in the work of Link (atomic D operator) and Schwarzschild (cover-based operator), which I reformulate in a Neo-Davidsonian event-based framework.
\end{abstract}

\section{Introduction}

Across languages, distributive items have different syntactic uses and different meanings. In English, each can be used in three essentially synonymous ways:

(1) a. Adnominal: The children saw two monkeys each.

b. Adverbial: The children each saw two monkeys.

c. Determiner: Each child saw two monkeys.

There are many terms for these three uses. Adnominal each is also called binominal or shifted; adverbial each is also called floated; and determiner each is also called prenominal. I will call adnominal and adverbial each distance-distributive items (DD items).

In German, adnominal and adverbial each are translated by one word, jeweils. Determiner each is translated by another one, jed-. I gloss DD items as DIST since, as we will see, they have a wider range of readings than each.

(2) a. Adnominal: Die Kinder haben [jeweils [zwei Affen]] gesehen. The children have DisT two monkeys seen.

b. Adverbial: Die Kinder haben [jeweils [zwei Affen gesehen]]. The children have DisT two monkeys seen.

c. Determiner: Jedes Kind hat zwei Affen gesehen. Each.sg.n child has two monkeys seen.

\footnotetext{
^ I am grateful to Anna Szabolcsi and to the audience of the 2011 Stuttgart workshop on quantification for helpful discussion.
} 
Though adverbial and adnominal jeweils take the same surface position in (2a) and $(2 \mathrm{~b})$, they can be teased apart syntactically, as shown in Zimmermann (2002). However, this distinction will play no role in this paper.

\section{Crosslinguistic Variation}

Zimmermann (2002) classifies about a dozen languages depending on whether the DD item can also function as a distributive determiner, as in English, or not, as in German. Across these languages, he observes that DD items which can also be used as determiners (e.g. each) always distribute over individuals, as determiners do. In contrast, many of those DD items which are formally distinct from determiners (e.g. jeweils) can also distribute over salient occasions (that is, chunks of time or space).

The best way to illustrate Zimmermann's generalization is to start by considering languages like German and Telugu, a Dravidian language. Both have DD items which look different from the distributive determiner. These DD items can distribute over individuals, but also (unlike in English) over spatial or temporal occasions, as long as context provides a salient set of such occasions. I call this the occasion reading. ${ }^{1}$

The following examples illustrate this pattern. Sentences (3) and (4) are ambiguous between a reading that distributes over individuals - the ones of which their plural subject consists, (3a)-(4a) - and one that distributes over occasions (3b)-(4b). While the former reading is always available, the latter requires a supporting context. That is, when (3) and (4) are uttered out of the blue, they only have the readings (3a)-(4a), while the readings (3b)-(4b) are only available in contexts where there is a previously mentioned or otherwise salient set of occasions, such as contexts in which the children have been to the zoo on several previous occasions.

(3) Die Kinder haben jeweils zwei Affen gesehen. (German)

The children have DisT two monkeys seen.

a. 'Each of the children has seen two monkeys.'

${ }^{1}$ The occasion reading corresponds to what Balusu (2005) calls the spatial key and temporal key readings. I leave open the question of whether the spatial and temporal cases should be distinguished as two separate readings. Another term for it is eventdistributive reading (Oh, 2001). Zimmermann (2002) uses the term adverbial reading for it. This term is misleading, because it suggests that only the adverbial use of jeweils can give rise to this reading. But as documented in Chapter 5 of Zimmermann (2002), adnominal jeweils can give rise to it as well. For example, in (i), jeweils is part of the subject DP and is therefore adnominal. However, as shown by the paraphrase, it distributes over occasions, not over individuals.

(i) Jeweils zwei Jungen standen Wache.

DisT two boys stood watch.

'Each time, two boys kept watch.' 
b. 'The children have seen two monkeys each time.'

(4) pilla-lu1 renDu renDu kootu-lu-ni cuus-ee-ru. (Telugu) kid-Pl two two monkey-Pl-Acc see-Past-3PPl.

a. 'Each of the children has seen two monkeys.'

b. 'The children have seen two monkeys on each occasion.'

Sentences (5) and (6) have singular subjects and only have an occasion reading. ${ }^{2}$ These sentences are odd out of the blue and require supporting context in the same way as readings (3b) and (4b) do. The Telugu sentences (4) and (6) are taken from Balusu (2005), who uses the terms participant key readings for distribution over individuals.

(5) Hans hat jeweils zwei Affen gesehen. (German)

Hans has Dist two monkeys seen.

'Hans has seen two monkeys on each occasion.'

(6) Raamu renDu renDu kootu-lu-ni cuus-ee-Du. (Telugu)

Ram two two monkey-Pl-Acc see-Past-3PSg.

'Ram has seen two monkeys on each occasion.'

While DD items in German and Telugu allow distribution both over individuals and over salient occasions, this is not the case for all DD items crosslinguistically. In many languages, adnominal DD items can only distribute over individuals. One example is English, where (7) can only mean (7a), not (7b).

(7) The children have seen two monkeys each.

a. Available: 'Each of the children has seen two monkeys.'

b. Unavailable: 'The children have seen two monkeys on each occasion.'

When adnominal each is used in a sentence like (8), whose subject is singular, distribution over individuals is not possible. Even with supporting context, no occasion reading surfaces, and the sentence as a whole is unacceptable.

(8) *John has seen two monkeys each.

Why does English each lack the occasion reading? We have seen in Section 1 that each also differs from jeweils in that only the former can also be used as a determiner. Zimmermann (2002) looks at a range of languages - French, Dutch, Norwegian, Icelandic, Italian, and Russian - in which an adnominal DD item can also be used as a determiner, and finds that the DD item lacks the occasion reading in all these languages. ${ }^{3}$ Based on this, he postulates a generalization which can be put as follows:

\footnotetext{
${ }^{2}$ Their other reading would involve vacuous distribution over only one individual and is presumably blocked through the Gricean maxim of manner "Be brief".

3 The French case is somewhat controversial. Adnominal chacun and determiner/adnominal chaque are not exactly identical, but Zimmermann (2002) argues (p. 44) that they are historically related and can still be considered formally identical.
} 
(9) Zimmermann's generalization: If a DD item can also be used as a distributive determiner, it lacks the occasion reading.

There are many languages besides German and Telugu whose DD items have occasion readings and cannot be used as determiners. Examples are Czech, Bulgarian, Korean, and Romanian (Zimmermann, 2002). However, the generalization goes only one way (the "if" cannot be strengthened to "if and only if"). In Japanese, the DD item sorezore cannot be used as a determiner and does not have an occasion reading (see Zimmermann (2002) for discussion). In Hungarian, distance-distributivity is expressed by reduplication (this is a common pattern across languages, see Gil (1982)), and determiners cannot be reduplicated. But unlike in Telugu, reduplication in Hungarian does not allow the occasion reading (Szabolcsi, 2010):
A gyerekek két-két majmot láttak. (Hungarian)
The children two-two monkey.acc saw.3pl
a. Available: 'Each of the children saw two monkeys.'
b. Unavailable: 'The children saw two monkeys on each occasion.'

The following requirements for a semantic analysis of distance-distributivity emerge. First, the synonymy of the determiner, adnominal and adverbial uses of each in English should be captured, ideally by essentially identical lexical entries. Second, the fact that DD items across all languages share some part of their semantics (namely the individual-distributive readings) should be represented, as well as the fact that some of them can additionally have occasion readings. Third, the analysis should clarify the connections between DD items and distributivity theory. Finally, there should be a way to capture the correlation expressed in (9). I now propose an analysis that fulfills these requirements. Section 3 presents distributivity operators; Section 4 relates them to DD items.

\section{Distributivity Operators in Event Semantics}

The following analysis is placed in the context of the general theory of distributivity developed in the work of Link (1987) and Schwarzschild (1996) on distributivity operators. Link postulates a silent VP-level operator that shifts a VP to a distributive interpretation, i.e. one that holds of any individual whose atomic parts each satisfy the unshifted VP. This so-called D operator is defined as in (11). The variable $x$ is resolved to a plural entity, the subject, and $y$ ranges over its atomic parts, i.e. the singular individuals of which it consists.

$$
\llbracket \mathrm{D} \rrbracket=\lambda P_{\langle e t\rangle} \lambda x \forall y[y \leq x \wedge \operatorname{Atom}(y) \rightarrow P(y)]
$$

The optional presence of the $\mathrm{D}$ operator derives the ambiguity between distributive and scopeless readings. For example, (12a) represents a scopeless reading and $(12 \mathrm{~b})$ a distributive reading. I use the term "scopeless" to refer both to collective and cumulative readings. The distinction between these two readings does not matter for this paper. See Landman (2000) for discussion. 
(12) a. The children saw two monkeys.

$\approx$ The children between them saw two monkeys. scopeless

b. The children D saw two monkeys.

$\approx$ The children each saw two monkeys.

distributive

I propose that DD items should be essentially thought of as versions of this D operator (cf. Link (1986) for a similar claim for German je, a short form of jeweils which lacks the occasion reading). Clearly, Link's D operator and each are similar, as can be seen from the paraphrase of (12b). I take adverbial each and similar DD items (e.g. Hungarian reduplication) to be D operators. As for DD items like jeweils and Balusu reduplication, we have seen that in special contexts, they can distribute over spatial and temporal intervals - arguably nonatomic entities. Link's D operator always distributes down to individual atoms and can therefore not be extended to these cases.

However, Schwarzschild (1996) argues on independent grounds that the D operator should be modified to allow for "nonatomic distributive" interpretations in a limited set of circumstances, namely whenever there is a particularly salient way to divide a plural individual. A good example of what Schwarzschild has in mind is provided by Lasersohn (1998). Shoes typically come in pairs, so a sentence like The shoes cost $\$ 50$ can be interpreted as saying that each pair of shoes (rather than each shoe) costs $\$ 50$. To model this kind of example, Schwarzschild modifies D and makes it anaphoric to a salient cover (a partition of a plural individual that allows overlap). C, the "cover variable", is free and anaphoric on the context. Schwarzschild assumes that $\mathrm{C}$ is a cover of the entire universe of discourse, but for most purposes one can instead think of $\mathrm{C}$ as a cover or a partition of the sum individual in question. In this case, $\mathrm{C}$ partitions the sum of shoes into pairs. Schwarzschild refers to his own version of the $\mathrm{D}$ operator as Part.

$$
\llbracket \text { Part }_{C} \rrbracket=\lambda P_{\langle e t\rangle} \lambda x \forall y[y \leq x \wedge \mathrm{C}(y) \rightarrow P(y)]
$$

This operator optionally applies to a VP and shifts it to a nonatomic distributive reading, as follows:

$$
\text { The shoes Part cost } \$ 50 \text {. }
$$

$\approx$ Each salient set of shoes costs $\$ 50$.

nonatomic distributive

It is of course possible to think of $\mathrm{D}$ as a special case of Part, namely the one that results when the variable $\mathrm{C}$ is resolved to the predicate Atom. However, I assume that both D and Part are present in the grammar. This assumption will allow us to capture the distinction between each and jeweils. The former corresponds to D and the latter corresponds to Part. This accounts for the fact that jeweils and its crosslinguistic relatives across languages has a wider range of readings than each does.

In count domains, distributivity over atoms is expected to be salient in almost all contexts and to obscure the presence of nonatomic distributive readings (Schwarzschild, 1996). It is therefore useful to look for nonatomic VP-level dis- 
tributivity in a noncount domain, such as time (Champollion, 2010). Here we find once again that the readings in question are available given appropriate contextual information or world knowledge. Example (15) is based on observations in Moltmann (1991). It is odd out of the blue because pills cannot be taken repeatedly, but it is acceptable in a context where the patient's daily intake is discussed. Example (16) is from Deo and Piñango (2011), and is acceptable because it is clear that snowmen are typically built in winter.

(15) The patient took two pills for a month and then went back to one pill.

(16) We built a huge snowman in our front yard for several years.

Since for-adverbials are otherwise not able to cause indefinites to covary (Zucchi and White, 2001), and since Part is dependent on a salient level of granularity just like (15) and (16) are, it is plausible to assume that a temporal version of Part is responsible for the distributive interpretation of these sentences (Champollion, 2010). The meaning of this temporal version can be paraphrased as daily in (15) and yearly in (16).

The original formulations of the operators in (11) and (13) can only "target" (i.e. distribute over parts of) the subject. Examples like (15) and (16) motivate a reformulation of the operators that allows them to target different thematic roles, including time. I will represent the relationship between $\mathrm{D}$ and the thematic role it targets through coindexation. For evidence that this relationship can be nonlocal, see Champollion (2010). This will allow us to capture the fact that DD items can also target different thematic roles (Zimmermann, 2002). For example, (17) can either involve two stories per boy or two stories per girl, depending on which noun phrase is targeted by each.

(17) The boys told the girls two stories each.

In the following, I assume a Neo-Davidsonian system loosely based on Carlson (1984) and Krifka (1989). Events, verbs and thematic roles are each assumed to be closed under sum formation. Verbs and their projections are all of type $v t$ (event predicates). Here is a sample entry of a verb.

$$
\llbracket \operatorname{see} \rrbracket=\lambda e{ }^{*} \operatorname{see}(e)
$$

This entry includes the star operator from Link (1983) as a reminder that the predicate is closed under sum formation. The star operator maps a set $P$ to the predicate that applies to any sum of things of which $P$ holds. It can be easily generalized to $n$-ary predicates and functions.

Noun phrases can be interpreted in situ (we will not need quantifier raising). Silent thematic role heads, which denote functions of type $\langle v e\rangle$ (event to individual), are located between noun phrases and verbal projections. I will often omit them in the LFs for clarity. The precise nature of the compositional process is not essential, but it affects the types of the lexical entries of DD items so let me make it concrete. I assume that the following type shifter applies first to the thematic role head, then to the noun phrase, and finally to the verbal projection. 


$$
\text { Type shifter: } \lambda \theta_{\langle v e\rangle} \lambda P_{\langle e t\rangle} \lambda V_{\langle v t\rangle} \lambda e[V(e) \wedge P(\theta(e))]
$$

This type shifter assumes that the noun phrase is of predicative type $\langle e t\rangle$, which is appropriate for indefinites. Definites are first shifted from type $e$ to type $\langle e t\rangle$ by the standard ident type shifter $(\lambda x \lambda y[y=x])$ so that (19) can apply. The type shifter (19) combines the noun phrase with its thematic role head and forms an event predicate modifier (type $\langle v t, v t\rangle)$. For example, after the noun phrases the children and two monkeys combine via (19) with the thematic roles $a g$ and th respectively, their denotation is as follows. I write $\bigoplus$ child for the sum of all children. I will write $2 M$ as a shorthand for $\lambda e\left[\left|{ }^{*} \operatorname{th}(e)\right|=2 \wedge{ }^{*} \operatorname{monkey}\left({ }^{*} \operatorname{th}(e)\right)\right]$.

$$
\begin{aligned}
& \llbracket[\text { agent }[\text { the children }]] \rrbracket=\lambda V \lambda e\left[V(e) \wedge{ }^{*} \operatorname{ag}(e)=\bigoplus \text { child }\right] \\
& \llbracket[\text { theme }[\text { two monkeys }]] \rrbracket=\lambda V \lambda e\left[V(e) \wedge 2 \mathrm{M}\left({ }^{*} \operatorname{th}(e)\right)\right]
\end{aligned}
$$

After the verb has combined with all its arguments, the event variable can be existentially bound if the sentence is uttered out of the blue. (If the sentence is understood with reference to a specific event, the event variable can instead be resolved to that event.) If the noun phrases combine directly with the verb, we get a scopeless reading:

$$
\llbracket \text { The children saw two monkeys } \rrbracket=\exists e\left[{ }^{*} \operatorname{ag}(e)=\bigoplus \operatorname{child} \wedge 2 \mathrm{M}\left({ }^{*} \operatorname{th}(e)\right)\right]
$$

To get a distributive reading, we use Link's D operator. Since VPs are event predicates, VP-level operators must be reformulated as event predicate modifiers. As described above, I assume that the $\mathrm{D}$ operator is coindexed with a thematic role $\theta$, its target. My entry is as follows: ${ }^{4}$

$$
\llbracket \mathrm{D}_{\theta} \rrbracket=\lambda V_{\langle v t\rangle} \lambda e\left[e \in{ }^{*} \lambda e^{\prime}\left[V\left(e^{\prime}\right) \wedge \operatorname{Atom}\left(\theta\left(e^{\prime}\right)\right)\right]\right]^{5}
$$

As an example, the distributive reading of (22) is derived like this:

$$
\begin{aligned}
& \llbracket \text { The children } D_{a g}[\text { saw two monkeys } \rrbracket \\
& \left.=\exists e e^{*} \operatorname{ag}(e)=\bigoplus \text { child } \wedge e \in\left[\llbracket D a g \rrbracket\left(\lambda e^{\prime}\left[{ }^{*} \operatorname{see}\left(e^{\prime}\right) \wedge 2 \mathrm{M}\left({ }^{*} \operatorname{th}\left(e^{\prime}\right)\right)\right]\right)\right]\right] \\
& \left.=\exists e e^{*} \operatorname{ag}(e)=\bigoplus \operatorname{child} \wedge e \in{ }^{*} \lambda e^{\prime}\left[{ }^{*} \operatorname{see}\left(e^{\prime}\right) \wedge 2 \mathrm{M}\left({ }^{*} \operatorname{th}\left(e^{\prime}\right)\right) \wedge \operatorname{Atom}\left(\operatorname{ag}\left(e^{\prime}\right)\right)\right]\right]
\end{aligned}
$$

This formula is true iff there is an event $e$ whose agent is the children, and which consists of seeing-two-monkeys events whose agents are atomic. Remember that events and thematic roles are closed under sum, so $e$ can be a plural event with a plural agent. The formula does not explicitly state that the seeing-two-monkeys events have children as agents. However, this fact is entailed by the assumption that thematic roles are closed under sum formation together with the assumption that the entities in the denotation of singular count nouns like child are atoms. Specifically, the existentially quantified event can only have the children as its agent if it consists of events whose individual agents are children.

\footnotetext{
${ }^{4}$ This definition is taken from Champollion (2010), except that PureAtom has been changed to Atom. This change is immaterial because we do not use impure atoms.

${ }^{5}$ This is not the only way to reformulate the D operator. See Lasersohn (1998) and Dotlačil (2011) for other proposals.
} 


\section{Each and Jeweils as Distributivity Operators}

Adverbial each is a VP modifier and can therefore be given the same entry as the D operator in (23). Adnominal and determiner each need to be type-shifted but both are defined in terms of $(23)$ :

(25) Adverbial: $\llbracket \operatorname{each}_{\theta} \rrbracket=\llbracket D_{\theta} \rrbracket=(23)$

(26) Adnominal: $\llbracket \operatorname{each}_{\theta} \rrbracket=\lambda M_{\langle v t, v t\rangle} \lambda V_{\langle v t\rangle} \lambda e\left[\llbracket D_{\theta} \rrbracket(M(V))(e)\right]$

$$
\text { Determiner: } \llbracket \text { each } \rrbracket=\lambda P_{\langle e t\rangle} \lambda \theta_{\langle v e\rangle} \lambda V_{\langle v t\rangle} \lambda e\left[\theta(e)=\bigoplus P \wedge \llbracket D_{\theta} \rrbracket(V)(e)\right]
$$

Adnominal each combines with a thematic-role-carrying noun phrase like (21). Determiner each combines first with a nominal and then with a theta role head. It is not coindexed with anything because it is not a DD item. In both cases, the result is a phrase of VP modifier type $\langle v t, v t\rangle$, which is also the type of $D_{\theta}$. Some intermediate steps of the derivations of (1) are shown in (28) and (29).

$$
\begin{aligned}
& \llbracket[[[\text { two monkeys }] \text { theme }] \text { each } a g] \rrbracket \\
& =\lambda V_{\langle v t\rangle} \lambda e\left[e \in\left[\llbracket D_{a g} \rrbracket\left(\lambda e^{\prime}\left[V\left(e^{\prime}\right) \wedge 2 \mathrm{M}\left({ }^{*} \operatorname{th}\left(e^{\prime}\right)\right)\right]\right)\right]\right] \\
& =\lambda V_{\langle v t\rangle} \lambda e\left[e \in{ }^{*} \lambda e^{\prime}\left[{ }^{*} \operatorname{see}\left(e^{\prime}\right) \wedge 2 \mathrm{M}\left({ }^{*} \operatorname{th}\left(e^{\prime}\right)\right) \wedge \operatorname{Atom}\left(\operatorname{ag}\left(e^{\prime}\right)\right]\right]\right. \\
& \llbracket[[\text { Each child }] \operatorname{ag}] \rrbracket \\
& \left.=\lambda V_{\langle v t\rangle} \lambda e{ }^{*} \operatorname{ag}(e)=\bigoplus \operatorname{child} \wedge \llbracket D_{a g} \rrbracket(V)(e)\right] \\
& \left.=\lambda V_{\langle v t\rangle} \lambda e^{*} \operatorname{ag}(e)=\bigoplus \operatorname{child} \wedge e \in^{*} \lambda e^{\prime}\left[V\left(e^{\prime}\right) \wedge \operatorname{Atom}\left(\operatorname{ag}\left(e^{\prime}\right)\right)\right]\right]
\end{aligned}
$$

The result of these derivations is always the same, which reflects their synonymy:

$$
\begin{aligned}
& \llbracket \text { The children each } a g \text { saw two monkeys } \rrbracket \\
& =\llbracket \text { The children saw two monkeys each } a g \rrbracket \\
& =\llbracket \text { Each child saw two monkeys } \rrbracket \\
& =(24)=\llbracket \text { The children } D_{a g} \text { saw two monkeys } \rrbracket
\end{aligned}
$$

We now come to the event-based reformulation of the Part operator. We obtain it by replacing Atom in (23) with a free variable C, which is assumed to be anaphoric on the context:

$$
\llbracket \operatorname{Part}_{\theta, C} \rrbracket=\lambda P_{\langle v t\rangle} \lambda e\left[e \in^{*} \lambda e^{\prime}\left[P\left(e^{\prime}\right) \wedge C\left(\theta\left(e^{\prime}\right)\right)\right]\right]
$$

Part takes an event predicate $P$ and returns a predicate that holds of any event $e$ which can be divided into events that are in $P$ and whose $\theta$ s satisfy the contextually salient predicate $\mathrm{C}$. Note that the definition of (31) entails that $\mathrm{C}$ is a cover over the value that $\theta$ maps $e$ to. (31) is also the lexical entry of adverbial jeweils. The same type shift as in (33) brings us from (31) to adnominal jeweils:

$$
\begin{aligned}
& \text { Adverbial: «jeweils } \theta, C \rrbracket=\llbracket \operatorname{Part}_{\theta, C} \rrbracket=(31) \\
& \text { Adnominal: } \llbracket \text { jeweils }_{\theta, C} \rrbracket=\lambda M_{\langle v t, v t\rangle} \lambda V_{\langle v t\rangle} \lambda e\left[\llbracket \operatorname{Part}_{\theta, C} \rrbracket(M(V))(e)\right]
\end{aligned}
$$

As in the case of the Part operator, the $\mathrm{C}$ parameter can be set to Atom so long as $\theta$ is set to a function whose range is a count domain, such as agent. In that case, jeweils distributes over individuals and is equivalent to each. 


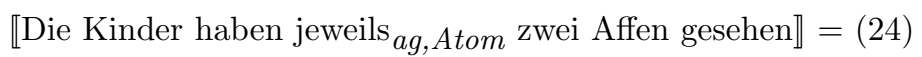

If - and only if - there is a supporting context, the anaphoric predicate $C$ can be set to a salient antecedent, and in that case $\theta$ is free to adopt values like $\tau$ (runtime). This leads to occasion readings:

$$
\begin{aligned}
& \llbracket \text { Die Kinder haben jeweils } \tau_{,}, C \text { zwei Affen gesehen } \rrbracket= \\
& { }^{*} \operatorname{ag}\left(e_{0}\right)=\bigoplus \text { child } \wedge e_{0} \in{ }^{*} \lambda e^{\prime}\left[{ }^{*} \operatorname{see}\left(e^{\prime}\right) \wedge 2 \mathrm{M}\left({ }^{*} \operatorname{th}\left(e^{\prime}\right)\right) \wedge \mathrm{C}\left(\tau\left(e^{\prime}\right)\right)\right]
\end{aligned}
$$

Suppose for example that (35) is uttered in a context where the children have been to the zoo three times - last Monday, last Wednesday and last Friday. I assume that the predicate that is true of these three times is salient in this context. I also assume that (35) is interpreted as referring to the sum of these three events, call it $e_{0}$. I have indicated this by resolving the event variable in (35) to $e_{0}$ rather than existentially quantifying over it. Runtime is closed under sum just like other thematic roles (that is, it is a sum homomorphism - see Krifka (1989)). So the runtime $\tau\left(e_{0}\right)$ of this event is the (discontinuous) sum consisting of last Monday, last Wednesday, and last Friday. Now (35) asserts that $e_{0}$ has the children as its agents; that it can be divided into subevents, each of whose runtimes is either last Monday, last Wednesday, or last Friday; and that these subevents are seeing-two-monkeys events. This is the occasion reading.

\section{Summary and Discussion}

The preceding analysis has captured the semantic similarities between DD items across languages, as well as their variation, by relating them to distributivity operators. A given DD item can be given the same lexical entry up to typeshifting regardless of its syntactic position. The parameters provided by our reformulation of the D and Part operators capture the semantic variation: DD items like each and Hungarian reduplication are hard-coded for distribution over atoms, which blocks distributivity over a noncount domain like time. DD items like jeweils and Telugu reduplication can distribute over noncount domains, but only if they can pick up salient nonatomic covers from context.

The remaining question is how to capture the correlation expressed in Zimmermann's Generalization (9). That is to say, why does a DD item which can also be used as a distributive determiner lack the occasion reading? Zimmermann himself proposes a syntactic explanation: Determiners must syntactically agree with their complement; DD each has a proform as complement, which acquires its agreement features from its antecedent, the target of each; only overt targets have agreement features. Alternatively, a semantic explanation seems plausible: Distributive determiners like English each are only compatible with count nominals (each boy, *each mud). Formally, this amounts to an atomicity requirement of the kind the D operator provides. This atomicity requirement can be seen as independent evidence of the atomic distributivity hard-coded in the entry (27) via the $\mathrm{D}$ operator (23). In other words, the DD item each inherits its atomicity requirement. Both types of explanations are compatible with this framework. 


\section{Bibliography}

Balusu, R. (2005). Distributive reduplication in Telugu. In Davis, C., Deal, A. R., and Zabbal, Y., editors, Proceedings of NELS 36.

Carlson, G. N. (1984). Thematic roles and their role in semantic interpretation. Linguistics, 22:259-279.

Champollion, L. (2010). Parts of a whole: Distributivity as a bridge between aspect and measurement. $\mathrm{PhD}$ thesis, University of Pennsylvania, Philadelphia.

Deo, A. and Piñango, M. M. (2011). Quantification and context in measure adverbs. In Ashton, N., Chereches, A., and Lutz, D., editors, Proceedings of SALT 21, pages 295-312.

Dotlačil, J. (2011). Fastidious distributivity. In Ashton, N., Chereches, A., and Lutz, D., editors, Proceedings of SALT 21, pages 313-332.

Gil, D. (1982). Distributive numerals. PhD thesis, University of California, Los Angeles.

Krifka, M. (1989). Nominal reference, temporal constitution and quantification in event semantics. In Bartsch, R., van Benthem, J., and van Emde Boas, P., editors, Semantics and contextual expression, pages 75-115. Foris, Dordrecht.

Landman, F. (2000). Events and plurality: The Jerusalem lectures. Kluwer, Dordrecht.

Lasersohn, P. (1998). Generalized distributivity operators. Linguistics and Philosophy, 21(1):83-93.

Link, G. (1983). The logical analysis of plurals and mass terms: A latticetheoretical approach. In Bäuerle, R., Schwarze, C., and von Stechow, A., editors, Meaning, use and interpretation of language, pages 303-323. de Gruyter, Berlin.

Link, G. (1986). Je drei Äpfel - three apples each: Quantification and the German 'je'. In Link (1998), pages 117-132.

Link, G. (1987). Generalized quantifiers and plurals. In Gärdenfors, P., editor, Generalized Quantifiers: Linguistic and Logical Approaches, pages 151-180. Reidel, Dordrecht.

Link, G. (1998). Algebraic semantics in language and philosophy. CSLI.

Moltmann, F. (1991). Measure adverbials. Linguistics and Philosophy, 14:629660.

Oh, S.-R. (2001). Distributivity in an event semantics. In Hastings, R., Jackson, B., and Zvolensky, Z., editors, Proceedings of SALT 11, pages 326-345. CLC Publications, Ithaca.

Schwarzschild, R. (1996). Pluralities. Kluwer, Dordrecht, Netherlands.

Szabolcsi, A. (2010). Quantification. Research Surveys in Linguistics. Cambridge University Press.

Zimmermann, M. (2002). Boys buying two sausages each: On the syntax and semantics of distance-distributivity. PhD thesis, University of Amsterdam.

Zucchi, S. and White, M. (2001). Twigs, sequences and the temporal constitution of predicates. Linguistics and Philosophy, 24:187-222. 


\title{
Cross-categorial donkeys
}

\author{
Simon Charlow (simon. charlow@nyu.edu) \\ Department of Linguistics, New York University
}

\begin{abstract}
Data from surprising sloppy readings of verb phrase ellipsis constructions argue that ellipsis sites can partially or totally consist of dynamically bound pronouns. I give an account, integrating Muskens' [11] CDRT with a focus-based theory of ellipsis and deaccenting (Rooth $[14,15])$. The result is essentially a hybrid of Hardt [6] and Schwarz [19] but manages greater empirical coverage than either.
\end{abstract}

\section{Surprisingly sloppy}

An influential theory of verb phrase (VP) ellipsis has it that elided VPs (' $\varepsilon$ ') and their antecedents (' $\alpha$ ') must share an interpretation/LF (Keenan [8]; Sag [17]; Williams [25]). If pronouns have bound and referential uses, this correctly predicts that (1a) is ambiguous between a strict reading (Chris thinks Simon is smart: $\llbracket \alpha \rrbracket^{g}, \llbracket \varepsilon \rrbracket^{g} \equiv \lambda x . x$ thinks Simon is smart) and a sloppy reading (Chris thinks Chris is smart: $\llbracket \alpha \rrbracket^{g}, \llbracket \varepsilon \rrbracket^{g} \equiv \lambda x . x$ thinks $x$ is smart). But requiring $\alpha$ and $\varepsilon$ to mean the same thing, though appealing, turns out to be too restrictive. Sloppy pronouns are sometimes bound only outside of $\varepsilon$ (cf. 1b) and sometimes lack a c-commanding antecedent altogether (cf. 1c).

(1) (a) Simon $\left[{ }_{\alpha}\right.$ thinks he's smart], and CHRIS $_{F}$ does $\varepsilon$ too.

(b) $\left[\mathrm{S}_{\alpha}\right.$ Bagels $\lambda_{i}$ I ${ }_{\alpha}$ like $\left.\left.t_{i}\right]\right]$

$\left[\mathrm{S}_{\varepsilon}\right.$ DONUTS $\lambda_{\mathrm{F}} \lambda_{j}$ I DON'T $_{\mathrm{F}}\left[\varepsilon\right.$ like $\left.\left.t_{j}\right]\right]$

(Evans [4])

(c) $\left[\mathrm{S}_{\alpha}\right.$ the cop who arrested $\mathrm{John}_{i}\left[{ }_{\alpha}\right.$ insulted $\left.\left.\operatorname{him}_{i}\right]\right]$

$\left[\mathrm{s}_{\varepsilon}\right.$ the cop who arrested BILL,$j$ DIDN'T $\mathrm{T}_{\mathrm{F}}\left[\varepsilon\right.$ insult him $\left.\left.{ }_{j}\right]\right]$ (Wescoat [24])

To deal with cases like (1b), Rooth [14] proposes a two-part theory of ellipsis: (i) $\alpha$ and $\varepsilon$ must be syntactically identical, but only up to variable names (and $F$ marks). (ii) A node dominating $\varepsilon$ is must also CONTRAST with a node dominating $\alpha$, in the sense of Definition $1{ }^{1}$ (CONTRAST prevents rank over-generation: "John likes him, and BILL does too" can't mean John likes Steve, and Bill likes Bill.)

Definition 1. CONTRAst $(\phi, \psi)$ at $g$ iff: $\llbracket \phi \rrbracket^{g} \neq \llbracket \psi \rrbracket^{g}$, and $\llbracket \psi \rrbracket^{g} \in\langle\phi\rangle^{g}$, with $\langle\cdot \cdot\rangle^{g}$ the standard Roothian (1985) function into focus sets:

. Focus values for non-F-marked terminals: $\langle\phi \phi\rangle^{g}=\left\{\llbracket \phi \rrbracket^{g}\right\}$

. Focus values for F-marked nodes: $\left\langle\phi_{\mathrm{F}}\right\rangle^{g}=\left\{x: x_{\tau(\phi)}\right\}$

- For any non-F-marked branching node $\phi$ dominating $\gamma$ and $\delta$, if $\llbracket \gamma \rrbracket^{g}\left(\llbracket \delta \rrbracket^{g}\right)$ is defined, $\left\langle\langle\phi\rangle^{g}=\left\{c(d): c \in\left\langle\langle\gamma\rangle^{g} \wedge d \in\left\langle\langle\delta\rangle^{g}\right\}\right.\right.\right.$

This accounts for (1b) $-\alpha$ and $\varepsilon$ are structurally identical modulo indices, and $\llbracket \mathrm{S}_{\alpha} \rrbracket^{g} \in\left\langle\left\langle\mathrm{S}_{\varepsilon}\right\rangle^{g}=\left\{f(\mathrm{I}\right.\right.$ like $\left.x): x_{e}, f_{t t}\right\}$ —but not for (1c). While, clearly, (1c)'s $\alpha$

\footnotetext{
${ }^{1}$ An idealization. Relations besides CONTRAST are often apt (Asher \& Lascarides [1]).
} 
and $\varepsilon$ are identical up to indices, no choice of any two nodes satisfies CONTRAST: since him $_{j}$ isn't c-commanded by a co-indexed expression, it must - assuming Reinhart's [12] view of the syntax-semantics interface, anyway-be interpreted referentially. This entails that, e.g., $\left\langle\left\langle\mathrm{S}_{\varepsilon}\right\rangle^{g}=\{\right.$ the cop who arrested $x$ insulted Bill: $\left.x_{e}\right\} . \llbracket \mathrm{S}_{\alpha} \rrbracket^{g}$ is not in this set. CONTRAST fails.

Yet CONTRAST must be satisfiable! Sentence (2) has a reading entailing that, for all $x$ other than Bill, I didn't say that the cop who arrested $x$ insulted $x$ (Tomioka [22]). Given a standard semantics for only (Definition 2), some LF for (2)'s S-node, call it ' $\mathcal{L}$ ', must be such that $\langle\mathcal{L}\rangle^{g}=\{$ the cop who arrested $x$ insulted $\left.x: x_{e}\right\}$. But $\mathcal{L}$ must also be available as an LF for (1c)'s $\mathrm{S}_{\varepsilon}$, the elliptical variant of S. Since (1c)'s $\llbracket \mathrm{S}_{\alpha} \rrbracket \in\langle\mathcal{L}\rangle^{g}$, CONTRAST must be satisfiable, after all.

(2) I only heard that $\left[\mathrm{S}\right.$ the cop who arrested $\mathrm{BILL}_{\mathrm{F}, i}$ insulted $\left.\operatorname{him}_{i}\right]$ !

Definition 2. $\llbracket$ only $\mathrm{VP} \rrbracket^{g}=\lambda x: \llbracket \mathrm{VP} \rrbracket^{g}(x) . \forall Q \in\left\langle\langle\mathrm{VP}\rangle^{g} \cdot Q(x) \rightarrow Q=\llbracket \mathrm{VP} \rrbracket^{g}\right.$

Sloppy elliptical VPs. Surprising sloppiness is cross-categorial. Sentence (3) can mean that when John has to clean, he doesn't want to clean (Hardt [6]; Schwarz [19]). But treating ellipsis as simple non-pronunciation of LF material yields the LFs in (3b), where $\alpha_{2}$ and $\varepsilon_{2}$ aren't even identical up to indices! Nor is CONTRAST satisfiable; (3b)'s $\mathrm{S}_{\varepsilon}$, for example, is associated with the focus set $\left\langle\mathrm{S}_{\varepsilon}\right\rangle^{g}=\left\{\right.$ if John has to $P, f$ (he wants to clean) $\left.: P_{e t}, f_{t t}\right\}$.

(3) (a) If John has to cook, he doesn't wANT to. If he has to CLEAN, he doesn't either.

(b) $\left[\mathrm{S}_{\alpha}\right.$ if John has to $\left[\alpha_{1}\right.$ cook] he doesn't $\left[\alpha_{2} \text { [WANT to }\right]_{\mathrm{F}}\left[\varepsilon_{1}\right.$ cook] $\left.]\right]$ $\left[\mathrm{S}_{\varepsilon}\right.$ if he has to $\left[\alpha_{3} \mathrm{CLEAN}_{\mathrm{F}}\right]$ he doesn't $\left[\varepsilon_{2}\right.$ want to $\left[\varepsilon_{3}\right.$ clean $\left.\left.]\right]\right]$

Yet, like (2), "I only heard that if John has to CLEAN he doesn't want to" has a covarying reading - such that for all $P_{\text {et }}$ other than clean, I didn't hear that if John has to $P$, he doesn't want to $P$ (cf. related claims in Kratzer [9]). So again there is reason to believe that, potentially, (3b)'s $\llbracket \mathrm{S}_{\alpha} \rrbracket^{g} \in \llbracket \mathrm{S}_{\varepsilon} \rrbracket^{g}$. But, again, how?

The scoping theory. Schwarz [19] argues that (a) syntactic binding (i.e. with LF c-command) underlies all sloppy readings, and (b) elided VPs are sometimes (but not always) null variable pro-VPs (' $P_{n}$ '). Moreover, he suggests, VPs can QR to positions of sentential scope. So, according to Schwarz, LFs like (4) underlie the sloppy reading of (3) (analogous LFs can be mooted for (1c) and (3)).

(4) $\left[\mathrm{S}_{\alpha} \operatorname{cook} \lambda_{1}\right.$ [if John has to $t_{1}$ he doesn't [ $\left.\left.\left.{ }_{\alpha}[\text { WANT to }]_{\mathrm{F}} P_{1}\right]\right]\right]$

$\left[\mathrm{S}_{\varepsilon}\right.$ CLEAN $_{\mathrm{F}} \lambda_{2}$ [if he has to $t_{2}$ he doesn't [ $\varepsilon$ want to $\left.\left.\left.P_{2}\right]\right]\right]$

Here, $\alpha$ and $\varepsilon$ are identical up to indices and F-marks. Moreover, $\llbracket \mathrm{S}_{\alpha} \rrbracket \in\left\langle\left\langle\mathrm{S}_{\varepsilon}\right\rangle=\{\right.$ if John has to $P$, he wants to $\left.P: P_{e t}, f_{t t}\right\}$. So the sloppy reading is generated.

But there are issues. (i) The proforms in (4) get bound from an $\bar{A}$-position, something Reinhart [12] deems possible only for traces. (ii) The account requires covert movement out of scope/extraction islands - including asymmetric QR out of conjunctions (against the Coordinate Structure Constraint), cf. (5). (iii) To explain (5b), NPs - not subject to overt movement - must QR, again across potentially unbounded distances (Elbourne [3]). (iv) Pro-VPs, if instantiated as variables, lack internal syntax; so it should be impossible to extract out of them, inconsistent with the grammatical sloppy reading of (5c) (Tomioka [23]). 
(5) (a) If I'm stressed and John says something awful, I get mad at him. If I'm stressed and BILL does, I DON'T.

(b) If you lose your visa, you get another. If you lose your PASSPORT, you DON'T.

(c) I bought everything I was SUPPOSED to and SOLD everything I WASN'T.

But most troubling for the scoping theory is that it fails to generate the correspondence reading of constructions like (6) - the one entailing that Sue waves to whoever Mary does (Rooth \& Partee [16]; Stone [20]). Just as no amount of QR gives donkey truth conditions for sentences like "if someone knocked, she $_{i}$ left", no amount of QR yields the correspondence reading of sentences like (6).

(6) If Mary waves to John or Bill, then SuE does too.

Hardt's dynamic theory. Hardt [6] gives a dynamic account of surprising sloppy readings using Muskens's [11] Compositional DRT (CDRT). I'll postpone the details of CDRT until the following section. For now, it suffices to note that Hardt assigns (3) the LF in (7), with $P_{n}$, as before, a phonologically null pro-VP.

(7) if $\mathrm{John}^{1}$ has to $\operatorname{cook}^{*, 2}$ he doesn't [want to $\left.P_{*}\right]^{3}$

if he ${ }_{1}$ has to clean*,4 he doesn't $P_{3}$

Superscripted indices correspond to the introduction of a discourse referent (dref); subscripted items denote previously introduced drefs. There is a dedicated index '*' which Hardt allows to be overwritten and dubs the "center". Informally, in (7) both $\alpha$ and $\varepsilon$ denote the property of wanting to $\sigma$, with $\sigma$ the current value of the center. Since clean $^{*}, 4$ overwrites $*$ with clean (roughly), $P_{3}$ evaluates to the property of wanting to clean, and the sloppy reading is derived.

Like the theory we began with, Hardt requires semantic identity of $\alpha$ and $\varepsilon$. This is why destructive update is crucial: for surprising sloppy configurations, it seems like the only way, in a dynamic theory, for $\alpha$ and $\varepsilon$ to denote identical properties! But this creates problems. For one, Hardt is forced to posit two indices on items $U$ that update the center. The reason: though $*$ may subsequently be overwritten, this shouldn't preclude subsequent "ellipsis" of $U$. But even with this complication, problems remain. As Sauerland [18] points out, there can be multiple surprising sloppy things of a single type (cf. 8, after Sauerland's ex. 10; NB: the indexing here merely indicates the intended reading). So Hardt's theory actually needs, in principle, an infinity of rewritable indices $*_{1}, *_{2}$, etc.

(8) When a $\operatorname{woman}_{i}$ buys a blouse $_{j}$ we $\left[{ }_{\alpha}\right.$ ask that she $_{i}$ try it ${ }_{j}$ on]

When a MAN $_{k}$ buys a $\operatorname{SHIRT}_{l}$ we DON'T [ $\varepsilon_{\varepsilon}$ ask that he $e_{k}$ try it on] $_{l}$

Hardt's account also makes heavy use of structure-less pro-VPs (again, this is difficult to square with extraction cases like (5c)) and lacks an account of correspondence readings (though one could be added). But the biggest issue with the theory is that focus is not implicated (remember, Hardt simply requires $\alpha$ and $\varepsilon$ to mean the same thing). There's at least two problems with this: (i) A story about surprising sloppy readings should also have something to say about surprising covarying association-with-focus readings. (ii) Sentences like John likes 
his mom, and Bill does too, but Sam doesn't utterly lack a reading on which Bill likes John's mom, and Sam likes Sam's mom ('strict-sloppy') (Fiengo \& May [5]). But Hardt generates that reading straightaway with the LF in (9a). Similarly, Mary's dad thinks she's smart, and Sue does too lacks a sloppy reading (Bos [2]). And again, Hardt over-generates with the LF in (9b).

(9) (a) John*,1 [likes his ${ }_{*}$ mom ${ }^{2}$. Bill ${ }^{3}$ does $P_{2}$ too. Sam*,4 doesn't $P_{2}$.

(b) Mary*,1's dad [thinks she ${ }_{*}$ is smart] ${ }^{2}$, and Sue*,3 does $_{2}$ too.

The unavailability of these readings falls out of a CONTRAST-based theory. In the first case, the strict reading of the Bill-clause corresponds to a proposition (viz. that Bill likes John's mom) not in the focus set associated with the sloppy reading of the Sam-clause - viz. $\left\{x\right.$ likes $x$ 's mom $\left.: x_{e}\right\}$. So if CONTRAST is operative here, the strict-sloppy reading is predicted bad. Likewise for the second example: the proposition that Mary's dad thinks Mary is smart isn't in $\{x$ thinks $x$ is smart $\left.: x_{e}\right\}$ (cf. also Bos [2]). So CONTRAst rules out that sloppy reading, as well.

Summing up. Schwarz's account of surprising sloppy readings incorporates focus but relies on an ad hoc variant of $\mathrm{QR}$ and fails to explain correspondence readings. Hardt's solution is dynamic and avoids these worries. But his reliance on semantic identity and (thus) destructive update in lieu of a focus-based theory means his account needs an infinity of rewritable indices and struggles with over-generation. Both theories have a paucity of structure at or inside ellipsis sites, making it difficult to see how extraction happens. What we need is a theory that references CONTRAST (or something like it), achieves covariation across focus alternatives despite a lack of syntactic binding, and allows "extraction out of" surprisingly sloppy items. I sketch such a theory in the next section.

\section{A theory}

The fragment. Following Hardt [6], I adopt a higher-order variant of Muskens' [11] Compositional DRT (CDRT). The underlying system is classical type logic with three primitive types: $e, t$, and $s$ (for 'states'). DRT boxes are syntactic sugar for $\lambda$-terms encoding the usual dynamic relations on states, as follows:

Definition 3. From DRT conditions to type logic formulae:

- $R_{\tau_{1} \rightarrow \ldots \rightarrow \tau_{n} \rightarrow t}\left(\alpha_{s \rightarrow \tau_{1}}\right) \ldots\left(\Omega_{s \rightarrow \tau_{n}}\right) \rightsquigarrow \lambda i . R(\alpha(i)) \ldots(\Omega(i))$

· $K \Rightarrow K^{\prime} \rightsquigarrow \lambda i . \forall j . K(i)(j) \rightarrow \exists k . K^{\prime}(j)(k)$

- $\neg K \rightsquigarrow \lambda i . \neg \exists j . K(i)(j)$

- $\alpha=\beta \rightsquigarrow \lambda i . \alpha(i)=\beta(i)$ or

$\alpha=\beta \rightsquigarrow \lambda i .(\lambda \hat{x} . \alpha(\hat{x})(i)=\lambda \hat{x} \cdot \beta(\hat{x})(i))$, whichever's defined ('=' only defined for 2 arguments of identical types; $\hat{x}$ a possibly empty sequence of arguments). ${ }^{2}$

Definition 4. Box sequencing (relational composition):

. $K ; K^{\prime} \rightsquigarrow \lambda i j . \exists k . K(i)(k) \wedge K^{\prime}(k)(j)$

Definition 5. Interpretation of boxes:

$\cdot\left[\nu_{1} \ldots \nu_{m} \mid \kappa_{1}, \ldots, \kappa_{n}\right] \rightsquigarrow \lambda i j . i\left[\nu_{1}, \ldots, \nu_{m}\right] j \wedge \kappa_{1}(j) \wedge \ldots \wedge \kappa_{n}(j)$

- $i\left[\nu_{1}, \ldots, \nu_{m}\right] j$ iff $i$ and $j$ differ at most in the values they assign to $1, \ldots, m$.

\footnotetext{
${ }^{2}$ The baroqueness here is due to variable dynamic properties (Definition 8).
} 
Definition 6. Merging lemma (ML): if $\nu_{1}^{\prime}, \ldots, \nu_{m}^{\prime}$ do not occur free in $\kappa_{1}, \ldots, \kappa_{l}$ : $\cdot\left[\nu_{1} \ldots \nu_{k} \mid \kappa_{1}, \ldots, \kappa_{l}\right] ;\left[\nu_{1}^{\prime} \ldots \nu_{m}^{\prime} \mid \kappa_{1}^{\prime}, \ldots, \kappa_{n}^{\prime}\right]=\left[\nu_{1} \ldots \nu_{k} \nu_{1}^{\prime} \ldots \nu_{m}^{\prime} \mid \kappa_{1}, \ldots, \kappa_{l}, \kappa_{1}^{\prime}, \ldots, \kappa_{n}^{\prime}\right]$

Definition 7. Truth in CDRT:

- $K$ is true at $i\left({ }^{(} \operatorname{True}_{i}(K)^{\prime}\right)$ iff $\exists j . K(i)(j) . K$ is true simpliciter iff $\forall i \exists j . K(i)(j)$.

I add two pieces to Muskens' basic system (call the extension 'CDRT' ${ }^{+}$). The first is the notion of a variable dynamic property - a box parametrized both to the usual arguments and incoming states (Hardt [6], Stone \& Hardt [21]). The second is a (externally) dynamic entry for disjunction - relational union, i.e. an instance of generalized disjunction (Rooth \& Partee [16]). Variable dynamic properties are an important part of the account of surprising sloppy readings. Dynamic disjunction is crucial for the account of correspondence readings (Stone [20]).

Definition 8. Variable dynamic properties:

- For any $\nu_{n}$ of type $s \rightarrow \tau_{1} \rightarrow \ldots \rightarrow \tau_{m} \rightarrow s \rightarrow s \rightarrow t$, and any (possibly empty) sequence of arguments $\hat{x}$ of length $m: \nu_{n}(\hat{x})=\lambda i j . \nu_{n}(i)(\hat{x})(i)(j)$

Definition 9. Box disjunction (externally dynamic):

- $K \sqcup K^{\prime} \rightsquigarrow \lambda i j . K(i)(j) \vee K^{\prime}(i)(j)$

Table 1. $\mathrm{CDRT}^{+}$fragment

\begin{tabular}{|c|c|c|}
\hline Expression(s) & Translation & Type \\
\hline $\mathrm{a}^{n}$ & $\lambda P Q \cdot\left[u_{n} \mid\right] ; P\left(u_{n}\right) ; Q\left(u_{n}\right)$ & $(e t)(e t) t$ \\
\hline the $_{n}$ & $\lambda P Q . P\left(u_{n}\right) ; Q\left(u_{n}\right)$ & $(e t)(e t) t$ \\
\hline another $_{m}^{n}$ & $\lambda P Q \cdot\left[u_{n} \mid u_{n} \neq u_{m}\right] ; P\left(u_{n}\right) ; Q\left(u_{n}\right)$ & $(e t)(e t) t$ \\
\hline every $^{n}$ & $\lambda P Q .\left[\mid\left(\left[u_{n} \mid\right] ; P\left(u_{n}\right)\right) \Rightarrow Q\left(u_{n}\right)\right]$ & $(e t)(e t) t$ \\
\hline $\mathrm{John}^{n}$ & $\lambda P .\left[u_{n} \mid u_{n}=\right.$ john $] ; P\left(u_{n}\right)$ & $(e t) t$ \\
\hline man & $\lambda v \cdot[\mid \operatorname{man}(v)]$ & et \\
\hline met & $\lambda \mathcal{Q} v \cdot \mathcal{Q}\left(\lambda v^{\prime} \cdot\left[\mid \operatorname{met}\left(v^{\prime}\right)(v)\right]\right)$ & $((e t) t) e t$ \\
\hline $\mathrm{he}_{n}, t_{n}$ & $\lambda P . P\left(u_{n}\right)$ & (et) $t$ \\
\hline$P_{n}$ & $P_{n}$ & $s($ et $)$ \\
\hline$R_{n}$ & $R_{n}$ & $s($ eet $)$ \\
\hline $\mathrm{X}^{\uparrow n}$ & $\lambda \hat{x} \cdot\left[\nu_{n} \mid \nu_{n}=\llbracket \mathrm{X} \rrbracket\right] ; \nu_{n}(\hat{x})$ & $\tau_{\mathrm{t}} \tau_{\mathrm{t}}$ \\
\hline if, when & $\lambda p q .[\mid p \Rightarrow q]$ & ttt \\
\hline and, $\mathrm{C}_{0}$ & $\lambda f g \hat{x} . f(\hat{x}) ; g(\hat{x})$ & $\tau_{\mathrm{t}} \tau_{\mathrm{t}} \tau_{\mathrm{t}}$ \\
\hline or & $\lambda f g \hat{x} . f(\hat{x}) \sqcup g(\hat{x})$ & $\tau_{\mathrm{t}} \tau_{\mathrm{t}} \tau_{\mathrm{t}}$ \\
\hline want to & $\lambda P . \operatorname{want}(P)$ & (et)et \\
\hline doesn't & $\lambda P v \cdot[\mid \neg P(v)]$ & (et) et \\
\hline
\end{tabular}

Table 1 gives the lexicon. The notational conventions are as follows: 'e' abbreviates ' $s \rightarrow e$ ', and ' $t$ ' abbreviates ' $s \rightarrow s \rightarrow t$ ' (the type of boxes). Types associate to the right; $\tau_{1} \tau_{2} \tau_{3}:=\tau_{1}\left(\tau_{2} \tau_{3}\right)$. ' $\tau$ ' is used both as a function into types and a variable over types; ' $\tau_{\mathrm{t}}$ ' stands for any type ending in $\mathrm{t}$. As before, ' $\hat{x}$ ' 
stands for a (possibly empty) sequence of arguments. Finally, subscripted terms are variable functions from states, sans serif proper names like 'john' are constant functions from states to individuals, and sans serif predicates like 'man' or 'met' are the familiar functions from individual(s) to truth values.

Much in Table 1 is as in Muskens, but there are several important add-ons (along with a couple minor embellishments like dynamic entries for John, the, and another). Variable dynamic properties - e.g. $P_{n}$ and $R_{n}$-were discussed above. Additionally, I've defined a family of ${ }^{\uparrow n}$ operators which type-shift constituents into dynamic binders. ${ }^{\uparrow n}$ is essentially a polymorphic dynamicizing identity function: $\llbracket \mathrm{X}^{\uparrow n} \rrbracket$ introduces a variable dynamic property $\nu_{n}$, ensures $\nu_{n}=\llbracket \mathrm{X} \rrbracket$, and otherwise behaves the same as $\llbracket \mathrm{X} \rrbracket$. I've also added a generalized entry for dynamic disjunction which disjoins any two expressions so long as they have the same type-ending-in-t. (Rooth \& Partee [16]). As for the syntax: it's implicit but straightforward (cf. Muskens [11]). For now, I assume with Muskens that object QPs needn't QR (cf. our entry for transitive verbs), although they can. (I'll come back to this when I consider extraction cases.)

(10) shows how the system treats a simple donkey anaphora case. As expected, the type logic translation is true (Definition 9) iff every man who knocked left.

(10) If a $a^{1}$ man knocked, he $e_{1}$ left.

$\llbracket \mathrm{a}^{1} \operatorname{man} \operatorname{knocked} \rrbracket=\left[u_{1} \mid\right] ;\left[\mid \operatorname{man}\left(u_{1}\right)\right] ;\left[\mid \operatorname{knocked}\left(u_{1}\right)\right]$

$={ }_{\mathrm{ML}}\left[u_{1} \mid \operatorname{man}\left(u_{1}\right), \operatorname{knocked}\left(u_{1}\right)\right]$

$\llbracket$ he $_{1}$ left $\rrbracket=\left[\mid \operatorname{left}\left(u_{1}\right)\right]$

$\llbracket$ if a $\mathrm{a}^{1}$ man knocked he $\mathrm{h}_{1}$ left $\rrbracket=\left[\mid\left[u_{1} \mid \operatorname{man}\left(u_{1}\right), \operatorname{knocked}\left(u_{1}\right)\right] \Rightarrow\left[\mid \operatorname{left}\left(u_{1}\right)\right]\right]$

$\rightsquigarrow \lambda i j . i[] j \wedge \forall k .\left(j\left[u_{1}\right] k \wedge \operatorname{man}\left(u_{1}(k)\right) \wedge \operatorname{knocked}\left(u_{1}(k)\right)\right) \rightarrow\left(\exists l . k[] l \wedge \operatorname{left}\left(u_{1}(l)\right)\right)$

Quiet VPs. I assume with Schwarz [19] that there are two ways for an XP $\varepsilon$ to go missing. The first ('DELETION') is the usual Roothian condition: $\varepsilon$ has a salient antecedent $\alpha$ with which it's syntactically identical up to indices and F-marks. The second ('BINDING') applies when $\varepsilon$ is a phonologically null or deaccented pro-XP (on this theory, English happens to lack non-pronominal pro-XPs).

We've seen ample reason to suppose that something like CONTRAST regulates DELETION. I haven't yet discussed whether unstressed (i.e. silent or deaccented) pro-XPs need to be licensed by CONTRAST, but it's clear they do. As noted previously, CONTRAST explains why "John likes him, and BILL does too" requires coreferential pronouns. But the exact same facts pertain to deaccented pro-forms (Rooth [14]). Neither "John likes him, and BILL likes him too" nor "Simon thinks he's smart, and CHRIS thinks he's smart too" has more interpretations than its elliptical counterpart. This follows if CONTRAST must relate a node dominating the unstressed pro-XP with some other node in the discourse.

So CONTRAST is relevant for both BINDING and DELETION. But it needs a dynamic reformulation: the version of CONTRAST I've been working with pulls the things it wants to compare out of their contexts of evaluation, in effect unbinding any dynamically bound variables (and CONTRAST is, in any case, defined for a system without assignments in the model). Moreover, as defined, $\operatorname{CONTRAST}(\phi, \psi)$ requires exact semantic identity between $\llbracket \psi \rrbracket$ and some $\kappa \in\langle\langle\phi\rangle$. But boxes yield extremely fine-grained denotations; two truth-conditionally equivalent boxes $K$ 
and $K^{\prime}$ can nevertheless differ in context change potential (cf. also Hardt's [6] fn. 12). But (11) indicates that CONTRAST should care only about truth-conditional import, not context change potential. So requiring semantic identity is too strict.

Together, these facts suggest a reformulation of contrast as a compositionally integrated presuppositional operator $\sim_{n}$ (after Rooth's [15] $\sim$ ) which is sensitive only to truth conditions and which itself gets dynamically bound.

(11) John met $\mathrm{a}^{1}$ man. Then BILL met $a^{2}$ man. / Then BILL did [e meet $\mathrm{a}^{2}$ man]. Definition 10. Local, dynamic reformulation of CONTRAST: $\llbracket \mathrm{X} \sim_{n} \rrbracket=\lambda \hat{x} i:\left[\exists \Gamma \in\{\delta(\hat{x}): \delta \in\langle\mathrm{X}\rangle\} . \operatorname{True}_{i}\left(\nu_{n}(\hat{x})\right) \rightarrow \operatorname{True}_{i}(\Gamma(\hat{x}))\right] \cdot \llbracket \mathrm{X} \rrbracket(\hat{x})(i)$

For $\llbracket \mathrm{X} \sim_{n} \rrbracket$ to be defined, all incoming states $i$ must be such that $\nu_{n}(i)$ entails (after being fed a possibly empty sequence of arguments $\hat{x}$ ) some value $\Gamma$ in $\langle\mathrm{X}\rangle$ (after also being fed $\hat{x}) .{ }^{3,4}$ Assuming definedness, $\llbracket \mathrm{X} \sim_{n} \rrbracket$ does not differ from $\llbracket \mathrm{X} \rrbracket$. Note that $\sim_{n}$ has to be bound (free variables are prohibited in (C)DRT) and that Definition 10 leads us to expect that it may even be donkey bound. We'll shortly see that correspondence readings offer instances of precisely that.

Let's see how the definition works in a simple case of pronominal deaccenting, (12a). (12b) is only defined for incoming states $i$ such that if $S_{2}$ is true at $i$, then some alternative in $\left.\left\langle\mathrm{S}_{\delta^{\prime}}\right\rangle\right\rangle$ is true at $i$. But box sequencing (Definition 4) guarantees that the only states fed to $(12 \mathrm{~b})$ are those output by (12c) - so they will all necessarily make $u_{1}$ a man who entered and $S_{2}$ the box $\left[u_{1} \mid \operatorname{man}\left(u_{1}\right)\right.$, entered $\left.\left(u_{1}\right)\right] .{ }^{5}$ Since presumably $\left\langle\left\langle\mathrm{S}_{\delta^{\prime}}\right\rangle=\left\{\left[\mid P\left(u_{1}\right)\right]: P_{e t}\right\}\right.$ (or something equivalent), $\left[\mid\right.$ entered $\left.\left(u_{1}\right)\right] \in\left\langle\left\langle\mathrm{S}_{\delta^{\prime}}\right\rangle\right\rangle$. And since all the states output by (12c) already assign $u_{1}$ to a man who entered, they will necessarily assign $u_{1}$ to someone who entered. So at all relevant $i$, it's impossible for $\operatorname{True}_{i}\left(S_{2}\right)$ to be true and $\operatorname{True}_{i}\left(\left[\mid\right.\right.$ entered $\left.\left.\left(u_{1}\right)\right]\right)$ to be false. The presupposition is met.

(12) (a) $\left[\mathrm{s}_{\alpha} \mathrm{a}^{1} \text { man entered }\right]^{\uparrow 2} ;\left[\mathrm{s}_{\delta}\left[\mathrm{s}_{\delta^{\prime}}\right.\right.$ he $\left.\left._{1} \mathrm{SAT}_{\mathrm{F}}\right] \sim_{2}\right]$

(b) $\llbracket \mathrm{S}_{\delta} \rrbracket=\lambda i:\left[\exists \Gamma \in\left\langle\left\langle\mathrm{S}_{\delta^{\prime}}\right\rangle \cdot \operatorname{True}_{i}\left(S_{2}\right) \rightarrow \operatorname{True}_{i}(\Gamma)\right] \cdot \llbracket \overline{\mathrm{S}_{\delta}} \rrbracket(i)\right.$

(c) $\llbracket\left[\mathrm{a}^{1} \text { man entered }\right]^{\uparrow 2} \rrbracket$

$\rightsquigarrow \lambda i j . i\left[u_{1}, S_{2}\right] j \wedge \operatorname{man}\left(u_{1}(j)\right) \wedge \operatorname{entered}\left(u_{1}(j)\right) \wedge S_{2}(j)=\llbracket \mathrm{a}^{1}$ man entered $\rrbracket$

Basic cases. I'm now ready to give LFs for (1c) and (3). Temporarily assuming definedness, (14a) derives $\llbracket \mathrm{S}_{\alpha_{13 \mathrm{~b}}} \rrbracket$, and $(14 \mathrm{~b})$ gives the meaning of $\llbracket \mathrm{S}_{\varepsilon_{13 \mathrm{~b}}} \rrbracket .^{6}$

${ }^{3}$ Recall that for variable dynamic properties, $\nu_{n}(\hat{x}) \rightsquigarrow \lambda i j . \nu_{n}(i)(\hat{x})(i)(j)$.

${ }^{4}$ A simplification. Implicational bridging requires contextual entailment (Rooth [14]).

${ }^{5}$ More accurately, at any $i, S_{2}(i)=\lambda j . i\left[u_{1}, S_{2}\right] j \wedge \operatorname{man}\left(u_{1}(j)\right) \wedge \operatorname{entered}\left(u_{1}(j)\right)$.

${ }^{6}$ Two notes: (i) I'm treating has to as vacuous. The reason: in DRT, drefs introduced under modals like has to are typically inaccessible outside the modal's box. But constant-y things (names, specific indefinites, VP-type meanings, etc.) should be accessible no matter where they're introduced (cf. Hardt's [6] fn. 13). It's possible to give a semantic definition of accessibility that handles our cases without outrageous meanings for traditionally externally static items (Stone \& Hardt [21]), but this requires a fully intensional semantics - way beyond what I can discuss here. (ii) I'm assuming $\sim_{n}$ operators can't be nested; hence the pro-form inside $\varepsilon$ is never responsible for any presuppositions beyond those due to ellipsis of $\varepsilon$. Though I'm not sure how defensible this is, I see no other way to make the account work. 
(13) (a) $\left.\left[\mathrm{S}_{\alpha} \text { the }_{0} \text { cop who [arrested John }\right]^{1}\right]^{\uparrow 2}\left[\left[{ }_{\alpha}\right.\right.$ INSULTED $\left.\left.\left._{\mathrm{F}} \operatorname{him}_{1}\right] \sim_{2}\right]\right]^{\uparrow 4}$ $\left[\left[\mathrm{S}_{\varepsilon}\right.\right.$ the $_{3}$ cop who arrested $\left(\text { BILL }^{5}\right)_{\mathrm{F}}$ DIDN'T $_{\mathrm{F}}\left[\varepsilon\right.$ insult him $\left.\left.\left.{ }_{5}\right]\right] \sim_{4}\right]$

(b) $\left[\mathrm{S}_{\alpha} \text { if } \mathrm{John}^{1} \text { [has to } \operatorname{cook}^{\uparrow 3}\right]^{\uparrow 5} \mathrm{he}_{1}$ doesn't $\left[\left[{ }_{\alpha} \mathrm{WANT}_{\mathrm{F}}\right.\right.$ to $\left.\left.\left.P_{3}\right] \sim_{5}\right]\right]^{\uparrow 6}$

$\left[\left[\mathrm{S}_{\varepsilon}\right.\right.$ if he $\mathrm{h}_{1}$ has to $\left(\mathrm{CLEAN}_{\mathrm{F}}\right)^{\uparrow 4} \mathrm{he}_{1}$ doesn't $\left[\varepsilon\right.$ want to $\left.\left.\left.P_{4}\right]\right] \sim_{6}\right]$

(14) (a) $\llbracket \mathrm{John}^{1}$ has-to $\operatorname{cook}^{\uparrow 3} \rrbracket=\left[u_{1} P_{3} \mid u_{1}=\right.$ john, $\left.P_{3}=\llbracket \operatorname{cook} \rrbracket\right] ; P_{3}\left(u_{1}\right)$

[he ${ }_{1}$ doesn't want to $P_{3} \rrbracket=\left[\mid \neg P_{3}\left(u_{1}\right)\right]$

【if John ${ }^{\uparrow 1}$ has-to cook ${ }^{\uparrow 3}$ he $_{1}$ doesn't [WANT to $]_{\mathrm{F}} P_{3} \rrbracket$ $=\left[\mid\left(\left[u_{1} P_{3} \mid u_{1}=\right.\right.\right.$ john, $\left.\left.\left.P_{3}=\llbracket \operatorname{cook} \rrbracket\right] ; P_{3}\left(u_{1}\right)\right) \Rightarrow\left[\mid \neg \operatorname{want}\left(P_{3}\right)\left(u_{1}\right)\right]\right]$

(b) 【if he 1 has-to $(\text { CLEAN })^{\uparrow 4}$ he $_{1}$ doesn't want to $P_{4} \rrbracket$

$=\left[\mid\left(\left[P_{4} \mid P_{4}=\llbracket \operatorname{clean} \rrbracket\right] ; P_{4}\left(u_{1}\right)\right) \Rightarrow\left[\mid \neg \operatorname{want}\left(P_{4}\right)\left(u_{1}\right)\right]\right]$

I omit the translations to type logic formulae here, but it's relatively straightforward to check that the resulting boxes are true iff (a) if John (has to) cook, he doesn't want to cook, and (b) if he (has to) clean, he doesn't want to clean.

So the meanings are correct. Now, I show that the conditions on ellipsis are satisfied. First: every silent XP is either a bound pro-form or has an identical-upto-indices antecedent, so each is an instance of DELETION or BINDING. Next: the presuppositions introduced by $\sim_{5}$ and $\sim_{6}$ are both met. The case of $\sim_{5}$ is like (12): in both, a dynamically bound pro-form contrasts with a constituent dominating the binder. Now $\left\langle\left\langle\alpha_{13 \mathrm{~b}}\right\rangle=\left\{f\left(P_{3}\right): f_{\text {(et) et }}\right\}\right.$. I assume has-to $\left(P_{3}\right) \in\left\langle\left\langle\alpha_{13 \mathrm{~b}}\right\rangle\right\rangle$. Since $\sim_{5}$ is bound by a constituent denoting has-to $(\llbracket \operatorname{cook} \rrbracket)$, and the definition of

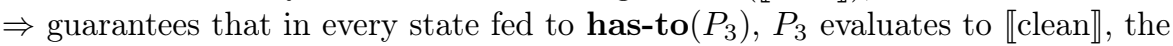
presupposition must be satisfied. As for $\sim_{6}$, I assume that $\llbracket \operatorname{cook} \rrbracket \in\left\langle\operatorname{CLEAN}{ }_{F}\right\rangle$, from which it follows that $\llbracket \operatorname{cook}^{\uparrow 4} \rrbracket \in\left\langle\left(\mathrm{CLEAN}_{\mathrm{F}}\right)^{\uparrow 4}\right\rangle$, from which it follows that $\left[\mid\left(\left[P_{4} \mid P_{4}=\llbracket \operatorname{cook} \rrbracket\right] ; P_{4}\left(u_{1}\right)\right) \Rightarrow\left[\mid \neg \operatorname{want}\left(P_{4}\right)\left(u_{1}\right)\right]\right] \in\left\langle\left\langle\mathrm{S}_{\varepsilon_{13 \mathrm{~b}}}\right\rangle\right.$. Since $(\mathrm{i}) \sim_{6}$ is bound to $\llbracket \mathrm{S}_{\alpha_{13 \mathrm{~b}}} \rrbracket$, (ii) sequencing entails that the $u_{1}$ 's free in $\mathrm{S}_{\varepsilon}$ always evaluate to john, and (iii) the two boxes in (15) have identical truth conditions, the presuppositions of $\sim_{6}$ must be satisfied at all possible incoming $i$.

(15) $\left[\mid\left(\left[u_{1} P_{3} \mid u_{1}=\right.\right.\right.$ john, $\left.\left.\left.P_{3}=\llbracket \operatorname{cook} \rrbracket\right] ; P_{3}\left(u_{1}\right)\right) \Rightarrow\left[\mid \neg \operatorname{want}\left(P_{3}\right)\left(u_{1}\right)\right]\right]$

$$
\left[\mid\left(\left[P_{4} \mid P_{4}=\llbracket \operatorname{cook} \rrbracket\right] ; P_{4}(\text { john })\right) \Rightarrow\left[\mid \neg \operatorname{want}\left(P_{4}\right)(\text { john })\right]\right]
$$

Mutatis mutandis, deriving truth conditions and checking definedness for (13a) works in an exactly analogous fashion.

Extraction. (16) gives LFs generating (5c)'s sloppy reading (I've split it into two sentences). Note that I've QR'ed the object. This (standard) move is forced by the (standard) assumption that the only possible antecedents for DELETION/BINDING are XPs: since the object starts in VP, the only way to generate ACD as DELETION of or BINDING by an XP is to scope the object out of VP (Sag [17]).

(16) $\left[\mathrm{S}_{\alpha} \mathrm{I}\left[\left[\text { every }^{0} \text { thing } \lambda_{2} \mathrm{I} \text { was }\left[{ }_{\alpha} \text { SUPPOSEDF } \text { to } R_{3} t_{2}\right]\right]\left[\lambda_{1} \text { bought } t_{1}\right]^{\uparrow 3}\right]\right]^{\uparrow 9}$ $\left[\left[\mathrm{S}_{\varepsilon} \mathrm{I}\left[\left[\right.\right.\right.\right.$ every ${ }^{8}$ thing $\lambda_{5}$ I WASN'TF $\left[\varepsilon\right.$ supposed to $\left.\left.\left.\left.\left.R_{4} t_{5}\right]\right]\left[\lambda_{6}\left[\operatorname{SOLDF}_{6} t_{6}\right]\right]^{\uparrow 4}\right]\right] \sim_{9}\right]$

Object $\mathrm{QR}$ is to a position under the subject, rather than to $\mathrm{S}$. This is independently motivated. Merchant [10] notes NPIs can participate in ACD, e.g. "I didn't read a damn thing you asked me to". Since the NPI needs to stay in the scope of VP-negation, ACD QR must at least potentially target VP rather than S.

How are the LFs in (16) interpreted? First, we need a way to quantify into VP. I'll adopt the following flexibly-typed rule for translating syntactic $\lambda$-operators: 
Definition 11. $\llbracket \lambda_{n} \mathrm{X} \rrbracket=\lambda \mathcal{Q} \hat{v} \cdot \mathcal{Q}\left(\lambda u_{n} \cdot \llbracket \mathrm{X} \rrbracket(\hat{v})\right)$

In $\mathrm{S}_{\alpha}, \llbracket \lambda_{1}$ bought $t_{1} \rrbracket$ translates to $\lambda \mathcal{Q} v \cdot \mathcal{Q}\left(\lambda u_{1}\right.$. [| bought $\left.\left.\left(u_{1}\right)(v)\right]\right)$. The ${ }^{\uparrow 3}$ operator shifts this to $\lambda \mathcal{Q} v$. $\left[R_{3} \mid R_{3}=\lambda \mathcal{Q} v \cdot \mathcal{Q}\left(\lambda u_{1}\right.\right.$. [ $\mid$ bought $\left.\left.\left.\left(u_{1}\right)(v)\right]\right)\right] ; R_{3}(\mathcal{Q})(v)$. So since $R_{3}$ is accessible inside $\mathcal{Q}$, the $R_{3}$ subsequent to "supposed to" is bound. The same goes, mutatis mutandis, for $\mathrm{S}_{\varepsilon}$. So adequate interpretations are generated. As for licensing: all silent material is either an instance of DELETION or BINDING. And $\sim 9$ 's presupposition is satisfied, which the reader is invited to check.

But, wait. Doesn't CONTRAst also have to license the silent pro-form $R_{3}$ ? Absolutely, which brings me to a slightly uncomfortable matter: since QR targets $\mathrm{S}_{\alpha}$ 's $V P$, it's hard to see which two nodes in $\mathrm{S}_{\alpha}$ could possibly be related by $\uparrow_{n} / \sim_{n}$ : the smallest constituent in which $t_{2}$ is bound already contains the subject pronoun "I"! Note that, while troubling, this doesn't seem like an issue for my proposal per se: QR to VP is what's creating the difficulty here, but Merchant's NPI ACD case shows that non-sentential QR is necessary. ${ }^{7}$

Correspondence readings, donkey $\sim_{n}$ binding. Here, finally, are the LF and interpretation of (6) (following Stone's [20] informal discussion):

(17) (a) If [[John or Bill] $\lambda_{4}$ [Mary [meets $\left.\left.\left.t_{4}\right]^{\uparrow 3}\right]^{\uparrow 6}\right]\left[\left[\operatorname{suEF} \operatorname{does} P_{3}(\right.\right.$ too $\left.\left.)\right] \sim_{6}\right]$

(b) $\llbracket$ Mary $\left[\text { meets } t_{4}\right]^{\uparrow 3} \rrbracket=\left[P_{3} \mid P_{3}=\lambda v\right.$. [| meets $\left.\left.\left(u_{4}\right)(v)\right]\right] ; P_{3}$ (mary)

$\left.\left.\llbracket\left[\text { Mary [meets } t_{4}\right]^{\uparrow 3}\right]^{\uparrow 6} \rrbracket=\left[S_{6} \mid S_{6}=\llbracket \text { Mary [meets } t_{4}\right]^{\uparrow 3} \rrbracket\right] ; S_{6}$

$\llbracket \lambda_{4}\left[\text { Mary }\left[\text { meets } t_{4}\right]^{\uparrow 3}\right]^{\uparrow 6} \rrbracket=\lambda \mathcal{Q} \cdot \mathcal{Q}\left(\lambda u_{4} \cdot\left[S_{6} \mid S_{6}=\llbracket\right.\right.$ Mary $\left.\left.\left[\text { meets } t_{4}\right]^{\uparrow 3} \rrbracket\right] ; S_{6}\right)$

$\llbracket$ John or Bill $\rrbracket=\lambda P . P($ john $) \sqcup P($ bill $)$

$\left.\llbracket\left[\text { John or Bill] } \lambda_{4} \text { [Mary [meets } t_{4}\right]^{\uparrow 3}\right]^{\uparrow 6} \rrbracket$

$=\left(\left[S_{6} \mid S_{6}=\left[P_{3} \mid P_{3}=\lambda v \cdot[\mid \operatorname{meets}(\right.\right.\right.$ john $\left.)(v)]\right] ; P_{3}$ (mary) $\left.) ; S_{6}\right) \sqcup$

$\left(\left[S_{6} \mid S_{6}=\left[P_{3} \mid P_{3}=\lambda v \cdot[\mid\right.\right.\right.$ meets $($ bill $\left.)(v)]\right] ; P_{3}$ (mary) $\left.] ; S_{6}\right)$

So the states output by the antecedent fix $P_{3}$ either to the property of meeting John or to the property of meeting Bill (this happens in slightly convoluted fashion since the introduction of $P_{3}$ is actually tucked inside the dynamic variable $S_{6}$ ). This guarantees truth conditions such that if Mary meets John, Sue meets John, and if Mary meets Bill, Sue meets Bill, exactly the meaning we're after.

Lastly, I show that (17a) is defined. The interesting bit here, which I alluded to previously, is is that $\sim_{6}$ acts like a donkey pronoun! Specifically, the states output by (17a)'s antecedent fix $S_{6}$ to one of two boxes: either «Mary [meets John $]^{\uparrow 3} \rrbracket$ or $\llbracket$ Mary $[\text { meets Bill }]^{\uparrow 3} \rrbracket$. Now if $\llbracket$ Mary $\rrbracket \in\left\langle\left\langle\operatorname{SuE}_{\mathrm{F}}\right\rangle\right.$, then $\left[\mid P_{3}\right.$ (mary) $] \in$ $\left\langle\operatorname{SuE}_{\mathrm{F}}\right.$ does $\left.P_{3}\right\rangle$. Since at each state $i$ output by the antecedent, $\operatorname{True}_{i}\left(S_{6}\right) \rightarrow$ $\operatorname{True}_{i}\left(\left[\mid P_{3}\right.\right.$ (mary) $\left.]\right)$, the presupposition is satisfied.

\section{Conclusion}

I've argued for a theory of cross-categorial surprising sloppy readings in which ellipsis sites may consist either in part or in whole of dynamically bound pro-forms. The account has three parts: the conditions regulating the distribution of elliptical sites and pro-XPs, a dynamicizing type-shifter ${ }^{\uparrow n}$, and a presuppositional, dynamically bound, alternative-sensitive CONTRAST operator $\sim_{n}$. Dynamic binding

\footnotetext{
7 One promising way forward is Heim's [7] formulas-based Roothian account of ACD. I see no reason to expect that Heim's theory couldn't be recast in to the present framework (quite the contrary), but I have to postpone a real investigation.
} 
is a more natural option for these cases than QR, and integration with a theory of focus avoids over-generation. Extraction cases are within reach (though it is not in the end clear how ACD is licensed), while dynamic disjunction generates correspondence readings and predicts the possibility of donkey-binding $\sim_{n}$.

\section{References}

[1] Asher, N., A. Lascarides. 2003. Logics of Conversation. Cambridge U. Press.

[2] Bos, J. 1994. Focusing Particles \& Ellipsis Resolution. In Verbmobil Report 61, Universitat des Saarlandes.

[3] Elbourne, P. 2001. E-type anaphora as NP deletion. Natural Language Semantics 9(3). 241-288.

[4] Evans, F. 1988. Binding into Anaphoric Verb Phrases. In Proceedings of ESCOL. OSU Working Papers in Linguistics.

[5] Fiengo, R., R. May. 1994. Indices and identity. Cambridge, MA: MIT Press.

[6] Hardt, D. 1999. Dynamic interpretation of verb phrase ellipsis. Linguistics and Philosophy 22(2). 185-219.

[7] Heim, I. 1997. Predicates or formulas? Evidence from ellipsis. In A. Lawson \& Eund Cho (eds.), Proceedings of SALT 7 19-221.

[8] Keenan, E. 1971. Names, quantifiers, and the sloppy identity problem. Papers in Linguistics 4(2), 211-232.

[9] Kratzer, A. 1991. The Representation of Focus. In A. von Stechow \& D. Wunderlich (eds.), Semantics: An International Handbook of Contemporary Research, 825832. Berlin: de Gruyter.

[10] Merchant, J. 2000. Antecedent Contained Deletion in Negative Polarity Items. Syntax 3, 134-15.

[11] Muskens, R. 1996. Combining Montague Semantics and Discourse Representation. Linguistics and Philosophy 19, 143-186.

[12] Reinhart, T. 1983. Anaphora and Semantic Interpretation: U. of Chicago Press.

[13] Rooth, M. 1985. Association with Focus: UMass, Amherst dissertation.

[14] Rooth, M. 1992a. Ellipsis redundancy and reduction redundancy. In S. Berman \& A. Hestvik (eds.), Proceedings of the Stuttgart Ellipsis Workshop, Stuttgart.

[15] Rooth, M. 1992b. A Theory of Focus Interpretation. Natural Language Semantics 1. $75-116$.

[16] Rooth, M., B. Partee. 1982. Conjunction, type ambiguity, and wide scope "or". In D. Flickinger et al. (eds.), Proceedings of the First West Coast Conference on Formal Linguistics, Stanford University, pp. 353-362.

[17] Sag, I. 1976. Deletion and Logical Form: MIT dissertation.

[18] Sauerland, U. 2007. Copying vs. structure sharing: a semantic argument. In Jeroen van Craenenbroeck (ed.), Linguistic variation yearbook 7, 27-51. John Benjamins Publishing Company.

[19] Schwarz, B. 2000. Topics in Ellipsis: UMass, Amherst dissertation.

[20] Stone, M. 1992. Or and anaphora. In Proceedings of SALT 2. 367-385

[21] Stone, M. \& D. Hardt. 1999. Dynamic discourse referents for tense and modals. In H. Bunt (ed.), Computational Semantics. Kluwer. 287-299.

[22] Tomioka, S. 1999. A sloppy identity puzzle. Natural Language Semantics 7(2). 217-241.

[23] Tomioka, S. 2008. A step-by-step guide to ellipsis resolution. In Kyle Johnson (ed.), Topics in ellipsis, 210-228. Cambridge University Press.

[24] Wescoat, M. 1989. Sloppy Readings with Embedded Antecedents. Stanford ms.

[25] Williams, E. 1977. Discourse and logical form. Linguistic Inquiry 8. 101-139. 


\title{
On wh-exclamatives and noteworthiness ${ }^{\star}$
}

\author{
Anna Chernilovskaya and Rick Nouwen \\ Utrechts Instituut voor Linguïstiek
}

\section{Introduction}

We explore a new approach to the semantics of wh-exclamatives, like (1).

(1) What a beautiful song John wrote!

We will aim for two things: (1) extend the empirical focus beyond English whatand how- exclamatives, to include exclamatives common in other languages that are based on other wh-words; (2) counter the common assumption that exclamative semantics needs to involve some kind of scalar mechanism.

Before we motivate and present our analysis, a word of caution is in order. To simplify matters for this short paper, we will be discussing the semantics of exclamatives like (1) in terms of truth-conditions. Such a move blatantly ignores the fact that an utterance of (1) counts as a speech act that comes with its own intricate and interesting properties, properties which will be quite different from those of an assertion. For the purpose of this short paper, however, we will remain agnostic as to what role the truth-conditions play in the pragmasemantics of exclamatives. (See Rett, 2011; Zanuttini and Portner, 2003, for extensive discussion.)

\section{Background: scalarity in exclamative semantics}

Rett 2011 proposes that the semantics of $w h$-exclamatives involves degree intensification (Cf. Castroviejo, 2006; Rett, 2008a,b, for related approaches). On her approach, the logical form of (1) will specify the (derived) degree predicate in (2). (See Rett's paper for details of the derivation.)

(2) $\quad \lambda d . \exists x[\operatorname{song}(x) \wedge \operatorname{wrote}(j, x) \wedge$ beautiful $(x, d)]$

According to Rett, an utterance of a wh-exclamative involves the speaker expressing that it is noteworthy that the degree property corresponding to the exclamative is instantiated by some value that exceeds the relevant contextual standard (in the case of (1) the standard of beauty w.r.t. songs).

\footnotetext{
* Both authors gratefully acknowledge the NWO VIDI grant that made this work possible. For stimulating discussion and helpful comments we would furthermore like to thank audiences in both Stanford and Chicago, where earlier versions of this paper were presented.
} 
If, as in (3-a), a wh-exclamative lacks an overt gradable adjective, a measurement operator $\lambda d \cdot \lambda x \cdot \mu_{\alpha}(x)=d$ is inserted. This operator basically plays the role of a silent adjective, where the relevant measurement dimension $\alpha$ is determined contextually. Via this operator, (3-a), too, ends up expressing a degree property, namely how $\alpha$, or specifically how beautiful or weird or complex, etc. John's song was. This is the predicate in (3-b). An utterance in (3-a), then, means that the speaker expresses that it is noteworthy that the degree of $\alpha$ of John's song exceeds the standard.

$$
\begin{aligned}
& \text { a. What a song John wrote! } \\
& \text { b. } \quad \lambda d \exists x\left[\operatorname{song}(x) \wedge \operatorname{wrote}(j, x) \wedge \mu_{\alpha}(x)=d\right]
\end{aligned}
$$

Rett's approach is to treat a wh-exclamative as a degree phenomenon and she argues that it could really be nothing else. In particular, she argues that the noteworthiness evaluation that is part of the wh-exclamation is necessarily directed at an instantiation of a degree predicate. She does so by turning to scenarios like the one in (4).

(4) Imagine that by some strange coincidence someone repeatedly picks out the same two cards (say, the $3 \diamond$ and the $6 \bigcirc$ ) from a (repeatedly reshuffled) pack of cards. Mary has seen this happen and now witnesses this person pick $3 \diamond$ and $6 \odot$ yet again.

Rett observes that it is now infelicitous for Mary to utter (5):

What cards he picked!

What is essential to this scenario is that no matter how noteworthy the events of picking these cards were, there is nothing particularly special about the cards that were picked. That is, there is no $\alpha$ such that $\mu_{\alpha}(3 \diamond \oplus 6 \oslash)$ returns a particularly high degree and this is why (5) is infelicitous. Rett concludes that wh-exclamatives are subject to a degree restriction: they always express that the degree to which something holds is deemed noteworthy by the speaker.

It is worth remarking that it is not necessary to interpret Rett's observation concerning (4) and (5) as saying something about the involvement of degrees in the semantics of $w h$-exclamatives, but that instead one could argue that it just shows that wh-exclamatives always involve some kind of scalar mechanism. For instance, we believe that the influential approach of Zanuttini and Portner (2003), although compositionally less specific, is in principle suitable for dealing with examples like (5) equally well (pace Rett's own assessment of that work). Zanuttini and Portner assume that the wh-word is associated to a partially ordered and restricted domain of quantification. Domains are restricted in that they consist of ordinary entities only. In wh-exclamatives this domain is then widened to also include entities that are somehow extra-ordinary, entities that exceed the part of the range of the partial order that is normally taken into account. In other words, for (3-a) the exclamative expresses that there is an 
entity that instantiates the predicate in (6) that is above and beyond the normal domain.

$$
\lambda x \cdot[\operatorname{song}(x) \wedge \operatorname{wrote}(j, x)]
$$

For instance, in the case of (6), there is widening from the normal domain of songs to a domain that also includes unusual songs (songs that are unusually beautiful, or unusually weird, or unusually complex, etc.) The exclamative then expresses that John's song is part of this widened part of the domain. In sum, Rett's degree approach and Zanuttini \& Portner's domain widening approach share an essential ingredient, namely scalarity.

\section{The scope of noteworthiness}

There is a caveat to the degree (or scalarity) restriction. Rett claims that in order to prevent her approach from predicting What cards he picked! to be felicitous in the card picking scenario of (4), the range of possible dimensions $\alpha$ needs to be restricted. In particular, it should not be possible to derive a degree predicate on the basis of how surprising, unexpected or noteworthy the picked cards were. ${ }^{1}$ This seems not entirely accurate to us. Recall that the scenario in (4) is set up in such a way that there is nothing special about the two cards, other than the fact that they keep on being picked. So, unlike (say) $A \oslash \oplus A \diamond \oplus A \boldsymbol{\phi} \oplus A \boldsymbol{\phi}$, the pair $3 \diamond \oplus 6 \odot$ lacks any noteworthy features. So even if we inserted an overt measurement relation based on noteworthiness, as in (7-a), we would still expect the exclamative What cards he picked! to be infelicitous in the given scenario. In fact, we predict the same if we inserted a silent measure of unexpectedness, as in (7-b), for it is the fact that these cards were picked that is unexpected. It seems to us that there is nothing intrinsically unexpected about the particular group of cards.

$$
\begin{array}{ll}
\text { a. } & \lambda d . \exists x\left[\operatorname{cards}(x) \wedge \operatorname{picked}(h, x) \wedge \mu_{\text {noteworthy }}(x)=d\right] \\
\text { b. } & \lambda d . \exists x\left[\operatorname{cards}(x) \wedge \operatorname{picked}(h, x) \wedge \mu_{\text {unexpected }}(x)=d\right]
\end{array}
$$

In sum, we conclude that there is no need to put a cap on the general scalarity involved in the wh-exclamatives we have seen so far. This conclusion point in the direction of our main argument: cases like (5)/(4) are not evidence of a degree (or scalarity) restriction, but rather point out that the noteworthiness evaluation is always directed at the referent of the wh-phrase. Call this the locality restriction: readings in which the $\mu$ applies to a structure that properly contains the wh-phrase, as in (8), are unavailable. Such degree predicates would incorrectly predict (5) to be felicitous in (4).

$$
\lambda d . \exists x\left[\mu_{\text {noteworthy }}(\wedge \operatorname{card}(x) \wedge \operatorname{picked}(h, x))=d\right]
$$

\footnotetext{
${ }^{1}$ A similar move in the domain widening approach would mean excluding such properties from determining the ordering on the domain.
} 


\section{Beyond English what-exclamatives}

Whilst English only has what- and how-exclamatives, languages like Dutch, German and Russian allow for exclamatives based on other wh-words too. The example in (9) presents a Dutch who-exclamative, and (10) a Dutch whichexclamative. There are similar examples in other languages, but not in English.

(9) Wie ik net gezien heb! who I just seen have
Welke vrouw ik net gezien heb! which woman I just seen have

The meaning of these examples shows a contrast with English what-exclamatives. It is infelicitous to utter (9) or (10) in response to seeing a woman with some noteworthy feature, e.g. an exceptionally tall woman. These exclamatives can be used when the very fact that the speaker just saw the woman in question is unexpected; for instance, as a reaction to seeing Mary, an in all senses absolutely normal woman, of whom everybody thought that she had left the country.

The approaches discussed in the previous study will have a hard time accounting for these examples. ${ }^{2}$ This is first of all because these exclamatives lack the readings that these approaches account for in the case of what-exclamatives. Moreover, the available readings are close to the readings that were meant to be excluded a possible interpretations of what-exclamatives. For instance, (11) is felicitous in the card picking scenario in (4).

$$
\begin{aligned}
& \text { Welke kaarten hij toen (weer) trok! } \\
& \text { which cards he then (again) pulled }
\end{aligned}
$$

As we discussed above, in the scenario there is no property such that $3 \diamond \oplus 6 \odot$ have this property to a particularly high degree. What appears to be needed to account for the fact that (11) is felicitous in the given scenario is to break with the locality restriction and assume that the exclamative expresses the degree predicate in (8). This is not unproblematic, for we need the locality restriction to avoid predicting the Dutch what-exclamatives in (12) to be felicitous in scenarios like (4), while in fact it behaves in complete parallel to its English counterpart.

$$
\begin{aligned}
& \text { Wat een kaarten hij toen (weer) trok! } \\
& \text { what a cards he then (again) pulled }
\end{aligned}
$$

\section{Proposal}

We propose that exclamatives directly express a noteworthiness evaluation and that no overt degree morphology or domain widening is needed. In other words, our approach lacks a degree or scalarity restriction. We furthermore categorise all wh-exclamatives in two distinct classes:

\footnotetext{
${ }^{2}$ Rett assumes that wh-exclamatives can only be formed with wh-words ranging over degrees, i.e. who-exclamatives are predicted to be ungrammatical. This is a fine prediction for English, but obviously not for other languages. Even if this restriction is lifted for such languages, the desired readings for examples like (9) and (10) are not derived.
} 
Type 1: expressing the noteworthiness of a referent of the wh-word

Type 2: expressing the noteworthiness of the proposition referenced in the exclamative

In addition to this, each $w h$-word introducing a $w h$-exclamative is specified for a kind of noteworthiness it can mark. For example, both English and Dutch what $a$-exclamatives are type 1 . Dutch who and which introduce wh-exclamatives of type 2 .

Some initial suggestive data for the type 1 /type 2 distinction is that type 1 exclamatives can typically be reduced while type 2 exclamatives can not. This would be expected since the latter but not the former are dependent on a larger propositional structure.

Type 1

Type 2

What a (beautiful) book!

Wat een (mooi) boek! what a (beautiful) book
(15) *Wie! who

(16) *Welk mooi boek! which beautiful book!

Moreover, in Dutch, word order seems to reflect the type $1 / 2$ distinction. Dutch has SVO for main clauses, with V2, and SOV for embedded clauses. Type 1 exclamatives are V2, whilst type 2 exclamatives are verb-final.

$$
\begin{aligned}
& \text { wat heeft-i een toetjes gemaakt! (type 1, V2) } \\
& \text { what a desserts has-he made! } \\
& \text { wie ik net gezien heb! (type 2, verb-final) } \\
& \text { who I just saw have }
\end{aligned}
$$

\subsection{Wh-exclamatives of type 1}

According to our proposal, the key ingredient to exclamative meaning is a noteworthiness evaluation. The meaning that we propose for (19-a) is in (19-b) (though we will refine this shortly):

$$
\begin{aligned}
& \text { a. What a song John wrote! } \\
& \text { b. } \exists x[\operatorname{song}(x) \wedge \operatorname{wrote}(j, x) \wedge \text { noteworthy }(x)]
\end{aligned}
$$

In some sense, our proposal is similar to that of Rett in that we insert a predicate that evaluates the wh-referent (for type 1 wh-exclamatives), but in our analysis it is always the same predicate, namely noteworthy. We think that roughly the following concept is behind the label noteworthy:

an entity is noteworthy iff its intrinsic characteristics (i.e. those characteristics that are independent of the factual situation) stand out considerably with respect to a comparison class of entities 
Examples of noteworthy things include the titanic, Frank Zappa's discography and blackberry, chicken liver and cauliflower cake. Also, $3 \diamond \oplus 6 \bigcirc$ is not noteworthy, and we will later use the fact that the proposition that these cards were picked several times in a row is noteworthy.

Given the characterisation above, (19-b) is true if and only if the song written by John stands out in some sense, compared to other songs. This can be because it is particularly good, or particularly weird, or particularly beautiful, etc.

Of course, noteworthy is a gradable predicate. We will take this into account by adopting a vague predicate approach to degree predicate (but nothing hinges on this). So we will write noteworthy $(x)(c)$ to express that among the members of comparison class $c, x$ will be deemed as noteworthy. The comparison class will normally be the class described by the wh-phrase.

$$
\exists x[\operatorname{song}(x) \wedge \operatorname{wrote}(j, x) \wedge \text { noteworthy }(x)(\lambda x . \operatorname{song}(x))]
$$

In case there is a gradable predicate present in the wh-phrase, this predicate will become part of the comparison class, as in (21) (where $c$ is the contextually determined comparison class for beautiful).

$$
\begin{array}{ll}
\text { a. } & \text { What a great song John wrote! } \\
\text { b. } & \exists x[\operatorname{song}(x) \wedge \operatorname{great}(x)(c) \wedge \text { wrote }(j, x) \wedge \\
& \text { noteworthy }(x)(\lambda x . \operatorname{great}(x)(c) \wedge \operatorname{song}(x))]
\end{array}
$$

Of course, the most salient reason for John's beautiful song to be a noteworthy beautiful song is that it was particularly beautiful, which accounts for the most natural reading of (21-a). However, there also exists a reading in which this whexclamative does not express how beautiful the song was, but that the beautiful song in question was noteworthy for some other reason (e.g. because it was very weird, unusually structured, or abnormally long):

Q: Did John write a beautiful song?

A: Yes he did, and whàt a beautiful song he wrote! It contained 36 verses!

$\mathrm{A}^{\prime}$ : Yes he did, and whàt a beautiful song he wrote! It has just one chord!

Note that we do not predict What cards he picked! to be felicitous in the cardpicking scenario of (4). This is because, as we remarked above, in that scenario there is nothing noteworthy about the cards themselves.

\subsection{Wh-exclamatives of type 2}

According to our proposal, Dutch who and which exclamatives differ from examples like (21-a) in that they do not involve the noteworthiness of the referent corresponding to the $w h$-phrase, but rather the noteworthiness of a proposition. For instance, for Dutch examples of the form Who/which I just saw! the interpretation in (23-a) is unavailable. Instead, the predicted reading is in (23-b), where the noteworthiness evaluation takes propositional scope. (We will leave 
the comparison class implicit. It suffices to assume it contains the speaker's experience.)

$$
\begin{aligned}
& \text { a. } \exists x[\operatorname{saw}(I, x) \wedge \text { noteworthy }(x)] \\
& \text { b. } \exists x[\operatorname{noteworthy}(\wedge \operatorname{saw}(I, x))]
\end{aligned}
$$

We will assume noteworthy to be factive. The form in (23-b) is true if and only if for some person, the true proposition that I saw this person stands out considerably.

\section{Discussion}

\subsection{No interaction with degree constructions}

In our proposal, wh-exclamatives are not a degree phenomenon in the sense of containing a mechanism that targets degree arguments of gradable predicates. The high degree reading of exclamatives is due to the fact that noteworthy objects are objects that stand out by possessing some attribute to an exceptionally high degree. But this high degree is lexically accessed, not compositionally. The result is that we predict that proper degree constructions, i.e. those that target degree slots of adjectives, will not stand in the way of forming a wh-exclamative. For instance, the degree slot in (24) is presumably saturated by extremely, witness the incompatibility of having further degree modification if extremely is there, as in (25). On a degree approach, it would be difficult to account for why (24) is felicitous, since extremely and what would target the same degree slot. (Cf. Castroviejo-Miro (2008) for discussion.)

(24) What an extremely nice man John is!

(25) a. *John is more extremely nice than Bill.

b. *John is too extremely nice.

c. ${ }^{*}$ How extremely tall is John? Answer: 7 feet.

In our proposal, however, the degree slot of nice in (24) plays no role in the exclamative semantics. We derive something along the lines of (26). (Here, we represent the function of extremely as the indicating that there is a degree that is higher than the standard by some considerable margin $(\gamma)$.

$$
\exists x \exists d[\operatorname{man}(x) \wedge \operatorname{nice}(x, d) \wedge d>s(\text { nice })+\gamma \wedge \text { noteworthy }(x)]
$$

\subsection{An extension to how-exclamatives}

In English, questions with how may form degree questions, as in (27-a). Given our proposal to abandon a degree semantics for wh-exclamatives, the question arises how we could account for exclamatives like (27-b).

$$
\begin{aligned}
& \text { a. How tall is John! } \\
& \text { b. How tall John is! }
\end{aligned}
$$


In particular, one might ask whether English how-exclamatives are type 1 or type 2. Although, we do not have any clearcut arguments for either choice, there is one reason to think that they are of type 1 , and that is because they can be reduced, as in How tall!, How bizarre!, etc. We will leave a more decisive argumentation for a choice between type 1 and type 2 to further research. It will be informative, however, to explore the two options a little bit.

How-exclamatives as type 1 If how-exclamatives are indeed of type 1, we get the following semantics for (27-b).

$$
\exists d[\operatorname{tall}(j, d) \wedge \text { noteworthy }(d)(c)]
$$

There are some problems with these truth-conditions. First of all, it is not clear what the comparison class $c$ should look like, but let us assume that this is just some set of degrees of tallness that makes contextual sense. Second, and more seriously, it is unclear when a degree is or is not noteworthy. For instance, it makes no sense to assume that (29) is true because people that tall are remarkable, for that would make $210 \mathrm{~cm}$ towers exclamation-worthy.

$$
\text { noteworthy }(210 \mathrm{~cm})(\mathrm{c})
$$

But it is not completely inconceivable that we can have attitudes to degrees. What we need is a notion of degree that expresses the relative positions entities take up in the relevant ordering. We could, for instance, use the universal scale of Bale (2008), where every entity is mapped to a universal degree representing its relative position in the (finite) weak order of entities under discussion. For now, however, we'll use a different mechanism originating from Klein (1980). We'll assume that how in exclamatives does not range over a fine-grained scale of degrees, but rather over a coarse-grained set of so-called degree functions. Instead of interpreting adjectives a degree relations, we take them to be relations between comparison classes and entities. Degree modifiers like very are manipulators of the comparison class argument (Klein, 1980; Kennedy and McNally, 2005).

$$
\llbracket \text { tall } \rrbracket=\lambda c \lambda x \cdot \operatorname{tall}_{c}(x) \quad \text { " } x \text { is tall in class } c "
$$

So, begin very tall just means being tall with respect to the class of tall individuals. Functions like that expressed by very are called degree functions. We can create an ordering of degree functions as follows.

$$
\begin{array}{ll}
\text { a. } & D_{0}=\lambda P \lambda c \lambda x \cdot P(c)(x) \\
\text { b. } & D_{1}=\lambda P \lambda c \lambda x \cdot P(P(c))(x) \\
\text { c. } & D_{2}=\lambda P \lambda c \lambda x \cdot P(P(P(c))(x) \\
\text { d. } & \text { for } n>0: D_{n}=\lambda P \lambda c \lambda x \cdot P\left(D_{n-1}(P)\right)(c)(x)
\end{array}
$$

The exclamative semantics could now be given in terms of such functions:

$$
\exists D[(D(\text { tall }))(c)(j) \wedge \text { noteworthy }(D)]
$$


Assume that things that are very very A are noteworthy, as are things that are very very very $\mathrm{A}$, etc. In other words, noteworthy $\left(D_{n}\right)$ is true for $n \geq 2$. This means that (27-b) is felicitous if John is very very tall. In this setup we now do not predict that towers of John's height are exclamation-worthy, since those towers will not count as very very tall.

Note that in this setup we have no problem with how-exclamatives that contain other degree manipulators as in (34-a), for which we would derive the meaning in (34-b).

$$
\begin{aligned}
& \text { a. How very tall John is! } \\
& \text { b. } \exists D\left[\left(D\left(D_{1}(\text { tall })\right)\right)(c)(j) \wedge \text { noteworthy }(D)\right]
\end{aligned}
$$

How-exclamatives as type 2 When we consider the option of how-exclamatives being of type 2, we get (35) for (27-b).

$$
\exists d[\operatorname{noteworthy}(\wedge \operatorname{tall}(j, d))]
$$

This is completely parallel to the semantics that Nouwen (2010) gives for (36-a), as in (36-b). (Assume that the predicate surprising is factive).

$$
\begin{aligned}
& \text { a. John is surprisingly tall. } \\
& \text { b. } \quad \exists d[\operatorname{surprisingly}(\wedge \operatorname{tall}(j, d))]
\end{aligned}
$$

The parallel is intuitively attractive. Both evaluative adverbs of degree like surprisingly and exclamatives seem to combine high degree with some sort of attitude. The page limit for this paper does not allow for a more thorough comparison of (27-b) and (36-a), nor for a proper evaluation of the semantics in $(35)$.

\section{Conclusion}

We have identified two kinds of wh-exclamatives: those that involve the noteworthiness of the wh-referent and those that involve a noteworthiness evaluation of the open proposition in the exclamative. Our approach simplifies the semantic mechanism and improves the empirical coverage over competing, scalar approaches. 


\section{Bibliography}

Bale, A. (2008). A universal scale of comparison. Linguistics and Philosophy, 31.

Castroviejo, E. (2006). Wh-Exclamatives in Catalan. Ph.D. thesis, Universitat de Barcelona.

Castroviejo-Miro, E. (2008). Adverbs in restricted configurations. In P. Cabredo Hofherr and O. Bonami, editors, Empirical issues in syntax and semantics, volume 7, pages 53-76.

Kennedy, C. and McNally, L. (2005). Scale structure, degree modification and the semantics of gradable predicates. Language, 81(2), 345-381.

Klein, E. (1980). A semantics for positive and comparative adjectives. Linguistics and Philosophy, 4, 1-45.

Nouwen, R. (2010). Degree modifiers and monotonicity. In P. Egré and N. Klinedinst, editors, Vagueness and Language Use. Palgrave McMillan.

Rett, J. (2008a). A degree account of exclamatives. In Proceedings of SALT $X V I I$.

Rett, J. (2008b). Degree modification in Natural Language. Ph.D. thesis, Rutgers.

Rett, J. (2011). Exclamatives, degrees and speech acts. Linguistics and Philosophy, Under review.

Zanuttini, R. and Portner, P. (2003). Exclamative clauses: at the syntaxsemantics interface. Language, 79(1), 39-81. 


\title{
Generalizing Monotonicity Inferences to Opposition Inferences
}

\author{
Ka-fat Chow \\ The Hong Kong Polytechnic University \\ kfzhouy@yahoo.com
}

\begin{abstract}
This paper generalizes the notion of monotonicities to opposition properties (OPs). Some propositions regarding the OPs of determiners will be proposed and proved. We will also define the notion of OP-chain and deduce a condition that enables us to determine the OPs of an iterated quantifier in its predicates based on the OPs of its constituent determiners.
\end{abstract}

Keywords: monotonicity inferences, opposition inferences, opposition properties, OP-chain, Generalized Quantifier Theory, Natural Logic

\section{Introduction}

Van Benthem (2008) characterized monotonicity inferences, an important type of Natural Logic inferences, as "inferences with inclusion premises" of the form "P $\leq Q \Rightarrow \varphi(P) \leq \varphi(Q)$ ". He also proposed the study on "inferences with exclusion premises" of the form " $P \leq \neg Q \Rightarrow \varphi(P) \leq \neg \varphi(Q)$ ", which we call "opposition inferences". In recent years, some scholars, e.g. MacCartney and Manning (2009), MacCartney (2009), Icard (2011), have started to study opposition inferences as a new type of Natural Logic inferences from different perspectives based on different frameworks. This paper is a study of opposition inferences as a generalization of monotonicity inferences based on the Generalized Quantifier Theory (GQT) framework.

\section{Basic Definitions}

First of all, let us review the definition of increasing monotonicity in the right argument for type $\langle 1,1\rangle$ generalized quantifiers (i.e. determiners) ${ }^{1}$.

\footnotetext{
${ }^{1}$ In what follows, we use $A$ and $B$ to denote the left and right argument of a determiner, respectively. We also use the symbol " $\leq$ " to denote the subset relation between sets as well as the entailment relation between propositions. Note that increasing monotonicity in the left argument can be defined analogously.
} 
Definition 1: Let $Q$ be a determiner, then $Q$ is increasing in the right argument iff for all $A, B, B^{\prime}, B \leq B^{\prime} \Rightarrow Q(A)(B) \leq Q(A)\left(B^{\prime}\right)$.

Note that an equivalent definition can be obtained by replacing both occurrences of " $\leq$ " by " $\geq$ " in the above definition. Based on Definition 1, we may denote increasing monotonicity figuratively by " $\leq \rightarrow \leq$ " (or equivalently " $\geq \rightarrow \geq$ "). Analogously, decreasing monotonicity may be denoted figuratively by " $\leq \rightarrow \geq$ " (or equivalently " $\geq \rightarrow \leq$ "). Now " $\leq$ " and " $\geq$ " are just two possible relations between sets / propositions. If we replace them by more general binary relations $R_{1}, R_{2}$ (written in prefix notation), we will obtain a more general definition ${ }^{2}$.

Definition 2: Let $Q$ be a determiner, then $Q$ is $R_{1} \rightarrow R_{2}$ in the right argument iff for all $A, B, B^{\prime}, R_{1}\left[B, B^{\prime}\right] \Rightarrow R_{2}\left[Q(A)(B), Q(A)\left(B^{\prime}\right)\right]$.

Hence, increasing and decreasing monotonicites are just special cases of Definition 2 with $R_{1}$ and $R_{2}$ instantiated as the "inclusion relations", i.e. $\leq$ and $\geq$.

Apart from "inclusion relations", we may also consider "exclusion relations". In this paper, we will consider two "exclusion relations" that are disjunctions of relations in the classical square of opposition: CC (standing for "contrary or contradictory") and SC (standing for "subcontrary or contradictory"). In classical logic, two propositions $p$ and $q$ satisfy the $\mathrm{CC}$ relation (denoted $\mathrm{CC}[p$, $q]$ iff they cannot be both true, and they satisfy the SC relation (denoted $\mathrm{SC}[p$, q]) iff they cannot be both false. More generally, we may adopt the following definitions so that these two relations are also applicable to sets: let $X$ and $X^{\prime}$ be sets or propositions,

$$
\mathrm{CC}\left[X, X^{\prime}\right] \Leftrightarrow X \leq \neg X^{\prime} ; \mathrm{SC}\left[X, X^{\prime}\right] \Leftrightarrow \neg X \leq X^{\prime}
$$

For example, we have CC[TEENAGER, ELDERLY] and SC[AGED-OVER50, AGED-BELOW-51] because an individual cannot be a teenager and an elderly simultaneously, whereas an individual must be either aged over 50 or aged below 51. According to (1), we also have

$$
\mathrm{CC}\left[X, X^{\prime}\right] \Leftrightarrow \mathrm{SC}\left[\neg X, \neg X^{\prime}\right]
$$

By instantiating $R_{1}$ and $R_{2}$ in Definition 2 as $\mathrm{CC}$ and $\mathrm{SC}$, we then have 4 possible properties of determiners: $\mathrm{CC} \rightarrow \mathrm{CC}, \mathrm{CC} \rightarrow \mathrm{SC}, \mathrm{SC} \rightarrow \mathrm{CC}$ and $\mathrm{SC} \rightarrow \mathrm{SC}$. These 4 properties will henceforth be called "opposition properties" (OPs).

\section{OPs of Determiners}

Our next task is to classify some commonly used determiners according to their OPs in the two arguments. For convenience, I will denote the sets of determiners possessing or not possessing a certain OP in a certain argument by placing a "+" or "-" sign on the left and right-hand sides of the name of the OP. For

\footnotetext{
${ }^{2}$ Similar property in the left argument can be defined analogously.
} 
instance, $-\mathrm{CC} \rightarrow \mathrm{CC}+$ denotes the set of those determiners that are $\mathrm{CC} \rightarrow \mathrm{CC}$ in the right but not left argument.

In what follows I first state and prove four propositions:

Proposition 1: A determiner $Q$ possesses a certain OP in its right argument iff each of its outer negation (denoted $\neg Q$ ), (right) inner negation (denoted $Q \neg$ ) and (right) dual (denoted $\left.Q^{d}\right)^{3}$ possesses a different OP in its right argument according to the following table:

\begin{tabular}{|c|c|c|c|}
\hline $\mathbf{Q}$ & $\neg \mathbf{Q}$ & $\mathbf{Q} \neg$ & $\mathbf{Q}^{\mathbf{d}}$ \\
\hline $\mathrm{CC} \rightarrow \mathrm{CC}$ & $\mathrm{CC} \rightarrow \mathrm{SC}$ & $\mathrm{SC} \rightarrow \mathrm{CC}$ & $\mathrm{SC} \rightarrow \mathrm{SC}$ \\
\hline $\mathrm{CC} \rightarrow \mathrm{SC}$ & $\mathrm{CC} \rightarrow \mathrm{CC}$ & $\mathrm{SC} \rightarrow \mathrm{SC}$ & $\mathrm{SC} \rightarrow \mathrm{CC}$ \\
\hline $\mathrm{SC} \rightarrow \mathrm{CC}$ & $\mathrm{SC} \rightarrow \mathrm{SC}$ & $\mathrm{CC} \rightarrow \mathrm{CC}$ & $\mathrm{CC} \rightarrow \mathrm{SC}$ \\
\hline $\mathrm{SC} \rightarrow \mathrm{SC}$ & $\mathrm{SC} \rightarrow \mathrm{CC}$ & $\mathrm{CC} \rightarrow \mathrm{SC}$ & $\mathrm{CC} \rightarrow \mathrm{CC}$ \\
\hline
\end{tabular}

Proof: Here we only prove the first row of the table. The remaining rows can be proved similarly. By Definition 2 and (1), $Q \in \mathrm{CC} \rightarrow \mathrm{CC}+$ iff

$$
\mathrm{CC}\left[B, B^{\prime}\right] \Rightarrow Q(A)(B) \leq \neg\left(Q(A)\left(B^{\prime}\right)\right)
$$

Now (3) is equivalent to

$$
\mathrm{CC}\left[B, B^{\prime}\right] \Rightarrow \neg(\neg Q(A)(B)) \leq \neg Q(A)\left(B^{\prime}\right)
$$

Substituting the arbitrary sets $B$ and $B^{\prime}$ by their negations and using (2) and the definitions of (right) inner negation and (right) dual, (3) and (4) can be rewritten as

$$
\begin{gathered}
\mathrm{SC}\left[B, B^{\prime}\right] \Rightarrow Q \neg(A)(B) \leq \neg\left(Q \neg(A)\left(B^{\prime}\right)\right) \\
\mathrm{SC}\left[B, B^{\prime}\right] \Rightarrow \neg\left(Q^{d}(A)(B)\right) \leq Q^{d}(A)\left(B^{\prime}\right)
\end{gathered}
$$

From (4)-(6), we have $\neg Q \in \mathrm{CC} \rightarrow \mathrm{SC}+, Q \neg \in \mathrm{SC} \rightarrow \mathrm{CC}+$ and $Q^{d} \in \mathrm{SC} \rightarrow \mathrm{SC}+$.

Proposition 2: Let $Q_{1}$ and $Q_{2}$ be determiners such that $Q_{1} \leq Q_{2}{ }^{4}$.

(a) If $Q_{2}$ is $\mathrm{CC} \rightarrow \mathrm{CC}(\mathrm{SC} \rightarrow \mathrm{CC})$ in an argument, then so is $Q_{1}$ in the same argument.

(b) If $Q_{1}$ is $\mathrm{CC} \rightarrow \mathrm{SC}(\mathrm{SC} \rightarrow \mathrm{SC})$ in an argument, then so is $Q_{2}$ in the same argument.

\footnotetext{
${ }^{3}$ Outer negation, (right) inner negation and (right) dual are as defined in Peters and Westerståhl (2006).

${ }^{4} Q_{1} \leq Q_{2}$ iff for all $A, B, Q_{1}(A)(B) \leq Q_{2}(A)(B)$. From this we can define the following: $Q_{1}=Q_{2}$ iff $Q_{1} \leq Q_{2}$ and $Q_{2} \leq Q_{1}$.
} 
Proof: Here we only prove part (a). Part (b) can be proved similarly. Suppose $Q_{2} \in \mathrm{CC} \rightarrow \mathrm{CC}+$ and $\mathrm{CC}\left[B, B^{\prime}\right]$. Let $\left\|Q_{1}(A)(B)\right\|=1$. Then since $Q_{1} \leq Q_{2}$, we have $\left\|Q_{2}(A)(B)\right\|=1$. But then we must have $\left\|Q_{2}(A)\left(B^{\prime}\right)\right\|=0$. By $Q_{1} \leq$ $Q_{2}$ again, we have $\left\|Q_{1}(A)\left(B^{\prime}\right)\right\|=0$. We have thus proved that $\mathrm{CC}\left[B, B^{\prime}\right] \Rightarrow$ $\mathrm{CC}\left[Q_{1}(A)(B), Q_{1}(A)\left(B^{\prime}\right)\right]$, i.e. $Q_{1} \in \mathrm{CC} \rightarrow \mathrm{CC}+$. The proofs for the cases $Q_{2} \in$ $+\mathrm{CC} \rightarrow \mathrm{CC}, \mathrm{SC} \rightarrow \mathrm{CC}+$ and $+\mathrm{SC} \rightarrow \mathrm{CC}$ are exactly the same.

Proposition 3: Let $Q_{1}$ be a symmetric determiner and $Q_{2}$ be a contrapositive determiner ${ }^{5}$.

(a) $Q_{1}$ possesses a certain $\mathrm{OP}$ in an argument iff $Q_{1}$ possesses the same OP in the other argument.

(b) $Q_{2}$ is $\mathrm{CC} \rightarrow \mathrm{CC}$ in an argument iff $Q_{2}$ is $\mathrm{SC} \rightarrow \mathrm{CC}$ in the other argument. $Q_{2}$ is $\mathrm{CC} \rightarrow \mathrm{SC}$ in an argument iff $Q_{2}$ is $\mathrm{SC} \rightarrow \mathrm{SC}$ in the other argument.

Proof: Here we only prove part (b). Part (a) can be proved similarly. Suppose $Q_{2} \in \mathrm{CC} \rightarrow \mathrm{CC}+$ and $\mathrm{SC}\left[A, A^{\prime}\right]$, which by $(2)$ is equivalent to $\mathrm{CC}\left[\neg A, \neg A^{\prime}\right]$. Let $\left\|Q_{2}(A)(B)\right\|=1$. By the contrapositivity of $Q_{2}$, this is equivalent to $\| Q_{2}(\neg B)$ $(\neg A) \|=1$. But then we must have $\left\|Q_{2}(\neg B)\left(\neg A^{\prime}\right)\right\|=0$. By the contrapositivity of $Q_{2}$ again, this is in turn equivalent to $\left\|Q_{2}\left(A^{\prime}\right)(B)\right\|=0$. We have thus proved that $\mathrm{SC}\left[A, A^{\prime}\right] \Rightarrow \mathrm{CC}\left[Q_{2}(A)(B), Q_{2}\left(A^{\prime}\right)(B)\right]$, i.e. $Q_{2} \in+\mathrm{SC} \rightarrow$ CC. Similarly, we can prove that if $Q_{2} \in+\mathrm{SC} \rightarrow \mathrm{CC}$, then $Q_{2} \in \mathrm{CC} \rightarrow \mathrm{CC}+$. The proofs for the cases $Q_{2} \in+\mathrm{CC} \rightarrow \mathrm{CC}, \mathrm{CC} \rightarrow \mathrm{SC}+$ and $+\mathrm{CC} \rightarrow \mathrm{SC}$ are exactly the same.

Proposition 4: On condition that $A \neq \emptyset^{6}$, (at least $r$ of $) \in \mathrm{CC} \rightarrow \mathrm{CC}+$ for $1 / 2<r<1 ;$ (more than $r$ of $) \in \mathrm{CC} \rightarrow \mathrm{CC}+$ for $1 / 2 \leq r<1 ;$ (exactly $r$ of $) \in$ $-\mathrm{CC} \rightarrow \mathrm{CC}$ for $0 \leq r \leq 1$.

Proof: Let $\|($ at least $r$ of $)(A)(B) \|=1$ for $1 / 2<r<1$ and $\mathrm{CC}\left[B, B^{\prime}\right]$. Then by (1), $B \leq \neg B^{\prime}$. Since (at least $r$ of) is right increasing, we have $\|$ (at least $r$ of $)(A)\left(\neg B^{\prime}\right) \|=1$, which is equivalent to $\|($ at most $1-r$ of $)(A)\left(B^{\prime}\right) \|=1$. Since $1 / 2<r<1$, this entails $\|($ less than $r$ of $)(A)\left(B^{\prime}\right) \|=1$, which is equivalent to $\|($ at least $r$ of $)(A)\left(B^{\prime}\right) \|=0$. We have thus shown that $\mathrm{CC}\left[B, B^{\prime}\right] \Rightarrow \mathrm{CC}[($ at least $r$ of $)(A)(B)$, (at least $r$ of $)(A)\left(B^{\prime}\right)$ ], i.e. (at least $r$ of $) \in \mathrm{CC} \rightarrow \mathrm{CC}+$ for $1 / 2<r<1$. The fact that (more than $r$ of $) \in \mathrm{CC} \rightarrow \mathrm{CC}+$ for $1 / 2 \leq r<1$ can be proved similarly.

To prove that (exactly $r$ of $) \in-\mathrm{CC} \rightarrow \mathrm{CC}$ for $0 \leq r \leq 1$, we devise a method for constructing counterexample models. Choose natural numbers $x$ and $y$ such that $x / y=r$. Construct two sets $A$ and $A^{\prime}$ such that $|A|=\left|A^{\prime}\right|=y$ and $A \cap A^{\prime}=$ $\emptyset$. Choose a subset $X$ of $A$ and a subset $X^{\prime}$ of $A^{\prime}$ such that $|X|=\left|X^{\prime}\right|=x$. Then set $U=A \cup A^{\prime}$ and $B=X \cup X^{\prime}$. It is easy to check that under this model,

\footnotetext{
${ }^{5}$ Symmetry and contrapositivity are as defined in Zuber (2006).

${ }^{6}$ In what follows, we assume that the truth condition of a proportional determiner involves $|A|$ in the denominator, e.g. $\|$ (at least $r$ of $)(A)(B) \|=1 \Leftrightarrow|A \cap B| /|A| \geq r$. Thus when $A=\emptyset$, these proportional determiners have no truth values.
} 
we have $\mathrm{CC}\left[A, A^{\prime}\right]$ and $\|($ exactly $r$ of $)(A)(B)\|=\|($ exactly $r$ of $)\left(A^{\prime}\right)(B) \|=1$. In other words, we do not have $\mathrm{CC}\left[(\right.$ exactly $r$ of $)(A)(B),($ exactly $r$ of $\left.)\left(A^{\prime}\right)(B)\right]$, thus completing the proof.

Based on the above propositions, we can now determine the OPs of some commonly used determiners. For example, let $1 / 2<r<1$ and $A \neq \emptyset$, then since (exactly $r$ of $) \leq$ (at least $r$ of), by Proposition 4 and Proposition 2(a), we immediately have (exactly $r$ of $)$, (at least $r$ of $) \in-\mathrm{CC} \rightarrow \mathrm{CC}+$. Similarly, since (exactly $r$ of $) \leq$ (more than $r-\varepsilon$ of $)$ where $\varepsilon$ represents an infinitesimal quantity such that $1 / 2 \leq r-\varepsilon<1$, by Proposition 4 and Proposition 2(a) again, we have (more than $r-\varepsilon$ of $) \in-\mathrm{CC} \rightarrow \mathrm{CC}+$. Replacing the arbitrary variable $r-\varepsilon$ by $r$, we can rewrite the last result as (more than $r$ of $) \in-\mathrm{CC} \rightarrow \mathrm{CC}+$ for $1 / 2 \leq r<1$. From the above, we can derive even more results.

Since the outer negation of "(at least $r$ of $)$ " is "(less than $r$ of )", by Proposition 1 , we have (less than $r$ of $) \in-\mathrm{CC} \rightarrow \mathrm{SC}+$ for $1 / 2<r<1$. Moreover, since the (right) inner negation and (right) dual of "(at least $r$ of )" are "(at most $1-r$ of )" and "(more than $1-r$ of )", respectively, by Proposition 1 again, we have (at most $1-r$ of $) \in-\mathrm{SC} \rightarrow \mathrm{CC}+$ and (more than $1-r$ of $) \in-\mathrm{SC} \rightarrow \mathrm{SC}+$ for $0<1-r<1 / 2$. Replacing the arbitrary variable $1-r$ by $r$, we can rewrite the last results as (at most $r$ of $) \in-\mathrm{SC} \rightarrow \mathrm{CC}+$ and (more than $r$ of $) \in-\mathrm{SC} \rightarrow \mathrm{SC}+$ for $0<r<1 / 2$.

A similar analysis for "(more than $r$ of $)$ " yields the following results: (at most $r$ of $) \in-\mathrm{CC} \rightarrow \mathrm{SC}+$ for $1 / 2 \leq r<1$; (less than $r$ of $) \in-\mathrm{SC} \rightarrow \mathrm{CC}+$ and (at least $r$ of $) \in-\mathrm{SC} \rightarrow \mathrm{SC}+$ for $0<r \leq 1 / 2$.

For "(exactly $r$ of $)$ ", its (right) inner negation may take two forms: "(all except $r$ of $)$ " and "(exactly $1-r$ of $)$ ". Thus, by Proposition 1, we have (all except $r$ of $) \in-\mathrm{SC} \rightarrow \mathrm{CC}+$ for $1 / 2<r<1$ and (exactly $1-r$ of $) \in-\mathrm{SC} \rightarrow \mathrm{CC}+$ for $0<1-r<1 / 2$. Replacing the arbitrary variable $1-r$ by $r$, we can rewrite the last result as (exactly $r$ of $) \in-\mathrm{SC} \rightarrow \mathrm{CC}+$ for $0<r<1 / 2$. Based on the last result and using the fact that the (right) inner negation of "(exactly $r$ of $)$ " is "(all except $r$ of )" and Proposition 1 again, we obtain (all except $r$ of $) \in-\mathrm{CC} \rightarrow \mathrm{CC}+$ for $0<r<1 / 2$.

We next consider the classical determiner "some". We have the relation: (at least $r$ of $) \leq$ some for $0<r \leq 1 / 2^{7}$, on condition that $A \neq \emptyset$. By virtue of a previous result and Proposition 2(b), we know that some $\in \mathrm{SC} \rightarrow \mathrm{SC}+$ on condition that $A \neq \emptyset$. Note that this condition is essential because when $A=\emptyset,\|\operatorname{some}(\emptyset)(B)\|=0$ for any $B$, and so we can never have $\operatorname{SC}\left[B, B^{\prime}\right]$ $\Rightarrow \neg$ some $(\emptyset)(B) \leq \operatorname{some}(\emptyset)\left(B^{\prime}\right)$. In other words, some $\notin \mathrm{SC} \rightarrow \mathrm{SC}+$ when $A=\emptyset$. As for the left argument of "some", by the symmetry of "some" and Proposition 3(a), we know that some $\in+\mathrm{SC} \rightarrow \mathrm{SC}$ subject to certain condition. One can easily find that this condition is $B \neq \emptyset$. The above facts will be represented

\footnotetext{
${ }^{7}$ Although we also have (at least $r$ of $) \leq$ some for $1 / 2<r<1$, (at least $r$ of) $\in$ $\mathrm{CC} \rightarrow \mathrm{CC}+$ in this range and this fact cannot be used to derive the OP of "some" by virtue of Proposition 2 .
} 
succinctly by: some $\in+\mathrm{SC} \rightarrow \mathrm{SC}+(B \neq \emptyset ; A \neq \emptyset)^{8}$. By using a similar line of reasoning and the fact that "no" is the outer negation of "some" and is a symmetric determiner, we can find that $n o \in+\mathrm{SC} \rightarrow \mathrm{CC}+(B \neq \emptyset ; A \neq \emptyset)$.

We next consider "every". Since "every" is the (right) dual of "some", by Proposition 1, we know that every $\in \mathrm{CC} \rightarrow \mathrm{CC}+$ subject to certain condition. This condition is $A \neq \emptyset$ because when $A=\emptyset$, $\|$ every $(\emptyset)(B) \|=1$ for any $B$, and so we can never have $\mathrm{CC}\left[B, B^{\prime}\right] \Rightarrow \operatorname{every}(\emptyset)(B) \leq \neg$ every $(\emptyset)\left(B^{\prime}\right)$. As for the left argument of "every", since "every" is contrapositive according to Zuber (2006), by Proposition 3(b), we know that every $\in+\mathrm{SC} \rightarrow \mathrm{CC}$ subject to certain condition. This condition is $B \neq U$ because when $B=U,\|\operatorname{every}(A)(U)\|=1$ for any $A$, and so we can never have $\mathrm{SC}\left[A, A^{\prime}\right] \Rightarrow \operatorname{every}(A)(U) \leq \neg$ every $\left(A^{\prime}\right)(U)$. The above facts will be represented succinctly by: every $\in+\mathrm{SC} \rightarrow \mathrm{CC} \cap \mathrm{CC} \rightarrow \mathrm{CC}+$ $(B \neq U ; A \neq \emptyset)$. By using a similar line of reasoning and the fact that " $n o t$ every)" is the outer negation of "every" and is a contrapositive determiner, we can find that (not every) $\in+\mathrm{SC} \rightarrow \mathrm{SC} \cap \mathrm{CC} \rightarrow \mathrm{SC}+(B \neq U ; A \neq \emptyset)$.

Note that in the above, we have only considered the case that a determiner possesses a single OP in its left / right argument. It is logically possible that a determiner may possess more than one OP in one or both of its arguments. However, to explore this possibility we would need more sophisticated notions and so this will be left for future studies.

Based on the above results, we can now derive valid inferential relations between quantified statements. For example, the following are instances exemplifying the facts every $\in \mathrm{CC} \rightarrow \mathrm{CC}+$ and some $\in \mathrm{SC} \rightarrow \mathrm{SC}+$ on condition that $A \neq \emptyset$ (given that CC[TEENAGER, ELDERLY], SC[AGED-OVER-50, AGEDBELOW-51]):

Example 1: (Condition: There is some member in this club.) CC[every member of this club is a teenager, every member of this club is an elderly]

Example 2: (Condition: There is some member in this club.) SC[some member of this club is aged over 50, some member of this club is aged below 51]

\section{OPs of Iterated Quantifiers}

An adequate theory on opposition inferences should achieve the following. Given an iterated quantifier composed of $\mathrm{n}$ constituent determiners ${ }^{9}$ in the form:

$$
Q_{1}\left(A_{1}\right)\left(\left\{x_{1}: \ldots Q_{n}\left(A_{n}\right)\left(\left\{x_{n}: B\left(x_{1}, \ldots x_{n}\right)\right\}\right) \ldots\right\}\right)
$$

\footnotetext{
${ }^{8}$ The conditions $B \neq \emptyset ; A \neq \emptyset$ are ordered such that the first (second) condition corresponds to the first (second) occurrence of " + " in $+\mathrm{SC} \rightarrow \mathrm{SC}+$.

${ }^{9}$ Iterated quantifiers refer to polyadic quantifiers constructed from monadic quantifiers by "iteration" (Peters and Westerståhl (2006)). In this paper we only consider iterated quantifiers constructed from determiners.
} 
we hope to determine the OPs of this iterated quantifier in the predicates $A_{1}, \ldots A_{n}, B$ based on the OPs of $Q_{1}, \ldots Q_{n}$. To this end, we first define the notion of "OP-chain":

Definition 3: Let $X$ be a predicate under an iterated quantifier. Suppose $X$ is within the $i_{k}$ argument of $Q_{k}(1 \leq k \leq n), i_{k-1}$ argument of $Q_{k-1}, \ldots i_{1}$ argument of $Q_{1}$, where each of $i_{k}, i_{k-1}, \ldots i_{1}$ is one of $\{$ left, right $\}$ and $Q_{k}, Q_{k-1}, \ldots Q_{1}$ are constituent determiners of the iterated quantifier ordered from the innermost to the outermost layers. Then $X$ has an OP-chain $\left\langle R_{k}, R_{k-1}, \ldots R_{0}\right\rangle$, where each of $R_{k}, R_{k-1}, \ldots R_{0}$ is one of $\{\mathrm{CC}, \mathrm{SC}\}$, iff $Q_{k}$ is $R_{k} \rightarrow R_{k-1}$ in the $i_{k}$ argument, $Q_{k-1}$ is $R_{k-1} \rightarrow R_{k-2}$ in the $i_{k-1}$ argument, ... $Q_{1}$ is $R_{1} \rightarrow R_{0}$ in the $i_{1}$ argument.

For instance, in the following iterated quantifier:

$$
\text { (at most 1/2 of })\left(A_{1}\right)\left(\left\{x_{1}: \operatorname{no}\left(A_{2}\right)\left(\left\{x_{2}: B\left(x_{1}, x_{2}\right)\right\}\right)\right\}\right)
$$

$A_{2}$ is within the left argument of "no" and right argument of "(at most 1/2 of )". Since $n o \in+\mathrm{SC} \rightarrow \mathrm{CC}$ on condition that its right argument is non-empty and (at most 1/2 of $) \in \mathrm{CC} \rightarrow \mathrm{SC}+$ on condition that its left argument is non-empty, $A_{2}$ has an OP-chain $\langle\mathrm{SC}, \mathrm{CC}, \mathrm{SC}\rangle$ on condition that $A_{1} \neq \emptyset \wedge\left\{x_{2}: B\left(x_{1}, x_{2}\right)\right\} \neq \emptyset$. Similarly, one can easily check that $B$ has an OP-chain $\langle\mathrm{SC}, \mathrm{CC}, \mathrm{SC}\rangle$ on condition that $A_{1} \neq \emptyset \wedge A_{2} \neq \emptyset$, while $A_{1}$ has no OP-chain.

To facilitate the discussion below, we first state a proposition.

Proposition 5: Let $P\left(x_{1}, \ldots x_{n}\right)$ and $P^{\prime}\left(x_{1}, \ldots x_{n}\right)$ be $n$-ary predicates and $\mathrm{R}$ be one of $\{\mathrm{CC}, \mathrm{SC}\}$, then $\mathrm{R}\left[P, P^{\prime}\right] \Rightarrow \mathrm{R}\left[\left\{x_{i}: P\left(y_{1}, \ldots y_{i-1}, x_{i}, y_{i+1}, \ldots y_{n}\right)\right\},\left\{x_{i}\right.\right.$ : $\left.\left.P^{\prime}\left(y_{1}, \ldots y_{i-1}, x_{i}, y_{i+1}, \ldots y_{n}\right)\right\}\right]$ for any $1 \leq i \leq n$ and any particular set of $y_{1}, \ldots y_{i-1}, y_{i+1}, \ldots y_{n}$.

Proof: Here we only prove the case in which $\mathrm{R}=\mathrm{CC}$. The case in which $\mathrm{R}=$ $\mathrm{SC}$ is similar. Suppose $\mathrm{CC}\left[P, P^{\prime}\right]$. By (1), this is equivalent to $P \leq \neg P^{\prime}$. Then for any particular set of $y_{1}, \ldots y_{i-1}, y_{i+1}, \ldots y_{n}$ and any arbitrary $x_{i}$, we have $P\left(y_{1}, \ldots y_{i-1}, x_{i}, y_{i+1}, \ldots y_{n}\right) \leq \neg P^{\prime}\left(y_{1}, \ldots y_{i-1}, x_{i}, y_{i+1}, \ldots y_{n}\right)$, and so we have $\left\{x_{i}: P\left(y_{1}, \ldots y_{i-1}, x_{i}, y_{i+1}, \ldots y_{n}\right)\right\} \leq\left\{x_{i}: \neg P^{\prime}\left(y_{1}, \ldots y_{i-1}, x_{i}, y_{i+1}, \ldots y_{n}\right)\right\}$. But $\left\{x_{i}: \neg P^{\prime}\left(y_{1}, \ldots y_{i-1}, x_{i}, y_{i+1}, \ldots y_{n}\right)\right\}$ can be rewritten as $\neg\left\{x_{i}: P^{\prime}\left(y_{1}, \ldots\right.\right.$ $\left.\left.y_{i-1}, x_{i}, y_{i+1}, \ldots y_{n}\right)\right\}$. Thus, by (1) again, we have $\operatorname{CC}\left[\left\{x_{i}: P\left(y_{1}, \ldots y_{i-1}, x_{i}\right.\right.\right.$, $\left.\left.\left.y_{i+1}, \ldots y_{n}\right)\right\},\left\{x_{i}: P^{\prime}\left(y_{1}, \ldots y_{i-1}, x_{i}, y_{i+1}, \ldots y_{n}\right)\right\}\right]$.

We can now deduce a condition for determining the OPs of the iterated quantifier (7) in its predicates based on the OPs of its constituent determiners. We focus on the predicate $B$ (the other predicates can be similarly treated). Let $B$ have an OP-chain $\left\langle R_{n}, R_{n-1}, R_{n-2}, \ldots R_{0}\right\rangle$ and $R_{n}\left[B, B^{\prime}\right]$. By Proposition 5 , we have $R_{n}\left[\left\{x_{n}: B\left(x_{1}, \ldots x_{n}\right)\right\},\left\{x_{n}: B^{\prime}\left(x_{1}, \ldots x_{n}\right)\right\}\right]$ for any $x_{1}, \ldots x_{n-1}$. Moreover, by the definition of OP-chain, $Q_{n}$ is $R_{n} \rightarrow R_{n-1}$ in the argument $\left\{x_{n}\right.$ : $\left.B\left(x_{1}, \ldots x_{n}\right)\right\}$, and so we have $R_{n-1}\left[Q_{n}\left(A_{n}\right)\left(\left\{x_{n}: B\left(x_{1}, \ldots x_{n}\right)\right\}\right), Q_{n}\left(A_{n}\right)\left(\left\{x_{n}\right.\right.\right.$ : 
$\left.\left.\left.B^{\prime}\left(x_{1}, \ldots x_{n}\right)\right\}\right)\right]$. The above reasoning can be seen as a kind of "upward derivation": from the $R_{n}$ relation at the $B$-level, we derive the $R_{n-1}$ relation at the $Q_{n^{-}}$ level. Now $Q_{n}\left(A_{n}\right)\left(\left\{x_{n}: B\left(x_{1}, \ldots x_{n}\right)\right\}\right)$ can be seen as an $(n-1)$-ary predicate (with $x_{1}, \ldots x_{n-1}$ as arguments). Thus, we can carry out the aforesaid upwardderivation again and derive a $R_{n-2}$ relation at the $Q_{n-1}$-level. The process of determining the OPs of the iterated quantifier (7) in $B$ is essentially a repetition of this upward derivation. After $n$ rounds of derivation, we will finally derive the $R_{0}$ relation at the $Q_{1}$ level. The net effect is thus $R_{n}\left(B, B^{\prime}\right) \Rightarrow R_{0}\left[Q_{1}\left(A_{1}\right)\left(\left\{x_{1}\right.\right.\right.$ : $\left.\left.\ldots Q_{n}\left(A_{n}\right)\left(\left\{x_{n}: B\left(x_{1}, \ldots x_{n}\right)\right\}\right) \ldots\right\}\right), Q_{1}\left(A_{1}\right)\left(\left\{x_{1}: \ldots Q_{n}\left(A_{n}\right)\left(\left\{x_{n}: B^{\prime}\left(x_{1}, \ldots\right.\right.\right.\right.\right.$ $\left.\left.\left.\left.\left.x_{n}\right)\right\}\right) \ldots\right\}\right)$ ], showing that the iterated quantifier is $R_{n} \rightarrow R_{0}$ in $B$.

The above derivation relies on the fact that $B$ has an OP-chain. This may not be the case either when at least one of $Q_{1}, \ldots Q_{n}$ possesses none of the OPs, or when the OPs possessed by $Q_{1}, \ldots Q_{n}$ do not form a chain. In either case, the absence of the OP-chain blocks the upward derivation. Based on the above discussion, we can thus formulate the following condition: let $X$ be a predicate under an iterated quantifier $Q$,

$$
Q \text { is } R_{k} \rightarrow R_{0} \text { in } X \text { iff } X \text { has an OP-chain }\left\langle R_{k}, \ldots R_{0}\right\rangle
$$

We now use (9) to determine the OPs of (8) in its predicates. Previously we have already found that $A_{2}$ and $B$ both have the OP-chain $\langle\mathrm{SC}, \mathrm{CC}, \mathrm{SC}\rangle$ subject to different conditions, whereas $A_{1}$ has no OP-chain. Thus, according to (9), we know that (8) is $\mathrm{SC} \rightarrow \mathrm{SC}$ in $A_{2}$ on condition that $A_{1} \neq \emptyset \wedge\left\{x_{2}: B\left(x_{1}, x_{2}\right)\right\} \neq \emptyset$ and $\mathrm{SC} \rightarrow \mathrm{SC}$ in $B$ on condition that $A_{1} \neq \emptyset \wedge A_{2} \neq \emptyset$. Moreover, (8) possesses none of the OPs in $A_{1}$. From the above result, we can derive the following (by letting $A_{1}=$ CLUB, $A_{2}=$ AGED-OVER-50, $A_{2}^{\prime}=$ AGED-BELOW-51, $B=$ ADMIT-AS-MEMBERS):

Example 3: (Condition: There is at least a club and every club admits somebody as members.) SC[at most $1 / 2$ of the clubs admit nobody aged over 50 as members, at most $1 / 2$ of the clubs admit nobody aged below 51 as members.]

The derivation process of (9) is not exclusively valid for (7). In fact the condition in (9) can also be applied to iterated quantifiers in a form different than (7). Consider the following:

$$
n o(A \cap\{x: \operatorname{some}(B)(\{y: C(x, y)\})\})(D)
$$

The above iterated quantifier represents a quantified statement whose subject contains a relative clause which is another quantified statement. Let's determine the OP of (10) in the predicate $B$ by using (9). Since $B$ falls within the left arguments of "some" and "no", which are $+\mathrm{SC} \rightarrow \mathrm{SC}$ and $+\mathrm{SC} \rightarrow \mathrm{CC}$, respectively, both on condition that their right arguments are non-empty, $B$ has an OP-chain $\langle\mathrm{SC}, \mathrm{SC}, \mathrm{CC}\rangle$. By (9), (10) is $\mathrm{SC} \rightarrow \mathrm{CC}$ in $B$ subject to the condition that $\{y: C(x, y)\} \neq \emptyset \wedge D \neq \emptyset$. From the above result, we can derive the following (by letting A = COMPANY, B = AGED-OVER-50, B' = AGED-BELOW-51, $\mathrm{C}=\mathrm{EMPLOY}, \mathrm{D}=$ GO-BANKRUPT): 
Example 4: (Condition: Every company employs somebody and some company went bankrupt.) CC[no company employing somebody aged over 50 went bankrupt, no company employing somebody aged below 51 went bankrupt]

Note that monotonicity inferences of iterated quantifiers are governed by the same condition as opposition inferences. We can define an analogous notion of MON-chain by replacing $\{\mathrm{CC}, \mathrm{SC}\}$ with $\{\leq, \geq\}$ in Definition 3 and modify the condition in (9) by replacing "OP-chain" with "MON-chain". The modified condition can then be used to determine the monotonicities of iterated quantifiers in its predicates.

For illustration, consider the iterated quantifier in (8) again. Let's determine the monotonicity of (8) in the predicate $A_{2}$. Since $A_{2}$ is within the left argument of " $n o$ " and right argument of "(at most 1/2 of)", and "no" is left decreasing while "(at most 1/2 of )" is right decreasing, $A_{2}$ has a MON-chain $\langle\leq, \geq, \leq\rangle$ (or equivalently, $\langle\geq, \leq, \geq\rangle$ ). According to the modified version of condition (9), we know that (8) is $\leq \rightarrow \leq$ (or equivalently $\geq \rightarrow \geq$ ), i.e. increasing, in $A_{2}$. This result is in accord with that obtained by using van Eijck (2007)'s "monotonicity calculus".

\section{Concluding Remarks}

According to van Eijck (2007), monotonicity inferences are an important type of inferences in modern Natural Logic. Even syllogistics, the most important type of classical inferences, are subsumable under monotonicity inferences. By proposing the study on "inferences with exclusion premises", van Benthem (2008) has opened up a new direction of studies on Natural Logic. This paper is an implementation and generalization of van Benthem (2008)'s proposal and a contribution to the studies on Natural Logic. We have proposed a number of results by which we can determine the OPs of determiners and iterated quantifiers composed of constituent determiners, and derive valid inferential relations between quantified statements.

Nevertheless, one may criticize that the inferential relations derived from the OPs of determiners are too weak. For instance, by (1) the inferential relation in Example 1 above can be rewritten as the following entailment:

Every member of this club is a teenager. $\Rightarrow$ Not every member of this club is an elderly.

Although valid, the conclusion above seems too weak because intuitively, one would expect that the proper conclusion of the above inference should be "No member of this club is an elderly".

However, entailment is not the only type of inferential relations. In some situations, we do need to establish some other types of inferential relations (such as the CC or SC relation) between sets / propositions. These situations do not only include solving logical puzzles, but also include linguistic uses. One such use is to determine the incompatibility between two sets / propositions. For instance, 
from the fact that every $\in-\mathrm{CC} \rightarrow \mathrm{CC}+{ }^{10}$, we know that "clubs every member of which is a teenager" and "clubs every member of which is an elderly" are incompatible, whereas "clubs of which every teenager is a member" and "clubs of which every elderly is a member" are not.

The determination of incompatibility can help us differentiate between entailments and implicatures, especially the "alternate-value implicatures" studied by Hirsch-berg (1975). For instance, in the following discourse, B's conclusion is a logical entailment inferred from A's utterance:

A: This is a club every member of which is a teenager.

B: So it is not a club every member of which is an elderly.

whereas in the following discourse, B's conclusion (inferred from A's utterance under suitable context) should be seen as an alternate-value implicature that is cancellable:

A: This is a club of which every teenager is a member.

B: So it is not a club of which every elderly is a member.

Note that the difference between the aforesaid two discourses is analogous to the difference between the following two discourses:

A: She is my enemy. B: So she is not your platonic friend.

A: She is my colleague. B: So she is not your platonic friend.

\section{References}

1. van Benthem, J.: A Brief History of Natural Logic. Technical Report PP-2008-05, Institute for Logic, Language and Computation (2008)

2. van Eijck, J.: Natural Logic for Natural Language. In: ten Cate, B.D. and Zeevat, H.W. (eds.), Logic, Language, and Computation; 6th International Tbilisi Symposium on Logic, Language, and Computation. Springer-Verlag, Berlin, 216-230 (2007)

3. Hirschberg, J.B.: A Theory of Scalar Implicature, PhD thesis, University of Pennsylvania (1985)

4. Icard, T.: Exclusion and Containment in Natural Language. Accepted to Studia Logica (2011)

5. MacCartney, B.: Natural Language Inference. PhD thesis, Stanford University (2009)

6. MacCartney, B. and Manning, C.D.: An extended model of natural logic. In: Proceedings of the Eighth International Conference on Computational Semantics (2009)

7. Peters, S. and Westerståhl, D.: Quantifiers in Language and Logic. Clarendon Press, Oxford (2006)

8. Zuber, R.: Symmetric and contrapositional quantifiers. Journal of Logic, Language and Information, Vol. 16, No 1, 1-13 (2006)

$\overline{10}$ Note that in Section 3, we have only established that every $\in \mathrm{CC} \rightarrow \mathrm{CC}+$. But since every $=($ exactly $0 \%$ of $)$, by Proposition 4 , we know that every $\in-\mathrm{CC} \rightarrow \mathrm{CC}$. 


\title{
Exclusive Updates! Brought to you by your local QUD
}

\author{
Elizabeth Coppock ${ }^{1}$ and David Beaver ${ }^{2}$ \\ 1 Heinrich Heine University, Düsseldorf \\ ${ }^{2}$ University of Texas at Austin
}

\begin{abstract}
We propose a dynamic semantics within a variant of standard type theory (Ty3; Beaver 2001) in which contexts include not only a common set of beliefs, but also a question under discussion (QUD) whose answers are information states consisting of world-assignment pairs, along with a strength ranking over such answers. The proposed framework satisfies several desiderata arising from the behavior of exclusives (e.g. only, just, mere and sole), including: (i) the possibility of presupposing a question; (ii) quantificational binding into such presupposed questions; (iii) the expressibility of presuppositional constraints regarding the strength ranking over the answers to the question under discussion; (iv) compositional derivation of logical forms for sentences.
\end{abstract}

\section{Introduction}

This paper offers a dynamic framework in which the contexts that are changed by Context Change Potentials contain, in addition to a set of facts and discourse referents, a question under discussion and a ranking over the answers. Its main application is in the analysis of exclusives including mere and only.

Our proposal for exclusives is based on Beaver and Clark's (2008) (semantically) static analysis of only, according to which it is presupposed that the prejacent is the weakest of the true answers to the QUD and at-issue that the prejacent is the strongest of those ( $S$ stands for an information state):

$$
\begin{aligned}
& \operatorname{ONLY}_{S}=\lambda p \cdot \lambda w: \operatorname{Min}_{S}(p)(w) \cdot \operatorname{MAX}_{S}(p)(w), \\
& \text { where } \operatorname{MiN}_{S}(p)=\lambda w \cdot \forall p^{\prime} \in \mathrm{CQ}_{S}\left[p^{\prime}(w) \rightarrow p^{\prime} \geq_{S} p\right] \\
& \text { and } \operatorname{MAX}_{S}(p)=\lambda w \cdot \forall p^{\prime} \in \mathrm{CQ}_{S}\left[p^{\prime}(w) \rightarrow p \geq_{S} p^{\prime}\right]
\end{aligned}
$$

By extending this idea to other exclusives, as proposed by Coppock and Beaver (2011), we can account for the equivalences in (2) and (3).

(2) a. John is only an employee. $\Longleftrightarrow$ b. John is a mere employee.

(3) a. Only John is an employee. $\Longleftrightarrow$ b. John is the sole employee.

We can also account for the fact that all exclusives have paraphrases with at least (expressing MIN) and at most (expressing MAX). For example, (2) entails John is at least/most an employee, and (3) entails At least/most John is an employee. 
Following Coppock and Beaver (2011), we propose that all exclusives express MAX/MIN and differ with respect to the question they presuppose. In (2), mere expresses upper and lower bounds on the true answers to the question What properties does John have?, and in (3), sole relates in the same way to the question Who is an employee? This explains why when mere is paraphrased by only, focus goes on the nominal property (as in (2)); for sole, focus goes on the subject of predication (as in (3)). Such constraints on the QUD are discourse presuppositions, which constrain the discourse context, rather than the common ground. A framework that makes it possible to express such presuppositions is therefore needed; indeed, this conclusion was reached independently by Jäger (1996) and Aloni et al. (2007) based on the apparent presupposition of a QUD by focus, and effects of questions on only's quantificational domain.

With adjectival exclusives in non-predicative constructions, these questions involve a variable that is bound by a quantifier outscoping the exclusive. For example, (4) relates to the question, What properties does $x$ have? where $x$ is the variable bound by the negative quantifier.

(4) No mere child could keep the Dark Lord from returning. [web ex.]

Evidence from NPI licensing shows, furthermore, that adjectival exclusives such as mere and sole typically take merely local scope. Generally, adjectival exclusives in e.g. the subject NP do not license NPIs in the VP: *A mere/sole child said anything, although NPIs can be licensed within the noun phrase: The *(sole/only) student who asked any questions got an A. Coppock and Beaver (2011) explain this by analyzing typical adjectival exclusives as follows:

$$
{\mathrm{G}-\mathrm{ONLY}_{S}}=\lambda P_{\langle e, p\rangle} \cdot \lambda X_{e} \cdot \mathrm{ONLY}_{S}(P(X))
$$

This entry ensures that the adjective has scope limited to the NP. Because there are $\lambda$-bound variables appearing in the argument of $\mathrm{ONLY}_{S}$, there are 'local' QUDs containing variables bound by quantifiers outscoping the exclusive. Binding into local QUDs is also necessary for only inside relative clauses:

(6) As a bilingual person I'm always running around helping everybody who only speaks Spanish. [web ex.]

The QUD that only relates to in (6) can be rendered as "What does $x$ speak?", where $x$ is bound by everybody. No extant compositional dynamic system allows for such questions, and the present work aims to fill that gap.

\section{Framework}

Because Beaver's (2001) dynamic semantics deals successfully with quantified presuppositions, we use this as starting point, and introduce QUDs and an answer strength ranking into the context.

Our ontological assumptions are as follows. We have three basic types, as in $\mathrm{Ty}_{3}$ (hence the name $\mathrm{Ty}_{3}$ ): $d$ for discourse markers (variables: $D, D^{\prime}$ ); $e$ for individuals $(x, y, z) w$ for worlds $\left(w, w^{\prime}\right)$. Complex types are built as follows: 
- Relational types: If $\tau_{1} \ldots \tau_{n}$ are types, then $\left(\tau_{1}, \ldots, \tau_{n}\right)$ is the type of an $n$-ary relation whose first element is of type $\tau_{1}$, etc. Special case:

- Set types: If $\tau$ is a type then $(\tau)$ is the type of sets (unary relations) containing elements of type $\tau$.

- Functional types: If $\sigma$ and $\tau$ are types then $\langle\sigma, \tau\rangle$ is the type of functions with domain elements type $\sigma$ and range elements of type $\tau$.

Certain complex types play an important role in the theory:

\begin{tabular}{lll}
\hline \hline Variable & Type & Interpretation \\
\hline$f, g, h$ & $\sigma=(d, e)$ & (extended) sequences (cf. Heim) \\
$I, J, K$ & $\iota=(w, \sigma)$ & information states \\
$Q$ & $(\iota)$ & questions: sets of information states \\
$R$ & $(\iota, \iota)$ & rankings \\
$S, S^{\prime}$ & $(\iota, \iota)$ & contexts \\
$C, C^{\prime}$ & $\Pi=((\iota, \iota),(\iota, \iota))$ & CCPs \\
$P, P^{\prime}$ & $\langle d, \Pi\rangle$ & lifted dynamic properties \\
\hline
\end{tabular}

The three components of the context have the following types:

- $\operatorname{INFO}_{S}$ : type $\iota=(w, \sigma)$, a set of world-assignment pairs

$-\mathrm{CQ}_{S}$ : type $(\iota)$, a set of answers, where each answer is an information state

$-\geq_{S}$ : type $(\iota, \iota)$, a partial order over information states

Under the assumption that the strength ranking does not rank answers other than those in the QUD, the QUD is recoverable from the strength ranking; it is its field (the union of its domain and its range), notated here with FIELD: ${ }^{3}$

(8) $\operatorname{FIELD}(R)=\{x \mid \exists y[y R x \vee x R y]\}$

So we define $\mathrm{CQ}_{S}$ as follows:

(9) $\quad \mathrm{CQ}_{S}=\operatorname{FIELD}\left(\geq_{S}\right)$

Likewise, the common ground is recoverable from the QUD. ${ }^{4}$ Since we are representing questions as sets of information states, we can recover the common ground by taking the union over all information states in the question:

(10) $\operatorname{INFO}_{S}=\bigcup \mathrm{CQ}_{S}=\bigcup \operatorname{FIELD}\left(\geq_{S}\right)$

Since all of the information that the context must provide is contained in the strength ranking, we can identify the context with the strength relation; $\geq_{S}=S$.

\footnotetext{
${ }^{3}$ Krifka (1999) represents alternative semantic values as relations corresponding to 'strength' in the same sense (perhaps 'argumentative value'; Ducrot 1980), and points out that the ordinary alternatives can be derived as the field of the ranking, defined as in (8).

${ }^{4}$ Jäger (1996) represents questions and information states as equivalence relations over possible worlds, which may be partial. "Hence each state nontrivially determines a certain proposition, which can be thought of as the factual knowledge shared by the conversants." That is the domain of the information state (which is the same as the field, since the information state is an equivalence relation).
} 


\section{Theory of exclusives}

Beaver and Clark's theory of only is formalized as follows (where infix notation is used for relations, with optional square brackets surrounding the relation):

(11) $\quad$ ONLY $=\lambda C \cdot\left\{\left\langle S, S^{\prime}\right\rangle \mid S[\operatorname{MIN}(C)] S \wedge S[\operatorname{MAx}(C)] S^{\prime}\right\}$

In the static definition of MAX and MIN, the denotation of the prejacent is one of the answers in the CQ. Because the meanings of sentences are CCPs, but the answers to questions are information states, we cannot use the denotation of the prejacent directly as the answer to the CQ whose strength is being compared to the strength of others. We must extract an information state from the CCP. We do this first by defining a static context that corresponds to the CCP thus:

(12) $\downarrow C=\{\langle I, J\rangle \mid\{\langle I, J\rangle\} C\{\langle I, J\rangle\}\}$

The propositional content of the prejacent is the information state corresponding to the static context corresponding to the CCP: $\mathrm{INFO}_{\downarrow}$. We then define MAX as follows: ${ }^{5}$

(13) $\operatorname{MAX}=\lambda C .\left\{\left\langle S, S^{\prime}\right\rangle \mid S^{\prime} \subseteq S \wedge \forall J \in \mathrm{CQ}_{S^{\prime}}\left[J \leq_{S^{\prime}} \mathrm{INFO}_{\downarrow C}\right]\right\}$

MIN is defined as follows:

(14) $\mathrm{MIN}=\lambda C .\left\{\left\langle S, S^{\prime}\right\rangle \mid S^{\prime} \subseteq S \wedge \forall J \in \mathrm{CQ}_{S^{\prime}}\left[\mathrm{INFO}_{\downarrow C} \leq S_{S^{\prime}} J\right]\right\}$

In (11), ONLY is defined to take a CCP $(C)$ and return another CCP (a relation between $S$ and $S^{\prime}$ ). The presuppositional nature of the MIN component is expressed by requiring that the input state $S$ be a reflexive point with respect to MIN and $C$. In (14), MAX is defined to take a CCP $C$ and provide another CCP relating contexts $S$ and $S^{\prime}$, where the CQ in $S^{\prime}$ is a subset of the CQ in $S$ containing only information states $J$ such that $J$ is as strong (according to the strength ranking in $S$ ) as the information state corresponding to $C{ }^{6}$

We use a type-raised (Geached) version of (11) for VP-only and adjectival exclusives as in (15) ( $D$ is a variable over discourse referents, and $P$ is a variable over dynamic properties, i.e. functions from discourse referents to CCPs):

$\mathrm{G}-\mathrm{ONLY}=\lambda P \lambda D .\left\{\left\langle S, S^{\prime}\right\rangle \mid S^{\prime} \subseteq S^{\prime} \wedge S[\operatorname{ONLY}(P(D))] S^{\prime}\right\}$

The lexical entry for mere further constrains G-ONLY by requiring a certain QUD:

(16) MERE $=\lambda P \lambda D .\left\{\left\langle S, S^{\prime}\right\rangle \mid S[\mathrm{ONLY}(P(D))] S^{\prime} \wedge \mathrm{CQ}_{S} \subseteq ? P^{\prime}\left[P^{\prime}(D)\right]\right\}$

(17) If $\nu$ is a variable of type $\alpha$ and $\phi$ is a CCP: $? \nu \phi=\left\{I \mid \exists x \in \mathcal{D}_{\alpha}\left[I=\mathrm{INFO}_{\downarrow \phi[\nu \rightarrow x]}\right]\right\}$

${ }^{5}$ The requirement that $S \subseteq S^{\prime}$ makes this a declarative update in Jäger's (1996) sense. An interrogative update would not affect the information state of the context, but would change how it is divided up into smaller information states in the Current Question.

${ }^{6}$ We must also assume that there is at least one answer in the CQ in the output state in order to get the inference to the prejacent. 
(18)

$$
? P^{\prime}\left[P^{\prime}(D)\right]=\left\{I \mid \exists P \in \mathcal{D}_{\langle d, \Pi\rangle}\left[I=\operatorname{INFO}_{\downarrow P(D)}\right]\right\}
$$

This entry ensures that in mere herring, mere ranges over a scale of properties, e.g. herring, octopus, caviar. ${ }^{7}$

\section{Examples}

\subsection{Predicative example}

Let us apply these ideas to the following examples:

(19) a. $\mathrm{He}_{7}$ is a mere child.

b. $\quad \mathrm{He}_{7}$ is only a child.

The meaning of child is fundamentally a function of type $\langle e,\langle w, t\rangle\rangle$; a function that returns true given an individual and a world if the individual is a child in the world. Call this $\langle e,\langle w, t\rangle\rangle$ function CHILD'. Following Beaver (2001, p. 180) in many respects, we can make this into a dynamic unary predicate as follows:

$$
\begin{aligned}
\text { CHILD }=\lambda D .\left\{\left\langle S, S^{\prime}\right\rangle\right. & \mid D \in \text { T-DOMAIN }\left(\operatorname{INFO}_{S}\right) \wedge \operatorname{INFO}_{S^{\prime}}= \\
& \left.\left\{\langle w, f\rangle \in \operatorname{INFO}_{S} \mid \forall x\left[\langle D, x\rangle \in f \rightarrow \operatorname{CHILD}^{\prime}(x)(w)\right]\right\}\right\}
\end{aligned}
$$

where (cf. Beaver 2001, p. 168, 170):

(21) T-DOMAIN $=\lambda I .\{D \mid \forall w \forall f[\langle w, f\rangle \in I \rightarrow D \in \operatorname{DOMAIN}(f)]\}$

(22) DOMAIN $=\lambda f .\{D \mid \exists x[\langle D, x\rangle \in f]\}$

The subscript 7 on he indicates that the pronoun is associated with discourse referent 7 . Thus the denotation of (19a) is (ignoring the presupposition that the referent is male):

(23) MERE(CHILD) $(7)$

$$
\begin{aligned}
& =\left\{\left\langle S, S^{\prime}\right\rangle \mid S[\operatorname{ONLy}(\operatorname{CHILd}(7))] S^{\prime} \wedge \mathrm{CQ}_{S} \subseteq ? P^{\prime}\left[P^{\prime}(7)\right]\right\} \\
& =\left\{\left\langle S, S^{\prime}\right\rangle \mid S[\operatorname{Min}(\operatorname{CHILD}(7))] S \wedge S[\operatorname{Max}(\operatorname{CHILD}(7))] S^{\prime} \wedge \mathrm{CQ}_{S} \subseteq ? P^{\prime}\left[P^{\prime}(7)\right]\right\}
\end{aligned}
$$

The denotation of (19b) is just slightly less specific, and it will turn out to be equivalent in this context: G-ONLY $(\mathrm{CHILD})(7)=\operatorname{ONLY}(\mathrm{CHILD}(7))$.

The $\left\langle S, S^{\prime}\right\rangle$ such that $S[\operatorname{MAx}(\operatorname{CHILD}(7))] S^{\prime}$ are those such that $S^{\prime} \subseteq S \wedge \forall J \in$ $\mathrm{CQ}_{S^{\prime}}\left[J \leq_{S^{\prime}} \operatorname{INFO}_{\downarrow} \operatorname{CHILD}(7)\right]$. Likewise, the $\left\langle S, S^{\prime}\right\rangle$ such that $S[\min (\operatorname{CHILD}(7))] S^{\prime}$ are those such that $S^{\prime} \subseteq S \wedge \forall J \in \mathrm{CQ}_{S^{\prime}}\left[\operatorname{INFO}_{\downarrow} \operatorname{CHILD}(7) \leq S_{S^{\prime}} 7\right] . \downarrow \operatorname{CHILD}(7)$ is the set of world-assignment pairs such that the assignment maps 7 only to individuals that are children in the world.

$$
\begin{aligned}
(24) & \downarrow \operatorname{CHILD}(7) \\
& =\{\langle I, J\rangle \mid\{\langle I, J\rangle\}[\operatorname{CHILD}(7)]\{\langle I, J\rangle\}\} \\
& =\{\langle I, J\rangle \mid \operatorname{INFO}\{\langle I, J\rangle\}[\operatorname{CHILD}(7)] \operatorname{INFO}\{\langle I, J\rangle\}\} \\
& =\{\langle I, J\rangle \mid \cup \operatorname{FiELD}(\{\langle I, J\rangle\})[\operatorname{CHILD}(7)] \cup \operatorname{FIELD}(\{\langle I, J\rangle\})\} \\
& =\{\langle I, J\rangle \mid I \cup J[\operatorname{CHILD}(7)] I \cup J\}
\end{aligned}
$$

\footnotetext{
${ }^{7}$ In contrast, the constraint placed by adjectival only requires the question to be What things have property $P$ ? where $P$ is the property denoted by the modified noun.
} 
This turns out to be $\{\langle I, J\rangle \mid \forall w \forall f[\langle w, f\rangle \in I \cup J \rightarrow \exists x[\langle 7, x\rangle \in f] \wedge \forall x[\langle 7, x\rangle \in$ $\left.\left.\left.f \rightarrow \operatorname{CHILD}^{\prime}(x)(w)\right]\right]\right\}$. The information state corresponding to this is as follows:

$$
\operatorname{INFO}_{\downarrow} \operatorname{CHILD}(7)=\left\{\langle w, f\rangle \mid \exists x[\langle 7, x\rangle \in f] \wedge \forall x\left[\langle 7, x\rangle \in f \rightarrow \operatorname{CHILD}^{\prime}(x)(w)\right]\right\}
$$

This is the information state that MIN and MAX require to be lower and upper bounds on the CQ, respectively.

Let us consider an example context. Assume that the CQ contains INFO $\downarrow$ ADULT(7) in addition to $\operatorname{INFO}_{\downarrow} \operatorname{CHILD}(7)$ (the former being set of world assignment pairs $\langle w, f\rangle$ such that $f$ maps 7 to an adult in $w)$. And the latter is stronger than the former. So the $\leq$ ranking of this state, and hence the state itself, is the following, where we abbreviate $\mathrm{INFO}_{\downarrow \mathrm{CHILD}(7)}$ as $I_{\mathrm{CHILD}(7)}$, etc.

$$
\left\{\left\langle I_{\mathrm{CHILD}(7)}, I_{\mathrm{CHILD}(7)}\right\rangle,\left\langle I_{\mathrm{ADULT}(7)}, I_{\mathrm{ADULT}(7)}\right\rangle,\left\langle I_{\mathrm{CHILD}(7)}, I_{\mathrm{ADUlT}(7)}\right\rangle\right\}
$$

This state satisfies MIN's criterion on the output state, because there are no answers in the CQ that are weaker than than $\downarrow$ CHILD(7). But it does not satisfy MAX's requirement on the output state, because there are stronger answers. So this state would be a possible input state for ONLY $(\operatorname{CHILD}(7))$, but not a possible output state. In the output state, to satisfy MAX, the possibility of 7 being an adult would have to be removed, leaving only:

$$
\left\{\left\langle I_{\mathrm{CHILD}(7)}, I_{\mathrm{CHILD}(7)}\right\rangle\right\}
$$

So, as far as MIN and MAX are concerned, (26) is a possible input state for He is a mere child and (27) is a possible output state.

Now the question is whether (26) and (27) as input and output contexts would also satisfy the CQ constraint: $\mathrm{CQ}_{S} \subseteq$ ? $P^{\prime}\left[P^{\prime}(7)\right]$, which is, again: $\{I \mid \exists P \in$ $\left.\mathcal{D}_{\langle d, \Pi\rangle}\left[I=\operatorname{INFO}_{\downarrow P(D)}\right]\right\}$. Since ADULt $\in \mathcal{D}_{\langle d, \Pi\rangle}, \operatorname{INFO}_{\downarrow} \operatorname{ADULT}(7)$ is in this set, so (26) is a possible input state for mere.

Suppose that the alternative were, instead of $\operatorname{ADULT}(7), \operatorname{CHILD}(8)$, where $\operatorname{CHILD}(8)$ is stronger than CHILD $(7)$. This would yield input and output contexts that would satisfy the MAX and MIN requirements, but it would not satisfy mere's constraint on the CQ. Note, however, that such input and output contexts would be appropriate for adjectival only, as in She is the only child (in the room).

\subsection{Argument NP example}

Now let us consider an example in which mere occurs within an argument NP:

(28) A mere child succeeded.

For this example, we need a theory of existential quantification. We will use the following definition of some (cf. Beaver 2001 p. 185), which needs to be complicated in order to deal with "internal dynamism" but is complicated enough for the present discussion.

(29) $\quad$ SOME $\left.=\lambda D \lambda P \lambda P^{\prime} .\left\{\left\langle S, S^{\prime}\right\rangle \mid \exists S_{i n} \exists S_{r e s} S[+i] S_{i n}[P(D)] S_{r e s}\left[P^{\prime}\right](D)\right] S^{\prime}\right\}$ 
where (Beaver 2001):

$(30)+=\lambda D \cdot\left\{\left\langle S, S^{\prime}\right\rangle \mid \neg D \in \mathrm{P}-\operatorname{DOMAIN}\left(\operatorname{INFO}_{S}\right)\right.$ $\left.\wedge \mathrm{INFO}_{S^{\prime}}=\left\{\langle w, f\rangle \mid \exists g\langle w, g\rangle \in \mathrm{INFO}_{S} \wedge g[\mathrm{ADD}(D)] f\right\}\right\}$

(31) P-DOMAIN $=\lambda I .\{D \mid \exists w \exists f[\langle w, f\rangle \in I \wedge D \in \operatorname{DOMAIN}(f)]\}$

$$
\begin{aligned}
\mathrm{ADD}=\lambda D\left\{\langle f, g\rangle \mid \exists x\left[g=\left\{\left\langle D^{\prime}, y\right\rangle \mid\right.\right.\right. & D^{\prime}=D \rightarrow x=y \\
& \left.\left.\left.\wedge\left(\neg D^{\prime}=D\right) \rightarrow\left\langle D^{\prime}, y\right\rangle \in f\right\}\right]\right\}
\end{aligned}
$$

So the meaning of (28) is:

(33) SOME(7)(MERE(CHILD))(SUCCEEDED)

$$
=\left\{\left\langle S, S^{\prime}\right\rangle \mid \exists S_{i n} \exists S_{\text {res }}\left[S[+7] S_{\text {in }}[\operatorname{MeRE}(\operatorname{CHILD})(7)] S_{\text {res }}[\operatorname{SuCCEEDED}(7)] S^{\prime}\right]\right\}
$$

Suppose that the model contains the individuals Alice (a), Bob (b), Charlie (c), and Dave (d) and one world $w$, where Alice and Bob are children and Charlie is an adult and Dave is frog, and Alice and Charlie succeeded.

(34) $\operatorname{ADULT}^{\prime}(c)(w), \operatorname{CHILD}^{\prime}(a)(w), \operatorname{CHILD}^{\prime}(b)(w), \operatorname{FROG}^{\prime}(d)(w)$, $\operatorname{SUCCEEDED}^{\prime}(a)(w), \operatorname{SUCCEEDED}^{\prime}(c)(w)$ and nothing else holds in $w$

The sentence is true in $w$, because there is a mere child, Alice, who succeeded.

The input to MERE(CHILD)(7) must be a context in which 7 is mapped to some individual. This is satisfied in $S_{i n}$, because 7 is added in the course of the first update. The +-formula requires that 7 is not defined in $\mathrm{INFO}_{S}$, and makes $\mathrm{INFO}_{i n}$ the set of world-sequence pairs such that the sequence maps 7 to some object. If $\operatorname{INFO}_{S}$ consists of one pair: $w$ and the empty sequence, then $\mathrm{INFO}_{\text {in }}$ contains the following pairs: $\langle w,[7 \mapsto a]\rangle,\langle w,[7 \mapsto b]\rangle,\langle w,[7 \mapsto c]\rangle,\langle w,[7 \mapsto d]\rangle$.

But we have a problem. MIN requires that there are no answers to the CQ that are weaker than $I_{\mathrm{CHILD}(7)}$. The addition of the discourse referent says nothing directly about the $\mathrm{CQ}$, but we have set things up so that taking the union of its elements must yield $\mathrm{INFO}_{i n}$ as just described. Any cover of that set of worldassignment pairs will satisfy that condition. The constraint on the CQ imposed by mere is that every answer to the CQ is an answer to the question, "what properties does 7 have?" so one possible cover would be: "7 is a child" (so 7 is mapped to a or b), "7 is an adult" (so 7 is mapped to c), "7 is a frog" (so 7 is mapped to d). If these answers are ranked in such a way that frogs and adults are at least as strong as children, then MIN's criterion is satisfied. But in most contexts, frogs are weaker than children.

The problem can be stated this way: the discourse referent is required to be new, so nothing can be known about it, but MIN requires it to be at least a child. A related problem is that we need to account for discourses like:

(35) A: Who succeeded?

B: A mere child did.

Here the CQ for B's utterance as a whole would appear to be Who succeeded? We need to have a separate local CQ for the NP. A third, related problem is that we need to account for the focus sensitivity of mere: 
(36) a. A mere PAPER by Beaver would not suffice (though a book by him would be OK).

b. A mere paper by BEAVER would not suffice (though a joint paper with Coppock would be an improvement).

Our solution is to temporarily introduce a new CQ that takes scope only within the restrictor of some, and remove it and restore the CQ to normal once we are "done," so to speak, with the restrictor, modulo any information that we have gained in the process of processing the restrictor. We propose a special mode of interpretation $\nu$ 'new', and propose the following definition for the special case where the expression $(F)$ is type $\langle d, \Pi\rangle$ :

$$
\begin{aligned}
\llbracket F \rrbracket^{\nu}=\lambda D \cdot\left\{\left\langle S, S^{\prime}\right\rangle \mid\right. & \exists S^{\prime \prime}, S^{\prime \prime \prime} \\
& \mathrm{INFO}_{S^{\prime \prime}}=\operatorname{INFO}_{S} \wedge \\
& \geq S^{\prime \prime} \subseteq \llbracket F \rrbracket^{A} \cdot D \wedge \\
& S^{\prime \prime}[\llbracket F \rrbracket(D)] S^{\prime \prime \prime} \wedge \\
& \left.\geq_{S^{\prime}}=\operatorname{RESTRICTION}\left(\geq_{S}, \operatorname{INFO}_{S^{\prime \prime \prime}}\right)\right\}
\end{aligned}
$$

where:

$-\llbracket F \rrbracket^{A}$ is the alternative semantic value of the expression $F$. We assume, following Krifka (1999), that the alternatives associated with words and phrases are (potentially) ranked, and represent them as the relations constituting the ranking. Thus, for example, $\llbracket$ child $\rrbracket^{A}$ might be $\{\langle\llbracket$ frog $\rrbracket, \llbracket$ frog $\rrbracket\rangle$, $\langle\llbracket$ frog $\rrbracket, \llbracket$ child $\rrbracket\rangle,\langle\llbracket$ child $\rrbracket, \llbracket$ child $\rrbracket\rangle,\langle\llbracket$ child $\rrbracket, \llbracket$ adult $\rrbracket\rangle,\langle\llbracket$ adult $\rrbracket, \llbracket$ adult $\rrbracket\rangle\}$. We assume a compositional mechanism for deriving alternative semantic values similar to the one proposed by Krifka (1999).

- The dot notation '.' denotes distributive function application; $\llbracket F \rrbracket^{A} \cdot D$ is the set of CCPs obtained by applying a member of $\llbracket F \rrbracket^{A}$ to $D$.

- RESTRICTION is a function that takes a ranking and an information state and returns a new ranking that is as similar as possible to the input ranking while incorporating the information in the information state. The information state will contain a subset of the worlds in the information state of the input ranking, but it might contain new variable assignments. The result should not contain any worlds that are not in the given information state, but it should include the new assignments.

The meaning of (28) becomes:

\section{(38) $\operatorname{SOME}(7)\left(\operatorname{MERE}\left(\llbracket \operatorname{child} \rrbracket^{\nu}\right)\right)(\operatorname{SuCCEEDED})$}

During the processing of the scope of some, the CQ temporarily becomes the question of what properties 7 has, and is then restored to the root-level question, presumably Who succeeded?

Updating with MAX produces a state res where stronger alternatives to $I_{\mathrm{CHILD}(7)}$ are ruled out. So $\mathrm{CQ}_{\text {res }}$ consists only of $I_{\mathrm{CHILD}(7)}$, and $\mathrm{INFO}_{\text {init }}$ consists of pairs whose first member is $w$ and whose second member is $a$ or $b$. Updating with the restrictor eliminates $\langle w,[7 \mapsto b]\rangle$ from the information state, since $b$ did not succeed. Hence, updating with B's response in (35) results in a state where 7 is mapped to a child (not a frog or an adult) who succeeded,. 


\section{$5 \quad$ NPI licensing}

Exclusives generally license NPIs within their semantic scope, but differ in scope, hence NPI licensing. Subject-modifying only takes scope over the sentence as a whole and hence licenses NPIs there, while mere has NP-scope.

(39) a. Only a child said anything.

b. $\quad *$ A mere child said anything.

The adjectival exclusives only and exclusive do not license NPIs in the VP either, although they do license NPIs in their syntactic scope:

(40) a. *The only author got any royalties.

b. The ${ }^{*}$ (only) student who asked any questions got an A.

(41) a. *The exclusive supplier of gas energy got any new contracts.

b. We are the ${ }^{*}$ (exclusive) supplier of any drink served here.

In order to account for these contrasts, we must establish that ONLY as defined above is an NPI-licesning environment. To do so, we define a dynamic notion of Strawson Downward Entailment (von Fintel, 1999) as follows:

(42) If $x \Rightarrow y$ and $f(x)$ and $f(y)$ are CCPs, then $f$ is dynamically Strawson downward-entailing iff $f(y)$ dynamically Strawson-entails $f(x)$.

(43) A CCP $C$ dynamically Strawson-entails another CCP $C^{\prime}$ iff for all $S, S^{\prime},\left[S[C] S^{\prime} \wedge S^{\prime}\right.$ ADMITs $\left.C^{\prime} \rightarrow S^{\prime}\left[C^{\prime}\right] S^{\prime}\right]$

The notion of admittance is defined thus:

(44) $\quad$ ADMits $=\left\{\langle S, C\rangle \mid \exists S^{\prime} S[C] S^{\prime}\right\}$

So our claim that ONLY produces a DSDE environment amounts to:

(45) If $x \Rightarrow y$ and $\operatorname{ONLY}(x)$ and $\operatorname{ONLY}(y)$ are CCPs, then for all $S, S^{\prime}$ :

$S[\mathrm{ONLY}(y)] S^{\prime} \wedge S[\mathrm{ADMITS}] \operatorname{ONLY}(x) \rightarrow S^{\prime}[\mathrm{ONLY}(x)] S^{\prime}$

We must also define $\Rightarrow$ in order to evaluate this claim. Because the $x$ and $y$ in our example are CCPs, we use a dynamic notion of entailment:

(46) $\quad$ entails $=\left\{\left\langle P, P^{\prime}\right\rangle \mid \forall S \forall S^{\prime}\left[S[P] S^{\prime} \rightarrow S^{\prime}[\right.\right.$ SATisfies $\left.\left.] P^{\prime}\right]\right\}$

(47) $\quad$ SATISFIES $=\{\langle S, P\rangle \mid S[P] S\}$

So what we want to show is:

(48) If $P$ [ENTAILs $] P^{\prime}$, then for all $S, S^{\prime}$ : $S[\mathrm{ONLy}(P)] S^{\prime} \wedge S[$ ADmits $] \operatorname{ONLy}\left(P^{\prime}\right) \rightarrow S^{\prime}\left[\operatorname{ONLy}\left(P^{\prime}\right)\right] S^{\prime}$ 
Let us consider two CCPs such that one ENTAILS the other: one for $\mathrm{He}_{7}$ likes kale, and another for $\mathrm{He}_{7}$ likes vegetables. Intuitively what we want to prove is that if he likes kale (the presupposition of Only he likes kale), then Only he likes vegetables implies Only he likes kale. In order to simplify things, let us represent the meaning of likes kale and likes vegetables as the $\langle e,\langle w, t\rangle\rangle$

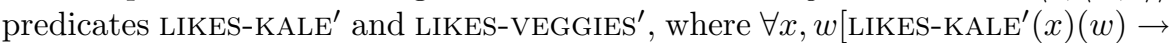
LIKES-VEGGIES $(x)(w)]$. The CCPs corresponding to He likes kale and He likes vegetables are LIKES-KALE(7) and LIKES-VEGGIES(7).

(49) LIKES-KALE(7) ENTAILS LIKES-VEGGies(7) iff: $\forall S \forall S^{\prime}\left[S[\operatorname{LiKES}-\operatorname{KALE}(7)] S^{\prime} \rightarrow S^{\prime}[\operatorname{LiKES}-\operatorname{VeggieS}(7)] S^{\prime}\right]$

It follows from what we have said above that this universal implication holds.

So if ONLY is DSDE, it should be the case that:

(50) For all $S, S^{\prime}: S[$ Only(LiKes-VEGgies(7)) $] S^{\prime} \wedge S[$ ADMits $]$ OnLy(LiKes-Kale(7)) $\rightarrow S^{\prime}[\mathrm{ONLY}(\operatorname{LiKES}-\operatorname{KALE}(7))] S^{\prime}$

The contexts that admit ONLY(LIKES-KALE(7)) are those contexts $S$ such that $S[\operatorname{Min}(\operatorname{LiKES}-\operatorname{Kale}(7))] S$, i.e. those $S$ such that $\forall J \in \mathrm{CQ}_{S}\left[I_{\text {LIKES-KALE(7) }} \leq_{S}\right.$ $J]$. Whether or not this holds of $S$ depends of course on the strength ranking over the alternatives. Because there is focus on the subject, the alternatives must correspond one-to-one with the set of individuals, and the ranking must be isomorphic to the part-of relation over individuals; so $a<a \oplus b$, etc. So stronger answers correspond to larger groups containing whatever individual 7 is mapped to. Now we learn that this individual is the only one who likes vegetables; the answers to the question of who likes vegetables are no stronger than the one corresponding to this individual. This means that there are no answers to the question of who likes kale that are stronger than the one corresponding to this individual.

Hence, ONLY as in (11) produces a dynamically Strawson Downward-Entailing environment in the sense of (42). In combination with an account of lexical type restrictions on different exclusives, which in turn predicts differences of scope, this explains differences among exclusives with respect to NPI licensing.

\section{Conclusion}

To summarize, we have proposed (i) that the meanings of sentences are CCPs that operate on contexts containing questions under discussions with ranked answers, making it possible for questions to be presupposed and for presuppositional constraints on questions and the rankings over their answers to be expressed; (ii) a mechanism for temporarily introducing 'local' questions under discussion, which enables quantificational binding into local questions; (iii) a dynamic notion of Strawson Downward Entailment, which makes it possible to capture the NPI licensing properties of exclusives. The framework is furthermore compositional, allowing for the compositional derivation of logical forms for sentences containing non-root-level question-sensitive operators. 


\section{Bibliography}

Aloni, M., Beaver, D., Clark, B., and van Rooij, R. (2007). The dynamics of topics and focus. In Aloni, M., Butler, A., and Dekker, P., editors, Questions in Dynamic Semantics, CRiSPI. Elsevier, Oxford.

Beaver, D. (2001). Presupposition and Assertion in Dynamic Semantics. CSLI Publications, Stanford.

Beaver, D. I. and Clark, B. Z. (2008). Sense and Sensitivity: How Focus Determines Meaning. Wiley-Blackwell, Chichester.

Coppock, E. and Beaver, D. (2011). Sole sisters. Paper presented at Semantics and Linguistic Theory (SALT 21).

Jäger, G. (1996). Only updates. In Dekker, P. and Stokhof, M., editors, Proceedings of the Tenth Amsterdam Colloquium, Amsterdam. ILLC, University of Amsterdam.

Krifka, M. (1999). At least some determiners aren't determiners. In Turner, K., editor, The Semantics/Pragmatics Interface from Different Points of View, pages 257-291. Elsevier, Oxford.

Krifka, M. (2001). Quantifying into question acts. Natural Language Semantics, 9:1-40.

von Fintel, K. (1999). NPI licensing, Strawson entailment, and contextdependency. Journal of Semantics, 16:97-148. 


\title{
Steedman's Temporality Proposal and Finite Automata
}

\author{
Tim Fernando \\ Trinity College Dublin, Ireland
}

\begin{abstract}
The proposal from Steedman 2005 that "the formal devices" required for temporality in linguistic semantics "are those related to representation of causality and goal-directed action" is developed using finite automata, implicit in which are notions of causality (labelled transitions) and goal-directed action (final/accepting states). Three strands in theories of aspect isolated in Binnick 2006 are examined: temporal relations, phases, and boundedness. The commonly held dichotomy between states and events is linked to that between programs and their runs, as strings representing events are extended to automata.
\end{abstract}

\section{Introduction}

In a wide-ranging study, Steedman (2005) proposes that "the so-called temporal semantics of natural language is not primarily to do with time at all" (as given say, by the real line $\mathbb{R}$ ) but that "the formal devices we need are those related to representation of causality and goal-directed action." Finite automata are simple candidates for such devices, with notions of causality and goal-directed action implicit in an automaton's transition table and accepting (final) states. Stepping back from automata, the strings which such automata may or may not accept are employed in Fernando 2004, 2008 to represent events of various kinds (for linguistic semantics). Does this step (back) not downplay and obscure, however, the notions of causality and goal-directed action offered by a finite-state approach? The present paper is an attempt to clarify these notions, linking the relationship between strings and automata to the dichotomy between events and states widely recognized (e.g. Kamp and Reyle 1993). The more general aim is to explain why Steedman's proposal - henceforth ST - is interesting, and how it might be implemented through finite-state methods $-\mathrm{ST}_{f a}$.

Motivation for ST can be found from discourse coherence, (1), down to tense and aspect, (2).

(1) Max fell. Mary pushed him.

(2) John [*has] left, but is back.

In (1), the push is most naturally understood as preceding the fall because (1) suggests a causal connection (e.g. Asher and Lascarides 2003), while in (2), the difference between the simple past and the present perfect (has left) is that under the latter, the result of John's departure persists through (2)'s utterance 
(incompatible with him back). In (1) and (2), time and its modelling by $\mathbb{R}$ are secondary to the changes or, as the case may be, non-changes that are communicated. Put crudely under $\mathrm{ST}_{f a}$, time is the result of running automata. A more moderate position is that time is conceived in ST to be relative (as opposed to absolute), the raison d'être of which is to place some set $E$ of events and states in some order. Fleshed out according to Russell and Wiener (e.g. Kamp and Reyle 1993), this relative conception of time can be brought in line with the $\mathrm{ST}_{f a}$-view of time as runtime (Fernando 2011a). Apart from their runs, however, do the automata merit a place in semantics? Consider the habitual $(3 \mathrm{a})$, in contrast to the episodic $(3 \mathrm{~b})$.

(3) a. Tess eats dal.

b. Tess is eating dal.

Not only can we assert (3a) and at the same time deny (3b), it is not entirely clear that the truth of (3a) at a world and time can be reduced to its episodic instances at that world. Under the rules-and-regulations view defended in Carlson 1995, generic sentences such as (3a) are true at a world and time only if at that world and time, "structures" exist that are not "the episodic instances but rather the causal forces behind those instances" (page 225). Can automata serve as such structures?

The present work sets out to make this plausible, based on a modelling of episodic sentences through strings representing runs of an automaton. This modelling is explained in the next two sections with an eye to the subsequent introduction (in the final section) of automata as causal forces. The plot, very briefly, is as follows. From a set $E$ of events and states (of interest), strings are formed on which a system of projections $\pi_{X}$ is defined that captures the Russell-Wiener construction for arbitrary finite subsets $X$ of $E$. Event-tokens (in $E$ ) with interval temporal extent bounded to the left by pre-states and to the right by poststates are generalized (beyond Russell-Wiener) to event-types that are analyzed in terms of temporal propositions subject to forces. Restricting the alphabet to a finite set paves the way for finite-state methods that

(i) transcend, in a precise sense recalled below, first-order logic, while keeping validity decidable, and

(ii) bounds the granularity at which meaning is described, yielding finitary fragments that contrast sharply with complete histories/possible worlds.

The resulting approach is allied with the "toolbox" conception of natural languages propounded in Cooper and Ranta 2008, centered around local use (and action), away from a monolithic truth (as in the representation of time through the real line $\mathbb{R})$.

\section{Strings for Russell-Wiener and beyond}

For quick orientation, it is instructive to represent a calendar year by the string

$$
s_{m o}:=\operatorname{Jan} \text { Feb } \cdots \text { Dec }
$$


of length 12 (with a month in each box), or (adding one of 31 days $d 1, d 2, \ldots d 31$ ) the string

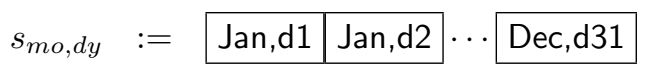

of length 365 (for a non-leap year). Unlike the points in the real line $\mathbb{R}$, a box can be split by enlarging the set $E$ of symbols we can put in it, as illustrated by

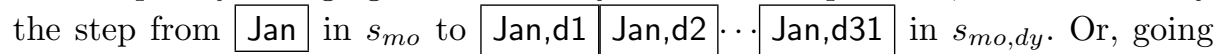
the opposite direction, from $s_{m o, d y}$ to $s_{m o}$, let us define two functions $\rho_{m o}$ and $b c$ that respectively, restricts $E$ to the months $m o=\{\mathrm{Jan}, \mathrm{Feb}, \ldots$ Dec $\}$

$$
\rho_{m o}\left(s_{m o, d y}\right)=\mathrm{Jan}^{31} \mathrm{Feb}^{28} \cdots \mathrm{Dec}^{31}
$$

and compresses a block $\alpha^{n}$ to $\alpha$

$$
b c\left(\mathrm{Jan}^{31} \mathrm{Feb}^{28} \cdots \mathrm{Dec}^{31}\right)=\mathrm{Dan}^{\mathrm{Feb}} \cdots \text { Dec }=s_{m o}
$$

so that $b c\left(\rho_{m o}\left(s_{m o, d y}\right)\right)=s_{m o}$. More precisely, for $X \subseteq E, \rho_{X}$ sees only the elements of $X$ (discarding non- $X$ 's)

$$
\rho_{X}\left(\alpha_{1} \alpha_{2} \cdots \alpha_{n}\right):=\left(\alpha_{1} \cap X\right)\left(\alpha_{2} \cap X\right) \cdots\left(\alpha_{n} \cap X\right)
$$

whereas block compression bc sees only change (discarding repetitions/stuttering)

$$
b c(s):= \begin{cases}b c\left(\alpha s^{\prime}\right) & \text { if } s=\alpha \alpha s^{\prime} \\ \alpha b c\left(\alpha^{\prime} s^{\prime}\right) & \text { if } s=\alpha \alpha^{\prime} s^{\prime} \text { with } \alpha \neq \alpha^{\prime} \\ s & \text { otherwise. }\end{cases}
$$

Let $b c_{X}$ be the composition $\rho_{X} ; b c$ mapping $s$ to

$$
b c_{X}(s):=b c\left(\rho_{X}(s)\right)
$$

so that

$$
\begin{array}{ll}
b c_{\{\mathrm{Jan}\}}\left(s_{m o, d y}\right)=b c_{\{\mathrm{Jan}\}}\left(s_{m o}\right)=\operatorname{Jan} \square & b_{\{\mathrm{d} 1\}}\left(s_{m o, d y}\right)=(\mathrm{d} 1 \square)^{12} \\
b_{\{\mathrm{Feb}\}}\left(s_{m o, d y}\right)=b c_{\{\mathrm{Feb}\}}\left(s_{m o}\right)=\mid \mathrm{Feb} & b_{\{\mathrm{d} 2\}}\left(s_{m o, d y}\right)=(\square \mathrm{d} 2)^{12} \square .
\end{array}
$$

We can delete any initial or final empty boxes by a function unpad, which we apply after $b_{X}$ to form $\pi_{X}$

$$
\pi_{X}(s):=\operatorname{unpad}\left(b_{X}(s)\right) .
$$

We can then say $e$ is an $s$-interval, and write $s \models \operatorname{interval}(e)$, if $\pi_{\{e\}}(s)$ is $e$

$$
s=\operatorname{interval}(e) \stackrel{\text { def }}{\Leftrightarrow} \pi_{\{e\}}(s)=e .
$$

Strings over the alphabet $\operatorname{Pow}(E)$ of subsets of $E$ relative to which each $e \in E$ is an interval have $\pi_{E}$-outputs in the language $\pi_{E}\left[\bigcap_{e \in E} \pi_{\{e\}}^{-1}[e]\right.$, which we abbreviate $\mathcal{L}_{\pi}(E)$

$$
\mathcal{L}_{\pi}(E):=\left\{\pi_{E}(s) \mid s \in \operatorname{Pow}(E)^{*} \text { and }(\forall e \in E) \pi_{\{e\}}(s)=e\right\} .
$$


For example, $\mathcal{L}_{\pi}(\{e\})$ consists of the single string $e$, whereas $\mathcal{L}_{\pi}\left(\left\{e, e^{\prime}\right\}\right)$ consists of 13 strings, one per interval relation in Allen 1983. Partitioning $\mathcal{L}_{\pi}\left(\left\{e, e^{\prime}\right\}\right)$ in terms of the relations $\prec$ of (complete) precedence and $\bigcirc$ of overlap used in the Russell-Wiener construction of time from $E$, we have

$$
\mathcal{L}_{\pi}\left(\left\{e, e^{\prime}\right\}\right)=\operatorname{Allen}\left(e \bigcirc e^{\prime}\right)+\operatorname{Allen}\left(e \prec e^{\prime}\right)+\operatorname{Allen}\left(e^{\prime} \prec e\right)
$$

where Allen $\left(e \bigcirc e^{\prime}\right)$ consists of the 9 strings in which $e$ overlaps $e^{\prime}$

$$
\text { Allen }\left(e \bigcirc e^{\prime}\right):=\left(e+e^{\prime}+\epsilon\right) e, e^{\prime}\left(e+e^{\prime}+\epsilon\right)
$$

(with empty string $\epsilon$ ), and Allen $\left(e \prec e^{\prime}\right)$ consists of the 2 strings in which $e$ precedes $e^{\prime}$

$$
\operatorname{Allen}\left(e \prec e^{\prime}\right):=\begin{array}{|l|l|l|l|}
\hline e & e^{\prime} & e & e^{\prime} \\
\hline
\end{array}
$$

and similarly for Allen $\left(e^{\prime} \prec e\right)$. In general, $\mathcal{L}_{\pi}(E)$ is larger than the set $\operatorname{RW}(E)$ of Russell-Wiener time orders over $E$ because no two Russell-Wiener times (within a single time order) can be related by inclusion (subset). For finite $E$, the discrepancy between $\mathrm{RW}(E)$ and $\mathcal{L}_{\pi}(E)$ can be traced to the language $\mathcal{M}(E)$ of strings over Pow $(E)^{*}$, no two symbols in which are related by inclusion

$$
\mathcal{M}(E):=\bigcup_{n \geq 1}\left\{\alpha_{1} \cdots \alpha_{n} \in \operatorname{Pow}(E)^{n} \mid \operatorname{not}(\exists i, j \in\{1, \ldots, n\}) \alpha_{i} \subset \alpha_{j}\right\} .
$$

$\mathrm{RW}(E)$ is the part of $\mathcal{L}_{\pi}(E)$ in $\mathcal{M}(E)$

$$
\left.\mathrm{RW}(E) \cong \mathcal{L}_{\pi}(E) \cap \mathcal{M}(E) \quad \text { (for finite } E\right) .
$$

To reconcile Russell-Wiener with $\mathcal{L}_{\pi}(E)$, an alternative to reducing $\mathcal{L}_{\pi}(E)$ is to neutralize the $\subseteq$-maximality requirement on RW-times by

(i) introducing for each $e \in E$, fresh "events" pre $(e)$ and post $(e)$ into an expansion $E_{ \pm}$of $E$

$$
E_{ \pm}:=E \cup\{\operatorname{pre}(e) \mid e \in E\} \cup\{\operatorname{post}(e) \mid e \in E\}
$$

and

(ii) delimiting occurrences of $e$ in a string by pre(e) to the left and by post $(e)$ to the right.

For instance, \begin{tabular}{|l|l|l|l|}
\hline & & $e^{\prime}$ & becomes
\end{tabular}

$$
\begin{array}{|l|l|l|}
\hline e, \operatorname{pre}\left(e^{\prime}\right) & \operatorname{post}(e), \operatorname{pre}\left(e^{\prime}\right) & \operatorname{post}(e), e^{\prime} .
\end{array}
$$

In general, given a string $s=\alpha_{1} \cdots \alpha_{n}$ of subsets $\alpha_{i}$ of $E$, we define its delimitation to be the string $s_{ \pm}=\alpha_{1}^{\prime} \cdots \alpha_{n}^{\prime}$ of subsets $\alpha_{i}^{\prime}$ of $E_{ \pm}$where

$$
\begin{array}{r}
\alpha_{i}^{\prime}:=\alpha_{i} \cup\left\{\operatorname{pre}(e) \mid e \in\left(\bigcup_{j=i+1}^{n} \alpha_{j}\right)-\bigcup_{j=1}^{i} \alpha_{j}\right\} \\
\cup\left\{\operatorname{post}(e) \mid e \in\left(\bigcup_{j=1}^{i-1} \alpha_{j}\right)-\bigcup_{j=i}^{n} \alpha_{j}\right\} .
\end{array}
$$




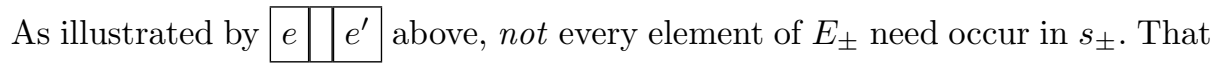
said, for every $s \in \mathcal{L}_{\pi}(E)$, if

$$
s_{ \pm}=\alpha_{1}^{\prime} \cdots \alpha_{n}^{\prime} \text { and } E_{s}:=\bigcup_{i=1}^{n} \alpha_{i}^{\prime}
$$

then for finite $E$,

(4) $s_{ \pm} \in \mathcal{L}_{\pi}\left(E_{s}\right) \cap \mathcal{M}\left(E_{s}\right)\left(\cong \operatorname{RW}\left(E_{s}\right)\right)$

as exactly one of $e, \operatorname{pre}(e)$ and post $(e)$ belongs to $\alpha_{i}^{\prime}$

$$
\left|\{e, \operatorname{pre}(e), \operatorname{post}(e)\} \cap \alpha_{i}^{\prime}\right|=1
$$

for all $e \in E$ and $1 \leq i \leq n$.

What if $E$ is infinite? In this case, we glue together approximations of $E$ given by the family $\operatorname{Fin}(E)$ of finite subsets of $E$. More precisely, $\operatorname{Fin}(E)$-indexed strings $\left(s_{X}\right)_{X \in F i n(E)}$ in which $s_{X}$ can be calculated as $\pi_{X}\left(s_{X^{\prime}}\right)$ for any $X^{\prime} \supseteq X$ are collected in the inverse limit of the system $\left(\pi_{X}\right)_{X \in F i n(E)}$ of functions

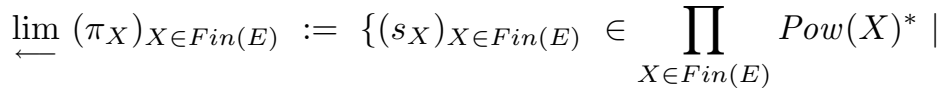

$$
\begin{aligned}
& \left.s_{X}=\pi_{X}\left(s_{X^{\prime}}\right) \text { whenever } X \subseteq X^{\prime} \in \operatorname{Fin}(E)\right\} \text {. }
\end{aligned}
$$

Every Russell-Wiener precedence relation $\prec$ on $E$ has a representation $\left(s_{X}^{\prec}\right)_{X \in F i n(E)}$ in this inverse limit with

$$
\prec=\left\{\left\langle e, e^{\prime}\right\rangle \in E \times E \mid s_{\left\{e, e^{\prime}\right\}}^{\prec} \in \operatorname{Allen}\left(e \prec e^{\prime}\right)\right\}
$$

and $s_{X}^{\prec}$ approximating the set $T_{\prec}$ of RW-times of $\prec$ on $E$ as follows. A finite subset $X$ of $E$ induces a notion $\approx_{X}$ of equivalence on $T_{\prec}$ that holds between RW-times $t$ and $t^{\prime}$ precisely if for all $e \in X$,

(i) $e \in t$ iff $e \in t^{\prime}$, and

(ii) $\left(\exists e^{\prime} \in t\right) e^{\prime} \prec e$ iff $\left(\exists e^{\prime} \in t^{\prime}\right) e^{\prime} \prec e$, and

(iii) $\left(\exists e^{\prime} \in t\right) e \prec e^{\prime}$ iff $\left(\exists e^{\prime} \in t^{\prime}\right) e \prec e^{\prime}$.

The idea is that (i) says $t$ and $t^{\prime}$ do not differ on $e$, (ii) on pre(e), and (iii) on $\operatorname{post}(e)$, as $\prec$ extends to RW-times $t$ and $t^{\prime}$ by existential quantification

$$
t \prec^{T} t^{\prime} \stackrel{\text { def }}{\Leftrightarrow}(\exists e \in t)\left(\exists e^{\prime} \in t^{\prime}\right) e \prec e^{\prime} .
$$

The number of $\approx_{X}$-equivalence classes cannot exceed the number $3^{|X|}$ of functions mapping $e \in X$ to one of $e, p r e(e)$ and post $(e)$. Whether or not $T_{\prec}$ is isomorphic to the real line $\mathbb{R}$, the relation $\prec_{X}$ on $\approx_{X}$-equivalence classes $U, U^{\prime}$ given by

$$
U \prec_{X} U^{\prime} \stackrel{\text { def }}{\Leftrightarrow}(\exists t \in U)\left(\exists t^{\prime} \in U^{\prime}\right) t \prec^{T} t^{\prime}
$$


is discrete (for $X$ finite), and representable as a string $\hat{s}$ over the alphabet $\operatorname{Pow}(X)$. Unpadding $\hat{s}$ yields the $X$-approximation $s_{X}^{\prec}:=\operatorname{unpad}(\hat{s}){ }^{1}$

The bounded granularity of $\approx_{X}$ (for finite $X$ ) is at odds with the infinite precision of real numbers, arbitrarily small increments in which lead to Sorites chains/arguments problematic for the vagueness of natural language. For the inverse limit construction above, the maps $\pi_{X}: \operatorname{Pow}(E)^{*} \rightarrow \operatorname{Pow}(X)^{*}$ can be restricted to $\pi_{X^{\prime}, X}: \operatorname{Pow}\left(X^{\prime}\right)^{*} \rightarrow \operatorname{Pow}(X)^{*}$, for $X \subseteq X^{\prime} \in \operatorname{Fin}(E)$, each of which is computable by a finite-state transducer. In other words, Russell-Wiener time can be pictured (in accordance with $\mathrm{ST}_{f a}$ ) as runtime up to a granularity bounded by the choice of a finite set of events. Enlarging the set of events refines the granularity. For instance, adding pre $(e)$ to the left of an event $e$ (to mark its past) and post (e) to its right (to mark its future) leads to refinements (suggested by Allen 1983) of the temporal relations $\bigcirc$ and $\prec$.

Given an event $e$, can we legitimately count pre $(e)$ or post $(e)$ as an event, as opposed to a state? Whether or not we can, their addition poses no obstacle to the Russell-Wiener construction. Where Russell-Wiener breaks down is over non-intervals. Not so, however, for the functions $\pi_{X^{\prime}, X}$. Although $s_{X}^{\prec} \in \mathcal{L}_{\pi}(X)$ for all $X \in \operatorname{Fin}(E)$ and Russell-Wiener precedence $\prec$ on $E$, the inverse limit above also has Fin $(E)$-indexed strings $\left(s_{X}\right)_{X \in F i n(E)}$ for which an $e \in X$ may fail to be an $s_{X}$-interval. Consider again the example of $s_{m o, d y}$ above, for which it is perfectly reasonable that $\mathrm{d} n$ is not an $s_{m o, d y}$-interval. Working with strings, we may relax the interval requirement to extend our event-based account of time into one of event-types, with events understood broadly to include states (delimiting events). In terms of Kripke semantics, Pow $(X)^{*}$ specifies not only Kripke frames (for event-tokens/time) but Kripke models with valuations for elements of $X$ construed as temporal propositions, also known as fluents. Indeed, the delimiting pre $(e)$-e-post $(e)$ configurations become the event nuclei of Moens and Steedman 1988 once we sharpen pre $(e)$ into $e$ 's preparatory process, and post $(e)$ into $e$ 's consequent state. Can we flesh out these phases with finite automata?

Regular languages (accepted by finite automata) enjoy extensive closure properties, perhaps the most striking expression of which is the Büchi-Elgot theorem characterizing regular languages in terms of Monadic Second-Order Logic (MSO; e.g. Thomas 1997). Various modal logics are expressible in MSO, includ-

${ }^{1}$ Because $\pi_{X}$ unpads (unlike $b c_{X}$ ), the string $s_{X}^{\prec}$ leaves out RW-times before all events in $X$, as well as after. We can repair this blemish by replacing $\pi_{X}$ with $b c_{X}$, as

(4) $s_{ \pm} \in \mathcal{L}_{\pi}\left(E_{s}\right) \cap \mathcal{M}\left(E_{s}\right)\left(\cong \mathrm{RW}\left(E_{s}\right)\right)$

holds for $s$ drawn not just from $\mathcal{L}_{\pi}(E)$ but from the larger language

$$
\left\{b_{E}(s) \mid s \in \operatorname{Pow}(E)^{*} \text { and }(\forall e \in E) s \models \operatorname{interval}(e)\right\}=(\square+\epsilon) \mathcal{L}_{\pi}(E)(\square+\epsilon) .
$$

An empty box $\square$ may then be attached to the start and/or end of a string in Allen $\left(e \prec e^{\prime}\right)$, and get filled upon delimiting. The projections $\pi_{X}$ abstract away information about boundedness retained by $b c_{X}$. This information is redundant if as in Allen 1983, all intervals are assumed bounded. 
ing well-known Priorean tense logics (with nominals interpretable as first-order objects, and other fluents as sets). Beyond the languages defined by truth $\models$ in these logics, there are relations such as $\pi_{X}$ that characterize not only $s$-intervals but also the temporal relations $\bullet \in\{\prec, \bigcirc, \succ\}$

$$
s=e \bullet e^{\prime} \stackrel{\text { def }}{\Leftrightarrow} \pi_{\left\{e, e^{\prime}\right\}}(s) \in \operatorname{Allen}\left(e \bullet e^{\prime}\right)
$$

(for $s$-intervals $e, e^{\prime}$ ). More in the next section, where we generalize $\pi_{X}$ to relations with outputs that may fall outside that of $\pi_{X}$ due to underspecification.

\section{Non-deterministic relations and underspecification}

The idea behind the present section is to derive satisfaction $\models$ of a formula ${ }^{2} \varphi$ from a relation $R_{\varphi}$ between strings $s$ and $s^{\prime}$ such that

$$
s R_{\varphi} s^{\prime} \quad \text { iff } \quad s^{\prime} \text { makes } \varphi \text { true at } s
$$

with inputs $s$ (fed to $R_{\varphi}$ ) as indices (to the left of $\models$ )

$$
s \models \varphi \quad \text { iff } \quad s \in \operatorname{domain}\left(R_{\varphi}\right)
$$

and outputs $s^{\prime}$ as extensions/denotations (Fernando 2011). For example, if $\varphi$ is $e \prec e^{\prime}$ then $R_{\varphi}$ can be $\pi_{\left\{e, e^{\prime}\right\}}$ restricted to outputs from Allen $\left(e \prec e^{\prime}\right)$. In stringing together sets of fluents, denotations develop the view of events as "intervals cum description" (van Benthem 1983) beyond pairs $\langle i, \varphi\rangle$ of descriptions $\varphi$ given by event-atoms (Pratt-Hartmann 2005) and intervals $i$, separate representations of which are rendered otiose by strings as descriptions (Fernando 2011b). The precise choice of denotations can be a delicate matter. One reason is underspecification, which in the present context takes the form of a resistance to treating disjunction as non-deterministic choice. Take overlap $\bigcirc$ on $s$-intervals $e$ and $e^{\prime}$

$$
s=e \bigcirc e^{\prime} \quad \text { iff } \quad \pi_{\left\{e, e^{\prime}\right\}}(s) \in \operatorname{Allen}\left(e \bigcirc e^{\prime}\right)
$$

which we can generalize to non-s-intervals $e, e^{\prime}$ as

$$
s \models e \bigcirc e^{\prime} \quad \text { iff } \quad s \sqsupseteq e, e^{\prime}
$$

where containment $\sqsupseteq$ is a (non-deterministic) relation that weakens componentwise inclusion $\unrhd$ between strings of the same length, pronounced subsumption,

$$
\alpha_{1} \cdots \alpha_{n} \unrhd \alpha_{1}^{\prime} \cdots \alpha_{m}^{\prime} \stackrel{\text { def }}{\Leftrightarrow} n=m \text { and } \alpha_{i} \supseteq \alpha_{i}^{\prime} \text { for } 1 \leq i \leq n
$$

to compare strings of different lengths

$$
s \sqsupseteq s^{\prime} \stackrel{\text { def }}{\Leftrightarrow}\left(\exists s^{\prime \prime}\right) s \unrhd s^{\prime \prime} \text { and } \operatorname{unpad}\left(s^{\prime}\right)=\operatorname{unpad}\left(s^{\prime \prime}\right)
$$

\footnotetext{
${ }^{2}$ For concreteness, the formula can (by Büchi-Elgot) be assumed to be from MSO.
} 
(paralleling the construction of $\pi_{X}$ from $b c_{X}$ in the the previous section, the deterministic analog of $\sqsupseteq, \unrhd)$. Formulas $\varphi$ for which satisfaction $\models$ is defined by the positive connectives $\wedge, \vee$ and $\exists$ can be expected to be $\sqsupseteq$-persistent in that

$$
\text { whenever } s \sqsupseteq s^{\prime} \text { and } s^{\prime} \models \varphi, s \models \varphi
$$

(e.g., let $\varphi$ be $e \bigcirc e^{\prime 3}$ ). On the other hand, the formula $e \prec e^{\prime}$ is demonstrably not $\sqsupseteq$-persistent (let $s^{\prime}$ be $e \quad e^{\prime}$ and $s$ be $\left.e \quad e, e^{\prime}\right)$. Even then, however, we might appeal to additional fluents $\operatorname{pre}\left(e^{\prime}\right)$, post $(e)$ and stipulate

$$
s \models e \prec e^{\prime} \quad \text { iff } \quad s_{ \pm} \sqsupseteq \operatorname{pre}\left(e^{\prime}\right) \operatorname{post}(e)
$$

provided we agree to equate $s$ with its delimitation $s_{ \pm}$. But the step from $s$ to $s_{ \pm}$goes against the partiality of information (behind underspecification). An alternative to (5) is to drop the delimitation $s_{ \pm}$, asserting

$$
s \models e \prec e^{\prime} \quad \text { iff } \quad s \sqsupseteq \operatorname{pre}\left(e^{\prime}\right) \text { post }(e)
$$

for $s$ satisfying suitable constraints on pre and post, short (to allow for underspecification) of setting $s=s_{ \pm}$. (These constraints exclude strings that $\sqsupseteq$-contain pre $(e), e$ or $\operatorname{post}(e), e$ or $\operatorname{post}(e), \operatorname{pre}(e)$ or any of the strings in

$$
\begin{array}{|l|l|l|}
\hline e & \|^{*} & \operatorname{pre}(e)+\operatorname{post}(e) \\
\hline
\end{array}
$$

for any e.) Making $\operatorname{pre}\left(e^{\prime}\right) \quad$ post $(e)$ the denotation of $e \prec e^{\prime}$ at such an $s$ steers clear of the trivial solutions $R_{\varphi}:=\{\langle s, \varphi\rangle|s|=\varphi\}$ or $\{\langle s, s\rangle \mid s \models \varphi\}$, to unwind $\varphi$ without simply returning the full input. An argument for defining $R_{e \prec e^{\prime}}$ one way or another may have less to do with the local concern for denotations of $e \prec e^{\prime}$ than with the more global picture of how the relations $R_{\varphi}$ fit together, for a range of different $\varphi$ 's. Insofar as denotations are proper parts of indices/inputs, they point to a piecewise approach to constructing indices using constraints (sometimes described as presuppositions).

\section{Peering inside the box(es)}

Having disputed the soundness of delimiting a string $s$ (to recognize underspecification in $s$ ), we face the question of how to bound fluents $\varphi$ (under pressure to draw inferences). One approach is to suppose there are forces acting for and against $\varphi$, and (similar to fluents pre $(e)$ and post $(e)$ delimiting $e$ ) fluents $f \varphi$ decreeing "there is a force for $\varphi$ " — at least, that is, in strings such that whenever f $\varphi$ occurs, $\varphi$ holds at the next moment unless some force opposes it. More precisely, let $\mathcal{F}_{\varphi}$ be the set of strings $s=\alpha_{1} \cdots \alpha_{n}$ such that

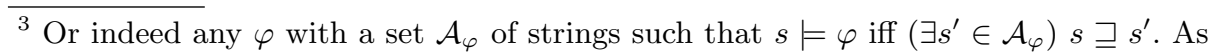
the relation $\sqsupseteq$ (like $\unrhd$ and unpad) can be computed by a finite-state transducer, the set of strings satisfying $\varphi$ is regular provided $\mathcal{A}[\varphi]$ is.
} 
for all $i \in\{2,3, \ldots, n\}, \quad \mathrm{f} \varphi \in \alpha_{i-1}$ implies $\varphi \in \alpha_{i}$ or $\mathrm{f} \bar{\varphi} \in \alpha_{i-1}$

where $\bar{\varphi}$ is the negation of $\varphi$ (whence f $\bar{\varphi}$ says "there is a force opposed to $\varphi$ "). $\mathcal{F}_{\varphi}$ can be expressed succinctly as

$$
\mathrm{f} \varphi\rceil \Rightarrow(\llbracket \varphi+\mathrm{f} \bar{\varphi}\rceil)
$$

using a binary operation $\Rightarrow$ that maps a pair of regular languages to a regular language (Fernando 2008, 2011). Closely related to $\mathcal{F}_{\varphi}$ is the regular language

$$
\varphi \rrbracket \Rightarrow(\square \varphi+\mathrm{f} \bar{\varphi} \mid)
$$

which intersected with

$$
\llbracket \varphi \Rightarrow(\varphi \mid+\mathrm{f} \varphi
$$

gives a regular language $\mathcal{I}_{\varphi}$ in which $\varphi$ is inertial — i.e., $\varphi$ persists forwards and backwards in the absence of forces on it. It is natural to view the languages $\mathcal{F}_{\varphi}$ and $\mathcal{I}_{\varphi}$ as constraints (satisfied by the strings belonging to them), associated with states (as opposed to events) represented by $\varphi$.

An example of a state that an inertial fluent may represent is given by the habitual (3a), repeated below, followed by the episodic (3b).

(3) a. Tess eats dal.

b. Tess is eating dal.

We must be careful not to confuse a string $s$ representing an instance of Tess eating dal with the fluent $\varphi$ representing the habit of Tess eating dal. We may, however, expect the string $s$ to belong to a language $\mathcal{L}_{\varphi}$ associated with $\varphi$. That is, $\mathcal{L}_{\varphi}$ sits alongside the sets $\mathcal{F}_{\varphi}$ and $\mathcal{I}_{\varphi}$ above as languages associated (in different ways) with $\varphi$. The obvious question is what is the status of such languages in our semantic theory?

I close with the suggestion that such languages are specifications of causal structures required by the rules-and-regulations view of Carlson 1995 to ground the truth of generic sentences. (These languages are underspecifications insofar as the causal structures are automata, but at present, I see no reason for that additional specificity.) Beyond $\mathcal{F}_{\varphi}, \mathcal{I}_{\varphi}$ and $\mathcal{L}_{\varphi}$, there are episodes in the sense of Moens and Steedman 1988 consisting of "sequences of causally or otherwise contingently related sequences of events" that (lest we confuse these with episodic instances at the world) are "more related to the notion of a plan of action or an explanation of an event's occurrence than to anything to do with time itself" (page 26). The intuition is that these languages are "resources for constructing local languages for use in particular situations," to quote Cooper and Ranta 2008 slightly out of context. A shift is made here from monolithic truth to use (or action) that is very much in line with (i) the focus in section 2 on maps for putting strings together, and (ii) the use in section 3 of input/output relations to interpret formulas. More questions are certainly left open than answered, an important issue being inference. For entailments between formulas $\varphi$ and $\varphi^{\prime}$ based 
on satisfaction $\models$ (i.e., the domains of $R_{\varphi}$ and $R_{\varphi^{\prime}}$ ), we can use a language $L$ to relativize the inclusion

$$
\begin{aligned}
\varphi \vdash_{L} \varphi^{\prime} \stackrel{\text { def }}{\Leftrightarrow} \quad(\forall s \in L) s \models \varphi \text { implies } s \models \varphi^{\prime} \\
\text { iff } \quad L \cap \operatorname{domain}\left(R_{\varphi}\right) \subseteq \operatorname{domain}\left(R_{\varphi^{\prime}}\right)
\end{aligned}
$$

which is decidable, provided all of $R_{\varphi}, R_{\varphi^{\prime}}$ and $L$ are regular. In fact, the inclusion remains decidable for $L$ context-free, as observed by Makoto Kanazawa. Regular or not, $L$ may consist of strings that represent episodic instances beyond those of any single world. It is one thing to say a causal force for $L$ exists at a world and time, another to spell out the consequences for the episodic instances at a world and time. This much is clear from (3a) versus (3b). And once causal forces are recognized as structures underlying generic sentences such as (3a), can we keep them out of the semantics of episodic sentences such as (3b)?

\section{References}

James F. Allen. Maintaining knowledge about temporal intervals. Communications of the ACM, 26(11):832-843, 1983.

Nicholas Asher and Alex Lascarides. Logics of Conversation. Cambridge University Press, 2003.

Johan van Benthem. The Logic of Time. Reidel, 1983.

Robert I. Binnick. Aspect and aspectuality. In The Handbook of English Linguistics, pages 244-268. Blackwell, 2006.

Gregory N. Carlson. Truth conditions of generic sentences: two contrasting views. In The Generic Book, pages 224-237. University of Chicago Press, 1995.

Robin Cooper and Aarne Ranta. Natural languages as collections of resources. In Language in Flux: Dialogue Coordination, Language Variation, Change and Evolution, College Publications, London, 2008.

Tim Fernando. A finite-state approach to events in natural language semantics. $J$. Logic and Computation, 14(1): 79-92, 2004.

Tim Fernando. Branching from inertia worlds. J. Semantics, 25(3): 321-344, 2008.

Tim Fernando. Regular relations for temporal propositions. Natural Language Engineering, 17(2): 163-184, 2011.

Tim Fernando. Finite-state representations embodying temporal relations. 9th International Workshop on Finite State Methods and Natural Language Processing, Blois, pages 12-20, 2011a.

Tim Fernando. Strings over intervals. Proceedings of TextInfer (Workshop on Textual Entailment, EMNLP 2011, Edinburgh), pages 50-58, 2011b.

Hans Kamp and Uwe Reyle. From Discourse to Logic. Kluwer, 1993.

Marc Moens and Mark Steedman. Temporal ontology and temporal reference. Computational Linguistics, 14(2): 15-28, 1988.

Ian Pratt-Hartmann. Temporal prepositions and their logic. Artificial Intelligence, 166: $1-36,2005$.

Mark Steedman. The Productions of Time. Draft tutorial notes about temporal semantics, Draft 5.0, May 2005 (http://homepages.inf.ed.ac.uk/steedman/papers.html).

Wolfgang Thomas. Languages, automata and logic. In Handbook of Formal Languages: Beyond Words, Volume 3, pages 389-455. Springer-Verlag, 1997. 


\title{
On Scales, Salience \& Referential Language Use

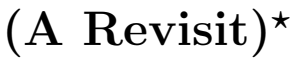

\author{
Michael Franke \\ Institute for Logic, Language \& Computation \\ Universiteit van Amsterdam
}

\begin{abstract}
Kennedy (2007) explains differences in the contextual variability of gradable adjectives in terms of salience of minimal or maximal degree values on the scales that these terms are associated with in formal semantics. In contrast, this paper suggests that the attested contextual variability is a consequence of a more general tendency to use gradable terms to preferentially pick out extreme-valued properties. This tendency, in turn, can be explained by demonstrating that it is pragmatically beneficial to use those gradable properties in referential descriptions that are perceptually salient in a given context.
\end{abstract}

\section{Scale Types, \& "Kennedy's Observation"}

A prominent line of current research in formal semantics links the meaning of gradable adjectives to degrees on scales (cf. Rotstein and Winter, 2004; Kennedy and McNally, 2005). In simplified terms, the denotation of a gradable adjective $A$ is taken to be a function $g_{A}: \operatorname{Dom}(\mathrm{A}) \rightarrow D$ that maps any applicable argument of $A$ to a degree $d \in D$, where $\langle D, \preceq\rangle$ is a suitably ordered scale of degrees. What is particularly interesting about this approach is that different adjectives may be associated with different kinds of scales. Standardly, one-dimensional scales are assumed and a distinction is made as to whether these are: (i) totally open (tall, short), (ii) totally closed (closed, open), or (iii) half-open (bent, pure). Scale types explain a number of otherwise puzzling observations, such as which adjectives can combine with which modifiers. E.g., the expression completely $A$ is felicitous only if $A$ has a totally or upper-closed scale with a maximal element: compare the felicitous completely closed with the awkward ? completely tall.

Scale types also influence the licensing conditions of utterances involving gradable adjectives in positive form. Generally speaking, a simple positive sentence like "object $x$ has property $A$ " is considered true whenever the contextually supplied minimal degree of $A$-ness, $c(A)$, is no higher than $g_{A}(x)$. However, the contextual standard of applicability $c(A)$ is also affected by the scale type (c.f. Kennedy, 2007): if there is a $\preceq$-maximal or -minimal degree contained in $\langle D, \preceq\rangle$,

\footnotetext{
* This paper reconsiders and complements an earlier paper on the same subject (Franke, 2011). I would like to thank the remaining G-Team (Gerhard Jäger, Roland Mühlenbernd, Jason Quinley), Joey Frazee and Graham Katz, as well as the anonymous reviewers for comments and discussion.
} 
then $c(A)$ is bound to this; otherwise it is to be retrieved more flexibly from the context of utterance. In more tangible terms, "Kennedy's observation" (1) says that closed-scale adjectives are used rather inflexibly to denote a minimal or maximal value on the associated scale (modulo the usual pragmatic slack where lack of precision is conversationally harmless), whereas open-scale adjectives allow for more contextual variability.

(1) “Kennedy's Observation":

\begin{tabular}{rll} 
scale type & contextual standard of applicability \\
\hline open & $\longleftrightarrow$ variable \\
closed & rigid, fixed to endpoints
\end{tabular}

For example, the contextual standard for the applicability of open-scale tall can vary considerably from one context (talking about jockeys) to another (talking about basketbal players), whereas that of closed-scale closed seems glued to the denotation of a minimal (zero) degree of openness.

\section{Explaining "Kennedy's Observation"}

Salience of Endpoints. Kennedy (2007) tries to explain the influence of scale topology on contextual usage conditions in terms of the salience of endpoints (2a) and a pragmatic principle called Interpretive Economy $(2 \mathrm{~b})$ which demands that pragmatic interpretation ought to make maximal use of the available semantic resources.

(2) "Kennedy's Explanation":

a. Salience Assumption:

End-points of closed scales are salient elements provided by the conventional semantic structure.

b. Interpretive Economy:

(Kennedy, 2007, (66), p.36) Maximize the contribution of the conventional meanings of the elements of a sentence to the computation of its truth conditions.

The idea seems quite natural: the evaluation of expression " $x$ is $A$ " requires us to fix a contextual standard $c(A)$; if $A$ is associated with a closed scale, then by (2a) the semantic structure supplies some outstanding element, which by $(2 \mathrm{~b})$ ought to be used to set $c(A)$; if $A$ is associated with an open scale, the semantic structure carries no such salient points and $c(A)$ can be set more variably.

We should be fully satisfied with neither (2a) nor (2b). Firstly, as for (2a), it is not clear a priori whether endpoints on closed scales not only appear salient to us because they are the preferred denotation of the corresponding natural language expressions. In that case, the attempted explanation would be circular. The crucial problem is that it is very hard to determine, conceptually or empirically, when an element of an abstract semantic structure is salient. Phrased more constructively, if salience is to play a role in an explanation of (1) it should 
better be an empirically informed notion of perceptual salience, i.e., of salience of objects of perception that stand out relative to others and attract attention more than others do. Secondly, we should not stop at the formulation of a pragmatic principle like $(2 \mathrm{~b})$ even if it seems plausible and yields the desired results, but continue to ask for a functional motivation: what is the added pragmatic value of the principle in question that enabled its evolution and sustenance?

Evolution of Pragmatic Standards. Potts (2008) addresses the latter issue. While adopting (2a), he seeks to explain (1), not via (2b), but instead by an evolutionary argument why speakers and hearers conventionally coordinate on endpoints as the contextual standard for the use of closed-scale adjectives. Towards this end, Potts consider a strategic game in which speaker and hearer simultaneously choose a standard of application for a closed-scale adjective. Payoffs are proportional to how close the players' choices are to each other, so that the maximal payoff ensues when players choose the same standard of application. Potts then shows that if a population initially has a slight bias towards choosing the endpoints (his way of implementing focality of endpoints), then the replicator dynamics (Taylor and Jonker, 1978) will eventually lead to all of the speakers and hearers of the whole population choosing endpoints as standards of application.

Potts' account has some shortcomings, unfortunately. For one, it fails to make clear what the particular pragmatic benefit of endpoint use is: it is just a consequence of the assumption that the to-be-explained outcome is already predominant in the population initially. What is more, Potts' account is either silent about or makes wrong predictions for adjectives with open scales. If we assume that the only thing that differentiates open-scale and closed-scale adjectives is the presence or absence of endpoints, then, looking at open-scale adjectives in the same way, we would simply drop the assumption that there is an initial bias in the population for a particular standard of comparison. But in that case the replicator dynamics will still eventually gravitate towards a single fixed standard of application, albeit not a focal endpoint, contrary to (1).

Extreme-Value Principle. In fact, adjectives with open scales, though more variable in their contextual standard of application, are not entirely unconstrained either. Take the open-scale adjective tall, for instance, and the question when an individual $x$ is called tall when compared with a group of individuals $Y$. Although the precise rule of application is a question of current empirical research (e.g. Schmidt et al., 2009), it seems fair to say that $x$ is more likely or more readily counted as tall, the more $x$ 's tallness falls within the extreme values of tallness within group $Y$. Usually it is not enough for $x$ to be just slightly taller than the average tallness in $Y$. Rather, the further away $x$ 's tallness is from the average or expected value of $Y$, the more readily it counts as tall.

If this is true, or at least not too far off-the-mark, then a different explanation suggests itself for (1). If there was a tendency for gradable expressions to be used preferably to describe extreme values, then it is to be expected that closed-scale terms will mostly be used for values close to the endpoints, while open-scale terms could be used for a wider range of values simply due to the open-endedness of 
the scale. In other words, I suggest that what we need not explain (1), but rather the principle in $(3)$.

\section{(3) Extreme-Value Principle:}

Gradable terms are preferably/usually used to describe extreme values, i.e., values far away from the median/mean of a given distribution.

The remainder of this paper is therefore concerned with two things: (i) a proof of concept that (3) indeed leads to a general association along the lines of (1), and (ii) an attempt of explaining (3) as a concomitant of pragmatic language use. Peaking ahead, the pragmatic rationalization for (3) that I will offer eventually is indeed superficially similar to Kennedy's explanation of (1) in (2), but conceptually different. My explanation of (3) involves a notion of salience of stimuli in context (4a), paired with an account of why the use of salience is actually beneficial in conversation $(4 \mathrm{~b})$.

(4) a. Salience of the Extreme:

Salience of a stimulus in a given context is proportional to its (apparent/subjectively felt) extremeness or outlieriness, i.e., to the extent that the stimulus appears unexpected or surprising against the background of the other stimuli in the context.

b. Benefit of the Extreme:

Describing those properties of objects that are salient is pragmatically advantageous for coordinating reference.

The main intuition that inspires (4) is this: terms are associated with extreme values because we use them, among other things, to identify referents, and for doing so the use of extreme values is a very natural and easy, yet surprisingly effective solution. ${ }^{1}$ In order to test this intuition, I propose a simple model of referential language use, to be introduced next.

\section{$3 \quad$ Referential Games}

A referential game is a game between a sender and a receiver, both of whom observe a context $c$ that consists of $n>0$ objects. One of these objects is the designated object $c_{o}$ that the sender wants to refer to. The receiver does not know which object that is. The goal is to describe the designated object by naming a property of $c_{o}$. For simplicity we assume that senders can choose only one property to describe $c_{o}$ with, but can indicate whether $c_{o}$ has a high or low degree of that property. If, after hearing the description, the receiver guesses the right referent, the game is a success for both players; if not, it's a failure.

\footnotetext{
${ }^{1}$ Elsewhere I tried to show that the use of extreme values would actually be detrimental if language was exclusively used to describe the actual degree of a given object as closely as possible (Franke, 2011). I focus here on the model of referential language use that was also discussed in that earlier work.
} 
More formally, let us assume that objects are represented as points in an $m$-dimensional feature space $\mathcal{F} \subseteq \mathbb{R}^{m}, m>0$. Each dimension of $\mathcal{F}$ corresponds to some gradable property: the value of dimension $j$ is the degree to which the object in question has property $j$. A context is thus a set of $n$ points in $\mathcal{F}$, which can easily be represented as an $n \times m$-matrix $c$. For example, the context in (5) contains three "objects", namely Hans, Piet and Paul, which are represented as a triple of features, namely their degrees of tallness, weight, and intelligence.

(5)

\begin{tabular}{lccl}
\hline & tallness & weight & intelligence \\
\hline Hans & 0.2 & -0.1 & 1.3 \\
Piet & -0.1 & 0.0 & 0.3 \\
Paul & 0.3 & -0.2 & 0.5 \\
\hline
\end{tabular}

To make a distinction between open and closed scales, it is reasonable to assume that open-scale features take values in $\mathbb{R}$, while closed-scale features take values on some closed interval of reals. But open and closed scales should also plausibly differ with respect to the probability that a particular degree is observed. To keep matters simple, assume that a random context is obtained by sampling independently $n$ random objects, and that a random object is obtained by sampling independently $m$ random degree values for the relevant properties. Finally, let us assume, rather naïvely, that degrees are sampled from the distributions in (6) (see also Figure 2).

(6)

\begin{tabular}{ll} 
scale type & distribution \\
\hline open & $\begin{array}{c}\text { normal distribution } \\
\text { (mean 0, standard deviation 1/3) }\end{array}$ \\
totally closed & uniform distribution on $[0 ; 1]$ \\
half-open & $\begin{array}{c}\text { truncated normal distribution on } \mathbb{R} \geq 0 \\
(\text { mean } 0.1, \text { standard deviation } 1 / 3)\end{array}$
\end{tabular}

Together this yields a unique probability density $\operatorname{Pr}(c)$ for each context $c$ (the exact nature of which will, however, not be of any relevance here). For each round of playing a referential game, a context is sampled with $\operatorname{Pr}(c)$ and from that context an object is selected uniformly at random as the designated one.

Finally, let the set of messages from which the sender can choose contain exactly one pair of antonymous terms for each property of the feature space. So, the set of messages is $M=\{1, \ldots, m\} \times\{$ low, high $\}$, where, e.g., $m=\langle j, l\rangle \in M$ has a conventional meaning saying that property $j$ is low. For example, if Hans is the designated object in context (5) above, the sender could describe him as being short or tall, skinny or fat, stupid or smart.

\section{Solving Referential Games}

Intuitively, I would describe Hans as the smart guy in the example above. (What about you?) This is because of his comparatively high value along that dimension, and his median values for the respective others. Does this intuition follow 
from an assessment of what is an optimal way of playing a referential game? Unfortunately it does not, which is why I suggest to instead look at a natural way of playing the game, namely by exploiting salience. But first: optimal play.

Optimal Solutions. Player behavior is captured in the notion of a (pure) strategy, as usual. A sender strategy is a function $\sigma: C \times\{1, \ldots, n\} \rightarrow M$ mapping a context and a designated object onto a message. A receiver strategy is a function: $\rho: C \times M \rightarrow\{1, \ldots, n\}$, mapping each context and each message onto an object. Given a context $c$ with designated object $o$, the utility of playing with a sender strategy $\sigma$ and receiver strategy $\rho$ is simply:

$$
\mathrm{U}(\sigma, \rho, c, o)= \begin{cases}1 & \text { if } \rho(c, \sigma(c, o))=o \\ 0 & \text { otherwise }\end{cases}
$$

The expected utility of $\sigma$ and $\rho$ is then just the averaged utility over all contexts and designated objects, weighted by the probability of occurrence:

$$
\begin{aligned}
\mathrm{EU}(\sigma, \rho) & =\int \operatorname{Pr}(c) \times \mathrm{EU}(\sigma, \rho, c) \mathrm{d} c, \text { where } \\
\mathrm{EU}(\sigma, \rho, c) & =\sum_{i=1}^{n} \frac{1}{n} \times \mathrm{U}(\sigma, \rho, c, i) .
\end{aligned}
$$

A usual, we say that $\langle\sigma, \rho\rangle$ is a Nash equilibrium iff (i) there is no $\sigma^{\prime}$ such that $\operatorname{EU}(\sigma, \rho)<\operatorname{EU}\left(\sigma^{\prime}, \rho\right)$ and (ii) there is no $\rho^{\prime}$ such that $\operatorname{EU}(\sigma, \rho)<\operatorname{EU}\left(\sigma, \rho^{\prime}\right)$. Call $\langle\sigma, \rho\rangle$ an optimal solution iff $\operatorname{EU}(\sigma, \rho, c)=\min \left(1, \frac{2 m}{n}\right)$ for all contexts $c$.

To understand this latter notion, let's take a step back and reflect on referential games. Interestingly, referential games can be considered an infinite collection of games $G_{c}$ one for each context $c$. These games are in fact standard Lewisean signaling games (Lewis, 1969): for fixed $c, G_{c}$ has a set of states (here: objects) that are drawn from a uniform distribution; it also has a set of messages; finally, the receiver tries to guess the actual state that only the sender knows. The maximum possible payoff attainable in each $G_{c}$ is $\min \left(1, \frac{2 m}{n}\right)$. This is because there are $2 m$ messages to encode $n$ states. If $n \leq 2 m$, perfect communication is possible; otherwise only $2 m$ of the $n$ states can be named successfully. Consequently, an optimal solution for a referential game is one that scores optimally in all $G_{c}$.

Since there is a pair of strategies that reaches the theoretically maximal communicative success in each $G_{c}$, it follows that an optimal solution for each referential game exists. Since there is always more than one optimal solution for any local game (we have always at least two messages, even if we have only one object), there are in fact infinitely many optimal solutions. Moreover, since an optimal solution is a pair of strategies that achieves maximal utility in every context, an optimal solution is in fact a Pareto-optimal Nash equilibrium. In other words, optimal solutions are the theoretically conceivable maximum, they exist and even abound.

Unfortunately, optimal solutions might be quite bizarre. Consider a simple, but non-trivial referential game with $n=3$ objects and $m=2$ open-scale properties. For concreteness, let us look at the infinite set of contexts in (7). 


\begin{tabular}{lcc}
\hline object & tallness & weight \\
\hline Hans & $p$ & 0.4 \\
Piet & $p-1$ & 0.3 \\
Paul & 1 & 4.5 \\
\hline
\end{tabular}

where $p \in \mathbb{N}^{\geq 3}$

One of the infinitely many optimal solutions to this game has the sender do the following: if $p$ is not a prime number, then Hans is referred to as the tall guy, Pieter the skinny guy, and Paul the fat guy; if $p$ is prime, then Paul is still identified as fat, but now Hans is called the the skinny guy, and Pieter the tall guy. We may happily assume that the receiver, since he knows the context as well, perfectly identifies the designated object in each case. Of course, it is a ludicrous idea to assume that humans can condition their language use on whether a numerical degree representation is prime or not. But that is not the point. The point is that optimal solutions for referential games are allowed to vary arbitrarily from one context to the other. In other words, it is not enough to know that there are optimal solutions (even if there are infinitely many), we would also need to ask whether any of these is human graspable and learnable in a reasonable way from finite observations.

A Natural Solution: Salience. Much could be said here about cross-contextual learning and rule-induction in an evolutionary game, but I do not want to go there. Instead, I would like to go straight forward and show that there is a fairly natural way of playing referential games that (i) presupposes hardly any rationality on the side of the agents, that (ii) arguably requires no learning at all, as it merely exploits the agents' cognitive make-up, but that still (iii) is sufficiently successful. The strategy I have in mind is one in which sender and receiver simply choose whatever is most salient from their own perspective: the sender chooses the most salient property of the designated object; the receiver chooses the most salient object with that property. Neither player thus reasons strategically about what the other player does. Players merely exploit a shared cognitive bias of perception. Still, statistically this rather myopic choice rule does fairly well and also leads to the selection of extreme values. Both of these claims will be backed up below by numerical simulations.

But let me first elaborate on the notion of salience that I would like to use, which is a notion of contextualized perceptual salience inspired by recent research on visual salience in terms of informativity or surprise (e.g. Rosenholtz, 1999; Itti and Koch, 2001; Bruce and Tsotsos, 2009; Itti and Baldi, 2009). The general idea is that, when presented with a scene, those things stand out that are unexpected. This may be due to sophisticated world-knowledge, but may also be due to much less sophisticated expectations raised by the immediate contextual environment. In the spirit of the latter, I suggest that how salient object $i$ 's having property $j$ to degree $c_{i j}$ is, is a measure of how unexpected, $c_{i j}$ appears against the background of the set $c_{j}^{-1}$ of degrees for property $j$ that occur in $c$. For example, given a context $c$, the set of degrees for property $j$ are a vector of numbers $c_{j}^{-1}$, one for each object. Such a vector could be a tuple 
like $\langle 2,3,1,1,2,1,37\rangle$ that could, for instance, represent abstractly the tallness of my 7 sons. Most values here lie around 1 or 2 , so that the one value of 37 looks suspiciously like an outlier. I suggest that for our purposes here we may assume that the more a degree looks like an outlier in context, the more it is perceptually salient (4a).

Indeed much work in statistics has been devoted to the issue of outlier detection (c.f. Ben-Gal, 2005, for overview). For simplicity, I explore here only one very manageable approach to outlier detection in terms of the (linear) distance between points in the feature space (c.f. Knorr and Ng, 1998; Ramaswamy et al., 2000). So define the salience of object $i$ having degree $c_{i j}$ for property $j$ as: ${ }^{2}$

$$
\operatorname{Sal}_{l i n}\left(c_{i j}, c\right)=\sum_{i^{\prime}}\left|c_{i^{\prime} j}-c_{i j}\right|
$$

Let $s$ be the saliency matrix for context $c$, given by $s_{i j}=\operatorname{Sal}_{l i n}\left(c_{i j}, c\right)$. The salience-based choice rules for sender and receiver are then simply this:

(8) Salience-based choice rules:

a. Sender: if $o$ is the designated object, select property $j^{*}$ uniformly at random from $\arg \max _{j} s_{o j}$; if $c_{o j^{*}} \geq \operatorname{median}\left(c_{j^{*}}^{-1}\right)$, send message $\left\langle j^{*}, h\right\rangle$, otherwise send $\left\langle j^{*}, l\right\rangle$;

b. Receiver: if $\left\langle j^{*}, h\right\rangle$ is the received message, select uniformly at random from $\arg \max _{i}\left\{s_{i j^{*}} \mid c_{i j^{*}} \geq \operatorname{median}\left(c_{j^{*}}^{-1}\right)\right\}$; if $\left\langle j^{*}, h\right\rangle$ is received, the same applies, except with $<$ in the set restriction.

The choice rule in (8) is rather successful (see Figure 1), despite the fact that players blindly maximize salience from their own perspective, without taking each other's strategy into account. Moreover, choices according to (8a) also corroborate (3). The values $c_{o j *}$ selected by (8a) are indeed extreme (Figure 2).

In conclusion, these simulation results give a proof-of-concept that it is possible to think of (3), and hence (1), as a concomitant of a pragmatically efficient language use. Crucial for this proposal is, of course, that the choice rules in (8) make use of a sense of salience that is shared by sender and receiver. Also it is important for an explanation of (3) that salience of a stimulus is, even if indirectly, linked to its extremity. But although both of these assumptions are, to my mind, defensible, the proposal has obvious shortcomings too. For example, it seems to predict that all pairs of antonymous gradable adjectives are noncomplementary and that even closed-scale adjectives allow for some contextual variation. To address these further issues it would be necessary to consider a more encompassing model of language use that not only considers referential descriptions in a shared and perfectly accessible immediate perceptual context.

\footnotetext{
${ }^{2}$ I stick to this notion here for continuity with earlier work (Franke, 2011), but I have also tested different, more standard notions of salience, with essentially the same results as reported here, such as in terms of (linear) distance from the median of $c_{j}^{-1}$, or in terms of multiples of its interquartile range.
} 
success optimal solution - average success of (8) in simulation

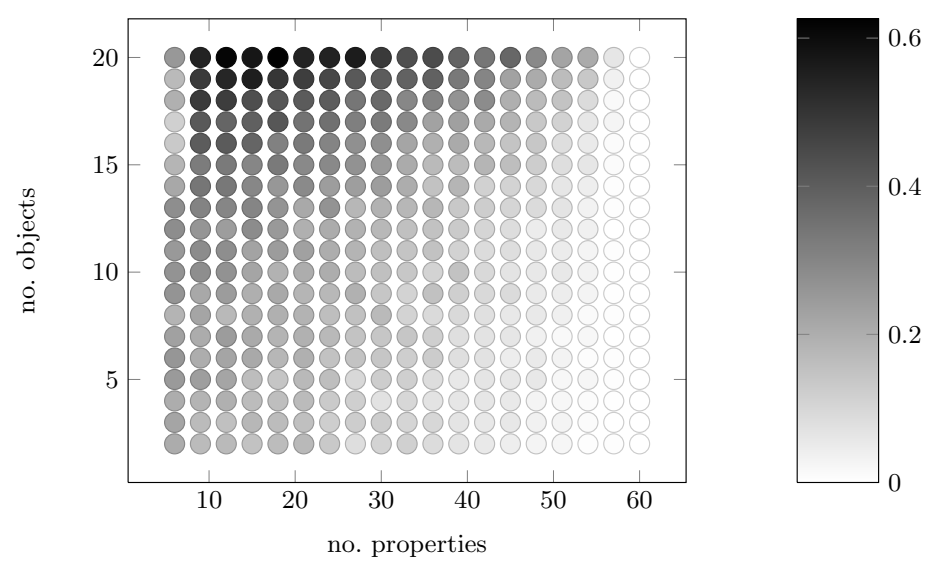

Fig. 1. Assessment of the success of choice rule (8). Each dot corresponds to a pair $n, m$ of context size and number of properties (with $\mathrm{m} / 3$ properties for open, closed, and half-open scales each). For each pair, (8) was applied to 500 random $n \times m$-sized contexts. The proportion of successful rounds was then subtracted from the theoretical maximum for the given $n$ and $m$. (8) often reached more than $80 \%$ of the theoretical optimum. With sufficient expressivity, i.e., large $m$, it even matches peak performance.
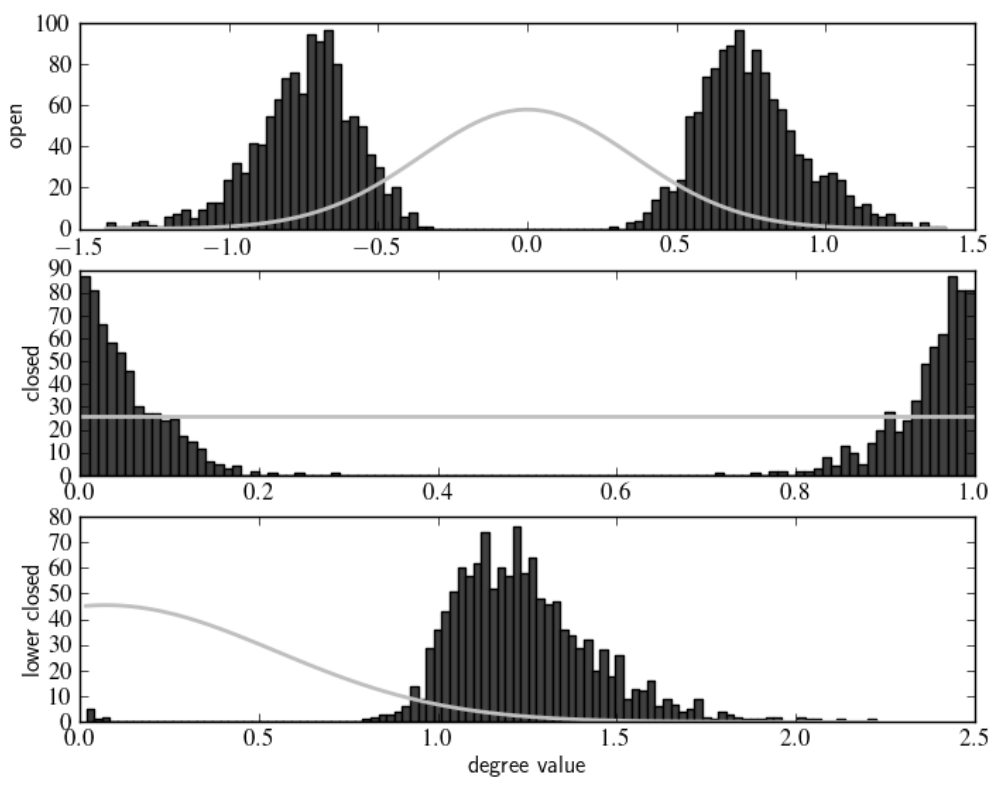

Fig. 2. Frequency with which degrees $c_{o j^{*}}$ were choosen by the choice rule in (8a) in 5000 randomly sampled contexts with $n=30$ and $m=24$ ( 8 properties each for open, closed, and half-open scales with prior distributions indicated by the light gray lines). 


\section{Bibliography}

Ben-Gal, I. (2005). Outlier detection. In Maimon, O. and Rockach, L., editors, Data Mining and Knowledge Discovery Handbook: A Complete Guide for Practitioners and Researchers, pages 131-148. Kluwer.

Bruce, N. D. B. and Tsotsos, J. K. (2009). Saliency, attention, and visual search: An information theoretic approach. Journal of Vision, 9(3):1-24.

Franke, M. (2011). Scales, salience and referential safety: The benefit of communicating the extreme. to appear in Proceedings of EvoLang IX.

Itti, L. and Baldi, P. (2009). Bayesian surprise attracts human attention. Vision Research, 49.

Itti, L. and Koch, C. (2001). Computational modelling of visual attention. Nature Reviews Neuroscience, 1:194-203.

Kennedy, C. (2007). Vagueness and grammar: The semantics of relative and absolute gradable adjectives. Linguistics and Philosophy, 30:1-45.

Kennedy, C. and McNally, L. (2005). Scale structure, degree modification, and the semantics of gradable predicates. Language, 81(2):345-381.

Knorr, E. M. and Ng, R. T. (1998). Algorithms for mining distance-based outliers in large datasets. In Proceedings of the 24th VLDB Conference, pages 392-403.

Lewis, D. (1969). Convention. A Philosophical Study. Harvard University Press.

Potts, C. (2008). Interpretive Economy, Schelling Points, and evolutionary stability. Manuscript, UMass Amherst.

Ramaswamy, S., Rastogi, R., and Shim, K. (2000). Efficient algorithms for mining outliers from large data sets. In Proceedings of the ACM SIGMOD International Conference on Management of Data.

Rosenholtz, R. (1999). A simple saliency model predicts a number of motion popout phenomena. Vision Research, 39:3157-3163.

Rotstein, C. and Winter, Y. (2004). Total adjectives vs. partial adjectives: Scale structure and higher-order modifiers. Natural Language Semantics, 12(3):259 288.

Schmidt, L. A., Goodman, N. D., Barner, D., and Tenenbaum, J. B. (2009). How tall is Tall? compositionality, statistics, and gradable adjectives. In roceedings of the Thirty-First Annual Conference of the Cognitive Science Society.

Taylor, P. D. and Jonker, L. B. (1978). Evolutionary stable strategies and game dynamics. Mathematical Bioscience, 40(1-2):145-156. 


\title{
On the Semantics and Pragmatics of Dysfluency
}

\author{
Jonathan Ginzburg ${ }^{1}$, Raquel Fernández ${ }^{2}$, and David Schlangen ${ }^{3}$ \\ 1 Univ. Paris Diderot, Sorbonne Paris Cité \\ CLILLAC-ARP (EA 3967), 75004 Paris, France \\ ${ }^{2}$ Institute for Logic, Language \& Computation \\ University of Amsterdam \\ P.O. Box 94242, 1090 GE Amsterdam, The Netherlands \\ 3 Faculty of Linguistics and Literary Studies \\ Bielefeld University \\ P.O. Box 1001 31, 33615 Bielefeld, Germany
}

\begin{abstract}
Although dysfluent speech is pervasive in spoken conversation, dysfluencies have received little attention within formal theories of dialogue. The majority of work on dysfluent language has come from psycholinguistic models of speech production and comprehension (e.g. [10, $3,1]$ ) and from structural approaches designed to improve performance in speech applications (e.g. $[14,8]$ ). In this paper, we present a detailed formal account which: (a) unifies dysfluencies (self-repair) with Clarification Requests (CRs), without conflating them, (b) offers a precise explication of the roles of all key components of a dysfluency, including editing phrases and filled pauses, (c) accounts for the possibility of self-addressed questions in a dysfluency.
\end{abstract}

\section{Introduction}

Although dysfluent speech is pervasive in spoken conversation, dysfluencies have received little attention within formal theories of dialogue. The majority of work on dysfluent language has come from psycholinguistic models of speech production and comprehension (e.g. $[10,3,1])$ and from structural approaches designed to improve performance in speech applications (e.g. $[14,8]$ ).

Recent psycholinguistic studies have shown that both the simple fact that a dysfluency is occuring and its content can have immediate discourse effects, which show in different behaviour of listeners. E.g., [1] found that "filled pauses may inform the resolution of whatever ambiguity is most salient in a given situation", and [2] found that in a situation with two possible referents, the fact that a description was self-corrected enabled listeners to draw the conclusion that the respective other referent was the correct one, before the correction was fully executed. Moreover, dysfluencies yield information: (1-a) entails (1-b) and defeasibly (1-c), which in certain settings (e.g. legal), given sufficient data, can be useful.

(1) a. Andy: Peter was, well he was fired.

b. Andy was unsure about what he should say, after uttering 'was'.

c. Andy was unsure about how to describe what happened to Peter. 


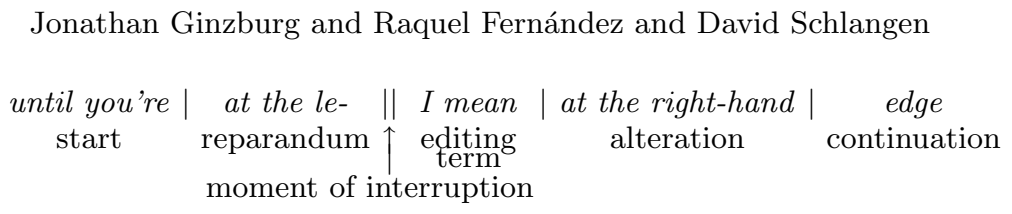

Fig. 1. General pattern of self-repair

In this paper, we present a detailed formal account within the framework KoS [7,6, for example] which: (a) unifies dysfluencies (self-repair) with Clarification Requests (CRs), without conflating them, (b) offers a precise explication of the roles of all key components of a dysfluency, including editing phrases and filled pauses, (c) accounts for the possibility of self-addressed questions in a dysfluency.

We start with background on dysfluencies and on KoS. We then describe in turn our accounts of the two main types of dysfluencies, and end with brief conclusions.

\section{Background}

\subsection{Dysfluencies: structure and taxonomy}

As has often been noted (see e.g. [10], and references therein for earlier work), speech dysfluencies follow a fairly regular pattern. The elements of this pattern are shown in Figure 1, annotated with the labels introduced by [14] (who was building on [10]). Of these elements, all but the moment of interruption and the continuation are optional.

We partition the dysfluencies in two: (i) backward-looking dysfluencies (BLDs), as in $(2-a, b)$ - the moment of interruption, which need not be followed by an explicit editing phrase, is followed by an alteration that refers back to an already uttered reparandum; (ii) forward-looking dysfluencies (FLDs), as in (2-c), where the moment of interruption is followed by a completion of the utterance which is delayed by a filled or unfilled pause (hesitation) or a repetition of a previously uttered part of the utterance (repetition).

(2) a. Flights to Boston I mean to Denver. (Shriberg 1994)

b. Have you seen Mark's erm earphones? Headphones. (British National Corpus, file KP0, 1. 369-370)

c. Show flights arriving in uh Boston. (Shriberg 1994)

\subsection{Dialogue GameBoards}

We start by providing background on the dialogue framework we use here, namely KoS (see e.g. $[7,6]$ ). On the approach developed in KoS, there is actually no single context - instead of a single context, analysis is formulated at a level of information states, one per conversational participant. The dialogue gameboard represents information that arises from publicized interactions. Its structure is given in $((3))$ - the $s p k r, a d d r$ fields allow one to track turn ownership, Facts represents conversationally shared assumptions, Pending and Moves 
represent respectively moves that are in the process of/have been grounded, QUD tracks the questions currently under discussion: ${ }^{4}$

$$
\text { DGBType }=\operatorname{def}\left[\begin{array}{l}
\text { spkr: Ind } \\
\text { addr: Ind } \\
\text { utt-time : Time } \\
\text { c-utt : addressing(spkr,addr,utt-time) } \\
\text { Facts : Set(Proposition) } \\
\text { Pending : list(locutionary Proposition) } \\
\text { Moves : list(locutionary Proposition) } \\
\text { QUD : poset(Question) }
\end{array}\right]
$$

The basic units of change are mappings between dialogue gameboards that specify how one gameboard configuration can be modified into another on the basis of dialogue moves. We call a mapping between DGB types a conversational rule. The types specifying its domain and its range we dub, respectively, the preconditions and the effects, both of which are supertypes of DGBType.

Examples of such rules, needed to analyze querying and assertion interaction are given in (4). Rule (4-a) says that given a question $q$ and $\operatorname{ASK}(\mathrm{A}, \mathrm{B}, \mathrm{q})$ being the LatestMove, one can update QUD with $q$ as QUD-maximal. QSPEC is what characterizes the contextual background of reactive queries and assertions. (4-b) says that if $q$ is QUD-maximal, then subsequent to this either conversational participant may make a move constrained to be $q$-specific (i.e. either About or Influencing $q) .{ }^{5}$

\footnotetext{
${ }^{4}$ We also note one fairly minor technical modification to the DGB field QUD, motivated in detail in $[4,6]$, assuming one wishes to exploit QUD to specify the resolution of non-sentential utterances such as short answers, sluicing, and various other fragments. QUD tracks not simply questions qua semantic objects, but pairs of entities: a question and an antecedent sub-utterance. This latter entity provides a partial specification of the focal (sub)utterance, and hence it is dubbed the focus establishing constituent (FEC) (cf. parallel element in higher order unification-based approaches to ellipsis resolution e.g. [5].) Thus, the FEC in the QUD associated with a wh-query will be the wh-phrase utterance, the FEC in the QUD emerging from a quantificational utterance will be the QNP utterance, whereas the FEC in a QUD accommodated in a clarification context will be the sub-utterance under clarification. Hence the type of QUD is InfoStruc, as defined in (i):
}

(i)

$$
\text { Info-struc }=\left[\begin{array}{l}
q: \text { Questn } \\
\text { fec }: \text { set(LocProp) }
\end{array}\right]
$$

${ }^{5}$ We notate the underspecification of the turn holder as 'TurnUnderspec', , an abbreviation for the following specification which gets unified together with the rest of the rule:

$$
\left[\begin{array}{ll}
\text { PrevAud }=\{\text { pre.spkr,pre.addr }\} & : \text { Set(Ind) } \\
\text { spkr } & : \text { Ind } \\
\text { c1 } & : \text { member }(\text { spkr, PrevAud }) \\
\text { addr } & : \text { Ind } \\
\text { 2 } & : \text { member(addr, PrevAud })
\end{array}\right]
$$




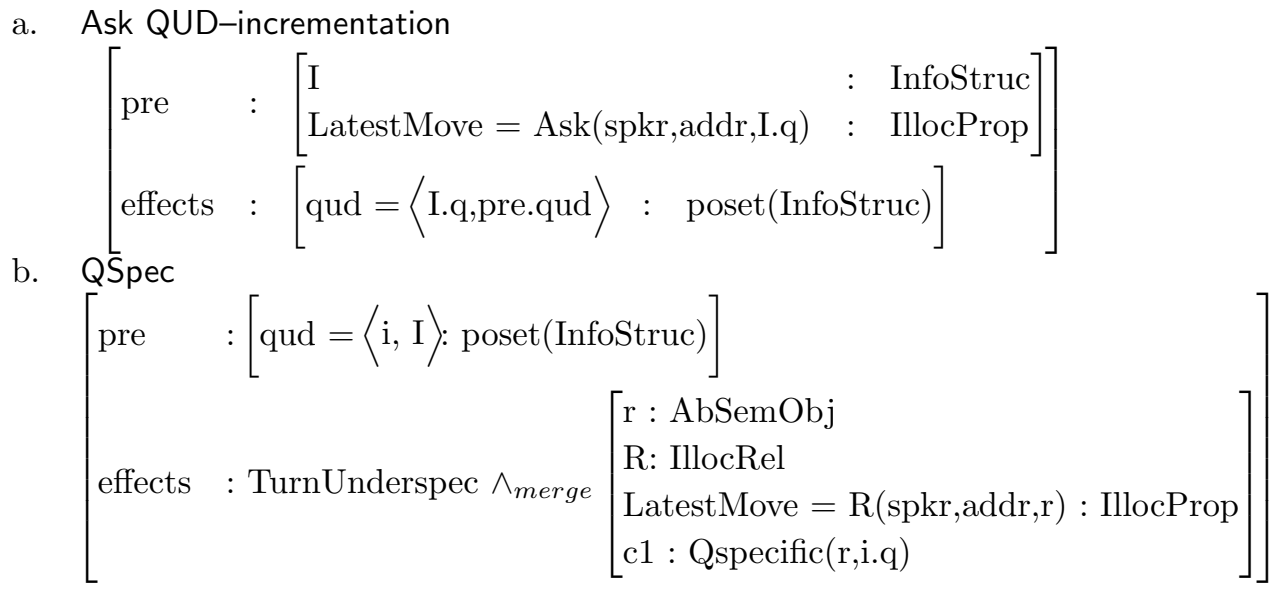

\subsection{Grounding and Clarification}

Given a setup with DGBs as just described and associated update rules, distributed among the conversationalists, it is relatively straightforward to provide a unified explication of grounding conditions and the potential for Clarification Requests (or CRification). in the immediate aftermath of a speech event $u$, Pending gets updated with a record of the form of (5) of type locutionary proposition (LocProp). Here $T_{u}$ is a grammatical type for classifying $u$ that emerges during the process of parsing $u$. The relationship between $u$ and $T_{u}$-describable in terms of the proposition $p_{u}$ given in (5) - can be utilized in providing an analysis of grounding/CRification conditions:

$$
p_{u}=\left[\begin{array}{l}
\text { sit }=\mathrm{u} \\
\text { sit-type }=\mathrm{T}_{u}
\end{array}\right]
$$

(6) a. Grounding: $p_{u}$ is true: the utterance type fully classifies the utterance token.

b. CRification: $p_{u}$ is false, either because $T_{u}$ is weak (e.g. incomplete word recognition) or because $u$ is incompletely specified (e.g. incomplete contextual resolution - problems with reference resolution or sense disambiguation).

We concentrate here on explicating the coherence of possible CRs. In the aftermath of an utterance $u$ a variety of questions concerning $u$ and definable from $u$ and its grammatical type become available to the addressee of the utterance. These questions regulate the subject matter and ellipsis potential of CRs concerning $u$ and generally have a short lifespan in context. To take one example, the non-sentential CRs in (7-a) and (7-b) are interpretable as in the parenthesized readings. This provides justification for the assumption that the context that emerges in clarification interaction involves the accommodation of an issue - one that for A's utterance in (7), assuming the sub-utterance 'Bo' is at issue, could be paraphrased as (7-c). The accommodation of this issue into QUD could be 
taken to license any utterances that are co-propositional with this issue, where CoPropositionality is the relation between utterances defined in (8). ${ }^{6}$ In other words, either a CR which differs from MaxQud at most in terms of its domain, or a correction - a proposition that instantiates MaxQud.

A: Is Bo leaving?

a. B: Bo? (= Who do you mean 'Bo'?)

b. B: Who? (= Who do you mean 'Bo'?)

c. Who do you mean 'Bo'?

d. B: You mean Mo.

CoPropositionality

a. Two utterances $u_{0}$ and $u_{1}$ are co-propositional iff the questions $q_{0}$ and $q_{1}$ they contribute to QUD are co-propositional.

(i) qud-contrib(m0.cont) is m0.cont if m0.cont : Question

(ii) qud-contrib(m0.cont) is ?m0.cont if m0.cont : Prop

(iii) $q_{0}$ and $q_{1}$ are co-propositional if there exists a record $r$ such that $q_{0}(r)=q_{1}(r)$.

Repetition and meaning-oriented CRs can be specified by means of a uniform class of conversational rules, dubbed Clarification Context Update Rules (CCURs) in ([6]). Each CCUR specifies an accommodated MaxQUD built up from a sub-utterance $\mathrm{u} 1$ of the target utterance, the maximal element of Pending (MaxPending). Common to all CCURs is a license to follow up MaxPending with an utterance which is co-propositional with MaxQud. (9) is a simplified formulation of one CCUR, Parameter identification, which allows $\mathrm{B}$ to raise the issue about A's sub-utterance $u 0$ : what did $A$ mean by u0? (9) underpins CRs such as those in (7).

$$
\begin{aligned}
& \text { Parameter identification: } \\
& {\left[\begin{array}{ll}
\text { pre } \quad:\left[\begin{array}{l}
\text { Spkr : Ind } \\
\text { MaxPending : LocProp } \\
\mathrm{u} 0 \in \text { MaxPending.sit.constits }
\end{array}\right] \\
\text { effects }:\left[\begin{array}{l}
\text { MaxQUD }=\left[\begin{array}{l}
\mathrm{q}=\lambda x \operatorname{Mean}(\mathrm{A}, \mathrm{u} 0, \mathrm{x}) \\
\text { fec }=\mathrm{u} 0
\end{array}\right]: \text { InfoStruc } \\
\text { LatestMove : LocProp } \\
\mathrm{c} 1: \text { CoProp(LatestMove.cont,MaxQUD.q) }
\end{array}\right]
\end{array}\right]}
\end{aligned}
$$

\section{From CRs to Dysfluency: Informal Sketch}

We argue that dysfluencies can and should be subsumed within a similar account, a point that goes back to [13]: in both cases (i) material is presented

\footnotetext{
${ }^{6}$ CoPropositionality for two questions means that, modulo their domain, the questions involve similar answers. For instance 'Whether Bo left', 'Who left', and 'Which student left' (assuming Bo is a student) are all co-propositional.
} 
publicly, (ii) a problem with some of the material is detected and signalled (= there is a 'moment of interruption'); (iii) the problem is addressed and repaired, leaving (iv) the incriminated material with a special status, but within the discourse context. Concretely for dysfluencies - as the utterance unfolds incrementally questions can be pushed on to QUD about what has happened so far (e.g. what did the speaker mean with sub-utterance u1?) or what is still to come (e.g. what word does the speaker mean to utter after sub-utterance u2?).

By making this assumption we obtain a number of pleasing consequences. We can:

- explain similarities to other-corrections: the same mechanism is at work, differentiated only by the QUDs that get accommodated.

- explain internal coherence of dysfluencies: ' $\# \mathrm{I}$ was a little bit + swimming' is an odd dysfluency, it can never mean 'I was swimming' in the way that 'I was a little bit + actually, quite a bit shocked by that' means 'I was quite a bit shocked by that'. Why coherence? Because 'swimming' is not a good answer to 'What did I mean to say when I said 'a little bit'?'.

- appropriateness changes implicate that original use unreasonable: examples like (10) involve quantity implicatures. These can be explicated based on reasoning such as the following: I could have said (reperandum), but on reflection I said (alteration), which differs only in filtering away the requisite entailment.

(10) it's basically (the $\mathrm{f}-\mathrm{+}$ a front) leg [implicature: no unique front leg]

\section{Dysfluency Rules}

As we have seen, there are various benefits that arrive by integrating CRs and dysfluencies within one explanatory framework. In order to do this we need to extend PENDING to incorporate utterances that are in progress, and hence, incompletely specified semantically and phonologically. Conceptually this is a natural step to make. Formally and methodologically this is a rather big step, as it presupposes the use of a grammar which can associate types word by word (or minimally constituent by constituent), as e.g. in Categorial Grammar [15] and Dynamic Syntax [9]. It raises a variety of issues with which we cannot deal in the current paper: monotonicity, nature of incremental denotations, etc.

For our current purposes, the decisions we need to make can be stated independently of the specific grammatical formalism used. The main assumptions we are forced to make concern where PENDING instantiation and contextual instantiation occur, and more generally, the testing of the fit between the speech events and the types assigned to them. We assume that this takes place incrementally. For concreteness we will assume further that this takes place word by word, though examples like (11), which demonstrate the existence of word-internal monitoring, show that this is occasionally an overly strong assumption.

$$
\text { From [11] We can go straight on to the ye-, to the orange node. }
$$


BLDs are handled by the update rule in $((12))$. This indicates that if $\mathrm{u} 0$ is a sub-utterance of the maximally-pending utterance, QUD may be updated so that the issue is 'what did A mean by $\mathrm{u} 0$ ', whereas the FEC is $u 0$, and the follow up utterance needs to be be co-propositional with MaxQud:

$$
\begin{aligned}
& \text { Backwards looking appropriateness repair: }
\end{aligned}
$$

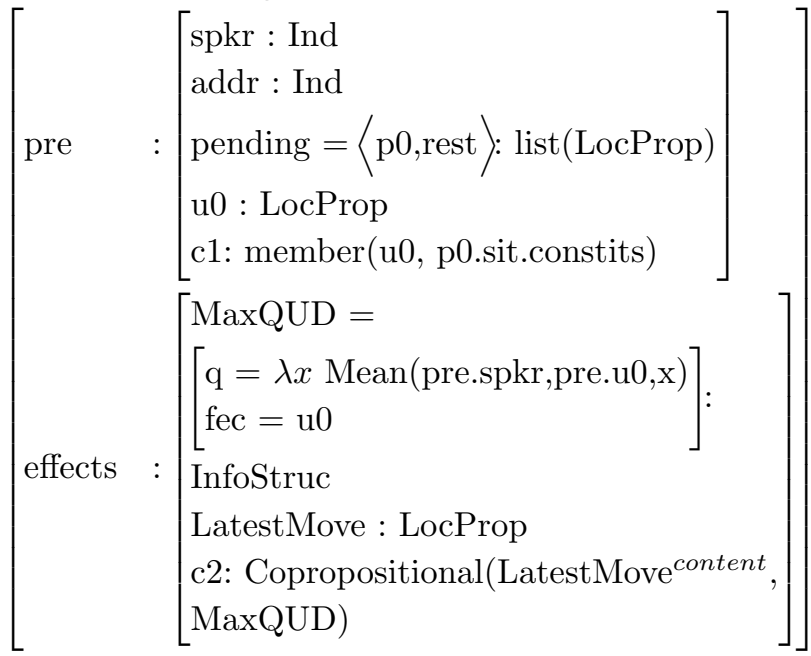

Given $((12)),(2 \mathrm{a}, \mathrm{b})$ can be analyzed as follows: in (2-a) the alteration 'I mean to Denver' provides a direct answer to the issue what did A mean with the utterance 'to Boston'; in (2-b) we analyze 'headphones' as a bare fragment ('short answer') which gets the reading 'I mean headphones' given the QUD-maximality of the issue what did A mean with the utterance 'earphones'.

Consider now (13). This differs from (2-a) in one significant way-a different editing phrase is used, namely 'no', which has distinct properties from 'I mean'.

$$
\text { From [11]: From yellow down to brown - no - that's red. }
$$

Whereas 'I mean' is naturally viewed as a syntactic constituent of the alteration, 'no' cannot be so analyzed. Arguably the most parsimonious analysis ${ }^{7}$ involves assimilating this use to uses such as:

a. [A opens freezer to discover smashed beer bottle] A: No! ('I do not want this (the beer bottle smashing) to happen')

b. [Little Billie approaches socket holding nail] Parent: No Billie ('I do not want this (Billie putting the nail in the socket) to happen')

This use of 'no' involves the expression of a negative attitude towards an event and would, in particular, allow 'no' to be used to express a negative attitude towards an unintended utterance event. We could analyze (13) as involving the utterance 'brown'. Following this, the rule (12) is triggered with the specification

\footnotetext{
${ }^{7}$ An extended version of this paper considers and rejects resolution based on a contextually available polar question or proposition.
} 
QUD.q = what did A mean by FEC? and the FEC = 'brown.' The analysis then proceeds like the earlier cases.

We specify FLDs with the update rule in (15) - given a context where the LatestMove is a forward looking editing phrase by A, the next speaker - underspecified between the current one and the addressee - may address the issue of what A intended to say next by providing a co-propositional utterance: ${ }^{8}$

$$
\begin{aligned}
& \text { Forward Looking Utterance rule: }
\end{aligned}
$$

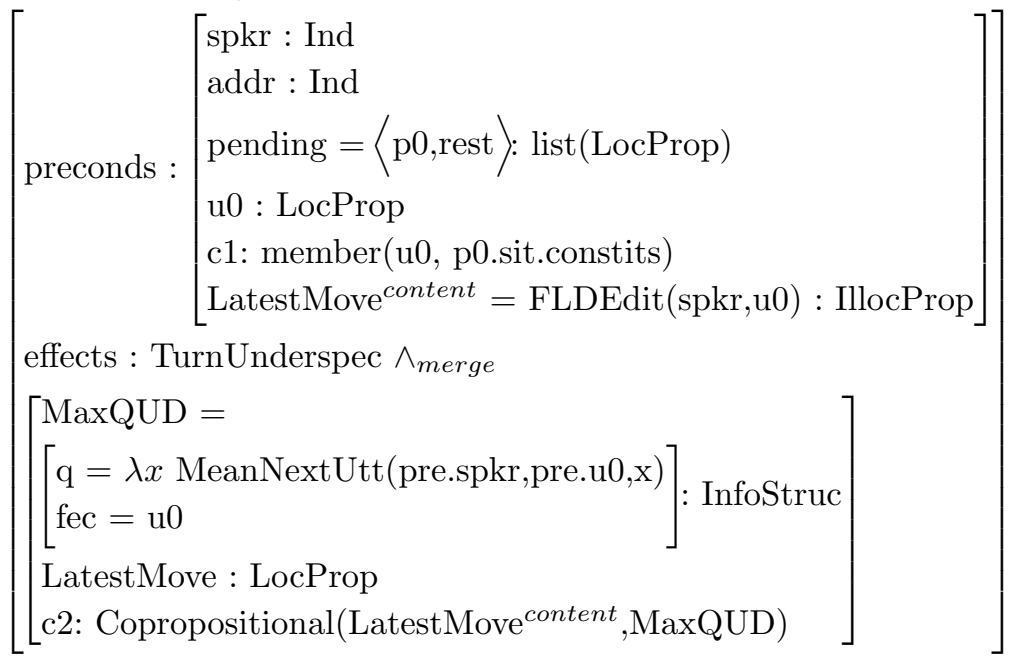

(15) differs from its BLD analogue, then, in two ways: first, in leaving the turn underspecified and second, by the fact that the preconditions involves the LatestMove having as its content what we describe as an FLDEdit move, which we elucidate somewhat shortly. Words like 'uh', 'thee' will be assumed to have such a force, hence the utterance of such a word is a prerequisite for an FLD. To make this explicit, we assume that 'uh' could be analyzed by means of the lexical entry in (16):

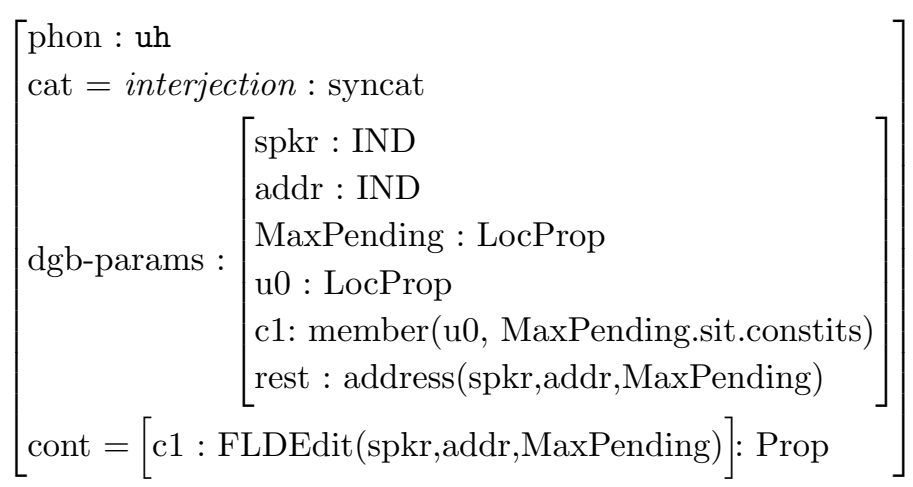

\footnotetext{
${ }_{8}$ This rule is inspired in part by Purver's rule for fillers, (91), p. 92, ([12]).
} 
We demonstrate how to analyze (17):

(17) From [14]: A: Show flights arriving in uh Boston.

After A utters $\mathrm{u} 0=$ 'in', she interjects 'uh', thereby expressing FLDEdit(A,B,'in'). This triggers the Forward Looking Utterance rule with MaxQUD.q $=\lambda x$ MeanNextUtt(A, 'in', $\mathrm{x})$ and FEC = 'in'. 'Boston' can then be interpreted as answering this question, with resolution based on the short answer rule.

Similar analyses can be provided for (18). Here instead of 'uh' we have a lengthened version of 'a', which expresses an FLDEdit moves:

(18) From [11]: A vertical line to a- to a black disk.

Let us return to consider what the predicate 'FLDEdit' amounts to from a semantic point of view. Intuitively, (19) should be understood as 'A wants to say something to B after u0, but is having difficulty (so this will take a bit of time)':

$$
\text { FLDEdit }(\mathrm{A}, \mathrm{B}, \mathrm{u} 0)
$$

This means we could unpack (19) in a number of ways, most obviously by making explicit the utterance-to-be-produced $u 1$, representing this roughly as in (20):

$$
\exists u 1[\operatorname{After}(\mathrm{u} 1, \mathrm{u} 0) \wedge \operatorname{Want}(\mathrm{A}, \operatorname{Utter}(\mathrm{A}, \mathrm{B}, \mathrm{u} 1))]
$$

This opens the way for a more 'pragmatic' account of FLDs, which we will sketch here, one in which (15) could be derived rather than stipulated. Once a word is uttered that introduces FLDEdit $(\mathrm{A}, \mathrm{B}, \mathrm{u} 0)$ into the context, in other words has an import like (20), this leads to a context akin to ones like (21), that license inter alia elliptical constructions like sluicing and anaphora:

a. A: A woman phoned. introduces issue: "who is the woman that phoned'.

b. A: Max drank some wine. introduces issue: "what wine did Max drink' .

Indeed a nice consequence of (15), whether we view it as basic or derived, is that it offers the potential to explain cases like (22) where in the aftermath of a filled pause an issue along the lines of the one we have posited as the effect of the conversational rule $((15))$ actually gets uttered:

a. Carol 133 Well it's (pause) it's (pause) er (pause) what's his name? Bernard Matthews' turkey roast. (BNC, KBJ)

b. Here we are in this place, what's its name? Australia.

c. They're pretty ... um, how can I describe the Finns? They're quite an unusual crowd actually. http://www.guardian.co.uk/sport/ 2010/sep/10/small-talk-steve-backley-interview

On our account such utterances are licensed because these questions are copropositional with the issue 'what did A mean to say after u0'. Such exam- 
ples also highlight another feature of KoS's dialogue semantics: the fact that a speaker can straightforwardly answer their own question, indeed in these cases the speaker is the "addressee" of the query. Such cases get handled easily in KoS because turn taking is abstracted away from querying: the conversational rule QSpec, introduced earlier as (4-b), allows either conversationalist to take the turn given the QUD-maximality of $q$.

Concluding Comment Finally, the account we provide has a strong methodological import: editing phrases like 'no' and 'I mean' select inter alia for speech events that include the discompetent products of performance. This means that the latter are also integrated within the realm of semantic competence.

Acknowledgements Raquel Fernández acknowledges support from NWO (MEERVOUD grant 632.002.001). David Schlangen acknowledges support from DFG (Emmy Noether Programme) Some portions of this paper were presented at Constraints in Discourse 2011 in Agay. We thank the audience there as well as the reviewers for Amsterdam Colloquium for their comments.

\section{References}

1. Bailey, K.G.D., Ferreira, F.: The processing of filled pause disfluencies in the visual world. In: van Gompel, R.P.G., Fischer, M.H., Murray, W.S., l. Hill, R. (eds.) Eye Movements: A Window on Mind and Brain, pp. 485-500. Elsevier (2007)

2. Brennan, S.E., Schober, M.F.: How listeners compensate for disfluencies in spontaneous speech. Journal of Memory and Language 44, 274-296 (2001)

3. Clark, H., FoxTree, J.: Using uh and um in spontaneous speech. Cognition 84, 73-111 (2002)

4. Fernández, R.: Non-Sentential Utterances in Dialogue: Classification, Resolution and Use. Ph.D. thesis, King's College, London (2006)

5. Gardent, C., Kohlhase, M.: Computing parallelism in discourse. In: IJCAI. pp. 1016-1021 (1997)

6. Ginzburg, J.: The Interactive Stance: Meaning for Conversation. Oxford University Press, Oxford (2012)

7. Ginzburg, J., Fernández, R.: Computational models of dialogue. In: Clark, A., Fox, C., Lappin, S. (eds.) Handbook of Computational Linguistics and Natural Language. Blackwell, Oxford (2010)

8. Heeman, P.A., Allen, J.F.: Speech repairs, intonational phrases and discourse markers: Modeling speakers' utterances in spoken dialogue. Computational Linguistics 25(4), 527-571 (1999)

9. Kempson, R., Meyer-Viol, W., Gabbay, D.: Dynamic Syntax: The Flow of Language Understanding. Blackwell, Oxford (2000)

10. Levelt, W.J.: Monitoring and self-repair in speech. Cognition 14, 41-104 (1983)

11. Levelt, W.J.: Speaking: From intention to articulation. The MIT Press (1989)

12. Purver, M.: The Theory and Use of Clarification in Dialogue. Ph.D. thesis, King's College, London (2004)

13. Schegloff, E., Jefferson, G., Sacks, H.: The preference for self-correction in the organization of repair in conversation. Language 53, 361-382 (1977)

14. Shriberg, E.E.: Preliminaries to a theory of speech disfluencies. Ph.D. thesis, University of California at Berkeley, Berkeley, USA (1994)

15. Steedman, M.: The Syntactic Process. Linguistic Inquiry Monographs, MIT Press, Cambridge (1999) 


\title{
Pragmatic Constraints on Gesture Use: The Effect of Downward and Non Entailing Contexts on Gesture Processing ${ }^{\star}$
}

\author{
Gianluca Giorgolo and Stephanie Needham \\ Institute of Cognitive Science, Carleton University, Ottawa, Canada, \\ gianluca_giorgolo@carelton.ca, sneedham@connect.carleton.ca
}

\begin{abstract}
We report on ongoing research on the semantic and pragmatic factors that influence the interpretation of co-speech spontaneous gestures. We extend the semantic theory introduced by Giorgolo in [1] by proposing a pragmatic principle that controls the felicitousness of spontaneous gestures in different linguistic contexts. The principle is based on the idea of rationality in communication and will result in an extension of the gricean Maxim of Quantity [2]. We also present an experiment that we designed to test the predictions of the combined semantico-pragmatic principles.
\end{abstract}

Keywords: gesture, multimodality, semantics, pragmatics, semanticspragmatics interface

\section{Introduction}

This paper is concerned with the identification of some of the principles that govern the interaction between language and gesture at the semantic level. The main contribution of this paper is the extension of the model for gesture semantics proposed by [1] to take into account pragmatic factors. Our extension is quite conservative as it is based on the assumption of rational behaviour in communication, a fairly standard assumption in current pragmatic theory [3]. This extensions allows us to make fairly strong predictions about the possibility of observing gestures in certain linguistic contexts. The paper presents the first results of an ongoing experiment designed to test these predictions.

In this paper, we will concentrate on the spontaneous manual gestures that typically accompany verbal language. More specifically we will focus on the class of gestures usually referred to as iconic gestures [4]. This class of gestures includes those hand movements that are used spontaneously by speakers and that visualize physical properties of the entities or the events referred to in the utterance. This type of gesture lacks a codified form of execution (in this

\footnotetext{
* This research is supported by an Early Researcher Award from the Ontario Ministry of Research and Innovation and NSERC Discovery Grant \#371969. The authors thank Sebastien Plante for acting in the experiment stimuli and Raj Singh and 
sense they are spontaneous and free form), even though they tend to be used consistently in the same stretch of discourse [5].

In Section 2 we present in more detail the framework proposed by [1] and discuss its limitations. Section 3 introduces the simple extension we propose to the framework and discusses some of the implications of this extension. The current state of the experimental work is discussed in Section 4. We conclude with Section 5 by discussing the significance of our findings and what they mean for a theory of gesture meaning.

\section{The Interpretation of Spontaneous Co-Speech Gestures}

Our starting point is the semantics for gesture proposed by Giorgolo [1]. This analysis assumes that gestures contribute to the interpretation of an utterance by providing additional information expressed in terms of an iconic representation. According to this analysis, the iconic representation is a process that identifies the salient spatial features of the referent of a gesture (be it an entity or an event) and that encodes them as visible actions. The representation identifies an equivalence class of spatial configurations that are indistinguishable from the virtual space created by the hand movements at a context dependent level of description (determined by the salient features picked for the gestural representation). In this semantic model the two modalities are interfaced by an adjunction operation that intersects the semantic content of the gesture with the content of its verbal anchor point (roughly the semantic constituent connected both temporally and semantically with the gesture).

The process can be visualized as the diagram in Figure 1. The speech component $\sigma$ of a multimodal utterance is interpreted in the usual way. Each verbal constituent is assigned an abstract object taken from an ontology $F$ of entities, events and truth values. A family of (partial) mappings Loc connects the ontology $F$ to a spatial ontology $S$ by linking each entity, event, property and relation to its spatial extension (a spatial region, a spatio-temporal region, a set of regions or a set of tuples of regions). On the other side, the gesture $\gamma$ is translated from a collection of motoric configurations into a virtual spatial object. This virtual space is then used to create the equivalence class of the spaces that are sufficiently similar to the represented one. The characteristic function of this class is taken to be the core meaning of the gesture. Finally the meaning contribution of the verbal component and the one provided by gesture are combined via a generalized meet operation.

This model makes already a number of strong predictions partially confirmed by experiments reported in [1]. For instance the model restricts the distribution of gestures by constraining their co-occurrence with verbal expressions. According to the model gestures can co-occur only with those linguistic constituents whose interpretation (under one of the Loc mappings) is of a type that can be intersected with the meaning of a gesture (basically the model restricts the verbal correlate of gestures to expressions that denote properties or relations). At the same time the model precludes the possibility of introducing discourse referents 


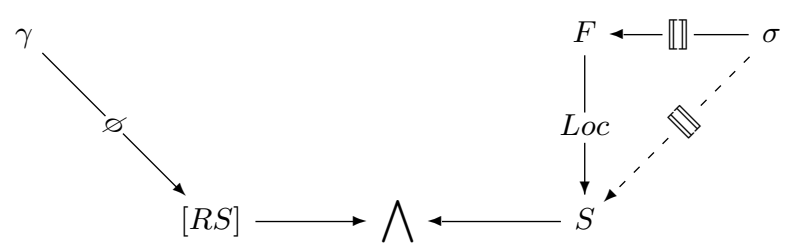

Fig. 1: Interpretation process for a multimodal utterance.

with gestures (see [6] for a different approach in which gestures have the possibility to introduce discourse referents and are in general treated as discourse segments).

However, in its current form, the model cannot predict the distribution of information between the two modalities. The extension we present in the next section allows us to make simple predictions of this kind. A full model of the distribution of information between modalities would require the introduction of more theoretical constructs such as a way of quantifying the expression of a piece of information in any specific modality. In this paper we present just a first attempt in this direction that does not require the introduction of any additional theoretical assumptions besides the notion of rationality in communication which is already independently motivated by the study of verbal language.

\section{Pragmatic Constraints on Gesture Interpretation}

Gesture is not the primary mode of communication in most cases. This is particularly true for the spontaneous gestures we are dealing with in this paper. Although we pay attention to the hands of our interlocutor, our attention is focused primarily on the facial area with quick glimpses at what the hands are doing ([7]). We expect that communication has evolved so that both listeners and speakers take into consideration this fact when they engage in a conversation.

We can couple this observation with the general rules governing communication that have been proposed when considering language in isolation. In particular the various incarnations of the Maxim of Quantity seem to be particularly related to the situation we are dealing with. In its most general form the maxim assigns a lower and upper bound to the amount of information that a contribution to the conversation should convey. The general idea is that the contribution should be such that it presents our interlocutor with enough information to move the conversation towards the desired goal (some ideal informational state) without including irrelevant or excessive details. By observing naturally occurring gestural data, one has pretty soon the impression that gestures often play the role of providing some more details about the situation under discussion without crossing the upper bound limit imposed by the Maxim of Quantity. In a sense gestures allow us to smuggle some more information into the conversation without making it too heavy. The freedom of adding more information, however, is 
combined with the lower saliency that information expressed gesturally shows. A speaker encoding a piece of information in gesture runs the risk of seeing that information disregarded by her interlocutor. We expect this fact to be built in the rules of communication and to determine how people use gestures.

We can express this rule in the following terms: encode a piece of information as a gesture only if it provides additional information moving you closer to your goal and if it is not necessary to achieve your goal. The idea is therefore that gestures can only monotonically increase the amount of information available and that they can do so only if their contribution is not vital for the success of the conversation. This rule allows us to preserve the validity of the Maxim of Quantity. With gesture we are apparently allowed to break it by introducing some information that often (if paraphrased in verbal terms) would make our contribution too heavy. At the same time we are not breaking the rule by marking this additional information as not particularly salient and by allowing our interlocutor to disregard it safely. ${ }^{1}$

If we take seriously the adjunct-like semantics for gesture we sketched above, we can find linguistic contexts in which the use of a gesture would contravene the rule just stated. The cases we focus on in this paper is the one of downward monotone and non-monotone contexts. Given the non propositional content of gestures we focus on the contexts induced by determiners and quantifiers. In those contexts deciding to use a gesture to convey information corresponding to an adjunction may result in a communicative failure. In fact in the case of a downward monotone context failing to integrate the gestural information may result on the part of the interlocutor in an interpretation that is too strict and that may even not include the state of information that is the goal of the speaker. Similarly in the case of a non monotone context the adjoined information may be crucial to determine the correct interpretation. This is never the case in an upward monotone context as any interpretation that includes the additional contribution of the gesture is in a sense a subset of the laxer interpretation without gesture. Another way of looking at this prediction is by considering the fact that only upward monotone contexts allow gesture to operate as an optional source of information that increases the overall amount of information in a monotonic way.

Our prediction is therefore that downward and non monotone contexts are bad candidates for the use of gestures. To show that this is the case we designed an experiment that takes advantage of the fact that listeners are attuned to the dispreference of speakers for using gestures in those contexts. We expect listeners to lack a strategy to interpret gestures in those contexts or to have at least a preference for not integrating them.

\footnotetext{
${ }^{1}$ There are linguistic expressions that play the role of moving saliency away from language and towards other media such as gesture. For example the use of deictics like "this" and "so" and similar phrases like "shaped like this" or "this big" create the effect of marking the gestural information as necessary for the communicative goals. We expect that in those cases this rule and the predictions depending on it would not apply.
} 


\section{Experiment}

The experiment is designed to measure whether subjects integrate or not the gestural information in their mental representation depending on the monotonicity of the linguistic contexts in which the gesture appears. The prediction is that preference for integration is dependent on the context and that the upward monotone contexts will show a strong preference for integrating gesture (around $75 \%$ according to previous measurements of [1]). On the other hand, downward and non monotone contexts will show a strong dispreference for an integrated interpretation.

\subsection{Experimental Setup}

We designed a set of stimuli to test our hypotheses. The experiment consisted of 12 stimuli grouped in three conditions corresponding to the three possible monotonic behaviours of determiners and quantifiers (upward entailing, downward entailing and non-entailing) and additionally subdivided according to the position occupied by the linguistic anchor point for the gesture (restrictor or predicate position of a determiner).

Each stimulus is a short audio and video recording of a confederate engaged in a seemingly "natural" conversation. The part of the conversation shown to the subject is a short monologue introducing a context ended with a quantified statement about the main topic of the monologue. The monologues are all constructed according to a single template. A class of entities or (possible) events is introduced together with a number of relevant subcategories. Each subcategory is identified by some physical property and an iconic gesture is associated with that property. The final statement contains a reference to the general category accompanied by a gesture associated with one of the subcategories. The clip is cut in such a way that the statement gives the impression of being the first part of a longer utterance. Subjects were asked to pick the most likely continuation of the monologue from a list of sentences. To clarify the setup we present here one of the stimuli used, the "spiders" stimulus. The monologue is reported below. The speech segments accompanied by a gesture are annotated with square brackets ([]) and with a tag identifying the gesture. The gestures are shown in Figure 2.

"Spiders" transcript: Last week I booked a flight down to South America 'cause I'm going there for vacation this summer and I was talking to some friends about - you know - what it's like there and one of my friends was telling me about the spiders there especially in the jungle and he was saying that there's these [LITTLE : little spiders] that are everywhere all the time and these [BIG : big fat spiders] that pretty much only come out at night and fortunately none of the [BIG : spiders] are actually poisonous...

The possible continuations for this stimulus are listed below: 
1. but the small ones are deadly poisonous

2. in fact some people get bitten on purpose by the small ones because they think it helps prevent heart diseases

3. so I definitely need to buy some serum in case I get bitten

4. the spiders living there are among the deadliest on the planet

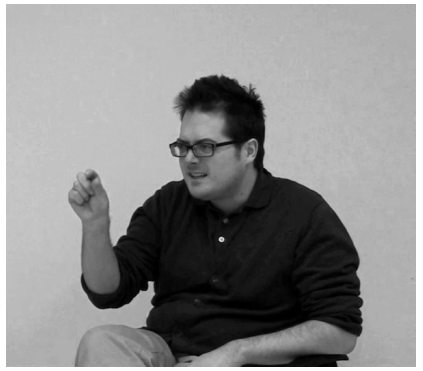

(a) LITTLE

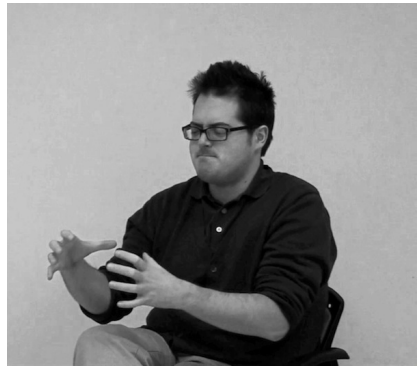

(b) BIG

Fig. 2: Still frames of the gestures used in the "spiders" stimulus.

The continuations are constructed in a way that allows us to use them to infer the interpretation that subjects assign to the stimuli. We assume that there are always two available interpretations: one that includes the gestural information and one constructed without it. To detect which interpretation is associated with each stimulus the continuations are designed to be compatible with only one of the two available interpretations and in contradiction with the other. In other words, given two interpretations $\Gamma$ and $\Delta$ each continuation $p$ is constructed in such a way that only one of $\Gamma \wedge p$ and $\Delta \wedge p$ is satisfiable.

However notice that, given the semantics of gesture, it is possible to construct this type of continuation only in the case of non-entailing contexts. In the case of upward and downward entailing contexts this is not possible. The reason in both cases is the fact that one interpretation entails the other. In the case of an upward entailing context $\Gamma[\cdot]^{\uparrow}$ we have that the common ground including the gestural information is entailed by the one that does not include it, in symbols $\Gamma[G(A)]^{\uparrow} \leq_{t} \Gamma[A]^{\uparrow}$, where $G$ is the denotation of a gesture and $A$ the denotation of the linguistic constituent that the gesture modifies. This follows from the definition of upward entailing function and from the intesective semantics of gestures (we have in general that $G(A) \leq A$, where $A$ is an object of some boolean type and $G$ a gesture modifying it). This means that whenever the addition of a proposition $p$ to a common ground $\Gamma[G(A)]^{\uparrow}$ is satisfiable so it is its addition to the weaker common ground $\Gamma[A]^{\uparrow}$ (this is a consequence of the fact that conjunction with a fixed proposition is an upward monotone operation). It is however possible to construct a proposition whose addition to $\Gamma[A]^{\uparrow}$ is satisfiable, while its addition to $\Gamma[G(A)]^{\uparrow}$ leads to a contradiction. In the case of downward entailing contexts we have the dual situation: given that $\Gamma[A]^{\downarrow} \leq_{t} \Gamma[G(A)]^{\downarrow}$ we can 
only find a proposition that is compatible with $\Gamma[G(A)]^{\downarrow}$ without being compatible with $\Gamma[A]^{\downarrow}$, as any proposition compatible with $\Gamma[A]^{\downarrow}$ will be compatible also with $\Gamma[G(A)]^{\downarrow}$ (where with compatible we mean that its conjunction with a proposition does not lead to a contradiction). Table 1 summarizes the types of continuations used in the different conditions to measure whether the gesture had been integrated in the interpretation or not. The types are codified as pairs that define whether the continuation is compatible (expressed by + ) or not ( expressed by -) with the interpretation that includes the information conveyed by gesture (first component of the pair) and the interpretation that does not include the gestural contribution (second component of the pair). Notice that this does not prevent us from distinguishing between the two interpretations as in both cases we expect to see a dispreference for the continuations compatible with a single interpretation (those that are not $\langle+,+\rangle$ ).

\begin{tabular}{lllll}
\hline $\begin{array}{l}\text { Gesture interpre- } \\
\text { tation }\end{array}$ & $\begin{array}{l}\text { Upward entailing } \\
\text { context }\end{array}$ & $\begin{array}{l}\text { Downward entail- } \\
\text { ing context }\end{array}$ & $\begin{array}{l}\text { Non-entailing con- } \\
\text { text }\end{array}$ \\
\hline Integrated & $\langle+,+\rangle$ & & $\langle+,-\rangle$ & $\langle+,-\rangle$ \\
Non integrated & $\langle-,+\rangle$ & $\langle+,+\rangle$ & $\langle-,+\rangle$ \\
\hline
\end{tabular}

Table 1: Compatibility of continuations with respect to integration of gesture in the interpretation. The pairs indicate whether the continuation used in the different condition is compatible $(+)$ or not (-) with the integrated (first component) and the non integrated (second component) gesture interpretation.

Returning to the "spiders" example, continuation 1 and 3 can be added to the common ground only if the gesture has been integrated in the interpretation (an effect similar to the one obtained by substituting the last sentence of the monologue with 'none of the big spiders are poisonous'). Continuation 2 is the only continuation compatible with the non-integration of the gesture, but at the same time it is also compatible with an integrated interpretation. The fact that none of the big spiders are poisonous does not entail that the small ones are, even though pragmatic factors may favor this interpretation (a fact that would make our result stronger).

To each list of continuations we added a distractor in the form of a sentence contradicting both the integrated and the non integrated interpretation (see continuation 4 in the "spiders" example). ${ }^{2}$

The experiment was run online with subjects recruited mainly among the undergraduate population of Carleton University. The instructions made no mention of gestures to avoid an unnatural focus of subjects on the manual modality. The sequence of stimuli was randomized as was the order in which the proposed

2 The distractor was added mainly to control for low quality entries in the results, given we could not control for particularly poor experimental conditions. 
continuations were presented. Subjects were allowed to watch each clip more than one time.

\subsection{Results}

We ran a first version of the experiment with 25 subjects. The results are summarized in Figure 3. As we can see the prediction is only partially confirmed by these results. The effect of the monotonicity of the linguistic context is clearly evident but it does not go in the direction predicted by our hypothesis. In upward entailing contexts we observe a clear preference for integrated interpretations, while in the case of downward entailing contexts the preference is for non-integrated interpretations. However non-entailing contexts seem to also trigger a preference for integrated interpretations. This is confirmed by a Tukey's HSD test: the mean preference of the upward monotone context condition is significantly different from the one of downward monotone contexts $(p=0.0000008)$, but it is not different from the mean preference of non monotone contexts $(p=0.317)$, and at the same time the mean preferences of downward monotone contexts are significantly different from non monotonic ones $(p=0.0019)$ while we expected these two condition two show a similar distribution of preferences.

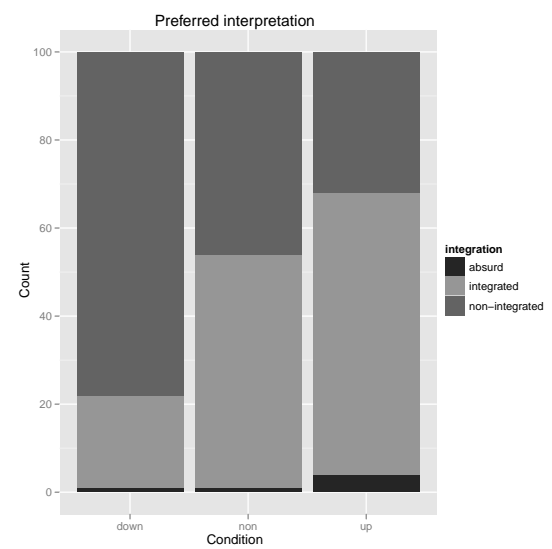

(a) Absolute count of chosen interpretation.

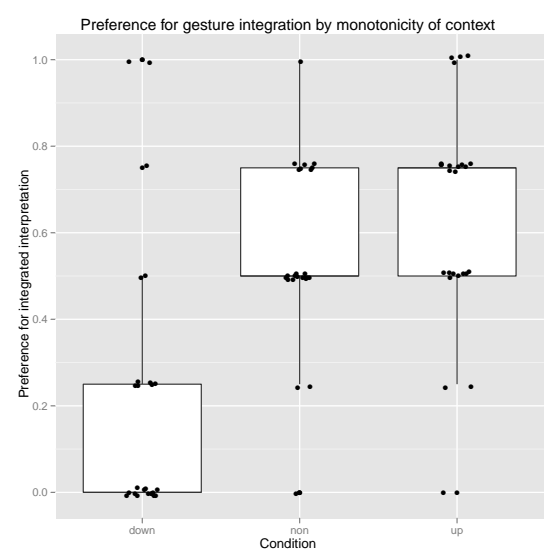

(b) Distribution of integrated interpretation by condition.

Fig. 3: Preferences for integrated and non-integrated interpretations for the first version of the experiment.

However by going through the stimuli again we realized that some of the non monotone tokens were not constructed according to the rules presented above. In particular the favoured answers in those cases were compatible with both an integrated and non integrated interpretation. To test this hypothesis and to 
justify the exclusion of the wrong test items, we ran another session of the experiment with 20 more subjects and corrected the list of continuations in one of the affected stimuli. Figure 4(a) shows the change in response with respect to the modified item. The switch to the non integrated interpretation is pretty clear. Figure 4(b) shows instead the distribution of the preferences for the integrated interpretation in the second experiment after removing the wrongly constructed stimuli. The distributions now show more clearly a behaviour close to our predictions. Performing a Tukey's HSD test shows the mean preference for the integrated interpretation in upward monotone contexts is significantly different from the one of downward and non monotone contexts ( $\mathrm{p}$-values respectively of 0.00013 and 0.00008 ), while the distribution of preference for integrated interpretations in downward and non-monotone contexts is to all purposes the same $(p=0.991)$, as our hypothesis predicted.

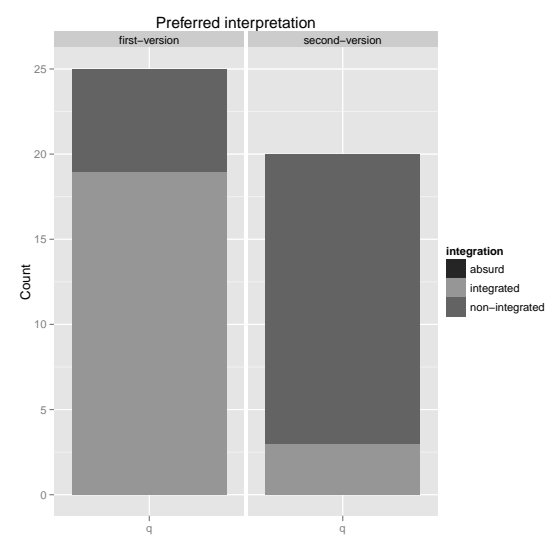

(a) Count of preferences for different interpretation in the two version of the incorrect stimulus.

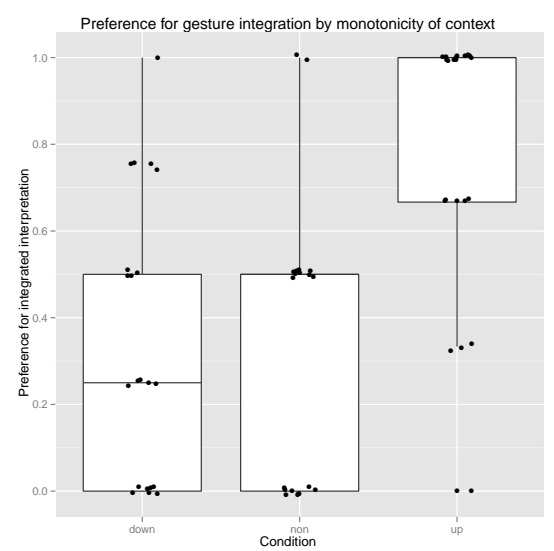

(b) Distribution of integrated interpretation by condition.

Fig. 4: Figure (a) shows the effect of the correction in the continuation for the selected non monotone context. Figure (b) shows the distribution of the preference for the integrated interpretation by condition in the second version of the experiment after removal of the incorrect stimuli.

\section{Conclusion}

We extended the semantics presented in [1] by adding a rule that deals with the pragmatic constraints of gesture use. The extension is based on the notion of rational behaviour in communication and more particularly on the Maxim of Quantity. This new framework makes strong and unexpected predictions about 
the possibility of encoding information as gesture in certain linguistic contexts. To test the validity of these predictions we designed an experiment that confirmed our expectations. The results are possibly compatible with other theories of gesture meaning. For instance it is possible to adapt the semantics proposed by Lascarides and Stone in [6] to deal with the data we collected. However such an adaptation would require very strong assumptions as it would be based on a theory of anaphoric-like connections between gesture and language. Our theory does not require any such stipulation as it is based on the simplest possible semantics we can associate with gesture and a general principle about human communication.

We are in the process of extending our empirical explorations to further confirm our hypotheses. In particular we want to use a different experimental setting that does not force our subjects to focus too much on logical operations. At the same time we want to confirm our expectations about the possibility of breaking the pragmatic rule we proposed by means of specific linguistic expressions.

\section{References}

1. Giorgolo, G.: Space and Time in Our Hands. PhD thesis, Utrecht Institute for Linguistics OTS, Utrecht: LOT publications 262 (2010).

2. Grice, H. P.: Logic and conversation, in P. Cole and J. Morgan (eds.), Syntax and Semantics, 3: Speech Acts, pp. 41-58, New York: Academic Press (1975).

3. Horn, L. R.: Implicature, in L. R. Horn and G. Ward (eds.), The Handbook of Pragmatics, Oxford: Blackwell Publishing (2004).

4. McNeill, D.: Hand and Mind. Chicago: University of Chicago Press (1992).

5. McNeill, D.: Gesture and Thought. Chicago: University of Chicago Press (2005).

6. Lascarides, A., M. Stone: A Formal Semantics Analysis of Gesture, Journal of Semantics (2009).

7. M. Mancas, F. Pirri and M. Pizzoli: Human-motion Saliency in Multi-motion Scenes and in Close Interaction, Proceedings of Gesture Workshop, Athens (2011). 


\title{
Sameness, Ellipsis and Anaphora
}

\author{
Daniel Hardt ${ }^{1}$, Line Mikkelsen ${ }^{2}$, and Bjarne Ørsnes ${ }^{3}$ * \\ 1 Copenhagen Business School, \\ dh.itm@cbs.dk, \\ 2 University of California at Berkeley, \\ mikkelsen@berkeley.edu, \\ 3 Freie Universität Berlin/Copenhagen Business School, \\ Bjarne.Dersnes@fu-berlin.de
}

\begin{abstract}
We compare explicit assertions of sameness with analogous elliptical and anaphoric expressions, and find striking differences in their interpretation. We account for those differences with a two part proposal: first, we propose that same is additive, similar to too. Second, same must take scope over a containing event-denoting expression. We give evidence that the scope-taking of same is subject to standard island constraints, and we also show that same always compares two event-denoting clauses that differ in a relevant property.
\end{abstract}

Keywords: Ellipsis, anaphora, same

\section{Introduction}

It is a truism that elliptical and anaphoric expressions are in some sense interpreted the same as the antecedent. For example, one widely cited work on ellipsis and anaphora poses as its central question: "Under what circumstances can bits of a syntactic structure be said to be the same as or different from other bits of a syntactic structure?" (Fiengo and May (1994)[p xi]). In this paper we examine expressions involving explicit assertions of sameness (do the same and the same $\mathrm{N})$, and we find that they differ in surprising ways from analogous elliptical and anaphoric expressions (VP ellipsis, do it/that/so and pronouns).

We observe that same always compares a described event with an antecedent event. The two events must be distinct, and the antecedent event description must be true in context. Our analysis is that same is additive and futhermore must take scope over a containing event-denoting expression. We show that this scope-taking is subject to standard island constraints on syntactic movement. We then examine differences between same and the additive particle too, and suggest that these differences have to do with the fact that same must compare events, and thus is subject to constraints on how events are individuated. We end with a brief discussion of related work on so-called "internal" readings of same.

\footnotetext{
* Thanks to Maribel Romero, Irene Heim, Maziar Toosarvandani and an anonymous reviewer for useful feedback and discussion.
} 


\section{Same vs. Ellipsis and Anaphora}

We begin with example (1);

(1) I feel it's important to vote in favor (although I don't have a vote). I appeal to my colleagues to do so/it/that/*the same, for the good of European citizens.

Here, do the same is infelicitous, while the related forms (do so/it/that) are felicitous. Intuitively, do the same is ruled out because the antecedent voting event did not actually occur. If the example is modified so that the antecedent event did occur, do the same is acceptable.

(2) I voted in favor. I appeal to my colleagues to do the same, for the good of European citizens.

Example (3) illustrates another case where do the same is ruled out where related forms are all acceptable.

(3) John caught a big fish, and he did VPE/so/it/that/*the same without any fishing equipment ${ }^{4}$.

Here, it seems that what rules out do the same is the very fact that it is referring to the same event; if the example is modified so that there are two different events, do the same is fine.

(4) John caught a big fish last week, and he did the same yesterday without any fishing equipment.

So, far we have seen that do the same describes an event that must differ from the antecedent event description, and second, the antecedent event description must be true in context.

We follow Carlson (1987) in claiming that same requires an event-based account. It is perhaps not surprising that the same is naturally described in terms of events when appearing as the object of $d o$, since it must describe an event (in this case, an action) in such cases. However in our view same always requires reference to events. To show this, we turn now to cases where same appears in ordinary NP's like the same book, as in the following examples (Irene Heim, p.c.):

(5) I read War and Peace on my last vacation, and I hope that you will read $\{$ it/the same book\} (next year).

(6) I never got around to reading War and Peace, but I hope that you will read $\left\{\mathrm{it} / *^{*}\right.$ the same book $\}$ (next year).

\footnotetext{
${ }^{4}$ In this example VPE (VP ellipsis) is acceptable, in addition to the other VP anaphora forms. VPE is not acceptable for example (1), presumably because of specific constraints on VPE when introduced by to in embedded clauses. See Johnson (2001); Lobeck (1995) for discussion.
} 
This contrast is very similar to the one observed between (1) and (2) - unless the antecedent event, reading War and Peace, actually happened, the same book is infelicitous, while the pronoun is acceptable. Example (7) affirms the contrast observed in (3): unlike a pronoun, same does not allow the antecedent event to be the same as the current event.

(7) I read War and Peace on my last vacation, and I read $\{$ it/*the same book\} in a single sitting.

Here, the same book is infelicitous, except perhaps on the reading where one has read War and Peace on two separate occasions.

These last two contrasts show that, even when same occurs in an ordinary, individual-denoting NP, it involves comparison of events. ${ }^{5}$ We conclude that same places two constraints on the event being described: first, the described event must be distinct from the antecedent event, and second, the antecedent event description must be true in context.

\section{Same is Additive}

In our view, the above observations suggest that same is additive, similar to too. This is supported by the following variants of (1) and (2) involving too:

(8) I feel it's important to vote in favor (although I don't have a vote). I appeal to my colleagues to do so/it/that *too, for the good of European citizens.

(9) I voted in favor. I appeal to my colleagues to do so/it/that too, for the good of European citizens.

Too patterns with same here, generating infelicity unless the antecedent event description is true in context. It is well known that too is "additive", in that it adds to the current context a clause that is closely related to an antecedent clause, but differing in some way. A recent characterization can be found in Singh (2008) [p 5] (see also Riester and Kamp (2010); Krifka (1999)):

(10) Let $\phi$ too $_{i}$ be an LF, with too co-indexed with $\mathrm{LF} \psi_{i}$. Then $c+\phi$ too $_{i}$ is defined iff:

(a) $[[\psi]] \neq[[\phi]]$, (b) $[[\psi]] \epsilon[[\phi]]^{F},($ c) $c+\psi=c$ (i.e. $\psi$ is true in $c$ )

(a) ensures that the antecedent is distinct from the phrase occurring with too

(b) identifies an "appropriately contrasting antecedent"

(c) ensures that the antecedent is true in context

\footnotetext{
${ }^{5}$ Note that we follow (Maienborn (2011)) in understanding the term "event" to cover processes and states as well as events in a narrow sense.
} 
We propose that same gives rise to the same conditions (a) - (c); furthermore, $\phi$ is always an event-denoting expression containing same. We state the conditions imposed by same as follows:

(11) Let same $_{i} \phi$ be an LF, with same co-indexed with LF $\psi_{i}$. Then $c+s a m e_{i}$ $\phi$ is defined iff:
(a) $[[\psi]] \neq[[\phi]]$,
(b) $[[\psi]] \epsilon[[\phi]]^{F}$,
(c) $c+\psi=c($ i.e. $\psi$ is true in $c)$

Returning to examples (1) and (2), we have

(12) $[\text { my colleagues do the same }(=\text { vote in favor })]_{V P}$

We assume that do the same is interpreted as vote in favor. With same adjoined to the containing VP, we have:

(13) $\left[\text { same }[\text { my colleagues vote in favor }]_{V P}\right]_{V P}$

Assuming there is focus on "colleagues", for (2):

$[[\phi]]=$ my COLLEAGUES vote in favor; $[[\phi]]^{F}=\mathrm{x}$ vote in favor; $[[\psi]]=\mathrm{I}$ vote in favor

(a) $[[\psi]] \neq[[\phi]]$ (ok)

(b) $[[\psi]] \epsilon[[\phi]]^{F}(\mathrm{ok})$

(c) $\mathrm{c}+\psi=\mathrm{c}(\psi$ true in current context) (ok)

For example (2), conditions (a) - (c) are all satisfied and the example is thus acceptable. For example (1), condition (c) is not satisfied - there is not an appropriately contrasting antecedent $\psi$ that is true in context.

Example (3) is ruled out by condition (a):

(14) $\quad[\text { john did the same (= caught big fish) }]_{V P}$ [same [john caught big fish]] fish

$\phi=\mathrm{JOHN}$ caught big fish; $[[\phi]]^{F}=\mathrm{x}$ caught big fish; $\psi=$ john caught big

a) $[[\psi]] \neq[[\phi]]$ (false)

b) $[[\psi]] \epsilon[[\phi]]^{F}(\mathrm{ok})$

c) $c+\psi=\mathrm{c}(\mathrm{ok})$

We turn now to the contrast in (5) and (6), repeated here:

(15) I read War and Peace on my last vacation, and I hope that you will read \{it/the same book\} (next year).

(16) I never got around to reading War and Peace, but I hope that you will read $\left\{\right.$ it $/ *^{*}$ the same book $\}$ (next year). 
For both (5) and (16), we have $\phi=$ YOU read War and Peace; $[[\phi]]^{F}=\mathrm{x}$ read War and Peace; $\psi=\mathrm{I}$ read War and Peace

a) $[[\psi]] \neq[[\phi]]$ (ok)

b) $[[\psi]] \in[[\phi]]^{F}(\mathrm{ok})$

For (5) the antecedent I read War and Peace is true in context (condition c), but not for (6), accounting for the difference in acceptablity. The account of (7) is completely parallel to that of (3). Note that this depends on same taking scope over an event-denoting constituent that contains the NP in which it appears. We turn now to this issue.

\section{Scope}

The most obvious difference between same and too stems from the fact that too is an adverb that can be overtly adjoined to a variety of constituents, including clauses. same is an adjective that moves covertly to take scope over a clausal element. Here we show that this covert movement is subject to island constraints. The example in (17) is a case in point.

(17) John knows why Mary killed a fish.

Harry knows why she did so/it/that, too.

*Harry knows why she did the same thing. ${ }^{6}$

The why clause is an island for movement being a wh-clause, thus same cannot take scope over the matrix clause; instead it takes scope over [she did the same thing]. This leads to a violation of our condition (a), distinctness. This problem does not arise with too, since it is adjoined to the matrix clause. Here the contrast between John and Harry satisfies distinctness.

Compare this with the example in (18): where same appears in an untensed VP:

(18) John asked Mary to catch a fish.

Harry asked her to VPE/do so/do it/do that, too.

Harry asked her to do the same thing.

We find (18) completely acceptable with same. This parallels the contrast found with wh-movement:

(19) *What does John know why Mary caught?

What did John ask Mary to catch?

What did John say Mary caught?

\footnotetext{
${ }^{6}$ Some of our examples have do the same while others have do the same thing. We treat them as being interpretively equivalent, and have nothing to say about what bears on the choice between them.
} 
The wh-clause is a barrier to movement, so movement out of the wh-clause is impossible. This explains why (17) is bad with same. However, (19) shows that movement is possible out of an untensed clause and out of a finite clause embedded under the bridge-verb say. This explains why (18) is acceptable with same and also why the example in (20) is acceptable with same.

(20) John said Mary caught a fish.

Harry said she did VPE/so/it/that, too.

Harry said she did the same thing.

Similar contrasts are found with other well-known island constraints:

\section{Complex NP constraint:}

(21) Peter rejected the claim that Mary had caught a fish.

Harry rejected the claim that she did VPE/so/it/that too.

* Harry rejected the claim that she did the same.

\section{Sentential Subject constraint:}

(22) That Mary caught a fish bothers Harry.

That she did VPE/so/it/that bothers Frank, too.

* That she did the same bothers Frank.

\section{Adjunct constraint:}

(23) Mary laughs when she catches a fish

Harry laughs when she does VPE/so/it/that, too.

* Harry laughs when she does the same thing.

In all of these examples with island environments we find that same is impossible or highly degraded. As an adjective same moves covertly to take scope over Mary had caught a fish or Mary catches a fish. But same cannot move out of the island. In all cases we find a violation of our principle (a): distinctness. The two compared events are not distinct. too, being an adverb that can adjoin to any clause, is less restricted in its scope. Thus, with too we can have a contrast in the referents of the matrix subjects, ensuring that the two compared events are sufficiently distinct.

\section{Individuation}

We have seen that same sometimes differs from too for a structural reason: since same must move to take scope over the material to be compared, it is subject to island constraints. Here we observe other cases where same differs from too: but in these cases we argue that the difference is semantic - we argue that same must compare events that differ in a relevant property, while too is more flexible. 
(24) Harry should vote, and

he will VPE/do so/do it/do that, too.

* he will do the same.

Here too is acceptable because it compares two propositions that differ in modality (should vs will). Modality, however, is not a property of events, so that it is not available as a basis for comparison with same. Tense on the other hand is a property of events, which explains the acceptability of same in the following example:

(25) Harry voted last year, and

he will next year, too.

he will do the same next year.

More generally, we expect the contrast requirement of same to track the Davidsonian conception of events in (26); from Maienborn (2011).

(26) Events are particular spatiotemporal entities with functionally integrated participants.

Only elements that individuate events (event type, participants, time and location) can satisfy the contrast requirement of same. For both same and too the contrast requirement is satisfied by the element expressing new or contrastive information, i.e. a focussed element. As a clause-level adjunct, too does not place any restrictions on what elements of that clause may be focussed. In contrast, same occurs as part of an anaphoric VP or NP, making certain constituents unavailable for focussing and contrast. This is illustrated by (27):

(27) I encourage all my colleagues to go to the meeting.

I encourage them to VOTE, too.

*I encourage them to do the same.

Here, too contrasts the VP's go to the meeting and vote. Same cannot do that here - it cannot contrast the VP that it appears within. Similarly, in (28), too contrasts the NP's early meeting and late meeting. But it is not possible for same to contrast the NP it appears within.

(28) I encourage all my colleagues to boycott the EARLY meeting.

I encourage them to boycott the LATE meeting, too.

*I encourage them to boycott the same LATE meeting.

In general, then, the contrasted element cannot appear in the same constituent as same. One interesting variation involves orphans (Culicover and Jackendoff (2005) [p 285-6]), as illustrated by (29).

(29) I encourage all my colleagues to boycott the EARLY meeting. I encourage them to do the same with the LATE meeting.

In Mikkelsen et al. (2011), it is argued that the NP the LATE meeting functions semantically as an argument to the VP, although it appears syntactically as an adjunct to VP. Being outside the VP anaphor, it can be focussed and being adjoined to VP, same can scope over it, explaning the felicity of (29). 


\section{Related Work}

Several authors have proposed that same can move to take scope over a clause. Heim (1985) briefly considers same and different, and argues that they can move to take clausal scope, much as she argues for comparative morphemes (see also Alrenga (2007) on comparative uses of same). Carlson (1987) also argues that same and different can take clausal scope, and furthermore argues that same and different always compare events. Carlson distinguishes between "internal" and "external" readings. In the current paper, we have dealt exclusively with external readings, that is, readings where there are two separate clauses to be compared. An internal reading arises in a clause that "provides its own context", as Carlson puts it [p 532]. An example of this is (30), discussed by Barker (2007):

(30) Anna and Bill read the same book.

The internal reading of (30) is described by Barker as follows: (30) is true "just in case there exists some book $x$ - any book $x$ - such that Anna read $x$ and Bill read $x "$ (Barker (2007)[p 2]). The internal reading in (30) relies on the existence of the conjoined NP Anna and Bill, which can be interpreted distributively. More generally, Barker argues that same must raise in the derivation of internal readings, and that movement is "parasitic" on the presence of another quantificational element (in the case of (30), a conjoined NP).

Both Carlson and Barker focus their attention on internal readings. In the current paper, we have restricted our attention to "external readings", in which the interpretation of same relies on an antecedent in prior discourse, and we have focused on differences with corresponding anaphoric and elliptical forms. Alrenga (2009) performs a similar comparison, arguing that for pronouns, the anaphoric link is stipulated, while for same-NP's it is asserted. This is based on three differences observed: first, same-NP's can appear with almost, second, they can appear in existential there-sentences, and third, they often carry certain existential implications. Both the observations and the proposed account of them are quite different from those of the current paper, and we will have to leave to future work the task of integrating these two perspectives.

Barker considers external readings briefly, but does not attempt to extend his account to them - in fact, he argues against doing this (Barker (2007) [p 6-9]). Similarly, in this paper we have not attempted to extend our account of external readings to apply to internal readings. However, in this section we make some observations about the relation between the two readings.

First, the internal readings are subject to the same island effects we have observed for external readings. This is observed by Carlson (1987)[p 534]: "the licensing NP must appear within the same 'scope domain' as the dependent expression". For example, in (17) above, same could not take scope over the matrix clause, making the external reading of same infelicitous. In (31), below, the same effect can be observed with an internal reading:

(31) * John and Harry know why Mary killed the same fish. 
Similar effects can be seen with internal versions of all of the island effects we have observed for external readings.

We also observed for external readings that the two events must differ in a particular event-property. Thus example (24) shows that a contrast between modals is not felicitous, since modality is not a property of events. The same is true of internal readings, as shown by the following contrast:

(32) * Harry necessarily reads the same book.

Harry usually reads the same book.

While we won't attempt any specific analysis of such internal readings, we suggest that the contrast in (32) is similar to that observed between (24) and (25). same compares events in terms of a distinction in properties, thus quantification over times (usually) can license an internal reading, while a modal quantification (necessarily) cannot.

Finally we have this observation: while Barker correctly observes that the internal reading of same requires a quantificational element that can function as a "trigger" for that reading, his notion of "parasitic" scope suggests that the movement of same is only possible when such a trigger is present. Discussing an example involving the quantificational element everyone, Barker notes, "The reason I call this parasitic scope is that the scope target for same does not even exist until everyone has taken scope."(Barker (2007)[p 21]) Our account of external readings casts doubt on this suggestion, since we have proposed that same moves in a similar way for external readings, in the absence of any such trigger.

\section{Conclusions}

Much of the literature on elliptical and anaphoric expressions rests on a notion of sameness: in some sense the elliptical or anaphoric expression is to be understood as the same as the antecedent. One might naturally expect, therefore, that explicit assertions of sameness would be interpretively indistinguishable from analogous elliptical or anaphoric expressions. In this paper we have seen that this is emphatically not the case; unlike their anaphoric and elliptical analogues, explicit sameness expressions require a comparison of two event-denoting expressions. The truth of the antecedent event-denoting clause is presupposed, and there is a requirement that the two events differ in a relevant property.

As far as we know, these facts have not been previously observed - however, we suspect that our account of these facts can be fruitfully related to a body of work accounting for so-called internal readings with same and related terms. While we have not attempted to extend our account to such internal readings, we have pointed out several points of commonality that we plan to explore in subsequent work. 


\section{Bibliography}

Alrenga, P. (2007). Dimensions in the semantics of comparatives. Ph. D. thesis, University of California Santa Cruz.

Alrenga, P. (2009). Stipulated vs. asserted anaphora. Paper presented at the 83rd annual LSA meeting in San Francisco, January 11.

Barker, C. (2007). Parasitic scope. Linguistics and Philosophy 30(4), 407-444.

Carlson, G. (1987). Same and different: Consequences for syntax and semantics. Linguistics and Philosophy 10(4), 531-565.

Culicover, P. W. and R. Jackendoff (2005). Simpler Syntax. Oxford University Press.

Fiengo, R. and R. May (1994). Indices and Identity. Cambridge, MA: MIT Press.

Heim, I. (1985). Notes on comparatives and related matters. Ms., University of Texas, Austin.

Johnson, K. (2001). What VP ellipsis can do, and what it can't, but not why. In M. Baltin and C. Collins (Eds.), The Handbook of Contemporary Syntactic Theory, pp. 439-479. Oxford: Blackwell.

Krifka, M. (1999). Additive particles under stress. In Proceedings of the Conference on Semantics and Linguistic Theory, Ithaca, NY: Cornell University, pp. 111-128.

Lobeck, A. (1995). Ellipsis: Functional Heads, Licensing, and Identification. Oxford: Oxford University Press.

Maienborn, C. (2011). Event semantics. In C. Maienborn, K. von Heusinger, and P. Portner (Eds.), Semantics: An International Handbook of Natural Language Meaning. De Gruyter Mouton.

Mikkelsen, L., D. Hardt, and B. Ørsnes (2011). Orphans hosted by VP anaphora. In Proceedings of 29th West Coast Conference on Formal Linguistics.

Riester, A. and H. Kamp (2010). Squiggly issues: Alternative sets, complex dps, and intensionality. In T. d. J. Maria Aloni, Harald Bastiaanse and K. Schulz (Eds.), Logic, Language and Meaning, pp. 374-383. Revised, Selected Papers from the 17th Amsterdam Colloquium.

Singh, R. (2008). VP-deletion, obligatory too, and focus semantics. Unpublished manuscript, MIT. 


\title{
As Simple as It Seems
}

\author{
Vincent Homer \\ ENS-IJN \\ 29, rue d'Ulm 75005 Paris, France
}

\begin{abstract}
Even when linearized after it, seem can take scope above the modal can in configurations that involve a downward-entailing expression. Though it is tempting to regard this syntax-semantic mismatch as idiomatic, there are strong reasons to aim for a compositional analysis. Not least among these reasons is the unifying property of the triggers of the scope reversal, namely downwardentailingness. The article claims that the main explanandum is the wide scope of seem not only over can, but also over the trigger. Reordering takes place, covertly: it is shown that seem is a mobile positive polarity item, which has the ability to raise above a potential anti-licenser. This movement permits aspectual configurations that are otherwise disallowed in the complement of seem. If it is on the right track, this analysis could pave the way for new insights on covert movement, complementation and subordinate tense.
\end{abstract}

Keywords: Polarity, Raising, Modal, Tense, Infinitive

\section{Introduction}

There is a case of syntax-semantics mismatch in English which has stirred interest since its introduction into contemporary linguistics (Quirk (1965), Langendoen (1970)) but remains ill-understood to this day.

(1) John can't seem to lose weight.

Paraphrasable as: It seems that John can't lose weight.

Scopal relations under this reading:

$\mathrm{SEEM} \gg \mathrm{NEG} \gg \mathrm{CAN}$

The phenomenon, known under the oversimplifying name of 'can't seem to' construction, consists in the reversal of the relative scope of seem (for some speakers, appear also) and can/could (in the schema below, $E_{D E}$ is a downward-entailing expression):

(2) Surface order (ignoring V-to-T movement):

$$
\begin{array}{ccccc}
E_{\mathrm{DE}} & \ldots & \text { can } & \ldots & \text { seem } \\
1 & & 2 & & 3 \\
\mathrm{SEEM} & \gg & \mathrm{E}_{\mathrm{DE}} & \gg & \mathrm{CAN} \\
3 & & 1 & & 2
\end{array}
$$$$
\text { Scopal relations: }
$$

Some conditions are necessary (though not sufficient). 1. Only can lends itself to the scope reversal (henceforth SR) with seem, but it only does so under certain construals (Property 1). These are identified as ability can by Langendoen (1970), but this is probably too restrictive, witness (3): 
(3) There can't seem to be enough vampire movies.

$\mathrm{SEEM} \gg \mathrm{NEG} \gg \mathrm{CAN}$

It is certain though that deontic and epistemic can are not involved. 2. The two verbs have to be relatively 'close' to each other (Property 2). 3. The reversal only occurs in the presence of an expression (henceforth the 'SR trigger') which denotes a DE function (1)-(4) (Property 3):

(4) \#John can seem to lose weight.

$* \mathrm{SEEM} \gg \mathrm{CAN}$

Not paraphrasable as: It seems that John can lose weight.

Furthermore, seem achieves wide scope over both the trigger and can (in that order) (2) (Property 4); contrary to what normally happens in present sentences (5), the predicate embedded under seem need not be stative, witness lose weight in (1) (Property 5).

(5) *John seems/doesn't seem to lose weight.

I ague that SR is not illusive (contra Jacobson (2006)): SR is due to some reordering, specifically to the covert movement of seem, which is positively polarized.

\section{Not an Idiom}

Restricting attention to the simple case where negation acts as a trigger, e.g. (1), one might get caught into thinking that SR is idiomatic. But there are a number of triggers besides negation: in fact, they form a substantial set, which is included in the familiar natural class of (Strawson) DE expressions:

(6) a. No one can seem to reach the website.

b. Few can seem to fathom how he could be so popular.

c. At most five people can seem to understand this.

d. John can never seem to speak in full sentences. [Jacobson 2006, ex. 7]

e. I just bought this lens, and I can rarely seem to get a clear picture.

f. I can hardly ever seem to find any good CD of English choral music.

g. Only John can seem to stomach watching reruns of the $6^{\text {th }}$ game of the 1986 Series.

They are thus characterized by their variability and their predictability: this allows us to eliminate the hypothesis that can, seem, and the trigger jointly form an idiom. Still, a possible rejoinder would be that there really are two parts to consider, a negatively polarized idiom can seem $(\mathrm{SEEM} \gg \mathrm{CAN}$ ) on the one hand, and some licenser (the trigger) on the other. This is a non-starter as well. In effect, the trigger can (and in fact must) take intermediate scope between the other two elements: in (6e) for example, the adverb rarely binds the closest situation variable in its scope, which is an argument of can, and hence quantifies over situations of being able to get a clear picture, not over seeming eventualities. (6e) is paraphrasable as (7a), not as (7b).

(7) a. It seems that I rarely can get a clear picture.

b. It seems upon rare occasions that I can get... 
Even if can seem were an idiom in its own right, it would be expected to combine with the rest of the sentence in a compositional way. Which means that the trigger would have to scope over the semantic construct can seem (SEEM $\gg$ CAN), contrary to fact. A proponent of the idiom line would then be forced to say that the trigger makes a non-compositional contribution to meaning in combination with can and seem. In other words, the three elements would have to form an idiom. But we have already rejected this possibility.

\section{Neg-raising}

It is important to eliminate a confound. Seem can achieve wide scope over negation and negative quantifiers by the semantic route of neg-raising. Think offers an example of a neg-raising predicate (NRP): a homogeneity inference, which is responsible for the effect, is attached to it (see Gajewski (2005) for details):

(8) John doesn't think that I understand French.

Paraphrasable as: John thinks that I don't understand French.

a. Assertion: It is not the case that John thinks that I understand French.

b. Homogeneity inference: John thinks that I understand French or John thinks that I don't understand French.

$\therefore$ John thinks that I don't understand French.

The neg-raising reading of a sentence containing a negated NRP is often favored, but it is not mandatory. Importantly, when it obtains, negation is syntactically higher than the NRP, it is not transported back into a low position: in fact, the examination of negraising suggests very strongly that movement of negation is simply impossible (Gajewski 2005). A distinctive property of NRPs is that only they pass the cyclicity test, as shown for want in (9). The test uses a negation and an NRP embedded under another NRP: it is passed when negation is interpreted below the lower NRP although it surfaces above the higher one (Horn 1978, 1989):

(9) I don't think that John wants to help me.

$\checkmark$ THINK $\gg$ WANT $\gg N E G$

Seem passes the test too, and is thus an NRP:

(10) I don't think that John seems to understand the situation. $\checkmark$ THINK $\gg S E E M \gg N E G$

The homogeneity inference has universal force in the scope of quantifiers (and is therefore assumed to be a presupposition by Gajewski (2005)). The quantifier over occasions rarely is interpreted above seem in (11), but due to homogeneity, its contribution seems to be equivalent to the combination of a high upward-entailing quantifier and of a low negation:

(11) On its op-ed page, the New York Times rarely seems to know that this nation is in major trouble.

a. Assertion: There are few occasions $\mathrm{z}$ on which the NYT seems at $\mathrm{z}$ to know that $\mathrm{p}(\mathrm{p}=$ this nation is in major trouble). 
b. Homogeneity inference: For each occasion $\mathrm{z}$, the NYT seems at $\mathrm{z}$ to know that $\mathrm{p}$ or the NYT seems at $\mathrm{z}$ not to know that $\mathrm{p}$.

$\therefore$ Paraphrasable as: It is very often the case that the NYT seems not to know that this nation is in major trouble.

But it is covert raising (syntax), not neg-raising (semantics), which explains the scope reversal SEEM $\gg$ CAN: the predicted neg-raised reading of (1) is (12) (due to the universal projection of the homogeneity inference associated with seem), but it is simply not available. Universal quantification over worlds accessible via an ability relation might be barred, witness the lack of ability must.

(12) In all worlds w' compatible with J.'s abilities in $\mathrm{w}^{*}$, it seems in w' that J. isn't losing weight.

\section{Movement}

We have seen that seem gives rise to neg-raising readings under DE expressions; these readings require seem to be syntactically under these expressions. But it also happens that seem is interpreted in a higher position than scope-bearing elements linearized before it, a fact that has not been documented yet, as far as I can tell.

We will need to distinguish two kinds of movements: (i.) seem can be interpreted in a higher position than certain elements, adverbs in particular (but the identity of the mover and the direction of the movement are unclear); (ii.) seem, qua positive polarity item (like deontic must and supposed) raises covertly out of the scope of a potential anti-licenser.

\subsection{Adverbial Expressions}

Because seem is a raising-to-subject verb, its wide scope of over quantified subjects can always be due to A-reconstruction. But adverbs are not usually assumed to A-move, let alone reconstruct. Therefore the fact that seem can outscope a number of adverbs, e.g. often, always, easily, that precede it on the surface, might suggest that it raises covertly past them:

(13) a. Context: Just looking at the hospital's visitors register, a doctor says... People often seem to visit the patient of room 32. SEEM $\gg$ OFTEN

b. Some of you guys easily seem to forget that football is a team sport.

$\mathrm{SEEM} \gg \mathrm{EASILY}$

Remarkably, the only option is surface scope when seem takes a tensed complement:

(14) a. It often seems that people visit this patient. ${ }^{*}$ SEEM $\gg$ OFTEN; OFTEN $\gg$ SEEM

b. It easily seems that some of you guys forget that football is a team sport.

$*$ SEEM $\gg$ EASILY; EASILY $\gg S E E M$

This fact is compatible with the view that adverbs are generated in the infinitive complement, moved overtly, and interpreted in a reconstructed position in (13a)-(13b). But the next section will describe facts that will make this conclusion hasty. 


\subsection{Seem is a Mobile PPI}

The deontic modal must is a mobile PPI (Property 3) (as claimed in Israel (1996, 2011), Iatridou \& Zeijlstra (2010)): this fact is established in Homer (2010a, 2010b). The property of must (should and supposed as well) that bears directly on the present discussion is its ability to raise covertly past an offending anti-licenser, e.g. negation (must is generated under negation, and raises covertly from this position; overt V-to-T is semantically idle, see Chomsky (2000)):

(15) a. John must $_{\text {deon }}$ n't leave.

MUST $\gg N E G ; * N E G \gg M U S T$

b. LF: $\left[\mathrm{John}_{1}\right.$ must $_{\text {deon } 2}$ not $\mathrm{t}_{2}\left[\mathrm{t}_{1}\right.$ leave $\left.]\right]$

Evidence for the movement of must $t_{\text {deon }}$ comes from occurrences of a quantified subject scopally sandwiched between raised must $_{\text {deon }}$ and a clausemate negation. I show in Homer (2010a) that the particular scopal configuration instantiated in (16) cannot obtain through a purely semantic route and has to be syntactic (in fact, must is not an NRP): the intermediate scope of quantified subjects is a test for movement:

(16) Context: The rules of this bowling game state that exactly one pin must remain standing, no matter which one...

Exactly one pin must $_{d e o n} n$ 't be knocked down. $\checkmark$ MUST $\gg$ EXACTLY_ONE $\gg N E G$

PPIs can be 'shielded' from an anti-licenser by interveners, e.g. every, always, conjunction, because-clauses, etc. Remarkably, when must $_{\text {deon }}$ is shielded, it cannot raise (compare with a single person, which is not an intervener):

(17) a. Not everyone must deon leave.

$*$ MUST $\gg N E G ; N E G \gg M U S T$

b. Not a single person must $t_{\text {deon }}$ leave.

MUST $\gg N E G ; * N E G \gg M U S T$

To sum up, certain intensional predicates (must, supposed, should) are positively polarized. And they scope out through covert movement when anti-licensed in their base position. This movement is blocked when unnecessary (due to shielding). I must add that it is also clause-bounded:

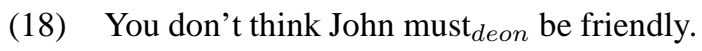

*MUST $\gg \mathrm{NEG} \gg \mathrm{THINK}$

The syntactic mechanism whereby PPIs acquire wide scope over potential anti-licensers is not to be confused with the semantic route to wide scope, viz. neg-raising. The two processes are distinct but not incompatible: nothing in principle precludes the conjunction of the two properties in a given predicate. Such is indeed the case of should andfor some speakers - of supposed (Homer 2010b). Such is also the case of seem, and I set out to show it.

In preamble, it bears saying that it is generally assumed, and wrongly so, that in order to be a PPI a given expression must be unable to be interpreted under a clausemate negation. In Homer (2011) I propose a theory of polarity item licensing which predicts that PPIs should exist that are licit in such a position. Licensing has three ingredients: (i.) polarity items are sensitive to the monotonicity of their syntactic environment (rather than to c-command by a DE expression), (ii.) to be licensed, a PI needs to find itself in at least one constituent that has the appropriate monotonicity w.r.t. its position (upward 
for a PPI, downward for an NPI), and (iii.) only certain constituents are eligible for the evaluation of the acceptability of PIs. For example, the presence of the Pol head, which hosts the polarity operator of the sentence (negation for negative sentences, and a positive operator for positive ones) is required in the constituents upon which the acceptability of some, a well-known PPI, is checked. Specifically, for each CP $\gamma$ that contains some, only the constituents that contain the Pol head of $\gamma$ are eligible. This condition on eligibility is PI-specific, and seems to be lexically determined. The contrast between (19a) and (19b) falls out from this hypothesis:

(19) a. John didn't understand something.

b. It is impossible that John understood something.

$* \mathrm{NEG} \gg \mathrm{SOME}$

IMPOS. $\gg$ SOME

In (19a), all eligible constituents are DE w.r.t. the position of some under negation, whereas in (19b), some is acceptable in at least one eligible constituent, e.g. the embedded TP. Just like must $_{\text {deon }}$, French devoir $_{\text {deon }}$ 'must' is a mobile PPI (Homer 2010a); but unlike must, it need not raise, which suggests that the smallest eligible constituents for its evaluation are smaller than the Polarity Phrase. This means that depending on which constituent is chosen for the evaluation (a constituent that contains negation vs. a constituent that doesn't), devoir either has to or cannot raise; this alternative gives rise to an illusive air of optionality:

(20) Jean ne doit deon $_{\text {pas }}$ faire de jogging. NEG $\gg$ DEVOIR; DEVOIR $\gg$ NEG Jean NEG must NEG do of jogging 'Jean need not jog/must not jog.'

Seem is similar to devoir. It can take scope above or below a clausemate negation. For clarity, I choose to add a presupposition trigger between negation and seem, viz. longer, yet. I assume these presupposition triggers to be fixed points; the make-up of the presupposition that gets computed is an index of the position where seem is licensed and interpreted, i.e. if seem is part of the presupposition, it is interpreted in the scope of the presupposition trigger; if not, it isn't:

(21) a. Context: James Bond is wending his way across a deserted warehouse; he trips over an unidentifiable body and says:

Well, this man no longer seems to be alive.

$\mathrm{SEEM} \gg \mathrm{NEG} \gg \mathrm{LONGER}$ Presupposition: This man was alive.

b. Uttered in 2006: In view of the latest recording, Bin Laden no longer seems to be dead.

Presupposition: Bin Laden used to seem to be dead.

(22) a. Uttered in 2006: In view of the latest recording, Bin Laden doesn't yet seem to be dead.

$\mathrm{SEEM} \gg \mathrm{NEG} \gg \mathrm{YET}$

Presupposition: Bin Laden is expected to die.

b. Context: Uttered right after a dictator's death by a member of his entourage (everyone in the clique strives to conceal his death from the public):

So far so good, he doesn't yet seem to be dead.

$\mathrm{NEG} \gg \mathrm{YET} \gg \mathrm{SEEM}$

Presupposition: He is expected to seem to be dead. 
The 'pin' test (16) corroborates the conclusion that seem has the ability to raise past negation:

(23) Context: There are as many guests as there are seats in an auditorium and the speaker knows it. The speaker takes a quick look and sees that exactly one seat is not taken...

Exactly one guest doesn't seem to have arrived. $\checkmark$ SEEM $\gg$ EXACTLY_ONE $\gg N E G$

The PPI hypothesis explains why seem only outscopes can when both can and seem are in the scope of a DE expression (the trigger of SR). Compare (24) and (25):

(24) a. \#John can seem to lose weight.

$* \mathrm{SEEM} \gg \mathrm{CAN}$

b. Context: John just rose from under the water. Incredible though it may sound. . . \#John can not seem to breathe for 2 minutes.

$*$ SEEM $\gg \mathrm{CAN} \gg \mathrm{NEG}$

(25) a. John can't seem to lose weight.

$\mathrm{SEEM} \gg \mathrm{NEG} \gg \mathrm{CAN}$

b. He can no longer seem to live without her. SEEM $\gg N E G \gg L O N G E R \gg C A N$

c. - How can I get my kids to donate old toys?

-When they can't yet seem to part with a favored item, try to respect their wishes but encourage them to look for other options to give.

$\mathrm{SEEM} \gg \mathrm{NEG} \gg \mathrm{YET} \gg \mathrm{CAN}$

We verify that seem not only can but has to outscope the DE expression that gives rise to SR (Property 4):

(26) Context: John had been bragging that someday he would levitate; and one day he rose above ground at a party, to his friends' amazement. But Peter later demonstrated to everyone that John used a mechanical trick at that party...

\#John can no longer seem to levitate.

SEEM $\gg \mathrm{NEG} \gg \mathrm{LONGER} \gg \mathrm{CAN}$

Paraphrasable as (only reading): John used to be able to levitate and he seems to have lost the ability.

Not as: It no longer seems that John can levitate.

This sets boundary conditions on any account of the phenomenon. And the PPI hypothesis is a natural way of explaining it. The shielding effect of every adds direct support to the PPI hypothesis:

(27) a. \#Not everyone can seem to lose weight.

$* \mathrm{SEEM} \gg \mathrm{NEG} \gg \mathrm{CAN}$

b. Not a single person can seem to lose weight.

$\mathrm{SEEM} \gg \mathrm{NEG} \gg \mathrm{CAN}$

The covert raising of must (should and supposed) is constrained: it appears to be clausebounded; likewise, seem cannot raise past a superordinate negation (28a), and also doesn't acquire maximal scope in the sentence (28b):

(28) a. \#You don't think John can seem to lose weight.*SEEM $\gg$ THINK $\gg N E G \gg C A N$

$*$ THINK $\gg \mathrm{NEG} \gg \mathrm{SEEM} \gg \mathrm{CAN}$

b. You think John can't seem to lose weight. *SEEM $\gg$ THINK $\gg N E G \gg C A N$

A superordinate negation doesn't trigger SR at all (28a). SR can occur in weakly negative environments, such as the one created by the strictly DE at most five (6c). The envi- 
ronment in the embedded clause in (28a) is one in which all NPIs, of all strengths, can be licensed, therefore it appears to be appropriately negative to precipitate SR whenever the acceptability of seem is evaluated in a constituent that contains this superordinate negation. The lack of SR must therefore be due to the presence of a clause boundary.

Lastly, when it takes a tensed complement, seem is unable to raise above a potential anti-licenser:

(29) Same context as (21a)...

\#Well, it no longer seems that this man is alive.

Presupposition: It used to seem that this man is alive.

We observed the same failure to raise in (14), hence by parity of reasoning, it is plausible that wide scope over adverbs (13) is due to a movement of the verb seem rather than to the reconstruction of the adverbs.

Since the syntactic route to wide scope is barred, it must be the semantic one (negraising) which gives rise to wide scope in (30) :

(30) It doesn't seem that this man is alive.

SEEM $\gg N E G$

\section{Temporal and Aspectual Properties of the Infinitive}

The infinitival complement of seem has two remarkable properties: (i.) its tense is identical with the matrix tense ((31d) conforms with this generalization, for it has a present perfect), and (ii.) when the matrix tense on seem is present, the main embedded predicate must be stative, or else receive a generic or habitual reading, just like predicates in present tense clauses (31e), hence the oddness of certain sentences where SR fails ((4), (27a), (28a), etc.); when the matrix tense is past, an embedded eventive is acceptable (31f):
a. John seems to be happy (*yes- terday/*tomorrow).
b. *John seems to fall.
c. *John seems to sleep.

d. John seems to have fallen.

e. John is happy/*falls/*sleeps.

f. John seemed to fall.

The 'can't seem' constellation provides the only exception to the second constraint (ii.): many of our examples contain non statives in the present, e.g. lose weight (1) or reach the website (6a). In the sentences that exhibit SR, the infinitive is undoubtedly c-selected by seem, not can, because of the presence of to. Irrespective of the particular hypothesis developed here (movement for polarity reasons), it seems inevitable that the source of the aspectual restriction under seem is not syntactic (pace Wurmbrand (2011) among many others) but semantic. This constraint remains to be explored, but one thing is already clear: in our perspective, once seem moves out, it no longer composes with the complement, but can does (Property 5). This is how the semantic constraint is respected when the complement contains an otherwise illicit non-stative. I emphasize that it wouldn't do to say that seem is a hedge (Jacobson 2006, Wurmbrand 2011), for it would then wrongly be expected to be able to take maximal scope (28b). 
The examination of seem calls for a revision of the received view on the temporalaspectual properties of the infinitival complement of seem, and of infinitives in general. This received view has an important shortcoming anyway: the problem it addresses is not well defined. For it seems ill-advised to place such a premium on the explanation of the properties of the infinitival complement of seem, given that the same puzzling contrast holds across complements, tensed or not:
a. *It seems that John falls.
b. It seemed that John fell.

The eventive predicate in (32b) is interpretable as denoting an eventuality simultaneous with the seeming eventuality; (nearly) everywhere else, past tense complements of past tense verbs cannot receive this interpretation. There has to be 'past-shifting': e.g. the time of the leaving is ordered before the time of the saying in (33) (see Stowell (2007); exceptions can be found in the complements of perception verbs, see examples of 'belief based on perception' by B. Partee, cited e.g. in von Stechow (2009)):

Peter said that John left the building.

\section{A New Distinction among Modals}

Given the clause-boundedness of the movement of modals, we are led to hypothesize that there is no clause boundary between can and seem in SR (Property 2). This prompts us to introduce a novel distinction among English root modals: can creates a monoclausal structure (Property 1), but only under certain construals. Therefore the syntax of can varies depending on its modal base and ordering source. Able does not enter into SR:

(34) \#John isn't able to seem to lose weight.

$* \mathrm{SEEM} \gg \mathrm{NEG} \gg \mathrm{ABLE}$

Able differs from can in that it takes a complement headed by to; it might also differ from it along the control/raising distinction; but it is too early to venture an explanation. Epistemic modals have been argued to create monoclausal structures (Homer 2010a); yet, they do not allow SR. It is possible that SR is blocked by Epistemic Containment, the constraint whereby an epistemic modal needs to outscope other scope-bearing elements (von Fintel \& Iatridou 2003).

\section{Conclusion}

The hypothesis that seem can move covertly to scope out of an anti-licensing environment provides a simple explanation to the scope reversal of can and seem. Ceasing to see the phenomenon as idiomatic brings new light on a number of grammatical facts, among which the temporal properties of infinitives. 


\section{References}

Chomsky, N.: Minimalist inquiries: The framework. In: Martin, R., Michaels, D., Uriagereka, J. (eds.) Step by Step: Essays On Minimalist Syntax in Honor of Howard Lasnik, pp. 89-155. MIT Press, Cambridge, Mass (2000)

von Fintel, K., Iatridou, S.: Epistemic containment. Linguistic Inquiry 34, 173-198 (2003)

Gajewski, J.: Neg-Raising: Polarity and Presupposition. Ph.D. thesis, MIT, Cambridge, Mass (2005)

Homer, V.: Epistemic modals: High ma non troppo. In: Proceedings of NELS 40 (2010a)

Homer, V.: Neg-raising and positive polarity: The view from modals. Ms., UCLA (2010b)

Homer, V.: Domains of polarity items. Ms., ENS-IJN (2011)

Horn, L.: Remarks on neg-raising. Syntax and Semantics 9, 129-220 (1978)

Horn, L.: A Natural History of Negation. University of Chicago Press (1989)

Iatridou, S., Zeijlstra, H.: Modals, negation and polarity. Talk given at Glow Asia 8 (2010)

Israel, M.: Polarity sensitivity as lexical semantics. Linguistics and Philosophy 19, 619-666 (1996)

Israel, M.: Grammar of Polarity: Pragmatics, Sensitivity and the Logic of Scales. Cambridge University Press (2011)

Jacobson, P.: I can't seem to figure this out. In: Birner, B.J., Ward, G. (eds.) Drawing the Boundaries of Meaning: Neo-Gricean Studies in Pragmatics and Semantics in Honor of Laurence R. Horn, Studies in Language Companion Series, vol. 80. John Benjamins Publishing Company (2006)

Langendoen, D.T.: The 'can't seem to' construction. Linguistic Inquiry 1(1), 25-35 (1970)

Quirk, R.: Descriptive statement and serial relationship. Language 41, 205-217 (1965)

von Stechow, A.: The (non)-interpretation of subordinate tense. Talk given at Georg-AugustUniversität Göttingen (2009)

Stowell, T.: The syntactic expression of tense. Lingua 117, 437-463 (2007)

Wurmbrand, S.: Tense and aspect in English infinitives. Ms., University of Connecticut. Storrs (2011) 


\title{
On the Non-Licensing of NPIs in the Only-Focus
}

\author{
I-Ta Chris Hsieh \\ University of Connecticut, Storrs, USA \\ i-ta.hsieh@uconn.edu
}

\begin{abstract}
This paper focuses on the ungrammaticality of NPIs in the focus associated with only. I first show that a naïve combination of the Strawson Downward Entailing (SDE) condition of NPI licensing (von Fintel 1999) and a Horn-style semantics of only (Horn 1969; a.o.) fails to predict that NPIs are ungrammatical in the focus associated only. To solve this problem, I suggest an analysis that appeals to a semantics of only implemented with the notion of innocent exclusion (Fox 2007) and a revision of the SDE condition that refers to assignment functions. The proposed analysis suggests that NPI licensing should be independent of the information provided by the discourse context.
\end{abstract}

Keywords: only, innocent exclusion, negative polarity item, NPI licensing

\section{Introduction}

Having a limited distribution, negative polarity items (NPIs) such as any and ever, as shown in (1), are grammatical in the scope of only. These items, as shown in (2) however, are ungrammatical in the focus ${ }^{1}$.

(1) a. Only [John $]_{\mathrm{f}}$ ate any vegetables. b. Only [Mary $]_{\mathrm{f}}$ has ever been to Paris.

(2) a. *Only [any student $]_{\mathrm{f}}$ ate vegetables.

b. *Only [that Mary has ever been to Paris $]_{\mathrm{f}}$ is new to John.

While the grammaticality of NPIs in the scope of only has received great attention (Horn 1996; von Fintel 1999; Wagner 2006; a.o.), their ungrammaticality inside the focus is not much discussed (Beaver 2004; Wagner 2006; a.o.).

The focus of this paper is the ungrammaticality of NPIs in the only-focus. Building on a Downward-Entailing-based (DE-based) approach of NPI licensing (Fauconnier 1975, 1979; Ladusaw 1979; von Fintel 1999; a.o.), I first show that a naïve combination of the Strawson Downward Entailing (SDE) condition (von Fintel 1999; a.o.) and a Horn-style semantics of only (Horn 1969; a.o.) fails to predict the ungrammaticality of NPIs inside the only-focus. To solve this problem along with the SDE approach, this paper suggests a solution that appeals to two crucial ingredients: one is a semantics of only implemented with the notion of innocent exclusion (Fox 2007), and the

1 Some seemingly counterexamples to this generalization are presented by Linebarger (1987) and Geurt and van der Sandt (2004). As pointed out by Horn (1996), Beaver (2004) and others, however, those examples should be analyzed as involving other licensors than only. 
other is a revision of the SDE condition that refers to assignment functions and is context independent.

To be more specific, by using the term 'context' in the introduction above, I refer to the contextual variable $C$, which is claimed in the current theories (von Fintel 1994; Rooth 1992; a.o.) to be syntactically present in quantificational sentences and sentences with focus. For instance, in (3) the contextual variable $C$ limits the quantificational domain to the salient individuals in the discourse context; in (4) the contextual variable $C$ introduces the alternative set.

(3) a. Every student had a good time. b. LF: [[every-C-student][had a good time]]

(4) a. Even [John $]_{\mathrm{f}}$ passed the exam. b. LF:[even-C [[John $]_{\mathrm{f}}$ passed the exam $\left.\left.]\right]\right]$ $C:=\{$ John passed the exam, Mary passed the exam, Bill passed the exam, ... $\}$

The claim I would like to make in this paper is that NPI licensing should be independent of the specification of $C$; an NPI is grammatical in a linguistic environment only if its SDE-ness is guaranteed solely by the lexically specified information, namely the presuppositions (definedness conditions) and the truth conditions. In an environment where an SDE inference is supported only with the implementation of information from the discourse context, NPIs cannot be licensed.

The rest of this paper is structured as the following. In section 2, I show that while a Horn-style semantics of only together with the SDE condition of NPI licensing captures the grammaticality of NPIs in the scope of only, such a combination fails to predict the ungrammaticality of these items in the focus. The proposed solution to this problem is given in section 3. Section 4 is the conclusion.

\section{$2 \quad$ NPIs and Only}

\subsection{NPIs in the Scope of Only and the SDE Condition}

Fauconnier $(1975,1979)$ and Ladusaw (1979) suggest that a downward entailing inference (i.e. an inference from a set to its subset) is crucial for an environment to license NPIs. For instance, a downward entailing inference is supported in the restrictor of the universal quantifier every but not that of the existential quantifier some (see (5)); NPIs hence are grammatical in the restrictor of every but not in that of some (see (6)). Here I adopt the notion of cross-categorial entailment $(\Rightarrow)$ given in (7).

(5) books on NPIs $\subseteq$ books

Every student who read a book passed the exam. =>

Every student who read a book on NPIs passed the exam.

Some student who read a book passed the exam.=/=>

Some student who read a book on NPIs passed the exam.

(6) Every/*some student who read any books on NPIs passed the exam.

(7) Cross-categorial entailment $(\Rightarrow)$

a. for any $\mathrm{p}, \mathrm{q}$ of type $\mathrm{t}, \mathrm{p}=>\mathrm{q}$ iff $\mathrm{p}=0$ or $\mathrm{q}=1$

b. for any $\mathrm{f}, \mathrm{h}$ of type $<\sigma, \mathrm{t}>, \mathrm{f}=>\mathrm{g}$ iff for all $\mathrm{x}$ of type $\sigma, \mathrm{f}(\mathrm{x}) \Rightarrow \mathrm{h}(\mathrm{x})$ 
A DE inference, however, is not intuitively supported in the scope of only despite the fact that NPIs are grammatical in this environment (see (1)). Take (8) for instance; it could be the case that nobody other than John ate vegetables but what John ate was not kale; in this case, the inference in (8) is not truth-preserving.

(8) kale $\subseteq$ vegetables

Only $[\mathrm{John}]_{\mathrm{f}}$ ate vegetables. $=/=>$ Only $[\mathrm{John}]_{\mathrm{f}}$ ate kale.

To account for the licensing of NPIs in the scope of only, von Fintel (1999) suggests that NPI licensing is subject to a weaker notion of entailment, which he dubbed as Strawson entailment; the premise Strawson-entails the conclusion iff the premise together with the presuppositions (i.e. definedness conditions) of the conclusion entail the conclusion. A licensing condition of NPIs based on Strawson Entailment is formalized as in (9).

(9) a. The SDE condition of NPI licensing (von Fintel 1999): An NPI is only grammatical if it is in the scope of $\alpha$ such that $\llbracket \alpha \rrbracket$ is SDE.

b. Strawson Downward Entailingness:

A function $f$ of type $<\sigma, \tau>$ is Strawson downward entailing (SDE) iff for all $\mathrm{x}$, $\mathrm{y}$ of type $\sigma$ such that $\mathrm{x} \Rightarrow \mathrm{y}$ and $\mathrm{f}(\mathrm{x})$ is defined: $\mathrm{f}(\mathrm{y}) \Rightarrow \mathrm{f}(\mathrm{x})$

The SDE condition in (9) together with a Horn-style semantics of only like (10) (Horn 1969; a.o.) provide a straightforward account for the licensing of NPIs in the scope of only. Here I assume that only takes as its first argument a contextually provided alternative set $C$ whose members are of the same type as that of the focalized constituent (Rooth 1985, 1992; a.o.); the focalized constituent then moves at LF to adjoin to only- $C$ and serves as the second argument of only ${ }^{2}$; a predicate that is created by $\lambda$-abstraction over the trace of the focalized constituent serves as the third argument (see (10a) $)^{3}$. Here I term the proposition that only quantifies in 'the prejacent'; for instance, the prejacent of Only [John $]_{\mathrm{f}}$ ate vegetables is John ate vegetables. Based on (10b), an only-sentence is defined only if its prejacent is true; if defined, it is true iff there is no alternative proposition that is true and not entailed by the prejacent. (11) is an example that demonstrates the semantics in (10). I further assume that $C$ is a free variable bearing an index and receives its value from a contextually provided assignment function $\mathrm{g}$, which maps an integer to a set of alternatives to the focus.

(10) a.

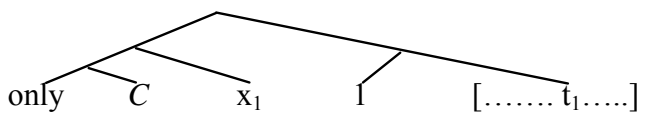

${ }^{2}$ In this paper I adopt a focus-movement approach for the syntax of an only-sentence (Wagner 2006; a.o.). Adopting a propositional approach (Rooth 1992; a.o.) instead, however, would not lead to different predictions regarding the issue discussed.

${ }^{3}$ I assume Intensional Functional Application (IFA; see (i); see Heim and Kratzer (1998)).

(i) Intensional Functional Application (IFA): If $\alpha$ is a branching node and $\{\beta, \gamma\}$ the set of its daughters, then, for any world $\mathrm{w}$ and assignment $\mathrm{g}$ : if $\llbracket \beta \rrbracket^{\mathrm{w}, \mathrm{g}}$ is a function whose domain contains $\left[\lambda \mathrm{w}^{\prime} \cdot \llbracket \gamma \rrbracket^{\mathrm{w}^{\prime}, \mathrm{g}}\right]$, then $\llbracket \alpha \rrbracket^{\mathrm{w}, \mathrm{g}}=\llbracket \beta \rrbracket^{\mathrm{w}, \mathrm{g}}\left(\lambda \mathrm{w}^{\prime}\right.$. $\left.\llbracket \gamma \rrbracket^{\mathrm{w}^{\prime}, \mathrm{g}}\right)$ 
b. for any $C_{<\sigma, t>}, \mathrm{x}_{\sigma}, \mathrm{P}_{<\mathrm{s},<\sigma \downarrow>}, \llbracket$ only $\rrbracket^{\mathrm{w}}(C)(\mathrm{x})(\mathrm{P})$ is defined only if $\mathrm{P}(\mathrm{w})(\mathrm{x})=1$; if defined, [only $\rrbracket^{\mathrm{w}}(C)(\mathrm{x})(\mathrm{P})=1$ iff $\forall \mathrm{y}_{\sigma}[\mathrm{y} \in C \& \mathrm{P}(\mathrm{w})(\mathrm{y})]$ :

$\left\{\mathrm{w}^{\prime}: \mathrm{P}\left(\mathrm{w}^{\prime}\right)(\mathrm{x})=1\right\} \subseteq\left\{\mathrm{w}^{\prime}: \mathrm{P}\left(\mathrm{w}^{\prime}\right)(\mathrm{y})=1\right\}$

(11) a. Only $[\mathrm{John}]_{\mathrm{f}}$ ate vegetables.

b. LF: [[only- $\left.\left.C_{8}\right] \mathrm{John}_{3}\right]\left[3\left[\mathrm{t}_{3}\right.\right.$ ate vegetables $\left.\left.]\right]\right]$

c. $\llbracket(11 \mathrm{a}) \rrbracket^{\mathrm{w}, \mathrm{g}}$ is defined only if $\llbracket$ ate vegetables $\rrbracket^{\mathrm{w}}(\mathrm{John})=1$; if defined, $\llbracket(11 \mathrm{a}) \rrbracket^{\mathrm{w}, \mathrm{g}}=1$ iff $\forall \mathrm{y}_{\mathrm{e}}[\mathrm{y} \in \mathrm{g}(8) \& \mathrm{P}(\mathrm{w})(\mathrm{y})]$ :

$\left\{\mathrm{w}^{\prime}: \llbracket\right.$ ate vegetables $\left.\rrbracket^{\mathrm{w}^{\prime}}(\mathrm{John})\right\} \subseteq\left\{\mathrm{w}^{\prime}: \llbracket\right.$ ate vegetables $\left.\rrbracket^{\mathrm{w}^{\prime}}(\mathrm{y})\right\}$

The semantics in (10) renders the scope of only (i.e. the third argument of only) SDE (in other words, 【only $\rrbracket^{\mathrm{w}}(C)(\mathrm{x})$ is an SDE function). Take (8) again for instance; while the premise Only John ate vegetables alone does not entail the conclusion only John ate kale, the premise together with the presupposition of the conclusion, namely John ate kale, entail the conclusion. The licensing of NPIs in the scope of only hence follows from the SDE condition (see (9)), which states that NPIs are only grammatical in SDE environments.

\subsection{NPIs in the Focus Associated with Only}

As shown in (2), unlike in its scope, NPIs are ungrammatical in the focus associated with only. While the combination of a Horn-style semantics of only like (10b) and the SDE condition in (9) correctly predicts the grammaticality of NPIs in the scope of only, they fail to capture the ungrammaticality of these items in the focus.

In order to show this, there are a couple of assumptions that need to be laid out. I assume that focus evokes a presupposition on the alternative set $C$ : $C$ must contain the focalized element $x$ and at least one other member than $x$ (Rooth 1992; a.o.). Furthermore, when checking the entailment relation between any two distinct propositions, $C$ should be kept constant in the premise and the conclusion; otherwise, there would be no constant context within which to access the downward or upward inference. Therefore, when checking the entailment relation between $(12 a, b)$, the alternative set $C$ should be kept constant in the premise and the conclusion and contain as its members both some student and some linguistics student.

Based on (10), the focus in an only-sentence is an SDE environment as well. As shown in (12), the premise (12a) contradicts the presupposition of the conclusion (12b); while (12a) asserts that no alternative proposition not entailed by its prejacent some student cried is true, $(12 \mathrm{~b})$ presupposes that its prejacent some linguistics student cried, one of the alternative propositions stronger than the prejacent of (12a), is true. Given that the premise (12a) together with the presupposition of the conclusion $(12 b)$ is a contradiction and a contradiction entails any propositions (see (7)), an SDE inference from (12a) to (12b) is valid (in $(12 \mathrm{a}, \mathrm{b}), \llbracket \Sigma \mathrm{P} \rrbracket^{\mathrm{W}}=\left[\lambda \mathrm{f}_{<\mathrm{e},<\mathrm{e}, \downarrow>} \mathrm{f}\left(\llbracket\right.\right.$ cried $\left.\left.\left.\rrbracket^{\mathrm{w}}\right)\right]\right)$.

(12) a. Only [some student $]_{\mathrm{f}}$ cried.

LF: [[only- $\left.C_{8}[\text { some student }]_{3}\right]\left[\Sigma \mathrm{P} 3\left[\mathrm{t}_{3}\right.\right.$ cried $\left.]\right]$

Presupposition: There is a student $\mathrm{x}$ such that $\mathrm{x}$ cried in $\mathrm{w}$. 
Assertion: $\forall \mathrm{f}_{<\mathrm{e},<\mathrm{e}, \downarrow>}\left[\mathrm{f} \in \mathrm{g}(8)\right.$ and $\left.\llbracket \Sigma \mathrm{P} \rrbracket^{\mathrm{w}}(\mathrm{f})=1\right]$ :

$\left\{\mathrm{w}^{\prime}:\right.$ there is a student $\mathrm{x}$ such that $\mathrm{x}$ cried in $\left.\mathrm{w}^{\prime}\right\} \subseteq\left\{\mathrm{w}^{\prime}: \llbracket \Sigma \mathrm{P} \rrbracket^{\mathrm{w}^{\prime}}(\mathrm{f})=1\right\}$

b. Only [some linguistics student $]_{\mathrm{f}}$ cried.

LF: [[only- $C_{8}$ [some linguistics student $\left.]_{3}\right]\left[{ }_{\Sigma \mathrm{P}} 3\left[\mathrm{t}_{3}\right.\right.$ cried $\left.]\right]$

Presupposition: There is a linguistics student $\mathrm{x}$ such that $\mathrm{x}$ cried in $\mathrm{w}$.

Assertion: $\forall \mathrm{f}_{<\mathrm{e},<\mathrm{e}, \mathrm{t}>}\left[\mathrm{f} \in \mathrm{g}(8)\right.$ and $\left.\llbracket \Sigma \mathrm{P} \rrbracket^{\mathrm{w}}(\mathrm{f})=1\right]:\left\{\mathrm{w}^{\prime}\right.$ : there is a linguistics student $\mathrm{x}$ such that $\mathrm{x}$ cried in $\left.\mathrm{w}^{\prime}\right\} \subseteq\left\{\mathrm{w}^{\prime}: \llbracket \Sigma \mathrm{P} \rrbracket^{\mathrm{w}^{\prime}}(\mathrm{f})=1\right\}$

c. $g(8):=\{$ some student, some linguistics student, .... $\}$

$\underline{(12 a)+\text { the presupposition of }(12 b)}=>(12 b)$

Contradiction

That the focus associated with only, based on a Horn-style semantics like (10), is SDE further leads to the prediction that NPIs are grammatical in this environment. As already shown in (2) however, this prediction is incorrect.

Given that the SDE-ness in the focus associated with only results from a contradiction between the premise and the presupposition of the conclusion and hence is trivial, one might try to save a Horn-style semantics like (10) from this wrong prediction by claiming that while NPI licensing is subject to SDE-ness, an environment that is trivially SDE cannot license NPIs. Such an analysis, however, cannot be an adequate solution. In the literature (e.g., Horn 1996; Ippolito 2008; a.o.), the claim that an onlysentence presupposes its prejacent has been challenged; many researchers have suggested that the presupposition triggered by only should be something weaker than the prejacent (see (13) for some of such proposals). Once the presupposition in (10b) is replaced by a weaker one like (13a, b), the focus associated with only would be nontrivially SDE, which again leads to the wrong prediction that NPIs are grammatical in this environment.

(13) a. Horn 1996: Existential Presupposition

【only $\rrbracket^{\mathrm{w}}(C)(\mathrm{x})(\mathrm{P})$ is defined only if $\exists \mathrm{y}[\mathrm{y} \in C$ and $\mathrm{P}(\mathrm{w})(\mathrm{y})=1]$

b. Ippolito 2008: Conditional Presupposition

$\llbracket$ only $\rrbracket^{\mathrm{w}}(C)(\mathrm{x})(\mathrm{P})$ is defined only if $\exists \mathrm{y}[\mathrm{y} \in C$ and $\mathrm{P}(\mathrm{w})(\mathrm{y})=1] \rightarrow \mathrm{P}(\mathrm{w})(\mathrm{x})$

Due to the controversy on the presupposition triggered by only, I suggest that the key to a fundamental solution should lie in its exclusive component. In the following, I suggest that a semantics of only implemented with the notion of innocent exclusion (Fox 2007) together with a revision of the SDE condition that refers to assignment functions provide a solution to the problem pointed above.

\section{The Proposal}

\subsection{Only and Innocent Exclusion}

Fox (2007) suggests that in an only-sentence, the alternatives that are excluded must be innocently excludable. In a nutshell, the set of innocently excludable alternatives (henceforth, I.E.) is the intersection of the maximal sets the conjunction of nega- 
tion of whose members is consistent with the prejacent. Take (14) for instance; assuming that the alternative set $C$ introduced in (14a) is (14b), the only innocently excludable alternative is John and Mary. The other two alternatives, namely John and Mary, cannot be in the same maximal set of excludable alternatives, for excluding both these two alternatives together would lead to contradiction to the prejacent (i.e. the conjunction of John did not cry and Mary did not cry contradicts to the prejacent John or Mary cried).

(14) a. Only [John or ${ }_{f}$ Mary] cried.

b. The alternative set C: $\{$ John, Mary, John or Mary, John and Mary\} Max. sets of excludable alternatives: $\{J o h n, J$. and M. $\},\{$ Mary, J. and M. $\}$ I.E.: $=\{$ John, John and Mary $\} \cap\{$ Mary, John and Mary $\}=\{$ John and Mary $\}$

Building on Fox's (2007) proposal, I suggest the semantics of only in (15), which is implemented with a cross-categorial notion of innocent exclusion ${ }^{4}$. Based on (15), the only-sentence (12a) translates as $\left(16 a^{\prime}\right)$.

(15) a. for any $C_{<\sigma, \downarrow}, \mathrm{x}_{\sigma}, \mathrm{P}_{<\mathrm{s},<\sigma \gg>,} \llbracket$ only $\rrbracket^{\mathrm{w}}(C)(\mathrm{x})(\mathrm{P})$ is defined only if $\mathrm{P}(\mathrm{w})(\mathrm{x})=1$; if defined, 【only $\rrbracket^{\mathrm{w}}(C)(\mathrm{x})(\mathrm{P})=1$ iff $\neg \exists \mathrm{y}_{\sigma} \in \operatorname{I}$.E. $(\mathrm{x}, C, \mathrm{P}): \mathrm{P}(\mathrm{w})(\mathrm{y})=1$

b. for any $\mathrm{x}$ of type $\sigma$ and its alternative set $C_{<\sigma, \downarrow}$ and any $\mathrm{P}_{<\mathrm{s},<\sigma, \downarrow>>}$, the set of innocent excludable alternatives to x w.r.t $C$ and $\mathrm{P}$ (henceforth, I.E.(x, $C$, P)) is defined as the following:

$$
\begin{aligned}
\text { I.E.(x, } C, \mathrm{P}):= & \cap\left\{C^{\prime} \subseteq C: C^{\prime} \text { is a maximal set in } C\right. \text { such that } \\
& \left.\cap\left(\left\{[\lambda \mathrm{w} . \neg \mathrm{P}(\mathrm{w})(\mathrm{y})]: \mathrm{y} \in C^{\prime}\right\} \cup\{[\lambda \mathrm{w}: \mathrm{P}(\mathrm{w})(\mathrm{x})]\}\right) \neq \varnothing\right\}
\end{aligned}
$$

(16) a. Only [some student $]_{\mathrm{f}}$ cried. LF: [[only- $\left.C_{8}[\text { some student }]_{3}\right]\left[{ }_{\Sigma \mathrm{P}} 3\left[\mathrm{t}_{3}\right.\right.$ cried $\left.]\right]$

$\mathrm{a}^{\prime}$. 【 (16a) $\rrbracket^{\mathrm{w}, \mathrm{g}}$ is defined only if there is a student $\mathrm{x}$ such that $\mathrm{x}$ cried in $\mathrm{w}$; if defined, $\llbracket(16 \mathrm{a}) \rrbracket^{\mathrm{w}, \mathrm{g}}=1$ iff $\neg \exists \mathrm{f}_{<\mathrm{e},<\mathrm{e}, \downarrow>} \in \mathrm{I}$.E.(some-stu, $\mathrm{g}(8),\left[\lambda \mathrm{w}^{\prime}\right.$. $\left.\left.\llbracket \Sigma \mathrm{P} \rrbracket^{\mathrm{w}^{\prime}}\right]\right)$ :

$$
\llbracket \Sigma \mathrm{P} \rrbracket^{\mathrm{W}}(\mathrm{f})=1
$$

The I.E. in $\left(16 a^{\prime}\right)$ is determined by the content of $g(8)$ and may vary from one discourse context to another. This is illustrated in the examples (17) and (18). Consider the discourse context in (17) first. In (17), the discourse context provides an alternative set $g(8)$ as in $\left(17 a^{\prime}\right)$; by asserting (16a) in this context, one excludes all the alternatives in $\mathrm{g}(8)$ other than the focus some student. In this case, the set of innocently excludable alternatives include all the members in $\mathrm{g}(8)$ other than some student.

(17) Discourse context: The college was holding a campus-wide reception for the new president. Faculty, staff, and students were all invited. You know the faculty, the staff, and you also know the students from linguistics. Nonethe-

4 The lexical entry for only and the original definition of innocent exclusion suggested in Fox (2007) are propositional (see (i))

(i) 【only $\rrbracket\left(\mathrm{A}_{<<\mathrm{s}, \mathrm{t}, \mathrm{t}}\right)\left(\mathrm{p}_{<\mathrm{s}, \mathrm{t}}\right)=[\lambda \mathrm{w}$ : $\mathrm{p}(\mathrm{w})=1 . \forall \mathrm{q} \in \mathrm{I}$.E. $(\mathrm{p}, \mathrm{A}) \rightarrow \mathrm{q}(\mathrm{w})=0]$

I.E. $(p, A)=\cap\left\{A^{\prime} \subseteq A: A^{\prime}\right.$ is a max. set in $A$ s.t. $A^{\prime} \cup\{p\}$ is consistent $\} ; A^{\prime}=\{\neg p: p \in A\}$ 
less, you didn't know the rest of the students by name. You saw that the faculty, staff and the linguistics students had no interest in the vegetables that were served, but one of the other students in the reception was eating them voraciously. The next day someone asks you who at the reception ate vegetables....

You: Only [some student $]_{\mathrm{f}}$ ate vegetables.

a. $g(8):=\{$ some student, some linguistics students, some faculty, some staff $\}$ max. set of excludable alt.: \{some ling. student, some faculty, some staff\} I.E.: = \{some linguistics student, some faculty, some staff $\}$

Now consider (18); this time the discourse context provides an alternative set $g(8)$ as in $\left(18 \mathrm{a}^{\prime}\right)$. In this context, the set of students is exhaustified by the set of linguistics students and the set of philosophy students. By asserting (16a) in this context, one merely excludes the alternatives some faculty and some staff but not the other two (namely some linguistics student and some philosophy student). In (18a'), given that the conjunction of negation of some linguistics student ate vegetables and that of some philosophy student ate vegetables contradicts to the prejacent some student ate vegetables, these two alternatives cannot be in the same maximal set of excludable alternatives. The set of innocently excludable alternatives hence merely contains the alternatives some faculty and some staff.

(18) Discourse context: The college was holding a reception to honor the students in linguistics and philosophy only. In addition to those students, the faculty and staff of the entire college were invited. While at this reception you saw that the faculty and staff showed no interest in the vegetables, someone you identified as being a student ate vegetables voraciously. The next day someone asks you who at the reception ate vegetables...

You: Only [some student $]_{\mathrm{f}}$ ate vegetables.

$a^{\prime} . g(8):=\{$ some stu, some phil. stu, some ling. stu, some faculty, some staff $\}$ (the set of students contains only linguistics and philosophy students) max. excludable alt. set:= some phil. stu, some faculty, some staff $\}$,

I.E.:=\{some faculty, some staff $\}$ \{some ling. stu, some faculty, some staff\}

Now with the semantics in (15), the focus associated with only (i.e. the second argument of only) is not always SDE. In the discourse context given in (17), (16a) contradicts the presupposition of (19a). Given that a contradiction entails any propositions, an SDE inference is valid in the only-focus under the context in (17) (see (20a); see also (19) for the meaning of (12b) based on (15)). On the other hand, in the discourse context in (18), an SUE rather than SDE inference is valid in the focus associated with only (see (20b)).

(19) a. Only [some linguistics student $]_{\mathrm{f}}$ cried.

LF: $\left[\left[\right.\right.$ only- $\left.C_{8}[\text { some linguistics student }]_{3}\right]\left[{ }_{\Sigma \mathrm{P}} 3\left[\mathrm{t}_{3}\right.\right.$ cried $\left.]\right]$ 
$a^{\prime}$. $\llbracket(19 b) \rrbracket^{\mathrm{N}, \mathrm{g}}$ is defined only if there is some linguistics student $\mathrm{x}$ such that $\mathrm{x}$ cried in $w$; if defined, $\llbracket(19 b) \rrbracket^{\mathrm{N}, \mathrm{g}}=1$ iff

$$
\left.\neg \exists \mathrm{f}_{<\mathrm{e},<\mathrm{e}, \mathrm{t}>} \in \mathrm{I} \text {.E.(some-ling-stu, } \mathrm{g}(8),\left[\lambda \mathrm{w}^{\prime} . \llbracket \Sigma \mathrm{P} \rrbracket^{\mathrm{w}^{\prime}}\right]\right): \llbracket \Sigma \mathrm{P} \rrbracket^{\mathrm{N}}(\mathrm{f})=1
$$

b. with the alternative set $\mathrm{g}(8)$ in $(17 \mathrm{a})$ :

I.E.(some-ling-stu, $\left.\mathrm{g}(8),\left[\lambda \mathrm{w}^{\prime} . \llbracket \Sigma \mathrm{P} \rrbracket^{\mathrm{w}^{\prime}}\right]\right):=\{$ some faculty, some staff $\}$

c. with the alternative set $\mathrm{g}(8)$ in $(18 \mathrm{a})$ :

I.E.(some-ling-stu, $\left.\mathrm{g}(8),\left[\lambda \mathrm{w}^{\prime} . \llbracket \Sigma \mathrm{P} \rrbracket^{\mathrm{v}^{\prime}}\right]\right):=\{$ some phil. stu., some faculty, some staff

(20) a. with the alternative set $g(8)$ in $(17 a)$ :

$(16 a)+$ the presupposition of $(19 a)=>(19 a)$

Contradiction

$(19 a)+$ the presupposition of $(16 a)=/=>(16 a)$

b. with the alternative set $\mathrm{g}(8)$ in $(18 \mathrm{a})$ :

$(16 a)+$ the presupposition of $(19 a)=/=>(19 a)$

$(19 a)+$ the presupposition of $(16 a)=>(16 a)$

\subsection{NPI Licensing, the SDE Condition, and Context (In)Dependency}

The second tool that is needed to account for the ungrammaticality of NPIs inside the only-focus is a licensing condition that is context-independent. As shown in (20), an SDE inference may be valid in the focus associated with only with certain specification for $C$ (i.e. the first argument of only). Based on the SDE condition in (10), we are led to the prediction that NPIs are grammatical in the focus associated with only in the discourse context that renders only- $C$ an SDE function (e.g., (17)). This prediction is not borne out; NPIs are ungrammatical in the focus of an only-sentence regardless the discourse context. A revision of the SDE condition in (10) hence is required.

Here I suggest a revision of the SDE condition as in (21). Based on (21), a syntactic object is qualified as an NPI licensor iff it denotes an SDE function with respect to any assignment function $\mathrm{g}$. Given that an assignment function $\mathrm{g}$ is given by the discourse context, (21) suggests that an NPI licensor should denote an SDE function across discourse contexts.

(21) The Revised SDE Condition: An NPI is only grammatical in the scope of $\alpha$ such that, for any assignment function $g, \llbracket \alpha \rrbracket^{\mathrm{g}}$ is SDE.

Based on the semantics of only in (15), only-C denotes an SDE function only with certain specification for $C$. Given that the focus associated with only is not always SDE with respect to a contextually provided assignment function g, NPIs are ungrammatical in this environment.

The analysis suggested above not only predicts the ungrammaticality of NPIs inside the focus associated with only but also that of these items in a DP-constituent containing the focus. As observed by Wagner (2006) and others, NPIs are ungrammatical in a DP-constituent containing the only-focus (see (22a)). Wagner (2006) and others suggest that at LF the whole DP-constituent that contains the focus is pie-pied and serves 
as the second argument of only (see (22b)); with this assumption, the ungrammaticality of NPIs in a DP-constituent containing the focus associated with only follows from the semantics in (15) and the SDE condition in (21): given that based on the semantics in (15), the second argument of only is not always an SDE environment with respect to an assignment function g, NPIs are ungrammatical in a DP-constituent containing the focus associated with only.

(22) a. *Only any inhabitant of [Twin Earth $]_{\mathrm{f}}$ met Particle Man.

b. LF: [[only-C [any inhabitant of Twin Earth $]_{3}\left[3\right.$ [ $\mathrm{t}_{3}$ met Particle Man]]]

\section{$4 \quad$ Concluding Remarks}

In this paper, I discuss the ungrammaticality of NPIs in the focus associated with only. I have shown that a naïve combination of a Horn-style semantics of only and the SDE condition of NPI licensing fails to predict the ungrammaticality of these items in the only-focus. I further suggest that this problem can be solved with a semantics of only with the implementation of the notion of innocent exclusion (Fox 2007) and a revision of the SDE condition that refers to assignment functions.

The proposal of this paper reaches the conclusion that NPI licensing is independent of the discourse context; for a linguistic expression to license an NPI, it must denote an SDE function without the implementation of the information provided by the discourse context. This, however, is not an uncontroversial claim; one challenge that this claims faces is the contrast between definite and indefinite superlatives with respect to NPI licensing. Herdan and Sharvit (2006) observe that although the distribution is limited, indefinite superlatives do exist (see (23b)); nevertheless, NPIs are only licensed in definite but not in indefinite superlatives (see (24)).

(23) a. This class has the best student. $\quad$ b. This class has a best student.

(24) This class has the/*a best student with any knowledge of French

To provide a unified semantics for -est in both definite and indefinite superlatives (see (23)), Herdan and Sharvit suggest the semantics in (25).

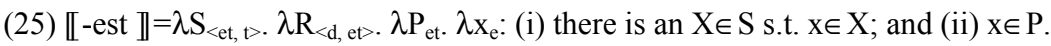
For some $X \in S$ such that $\mathrm{X} \in X$, there is a degree $d$ such that $\{z: R(d)(z)=1$ and $\mathrm{P}(\mathrm{z})=1\}=\{\mathrm{x}\}$

Based on this semantics, the superlative morpheme $\llbracket$-est $\mathbb{( S})(\mathrm{R})$ is not an SDE function. As Herdan and Sharvit (2006) point out however, an SDE inference, based on (25), is supported in the restrictor P of -est when $\mathrm{S}$ is a singleton set. Given that this is only guaranteed in a definite superlative due to the uniqueness requirement of the, NPIs are grammatical only in definite superlatives.

The contrast in (23) poses a challenge to the conclusion drawn from the discussion above, for based on Herdan and Sharvit's analysis, NPIs in definite superlatives are licensed because of a unique specification for the contextual variable S. In Hsieh (2011), I suggest that although further investigation on the (anti-)uniqueness require- 
ment in (in)definite superlatives is required, the claim that NPI licensing is contextindependent may be maintained if an environment-based approach of NPI licensing (Heim 1984; Zwarts 1996; Gajewski 2007; a.o.), rather than an operator-based one, is adopted. I thereby refer the reader to Hsieh (2011) for more details and leave this issue for future research.

\section{References}

1. Beaver, David. 2004. Five only pieces. Theoretical Linguistics 30: 45-64.

2. Fauconnier, Gilles. 1975. Polarity and the Scale Principle. Chicago Linguistics Society 11.

3. Fauconnier, Gilles. 1979. Implication Reversal in a Natural Language. Formal Semantics and Pragmatics for Natural Languages, ed. by Franz Guenthner and S. J. Schmidt, 289302: Dodrecht.

4. von Fintel, Kai. 1994. Restrictions on Quantifier Domains. Ph.D. Dissertation, Umass Amherst.

5. von Fintel, Kai. 1999. NPI-licensing, Strawson entailment, and context dependency. Journal of Semantics 16: 97-148.

6. Fox, Danny. 2007. Free Choice Disjunction and the Theory of Scalar Implicature. Presupposition and Implicature in Compositional Semantics, ed. by Uli Sauerland and Penka Stateva. New York, NY: Palgrave Macmillan.

7. Gajewski, Jon. 2007. Neg-raising and polarity. Linguistics and Philosophy 30. 289-328.

8. Geurt, Bart, and Rob van der Sandt. 2004. Interpreting focus. Theoretical Linguistics 30: 1-44.

9. Heim, Irene. 1984. A Note on Negative Polarity Items and DE-ness. NELS 14.

10. Heim, Irene, and Angelika Kratzer. 1998. Semantics in Generative Grammar. Oxford: Blackwell

11. Herdan, Simona, and Yael Sharvit. 2006. Definite and nondefinite superlatives and NPI licensing. Syntax 9: 1-31.

12. Horn, Laurence R. 1969. A presuppositional analysis of only and even. Chicago Linguistics Society 5.

13. Horn, Laurence R. 1996. Exclusive company: Only and the dynamics of vertical inference. Journal of Semantics 13: 10-40.

14. Hsieh, I-Ta Chris. 2011. On the non-licensing of NPIs in the only-focus. Talk given in Harvard University.

15. Ippolito, Michela. 2008. On the meaning of only. Journal of Semantics 25: 45-91.

16. Ladusaw, William A. 1979. Polarity Sensitivity as Inherent Scope Relations. Ph.D Dissertation: The University of Texas at Austin.

17. Linebarger, Marcia C. 1987. Negative polarity and grammatical representation. Linguistic and Philosophy 10: 325-87.

18. Rooth, Mats. 1985. Association with Focus. Ph.D. Dissertation: Umass, Amherst

19. Rooth, Mats. 1992. A theory of focus interpretation. Natural Language Semantics 1: 75116.

20. Wagner, Michale. 2006. Association by movement: Evidence from NPI-licensing. Natural Language Semantics 14: 297-324.

21. Zwarts, Frans. 1996. Facets of Negation. Quantifiers, Logic and Language, ed. by Jaap van der Does and Jan van Eijck, 385-421. Standford: CSLI Publication. 


\title{
Now: A Discourse-Based Theory
}

\author{
Julie Hunter \\ l'Université de Pau et des Pays de l'Adour and \\ l'EHESS/l'Institut Jean-Nicod, Paris
}

In general, now is interpreted as the utterance time and cannot refer to a time made salient in the discourse in the way that a third person pronoun can refer to an individual made salient in the discourse:

(1) I like to think about my grandmother. I always had a great time with her.

(2) I like to think back on the summer of '97. I was so happy ${ }^{*}$ now.

Yet there are exceptions (cf. Banfield 1982, Hunter 2010, Kamp \& Reyle 1993, Lee \& Choi 2009, Predelli 1998, Recanati 2004, Schlenker 2004). In the following examples, now denotes a time that lies in the past of the utterance time and is introduced at some prior point in the discourse:

(3) Five months later, I sat with her as she lay in bed, breathing thin slivers of breath and moaning... I was alone in her bleak room. Alone, because there was none of her in it, just a body that now held no essence of my mum. ${ }^{1}$

(4) The letter is marked "personal and private" and is addressed to President Franklin D. Roosevelt's secretary, Grace Tully, who was with the ailing chief executive in Warm Springs, Ga., that Thursday in 1945. The writer was Lucy Mercer Rutherfurd, who decades before had been FDR's mistress and who now was making arrangements for what would be their last fateful meeting at the president's rural retreat. ${ }^{2}$

(3) is taken from an article in which the author describes her mother's struggles with Alzheimer's. Throughout the article, it is clear that the author is recounting past events. Her use of now does not denote the utterance time in any sense; it rather denotes a time in the past at which she visited her ailing mother. The two sentences in (4) are about a letter to FDR that was acquired by the National Archives. The author of the article describes the writing of the letter as an event in the past and clearly distances that event from the time of the acquisition. Still, he can use now to denote the time of the past letter writing event.

This paper offers a semantic theory of anaphoric uses of now; that is, uses of now in which now refers to a time introduced in discourse. Contrary to existing theories of now, I argue that the interpretation of an anaphoric use of now is determined by the rhetorical structure of the discourse in which the token of now figures. The details of my theory are presented in Segmented Discourse Representation Theory (Asher \& Lascarides 2003).

\footnotetext{
1 'Her misery was now so deep, her existence so shallow - Fiona Phillips on dealing with Alzheimer's', from Daily Mail, August 28, 2010.

2 'What was for FDR's eyes only is now for yours', The Washington Post, 07.29.2010.
} 


\section{Previous discourse accounts}

Kamp \& Reyle (1993) recognize that now can be used anaphorically to refer to a time introduced in discourse. However, now can only be so used, they claim, to modify past tense clauses that describe states. The idea underlying this distinction is that clauses describing states exploit the incoming reference time while clauses describing events introduce reference times of their own. While I cannot elaborate on Kamp \& Reyle's view here, the important point is that because state-denoting clauses exploit incoming reference points, they are able to shift the temporal perspective point (TPpt) of a discourse, where the TPpt is the time relative to which events are described as unfolding. Event denoting sentences, because they do not exploit incoming reference times, do not shift the TPpt. As now depends on the TPpt of a discourse, according to Kamp \& Reyle, it can refer to a past time only when the TPpt has been shifted to the past by a state-denoting sentence.

Kamp \& Reyle's account does not do justice to the data on now, however. First, now can be used to modify event denoting clauses.

(5) That was the kind of people in whom Paul had become so interested, and to whom he now wrote his letter. ${ }^{3}$

(6) Before being dipped into the liquid air, it would not burn; but now it exploded, it was consumed so rapidly. ${ }^{4}$

A second problem stems from the fundamental claim that clauses that describe events shift the time relative to the input context while clauses that describe states inherit the time of the incoming context. A third problem is that Kamp \& Reyle use a discourse theory that updates the temporal perspective point for a discourse sentence by sentence, or perhaps clause by clause, without taking into account the relations between these sentences or clauses. ${ }^{5}$ What we see when we look at data on now is that (a) a past tense clause modified by now may fail to stand in a temporal relation to the clause that has come before it, regardless of its aspect, and (b) even if there is a relation between the two clauses, this relation may not be enough to determine the interpretation of now because sometimes the time denoted by now is the time introduced by a clause much earlier in the discourse. Such long distance relationships can hold regardless of the aspect of the clause modified by now.

When $t_{\beta}$ is independent of $t_{\alpha}$ : Kamp \& Reyle hold that the time denoted by a clause $\beta$ depends on the tense and aspect of $\beta$ together with a reference point

\footnotetext{
${ }^{3}$ From, 'The Story of the New Testament,' by Edgar J. Goodspeed,

4 'Liquid Air Experiments' in The New York Times, May 13, 1899.

${ }^{5}$ While I do not have the space to go into their theory here, Lee \& Choi (2009) also suffers from the second and third problems for Kamp \& Reyle's view. Their treatment of aspect is more nuanced than Kamp \& Reyle's, but their account retains at bottom the division between state and event denoting clauses as well as a discourse theory that simply updates the reference point clause by clause.
} 
introduced by the previous sentence. If $\alpha$ is the clause preceding $\beta, \beta$ should stand in a temporal relation with $\alpha$. Yet sometimes, a clause immediately preceding $\beta$ can denote a time that is completely irrelevant to the interpretation of $\beta$.

(7) Asked in 2012

a. [Why was the left so much more accepting of the 2011 budget than of the 2010 budget? $]_{\chi}$

b. [First, many on the left took a cue from conservatives, $]_{\gamma}[$ who had assailed the 2011 budget as falling far short of the cutting that was needed. $]_{\alpha}$

c. [Second, Mr. Obama was now in better standing with liberals than he had been in 2010$]_{\beta}$ [having recently repealed the "don't ask, don't tell" policy. $]_{\eta}$

Kamp \& Reyle predict that because now modifies a stative clause in $\beta$, it should refer to the temporal perspective point determined by the discourse through clause $\alpha$. Because $\alpha$ is in the past perfect, it in turn exploits the time introduced by the simple past clause $\gamma$, thereby making $t_{\gamma}$ (the time denoted by $\gamma$ ) the temporal perspective point. $t_{\gamma}$, of course, must have started before $t_{\chi}$ - the left first took the cue and then accepted the budget - but given that it comes after the conservative reaction to the president's budget, we know that $t_{\gamma}$ did not begin too long before the beginning of $t_{\chi}$. (I assume the question presupposes that the left was more accepting of the 2011 budget.) Intuitively, $t_{\beta}$ is independent of $t_{\alpha}$ and, therefore, $t_{\gamma}$; now refers to the time at which the left accepted the budget not the time at which they took a cue from conservatives. The tense and aspect of the sentences in (7) do not alone determine a temporal relation between $\gamma$ (or $\alpha$ ) and $\beta$, though the discourse does enforce a temporal relation between $\beta$ and $\chi: t_{\beta}$ overlaps $t_{\chi}$ and because $\beta$ is presented as an answer, or in this case an explanation, of $\chi, t_{\beta}$ must begin before the beginning of $t_{\chi}$. If we view (7) as a question with multiple independent answers, as opposed to a mere sequence of sentences, the temporal relation between $\beta$ and $\chi$ on the one hand coupled with the temporal independence of $\gamma / \alpha$ and $\beta$ on the other is no surprise.

The following example demonstrates the same point as (7), though the discourse has a different rhetorical structure.

(8) a. [When Mr. Kaine agreed to run the DNC in 2009 $]_{\chi}$ — [even while finishing his last year as governor $]_{\gamma}-[\text { his closest advisers were stunned }]_{\eta}$ [and they counseled him to renege. $]_{\alpha}$

b. [Now Mr. Kaine was facing an unwanted repeat of the same, uncomfortable situation. $]_{\beta}{ }^{6}$

Again, because $\beta$ describes a state using the past perfect, it should set the time of $\alpha$ as the temporal perspective point and then inherit that time. Yet intuitively, now in $\beta$ refers to a time introduced in the discourse prior to the introduction

\footnotetext{
${ }^{6}$ Variation on example from 'Will Obama Ask Kaine to Seek Virginia Senate Seat?' by Michael D. Shear, The New York Times, February 10, 2011. Original did not contain $\alpha ; \beta$ contained may face in the place of was facing.
} 
of clauses $\chi-\alpha$, which describe eventualities holding well in the past of $t_{\beta}$.

When $t_{\beta}$ is the time denoted by a clause preceding $\alpha$ : Even when a clause $\beta$ is temporally related to the preceding clause $\alpha$, Kamp \& Reyle can still make the wrong predictions. They predict, for example, that event denoting clauses in the simple past, like $\beta$ in (9) below, will denote a time other than that denoted by the preceding clause. But if clause $\alpha$ maintains the time $t_{\chi}$ of $\chi$, then Kamp $\&$ Reyle predict that $\beta$ will not refer to $t_{\chi}$, contrary to intuitions (the result is the explosion). If $\alpha$ does not maintain the time of $\chi$ then their account still falls short because it predicts only that $\beta$ refers to a time other than that of $\alpha$, not the stronger claim that it refers to $t_{\chi}$.

(9) a. [The scientist dipped the felt into liquid air $]_{\gamma}$ [and the result was astonishing $]_{\chi}$.

b. [Normally it would not burn, $]_{\alpha}$ [but now it exploded, it was consumed so rapidly. $]_{\beta}$

While Kamp \& Reyle are right that a past tense use of now must refer to a time already available in the discourse and that this time will not in general be made available by the preceding clause if the now modified clause denotes an event, (9) shows that now need not find its referent in the preceding clause. Kamp \& Reyle's prediction that now cannot modify past tense clauses describing past events - a prediction discredited by examples like (9) - is explained in part by the fact that they only considered the temporal relation between a clause $\beta$ and the temporal perspective point used by the previous clause $\alpha$.

\section{Rhetorical contexts and now}

The temporal relations in a text-which determine the time to which a past use of now will refer-are not determined, at least not entirely, by the tense and aspect of individual sentences together with the order in which they appear in the text. To make predictions about the interpretation of now in past tense clauses, we need a theory that allows a clause $\beta$ to stand in a temporal relation to a clause $\chi$ even if there is a clause $\alpha$ introduced in the discourse between $\chi$ and $\beta$ such that (a) $\alpha$ stands in no temporal (or rhetorical) relation to $\beta$ or (b) the temporal or rhetorical relation between $\alpha$ and $\beta$ is not sufficient for determining the time of $\beta$. We need the structure offered by a theory of rhetorical contexts and relations between clauses in a discourse. I will show that in particular, the temporal relations offered by Segmented Discourse Representation Theory (Asher \& Lascarides 2003), or SDRT, can be used to make more accurate predictions about the interpretation of now than can theories of tense and aspect alone.

To capture the semantics of now, we need a theory of discourse content that uses structured contexts. I begin with Kamp \& Reyle's Discourse Representation Theory and add structure to discourse contexts in two ways. First, to each DRS, $\mathrm{I}$ add a level, call it $\mathrm{K}_{0}$, that represents information about utterance events. $\mathrm{K}_{0}$ is the most global level of a given DRS $\mathrm{K}$; the content of utterances, i.e., the content that is normally treated by discourse theories like DRT, is added 
to sub-contexts of this 'extra-linguistic' level. The notion of $\mathrm{K}_{0}$ is introduced in Hunter (2010) in order to handle indexicals, among other expressions, and we need it to handle examples in which now picks out the time of utterance rather than a time introduced in discourse. ${ }^{7}$

Second, I expand on Hunter (2010)'s contexts by adding rhetorical structure as developed by Asher \& Lascarides (2003) and use Asher \& Lascarides' semantics for discourse relations. We start by dividing a discourse into elementary discourse units or EDUs, where an EDU is a minimal unit in a discourse that can stand in a rhetorical relation with another unit - EDUs are, in a sense, the 'words' of a discourse. Next, each EDU is represented as a DRS. Finally, each DRS for each EDU is related to another EDU, or chunk of EDUs, via a rhetorical relation. Both the content of the segmented DRSs and the relations between them are recorded in our contexts below level $\mathrm{K}_{0}$.

With our structured discourse contexts in place, I propose that we treat now as an anaphoric, presuppositional expression along the lines of van der Sandt (1992). While I will not provide a complete motivation for this proposal here - see Hunter (2010), Hunter \& Asher (2005), Maier (2009), Roberts (2002), and Zeevat (1999) for arguments - the general idea is that now triggers a presupposition that must be bound to, or otherwise satisfied by, an antecedent time. Now, like other indexicals, does not bring along its own interpretation, but depends on the incoming context - generally the $\mathrm{K}_{0}$ level of the incoming context - to provide one. The fact that it is up to the incoming context to provide an antecedent for standard indexicals is seen most clearly with you and that, which can fail to refer if the context does not provide an antecedent.

Now can find its antecedent time either from the extra-linguistic context, $\mathrm{K}_{0}$, or from the discourse context, $\mathrm{K}_{1}-\mathrm{K}_{n}$. Now exhibits a strong preference for resolution to the utterance time, but this preference can be over-ridden when resolution at $\mathrm{K}_{0}$ is blocked. In the examples considered here, the past tense blocks resolution at the extra-linguistic level. To capture now's preference for resolution at $\mathrm{K}_{0}$, I use the operator $\uparrow$ introduced in Hunter \& Asher (2005), which requires material in its scope to be resolved at the highest context possible. Given DRT's treatment of existential formulas as introducers of discourse referents, the presupposition of now will look like this:

(10) $\uparrow \exists t(t=?)$

The question mark signals that $t$ is anaphoric and needs to be identified with a discourse referent for a time already available in the context in which the presupposition of now is triggered.

Now, like other indexicals and third person pronouns, is incapable of local accommodation. It always requires that there be a super-ordinate time that it can treat as the 'current' time. Even when it refers to the utterance time, its presupposition is triggered in a sub-context of $\mathrm{K}_{0}$ and then bound in $\mathrm{K}_{0}$. When now cannot be resolved to the utterance time, I claim that it is resolved to the time of its immediately super-ordinate antecedent clause, i.e. the clause to

\footnotetext{
${ }^{7}$ See Hunter (2010) and Maier (2009) for a motivation of such structured contexts.
} 
which it is linked by a subordinating discourse relation, such that the relation between the time of the now modified clause and its super-ordinate antecedent is as close to identity as the rhetorical relations into which the now-modified clause enters will allow. This feature of now's semantics has the following consequences:

Now restricts the temporal relations predicted by SDRT: Now restricts the temporal relations that a theory of rhetorical structure like SDRT predicts will hold between a subordinate clause and its super-ordinate antecedent. For example, if a clause $\beta$ explains a clause $\alpha$, SDRT allows that the time of $\beta\left(t_{\beta}\right)$ might start well before $t_{\alpha}$ and it might even end before $t_{\alpha}$ begins.

(11) $[\text { I hit him today }]_{\alpha}[\text { because he hit me last week. }]_{\beta}$

In (11), the event described in $\beta$ began and ended before the event described in $\alpha$ began. If $\beta$ is modified by now, however, $t_{\beta}$ must overlap $t_{\alpha}$ and it must start immediately before $t_{\alpha}$.

(12) $[\text { I hit him }]_{\alpha}$ [because he (*now) hit me. $]_{\beta}$

(13) a. [This became apparent in Darwin's reaction to Jenkin's critique $]_{\gamma} \ldots$

b. [Darwin gave up his original assumption that evolution occurred best in small, isolated populations $]_{\chi}$,

c. [because he now feared that small populations would not throw up enough individual variants for selection to be effective. $]_{\beta}$

In (12), the cause ended before the effect began, so now is not licensed. In (13), the cause $(\beta)$ immediately brought about its effect $(\chi)$ and $t_{\chi}$ overlaps $t_{\beta} \cdot \chi$ and $\beta$ together elaborate on Darwin's reaction to Jenkin's critique, introduced in $\gamma$. The semantics of ELABORATION require that $t_{\chi}$ and $t_{\beta}$ together be included in $t_{\gamma}$.

$\operatorname{COOR}(\alpha, \boldsymbol{n o w}-\beta)$, then $\operatorname{SuB}(\chi,(\alpha, \boldsymbol{n o w}-\beta))$ : If a past-tense, now-modified clause $\beta$ is related to a preceding clause $\alpha$ via a coordinating relation, the whole unit $(\alpha+\beta)$ will be subordinate to another clause $\chi$ whose time will serve as now's antecedent. In (4), $\alpha$ and $\beta$ provide information about the same individual and so would be related via CONTINUATION, a coordinating relation in SDRT, while the unit $\alpha+\beta$ would be related to $\chi$ by BACKGROUND, a subordinating relation.

(4) a. [The letter is marked "personal and private" and is addressed to FDR's secretary... $]_{\eta}$

b. [The writer was Lucy Mercer Rutherfurd, $]_{\chi}$

c. [who decades before had been FDR's mistress $]_{\alpha}$ [and who now was making arrangements for what would be their last fateful meeting at the president's rural retreat. $]_{\beta}$

The semantics of BACKGROUND in SDRT allow that if a clause $\beta$ is subordinate to a clause $\chi$ via BACKGROUND, then $t_{\beta}$ can start well before $t_{\chi}$. Unlike EXPLANATION, however, $t_{\chi}$ must always be included in $t_{\beta}$. When we add now to $\beta$, temporal overlap is taken care of by the semantics of BACKGROUND, but $t_{\beta}$ must start when $t_{\chi}$ starts. Because $\chi$ elaborates on or provides background for 
$\eta$ by providing information about who wrote the letter, $\chi$ in turn inherits $\eta$ 's time. I assume that since $\chi$ and $\eta$ are both about the writing of the letter under discussion, the time that they both denote is the time of the letter writing event. Now is thus interpreted as the time of the letter writing event, as desired.

Now can be used as a modifier of past tense sentences in narratives without an explicit super-ordinate antecedent.

(14) But Rokiroki, exerting all his strength, gripped the strangers wrists so that he could not draw his hatchet. And now he called again to his little daughter, who stood trembling on the bank above. ${ }^{8}$

However, even if the antecedent is not explicit, SDRT posits topics for narrations, so now in (14) will have a super-ordinate antecedent determined by the discourse topic. Now, like next and then, is easily used to modify a sentence related to another sentence via NARRATION because all of the sentences that figure in a narration elaborate on the topic event. Because they all elaborate on the topic, they must all share in the topic time, but none can be identical to the topic time because the semantics of narration ensure that there is no temporal overlap between two clauses related by NARRATION.

Now can be used to enforce a temporal break: Suppose a now-modified clause $\beta$ is in a coordinating relation with a clause $\alpha$ where $\alpha+\beta$ is subordinate to another clause $\chi$ as described above. If the time of $\alpha$ is different from the time of $\chi$ and the rhetorical relation between $\alpha$ and $\beta$ does not enforce a temporal break between $\alpha$ and $\beta$ then now will be licensed to enforce a break and a return to the time of $\chi$. The felicity of (4), for example, is greatly aided by now because CONTINUATION does not impose a temporal relation between its arguments. With now, it is clear that while on the one hand, the now-modified clause is still providing background, it's providing information about what was going on at the time of the letter writing event (indirectly) introduced in $\chi$, not at the time of $\alpha$. Similar remarks can be made for (9):

(9) a. [The scientist dipped the felt into liquid air $]_{\gamma}$ [and the result was astonishing $]_{\chi}$.

b. [Normally it would not burn, $]_{\alpha}$ [but now it exploded, it was consumed so rapidly. $]_{\beta}$

The example is much clearer with now because now helps to separate the time of $\beta$ from the time of $\alpha$ and to enforce a tie between $t_{\beta}$ and $t_{\chi}$.

Now can be omitted in certain subordinating structures: When a clause $\beta$ elaborates on a clause $\alpha$, for example, it is ensured by the semantics of ELABORATION that $t_{\beta}$ is included in $t_{\alpha}$. Sometimes, it is also clear that $t_{\alpha}$ is included in $t_{\beta}$ whether or not $\beta$ is modified by now. This is the case in (3):

(3) [Five months later, $]_{\eta}[\text { I sat with her as she lay in bed }]_{\gamma} \ldots[$ I was alone in her bleak room. $]_{\chi}[\text { Alone, because there was none of her in it, }]_{\alpha}$ [just a body that (now) held no essence of my mum. $]_{\beta}$

\footnotetext{
${ }^{8}$ From Legends of the Maori: The Tale of Rokiroki-A Memory of the Mokau,
} 
$\beta$ does not figure in a complex subordinate unit, i.e. $\beta$ is not related via a coordinating relation to any other discourse units, and there are no markers such as for example to suggest that $t_{\beta}$ is only properly included in $t_{\alpha}$. Regardless of whether now is used in (3), it is understood that $t_{\beta}$ is $t_{\alpha} \cdot{ }^{9}$ In this case, the requirements of now are already satisfied by $\beta$, so now can be omitted without affecting the truth conditions. This observation can be generalized to other subordinating relations: if a clause $\beta$ is subordinate to a clause $\alpha$ via BACKGROUND or EXPLANATION and $t_{\alpha}$ and $t_{\beta}$ are as close to identical as allowed by the semantics of these relations without now, then the requirements of now are satisfied and now can be omitted without changing the truth conditions for the discourse.

\section{A note about contrast and change of state}

If we remove now from (3), the truth conditions of the example do not change, but something is nonetheless lost. Now suggests that the state described in $\beta$ began recently; the change from the author's mother's body having an essence to its not holding an essence is important for the story the author is recounting and the use of now reinforces this theme. Similarly, in (13), now makes it clear that Darwin did not always have the fear described in the now-modified clause. Now suggests a change in Darwin's thinking and so aids the tie between the now modified clause and its antecedent, which mentions the catalyst for the change in Darwin's thinking. As a final illustration, now in (9) emphasizes that fact that the felt's exploding is a new event and so reinforces the contrast that holds between the now modified clause and the preceding clause.

As Hunter (2010), Lee \& Choi (2009), and Recanati (2004) have observed, now, at least when it modifies past tense clauses, often signals a recent change or a contrast of some sort. In opposition to Hunter (2010) and Recanati (2004), however, I maintain that this effect does not arise from a semantic requirement that the eventuality described by the now-modified clause be contrasted, either explicitly or implicitly, with some other eventuality. Rather, the contrastive feel of so many now examples is a pragmatic effect that arises naturally from the semantics that I have laid out so far together with certain features of a discourse. There are multiple reasons to resist the claim that now requires a contrast or a change of state. First, even in examples that have a contrastive feel, it is often difficult to say in what sense these examples contain a contrast. It is certainly not the case that the now-modified clause must be related to another clause via the CONTRAST relation defined in SDRT, for example. Amongst the English examples that I have discussed in this article, only in (9) would SDRT say that the now-modified clause is an argument for CONTRAST.

\footnotetext{
9 The chances are high that the author's mother's room contained an essence-less body long before the time at which the author paid the visit under discussion in $\alpha$. Nevertheless, the discourse only demands that $t_{\alpha}$ be the same as $t_{\beta}$. The discourse could be true in a scenario in which the mother's body lost its essence at exactly the moment that the author walked through the door to pay the visit mentioned in $\alpha$. While this scenario is implausible, it is allowed by the discourse structure.
} 
Second, there are many examples in which now modifies a past tense clause that do not give rise to a contrastive effect.

(15) [2011 was a great year for computer science.] In attacking the problem of the ambiguity of human language, computer science was now closing in on what researchers refer to as the "Paris Hilton problem". ${ }^{10}$

(14) But Rokiroki, exerting all his strength, gripped the strangers wrists so that he could not draw his hatchet. And now he called again to his little daughter, who stood trembling on the bank above.

In (15), it is obvious from the context that computer science was not closing in on the Paris Hilton problem before whatever time serves as now's antecedent. In this sense, there is a kind of opposition implicit between the time at which computer science is said to have been closing in on the Paris Hilton problem and the times before. Nevertheless, (15) does not have a contrastive feel because the change of state is not at issue in the discourse. Now simply serves to emphasize the period under discussion in the discourse and other times are not relevant. Note that we could replace now in this example with at this time and the implicit opposition between the time of the eventuality described by the now-modified clause and previous times would still be there. Yet we would not for this reason want to build a requirement of contrast into the semantics of at this time. In (14), now again signals a change from one eventuality to another, but again, this is not a reason to argue that now requires a contrast between two times. For one thing, now could not felicitously be replaced by but, a marker for contrast in discourse theories like SDRT. For another, and now could be replaced by next or and then and the discourse would have the same effect of signalling a shift from one eventuality to another. But as with at this time, we would not want to argue that next or then requires a contrast.

The contrastive feel of examples involving past tense uses of now is better explained as a natural consequence of the semantics of now combined with certain features of the discourse in which the now modified clause figures. Now inherits the time of its super-ordinate antecedent. Sometimes this feature of now's semantics allows it to play an indispensable structuring role in a discourse. Other times, now is not needed to structure a discourse, but simply serves to emphasize the temporal relation between the clause it modifies and its antecedent. Now's semantics stop here and will not give rise to a contrastive effect on their own. If, however, within the discourse, the now-modified clause and its antecedent clause fall on one side of a larger contrastive structure, then the fact that now emphasizes the temporal relation between the clause that it modifies and its antecedent will naturally give rise to an emphasis on the temporal nature of the contrasted eventualities. In (6) and (9), the now-modified clause enters into a local contrast relation with the previous clause in the discourse. Because the use of now emphasizes the temporal nature of one side of the contrast, the temporal

${ }^{10}$ From 'A Fight to Win the Future: Computers vs. Humans' by John Markoff for The New York Times, Feb. 14, 2011. The original text contains is in the place of was. 
nature of the other side is brought to light. In (3) and (13), the discourse is about a change of a body state and a change in a set of beliefs, respectively. In both cases, the discourse sets up a much higher-level contrastive structure. Again, now serves to emphasize the temporal relations on one side of this structure, which naturally gives rise to a 'then' vs. 'now' reading of the contrastive structure. ${ }^{11}$

\section{References}

Afantenos, S., et al: An empirical resource for discovering cognitive principles of discourse organization: the ANNODIS corpus, Proceedings of LREC 2012 (submitted) (2012)

Asher, N., Lascarides, A.: The Logics of Conversation. Cambridge University Press (2003)

Banfield, A.: Unspeakable Sentences: Narration and Representation in the Language of Fiction. Routledge \& Kegan Paul, London (1982)

Doron, E.: Point of view as a factor of content. In: Moore, S., Wyner, A. (eds.) Proceedings of SALT I. CLC Publication, Ithaca, NY (1991)

Hunter, J.: Presuppositional Indexicals. Ph.D. thesis, The University of Texas (2010)

Hunter, J., Asher, N.: A presuppositional account of indexicals. In: Dekker, P., Franke, M. (eds.) The Proceedings of the $15^{\text {th }}$ Amsterdam Colloquium. pp. 119-124 (2005)

Kamp, H.: Formal properties of now. Theoria 37, 227-273 (1971)

Kamp, H., Reyle, U.: From Discourse to Logic. Kluwer Academic Publishers (1993)

Kaplan, D.: Demonstratives. In: Almog, J., Perry, J., Wettstein, H. (eds.) Themes from Kaplan. Oxford University Press, USA (1989)

Lee, E., Choi, J.: Two nows in korean. Journal of Semantics 26 (2009)

Maier, E.: Proper names and indexicals trigger rigid presuppositions. Journal of Semantics 23, 253-315 (2009)

Predelli, S.: Utterance, interpretation and the logic of indexicals. Mind $\mathscr{E}$ Language 13(3), 400-414 (1998)

Recanati, F.: Indexicality and context shift (manuscript), Harvard University (2004)

Roberts, C.: Demonstratives as definites. In: van Deemter, K., Kibble, R. (eds.) Information Sharing. CSLI Press (2002)

Rooth, M.: A theory of focus interpretation. Natural Language Semantics 1, 75-116 (1992)

van der Sandt, R.: Presupposition projection as anaphora resolution. Journal of Semantics 9, 333-377 (1992)

Schlenker, P.: Context of thought and context of utterance. Mind $\mathcal{E}$ Language 19(3), 279-304 (2004)

Zeevat, H.: Demonstratives in discourse. Journal of Semantics 16, 279-313 (1999)

\footnotetext{
$\overline{11}$ Another factor that might encourage a contrastive reading of now is focus. Following the work of Rooth (1992), a focused element in a sentence gives rise to a set of alternatives, which in turn gives rise to a contrast. How exactly focus affects the interpretation of now would be an interesting topic for further study, though I doubt that focus affects the fundamentals of the theory that I am presenting here.
} 


\title{
Obligatory implicatures and grammaticality
}

\author{
Natalia Ivlieva \\ MIT \\ ivlieva@mit.edu
}

\begin{abstract}
The paper explores some puzzling data on agreement with disjunctive noun phrases in Russian. I show that the data can be nicely explained by the theory of implicatures. The proposed analysis raises a more general question, namely whether scalar implicatures of a sentence can ever lead to ungrammaticality. In many theories of scalar implicatures it is predicted to be impossible. I argue that the answer is yes, but that happens in a limited number of cases, namely when a scalar implicature of a given sentence has to be calculated but potentially leads to a contradiction if conjoined with another scalar implicature of the sentence, importantly the one which cannot be disregarded due to relevance.
\end{abstract}

Keywords: Scalar Implicature Calculation, Formal Alternatives, Dependent Plurals, Number Agreement.

\section{The puzzle}

The core observation for this paper comes from Russian number agreement with disjunctive noun phrases. Typically, when the subject is a disjunction and both disjuncts are singular, plural agreement on the verb is out, as shown in (1):

(1) Bill ili Fred prišel-Ø/*-i.

Bill or Fred came-SG/*-PL

'Bill or Fred came.'

However, as first noted in [3], plural agreement with singular disjuncts is not always ungrammatical. Specifically, it becomes an option under modals and frequency adverbials, for example, an adverbial 'every day', as in (2):

(2) Každyj vtornik Bill ili Fred prixodil-Ø/-i k Saše. every Tuesday Bill or Fred came-SG/-PL to Sasha 'Every day Bill or Fred came to Sasha.'

Number agreement makes a semantic difference in (2). When the verbs agrees in singular, the disjunction can have either wide or narrow scope with respect to the quantificational adverbial, but when the agreement is plural, the wide scope 362 interpretation of disjunction is ruled out. So, in a scenario where every day the same

adfa, p. 1, 2011.

(C) Springer-Verlag Berlin Heidelberg 2011 
person was coming to Sasha, but the speaker is unsure which one, only singular agreement is possible, as demonstrated by (3):

(3) Každyj vtornik Bill ili Fred prixodil ${ }_{\mathrm{SG}} / *_{-} \mathrm{i}_{\mathrm{PL}} \mathrm{k} \mathrm{S}$. — ja ne pomnju kto imenno.

'Every Tuesday Bill or Fred came to S., but I don't remember who exactly.'

In the following sections, I am going to show that the ungrammaticality of plural agreement in (1) and (3) follows from independently motivated principles of scalar implicature calculation. To do so, I will first show that the plural agreement with disjunction is a special case of dependent plurality that has been analyzed as a scalar phenomenon in [11].

\section{Zweig's dependent plurality}

Dependent plurality is phenomenon observed in English when a bare plural appears in the scope of another plural leading to non-trivial semantic consequences. Although at first glance this description doesn't seem to extend straightforwardly to Russian agreement data, the semantic effects are quite on a par.

Here is an example of a dependent plural reading. The sentence in (4a) doesn't necessarily mean that any of my friends attends more than one school. It may be the case that each of my friends attends just one good school, but at the same time it is required that more than one school were referred to overall. If all of my friends attend the same school, (4b) must be used:

(4) a. My friends attend good schools.

b. My friends attend a good school. (examples from [11])

Zweig's proposal in [11] is that bare plurals do not have the 'more than one' component as part of their meaning, i.e. they are number neutral predicates truthconditionally, but the 'more than one' component arises as a scalar implicature, based on the scale-mate relationship between the bare plural and its singular alternative.

An independent piece of evidence in favor of viewing 'more than one' as a scalar implicature comes from the fact that precisely this component disappears in environments where known scalar implicatures do too, for example, under negation:

(5) John doesn't own dogs. (see [10], [11] etc.)

According to [11], implicature calculation takes place at the level of an event predicate, namely before event closure is applied. The event predicate with a plural variable is weaker than its singular counterpart, giving rise to a scalar implicature. This assumption is crucial for the accounting for the multiplicity implicature, since after event closure applies, plural and singular alternatives become equivalent, so the multiplicity implicature cannot be generated any more.

There is a striking similarity between the reading of (2) with plural agreement and the dependent plural reading of (4a). Namely, the sentence (2) with plural agreement 
requires that each Tuesday at least one of the guys come to Sasha, while at the same time it is inappropriate if each Tuesday, it is the same person who is coming (in that case singular agreement must be used). One could say that plural agreement makes dependent plural reading obligatory.

\section{The Proposal}

As we just saw, Russian disjunctions behave similar to English indefinite NPs (either singular or plural). To capture this correlation, I will make the following assumptions:

- Disjunction is a GQ consisting of a covert existential quantifier and a predicate [A or B].

- Predicate $[\mathrm{A}$ or B] can be singular or plural, triggering singular or plural agreement on the verb respectively.

- The plural feature denotes the closure of the predicate under sum formation. The predicate $[\mathrm{A}$ or $\mathrm{B}]$ having a plural feature will have the following denotation:

(6) $\llbracket(\mathrm{A}$ or $\mathrm{B})-P L \mathbb{} \rrbracket *(\lambda \mathrm{x} . \mathrm{x}$ is $\mathrm{A}$ or $\mathrm{X}$ is $\mathrm{B})=\lambda \mathrm{x}$. $\mathrm{x}$ is $\mathrm{A}$ or $\mathrm{x}$ is $\mathrm{B}$ or $\mathrm{x}$ is $\mathrm{A} \oplus \mathrm{B}$

It seems reasonable to hypothesize that sentences like (1) or (2) have two implicatures: a multiplicity implicature (MI) generated by plural feature (following Zweig's logic) and an exclusivity implicature (EI) generated by a scalar item or. In case of (1), repeated below as (7), the two implicatures look as shown in (8):

(7) $*[\text { Bill or Fred }]_{\mathrm{PL}}$ came.

(8) a. Bill or Fred came and it's not true that only one of them came (MI) = $=$ Bill and Fred came.

b. Bill or Fred came and it's not true that both Bill and Fred came (EI)

It is obvious that these two implicatures contradict each other, and this is precisely where the plural agreement (which reflects the plural feature on disjunction) is ungrammatical.

In cases like $(2) /(9)$ the situation is different:

(9) Every Tuesday [Bill or Fred $]_{\mathrm{PL}}$ came.

The two implicatures we get in this case are given below:

(10) a. Every Tu. Bill or Fred came and it's not true that every Tu. Bill came and it's not true that every Tuesday Fred came (MI)

b. Every Tu. B. or F. came and it's not true that every Tu. B. and F. came. (EI) 
In this case, the two implicatures are consistent with each other, giving rise exactly to the dependent plural reading: both boys have to come overall, but on no Tuesday, both boys have to come.

In order to formalize this intuition, I will explicitly lay out my assumptions on the implicature calculation process. First, I assume that scalar implicatures are brought about by a covert exhaustivity operator EXH akin to 'only' (see [7], for example):

(11) $\left[\left[\mathrm{EXH}_{\mathrm{ALT}}\right]\right]=\lambda \mathrm{P}_{<\mathrm{s}, \mathrm{t} .} . \lambda \mathrm{e} . \mathrm{P}(\mathrm{e}) \& \forall Q \in \mathrm{ALT} \& \mathrm{Q} \subseteq \mathrm{P}:[\neg \mathrm{Q}(\mathrm{e})]$

Second, I assume, following [9], that the set of alternatives for a sentence with two occurrences of a scalar item $\varphi(\mathrm{X}, \mathrm{Y})$, where $\mathrm{X}$ is an element of the scale $\mathrm{Q}_{\mathrm{X}}$ and $\mathrm{Y}$ an element of the scale $Q_{Y}$, is defined as follows:

(12) $\operatorname{Alt}(\varphi(X, Y))=\left\{\varphi\left(X^{\prime}, Y^{\prime}\right) \mid X^{\prime}\right.$ an element of $Q_{X}, Y^{\prime}$ an element of $\left.Q_{Y}\right\}$

Third, I follow [11] in assuming that implicature calculation happens before the event closure.

Also, I adopt the idea that plural and singular are scalar alternatives with singular being the strongest element of the scale.

On top of these assumptions, I would like to add a new assumption, namely the predicative OR ([A or B]) has non-boolean conjunction $[\mathrm{A} \oplus \mathrm{B}]$ defined in (13) as its non-weaker alternative:

$$
\left[\mathrm{A}_{<\mathrm{e}, \mathrm{t}} \oplus \mathrm{B}_{<\mathrm{e}, \mathrm{t}}\right]=\lambda \mathrm{x}_{\mathrm{e}} \cdot \exists \mathrm{y}_{\mathrm{e}}, \mathrm{z}_{\mathrm{e}}[\mathrm{x}=\mathrm{y} \oplus \mathrm{z} \& \mathrm{~A}(\mathrm{y}) \& \mathrm{~B}(\mathrm{z})] \quad(\mathrm{cf} .[6])
$$

Below I give some examples from Krifka's [6] which serve as an evidence for the existence of non-boolean conjunction which applies to $<\mathrm{e}, \mathrm{t}>$ predicates (for the details I refer the reader to Krifka's paper):

(14) John and Mary are husband and wife.

(15) The flag is green and white.

Now let's see how these assumptions taken together allow us to explain for the data in $(1) /(7)$ and $(2) /(9)$. First, let's examine a "non-quantificational" case repeated below:

(16) $*[\text { Bill or Fred }]_{\mathrm{PL}}$ came.

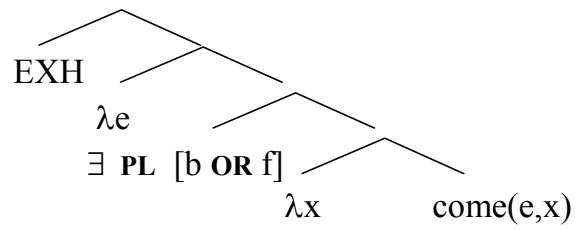

Before existential closure applies, our sentence denotes the following: 
As shown in the LF, we are dealing with two scalar items: predicative OR and PL associated with two scales $<\mathrm{OR}, \mathrm{AND}>$ and $<\mathrm{PL}, \mathrm{SG}>$ respectively.

Based on the assumption in (12), the set of scalar alternatives for (17) consists of (18) and (19) (the [SG, AND] alternative is contradictory):

(18) $\lambda \mathrm{e} . \exists \mathrm{x}[\mathrm{x}=\mathrm{b}$ or $\mathrm{x}=\mathrm{f} \& *$ came $(\mathrm{e}, \mathrm{x})]$

(19) $\lambda \mathrm{e} . \exists \mathrm{x}[\mathrm{x}=\mathrm{b} \oplus \mathrm{f} \& *$ came $(\mathrm{e}, \mathrm{x})]$

[PL, AND]

The result of exhaustification of (17) with respect to the alternatives given above is shown in (20):

(20) $\lambda \mathrm{e} . \exists \mathrm{x}[\mathrm{x}=\mathrm{b}$ or $\mathrm{x}=\mathrm{f}$ or $\mathrm{x}=\mathrm{b} \oplus \mathrm{f} \& *$ came $(\mathrm{e}, \mathrm{x})] \&$

$$
\begin{aligned}
& \& \neg \exists \mathrm{x}\left[\mathrm{x}=\mathrm{b} \text { or } \mathrm{x}=\mathrm{f} \&{ }^{*} \text { came }(\mathrm{e}, \mathrm{x})\right] \& \\
& \& \neg \exists \mathrm{x}[\mathrm{x}=\mathrm{b} \oplus \mathrm{f} \& * \text { came }(\mathrm{e}, \mathrm{x})]
\end{aligned}
$$

(20) is contradictory, which I take to be the reason of the ungrammaticality of (1)/(7).

Now let's turn to the "quantificational" case repeated below:

(21) Every Tuesday [Bill or Fred $]_{\mathrm{PL}}$ come.

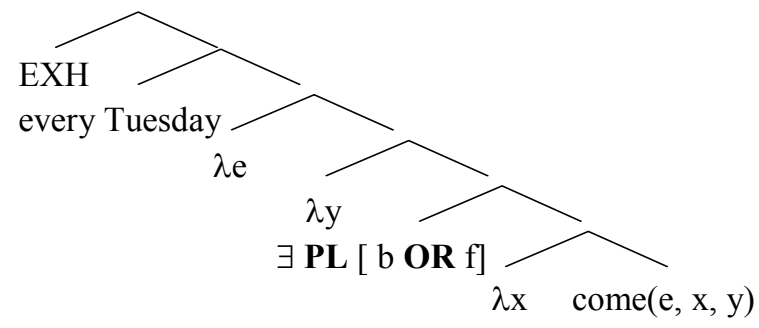

In order to make the analysis work, we will need to adopt the following denotation for the adverbial universal quantifier (based on [5]):

(22) $[[$ every Tuesday $]]=$

$$
=\lambda \mathrm{P} . \lambda \mathrm{e} . \exists \mathrm{Y}\left[* T u(\mathrm{Y}) \& * \mathrm{P}(\mathrm{e})(\mathrm{Y}) \& \forall \mathrm{y}\left[T u(\mathrm{y}) \rightarrow \exists \mathrm{e}^{\prime}: \mathrm{e}^{\prime} \subset \mathrm{e} \& \mathrm{P}\left(\mathrm{e}^{\prime}\right)(\mathrm{y})\right]\right]
$$

It's important to point out that the denotation we will adopt consists of two parts namely, besides universal quantification over events, it also introduces a big event which is the sum of smaller events we quantify over. This will be very crucial for accounting for $(2) /(21)$.

Before existential closure the sentence will denote the following:

(23) $\lambda \mathrm{e} . \exists \mathrm{Y}[* T u .(\mathrm{Y}) \& \exists \mathrm{x}[\mathrm{x}=\mathrm{b}$ or $\mathrm{x}=\mathrm{f}$ or $\mathrm{x}=\mathrm{b} \oplus \mathrm{f} \& * \operatorname{come}(\mathrm{e}, \mathrm{x}, \mathrm{Y})] \&$ $\& \forall \mathrm{y}\left[T u .(\mathrm{y}) \rightarrow \exists \mathrm{e}^{\prime}\left[\mathrm{e}^{\prime} \subset \mathrm{e} \& \exists \mathrm{x}\left[\mathrm{x}=\mathrm{b}\right.\right.\right.$ or $\mathrm{x}=\mathrm{f}$ or $\left.\left.\left.\left.\mathrm{x}=\mathrm{b} \oplus \mathrm{f} \&{ }^{*} \operatorname{come}\left(\mathrm{e}^{\prime}, \mathrm{x}, \mathrm{y}\right)\right]\right]\right]\right]$ 
The alternatives of (24) are given below:

(24) a. $S G$-alternative:

$\lambda$ e. $\exists \mathrm{Y}[* T u .(\mathrm{Y}) \& \exists \mathrm{x}[\mathrm{x}=\mathrm{b}$ or $\mathrm{x}=\mathrm{f} \& * \operatorname{come}(\mathrm{e}, \mathrm{x}, \mathrm{Y})] \&$

$\& \forall \mathrm{y}\left[T u .(\mathrm{y}) \rightarrow \exists \mathrm{e}^{\prime}\left[\mathrm{e}^{\prime} \subset \mathrm{e} \& \exists \mathrm{x}\left[\mathrm{x}=\mathrm{b}\right.\right.\right.$ or $\left.\left.\left.\left.\mathrm{x}=\mathrm{f} \&{ }^{*} \operatorname{come}\left(\mathrm{e}^{\prime}, \mathrm{x}, \mathrm{y}\right)\right]\right]\right]\right]$

b. $A N D$-alternative:

$\lambda$ e. $\exists \mathrm{Y}\left[{ }^{*} T u .(\mathrm{Y}) \& \exists \mathrm{x}\left[\mathrm{x}=\mathrm{b} \oplus \mathrm{f} \&{ }^{*}\right.\right.$ come $\left.(\mathrm{e}, \mathrm{x}, \mathrm{Y})\right] \&$

$\& \forall \mathrm{y}\left[T u .(\mathrm{y}) \rightarrow \exists \mathrm{e}^{\prime}\left[\mathrm{e}^{\prime} \subset \mathrm{e} \& \exists \mathrm{x}\left[\mathrm{x}=\mathrm{b} \oplus \mathrm{f} \& * \operatorname{come}\left(\mathrm{e}^{\prime}, \mathrm{x}, \mathrm{y}\right)\right]\right]\right]$

The result of exhaustification of (23) with respect to these alternatives is in (25):

(25) $\lambda \mathrm{e} . \exists \mathrm{Y}\left[{ }^{*} T u .(\mathrm{Y}) \& \exists \mathrm{x}[\mathrm{x}=\mathrm{b}\right.$ or $\mathrm{x}=\mathrm{f}$ or $\mathrm{x}=\mathrm{b} \oplus \mathrm{f} \& * \operatorname{come}(\mathrm{e}, \mathrm{x}, \mathrm{Y})] \&$

$\& \forall \mathrm{y}\left[T u .(\mathrm{y}) \rightarrow \exists \mathrm{e}^{\prime}\left[\mathrm{e}^{\prime} \subset \mathrm{e} \& \exists \mathrm{x}\left[\mathrm{x}=\mathrm{b}\right.\right.\right.$ or $\mathrm{x}=\mathrm{f}$ or $\left.\left.\left.\left.\mathrm{x}=\mathrm{b} \oplus \mathrm{f} \& *^{*} \operatorname{come}\left(\mathrm{e}^{\prime}, \mathrm{x}, \mathrm{y}\right)\right]\right]\right]\right] \&$

$\& \neg[\exists \mathrm{Y}[* T u .(\mathrm{Y}) \& \exists \mathrm{x}[\mathrm{x}=\mathrm{b}$ or $\mathrm{x}=\mathrm{f} \& * \operatorname{come}(\mathrm{e}, \mathrm{x}, \mathrm{Y})] \&$

$\& \forall \mathrm{y}\left[T u .(\mathrm{y}) \rightarrow \exists \mathrm{e}^{\prime}\left[\mathrm{e}^{\prime} \subset \mathrm{e} \& \exists \mathrm{x}\left[\mathrm{x}=\mathrm{f}\right.\right.\right.$ or $\left.\left.\left.\left.\left.\mathrm{x}=\mathrm{b} \& \operatorname{come}\left(\mathrm{e}^{\prime}, \mathrm{x}, \mathrm{y}\right)\right]\right]\right]\right]\right] \&$

$\& \neg\left[\exists \mathrm{Y}\left[* T u .(\mathrm{Y}) \& \exists \mathrm{x}\left[\mathrm{x}=\mathrm{b} \oplus \mathrm{f} \&{ }^{*} \operatorname{come}(\mathrm{e}, \mathrm{x}, \mathrm{Y})\right] \&\right.\right.$

$\left.\left.\& \forall \mathrm{y}\left[T u .(\mathrm{y}) \rightarrow \exists \mathrm{e}^{\prime}\left[\mathrm{e}^{\prime} \subset \mathrm{e} \& \exists \mathrm{x}\left[\mathrm{x}=\mathrm{b} \oplus \mathrm{f} \&{ }^{*} \operatorname{come}\left(\mathrm{e}^{\prime}, \mathrm{x}, \mathrm{y}\right)\right]\right]\right]\right]\right]=$

$\lambda \mathrm{e} . \exists \mathrm{Y}\left[{ }^{*} T u .(\mathrm{Y}) \& \exists \mathrm{x}[\mathrm{x}=\mathrm{b}\right.$ or $\mathrm{x}=\mathrm{f}$ or $\mathrm{x}=\mathrm{b} \oplus \mathrm{f} \& * \operatorname{come}(\mathrm{e}, \mathrm{x}, \mathrm{Y})] \&$

$\& \forall \mathrm{y}\left[T u .(\mathrm{y}) \rightarrow \exists \mathrm{e}^{\prime}\left[\mathrm{e}^{\prime} \subset \mathrm{e} \& \exists \mathrm{x}\left[\mathrm{x}=\mathrm{b}\right.\right.\right.$ or $\mathrm{x}=\mathrm{f}$ or $\left.\left.\left.\left.\mathrm{x}=\mathrm{b} \oplus \mathrm{f} \&{ }^{*} \operatorname{come}\left(\mathrm{e}^{\prime}, \mathrm{x}, \mathrm{y}\right)\right]\right]\right]\right] \&$

a. $\neg \exists \mathbf{Y}[* \boldsymbol{T u} .(\mathbf{Y}) \& \exists \mathbf{x}[\mathbf{x}=\mathbf{b}$ or $\mathbf{x}=\mathbf{f} \& *$ come $(\mathrm{e}, \mathrm{x}, \mathrm{Y})]] \vee$

$\vee \neg\left[\forall \mathrm{y}\left[T u .(\mathrm{y}) \rightarrow \exists \mathrm{e}^{\prime}\left[\mathrm{e}^{\prime} \subset \mathrm{e} \& \exists \mathrm{x}\left[\mathrm{x}=\mathrm{f}\right.\right.\right.\right.$ or $\left.\left.\left.\left.\mathrm{x}=\mathrm{b} \&{ }^{*} \operatorname{come}\left(\mathrm{e}^{\prime}, \mathrm{x}, \mathrm{y}\right)\right]\right]\right]\right] \quad M I$

b. $\neg \exists \mathrm{Y}[* T u .(\mathrm{Y}) \& \exists \mathrm{x}[\mathrm{x}=\mathrm{b} \oplus \mathrm{f} \& * \operatorname{come}(\mathrm{e}, \mathrm{x}, \mathrm{Y})]] \vee$

$\vee \neg\left[\forall \mathbf{y}\left[T u .(y) \rightarrow \exists \mathrm{e}^{\prime}\left[\mathrm{e}^{\prime} \subset \mathrm{e} \& \exists \mathrm{x}\left[\mathrm{x}=\mathrm{b} \oplus \mathrm{f} \& * \operatorname{come}\left(\mathrm{e}^{\prime}, \mathrm{x}, \mathrm{y}\right)\right]\right]\right]\right]$

The conjunction of the assertion with the first disjunct in (25b) and the second disjunct in $(25 \mathrm{c})$ gives the meaning we are after. To be clear, let's give informal paraphrases of what we got in (25).

The first disjunct in (25b) can be paraphrased as below:

"It's not true that there is $\mathrm{x}$ such that $\mathrm{x}$ is atomic and it is either Bill or Fred which is the agent of the big coming event - that guarantees that the agent of the big event will be the sum of Bill and Fred, which excludes the situation in which it is the same person who is coming every Tuesday."

The paraphrase of the second disjunct in $(25 \mathrm{c})$ is:

"It's not the case that for every Tuesday there is an event the agent of which is the sum of Bill and Fred - that guarantees that there must be days on which only one of them comes." 
Now assume that the first disjunct in $(25 \mathrm{c})$ is true. The truth of the first disjunct in (25c) is incompatible with neither disjunct in (25b). So as we just showed, the LF in (25) doesn't predict any other possible meanings for the sentence in (2)/(21).

\section{Implications for the theory of scalar implicatures}

The analysis we proposed in the previous section raises a question: can the scalar implicatures of a sentence be a reason for its ungrammaticality?

It is well-known that in general scalar implicatures are not obligatory, they can be cancelled, as illustrated below:

(26) Peter or John came. In fact, both Peter and John came.

If the implicature generated by disjunction (It's not the case that both Peter and John came) was obligatory, we would get a contradictory sequence of sentences, but the common intuition is that it is not.

One possible way of thinking about that is the following. Following [8], I will assume that the exhaustification operator is obligatory. But certain alternatives are subject to relevance. If they are not relevant, we are allowed to prune them and thus whether we get a certain implicature or not is a matter of whether we prune alternatives or not.

So cases of implicature cancellation like the one in (26) can be analyzed as follows: the $A N D$-alternative ('Bill and Fred came') is not relevant, thus no implicature is generated and no contradiction arises.

It is also known that certain implicatures are obligatory, i.e. noncancellable. For example, as was shown in [2], the implicature associated with plural is obligatory, cf. oddness of the sequence in (27):

(27) I saw boys. \# In fact, I saw only one boy.

The way of stating that could be that certain alternatives are not subject to relevance and they cannot get pruned. In our case, we will have to say that alternatives to plural cannot get pruned, thus obligatorily leading to a multiplicity implicature.

Now let's come back to the explanation of the ungrammaticality of (28):

$$
*[\text { Bill or Fred }]_{\mathrm{PL}} \text { came. }
$$

In the previous section, I argued that the ungrammaticality is due to the conflict between implicatures which are generated by two scalar items: PL and OR.
(29) $\lambda \mathrm{e} . \exists \mathrm{x}[\mathrm{x}=\mathrm{b}$ or $\mathrm{x}=\mathrm{f} \& *$ came $(\mathrm{e}, \mathrm{x})]$
[SG, OR]
(30) $\lambda \mathrm{e} . \exists \mathrm{x}[\mathrm{x}=\mathrm{b} \oplus \mathrm{f} \& *$ came $(\mathrm{e}, \mathrm{x})]$
[PL, AND] 
But as I said above, the status of these implicatures must be different: the one generated by the plural cannot get pruned, whereas the one generated by or is in fact prunable.

Note that if we were able to prune the $A N D$-alternative, we wouldn't get a contradiction and the sentence would be grammatical, meaning 'Bill and Fred came'. Note that this is exactly the and-alternative to our sentence.

The fact that the sentence is ungrammatical suggests that the and-alternative cannot be pruned, which may be a reflex of the principle in (31), cf. similar ideas in [4]:

(31) It is impossible to prune a certain alternative of a sentence if the result of its exhaustification with respect to the remaining subset of alternatives gives the meaning which is equivalent to that alternative.

This generalization can be related to relevance and its properties. It has been argued in [4] that relevance is closed under negation and conjunction. It is also assumed that the prejacent of the EXH operator is always relevant.

So when we exhaustify $[p \vee q]_{P L}$ with respect to the singular alternative $(p \vee q)_{S G}$, what we get looks schematically as follows: $(p \vee q)_{\mathrm{PL}} \& \neg(p \vee q)_{\mathrm{SG}}$. As relevance is closed under negation and conjunction, the result we got is relevant, but that's equivalent to $[\mathrm{p} \& \mathrm{q}]$ (which in turn is equivalent to the and-alternative), so the andalternative cannot be not relevant and hence cannot be pruned.

The following generalization describes how grammaticality is related to implicature calculation:

(32) Ungrammaticality arises in those cases when implicatures of a sentence lead to a contradiction and there is no possibility of obviating the contradiction by pruning alternatives which give rise to those implicatures (either due to the requirements of certain scalar items or to the principle in (31)).

The difference between cases of implicature cancellation like those in (26) and our cases falls out: in cases like (26), the contradiction can be obviated by pruning "malicious" alternatives, as nothing blocks pruning them in those cases. In our cases the contradiction created by implicatures cannot be saved by pruning of alternatives due to the requirements of plural (alternatives to plural cannot be pruned) and the constraint on pruning (impossibility of pruning singular alternative leads to the meaning equivalent to the and-alternative, thus it cannot be pruned).

One more phenomenon which is covered by that generalization is the distribution of free choice items like any, namely the fact that free choice items are bad in episodic sentences as shown in (33) as opposed to modal environments (34):

*John read any book.

(34) You are allowed to read any book.

In [1], Chierchia accounts for the ungrammaticality of sentences like (33) in the following way: on the one hand, FC items (we can schematically represent them as 
disjunctions: $\mathrm{a} \vee \mathrm{b}$ ) obligatorily activate domain alternatives $(\mathrm{a}, \mathrm{b})$; on the other hand, they also have a scalar implicature $(a \& b)$. In episodic sentences these two implicatures clash, thus leading to ungrammaticality. Modals are able to resolve this conflict, making the implicatures consistent.

However, Chierchia doesn't explain why the scalar implicature is obligatorily calculated in this case - he uses a stipulation saying that if D-alternatives are active, scalar alternatives cannot be not active.

We can give it more of a principled explanation now.

Domain alternatives (similar to alternatives to plural) cannot be pruned. But the scalar one is in principle prunable. But notice if we pruned it, the result of secondlevel exhaustification ${ }^{1}$ of $a \vee b$ with respect to the domain alternatives would give us the meaning which is equivalent to the $A N D$-alternative (as shown in (35)), thus the constraint in (31) would apply, blocking the $A N D$-alternative from being pruned.

$$
\begin{aligned}
& \mathrm{EXH}_{\mathrm{ALT}}(\mathrm{p} \vee \mathrm{q})=\mathrm{p} \vee \mathrm{q} \& \neg \mathrm{EXH}(\mathrm{p}) \& \neg \mathrm{EXH}(\mathrm{q})=\mathrm{p} \vee \mathrm{q} \& \neg(\mathrm{p} \& \neg \mathrm{q}) \& \neg(\mathrm{q} \& \neg \mathrm{p}) \\
& =\mathrm{p} \& \mathrm{q}
\end{aligned}
$$

\section{Conclusions}

In this paper, I raised the question of whether scalar implicatures can ever lead to ungrammaticality. Based on the puzzling data on agreement with disjunctions, I argued that this happens in those cases when implicatures of a sentence lead to contradiction which cannot be obviated by pruning alternatives which give rise to those implicatures (either due to the requirements of certain scalar items or to constraint on pruning stated in (31)).

\section{References}

1. Chierchia, G.: Meaning as an Inferential System: Polarity and Free Choice phenomena (in prep.)

2. Chierchia, G., Fox, D., and Spector, B.: The Grammatical View of Scalar Implicatures and the Relationship between Semantics and Pragmatics. In: Pornter, P., Maienborn, C., von Heusinger, K. (eds.) An International Handbook of Natural Language Meaning. Mouton de Gruyter (to appear)

3. Crockett, D. B.: Agreement in Contemprorary Standard Russian. Slavica Publishers, Cambridge MA (1976)

4. Fox, D., Katzir, R.: On the Characterization of Alternatives. Natural Language Semantics 19(1):87-107.

5. Kratzer, A.: The Event Argument (in prep)

\footnotetext{
${ }^{1}$ Note that the first-level exhaustification would lead to contradiction in this case. But according to Chierchia, recursive exhaustification is an option which is always available. So what we do is we exhaustify the prejacent with respect to pre-exhaustified set of alternatives $\{\operatorname{EXH}(\mathrm{p})=\mathrm{p} \& \neg \mathrm{q}, \operatorname{EXH}(\mathrm{q})=\mathrm{q} \& \neg \mathrm{p}\}$. For details, I refer the reader to [1].
} 
6. Krifka, M.: Boolean and Non-Boolean And. In Kálman, L. and Polos, L. (eds.): Papers from the Second Symposium on Logic and Language. Akadémiai Kiadó, Budapest (1990)

7. Krifka, M.: The Semantics and Pragmatics of Polarity Items. Linguistic Analysis, 25: 209257 (1995)

8. Magri, G.: A Theory of Individual-Level Predicates based on Blind Mandatory Implicatures. MIT dissertation (2009)

9. Sauerland, U. Scalar Implicatures in Complex Sentences. Linguistics and Philosophy 27: 367-391 (2004)

10. Sauerland, U., Andersen, J., Yatsushiro, K.: The plural involves comparison. In: Kesper, S., Reis, M. (eds.) Linguistic evidence. Mouton de Gruyter, Berlin (2005)

11. Zweig, E.: Number-Neutral Bare plurals and the Multiplicity Implicature, Linguistics and Philosophy 32(4): 353-407 (2009) 


\title{
Only Only ? An Experimental Window on Exclusiveness ${ }^{\star}$
}

\author{
Jacques Jayez ${ }^{1}$ and Bob van Tiel ${ }^{2}$ \\ 1 ENS de Lyon and $1 \mathrm{SC}_{2}$, CNRS, Lyon, France \\ 2 Radboud University, Nijmegen, Netherlands
}

\begin{abstract}
In a recent book, David Beaver and Brady Z. Clark argue that only does not presuppose the proposition in its scope, contrary to the 'standard' theory articulated in (Horn 1969), and propose a new semantic representation for only. Their rejection of the standard theory is based on linguistic observations and a survey test. We adduce new experimental evidence suggesting that (i) dropping the standard theory altogether might be too radical a move and (ii) the very idea that the behaviour of only derives from a combination logical form + context, even if it proves ultimately adequate, cannot be taken as granted.
\end{abstract}

\section{Introduction}

According to Horn's (1969) famous analysis, Only $p$, where $p$ is a proposition, presupposes that its prejacent, $p$, is true and asserts that, for some salient set of alternatives to $p, \operatorname{Alt}(p)$, every member of $\operatorname{Alt}(p)$ different from $p$ is false. So, (1a) presupposes that Paul smokes and asserts that no other person from a salient set does. (1b) shows that only behaves like a standard presupposition trigger. The presupposition that Paul smokes projects under sentential negation and interrogation.

(1) a. Only Paul smokes.

b. It is not true that only Paul smokes. / Does only Paul smoke?

Beaver and Clark (2008) (B\&C) raise a number of objections against this approach and argue that only presupposes that the prejacent is the weakest proposition on a scale and asserts that it is the strongest proposition on the same scale. 'Weak' and 'strong' are not defined in terms of logical entailment, but in a more liberal way, which is compatible with scales based on degrees of importance or cardinals.

In this paper, we reconsider the empirical and experimental evidence laid out by $\mathrm{B} \& \mathrm{C}$ to back up their claim. We show that it is problematic in certain respects and that it does not support the view that the observations for only can be derived from the scalar semantics they propose, essentially because other presuppositional triggers with a quite different semantics behave in the same

* We gratefully acknowledge the financial support of European Science Foundation, ESF travel grant Euro-Xprag 4273 
way and a semantically very similar trigger (seulement in French) behaves quite differently.

In sections 2.1, 2.2 and 2.3, we present B\&C's arguments and theory. In section 2.4, we show that their linguistic arguments are not conclusive. In section 3 , we present the results of a new experimental approach and discuss them in section 4 , showing that they undermine the very idea that only is a special item, whose particular behaviour is a reflection of its logical form.

\section{Beaver and Clark's Approach}

\section{$2.1 \quad$ Linguistic Observations}

$\mathrm{B} \& \mathrm{C}$ observe that there are cases in which the prejacent is not preserved under negation. Consider (2), their example 9.42.c. It is clear that the speaker does not believe that the person in question is a blond bimbo with no brains.

(2) She's one of the first that really represents the country and isn't only some blond bimbo with no brains.

Another piece of evidence in the same direction is provided by examples like (3), which compares cardinals and does not entail that Mary invited Susan and Paul, since she invited their six cousins instead.

(3) Last year, Mary invited Susan and Paul. This year, she did not invite only Susan and Paul, but preferred to invite their six cousins.

B\&C note that such examples run counter to Horn's (1969) initial proposal and to Robert's (2006) defence of the standard theory as well as to Ippolito's (2008) implicative theory, which assumes that if some proposition is true in the set of alternatives associated with Only $p$, then $p$ is true. Under that analysis, for instance, the prejacent of (2) is predicted to project, because the set of alternatives contains the proposition described by 'She is one of the first that really represents the country'.

\subsection{The Tequila Test}

In order to show that the prejacent of only is more 'fragile' than presuppositions of other triggers, $\mathrm{B} \& \mathrm{C}$ devised an experiment based on the interpretation of a little story:

One year there were 90 students in Arroyo.

30 drank Tequila and nothing else.

30 drank non-alcoholic beverages and nothing else.

30 drank everything, no matter what.

Subjects had to answer the following two questions: How many students didn't only drink Tequila (VP-only) and How many students didn't drink only Tequila (VP-only). They had to pick one of the answers: '30', '60' and 'Don't know'.

Suppose that the subjects follow the standard theory. In that case, they should choose the ' 30 ' answer, since they would actually answer the following 
question: 'For how many $x$ is it true that $x$ drank Tequila (the prejacent) and false that $x$ didn't drink anything else?'. The third set is then the only correct answer. If, on the contrary, the prejacent is not preserved, subjects would answer the following question: 'For how many $x$ is it true or false that $x$ drank Tequila (the prejacent) and false that $x$ didn't drink anything else?'. In that case the second and third sets are good candidates. Assuming that the first set is not a correct candidate, no matter if the prejacent is preserved or not, we see that the Tequila test might theoretically act as a separator between the two interpretations (with/without the prejacent).

$\mathrm{B} \& \mathrm{C}$ report that, out of 41 participants, 17 chose answer ' 30 ' for VP-only and 9 for NP-only, whereas 23 chose answer ' 60 ' for VP-only and 31 for NP-only. Because the subjects were not divided into two independent or paired samples, it is difficult to interpret these results in a reliable way, but they seem problematic for the standard theory. The number of ' 60 ' answers is particularly high for NPonly. It is possible to run a McNemar's test on the results, under the assumption that the subjects are 'coherent', that is, that the subjects who chose '30' for NPonly are a subset of those who chose ' 30 ' for VP-only and that the subjects who chose '60' for VP-only still chose '60' for NP-only. In that case, the difference between the two positions for only is significant at the 0.05 threshold (p-value $\approx 0.012){ }^{3}$

In addition to only, $\mathrm{B} \& \mathrm{C}$ used a comparable testing procedure for four other presupposition triggers: stop, realize, regret and their. The results are clearly different than for only. For instance, with stop, realize and regret, 9, 12 and 10 subjects out of a total of 13 , choose the answer that is compatible with the projection of the presupposition. These results might be taken to suggest that only is special, at least as regards the projection of the prejacent under negation.

\subsection{The Proposal}

$\mathrm{B} \& \mathrm{C}$ propose to amend the standard theory by exploiting the scalar character of only. Only presupposes that the prejacent is at most as strong and asserts that it is at least as strong as any true alternative. More precisely, we have (4).

(4) Only $p$ presupposes (asserts) that for every proposition $q$ in an appropriate set of alternatives to $p, A L T(p)$, if $q$ is true then $p$ is at most (at least) as strong as $q$, in symbols:

only $p$ presupposes the proposition defined by $\lambda w \forall q \in A L T_{\sigma}(p)(w=q \Rightarrow$ $\left.q \geq_{\sigma} p\right)$, and asserts the propositions defined by $\lambda w \forall q \in A L T_{\sigma}(p)(w \models$ $\left.q \Rightarrow q \leq_{\sigma} p\right)$, where $\sigma$ is the belief state of the speaker.

Let us see what happens with (1a), assuming that the set of alternatives is calculated on the basis of a form ' $x$ smokes', where $x$ ranges over a set of possible

\footnotetext{
${ }^{3} \mathrm{~B} \& \mathrm{C}$ report a non-significant result for a chi-square test. The problem is twofold: if the subjects are coherent, in the sense considered here, the chi-square is not a good indicator. If they are not coherent, to a degree that falsifies our assumption, the question is more complex because the shift in perception that this incoherence suggests has to explained.
} 
persons or groups, and that they are ordered with respect to entailment. The presupposition eliminates worlds in which 'Paul smokes' is stronger than some alternative true at the same world. The common ground is then updated with the main content. This move eliminates worlds in which there is a proposition of the form ' $a$ smokes' which is stronger than 'Paul smokes', for instance it eliminates worlds in which Paul and Mary or Paul and John smoke. The net result is a set of worlds where, for each true proposition $q \in A L T_{\sigma}(p), q={ }_{\sigma} p$. If we apply a negation to Only Paul smokes, the presupposition is (normally) preserved but the main content is negated. So, the negated sentence asserts the proposition corresponding to $\lambda w \exists q \in A L T_{\sigma}(p)\left(w \mid=q \& q>_{\sigma} p\right)$, in other terms, the proposition that Paul and someone else smoke.

When alternatives are not ordered with respect to entailment, a different result can obtain. For instance, if a cardinality-based ordering is used, the presupposition is the proposition that the prejacent concerns at most as many individuals as any true alternative. This delivers the required reading for (3). The negated main content entails that Mary invited more persons than just two. However, it does not entail that the guests include Susan and Paul, since the alternatives are compared on the basis of cardinality and not of entailment.

Summarising, B\&C's approach consists in (i) replacing the prejacent with a condition on the relative strength of the prejacent and its competitor and (ii) using the opposite condition as the main content. The derivation of the prejacent is an effect of the interaction between the two constraints, not an intrinsic semantic property of only.

\subsection{Preliminary Discussion}

Two points are in need of clarification. First, if the ordering is based on cardinality, what happens with a sentence like Mary invited only Susan and Paul? Intuition says that Mary invited Susan and Paul, but this is not a direct consequence of the formal part of the theory, because it is compatible with a situation where Mary invited two persons, (partly) different from Susan and Paul. In that case, every true alternative is equivalent to 'Mary invited Susan and Paul'. This possibility is excluded because it would be totally misleading. Suppose a speaker wants to address the question How many persons did Mary invite? by conveying two pieces of information, (1) that Mary invited only two persons and (2) that they were John and Sandy. If that speaker mentioned Susan and Paul as guests, she would just sound incoherent, because nobody would just ignore the names Susan and Paul in order to get the intended message 'only two'. See the contrast in $(5)$.

$$
\begin{aligned}
& \text { A - How many persons did Mary invite? } \\
& \text { B1 -Only two persons. John and Sandy. } \\
& \text { B2 -?? Only Susan and Paul. John and Sandy. }
\end{aligned}
$$

But how is it that (3) is unproblematic? An obvious answer is that it is relevant to mention Susan and Paul because they were invited last year and there is a 
contrast with the current situation. In the absence of a salient contrast, (3) is infelicitous as an answer to a how many question.

(6) [Context: B does not know that Mary had previously invited Susan and Paul]

A - How many persons did Mary invite?

B - \# She invited six persons. She didn't invite only Susan and Paul.

Examples like (3) are taken into account in any theory of presupposition and thus do not seem to be specific to only. Suppose that B has been a chain-smoker for years and has suddenly decided to quit. The beginnings are difficult and, when A meets B, B is particularly nervous and tired. One year after, A meets B again and is surprised at how B looks better. In a dialogue like (7), B does not presuppose that he has been smoking recently. A similar observation holds for (2), see (8). Both examples are based on a contrast between different time periods or individuals.

(7) A - I'm glad to see that you are much better than last time!

B - Well, unlike last year, I'm not quitting smoking. Fortunately, it's behind me now! It has been six months since I have stopped smoking completely.

(8) [Context: it is common belief that John never smoked. B is trying to quit.]

A - John seems to be much more relax than you are.

B - HE didn't stop smoking a week ago!

On the whole, B\&C's empirical observations are not as conclusive as one may wish because they do not seem to be restricted to only, but concern rather the pragmatic conditions on the felicity of presupposing. So, it is not clear that a specific theory should be constructed for only on the basis of examples such as (2) or (3). ${ }^{4}$ Still, the results of the Tequila test separate only from other presupposition triggers. We now turn to these experimental data.

\section{An Experimental Approach}

The Tequila test of $\mathrm{B} \& \mathrm{C}$ is affected by two problems. First, it does not satisfy some usual requirements for a survey test, e.g. on the number of subjects, the independence or pairing of samples and the presence of fillers. Second, it is based on the calculation of the size of certain subsets, a fact which might have an effect on the answers. If we have a set of people drinking only Tequila, the complement of this set includes those people who drank Tequila and something else and those people who drank something different from Tequila. It is unclear whether subjects interpreted the question How many students didn't (only) drink (only) Tequila? as bearing on the adverb or as a complementation question.

\footnotetext{
${ }^{4}$ A similar remark applies to Ippolito's $(2008,50-52)$ discussion about it's possible that only $p$. As shown in (Herburger 2000, 95) the observations that would tend to show that the prejacent is suspended are not specific to only or to presuppositions. B\&C mention Herburger's work and add further examples that suggest that only is not the main factor in those cases (Beaver and Clark 2008, 245-246).
} 


\subsection{The Basic Protocol}

In order to remedy some of these problems, we changed the design of the Tequila test. Instead of using numbers, we used characters, who are differentiated by their actions or situation. Typically a subject had to go through sequences like the following and tick one of the boxes.

Fig. 1. Two target stimuli

\begin{tabular}{|l|l|}
\hline Only & Other triggers \\
\hline Three people were in the cafeteria & Three people are riding a bus \\
A drank orange juice and nothing else & A had a job at the bank but quit \\
B drank coffee and nothing else & B never had a job in her life \\
C drank orange juice and coffee & C has a job at the bank and still works there \\
\hline Who didn't drink only orange juice? & Who didn't resign from the bank? \\
$\square$ C & $\square$ C \\
$\square$ C and B & $\square$ C and B \\
$\square$ I don't know & $\square$ I don't know \\
\hline
\end{tabular}

We ran experiments in three languages: Dutch, English and French. The target stimuli were interspersed with filler stimuli in the same vein but using various quantifiers such as at most three or often. The stimuli and the attribution of the actions/situations to A, B and C were pseudo-randomised. We had 16 presupposition triggers and 16 fillers for Dutch, the same numbers for English and 15 triggers and 23 fillers for French. The triggers include focus particles like only or also, factives like know or regret, implicatives like manage or succeed, aspectuals like stop or start and definites like the or all. For English, subjects were recruited through Amazon MTurk. They were university students for Dutch and French. After we got the results, we decided to eliminate the démissionner ('resign') case from the French data, because the French little story associated with it was possibly problematic.

\subsection{The image-based Protocol}

When it turned out that the results for English were markedly distinct from those for Dutch and French, as explained in the next section, we decided to run an additional experiment for English speakers. Subjects were presented with series of three images and had to answer the same sort of question as for the test based on purely linguistic stimuli. An example of stimulus is shown in Figure 2.

There were 25 subjects who had to check 6 triples of images, including 1 target stimulus featuring only and 5 fillers again using quantifiers. The stimuli were pseudo-randomised as in the other experiments. 
Fig. 2. An image-based target stimulus

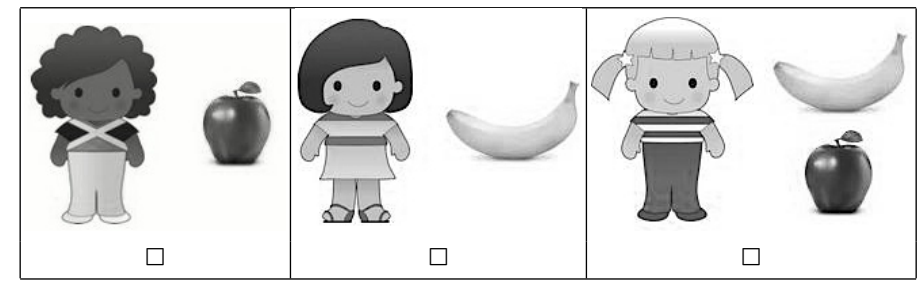

Who does not have only an apple?

\subsection{Results}

In a nutshell, the observations collected for English support B\&C's approach but the observations for Dutch and French do not.

The comparison between English triggers is summarised graphically in figure 3. The left (black) column represents the percentage of ' $\mathrm{B}$ ' answers in figure 1, the middle (white) column the percentage of 'B and C' answers and the right one (grey) the 'I don't know' answers. Because there are very few 'I don't know' answers, it is possible to binarize the results by dividing the answers into ' $\mathrm{B}$ '-type versus others ('B and C' and 'I don't know'). The dependent variable is then the proportion of ' $\mathrm{B}$ ' answers with respect to languages and types of stimuli, e.g. implicatives, factives, etc. There are several ways to analyse such data. One may run a McNemar test on every pair of stimuli and have a fine-grained image of the comparative distributions of answer. One may also use logistic regression, since the response is binary. Finally, another option is to cluster the items with respect to the binary response.

We first illustrate the case of logistic regression. Using the lme4 package in $\mathrm{R}$, we fitted a simple model of mixed logistic regression, having the subjects as random effect and adding a post hoc comparison based on the multcomp package. The results are summarised in figure 3 for English only. The response for this item is compared to the overall response for different classes. The difference is significant for factive and focus elements and non-significant for other categories.

Fig. 3. English triggers

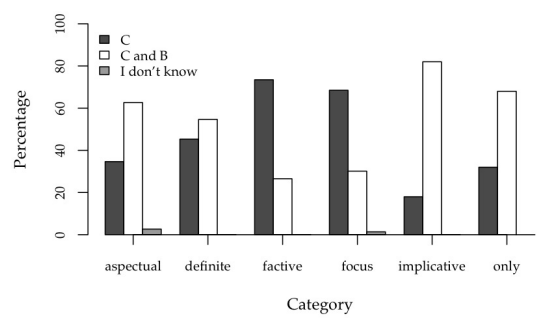

$\mathrm{z}$ value $\operatorname{Pr}(>|\mathrm{z}|)$

vs. aspectual $-0.261 \quad 0.99983$

vs. definite $\quad-1.249 \quad 0.80606$

$\begin{array}{lll}\text { vs. factive } & -3.892 & 0.00139^{* *}\end{array}$

vs. focus $\quad-3.2890 .01233^{*}$

vs. implicative $1.419 \quad 0.70749$ 
The pictorial task for English illustrated in figure 2 gave totally consonant results. $72 \%$ of speakers chose the 'B and C' answer. So it seems unlikely that the nature of the test was an issue.

For Dutch and French, the counterparts of only, alleen and seulement, do not behave like in English. The two relevant histograms and the post hoc contrasts are shown in figure 4 . In addition, the post hoc contrasts on a simple logistic regression with the response binary variable restricted to the only case show that Dutch and French are not significantly different whereas they are both different from English $(\operatorname{Pr}(>|z|)=0.93,0.0004,0.0008)$.

We see that Dutch and French are similar except for implicatives. Since we had a difference for implicative stimuli between the two languages ${ }^{5}$, we fitted two other separate models for the two cases of Dutch ('succeed' and 'manage'). The difference with French turned out to stable. Another, less marked, difference concerns the definite article. This difference cannot be reliably taken into account, because of a difference in the structure of the stimuli ('a professor' in Dutch versus 'one of his professors' in French).

Fig. 4. Dutch and French triggers
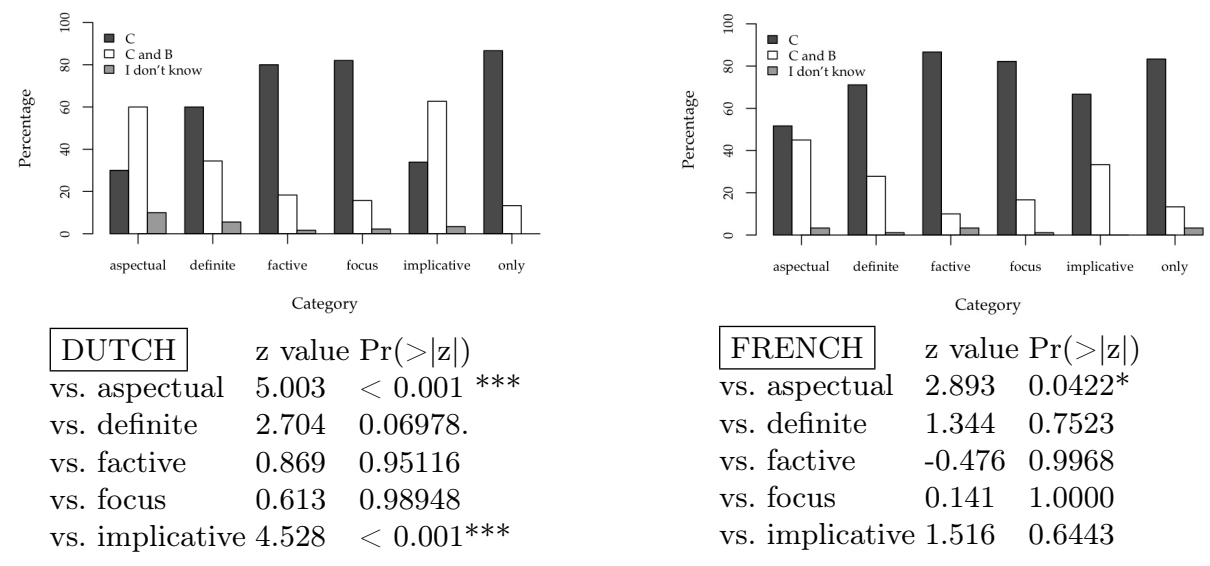

Probabilistic clustering with the pvclust package allows one to sharpen the general conclusions provided by using the mixed models, see figure 5. Only was found different from factives in the mixed model. Similarly, the 'regret', 'know', 'discover' and 'realize' nodes are far apart from the 'only' node in the cluster. This is true to a lesser extent for 'anymore', 'also', 'again', which form the focus category minus 'only'. Similar correspondences hold for Dutch and French clusters.

\footnotetext{
${ }^{5}$ This is due to the fact that, in French, réussir à ('succeed', 'manage') contains the same verb as réussir + NP ('pass', an exam for instance) and that succeed and manage are translated by réussir.
} 
Fig. 5. Clustering for English English w.r.t. ONE (pvclust)

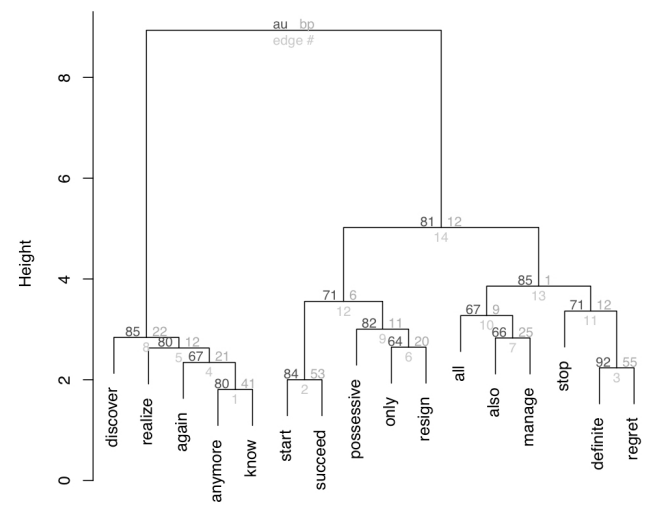

\section{Discussion and perspectives}

The language-based and image-based results for English are consonant with B\&C's observations for the part of the Tequila test that concerns only. However, we did not find the same results for other triggers. Figure 3 shows no difference between only and the case of aspectuals, implicatives, or definites. This is unexpected if the relation of only to its prejacent is specific. Moreover, if the fact that the prejacent is suspended under negation is taken as an indication that only does not presuppose its prejacent, it seems that we cannot escape the same conclusion for the triggers that pattern with only.

One might wonder whether the observed profiles coincide with the 'weak' versus 'strong' trigger distinction in (Abusch 2010). Abusch contrasts examples like those in (9). Win, which presupposes a participation in the competition, allows for the suspension of its presupposition and is, in this respect, 'weak', in contrast to again, which is a 'strong' trigger

(9) a. I don't know whether John finally participated in the race, but if he won it he may be very proud!

b. ?? I don't know whether John won this race before, but if he won again, he may be very proud!

Again is one of the strong focal elements compared with only and it is indeed clearly separated from it in the clustering (figure 5). This extends to also. Unfortunately, the parallelism breaks down when it comes to aspectuals and factives, which are presumably weak. Start is akin to only whereas stop is in a distinct subgroup. Discover, realize and know are also separated from only. In Dutch, again is close to only, as is anymore in French. Overall, the data do not correspond to a systematic weak/strong distinction.

An important issue in the semantics of exclusives is their scalar character. It is well-known that only is scalar at least in that it can be interpreted as 
entailing that the degrees above or below a certain threshold, expressed by the prejacent, are not reached. The French seulement has the same property, see (Beyssade 2010), whereas alleen is not scalar, see (10). However, both items resist suspension of the prejacent.

(10) a. Paul is only a first-year student ( $\Rightarrow$ he is not a second/third/dotsyear student)

b. Paul est seulement un étudiant de première année

c. *Paul is alleen een eerstejaars student.

The reported observations raise a more general question. It is often (partly) implicitly assumed that the distribution of triggers should be a reflection of their formal semantics, because assuming the contrary would lead us to renounce any explanation. In our opinion, this dilemma between calculation from a logical form and sterility lacks serious foundations. The high cross-linguistic variability of certain triggers, which sound otherwise quite comparable, comes as some surprise under this view, but remains compatible with an approach that is not (entirely) representational, where triggers, in addition to a descriptive content (main content + presupposed content) have a statistical profile with respect to, say, suspension under negation or other environments. This profile does not necessarily derive from something else.It could be an 'intrinsic' property of the item, that is, a property stabilised after some learning.

In future work, we intend to tighten the experimental conditions, by having a homogeneous pool of subjects in the three languages and controlling the stimuli and the choice of answers even more carefully. We are also planning two new experiments, one using reaction times, in order to determine whether there is any correlation between the ' $\mathrm{B}$ and $\mathrm{C}$ ' answer and the choice duration. We also intend to test whether the observed difference might be connected with the 'loneliness' flavour associated with seulement and alleen, which both provide adjectives meaning 'alone', in contrast to English (*Paul is only). To this aim, we will turn to languages similar to English in this respect (e.g. Chinese).

\section{References}

Abusch, D. (2010). Presupposition triggering from alternatives. Journal of Semantics $27,37-80$.

Beaver, D. and Clark, B.Z. (2008). Sense and Sensitivity. How Focus Determines Meaning. Wiley-Blackwell, Chichester.

Beyssade, C. (2010). Seulement et ses usages scalaires. Langue Française 165, 103-124.

Herburger, E. (2000). What Counts: Focus and Quantification. Cambridge (MA): MIT Press.

Horn, L. (1969).A Presuppositional Analysis of only and even. In Papers from the Fifth Regional Meeting of the Chicago Linguistics Society, 98-107.

Ippolito, M. (2008). On the Meaning of Only. Journal of Semantics 25, 45-91.

Roberts, C. (2006). Only, presupposition and implicature. MS., Ohio State University, http://ling.osu.edu/ croberts/only.pdf. 


\title{
Computing quantifier scope with witness sets
}

\author{
Udo Klein
}

Bielefeld University

\section{Introduction}

One of the basic claims of Generalized Quantifier Theory ${ }^{1}$ is that natural language determiners like most, every, some but not all, ... denote binary functions from subsets of the domain of individuals $D$ into the set of truth-values (or equivalently, binary relations between subsets of $D$ ). In this paper I challenge this view by developing an alternative theory of quantification where determiners denote unary functions from subsets of $D$. The basic idea, developed in section 2 , is that if a determiner denotation is applied to a subset $R$ (mnemonic for restrictor set) of $D$, the value is a pair $\langle R, W\rangle$, where $W$ is the set of subsets of $R$ which satisfy the condition imposed by the determiner. These subsets will be called witness

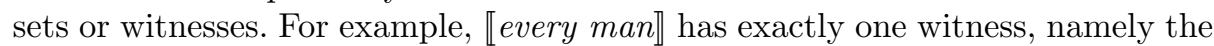

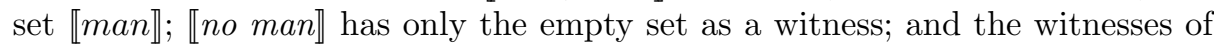

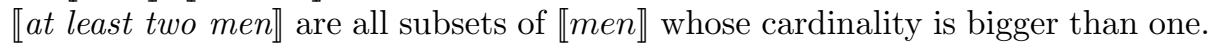

Next, we need to specify (i) how the semantic composition of predicates and determiner phrase denotations proceeds, and (ii) how the different scope dependencies are computed. Since semantic composition is not driven by the denotation types, determiner phrase denotations can compose with the predicate in any order. The specification of the scope dependencies can be done in two different ways. The scope of a determiner phrase can either be fixed in the process of semantic composition, or it can be decoupled from semantic composition, by leaving the scope relations unspecified during semantic composition, and specifying them afterwards by means of so-called expansion operations. In section 3 I briefly sketch the first option, before turning in section 4 to the much more challenging second option. Section 5 concludes.

Given the determiner denotation type and the proposed principles of semantic composition, this theory predicts that the set of entities which are in the scope set but not the restrictor set is always irrelevant for the truth-conditions, providing a novel explanation for conservativity. Further, if scope dependencies are decoupled from semantic composition, and determined instead by applying so-called expansion operations to underspecified denotations, we capture (unlike most other theories of scope underspecification) (i) what the possible readings all have in common, and (ii) the intuitive idea that specifying the scope dependencies should amount to adding information, as opposed to choosing an element (a fully specified reading) from a set of fully specified readings (the denotation of the underspecified reading).

\footnotetext{
${ }^{1}$ Cf. Peters and Westerståhl (2006) for a comprehensive treatment of Generalized Quantifier Theory.
} 


\section{Restricting determiner denotation type}

In Generalized Quantifier Theory, determiners like for example every, most, no denote binary functions from subsets of the domain to truth-values (or equivalently relations between subsets of the domain). Determiner phrases like every man, most girls, no student denote unary functions from subsets of the domain to truth-values. Here we shall depart from this analysis, and propose instead that (i) determiners are unary functions, taking as their single argument the restrictor set, and (ii) determiner phrases refer to pairs $\langle R, W\rangle$ consisting of a restrictor set $R$, and the set $W$ of subsets of $R$, called witness sets. To illustrate, let the domain be $D=\left\{p_{1}, p_{2}, p_{3}, p_{4}, p_{5}, r_{1}, r_{2}, r_{3}, r_{4}\right\}$, and let the denotations of paper and referees be $\llbracket$ paper $(s) \rrbracket=\left\{p_{1}, p_{2}, p_{3}, p_{4}, p_{5}\right\}$ and $\llbracket$ referees $\rrbracket=\left\{r_{1}, r_{2}, r_{3}, r_{4}\right\}$, respectively.

$$
\begin{aligned}
& \llbracket \text { every } \rrbracket(A) \\
& :=\langle A,\{X: X \subseteq A \wedge X=A\}\rangle=\langle A,\{A\}\rangle \\
& \llbracket \text { every } \rrbracket(\llbracket \text { referee } \rrbracket) \\
& =\left\langle\left\{r_{1}, r_{2}, r_{3}, r_{4}\right\},\left\{\left\{r_{1}, r_{2}, r_{3}, r_{4}\right\}\right\}\right\rangle \\
& \llbracket \text { more than half the } \rrbracket(A) \quad:=\langle A,\{X: X \subseteq A \wedge|A-X|<|A \cap X|\}\rangle \\
& \llbracket \text { more than half the } \rrbracket\left(\left\{r_{1}, r_{2}, r_{3}, r_{4}\right\}\right)=\left\langle\left\{r_{1}, r_{2}, r_{3}, r_{4}\right\},\left\{\left\{r_{1}, r_{2}, r_{3}\right\},\left\{r_{1}, r_{2}, r_{4}\right\}\right. \text {, }\right. \\
& \left.\left.\left.\left\{r_{1}, r_{3}, r_{4}\right\},\left\{r_{2}, r_{3}, r_{4}\right\},\left\{r_{1}, r_{2}, r_{3}, r_{4}\right\}\right\}\right\rangle\right\rangle
\end{aligned}
$$

Given a determiner phrase $D P$ with $\llbracket D P \rrbracket=\langle R, W\rangle$, the negation $\llbracket$ not $D P \rrbracket$ consists of the same restrictor set $R$ and the set of subsets of $R$ which are not in $W$, i.e. $\langle R, \wp(R)-W\rangle$, which shall be represented as $\langle R, \bar{W}\rangle$. To illustrate again:

$$
\begin{aligned}
\llbracket \text { not every } \rrbracket(A) & :=\langle A,\{X: X \subseteq A \wedge X \neq A\}\rangle \\
\llbracket \text { not every } \rrbracket(\llbracket \text { referee } \rrbracket) ~ & \left\langle\left\{r_{1}, r_{2}, r_{3}, r_{4}\right\},\left\{\varnothing,\left\{r_{1}\right\},\left\{r_{2}\right\},\left\{r_{3}\right\},\left\{r_{4}\right\},\left\{r_{1}, r_{2}\right\},\left\{r_{1}, r_{3}\right\},\right.\right. \\
& \left\{r_{1}, r_{4}\right\},\left\{r_{2}, r_{3}\right\},\left\{r_{2}, r_{4}\right\},\left\{r_{3}, r_{4}\right\},\left\{r_{1}, r_{2}, r_{3}\right\},\left\{r_{1}, r_{2}, r_{4}\right\}, \\
& \left.\left.\left\{r_{1}, r_{3}, r_{4}\right\},\left\{r_{2}, r_{3}, r_{4}\right\}\right\}\right\rangle \\
\llbracket= & \langle A,\{X: X \subseteq A \wedge|X| \neq 2\}\rangle \\
\llbracket \text { not exactly two } \rrbracket(A) \quad & \left\{r_{1}, r_{2}, r_{3}, r_{4}\right\},\left\{\emptyset,\left\{r_{1}\right\},\left\{r_{2}\right\},\left\{r_{3}\right\},\left\{r_{4}\right\},\left\{r_{1}, r_{2}, r_{3}\right\},\right. \\
\llbracket \text { not exactly two } \rrbracket(\llbracket \text { referees } \rrbracket)= & \left.\left.\left\{r_{1}, r_{2}, r_{4}\right\},\left\{r_{1}, r_{3}, r_{4}\right\},\left\{r_{2}, r_{3}, r_{4}\right\},\left\{r_{1}, r_{2}, r_{3}, r_{4}\right\}\right\}\right\rangle
\end{aligned}
$$

The conjunction of two DP denotations $\langle R, W\rangle$ and $\left\langle R^{\prime}, W^{\prime}\right\rangle$ is the pair consisting of the union $R \cup R^{\prime}$ of the two restrictor sets, and the set of pairwise unions of witness sets in $W$ and $W^{\prime}$, i.e. $\langle R, W\rangle \wedge\left\langle R^{\prime}, W^{\prime}\right\rangle=\left\langle R \cup R^{\prime},\left\{w \cup w^{\prime}: w \in\right.\right.$ $\left.\left.W \wedge w^{\prime} \in W^{\prime}\right\}\right\rangle$. For example, the denotation of every referee and at most one paper is:

$$
\begin{aligned}
\llbracket \text { every referee and at most one paper } \rrbracket= & \llbracket \text { every referee } \rrbracket \wedge \llbracket \text { at most one paper } \rrbracket \\
= & \left\langle\llbracket \text { paper } \rrbracket,\left\{\emptyset,\left\{p_{1}\right\},\left\{p_{2}\right\},\left\{p_{3}\right\},\left\{p_{4}\right\},\left\{p_{5}\right\}\right\}\right\rangle \\
= & \langle\llbracket \text { referee } \cup \llbracket \text { paper } \rrbracket, \\
& \left\{\left\{r_{1}, r_{2}, r_{3}, r_{4}\right\},\left\{r_{1}, r_{2}, r_{3}, r_{4}, p_{1}\right\},\left\{r_{1}, r_{2}, r_{3}, r_{4}, p_{2}\right\},\right. \\
& \left\{r_{1}, r_{2}, r_{3}, r_{4}, p_{3}\right\},\left\{r_{1}, r_{2}, r_{3}, r_{4}, p_{4}\right\}, \\
& \left.\left.\left\{r_{1}, r_{2}, r_{3}, r_{4}, p_{5}\right\}\right\}\right\rangle
\end{aligned}
$$


The exclusive disjunction of two DP denotations $\langle R, W\rangle$ and $\left\langle R^{\prime}, W^{\prime}\right\rangle$ is the pair consisting of the union $R \cup R^{\prime}$ of the two restrictor sets, and the union of the sets $W$ and $W^{\prime}$ of witness sets, i.e. $\langle R, W\rangle \wedge\left\langle R^{\prime}, W^{\prime}\right\rangle=\left\langle R \cup R^{\prime}, W \cup W^{\prime}\right\rangle$. The denotation of every referee or at most one paper is:

$$
\begin{aligned}
\llbracket \text { every referee or at most one paper } \rrbracket= & \llbracket \text { every referee } \rrbracket \vee \llbracket \text { at most one paper } \rrbracket \\
= & \left\langle\llbracket \text { referee } \rrbracket,\left\{r_{1}, r_{2}, r_{3}, r_{4}\right\}\right\rangle \vee \\
& \left\langle\llbracket \text { paper } \rrbracket,\left\{\emptyset,\left\{p_{1}\right\},\left\{p_{2}\right\},\left\{p_{3}\right\},\left\{p_{4}\right\},\left\{p_{5}\right\}\right\}\right\rangle \\
= & \langle\llbracket \text { referee } \rrbracket \cup \llbracket \text { paper } \rrbracket, \\
& \left.\left\{\left\{r_{1}, r_{2}, r_{3}, r_{4}\right\}, \emptyset,\left\{p_{1}\right\},\left\{p_{2}\right\},\left\{p_{3}\right\},\left\{p_{4}\right\},\left\{p_{5}\right\}\right\}\right\rangle
\end{aligned}
$$

Unlike in Generalised Quantifier Theory, the semantics of the determiner does not make reference to the scope set but only to the restrictor set. This has an important consequence, namely that the number of possible determiner denotations is considerably reduced, compared to Generalised Quantifier Theory. Given a universe of discourse $D$ containing exactly two entities and the set $T=\{0,1\}$ of truth-values, there are four subsets of $D, 2^{4}=16$ functions from subsets of $D$ into $T$ (i.e. 16 possible determiner phrase denotations), and $2^{16}=65536$ functions from subsets of $D$ into DP denotations (i.e. 65536 possible determiner denotations). Out of these only 512 determiner denotations are conservative, where a determiner denotation $D$ is conservative iff $D(A)(B) \leftrightarrow D(A)(A \cap B)$. To illustrate, the determiner denotation $\llbracket m o s t \rrbracket$ is a conservative function, since the sentence Most students smoke is true (in a model M) if and only if Most students are students who smoke. is true (in M). Based on the fact that no clear example of a determiner $D E T$ has yet been found, where the equivalence $\llbracket D E T \rrbracket(A)(B) \leftrightarrow D(A)(A \cap B)$ does not hold, Barwise and Cooper (1981) and Keenan and Stavi (1986) have hypothesized that all natural language determiner denotations are conservative. If, on the other hand, determiners are functions from subsets $R$ of $D$ into pairs $\langle R, W\rangle$, where $W$ is a set of subsets of $R$, i.e. $W \subseteq \wp(R)$, then there are exactly 512 possible determiner denotations. To see this, note that given the same universe of discourse $D=\{a, b\}$ there are $n_{1} \times n_{2} \times n_{4} \times n_{4}$ possible determiner denotations, where $n_{1}=2$ is the number of possible witnesses given the restrictor set $\varnothing$ (the first witness is $\varnothing$, and the second is $\{\varnothing\}), n_{2}=4$ is the number of possible witnesses given the restrictor set $\{a\}$ (namely $\varnothing,\{\varnothing\},\{\{a\}\}$ and $\{\varnothing,\{a\}\}$ ), $n_{3}=4$ is the number of possible witnesses given the restrictor set $\{b\}$ (namely $\varnothing,\{\varnothing\},\{\{b\}\}$ and $\{\varnothing,\{b\}\}$ ), and $n_{4}=16$ is the number of possible witnesses given the restrictor set $\{a, b\}$ (namely all the subsets of $\{\varnothing,\{a\},\{b\},\{a, b\}\}$. So there are $2 \times 4 \times 4 \times 16=512$ possible DET denotations, showing that the present proposal considerably reduces the class of possible DET denotations. Assuming that the denotation type fixes the search space for the learner, the reduction of the class of possible DP denotations is attractive, as it narrows down the search space and thus simplifies the learnability problem (other things being equal). 


\section{$3 \quad$ Fixing quantifier scope during semantic composition}

To illustrate the basic idea, note that the truth-condition of the sentence Most dogs sleep. can be formulated as follows: there is a witness set $X$ of $\llbracket m o s t$ dogs $\rrbracket$ such that for all elements $x$ in $\llbracket \operatorname{dog} \rrbracket \rrbracket$ it holds that $x$ is an element of the witness set $X$ if and only if $x$ sleeps. And the truth-condition for the subject-widescope reading of Most dogs chased some cat. is that there is a witness set $X$ of

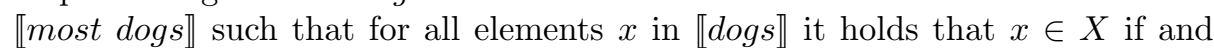
only if there is a witness set $X^{\prime}$ of $\llbracket$ some cat $\rrbracket$ such that for all elements $x^{\prime}$ of $\llbracket c a t \rrbracket$ it holds that $x^{\prime} \in X^{\prime}$ if and only if $x$ chased $x^{\prime}$.

We shall assume that the denotation of chased is the basic (open) formula chase $^{\prime}\left(x_{1}, x_{2}\right)$, and that the determiner phrase denotations can compose with it in any order. The scope relations are determined by the order in which the determiner phrases compose, with the phrase composing first having narrowest scope. To 'record' this information, we assume a so-called scope dependency sequence, which in the case of basic formulas is the empty string, and which gets prefixed with the index of the variable that gets replaced by a determiner phrase.

(1) Terms:

a. If $t$ is a variable, then $t$ is a term.

b. If $t=\operatorname{det}(c n)$, where $\operatorname{det} \in\{$ most, no, every, exactly two,... $\}$ and cn $\in\{$ man, boy, girl,...\}, then $t$ is a (quantified) term.

c. If $t$ is a term, then so is $\operatorname{not}(t)$.

d. If $t_{1}, t_{2}$ are terms, then so is and $\left(t_{1}, t_{2}\right)$

(2) Basic formulas:

a. Let $R$ be an $n$-ary relation symbol and let $x_{1}, \ldots, x_{n}$ be pairwise distinct variables. Then $\left\langle R\left(x_{1}, \ldots, x_{n}\right), \epsilon\right\rangle$ is a formula relative to the empty scope dependency $\epsilon$ (where $\epsilon$ is the empty string).

b. Let $\left\langle R\left(x_{1}, \ldots, x_{n}\right), \epsilon\right\rangle$ be a formula. Then the semantic value of $\left\langle R\left(x_{1}, \ldots, x_{n}\right), \epsilon\right\rangle$ relative to model $M$ and assignment function $g$ is:

$$
\llbracket\left\langle R\left(x_{1}, \ldots, x_{n}\right), \epsilon\right\rangle \rrbracket^{M, g}=1 \text { iff }\left\langle g\left(x_{1}\right), \ldots, g\left(x_{n}\right)\right\rangle \in I(R)
$$

(3) Quantified formulas:

a. Let $\left\langle R\left(t_{1}, \ldots, t_{t-1}, x_{i}, t_{t+1}, \ldots, t_{n}\right), \delta\right\rangle$ be a formula, and let $q$ be a quantified term. Then $\left\langle R\left(t_{1}, \ldots, t_{t-1}, q, t_{t+1}, \ldots, t_{n}\right),\langle i, \delta\rangle\right\rangle$ is a formula for any $1 \leq i \leq n$.

b. $\quad$ Let $\llbracket q \rrbracket^{M, g}=\langle R, W\rangle$. Then:

$$
\begin{aligned}
& \llbracket\left\langle R\left(t_{1}, \ldots, q, \ldots, t_{n}\right),\langle i, \delta\rangle\right\rangle \rrbracket^{M, g}=1 \text { iff } \\
& \exists Y \in W . \forall y \in R .\left[y \in Y \leftrightarrow \llbracket\left\langle R\left(t_{1}, \ldots, x_{i}, \ldots, t_{n}\right), \delta\right\rangle \rrbracket^{M, g\left[x_{i} / y\right]}=1\right]
\end{aligned}
$$




\section{Decoupling scope determination from semantic composition}

\subsection{Reformulating verb denotation and semantic composition}

In what follows we shall separate the analysis of semantic role assignment from the analysis of the resolution of scope dependencies. The main motivation for this is the observation that in many languages the assignment of semantic roles is (i) encoded grammatically and therefore (ii) independent of the context of use, whereas the resolution of scope dependencies is often (i) not encoded grammatically, and moreover (ii) dependent on the particular lexical items involved as well as the particular context of use. ${ }^{2}$ Semantic role assignment will be achieved by SEMANTIC COMPOSITION RULES, whereas scope dependencies are determined by the way in which EXPANSION RULES apply.

The semantic composition rules combining DP and verb (phrase) denotations assign a semantic role of the verb (phrase) denotation to the DP denotation. Given a verb like give, we shall assume that it assigns three (generalised) semantic roles, which we simply name 1,2 and 3, so that the person being assigned the role 1 gives the entity assigned the role 2 to the person assigned the role 3 . If a DP denotation $\llbracket D P \rrbracket$ is assigned the (generalised) semantic role 2, we shall record this information by adding the pair $\langle 2, \llbracket D P \rrbracket\rangle$ to the verb (phrase) denotation. This requires the verb (phrase) denotation to be analyzed as a pair consisting of a relation $R$ and a store $S$. To illustrate, consider:

(4) More than half the referees read at least three papers.

The denotation of this clause can be computed in two different ways. One possibility is to combine the verb and direct object denotations first, and then combine the resulting denotation with the subject denotation.

$\mathcal{O}_{2}(\langle\llbracket$ read $\rrbracket, \emptyset\rangle, \llbracket$ at least three papers $\rrbracket)=\langle\llbracket$ read $\rrbracket,\{\langle 2, \llbracket$ at least three papers $\rrbracket\rangle\}\rangle$

$\mathcal{O}_{1}(\langle\llbracket r e a d \rrbracket,\{\langle 2, \llbracket$ at least three paper $s \rrbracket\rangle\}\rangle, \llbracket$ more than half the referees $\rrbracket)=$ $\langle\llbracket$ read $\rrbracket,\{\langle 1, \llbracket$ more than half the referees $\rrbracket\rangle,\langle 2, \llbracket$ at least three papers $\rrbracket\rangle\}\rangle$

The second possibility is to combine the subject with the verb first, and then combine the resulting denotation with the object denotation:

$\mathcal{O}_{1}(\langle\llbracket r e a d \rrbracket, \emptyset\rangle, \llbracket$ more than half the referees $\rrbracket)=\langle\llbracket$ read $\rrbracket,\{\langle 1, \llbracket$ more than half the referees $\rrbracket\rangle\}\rangle$

$\mathcal{O}_{2}(\langle\llbracket$ read $\rrbracket,\{\langle 1, \llbracket$ more than half the referees $\rrbracket\rangle\}\rangle, \llbracket$ at least three papers $\rrbracket)=$ $\langle\llbracket$ read $\rrbracket,\{\langle 1, \llbracket$ more than half the referees $\rrbracket\rangle,\langle 2, \llbracket$ at least three papers $\rrbracket\rangle\}\rangle$

\footnotetext{
${ }^{2}$ Despite this observation, there are a number of theories of quantification which do not separate semantic role assignment from the resolution of scope dependencies. For example, in Heim and Kratzer (1998) both the semantic role of a DP denotation as well as its scope is fixed at the same point in the derivation, namely when the DP denotation is combined with a property.
} 
Given a (finite) set S of semantic roles (which, for convenience, are represented by means of integers), the SEMANTIC COMPOSITION RULE (schema) is defined as follows:

$$
\mathcal{O}_{i}(\llbracket D P \rrbracket,\langle R, S\rangle)=\langle R, S \cup\{\langle i, \llbracket D P \rrbracket\rangle\}\rangle
$$

for every semantic role $i \in \mathrm{s}$.

Given these semantic composition rules, the DP denotations can combine with the verb denotation in any order, without having to type-lift either DP or verb denotation (as required in type-driven approaches to semantic composition). Semantic composition theories that are equally flexible without requiring typeshifting include among others the recent approaches in Beaver and Condoravi (2007) and Eckardt (2010). Unlike e.g. in Hendriks (1993), the combination of verb and DP denotations does not require (i) the DP denotations to combine in a certain order, (ii) the scope dependencies to be fixed beforehand, and (iii) the verb denotation to be lifted/expanded to the worst case (in order to anticipate all possible DP denotations). Unlike in Cooper (1983), the result of combining DP and verb denotations is not the set of all possible readings, but a relationstore pair capturing what all possible expansions have in common, namely the verb denotation and the assignment of semantic roles to DP denotations.

\subsection{Expansion}

To complete our toy model, assume that

$$
\begin{aligned}
\llbracket \text { read } \rrbracket=\{ & \left\langle r_{1}, p_{1}\right\rangle,\left\langle r_{1}, p_{2}\right\rangle,\left\langle r_{1}, p_{3}\right\rangle,\left\langle r_{1}, p_{5}\right\rangle,\left\langle r_{1}, b_{1}\right\rangle,\left\langle r_{1}, b_{2}\right\rangle, \\
& \left\langle r_{2}, p_{1}\right\rangle,\left\langle r_{2}, p_{2}\right\rangle,\left\langle r_{2}, p_{4}\right\rangle,\left\langle r_{2}, b_{2}\right\rangle, \\
& \left\langle r_{3}, p_{2}\right\rangle,\left\langle r_{3}, p_{3}\right\rangle,\left\langle r_{3}, p_{4}\right\rangle,\left\langle r_{3}, b_{1}\right\rangle, \\
& \left\langle r_{4}, p_{4}\right\rangle,\left\langle r_{4}, b_{1}\right\rangle,\left\langle r_{4}, b_{2}\right\rangle, \\
& \left.\left\langle s_{1}, p_{1}\right\rangle\right\}
\end{aligned}
$$

which, for ease of readability, will be represented as:

$$
\begin{aligned}
& r_{1} \rightarrow p_{1}, p_{2}, p_{3}, p_{5}, b_{1}, b_{2} \\
& r_{2} \rightarrow p_{1}, p_{2}, p_{4}, b_{2} \\
& r_{3} \rightarrow p_{2}, p_{3}, p_{4}, b_{1} \\
& r_{4} \rightarrow p_{4}, b_{1}, b_{2} \\
& s_{1} \rightarrow p_{1}
\end{aligned}
$$

$$
\begin{aligned}
& r_{1}, r_{2}, s_{1} \rightarrow p_{1} \\
& r_{1}, r_{2}, r_{3} \rightarrow p_{2} \\
& r_{1}, r_{3} \rightarrow p_{3} \\
& r_{2}, r_{3}, r_{4} \rightarrow p_{4} \\
& r_{1} \rightarrow p_{5} \\
& r_{1}, r_{3}, r_{4} \rightarrow b_{1} \\
& r_{1}, r_{2}, r_{4} \rightarrow b_{2}
\end{aligned}
$$

So in this toy model, the sentence (5) denotes (6):

(5) More than half the referees read at least three papers.

(6) $\quad$ $\llbracket$ read $\rrbracket,\{\langle 1, \llbracket$ more than half the referees $\rrbracket\rangle,\langle 2, \llbracket$ at least three papers $\rrbracket\rangle\}\rangle$

(6) is an underspecified denotation which (i) expresses what all the possible specified readings have in common, while (ii) leaving the scope dependencies 
unspecified. The scope dependencies are specified by applying expansion operations to this underspecified denotation. The idea behind the sequential expansion operation, which was initially inspired by Akiba (2009), is to add tuples to the relation $R$ of a clause denotation $\langle R, S\rangle$. To illustrate, the expansion of the second

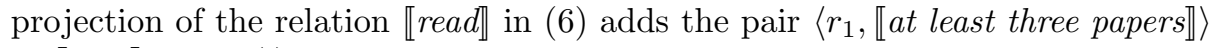
to $\llbracket$ read $\rrbracket$, since (i) $r_{1}$ stands in the reading relation to the papers $p_{1}, p_{2}, p_{3}, p_{5}$, (ii) the set $\left\{p_{1}, p_{2}, p_{3}, p_{5}\right\}$ is a witness set of «at least three paper $₫ \rrbracket$, and (iii)

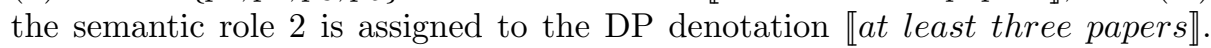
Further, $r_{2}$ reads the papers $p_{1}, p_{2}, p_{4}$, and since $\left\{p_{1}, p_{2}, p_{4}\right\}$ is also a witness

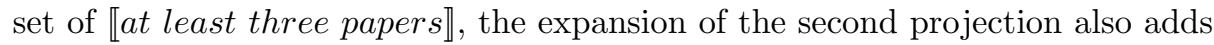
$\left\langle r_{2}, \llbracket\right.$ at least three papers $\left.\rrbracket\right\rangle$ to $\llbracket$ read $\rrbracket$. Finally, $r_{3}$ read the papers $p_{2}, p_{3}, p_{4}$, and

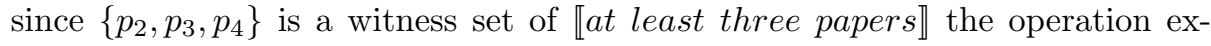

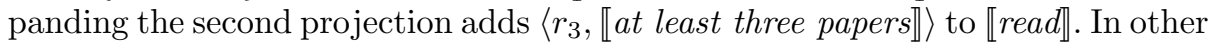
words, the expansion of the second projection makes explicit the information that $r_{1}, r_{2}$ and $r_{3}$ read at least three papers each. Based on this, the expansion of the

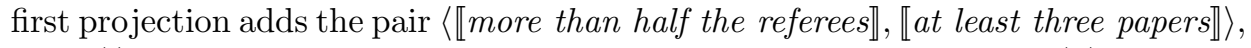
since (i) the only referees who read at least three papers are $r_{1}, r_{2}, r_{3}$, (ii) the set $r_{1}, r_{2}, r_{3}$ is a witness set of the subject DP denotation $\llbracket$ more than half the referees $\rrbracket$,

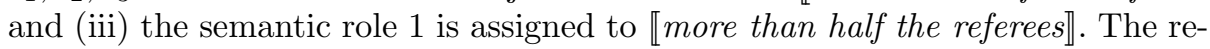
sulting denotation, then, includes the following pairs:

\begin{tabular}{|c|c|}
\hline$r_{1}$ & $\rightarrow p_{1}, p_{2}, p_{3}, p_{5}, b_{1}, b_{2}$ \\
\hline$r_{2}$ & $\rightarrow p_{1}, p_{2}, p_{4}, b_{2}$ \\
\hline$r_{3}$ & $\rightarrow p_{2}, p_{3}, p_{4}, b_{1}$ \\
\hline$r_{4}$ & $\rightarrow p_{4}, b_{1}, b_{2}$ \\
\hline$s_{1}$ & $\rightarrow p_{1}$ \\
\hline$r_{1}$ & $\rightarrow \llbracket$ at least three papers \\
\hline$r_{2}$ & $\rightarrow \llbracket$ at least three papers $\rrbracket$ \\
\hline $\begin{array}{l}r_{3} \\
\| \text { more than half the referees } \rrbracket\end{array}$ & $\begin{array}{l}\rightarrow \llbracket \text { at least three papers } \rrbracket \\
\rightarrow \llbracket \text { at least three papers }\end{array}$ \\
\hline
\end{tabular}

So expanding first the second projection and then the first projection resulted

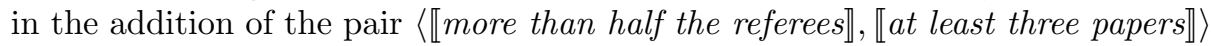
to $\llbracket r e a d \rrbracket$. In this case we shall say that the sentence is true in the given model $\mathrm{M}$ relative to expanding the second projection and then the first projection, i.e. under the reading where the subject has wide scope.

However, if we expand the first projection before the second projection, this pair cannot be added, as shown in the next subsection, and therefore the sentence is false in this model relative to the inverse order of expansion.

The evaluation of the inverse reading begins with the expansion of the first projection of $\llbracket r e a d \rrbracket$. To see more clearly which entities stands in the reading relation to a given entity, we use the following representation of $\llbracket$ read $\rrbracket$ : 


$$
\begin{array}{ll}
r_{1}, r_{2}, s_{1} & \rightarrow p_{1} \\
r_{1}, r_{2}, r_{3} & \rightarrow p_{2} \\
r_{1}, r_{3} & \rightarrow p_{3} \\
r_{2}, r_{3}, r_{4} & \rightarrow p_{4} \\
r_{1} & \rightarrow p_{5} \\
r_{1}, r_{3}, r_{4}, s_{1} \rightarrow b_{1} \\
r_{1}, r_{2}, r_{4}, r_{2} \rightarrow b_{2}
\end{array}
$$

As can be seen, the paper $p_{1}$ is read by exactly two referees, namely $r_{1}$ and $r_{2}$. Since in our model $\left\{r_{1}, r_{2}\right\}$ is not a witness of $\llbracket$ more than half the referees $\rrbracket$, the expansion of the first projection cannot add the pair $\left\langle\llbracket\right.$ more than half the referees $\left.\rrbracket, p_{1}\right\rangle$ to $\llbracket r e a d \rrbracket$. However, the paper $p_{2}$ is read by the referees $r_{1}, r_{2}$ and $r_{3}$, and since

\begin{tabular}{|c|c|}
\hline$r_{1}, r_{2}, s_{1}$ & $\rightarrow p_{1}$ \\
\hline$r_{1}, r_{2}, r_{3}$ & \\
\hline$r_{1}, r_{3}$ & \\
\hline$r_{2}, r_{3}, r_{4}$ & \\
\hline$r_{1}$ & \\
\hline$r_{1}, r_{3}, r_{4}, s_{1}$ & \\
\hline $\begin{array}{l}r_{1}, r_{2}, r_{4}, r_{2} \\
\llbracket \text { more than half the referees } \rrbracket \\
\llbracket \text { more than half the referees } \rrbracket \\
\llbracket \text { more than half the referees } \rrbracket \\
\llbracket \text { more than half the referees }\end{array}$ & $\rightarrow$ \\
\hline
\end{tabular}

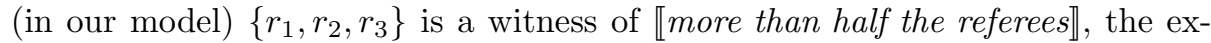
pansion of the first projection adds the pair $\left\langle\llbracket\right.$ more than half the referees $\left.\rrbracket, p_{2}\right\rangle$ to $\llbracket r e a d \rrbracket$. Further, $p_{4}, b_{1}$ and $b_{2}$ are read by $\left\{r_{2}, r_{3}, r_{4}\right\},\left\{r_{1}, r_{3}, r_{4}\right\}$ and $\left\{r_{1}, r_{2}, r_{4}\right\}$ respectively. Since these sets are witnesses of $\llbracket$ more than half the referees》, the three pairs $\left\langle\llbracket\right.$ more than half the referees $\left.\rrbracket, p_{4}\right\rangle,\left\langle\llbracket\right.$ more than half the referees $\left.\rrbracket, b_{1}\right\rangle$ and $\left\langle\llbracket\right.$ more than half the referees $\left.\rrbracket, b_{2}\right\rangle$ are added to $\llbracket$ read $\rrbracket$, resulting in:

As can now be seen, if the first projection is expanded first, there are only two papers which are being read by $\llbracket$ more than half the referees $\rrbracket$, namely $p_{2}$ and $p_{4}$. But

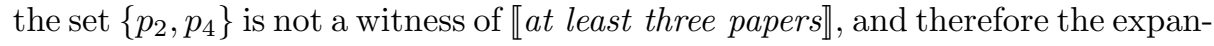

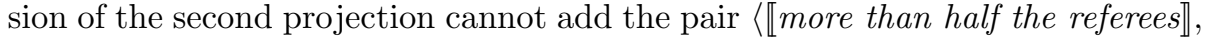
$\llbracket$ at least three papers $\rangle$. This shows that the sentence (5) is not true in this model relative to the inverse scope reading (analyzed as expanding the first projection of $\llbracket r e a d \rrbracket$ before the second projection).

Consider next the following sentence containing the downward entailing expression no referee. ${ }^{3}$

(7) No referee read every paper.

Note that in our toy model this sentence is true under the direct scope reading. For this to come out right, the expansion of the second projection before the first

${ }^{3}$ Within the present theory, a DP denotation is downward entailing iff the set of witness sets contains $\varnothing$. 
projection should result in the addition of the pair $\langle\llbracket n o$ referee $\rrbracket$, «every paper $\rrbracket\rangle$

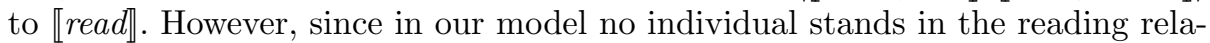
tion to every paper, the expansion strategy employed so far does not add any

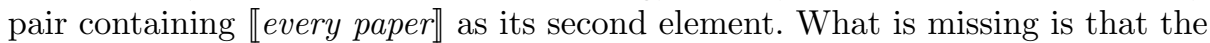
expansion operator also adds negative information, i.e. information about what

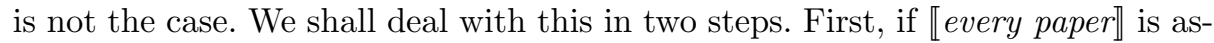
signed the semantic role 2, and an individual $x$ stands in the reading relation to

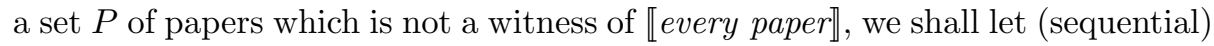
expansion add the pair $\langle x, \llbracket n o t$ every paper $\rrbracket\rangle$.

If every referee stands in the reading relation to $\llbracket$ not every paper , that is just another way of saying that no referee read every paper. Therefore, the second step in dealing with negative information in our example is to let the expansion of the first projection add the pair $\langle\llbracket n o$ referee $\rrbracket$, every paper $\rrbracket\rangle$, provided that the following condition on the expansion of the first projection is met: (i) the set of referees standing in the reading relation to $\llbracket$ not every paper $\rrbracket$ is not a witness

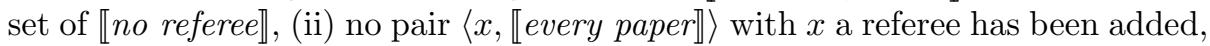
and (iii) the semantic role 1 is assigned to a downward entailing DP denotation. Since this condition is indeed satisfied, expansion of the first projection results in the addition of the pair $\langle\llbracket$ no referee $\rrbracket$, «every paper $\rrbracket\rangle$, showing that the sentence (7) is true in our model under the direct scope reading.

\subsection{Formalizing expansion and truth}

(8) Definition (sequential $i$-expansion):

Let $\llbracket \phi \rrbracket=\left\langle V,\left\{\left\langle i,\left\langle R_{i}, W_{i}\right\rangle\right\rangle: 1 \leq i \leq n\right\}\right\rangle$ be the denotation of an $n$-ary formula $\phi, \sigma[i / Y]$ be the result of replacing the $i$-the element of $\sigma$ by $Y$, let $\sigma_{i}^{V}=\left\{\pi_{i}(\tau): \tau \sim_{i} \sigma \wedge \tau \in V\right\}$, and let $X_{\sigma}=\sigma_{i}^{V} \cap R_{i}$ for any $n$-ary sequence $\sigma .^{4}$ Then the sequential $i$-expansion $\operatorname{EXP}_{i}^{S E Q}(\llbracket \phi \rrbracket)$ of $\llbracket \phi \rrbracket$ is the pair $\left\langle V^{\prime}, S\right\rangle$ where $V^{\prime}$ is the smallest set satisfying the following conditions:

1. $V \subseteq V^{\prime}$

2.for all $\sigma \in D^{n}$ :

(a) $X_{\sigma} \in W_{i} \rightarrow \sigma\left[i /\left\langle R_{i}, W_{i}\right\rangle\right] \in V^{\prime}$

(b) $X_{\sigma} \notin W_{i} \rightarrow \sigma\left[i /\left\langle R_{i}, \overline{W_{i}}\right\rangle\right] \in V^{\prime}$

3.for all $\sigma \in V$ with $\pi_{i}(\sigma) \in D$ and $\pi_{j}(\sigma) \notin D(1 \leq j \leq n$ and $j \neq i)$

(a) $X_{\sigma} \in W_{i} \rightarrow \sigma\left[i /\left\langle R_{i}, W_{i}\right\rangle\right] \in V^{\prime}$

(b) $\left(X_{\sigma} \notin W_{i} \wedge X_{\sigma} \neq R_{i}\right) \rightarrow \sigma\left[i /\left\langle R_{i}, \overline{W_{i}}\right\rangle\right] \in V^{\prime}$

(c) $\left(X_{\sigma} \notin W_{i} \wedge X_{\sigma}=R_{i} \wedge \emptyset \in W_{i}\right) \rightarrow \sigma\left[i /\left\langle R_{i}, W_{i}\right\rangle, j /\left\langle R_{j}, \overline{W_{j}}\right\rangle\right] \in V^{\prime}$

(d) $\left(X_{\sigma} \notin W_{i} \wedge X_{\sigma}=R_{i} \wedge \emptyset \notin W_{i}\right) \rightarrow \sigma\left[i /\left\langle R_{i}, \overline{W_{i}}\right\rangle, j /\left\langle R_{j}, \overline{W_{j}}\right\rangle\right] \in V^{\prime}$

So if $X_{\sigma}$ (the set of entities in $R_{i}$ for which there is a $\tau \in V$ with $\sigma \sim_{i} \tau$ ) is a witness set of the denotation assigned the $i$-th semantic role, then the sequence $\sigma\left[i /\left\langle R_{i}, W_{i}\right\rangle\right]$ is added to $\mathrm{V}$ (clauses (2a) and (3a)). If, however, $X_{i}$ is

${ }^{4} \tau \sim_{i} \sigma$ iff $\tau$ differs from $\sigma$ at most in the $i$-th element; $\pi_{i}(\sigma)$ is the $i$-th element of the sequence $\sigma$. 
not a witness set (i.e. $X_{i} \notin W_{i}$ ), we need to distinguish four cases. Clause (2b) accounts for the addition of $\left\langle r_{1}\right.$, \not every paper $\left.\rrbracket\right\rangle$ in $(7)$, when the second projection of the clause denotation is expanded. Clause (3c) accounts for the addition of $\langle\llbracket$ no referee $\rrbracket$, every paper $\rrbracket\rangle$ when the first projection is expanded. To illustrate the need for the remaining clauses, consider expanding the third projection, then the second projection and finally the first projection of the denotation of No boy gave exactly two girls exactly three flowers.. If $\llbracket$ gave $\rrbracket=\emptyset$, then by clause $(2 \mathrm{~b}), 3$-expansion adds $\langle x, y$, \not exactly three flowers $\rrbracket\rangle$ for every $x, y \in D$. If, further, the model does not contain exactly two girls, then by clause (3d) the 2-expansion will add $\langle x, \llbracket$ not exactly two girls $\rrbracket$, exactly three flowers $\rrbracket\rangle$ for every $x \in D .^{5}$ Finally, by clause (3c), the 1-expansion adds the triple

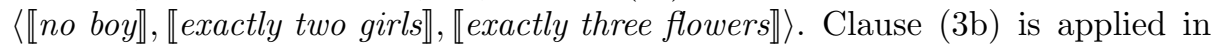
the 2-expansion of the same sentence in a model where one boy (say $b_{1}$ ) gives one girl $\left(g_{2}\right)$ three flowers, and another boy $\left(b_{2}\right)$ gives another girl $\left(g_{2}\right)$ three flowers. In this case, 3-expansion adds both $\left\langle b_{1}, g_{1}\right.$, «exactly three flowers $\rrbracket$ and $\left\langle b_{2}, g_{2}, \llbracket\right.$ exactly three flowers $\rrbracket$, and 2-expansion adds by clause (3b) the triples

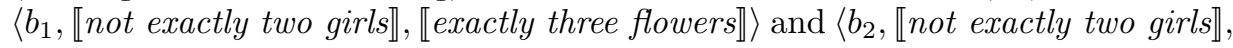

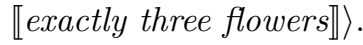

(9) Definition (truth relative to sequence of expansions):

An $n$-ary formula $\phi$ with $\llbracket \phi \rrbracket=\left\langle V,\left\{\left\langle i, X_{i}\right\rangle: 1 \leq i \leq n\right\}\right\rangle$ is true relative to a sequence of expansions $\left\langle\alpha_{1}, \ldots, \alpha_{m}\right\rangle, 0 \leq m$ iff

$$
\left\langle X_{1}, \ldots, X_{n}\right\rangle \in \pi_{1}\left(\alpha_{1}\left(\alpha_{2}\left(\ldots \alpha_{m}(\llbracket \phi \rrbracket)\right)\right)\right)
$$

\section{Conclusion}

To conclude, I briefly highlight two important features of the present proposal and relate them to other proposals.

Theories of semantic scope underspecification can be divided into two types, depending on whether they are based on the notion of satisfaction or on the notion of derivation. ${ }^{6}$ The central tenet of satisfaction-based theories of scope underspecification is that the relation between a representation SR with fully specified scope relations and a representation UR with underspecified scope relations is one of satisfaction: SR is a possible specification of underspecified representation UR iff SR satisfies UR. ${ }^{7}$ On the other hand, the central idea of

\footnotetext{
${ }^{5}$ If the model contains exactly two girls, then by clause (3a) the 2-expansion adds $\langle x, \llbracket$ exactly two girls $\rrbracket$, $\llbracket$ not exactly three flowers $\rrbracket$ for every $x \in D$.

${ }^{6}$ See Egg (2011) for a survey of theories of underspecification.

${ }^{7}$ For example, in Hole Semantics introduced in Bos (1996) an object-level formula SR (e.g. a formula of Predicate Logic) is a possible specification of an underspecified meta-level representation UR (consisting of a set of metavariables called holes, a set of labeled formulas possibly containing holes, and a set of constraints on the relation between holes and labeled formulas) iff the bijective mapping between holes and labels that characterizes the object-level formula sR satisfies the constraints of the underspecified representation UR.
} 
derivation-based theories of underspecification is that the relation between a fully specified representation SR and an underspecified representation UR is one of derivation: SR is a possible specification of an underspecified representation UR iff SR can be derived from UR. ${ }^{8}$ What both types of theories have in common

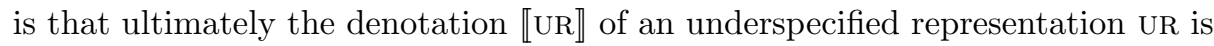
the set of denotations $\left\{\llbracket \mathrm{SR}_{1} \rrbracket, \ldots, \llbracket \mathrm{SR}_{n} \rrbracket\right\}$ of those fully specified representations $\mathrm{SR}_{1}, \ldots, \mathrm{SR}_{n}$ which (depending on the type of underspecification theory) either (i) satisfy UR or (ii) can be derived from UR.

According to this perspective on underspecification, then, an underspecified representation denotes a set of interpretations (readings), while the (partial or full) specification of an underspecified denotation amounts to narrowing down the set of interpretations. Arguably, this perspective on underspecification has two shortcomings. First, since the denotation of the underspecified representation is a set of fully specified readings, it does not capture what the different readings have in common. That is, this type of denotation for underspecified representations does not capture the fact that all readings involve the same verb denotation, and the same assignment of semantic roles to the determiner phrase denotations. Secondly, the denotation of the underspecified representation is not in any sense part of the denotation of each specified representation; on the contrary, there is a clear sense in which the denotations of the specified representations are part of the denotation of the underspecified representation, being elements of the underspecified denotation. In this paper I have propose an alternative theory of quantification and scope underspecification, where (i) the denotation of the underspecified representation does indeed capture what the possible readings all have in common, and (ii) the specification of an underspecified denotation amounts to adding information, as opposed to choosing an element (a specific reading) out of a set of specific readings (the underspecified denotation).

Unlike in GQT, conservativity follows (i) from the restriction of the denotation type of determiners, and (ii) from definition of quantified formulas in (3). To see this, note that the truth-conditions for sentences containing non-conservative determiners cannot be derived in the present theory. To illustrate, assume the non-conservative determiner rouf (mnemonic for the inverse of 'four'), which denotes the following binary function:

$$
\llbracket \text { rouf } \rrbracket(A)(B)=1 \text { iff }|B-A|=4
$$

In the present theory every determiner can be seen as imposing a condition on which subsets of the restrictor set are witnesses. However, the semantic contribution of rouf makes essential reference to two sets, and cannot therefore be formulated as a condition on which subsets of the restrictor set are witnesses.

\footnotetext{
${ }^{8}$ For example, Ambiguous Predicate Logic (APL) introduced in Jaspars and van Eijck (1996) allows for formulas of Predicate Logic to be prefixed with a structured list of scope-bearing operators, which essentially represent a partial ordering on the scopebearing operators. Further, APL defines a rewrite relation on formulas, such that SR is a possible specification of an underspecified representation UR iff it is possible to rewrite UR into SR by means of the specified rewrite relation.
} 


\section{Bibliography}

Akiba, Ken. 2009. A New Theory of Quantifiers and Term Connectives. Journal of Logic Language and Information 18:403-431.

Barwise, Jon, and Robin Cooper. 1981. Generalized quantifiers and natural language. Linguistics and Philosophy 4:159-219.

Beaver, David, and Cleo Condoravi. 2007. On the logic of verbal modification. In Proceedings of the 16th amsterdam Colloquium, ed. Maria Aloni, Paul Dekker, and Floris Roelofsen, 6-12. Amsterdam: University of Amsterdam.

Bos, Johan. 1996. Predicate logic unplugged. In Proceedings of the tenth amsterdam colloquium, ed. Paul Dekker and Martin Stokhof, 133-143. Amsterdam, Netherlands.

Cooper, Robin. 1983. Quantification and syntactic theory. Dordrecht: Reidel.

Eckardt, Regine. 2010. A logic for easy linking semantics. In Proceedings of the 17th amsterdam colloquium, 274-283. Berlin: Springer-Verlag.

Egg, Markus. 2011. Semantic underspecification. In Semantics: an international handbook of natural language meaning, ed. Claudia Maienborn, Klaus von Heusinger, and Paul Portner, 533-572. Berlin: de Gruyter.

Heim, Irene, and Angelika Kratzer. 1998. Semantics in generative grammar. Oxford: Blackwell.

Hendriks, Herman. 1993. Studied flexibility. categories and types in syntax and semantics. Ph.D. thesis, University of Amsterdam.

Jaspars, Jan, and Jan van Eijck. 1996. Underspecification and reasoning. In Building the Framework, 266-287. FraCaS Deliverable D15.

Keenan, Edward, and Jonathan Stavi. 1986. A semantic characterization of natural language determiners. Linguistics and Philosophy 9:253-326.

Peters, Stanley, and Dag Westerståhl. 2006. Quantifiers in Language and Logic. Oxford: Oxford University Press. 


\title{
On the Grounding Status of Appositive Relative Clauses
}

\author{
Todor Koev \\ Department of Linguistics, Rutgers University \\ tkoev@eden.rutgers.edu
}

\begin{abstract}
The prevalent assumption in the literature is that appositive relative clauses (ARCs) contribute asserted but backgrounded content (see Böer \& Lycan [4], Chierchia \& McConnell-Ginet [5], Bach [3], Dever [7], Potts [14], AnderBois et al. [2]). In this paper I demonstrate that ARCs are not inherently backgrounded. Rather, clause-medial ARCs are backgrounded whereas clause-final ARCs behave like regular foregrounded content. I propose a uniform semantics for ARCs which derives their grounding status from whether they are processed before or after the content contributed by the main clause is accepted by the addressee. The idea is formally implemented in Dynamic Predicate Logic (Groenendijk \& Stokhof [11]) enriched with propositional variables (AnderBois et al. [2]).
\end{abstract}

Keywords: appositive relative clauses, backgrounded content

\section{Introduction}

There are two main theories for the semantics of appositive relative clauses (ARCs). On one theory, ARCs are clausal conjuncts and as such are part of the proposition contributed by the whole sentence (see Frege [9], Böer \& Lycan [4], Schlenker [16], AnderBois et al. [2]). On another theory, ARCs contribute independent propositions (see Chierchia \& McConnell-Ginet [5], Bach [3], Dever [7], Potts [14]). On the former theory, sentences with ARCs are interpreted conjunctively and have a single truth value. On the latter theory, sentences with ARCs represent two-dimensional semantic objects that have two truth values: one contributed by the main clause and another contributed by the ARC. Both theories place extra conditions on the way ARCs are interpreted ${ }^{1}$ : ARCs are typically assumed to contribute asserted but backgrounded content, i.e. asserted content that is not part of the main point of the utterance. ${ }^{2}$ In this respect ARCs differ from main clauses, which contribute asserted and foregrounded content.

In this paper I argue against the claim that ARCs are inherently backgrounded. Rather, what rather seems to be the case is that the grounding status of ARCs depends on their linear position in the clause. ARCs are backgrounded

\footnotetext{
${ }^{1}$ Although Frege [9] is an exception.

${ }^{2}$ Backgrounded content is also known as not at-issue content.
} 
when they occur clause-medially; however, when they occur clause-finally, ARCs behave like regular foregrounded content. ${ }^{3}$ Building on previous accounts (see AnderBois et al. [2] and Murray [13], both of which elaborate on Stalnaker [18]), I assume that backgrounded and foregrounded content differ in how they enter the context set. Content contributed by ARCs imposes conditions on the current context set, i.e. directly restricts the context set. In contrast, content contributed by main clauses puts forward an update proposal, a subset of the current context set, which, if accepted, becomes the new context set. My analysis treats ARCs as conjuncts and interprets them at their surface position. Clause-medial ARCs are interpreted before the update proposal introduced by the main clause has been accepted; hence, they restrict the old context set and thus are backgrounded. Clause-final ARCs can be interpreted after the update proposal has been accepted; hence, they restrict the new context set. Even though not part of the proposal, clause-final ARCs end up restricting the same set of worlds as the one representing the new proposal and thus behave like foregrounded content. These ideas are formally implemented in Dynamic Predicate Logic (Groenendijk \& Stokhof [11]) enriched with propositional variables representing the context set and the update proposal (AnderBois et al. [2]).

The paper is structured as follows. Section 2 defines some terminology. Section 3 presents the main empirical facts on the basis of two tests for backgroundedness: the Direct Reply Test and the Answerability Test. Section 4 presents the formal account. Section 5 provides further evidence for the account coming from sentences with clause-final adjuncts. Section 6 concludes the discussion and situates the proposal in the broader context of apposition and clausal types.

\section{Preliminaries}

Before diving into the discussion, let us distinguish between clause-medial vs. clause-final ARCs. I propose the following definition (to be revised): An ARC is called clause-medial/clause-final if it occurs within/outside the smallest potential clause that contains the anchor of the ARC. To illustrate, the ARC in (1) is clause-medial because the smallest clause that contains the anchor of the appositive, i.e. "Maradona," also contains the ARC. The ARC in (2) is clausefinal because the smallest potential clause that contains the anchor excludes the ARC.

(1) Maradona, who scored a goal with his hand, won the Golden Boot.

(2) Sally admires Maradona, who scored a goal with his hand.

It is important to note that the above definition is not meant to imply any claims about the constituency of ARCs. ${ }^{4}$ It merely emphasizes the linear position of ARCs on which the semantics proposed below is based.

\footnotetext{
3 This fact is noted but not explained in Cornilescu [6] and AnderBois et al. [2].

${ }^{4}$ See de Vries [19] for an overview of the various syntactic theories for ARCs.
} 
I would like to also distinguish backgroundedness from projection, the ability of certain implications to arise even when their triggers occur in non-assertive contexts. The two categories are rarely distinguished and some authors try to explain projection in terms of backgroundedness (e.g. Simons et al. [17]). Even though there is a significant overlap between backgroundedness and projectionpresupposition being the prime example that comfortably fits both categoriesthe two categories are distinct. First, meanings can be backgrounded without projecting. E.g., the embedded clause of "Jack imagined that it was raining outside" behaves like backgrounded content: it cannot be directly challenged by "That's not true" and it cannot answer the question "Is it raining outside?" (see Section 3 for a detailed discussion of those two tests for backgroundedness). Also, as Simons et al. [17] themselves observe, there seem to be meanings that project but are not backgrounded, e.g. clause-final ARCs. ${ }^{5}$ Whatever the final verdict on the intricate interaction between backgroundedness and projection might be, this paper will only focus on the grounding status of ARCs. Thus, the explanations proposed here need not necessarily extend to the projective properties of ARCs.

Finally, let me comment on the way I mark acceptability judgments. As is standard in the literature, I use ?, ?? and \# to mark a decreasing degree of acceptability. For theoretical purposes, I treat unmarked sentences and ?sentences as acceptable and ??- and \#-sentences as unacceptable.

\section{Two Tests for Backgroundedness}

In this section I discuss two tests for backgroundedness, both of which underscore the inability of backgrounded content to address the main topic of the conversation, or, in Roberts' [15] terms, the question under discussion. It is demonstrated that clause-medial and clause-final ARCs pattern differently: the former as backgrounded and the latter as foregrounded content.

\subsection{The Direct Reply Test}

Since backgrounded content is not part of the primary assertion of a sentence, one would expect that such content is not directly targetable in subsequent discourse. I call this the Direct Reply Test. ${ }^{6}$

(3) Direct Reply Test

Backgrounded content cannot be directly targeted by the hearer, e.g. by replies like "Yes," "No," "That's not true," etc.

One should note that backgrounded content can be targeted but only indirectly. Indirect targeting has a more severe effect: it disrupts the natural flow

\footnotetext{
${ }^{5}$ See Simons et al. [17] for more discussion.

${ }^{6}$ This test is sometimes called the cancellation/deniability test. Since it does not necessarily involve negative replies, I prefer to call it the Direct Reply Test.
} 
of discourse. It also involves different grammatical tools, including "Hey, wait a minute," "actually," "well," etc.

Clause-medial ARCs pass the Direct Reply Test and thus are backgrounded.

(4) (cf. Amaral et al. [1])

A: Edna, who is a fearless leader, started the descent.

B: \#No, she isn't. She is a coward.

In contrast, clause-final ARCs fail the Direct Reply Test. In this they behave like foregrounded content.

(5) A: Jack invited Edna, who is a fearless leader.

B: No, she isn't. She is a coward.

The same contrast crops up in cases in which the main clause and the ARC are of a different sentence type. ${ }^{7}$ Let us look at cases in which one of the clauses is a declarative and the other an interrogative. (The example in $(6 \mathrm{~A})$ is due to McCawley [12].)

(6) A: Has John, who was talking to Mary a minute ago, gone home?

B: No, he hasn't. He is still at the party.

B': \#No, he wasn't. He was talking to Stacy.

(7) A: Did you see John, who was talking to Mary a minute ago?

B: No, I didn't. I had no idea he was at the party.

B': ??No, he wasn't. He was talking to Stacy.

(8) A: Marcia, who you wanted to meet, didn't you?, has just arrived.

B: No, she hasn't. She is still in San Francisco.

B': ??No, I didn't. I wanted to meet Sarah.

(9) A: Jack invited Marcia, who you wanted to meet, didn't you?

B: ??No, he didn't. He invited Sarah.

B': No, I didn't. I wanted to meet Sarah.

One can make sense of this paradigm as follows. Only one direct reply is felicitous in each case: the addressee is not given the choice of directly replying to either the declarative or the interrogative clause. Clause-medial ARCs, whether declarative or interrogative, are backgrounded (7), (9). When the ARC occurs clause-finally, it is foregrounded. If we make the reasonable assumption that answering a question has a higher urgency in discourse than replying to an assertion, we can explain why replying to the assertion in (8) and (10) is blocked. In short, the generalization from above is upheld in these cases too.

\footnotetext{
${ }^{7}$ McCawley [12] claims that there are two independent speech acts performed in such cases.
} 


\subsection{The Answerability Test}

Since backgrounded content cannot address the main topic of conversation, it is expected that it cannot answer questions. I call this test the Answerability Test.

(10) Answerability Test

Backgrounded content cannot be employed by interlocutors to answer questions.

Both types of ARCs fail the Answerability Test when a singleton question is asked (the examples below are based on AnderBois et al. [2]).

(11) A: What disease did Tammy's husband have?

B: ??Tammy's husband, who had prostate cancer, was treated at the Dominican Hospital.

(12) A: What disease did Tammy's husband have?

B: ??The doctors of the Dominican Hospital treated Tammy's husband, who had prostate cancer.

However, there seems to be a general problem with the answers in (11B) and (12B): the information contributed by the main clause is not relevant to the question being asked. A way to come around this problem is to frame the dialogue in such a way that it involves conjoined questions. ${ }^{8}$ In such contexts the contrast between clause-medial and clause-final ARCs reemerges. Clause-medial ARCs pass the Answerability Test and hence are backgrounded.

(13) A: What did Paula bring and when did she leave the party?

B: ??Paula, who brought cookies, left after midnight.

Clause-final ARCs fail the Answerability Test and once again behave like foregrounded content.

(14) A: Who did you meet at the party and what did they bring?

B: I met Paula, who brought cookies.

\subsection{Intermediate conclusions}

I conclude that clause-medial ARCs are heavily restricted in their uses: they cannot address the main topic of conversation, i.e. represent backgrounded content. In contrast, clause-final ARCs can serve any pragmatic purpose that main clauses can serve. In that respect they behave like foregrounded content.

\footnotetext{
${ }^{8}$ I owe this observation to Veneeta Dayal.
} 


\section{Formal Account}

In Stalnaker's [18] view, assertion (of foregrounded content) is an attempt to reduce the context set and the attempt succeeds only if there are no objections on the side of the addressee. One could then argue that foregrounded content is introduced first as a proposal to restrict the context set and then the proposal is accepted by the addressee (see Groenendijk \& Roelofsen [10], Farkas \& Bruce [8]). This two-step procedure is what makes foregrounded content directly accessible to the addressee. However, backgrounded content is intuitively not directly accessible to the addressee. This intuition could be modeled by the assumption that asserting backgrounded content involves only one step which imposes a restriction on the current context set (see AnderBois et al. [2], Murray [13]). Building on the particular implementation of those ideas proposed in AnderBois et al. [2], the current account derives the contrast between clausemedial and clause-final ARCs from the relative order in which foregrounded and backgrounded content are processed.

\subsection{Formal Background}

Dynamic Predicate Logic (DPL; see Groenendijk \& Stokhof [11]) shares the syntax of first-order logic but has a different semantics. Most importantly, existential quantifiers can bind variables not only in their syntactic scope but also in subsequent conjuncts. Formulas of DPL are interpreted as relations between two information states, represented as assignment functions. AnderBois et al. [2] enrich classical DPL with propositional variables, interpreted as sets of possible worlds. Lexical relations represent atomic formulas that are relativized to propositions in the sense that the formula needs to hold in every world of that proposition. ${ }^{9}$

$$
\langle g, h\rangle \in \llbracket R_{p}\left(u_{1}, \ldots, u_{n}\right) \rrbracket \text { iff } g=h \& \forall w \in h(p):\left\langle h\left(u_{1}\right), \ldots, h\left(u_{n}\right)\right\rangle \in \llbracket R \rrbracket^{w}
$$

Atomic formulas which do not represent lexical relations are interpreted as in classical DPL, i.e. without being relativized to propositions. ${ }^{10}$ The rest of the semantics is the same as in classical DPL. I briefly state the rules for formulas that will be used in the examples below. Conjunction is interpreted as relational composition (16). Random assignment, represented as a separate formula, assigns a new value to its variable and keeps all other variable values intact (17).

$$
\begin{aligned}
& \langle g, h\rangle \in \llbracket \phi \wedge \psi \rrbracket \text { iff } \exists k:\langle g, k\rangle \in \llbracket \phi \rrbracket \&\langle k, h\rangle \in \llbracket \psi \rrbracket \\
& \langle g, h\rangle \in \llbracket \exists u \rrbracket \text { iff } \forall v: v \neq u \Rightarrow g(v)=h(v)
\end{aligned}
$$

A formula $\phi$ is true in a model $M$ and with respect to an assignment function $g$ if and only if there is an assignment $h$ such that $\langle g, h\rangle \in \llbracket \phi \rrbracket^{M}$.

\footnotetext{
${ }^{9}$ Whenever possible, I omit reference to models.

10 Below, I use the identity and the subset relations, interpreted in an obvious way.
} 
Asserting a sentence without appositives amounts to the following. First, a proposal is put forward by introducing a new propositional variable that is included in the current context set and is a candidate for the new context set. Second, the descriptive content is introduced, where lexical relations are relativized to the proposal proposition. Finally, the proposal is accepted by resetting the value of the context set to that of the proposal proposition. This is illustrated in (18), where $p$ represents the proposal proposition and $p^{c s}$ is a distinguished variable representing the current context set at any stage of the conversation.

(18) a. Edna is a fearless leader.

b. $\underbrace{\exists p \wedge p \subseteq p^{c s}}_{\text {proposal }} \wedge \underbrace{\exists x \wedge x=\text { edna } x \text { fearless.leader } p(x)}_{\text {descriptive content }} \wedge \underbrace{\exists p^{c s} \wedge p^{c s}=p}_{\text {acceptance }}$

\subsection{Account}

The context set $p^{c s}$ represents the information that is taken for granted among speech participants and the proposal proposition, say $p$, represents the current topic of conversation. Following AnderBois et al. [2], I make the crucial assumption that content contributed by ARCs is relativized not to $p$ but directly to $p^{c s}$. The basic idea is that backgrounded content restricts $p^{c s}$ whereas foregrounded content restricts $p$.

The incremental processing of sentences with clause-medial ARCs explains why such ARCs are backgrounded. Clause-medial ARCs are processed before the proposal has been accepted and thus impose a condition on the old context set. They do not restrict the proposal proposition, i.e. the topic of conversation, and are backgrounded. This is illustrated below, where the acceptance update, underlined for convenience, follows the conjunct contributed by the ARC.

(19) a. Edna, who is a fearless leader, started the descent.

b. $\exists p \wedge p \subseteq p^{c s} \wedge \exists x \wedge x=e d n a \wedge$ fearless.leader $p^{c s}(x) \wedge$ start.descent $t_{p}(x) \wedge$ $\exists p^{c s} \wedge p^{c s}=p$

I claim that the same strategy can explain why clause-final ARCs behave like foregrounded content. For this, one additional assumption is added: let us allow for the possibility that the acceptance update optionally precedes the conjuncts contributed by clause-final ARCs. ${ }^{11}$ Given this assumption, there are two possibilities for the grounding status of clause-final ARCs. When the acceptance update comes at the very end, clause-final ARCs are backgrounded similarly to clause-medial ARCs. This option accounts for the intuition that clause-final ARCs need not be foregrounded; e.g. they can contribute information that is not picked up in subsequent discourse. More importantly, when the acceptance update precedes the information contributed by the ARC, the ARC imposes a condition on the new context set. Even though not part of the proposal proposition, clause-final ARCs end up restricting the same set of worlds as the ones

${ }^{11}$ This assumption seems intuitive because in some sense the addressee has finished processing the main clause before she starts processing the appositive. 
characterized by the proposal proposition and thus behave like foregrounded content. These two options for clause-final ARCs are illustrated on the example in (20a). In (20b) the ARC restricts the context set before it has been reset to the proposal proposition and is thus backgrounded. In (20c) the ARC further restricts the context set after it has been reset to the proposal proposition and behaves like foregrounded content.

(20) a. Jack invited Edna, who is a fearless leader.

b. $\exists p \wedge p \subseteq p^{c s} \wedge \exists x \wedge x=$ jack $\wedge \exists y \wedge y=$ edna $\wedge$ invite $_{p}(x, y) \wedge$ fearless.leader $p^{c s}(y) \wedge \exists p^{c s} \wedge p^{c s}=p$

c. $\exists p \wedge p \subseteq p^{c s} \wedge \exists x \wedge x=$ jack $\wedge \exists y \wedge y=$ edna $\wedge$ invite $_{p}(x, y) \wedge$ $\exists p^{c s} \wedge p^{c s}=p \wedge$ fearless.leader $p^{c s}(y)$

In short, the puzzling differences in grounding status between clause-medial and clause-final ARCs are amenable to a uniform account if we pay close attention to the order in which foregrounded and backgrounded content is processed.

\section{Further Support for the Account: Clause-final Adjuncts}

The current account turns out to predict that if a constituent contributes old information, i.e. information that is already contained in the current context set, it should be able to follow an ARC without disrupting its foregrounded status. This, it seems, is exactly what we find with clause-final adjuncts. The ARC in (21B), where the locative adverbial is informative, is much harder to directly challenge than the ARC in (22B), where the same adverbial is old information (and spoken with flat intonation). Similarly, the ARC in (23B), where the adverbial is new information, is not able to address questions but the ARC in (24B), where the adverbial is previously given, can marginally do so.

(21) A: So, what happened next?

B: Howard met Bernadette, who is Canadian, at the Vancouver airport.

C: ??Not really—Bernadette was born in the US.

(22) A: Who did Howard meet at the Vancouver airport?

B: He met Bernadette, who is Canadian, at the Vancouver airport.

$\mathrm{C}$ : ?Not really-Bernadette was born in the US.

(23) A: Who did you meet and what did they bring?

B: \#I met Jimmy, who brought tuna salad, in the hallway.

(24) A: Who did you meet in the hallway and what did they bring?

B: ?I met Jimmy, who brought tuna salad, in the hallway.

The foregrounded status of clause-final ARCs thus falls out as a special case of the broader generalization that ARCs become foregrounded only if they are the last informative constituent in the clause. 
On the current account, the data above can be explained as follows. When a clause-final adverbial contributes new information, this information is part of the proposal and the acceptance needs to come last. This has as a consequence that any ARC that precedes the adverbial will be backgrounded. When, however, a clause-final adverbial contributes old information, this information is not part of the proposal and the acceptance can precede the update contributed by the ARC. This has as a consequence that the ARC behaves like foregrounded content, similarly to genuinely clause-final ARCs. Those two options are illustrated on the sentence in $(25 \mathrm{a})$, where the adjunct contributes new information in $(25 \mathrm{~b})$ but old information in $(25 \mathrm{c})$.

(25) a. Howard met Bernadette, who is Canadian, at the Vancouver airport.

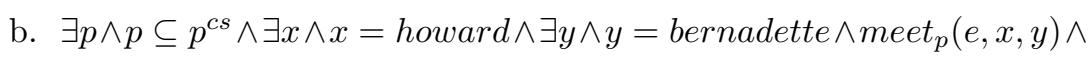
canadian $_{p^{c s}}(y) \wedge$ at.vancouver.airport $p(e) \wedge \exists p^{c s} \wedge p^{c s}=p$

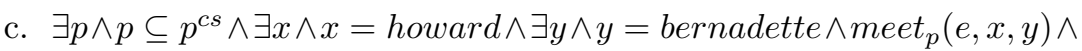
$\exists p^{c s} \wedge p^{c s}=p \wedge$ canadian $_{p^{c s}}(y) \wedge$ at.vancouver.airport $p(e)$

We could uphold the terminology of clause-medial vs. clause-final ARCs if we revise the definition from Section 2 along the following lines: An ARC is called clause-final if it occurs either outside or else is the last informative constituent within the smallest potential clause that contains the anchor of the ARC. An ARC is called clause-medial if it is not clause-final.

\section{Conclusion and Beyond}

In this paper I have shown that clause-medial ARCs are backgrounded whereas clause-final ARCs can be foregrounded. This shift in grounding status was explained by (i) the assumption that ARCs impose a condition on the current context set and (ii) the relative order in which ARCs and foregrounded content are processed. The current proposal lends further support for the conjunctive theory of ARCs as two-dimensional theories predict no difference in grounding status between clause-medial and clause-final ARCs. At the same time, it maintains a uniform semantic mechanism for both clause-medial and clause-final ARCs. My hope is that paying attention to the conditions under which ARCs shift their grounding status would bring us closer to an explanation of the notion of backgroundedness.

Still, further questions remain. First, how do other clauses behave with respect to backgroundedness? Assuming that ARCs have a main clause-like status (see de Vries [19] and literature cited therein), the main vs. subordinate clause divide does not seem to correctly characterize the foregrounded vs. backgrounded content divide. E.g., restrictive relative clauses (unless inside a definite DP, in which case they contribute presupposed content) behave like foregrounded asserted content independently from their surface position: the relative clause in both "A woman who works for Apple invited me for dinner" and "I had dinner 
with a woman who works for Apple" seems to fail both tests for backgroundedness. Second, can other types of appositives shift their grounding status depending on linear position? Nominal appositives apparently cannot: both "Mike Stewart, a comedian from New Jersey, was at the party last night" and "I talked to Mike Stewart, a comedian from New Jersey" seem to pass the two tests for backgroundedness, a fact that might be due to their non-clausal status. I leave both questions to further research.

\section{References}

1. Amaral, P., Roberts, C., Smith, E.A.: Review of The Logic of Conventional Implicatures by Chris Potts. Linguistics and Philosophy 30, 707-749 (2007)

2. AnderBois, S., Brasoveanu, A., Henderson, R.: Crossing the Appositive/At-issue meaning Boundary. In: Proceedings of SALT 20, 328-346. elanguage (2010)

3. Bach, K.: The Myth of Conventional Implicature. Linguistics and Philosophy 22, 327-366 (1999)

4. Böer, S., Lycan, W.: The Myth of Semantic Presupposition. Indiana University Linguistics Club, Bloomington: IN (1976)

5. Chierchia, G., McConnell-Ginet, S.: Meaning and Grammar: An Introduction to Semantics. MIT Press, Cambridge: MA (1990)

6. Cornilescu, A.: Non-Restrictive Relative Clauses, An Essay in Semantic Description. Revue Roumaine de Linguistique 26, 41-67 (1981)

7. Dever, J.: Complex Demonstratives. Linguistics and Philosophy 24, 271-330 (2001)

8. Farkas, D.F., Bruce, K.B.: On Reacting to Assertions and Polar Questions. Journal of Semantics 27, 81-118 (2001)

9. Frege, G.: Über Sinn und Bedeutung. Zeitschrift für Philosophie und philosophische Kritik 100, 25-50 (1892)

10. Groenendijk, J., Roelofsen, F.: Inquisitive Semantics and Pragmatics. Stanford Workshop on Language, Communication and Rational Agency, May 30-31, 2009 (2009)

11. Groenendijk, J., Stokhof, M.: Dynamic Predicate Logic. Linguistics and Philosophy 14, 39-100 (1991)

12. McCawley, J.D.: The Syntactic Phenomena of English. Vol. 2. The University of Chicago Press, Chicago (1988)

13. Murray, S.: Evidentiality and the Structure of Speech Acts. Unpublished Dissertation, Rutgers University (2010)

14. Potts, C.: The Logic of Conventional Implicatures. OUP, New York (2005)

15. Roberts, C.: Information Structure: Towards an Integrated Formal Theory of Pragmatics. In: Yoon, J.H., Kathol, A. (eds.) OSU Working Papers in Linguistics. vol. 49, pp. 91-136. The Ohio State University, Department of Linguistics (1996)

16. Schlenker, P.: Supplements Within a Unidimensional Semantics. In: Aloni, M., Schulz, K. (eds.) Proceedings of the Amsterdam Colloquium 2009. pp. 62-70. Springer, Heidelberg (2009)

17. Simons, M., Tonhauser, J., Beaver, D., Roberts, C.: What Projects and Why. In: Proceedings of SALT 20, 309-327. elanguage (2010)

18. Stalnaker, R.: Assertion. In: Cole, P. (ed.) Syntax and Semantics 9: Pragmatics. LNCS, pp. 315-332. Academic Press, New York (1978)

19. de Vries, M.: The Syntax of Appositive Relativization: On Specifying Coordination, False Free Relatives, and Promotion. Linguistic Inquiry 37, 229-270 (2006) 


\title{
Definiteness in Superlatives
}

\author{
Sveta Krasikova \\ University of Konstanz \\ svetlana.krasikova@uni-konstanz.de
}

\begin{abstract}
A new analysis of superlatives is presented, which assigns two functions to the definite article. It reflects the definiteness of the DP in absolute superlatives, and the definiteness of the DegP in comparative superlatives. The analysis accounts for the distribution of the in attributive, amount, predicative and adverbial superlatives in English.
\end{abstract}

Keywords: superlatives, definiteness, absolute-comparative ambiguity

\section{Introduction}

Most semantic theories of superlatives are concerned with resolving the comparativeabsolute ambiguity superlative sentences are notorious for. Comparative superlatives are considered a thorny case because their semantic indefiniteness seems to clash with the presence of the definite article. The standard solution is to replace the by an abstract $A$ in such cases.

However, the empirical landscape is much more intricate. The definite article is optional in some superlatives, e.g. with superlative adverbs. In amount superlatives, it disambiguates between comparative and proportional readings. For example, the bare superlative in (1) is interpreted as (1-a), and the insertion of the leads to the interpretation in (1-b) .

(1) John climbed (the) most mountains.

a. John climbed more than half of the mountains.

b. John climbed more mountains than anyone else.

In this work, we aim at a more accurate account of the contribution of the definite article in superlatives, which can explain the observed distribution and establish a correlation between the superlative meaning and definiteness.

The paper is structured as follows. In section 2 and 3 we review the existing analyses of superlatives and motivate a new approach, which is developed in section 4 and compared to other theories in section 5. Section 6 discusses the predictions of the new analysis on the distribution of the definite article.

* I am grateful to Arnim von Stechow, Doris Penka, Irene Heim, Maribel Romero, Brian Leahy for very insightful comments and the discussion of various aspects of this work. The errors are all mine. Support for this research was provided by the 


\section{Absolute and Comparative Superlatives}

Adjectival superlatives are known to be ambiguous between an absolute and a comparative reading. ${ }^{1}$ For example, sentence (2-a) can be used to convey that John climbed a mountain that is higher than any other mountain, cf. (2-b). On the other hand, (2-a) can be used to compare John's achievement to the other people's achievements, cf. (2-c).

(2) a. John climbed the highest mountain.

b. John climbed Mount Everest.

c. John climbed a higher mountain than anyone else.

Comparative readings are not so easily distinguishable from absolute ones. In the example at hand, we are ultimately comparing the heights of mountains in both cases. The comparative reading may therefore be thought of as a case of the absolute one in which the mountains compared are the ones climbed by the salient people. There are two strands in the literature, depending on whether comparative readings are subsumed under absolute ones or not. I will refer to them as pragmatic and structural approaches (PA and SA, henceforth).

Before we look at the difference between PA and SA, let us identify their common features. Be it pragmatic or structural, any existing analysis assumes that the superlative meaning is contributed by the superlative morpheme which is restricted by a contextual variable corresponding to the comparison class. According to the lexical entry in (3) from [4], -est applies to a comparison class $C$ and a gradable predicate $A$ to return a predicate of individuals that is true of those entities whose $A$-ness degrees exceeds $A$-ness degrees of everyone else in $C$. Thus, the role of the superlative is to existentially bind the degree variable projected by the gradable predicate and restrict it by an appropriate type of comparison. $C$ is also presupposed to consist of entities to which $A$ applies. ${ }^{2}$

$$
\begin{array}{ll}
\text { a. } & \llbracket \text {-est }=\lambda C \in D_{e t} \lambda A \in D_{d(e t)} \lambda x \in D_{e} \exists d[A(d)(x) \wedge \\
& \forall y[y \in C \wedge y \neq x \rightarrow \neg A(d)(y)]] \\
\text { b. } \quad \llbracket \text {-est } \rrbracket(C)(A)(x) \text { is only defined iff } \forall y \in C[\exists d[A(d)(x)]]
\end{array}
$$

PA ([4], [2], [8], [9]) maintain the view that to resolve the ambiguity, it suffices to pragmatically fix the value of $C$. The corresponding analysis of (2-a) is outlined in (4), where (4-a) is the assumed LF, (4-b) is the meaning of the NP, (4-c) are the resulting truth conditions, and (5-a) and (5-b) are the value assignments of $C$ under the absolute and the comparative reading, respectively.

$$
\begin{aligned}
& \text { a. John climbed [the [-est } \mathrm{C}[\text { high mountain }]]] \\
& \text { b. } \llbracket \text { high } \rrbracket(\llbracket \text { mountain } \rrbracket)=\lambda d \lambda x \text { is a mountain } \wedge \text { height }(x) \geq d \\
& \text { c. John climbed the unique } x, \text { s.t. } x \text { is a mountain } \wedge \exists d[\text { height }(x) \geq d \\
& \wedge \forall y[y \in C \wedge y \neq x \rightarrow \neg[y \text { is a mountain } \wedge \text { height }(y) \geq d]]]
\end{aligned}
$$

\footnotetext{
${ }^{1}$ This observation goes back to [7]

${ }^{2}$ I disregard other presuppositions usually assumed to be triggered by the superlative as they are orthogonal to the present discussion.
} 
(5) a. $\quad \mathrm{C}_{A B S}=\{x: x$ is a salient mountain $\}$

b. $\mathrm{C}_{C O M P}=\{x: x$ is a mountain climbed by a salient individual $\}$

SA ([10], [4]) agree that ambiguity is related to the value of the comparison class, but argue that the latter depends on the structural position of -est at LF. Thus, to derive the comparative reading of (2-a), -est is interpreted DPexternally. Its movement creates the right kind of property for the derivation of the comparative reading, namely the relation holding between an individual $x$ and a degree $d$ if $x$ climbed a $d$-high mountain. Correspondingly, it is not mountains but mountain climbers that are compared, and the resulting value of $C_{C O M P}$ in (6-c) is different from the one in (5-b). ${ }^{3}$ The superlative DP is assumed to be indefinite, which accords with the mobility of - est . ${ }^{4}$ Accordingly, an abstract indefinite determiner $A$ heads the DP. Note that the uniqueness requirement contributed by -est renders the definite article superfluous, so the replacement of the by $A$ does not have a considerable semantic effect.

(6) a. John [-est C] [ $\lambda d \lambda x \times$ climbed [A d high mountain $]]$

b. $\exists x[x$ is a mountain $\wedge$ John climbed $x \wedge \exists d[$ height $(x) \geq d \wedge \forall y[y \in C \wedge$ $y \neq$ John $\rightarrow \neg \exists z[z$ is a mountain $\wedge y$ climbed $z \wedge$ height $(z) \geq d]]]]$

c. $\quad \mathrm{C}_{\text {COMP }}=\{x: \exists d[x$ climbed a $d$-high mountain $\}$

The main argument in favour of SA, and contra PA, is the availability of the so called upstairs de dicto readings in intensional superlatives. Consider sentence (8) uttered in the context in (7).

(7) - How high a mountain do you need to climb?

John: - I need to climb a $6000 \mathrm{~m}$ high mountain.

Bill: - I need to climb a $4000 \mathrm{~m}$ high mountain.

Mary: - I need to climb a $3000 \mathrm{~m}$ high mountain.

(8) John needs to climb the highest mountain.

Two things are certain: the DP receives a de dicto interpretation, and we are dealing with a comparative superlative. However, the task of providing a value for $C$ under PA has proved a challenge in such cases. No matter how we constrain the mountains in $C$ and whether we replace the by an abstract indefinite article, the uniqueness requirement on -est leads to a definite interpretation, which is incompatible with the given scenario, see [4]. SA do not have this problem. The relevant interpretation can be derived by assigning -est wide scope relative to need, cf. (9-a). This move guarantees that $C$ corresponds to the set in (9-b), which does not create a problem in combination with the uniqueness requirement.

(9) a. John [-est C] $[\lambda d \lambda x \times$ needs to climb [A d high mountain $]]$ b. $\quad \mathrm{C}=\{\mathrm{x}: \exists d[x$ needs to climb a $d$-high mountain $]\}$

\footnotetext{
${ }^{3}$ Both PA and SA concur that focus plays a role in promoting this or that reading. That is, if John is focussed in (2-a), the contextual variable is assigned $C_{C O M P}$ as its value. We will return to the issue of focus in section 4 .

${ }^{4}$ Movement out of a definite DP is subject to syntactic island constraints.
} 
We can draw two lessons from this kind of sentences. First, unless we make sure that we technically compare different kinds of entities under an absolute and a comparative reading, the uniqueness requirement clashes with the indefiniteness of the DP in comparative superlatives. Second, the definite article, though merely superfluous in other kinds of superlatives, is at odds with an indefinite interpretation of the upstairs de dicto superlatives.

\section{Distribution of the Definite Determiner}

The previous section revealed a weakness of existing approaches. They don't assign any role to the. Moreover, the appears incompatible with an apparently indefinite interpretation of some comparative superlatives. Szabolsci [10] is one of the first to conclude that only comparative superlatives are indefinite. ${ }^{5}$ Along with some syntactic tests, she considers how superlatives behave in "definiteness effect contexts". It turns out that comparative superlatives may occur in such contexts, while absolute superlatives are unacceptable. For example, putting focus on John in (10-c) promotes the comparative reading and creates a contrast to (10-b), which is as bad as the variant with a non-superlative definite in (10-a).

(10) a. *John has the sister.

b. John has the smartest sister.

c. JOHN has the smartest sister.

However, the occurrence of the in superlatives does not seem to correlate with their definiteness. Three classes of English superlatives may be distinguished relative to how the is distributed.

First, attributive superlatives require the presence of the article regardless of the interpretation available, cf. (2-a). Adopting Szabolsci's generalisation that absolute superlatives are definite and comparative ones are indefinite, it looks as if the were interpreted in the former and were spurious in the latter case.

Second, in amount superlatives, which are indefinite under comparative and proportional readings, the has been observed to play a disambiguating role, cf. [3]. (11) and (12) are examples of a comparative and a proportional reading of amount superlatives, the paraphrases given in (11-c) and (12-c) follow the analysis in Hackl [3].

(11) a. John climbed the most mountains.

b. John climbed more mountains than anyone else.

c. There is a plurality of mountains John climbed whose cardinality exceeds the cardinalities of pluralities of mountains others climbed.

(12) a. John climbed most mountains.

b. John climbed more than half of the mountains.

c. There is plurality of mountains that John climbed that exceeds the cardinalities of all non-overlapping pluralities of mountains.

\footnotetext{
${ }^{5}$ See $[8]$ for an alternative view.
} 
Finally, adverbial and predicative superlatives do not require the definite article, cf. (13). The optionality of the is not disambiguating in this case. In fact, neither of the constructions can exhibit the relevant kind of ambiguity. Adverbial superlatives can be ambiguous, but comparison classes associated with different readings all involve different kinds of eventualities, modified by the adverb. In predicative superlatives, there is no room for variation either: alternatives in comparison classes are always shaped after the subject.

$$
\begin{aligned}
& \text { a. John ran (the) fastest. } \\
& \text { b. John is (the) tallest. }
\end{aligned}
$$

There is a fair amount of variation in how superlatives are formed across languages. However, the fact that the presence of the definite determiner does not reflect the definiteness of the superlative DP holds across languages. For example, in German the definite article can never be optional, regardless of the reading and the kind of superlative, cf. (14). Note that (14-a) and (14-b) have both an absolute/proportional and a comparative reading.
a. Hans hat *(den) höchsten Berg bestiegen.
Hans has the highest mountain climbed
b. Hans hat (die) meisten Berge bestiegen
Hans has the most mountains climbed
c. Hans ist $*(\mathrm{am})$ schnellsten gelaufen.
Hans is at.the fastest run
d. Hans ist * (am) schnellsten.
Hans is at.the fastest

To give an intermediate summary, most of the existing theories are successful in accounting for the differences in absolute and comparative superlatives; however, the presence of the definite article remains a mystery under any approach. The distribution of the does not seem incidental, considering its role in amount superlatives, but it does not reflect the definiteness of the superlative DP in all cases. Ideally, we would like to understand why comparative superlatives are marked for definiteness despite their semantic indefiniteness, and why some languages make the definite article optional in some contexts.

\section{Definite DP and Definite DegP}

We would like to suggest that the distribution of the definite article is complicated by the fact that it plays a double role in superlatives. It either heads a definite determiner phrase, or provides a definite standard of comparison, i.e. heads a definite degree phrase. In absolute superlatives, which behave as definites, the requires the uniqueness of the referent of the superlative DP. In comparative superlatives, it requires the uniqueness of the degree to which the gradable predicate relates an arbitrary individual. Let us first look at the least controversial, absolute case. 


\subsection{DP-internal Superlatives}

We assume that absolute and proportional superlatives involve an abstract superlative degree modifier whose presence is licensed by the uninterpretable superlative morphology on the adjective. Let us call this modifier SUP. The following definition is based on the entry for the superlative morpheme given in (3).

$$
\begin{aligned}
& \llbracket \mathrm{SUP} \rrbracket=\lambda A \in D_{d(e t)} \lambda P \in D_{e t} \lambda x \in D_{e} P(x) \wedge \exists d[A(d)(x) \wedge \\
& \forall y[P(y) \wedge y \neq x \rightarrow \neg A(d)(y)]]
\end{aligned}
$$

According to this definition, SUP applies to a gradable predicate $A$ and a predicate $P$, expressed by the head noun, to return a predicate holding of $x$ iff $x$ is true of $P$, and has $A$ to a degree to which no other entity in the extension of $P$ does. Line (16) illustrates the application of SUP to highest mountain in the derivation of the absolute reading of (2-a).

$$
\begin{aligned}
& \llbracket \mathrm{SUP} \rrbracket(\llbracket \text { highest } \rrbracket)(\llbracket \text { mountain } \rrbracket)=\lambda x \quad x \text { is a mountain } \\
& \wedge \exists d[\text { height }(x) \geq d \wedge \forall y[y \text { is a mountain } \wedge x \neq y \rightarrow \text { height }(y)<d]]
\end{aligned}
$$

Due to the uniqueness requirement of SUP, the resulting predicate in (16) can only be true of one mountain. Consequently, a principle in the spirit of "maximise presuppositions" requires that the DP be realised as definite. The definite article therefore emphasises that the referent of the superlative DP is unique. In (17) we sketch the complete LF and the truth conditions for the absolute reading of $(2-\mathrm{a})$.

$$
\begin{aligned}
& \text { a. John climbed [the [SUP highest mountain]] } \\
& \text { b. John climbed the unique } x \text {, such that } x \text { is a mountain } \wedge \\
& \exists d[\text { height }(x) \geq d \wedge \forall y[y \text { is a mountain } \wedge x \neq y \rightarrow h e i g h t(y)<d]]
\end{aligned}
$$

In proportional amount superlatives, the uniqueness condition on SUP does not have the same effect. Since we are dealing with plurals, there may be several pluralities falling under the superlative description. The use of the definite article would therefore be unmotivated and the DP is realised as a bare plural, see (18).

$$
\begin{array}{ll}
\text { a. } & \text { John climbed [A [SUP most mountains }]] \\
\text { b. } & \exists X[X \text { are mountains } \wedge \text { John climbed } X \wedge \exists d[\operatorname{card}(X) \geq d \wedge \\
& \forall Y[Y \text { are mountains } \wedge X \text { does not overlap } Y \rightarrow \operatorname{card}(Y)<d]]]
\end{array}
$$

This derivation is almost identical to the PA derivation given in (4). The difference is that (4-a) is unspecified, corresponding to the absolute or the comparative reading depending on the value of $\mathrm{C}$. (17-a) is not ambiguous in the same sense and is meant to represent the absolute reading only.

\subsection{Superlatives as Plural Definites}

We propose that comparative superlatives involve a different kind of degree binding. While the degree argument of the gradable predicate is existentially bound 
by SUP in absolute superlatives, it is saturated by a definite degree description in comparative superlatives. We assume the structure in (19) for the comparative reading of (2-a). The definite article restricted by a contextual variable $C$ fills the degree argument slot of highest, whose morphology is again not interpreted. The resulting predicate can be intersectively combined with the head noun. The entire DP is realised as definite due to the definiteness of the DegP.

$$
\left[{ }_{N P}\left[{ }_{A P}\left[{ }_{D e g P} \text { the } \mathrm{C}\right] \text { highest }\right] \text { mountain }\right]
$$

To spell out the analysis of (2-a), we need to know how $C$ receives its value, and what the contribution of the is. Following Szabolsci's insight, which guided most of the existing theories of superlatives, we propose that the value of $C$ is determined by the focus structure of the sentence. For concreteness, assume that focus is evaluated by Rooth's squiggle operator ([6]), which comes with a variable corresponding to the focus semantic value of the constituent in its scope. The value of the free variable $C$ on the definite determiner is restricted to be a subset of the value of the variable introduced by the squiggle. Suppose, the focus falls on the subject in (2-a), then $C$ ends up a subset of the set of degree sets defined in (20-b).

$$
\begin{array}{ll}
\text { a. } & [\lambda d \text { JOHN climbed [ A d highest mountain }] \sim C] \\
\text { b. } & C=\{D: \exists x[D=\lambda d x \text { climbed a } d \text {-high mountain }]\}
\end{array}
$$

This mechanism of providing a value for $C$ is reminiscent of Heim's analysis of superlative as a focus sensitive operator restricted by a comparison class consisting of degree sets, cf. [4]. Like in Heim's analysis, representing comparison classes in terms of degrees may lead to an "information loss" problem. ${ }^{6}$ For example, if the highest mountains John and Mary happen to have climbed are both 6000 $\mathrm{m}$ high, the degree sets corresponding to Mary and John are indistinguishable. To avoid this, we follow [5], who deals with the same problem in the analysis of only, and intensionalise the focus value, so that it consists of degree properties.

$$
C=\{D: \exists x[D=\lambda w \lambda d x \text { climbed a } d \text {-high mountain in } w]\}
$$

Turning to the contribution of the, we assume that it applies to a set of degree properties $C$ and returns the unique maximal element, as the following definition shows.

$$
\llbracket \text { the } \rrbracket=\lambda w \lambda C \in D_{(d t) t} \iota D\left[C(D) \wedge \forall D^{\prime}\left[C\left(D^{\prime}\right) \rightarrow D(w) \subseteq D^{\prime}(w)\right]\right]
$$

Following a proposal in [1], we treat the extension of the resulting property as a plurality of degrees. Beck assumes that a set of degrees may saturate the degree argument of some degree predicate by acting as a plurality of degrees interpreted distributively. To derive distributive readings, she introduces Link's star operator, to the effect that the plurality of degrees receives a sentential scope. We adopt this proposal here. The definite article together with the restricting

${ }^{6} \mathrm{Im}$ grateful to Arnim von Stechow and Maribel Romero (p.c.) for pointing this out to me and discussing the solution. 
property $C$ moves out of the DegP of the adjective and combines with the degree property that has been modified by $*$. Given the standard definition of *, which in this case turns a degree set into its power set, the resulting truth conditions boil down to (23-b).

$$
\begin{array}{ll}
\text { a. } & \text { [the } \mathrm{C}] *[\lambda d \text { JOHN climbed }[\mathrm{A} d \text { highest mountain }] \sim \mathrm{C}] \\
\text { b. } & \exists x[x \text { is a mountain in } w \wedge \text { John climbed } x \text { in } w \wedge \\
\forall d[d \in \llbracket \text { the } \mathrm{C} \rrbracket(w) \rightarrow \operatorname{height}(x) \geq d]]
\end{array}
$$

To see that this outcome is correct, consider the predictions of this analysis in three different scenarios. First, suppose that John climbed a $6000 \mathrm{~m}$ high mountain, Mary climbed a $5000 \mathrm{~m}$ high mountain and Bill climbed a $4000 \mathrm{~m}$ high mountain. Then the definite article returns the property $[\lambda w \lambda d$ John climbed a $d$-high mountain] and the sentence is predicted true. If John and Mary each climbed a $6000 \mathrm{~m}$ high mountain and Bill climbed a $4000 \mathrm{~m}$ high mountain, the definite article applied to $C$ returns undef. Finally, if Mary climbed a $6000 \mathrm{~m}$ high mountain, John climbed a $5000 \mathrm{~m}$ high mountain and Bill climbed a 4000 $\mathrm{m}$ high mountain, the sentence comes out false. For completeness, we also sketch the analysis of the comparative amount superlative (11-a) in (24).

$$
\begin{array}{ll}
\text { a. } & \text { [the } \mathrm{C}] *[\lambda d \text { JOHN climbed }[\text { A } d \text { most mountains }] \sim \mathrm{C}] \\
\text { b. } & C=\{D: \exists x[D=\lambda w \lambda d \exists Y[Y \text { are mountains in } w \\
& \left.\left.\left.\wedge x \text { climbed } Y \text { in } w \wedge \operatorname{card}_{w}(Y) \geq d\right]\right]\right\} \\
\text { c. } & \exists X[X \text { are mountains in } w \wedge \text { John climbed } X \text { in } w \wedge \\
& \left.\forall d\left[d \in \llbracket \text { the } \mathrm{C} \rrbracket(w) \rightarrow \operatorname{card}_{w}(X) \geq d\right]\right]
\end{array}
$$

\section{Comparison of Approaches}

The current approach distinguishes between absolute and comparative superlatives structurally. In comparative superlatives, the definite article provides a plurality of degrees to saturate the degree position of the gradable predicate. We assumed that it is interpreted distributively and its restriction is provided by the focus. This analysis shares with SA the ability to derive the right truth conditions for upstairs de dicto readings. By moving the definite term over the modal, we create the comparison class $C$ that contains properties holding of $d$ in $w$ iff some salient individual climbs a $d$-high mountain in every world accessible from $w$, cf. (25-b). If John happens to have a requirement exceeding that of Bill or Mary, the sentence is correctly predicted true, cf. (25-c). Thus, the analysis does not face the problem of PA with the uniqueness requirement in intensional contexts.

(25) a. $\quad[$ the $\mathrm{C}] *[\lambda d$ need JOHN climbed $[\mathrm{A} d$ highest mountains $] \sim \mathrm{C}]$

b. $\quad\left\{D: \exists x\left[D=\lambda w \lambda d \forall w^{\prime} \in A c c_{w}\left[x\right.\right.\right.$ climbed a $d$-high mountain in $\left.\left.\left.w^{\prime}\right]\right]\right\}$

c. $\forall d\left[d \in \llbracket\right.$ the $\mathrm{C} \rrbracket(w) \rightarrow \forall w^{\prime} \in A c c_{w}\left[\exists x\left[x\right.\right.$ is a mountain in $w^{\prime}$

$\wedge$ John climbed $x$ in $w^{\prime} \wedge$ height $\left.\left.\left.w_{w^{\prime}}(x) \geq d\right]\right]\right]$ 
The second advantage is that under the present approach the definite article makes a meaningful contribution to the analysis of a superlative construction. Absolute superlative DPs are analysed as semantically definite, the acting as a definite determiner in the presence of SUP. Comparative superlative DPs are not necessarily definite, the reflects the definiteness of the degree argument.

Regarding the definiteness of the DP, the present approach reveals more flexibility than SA. [8] point out that PA and SA predict different truth conditions in certain contexts. They discuss two situations that can be referred to as "two-equally-high-mountains" and "the-same-mountain" scenarios. In the former, John is assumed to have climbed two equally high mountains, which are higher than any other mountains. In the latter, John and Bill are assumed to have climbed the same mountain, which happens to be the highest. Sharvit and Stateva point out that SA's predictions in these cases are not quite adequate. In the "two-equally-high-mountains" scenario, (2-a) comes out true under SA; and in the "the-same-mountain" scenario it is predicted false. Since the superlative DP may but need not be definite under the present analysis, the "two-equallyhigh-mountains" scenario predicts that the sentence is either true or undefined under the comparative reading, which reflects Sharvit and Stateva's judgments, who report that their informants hesitate to assign the sentence a truth value in this scenario. In the "the-same-mountain" scenario, the sentence is undefined under the comparative reading and true under the absolute reading, which accords with the fact that the sentence is usually judged misleading in such cases.

\section{Optionality}

The present approach predicts that the definite article is optional in amount superlatives, which are indefinite regardless of the superlative reading available. Though right for English, this prediction is not borne out for German and other languages where the definite article is never omitted. Unlike English, German marks superlatives as definite even when the DegP and the DP are semantically indefinite. A possible explanation is that in languages like German the uniqueness requirement on the superlative modifier SUP introduces the feature 'definite' which projects to the DP level and is responsible for the insertion of the article. In English, SUP lacks the feature 'definite' and the article is optional in absolute superlatives with indefinite DPs.

For adverbial superlatives both an absolute and a comparative construal are possible and derive the same truth conditions, cf. (26) and (27) . For the absolute case, we can assume a covert contextual variable that plays the role of the head noun, see (27-c). Consequently, the is predicted optional in English adverbial superlatives.

$$
\begin{array}{ll}
\text { a. } & \text { the } \mathrm{C}] *[\lambda d \text { JOHN ran }[\mathrm{A} \mathrm{d} \text { fastest }] \sim \mathrm{C}] \\
\text { b. } \exists e[e \text { is running by John } \wedge \forall d[d \in \llbracket \text { the } \mathrm{C} \rrbracket(w) \rightarrow \operatorname{speed}(e) \geq d]] \\
\text { c. } \mathrm{C}=\{D: \exists x[D=\lambda w \lambda d \exists e[e \text { is running by } x \wedge \operatorname{speed}(e) \geq d]]\} \\
\text { a. John ran [SUP fastest } \mathrm{C}]
\end{array}
$$




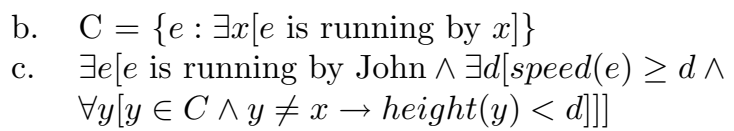

Predicative superlatives are always absolute. The present analysis predicts that if the definite article is missing, the resulting property does not necessarily apply to a unique referent. As the relevant data are subtle, we do not evaluate this prediction here but leave this for a future occasion.

$$
\begin{aligned}
& \text { a. John is [the [SUP tallest C] } \\
& \text { b. John is [SUP tallest C] }
\end{aligned}
$$

\section{Conclusion}

We argued that the definite article in superlatives may reflect definiteness at the DP as well as DegP level. According to the present approach, only absolute attributive superlatives are genuinely definite; in comparative superlatives, the marks the definiteness of the degree argument, and does not necessarily reflect the definiteness of the superlative DP. This analysis is not only successful in deriving all possible readings of superlatives, it also accounts for the distribution of the in different kinds of superlatives.

\section{References}

1. Beck, Sigrid: Lucinda Driving Too Fast Again - the Scalar Properties of Ambiguous Than-Clauses. University of Tbingen. Unpublished manuscript (2010)

2. Farkas, Donka and Katalin E. Kiss: On the Comparative and Absolute Readings of Superlatives. Natural Language and Linguistic Theory 18(3), 41755 (2000)

3. Hackl, Martin: On the Grammar and Processing of Proportional Quantifiers: 'Most' Versus 'More Than Half'. Natural Language Semantics 17, 63-98 (2009)

4. Heim, Irene: Notes on Superlatives. MIT. Unpublished manuscript (1999)

5. Rooth, Mats: Association With Focus. Ph.D. Dissertation. University of Massachusetts at Amherst (1985)

6. Rooth, Mats: A Theory of Focus Interpretation. Natural Language Semantics 1(1), 75116 (1992)

7. Ross, John Robert: A Partial Grammar of English Superlatives. MA thesis. University of Pennsilvania (1964)

8. Sharvit, Yael and Penka Stateva: Superlative Expressions, Context and Focus. Linguistics and Philosophy 25, 453505 (2002)

9. Stateva, Penka: How Different Are Different Degree Constructions. PhD Dissertation. University of Connecticut (2002)

10. Szabolsci, Anna: Comparative Superlatives. In: Fukui, N., Rapoport, T. and Sagey, E. (eds.) MIT WPL 8, 245-66 (1986) 


\title{
The Accommodation Potential of Implicative Verbs
}

\author{
Noor van Leusen \\ Radboud University, Nijmegen, the Netherlands \\ n.vanleusen@phil.ru.nl
}

\begin{abstract}
We present an analysis of implicative verbs, ${ }^{1}$ complementtaking verbs which induce entailment-like inferences, but which are also claimed to trigger presuppositions. What is presupposed, however, is much more variable than with e.g. factive verbs. Sketching a formal treatment in Logical Description Grammar we consider the role of pragmatic reasoning and accommodation in deriving these presuppositions.
\end{abstract}

Keywords: Implicative verbs, presupposition, accommodation, LDG

\section{Introduction}

Since Karttunen's work in the seventies [5], implicative verbs in English, such as 'to manage to', 'to forget to', 'to happen to', or 'to force to', etc., have become known for their characteristic inference pattern. On the one hand, they are claimed to entail or implicate the truth or falsity of their complement sentence, depending on the polarity of the embedding construction. For instance, we have

(1) a. Jim managed to button his coat. $\rightarrow$ Jim buttoned his coat.

b. Jim did not manage to button his coat.

Jim did not happen to manage to button his coat.

$\rightarrow$ Jim did not button his coat.

Various subclasses of implicative verbs can be distinguished. Following [9], 'to manage to' might be called a two-way implicative, because it triggers an entailmentlike inference both in its unnegated and its negated form; moreover, it may be called affirmative because it implies the truth of the complement sentence in its bare, unnegated form.

On the other hand, implicative verbs are often claimed to evoke presuppositions. Verbs of success or failure are taken to presuppose that there is or was an attempt on the subject's part to achieve the state or event described by the complement sentence. 'To manage to' it is also often assumed to be presuppose

\footnotetext{
${ }^{1}$ This research was supported by a grant from the Netherlands Organisation for Scientific Research (NWO), which is gratefully acknowledged.
} 
that there is some difficulty to be overcome in order to achieve the complement state or event. Thus, both (1a.b) would presuppose ${ }^{2}$, variously,

$(2) \leadsto$ Jim made an attempt to button his coat. $\leadsto$ It was difficult for Jim to button his coat.

Verbs of forgetting or remembering are often taken to presuppose that there is or was an obligation on the subject's part to achieve the state or event described by the complement sentence. More tentatively, it is suggested that these verbs evoke the supposition that the subject intends to achieve the complement state or event, or that, less specifically, there is an expectation that he or she will achieve it, or ought to do so.

Moreover, most implicatives evoke what may be called characteristic causal or explanatory presuppositions: reasons why the state or event denoted by the complement sentence is or is not achieved in the case at hand. This comes out perhaps most clearly in cases such as (3) where the implicative is negated,

(3) Ed didn't manage / remember / bother / dare / happen to open the door.

all of which imply that Ed did not open the door but presuppose a different reason for this fact. Ed did not make a sufficient effort or was not sufficiently skilled to open the door, he did not keep in mind his plan or obligation to close it, he did not care enough or did not take the trouble, he did not have sufficient courage, or somehow the right circumstances did not apply.

While most of the early descriptive literature $[5,3]$ and some more recent papers $[8,9]$ concentrate on the entailments or implications of sentences with implicative verbs, the focus of this paper is on the presuppositional side of implicatives. In particular, we are concerned with the variability of their presuppositions - if indeed that is what they are. We will investigate how these inferences may come about, and consider what that means for the interaction between general pragmatic reasoning and the satisfaction of lexical presuppositional conditions in a dynamic semantic perspective on sentence meaning.

Existing treatments of implicatives within the dynamic semantic paradigm, e.g. [2], tend to treat them exclusively as presupposition triggers and often only provide an analysis of the bench-mark case 'to manage to'. We aim to improve on this in the following way. Firstly, if we want to account for the presuppositions of implicatives, we cannot ignore looking at their implications or entailments too. It is on the basis of their full inferential signature that we may establish the semantic-pragmatic meaning of implicative sentences, and tease apart their semantic content from the requirements on context they induce. What is presupposed then follows from the interaction with general pragmatic reasoning. Secondly, it is fruitful to look at implicatives other than 'to manage to', which happens to be one that induces virtually uncancellable implications both in its bare and negated form. Not all implicative verbs are like that, and we will zoom in on a slightly weaker instance, namely 'to remember to'.

\footnotetext{
${ }^{2}$ In some papers, notably [7], these inference are classified as conventional implicatures.
} 
Finally, the status of the inferences under discussion as presuppositions can be called into question. While some of them may well be viewed as scalar and conversational implicatures, we propose that most of them are 'pragmatic presuppositions' (Stalnaker), or 'secondary inferences' that result from the need to satisfy and explain a basic appropriateness condition of the implicative verb. They constitute accommodated material in the wide sense of [12].

Our analysis is couched in Logical Description Grammar for discourse [11, 10], an incremental model of language interpretation which combines underspecification with discourse theory, and employs compositional DRT as semantic representation language. Crucially, it supports a liberal notion of accommodation, where what is accommodated can be more than what is minimally required in terms of logical strength to satisfy presuppositional constraints and other conditions on contexts. What comes out as a most preferred context specification follows from interaction with the interpreting agent's common knowledge and pragmatic reasoning. Thus, on the basis of a sufficiently general requirement on context, a variety of more specific suppositions can be abduced.

We start off with a sketch of the LDG formalism, highlighting just what is relevant for the topic at hand. Our analysis of implicative verbs is laid down in subsequent sections. For reasons of space, we concentrate on the core proposal and economize on discussion of data.

\section{Discourse Meaning and Context Specification in LDG}

The LDG framework of discourse interpretation consists of a description grammar, which embodies a language user's linguistic knowledge, a representation of his world knowledge and beliefs, and a preference system, which models his capacity to assign preferences over different potential interpretations of a discourse and to draw default inferences. In processing a discourse, the language user incrementally constructs a discourse description from input sentence descriptions. It describes the specific syntactic, semantic, and pragmatic properties of the discourse.

There are two levels of analysis: the level of the descriptions and the level of their verifying trees. Descriptions are sets of statements in a logical language (classical type logic, in our case). Verifying trees are fully specified linguistic objects. For sentences and discourses alike, they are tree structures, decorated with syntactic labels, semantic values, and local contexts. Natural language interpretation is a reasoning task, in which the hearer infers what tree structures may verify the discourse description given his grammar and nonlinguistic knowledge. As descriptions can be partial, they may underspecify the syntactic, semantic, or pragmatic properties of the linguistic object they describe, hence fit more than a single verifying structure. Each verifying tree comes with a potential reading of the sentence or discourse. One or more of these may come out as most preferred readings.

See $[11,10]$ for an explication of the incrementality of the formalism and the specifics of the parsing process. A central feature of the semantic dimension 
of the model is that it makes available local contexts as a parameter in the compositional semantics. Each node $k$ in a sentence or discourse tree comes not just with the usual syntactic categories and semantic values $\left(\sigma_{k}\right)$, but also carries a local context $\left(\Gamma_{k}\right)$. Local contexts are constructed from semantic values in the style of Karttunen (1974). The local context of the root of a discourse tree is identified with $B$, the implicit background to the discourse. $B$ stands for whatever is taken for granted or pragmatically presupposed by the discourse participants in the view of the interpreting agent.

The description grammar specifies a few general conditions on local contexts, and elements the lexicon may introduce specific ones. Typically, context-sensitive elements such as anaphors and presupposition triggers contribute the latter. To illustrate, (4) shows a picture of a lexical description of the definite description 'the door' in LDG. In our analysis it contributes a familiarity condition on its referent and a suitability condition formulated in terms of its descriptive content. The first requires that the (underspecified) discourse marker $\sigma_{k}^{\pi}$ is included in the universe of the relevant local context, the second that the local context entails that the property expressed by the nominal part of the definite description applies to the referent.

(4)

$$
\left[\sigma_{k} \mid\right] \sqsubseteq \Gamma_{k}, \Gamma_{k} \curvearrowleft\left[\mid w_{r}: \text { door } \sigma_{k}^{\pi}\right]
$$

Semantic values are in a variant of compositional DRT put forward in [11]. $\sigma_{k}^{\pi}$ is a semantic value of type $\pi$ (type register), i.e. an underspecified discourse marker. [..|...] is a DRS, to the left of the | sign is the universe, to the right the conditions. We use $o_{k}, u_{k}, w_{r}, \ldots$ for different types of discourse markers; ${ }^{3}$ a condition ' $w_{r}$ : door $u_{3}$ ' should be read as 'the occupant of $u_{3}$ is a door in the world occupying $w_{r}$ '. The operator $\oplus$ merges DRSs, $\sqsubseteq$ denotes inclusion and $\leftleftarrows$ denotes entailment.

The motivation of this particular treatment of definites is irrelevant here, what matters is that the semantic and pragmatic contribution of any given clause is defined in terms of the composition of its semantic value plus the collected constraints on local contexts of its components. In particular, discourse meaning is defined as $B \oplus \sigma_{r}$, i.e., what was 'taken for granted' updated with 'what was said' (i.e. bare semantic content), where all the syntactic, semantic and pragmatic conditions collected in the discourse description must be satisfied, including the constraints on local contexts. For example, the meaning of the out-of-the-blue sentence in (5a) is $B \oplus\left[\mid w_{r}: o_{0}\right.$ closed $\left.\sigma_{2}\right]$, where, among others, the conditions in $(5 \mathrm{c})$ and $(5 \mathrm{~d})$ must be satisfied.

\footnotetext{
${ }^{3} u_{k}$ represent new referents (generated in the discourse); $o_{k}$ represent old referents in the sense that they belong to the general background $B$, they are interpreted referentially rather than existentially; $w_{r}$ is a discourse marker that can store worlds.
} 
(5) a. Ed closed the door.

b. $\sigma_{r}=\left[\mid w_{r}: o_{0}\right.$ closed $\left.\sigma_{3}\right]$

c. triggered by the name: $\left[o_{0} \mid w_{r}: E d o_{0}\right] \sqsubseteq B$

d. triggered by the definite description: $\left[\sigma_{3} \mid\right] \sqsubseteq B, B \curvearrowleft\left[\mid w_{r}\right.$ : door $\left.\sigma_{3}\right]$

The hearer may satisfy (5c) and (5d) by assuming or accepting $\left[o_{0} \mid w_{r}: E d o_{0}\right] \sqsubseteq$ $B$ and $\left[u_{7} \mid w_{r}\right.$ : door $\left.u_{7}\right] \sqsubseteq B$ (where $u_{7}$ is an arbitrary fresh discourse marker). By further reasoning the hearer may obtain (6) as final discourse meaning:

(6) $B \oplus\left[w_{r}, o_{0}, u_{7} \mid w_{r}: E d o_{0}, w_{r}: o_{0}\right.$ closed $u_{7}, w_{r}:$ door $\left.u_{7}\right]$

Note that this is obtained on the basis of accommodation: the hearer adjusts his representation of the implicit background in order to satisfy linguistically generated requirements.

Crucially, local contexts in LDG are underspecified objects; accommodation is simply the (partial) specification of local contexts as it results from the satisfaction of constraints stated on them in the grammar or discourse description. The implicit background is a largely underspecified body of information which gets to be described bit by bit in the course of the interpretation process. We call this 'context specification'. ${ }^{4}$

An interpreting agent may freely abduce background information in order to satisfy constraints, selecting a context scenario which is most likely to explain what is presupposed, and consistent with what he considers to be common knowledge and the speaker's beliefs and intentions in making his assertion. What is accommodated in any given case is not just a matter of satisfying presuppositional conditions, it depends on the total of the hearer's beliefs and preferences. Pragmatic reasoning is indispensible for generating preferences over accommodation options. We asume the preference system of the model implements a form of defeasible reasoning about what the speaker is presumably committing to given his beliefs and intentions and what he said so far. The pragmatic rules are not formally specified, but we consider their impact in the interpretation process.

\section{Reasoning towards Culmination}

Right from the birth of 'implicative verbs' as a distinct class in [5], there has been discussion about the strength or status of the derived complements. In recent work [9], Karttunen and co-authors point out that "it can be difficult to distinguish entailments that is, what the author is actually committed to, and conversational implicatures, that is, what a reader/hearer may feel entitled to infer." Indeed, judgements may vary. For instance, 'to remember to' is viewed as a two-way implicative in [9], but we prefer to categorise it as a one-way implicative, in line with Horn [4]. Consider

(7) a. Martha remembered to turn out the lights.

\footnotetext{
${ }^{4}[10]$ section 6.4 provides a treatment which handles local and intermediate accommodation as well.
} 
b. Martha turned out the lights.

c. Martha didn't remember to turn out the lights.

d. Martha didn't turn out the lights.

e. (i) ... so I had to remind her.

(ii) ... but luckily she brushed against the switch.

While (7a) implies (7b) in a strong sense, (7c) only 'invites the inference' in (7d). As can be seen from the continuations in (7e), the inference is defeasible. Horn categorises it as a subspecies of implicature.

The strong inferences can be recognised by their resistance to cancellation:

(8) a. \# Though Jim managed to button his coat, he did not button it.

b. ?? Martha remembered to call the dean, but her collegues prevented it.

For various reasons, however, it can be hard to determine whether the inference arises and is, either or not, cancelled. One is that some verbal constructions, e.g. 'to be able to', are ambiguous between implicative and nonimplicative senses. In other cases, strong contrastive marking may override the implicative inference, or select a nonimplicative sense of the construction. Finally, the inferences are temporally specific [3]; they inherit the temporal, spatial and modal setting of the implicative predicate. To illustrate, (9a) constitutes no evidence of cancellation: the continuation in (9a) is simply consistent with the inference $(9 \mathrm{~b}) .{ }^{5}$

(9) a. Martha didn't remember to turn out the lights $\left(t_{1}\right)$, but she turned them out later on $\left(t_{2}, t_{1}<t_{2}\right)$.

b. Martha didn't turn out the lights (at $t_{1}$ )

Moreover, the inferences vary with the tense and aspect of the implicative predicate. The clearcut 'actuality entailments' evoked with simple past predicates do not surface with a generic or progressive use of the same verb. Arguably, these evoke generic and progressive implicative inferences.

Analysis As [8] observes, implicatives always occur as part of a sequence of verbs expressing a single process. We may add that in general, that process is resultative. The implicative verb refers to an attitude or state of the subject or an activity he is involved in, which in the view of the speaker instigates, causes, or culminates in the state or event referred to by the complement sentence. In line with Karttunen's intuitive analysis in [5], we suggest that in the context of interpretation, the implicative state or activity figures as a sufficient and/or necessary condition for the achievement or culmination (or lack thereof) of the complement state or event. This explains the implicative inferences.

We'll come to a formal implementation of this shortly, but like to emphasize that, ultimately, pragmatics is what drives these inferences and what explains why implicative verb senses come into existence in a language. There is a general pragmatic drift to expect that a process culminates or a purpose is achieved or an intention made true, when a speaker asserts that a precondition for the result

\footnotetext{
${ }^{5}$ Perhaps a two-way analysis of 'to remember to' can be defended along these lines.
} 
or goal is satisfied or that an intentional act took place. And when the speaker conveys that that precondition is not fulfilled, we expect that the result or the goal is not achieved or the intention not obtained. In the course of time, for some verbs the implicativeness can become conventionalised and part of their lexical description. Specialisation may take place, resulting in groups of semantically closely related verbs which differ only in that the implication they evoke is optional or obligatory. From a typological perspective it is to be expected that verbs which express the same core meaning across languages may differ in the strength of the implicative inference.

\section{The Implicative Condition}

We propose that implicative verbs in LDG lexically introduce an implicative condition, a requirement on their local context which defines the resultative, causal or conditional relation between the state or action the implicative verb refers to, and the state or event described by the complement sentence. In combination with the semantic content of the sentence containing the verb, it accounts for the implicative inferences in context. The one-way implicative 'to remember to' carries, we propose, a sufficient condition: remembering to close the door instigates a door-closing. Suppose a hearer computes the discourse meaning of (10a). At some point he may have inferred (10b), while the implicative condition (10c) must still be satisfied.

(10) a. Ed remembered to close the door.

b. $B \oplus\left[w_{r} o_{0} t_{1} \mid w_{r}: E d o_{0}\right.$, $w_{r}$ : remember.at $\left(o_{0}, \lambda w, t\left[\mid w: o_{0}\right.\right.$ close.door.at $\left.\left.\left.t\right], t_{1}\right), t_{1}<n\right]$

c. Implicative condition, contributed by the tensed verb:

$$
\begin{aligned}
& B \models \\
& {\left[\mid\left[t_{1} \mid w_{r}: \text { remember.at }\left(o_{0}, \lambda w, t\left[\mid w: o_{0} \text { close.door.at } t\right], t_{1}\right), t_{1}<n\right] \Rightarrow\right.} \\
& \left.\left[t_{2} \mid w_{r}: o_{0} \text { close.door.at } t_{2}, t_{1} \bigcirc t_{2}, t_{2}<n\right]\right]
\end{aligned}
$$

Various accommodation options arise. The one shown in (11a) can be excluded because it sinns against a grammatical constraint: it results in an improper implicit background DRS. The other two are viable options.

(11) a. $\left[t_{2} \mid w_{r}: o_{0}\right.$ close.door.at $\left.t_{2}, t_{1} \bigcirc t_{2}, t_{2}<n\right] \sqsubseteq B$

b. $\left[\mid\left[t_{1} \mid w_{r}\right.\right.$ :remember.at $\left(o_{0}, \lambda w, t\left[\mid w: o_{0}\right.\right.$ close.door.at $\left.\left.\left.t\right], t_{1}\right), t_{1}<n\right] \Rightarrow$ $\left[t_{2} \mid w_{r}: o_{0}\right.$ close.door.at $\left.\left.t_{2}, t_{1} \bigcirc t_{2}, t_{2}<n\right]\right] \sqsubseteq B$

c. $\left[\mid\left[t_{1} \mid w_{r}\right.\right.$ :remember.at $\left(o_{0}, \lambda w, t\left[\mid w: o_{0}\right.\right.$ close.door.at $\left.\left.\left.t\right], t_{1}\right), t_{1}<n\right] \Leftrightarrow$ $\left[t_{2} \mid w_{r}: o_{0}\right.$ close.door.at $\left.\left.t_{2}, t_{1} \bigcirc t_{2}, t_{2}<n\right]\right] \sqsubseteq B$

Considering what would explain the speaker's use of an implicative in the given context, the hearer might assume that he simply intends to describe a state of affairs and takes for granted that Ed remembering to close the door in the situation at hand is sufficient to make him close it. Thus the hearer would accept option (11b). However, he may go on to ask what makes the speaker mention Ed's 
remembering at all, if he could have said right away that Ed opened the door. Quite possibly because in the situation under discussion, the speaker considers it a necessary requirement as well: without remembering to close the door Ed would not have closed it. If, in addition, Ed's remembering is relevant in the sense that it is an open issue in the discourse whether he did or not, the speaker has good reasons to mention it. Now the hearer accepts (11c).

Whatever the hearer's choice is, the indefeasibility of the implicative inference with the bare positive use of the verb is accounted for because there is no grammatical interpretation in which the implicative condition is not satisfied. With the negated form, however, only the interpretation corresponding to option (11c) predicts the implicative inference. We suggest that option (11c) is the preferred one in principle. ${ }^{6}$ The implicative inference is then predicted to arise with the negated form in out-of-the-blue use and in linguistic contexts such as (7e.i) which reinforce the inference. But when it is disconfirmed, as in (7e.ii), the hearer selects accommodation option (11b). Typically, the continuation in (7e.ii) conveys that there is another action beside remembering that would result in Martha turning out the lights on the relevant occasion. So the speaker does not consider Martha's remembering a necessary requirement. The choice of accommodation options accounts for the defeasibility of the implicative inference.

Because the implicative condition is 'presuppositional', i.e. a requirement on local context, the resultative or conditional link constitutes non-at issue information. It will commonly project to global context, unless forced to accommodate locally, e.g. through metalinguistic denial. As such it is employed by the hearer in the computation of discourse meaning. More complex examples in which one implicative construction embeds another will be interesting test-cases. If the various preconditions project to global context, the implicative inferences evoked by the sentence should follow from their interaction in the spirit of [6].

Furthermore, the implicative condition is lexically introduced. The constraint fires only when there is an occurrence of the implicative verb in the discourse, and it is context-dependent. In no sense does an assertion of ' $S$ ' mean the same thing as an assertion of e.g. 'Ed remembered to S'. The fact that the condition is stated in the domain of locality of the implicative verb has some important consequences, which space restrictions allow us only to mention here. One is that entailments which hold locally do not necessarily surface globally. Thus, it can be explained that when triggered in the scope of e.g. modal and generic operators the implicative inference is not evoked. Another is that it solves the notorious 'binding problem', detected in [7].

Every implicative verb comes with its own semantic content and characteristic implicative condition. In the case of bench-mark 'to manage to', we follow the general trend and assume that its semantic content says that the complement state or event succeeds. Given a proper meaning definition of the predicate succeed.in.at the entailments with both the positive and the negated use of the verb follow from this straight away, and are predicted to be indefeasible. Instead

\footnotetext{
${ }^{6}$ In terms of pragmatic rules, it provides a 'better explanation' of the speaker's use of an implicative.
} 
of the usual attempt/difficulty-presupposition, however, we'll have an implicative condition which says that the complement state or event succeeds only if the subject individual makes a certain effort to obtain that result:

(12) a. Someone managed to close the door.

b. $B \oplus\left[w_{r} t_{1} u_{1} \mid w_{r}\right.$ : human $u_{1}$,

c. $B \oplus\left[u_{1} \mid w_{r}:\right.$ human $\left.u_{1}\right] \curvearrowleft$

$$
\begin{aligned}
& {\left[\mid\left[t_{1} \mid w_{r}: \text { succeed.in.at }\left(u_{1}, \lambda w, t\left[\mid w: u_{1} \text { close.door.at } t\right], t_{1}\right), t_{1}<n\right] \Rightarrow\right.} \\
& {\left[t_{2} \mid w_{r}: \text { put.effort.d.in.at }\left(u_{1}, \lambda w, t\left[\mid w: u_{1} \text { close.door.at } t\right], t_{2}\right),\right.} \\
& \left.\left.\quad t_{2} \bigcirc t_{1}, t_{2}<n\right]\right]
\end{aligned}
$$

This differs minimally from standard treatments in that the requirement that an effort be made is a postcondition on the succes of the complement state or event, and is formulated in somewhat less specific terms than attempt or difficulty.

\section{Context Specification. What is presupposed?}

While any specific implicative verb can carry additional presuppositions, we are interested to see how far the implicative condition may take us in accounting the presuppositions claimed to be evoked by implicatives, given interaction with pragmatic reasoning. Remember the presuppositions are a diverse lot; focusing on 'to manage to' we will go through them one by one.

The implicative condition accounts directly for the characteristic causal or explanatory presuppositions mentioned in the introduction. Satisfying the implicative condition in (12c), the hearer may accommodate that for anybody to succeed in closing the door on the particular occasion under discussion, he must make a certain effort to do so, and making the effort will cause the closing of the door. If we are told that somebody did not succeed in closing the door, then not having made that effort is a likely explanation of why he didn't. Thus, the satisfaction of the implicative condition induces the accommodation of explanatory suppositions.

Now for the attempt suppositions, cf. (2). The hearer may very well satisfy the implicative condition by accommodating that opening the door on this occasion requires an intentional, active attempt to do so on the agent's part. This is a plausible instance of putting in a certain effort. Arguably, the inference is defeasible with a positive use of the verb in examples like the following. What is preserved is a weaker inference which signals that the implicative condition is still minimally satisfied.

(13) Without intending it, Bill managed to insult the dean. t $\rightarrow$ Bill attempted to insult the dean.

$\leadsto$ Bill was involved in some effort which resulted in insulting the dean.

As suggested above, with the negated use of the implicative (and neutral stress pattern), as in 
(14) Jack didn't manage to convince the dean.

the hearer is likely to accommodate that subject did not make the effort necessary to cause convincing the dean. The observation is, however, that something more specific is accommodated, namely that Jack did make an effort, though an insufficient one. He tried to convince the dean but failed. We propose that this strengthened interpretation results from (Gricean) scalar reasoning, based on either a Horn scale 〈try to, manage to〉 where 'to manage to' is the informationally stronger alternative, or on the relation of asymmetric entailment between DRSs established in the relevant local context due to the implicative condition. In context, 'Jack did not put sufficient effort into convincing the dean' is a stronger alternative than 'Jack did not succeed in convincing the dean.' Why didn't the speaker assert the stronger alternative? Presumably because he does not believe that Jack did not put sufficient effort into convincing the dean. If the speaker is informed about the matter, it follows that he believes that Jack tried to convince the dean (the strong scalar implicature). Defeasibility of the inference in constructions such as (15) is thus predicted.

(15) Jack didn't manage to convince the dean, in fact, he didn't even try.

The difficulty suppositions are naturally accounted for as an accommodation effect of satisfying the implicative condition. If it requires an effort on Jack's part to convince the dean, then probably there is some difficulty or challenge involved in this, which explains why the effort is needed. A most preferred context does not just make the implicative condition true but also likely or plausible in the situation under discussion. Testing in projection and cancellation contexts reveals that the difficulty suppositions are generally persistent, often generic, but always adapted to the situation under discussion given common knowledge. The difficulty may be specific to the subject given his opportunities and skills, as in (2), there can be a difficulty in the type of action or event to be achieved for anybody in general, or it can be completely contingent on the situation at hand. All of this is to be expected when the inference is not hard-wired as a linguistic presupposition, but rather the product of context specification. Finally, the implicative condition is sufficiently unspecific to account for cases like the following in which the effort made by the subject on the event referred to does not involve any concrete difficulty.

(16) John generally runs 10 miles per hour. Small wonder he managed to run 10 kilometers in 45 minutes yesterday.

$\chi_{\rightarrow}$ It was difficult for John to run 10 kilometers in 45 minutes yesterday.

$\sim$ It is difficult for most people to run 10 kilometers in 45 minutes.

$\leadsto$ For John to run 10 kilometers in 45 minutes requires a certain effort.

A discussion of suppositions of expectation or norm and those of obligation attached to 'to remember to' shall have to wait for another occasion. We hope to have shown that an analysis in terms of implicative condition, pragmatic reasoning and accommodation effect is well-suited to account for the variability and defeasibility of the inferences under discussion. 


\section{Conclusion}

We looked into the inferential behavour of implicative verbs in context, and argued that they contribute a presuppositional constraint that characterises the process they describe as resultative, culminating in the state or event described by the complement sentence. It was proposed that what are often claimed to be presuppositions may well be viewed as implicatures, or secondary inferences resulting from the need to satisfy and explain the implicative condition of the verb. The analysis presupposes a liberal notion of accommodation, where pragmatic reasoning rules serve to provide best explanations of the speaker's assertions.

Further research is needed, especially the interactions with tense, aspect and modality must be spelled out. More extensive testing of the projection behaviour of inferences and cancellation contexts is needed. Cross-linguistic research will be helpful to get a grasp on the diversity of implicitive word senses within the language system. Moreover, the explicit specification of pragmatic reasoning rules that justify the selection of accommodation options constitutes a theoretical challenge. The investigation of implicative verbs will be of interest for the theory of presupposition as well as the theory of pragmatics/accommodation. If implicative verbs are to be members of the family of presupposition triggers, or perhaps of the broader family of projective elements, a rich interpretational system is called for. This might be LDG for discourse, or a model of comparable strength such as [1].

\section{References}

1. Asher, N., Lascarides, A.: Logics of Conversation. Cambridge University Press, Cambridge (2003)

2. Beaver, D.I.: Presupposition and Assertion in Dynamic Semantics. CSLI Publications, Stanford, California (2001)

3. Givón, T.: The time-axis phenomenon. Language 49(4), 890-925 (1973)

4. Horn, L.R.: On the Semantic Properties of the Logical Operators in English. Ph.D. thesis, University of California at Los Angeles (1972)

5. Karttunen, L.: Implicative verbs. Language 47(2), 340-358 (June 1971)

6. Karttunen, L.: The logic of English predicate complement constructions. distributed by the Indiana University Linguistics Club (1971)

7. Karttunen, L., Peters, S.: Conventional implicature. Syntax and Semantics 11 (1979)

8. Luzón Marco, M.J.: A semantic-syntactic study of implicative verbs based on corpus analysis. Estudios Inglese de la Universidad Complutense 7, 69-87 (1999)

9. Nairn, R., Condoravdi, C., Karttunen, L.: Computing relative polarity for textual inference. In: Proceedings of ICoS-5 (2006)

10. van Leusen, N.: Description Grammar for Discourse. Ph.D. thesis, Radboud University Nijmegen (2007)

11. van Leusen, N., Muskens, R.: Construction by Description in Discourse Representation. In: Peregrin, J. (ed.) Meaning, the Dynamic Turn. Elsevier (2003)

12. Thomason, R.: Accommodation, meaning, and implicature: Interdisciplinary foundations for pragmatics. In: Cohen, P.R., Morgan, J., Pollock, M.E. (eds.) Intentions in Communication, chap. 16. MIT Press (1990) 


\title{
Inflection and Derivation: How Adjectives and Nouns Refer to Abstract Objects
}

\author{
Louise McNally $^{1} \&$ Henriëtte de Swart ${ }^{2}$ \\ ${ }^{1}$ Universitat Pompeu Fabra, Barcelona, Spain \\ ${ }^{2}$ Utrecht University, Utrecht, The Netherlands
}

\begin{abstract}
The study of nominalizations raises foundational questions about the relation between adjectives and nouns in natural language and the differences in their semantics. In this talk, we present a syntactic and compositional semantic analysis of a previously unanalyzed type of inflected adjective in Dutch and contrast it with the semantics of the corresponding uninflected forms and deadjectival nominalizations. We use a rather sparse ontology, but assign a special relational semantics to nominalized inflected adjectives. We then briefly compare our analysis of the inflected forms to that of $l o$-marked adjectives in Spanish (Villalba 2009) to shed light on reference to abstract objects in natural language more generally.
\end{abstract}

Keywords. adjective semantics, noun semantics, abstract objects, nominalization, inflected adjectives, kinds, properties

\section{Inflection, Derivation and Reference to Abstract Objects}

Adjective inflection in Dutch depends in part on the gender of the noun that the adjective co-occurs with. Dutch has two definite articles: het (glossed here as 'the sg $^{\prime}$ '), which is singular and neuter; and de (glossed here as 'the'), which combines with all non-neuter (i.e. masculine and feminine) singular nouns, as well as all plural nouns. It has one (singular) indefinite article een 'a', which is used for all nouns. When a neuter noun appears with the indefinite article, any accompanying adjective appears in the basic uninflected form:

(1) Ik kocht een groen boek I bought a green $\varnothing$ book

[neuter noun, indefinite]

'I bought a green book.'

In contrast, the adjective appears in its inflected form in definite DPs containing neuter nouns (2a), as well as in both definite and indefinite DPs containing non-neuter nouns $(2 b)$, in plurals, etc.:

(2) a. Het groene boek was mooi. the sg.n $_{\text {green }}$ [+-e] $_{\text {book was nice }}$

'The green book was nice.'

b. Ik heb een/de groene tafel gekocht

I have a/ the green $\left[\begin{array}{l}{[+-\mathrm{e}]} \\ \text { table bought }\end{array}\right.$

'I bought a/the green table.' [neuter noun, definite]

[non-neuter noun, indef/def]

All adjectives show this alternation, except when they end in //ə// (roze 'pink', mauve 'mauve'), or - $a$ (lila, 'lilac'), or -en (gouden 'golden') which we therefore leave out of this discussion.

The focus of this paper is on the nominalized use of inflected adjectives $(3 b, 4 b)$, in contrast to deadjectival nominalizations $(3 \mathrm{c}, 4 \mathrm{c})$, and uninflected forms $(3 \mathrm{a}, 4 \mathrm{a})$ to refer to abstract objects like colors and tastes. Note the subtle meaning differences between these examples, manifest in the corresponding translations:

(3) a. Misschien kun je het rood van de aardbeien nog een beetje roder maken? maybe can you the red of the strawberries yet a bit redder make 'Maybe you can change the red (shade) of the strawberries so that it is a bit redder?' 
b. Het rode van de aardbeien, het witte van de mascarpone en de slagroom en het groene the red ${ }_{[+-e]}$ of the strawberries, the white ${ }_{[+-e]}$ of the mascarpone and the cream and the green mintblaadje kleurden prachtig bij elkaar.

mint-leaf.dim colored beautifully with each-other

'The red (aspect) of the strawberries, the white (aspect) of the mascarpone and the cream, and the green mint leaf are a wonderful color combination.'

c. De roodheid van de huid kan achterliggende oorzaken hebben

the redness of the skin can deeper causes have

'The redness of the skin can have deeper causes.'

(4) Het bitter van olijven, het zout van ansjovis en het milde zuur van komkommers the bitter of olives, the salt of anchovies and the mild sour of cucumbers

'the bitter (taste) of olives, the salt (taste) of anchovies and the mild sour (taste) of cucumbers'

Het bittere in het bier is een mooi contrast met het zoete van de mout.

the bitter $_{[+-e]}$ in the beer is a nice contrast with the sweet ${ }_{[+-e]}$ of the malt

'The bitter (aspect) of the beer is a nice contrast with the sweet (aspect) of the malt.'

d. Ik moest, net als Hollandse kindertjes, wennen aan de bitterheid van witlof

I must.pst, just like Dutch children.dim, get-used to the bitterness of Belgian-endive

'Like all Dutch children, I had to get used to the bitterness of Belgian endives.'

The Dutch inflected form in (3b) and (4b) is strikingly similar to lo-marked adjective (5b) in Spanish (cf. [12]), which establishes a contrast with nominalized adjectives taking the article el (5a), and derived nominals (5c):

(5) a. ¿Conoce usted más blancos que los esquimales?...El blanco de los esquimales...

know you more whites than the Eskimos? ... the white of the Eskimos

'Do you know more (shades of) white than the Eskimos?...The white of the Eskimos...

b. ...los acantilados negros constrastan bellamente con lo blanco de las dunas de arena

the cliffs black contrast beautifully with the white of the dunes of sand

'...the black cliffs contrast beautifully with the white (aspect) of the sand dunes.'

c. La blancura del papel es también una cuestión de gusto.

the whiteness of-the paper is also a matter of taste

'The whiteness of paper is also a matter of taste.'

In contrast, English lacks the inflected form, and only shows the contrast between the red and the redness. For (b) either a paraphrase (see the above translations) or in some cases the derived nominalized form is used.

The nominalized use of inflected adjectives in Dutch is rather frequent with abstract adjectives (het vreemde van dit boek 'the strange thing about this book', het gezonde van biologisch voedsel 'the healthy thing about biological food'), but rare with concrete adjectives such as dicht 'closed': ?*het dichte van deze doos 'the closed $_{[+-e]}$ of this box'. Not all adjectives allow modification by -heid to form a nominalization, or have uninflected nominal counterparts. We will focus on triplets like those in (3)-(4), which allow us to investigate the full range of forms and meanings. We use examples in the domain of color and taste terms for illustration, with the goal of addressing two questions:

Q1: What is the difference between the semantics of the three forms in (3a-c) and (4a-c)?

Q2: How is this semantics related to the morphosyntax of the three forms?

We use an exploration of $\mathbf{Q 1}$ and $\mathbf{Q 2}$ in the case of Dutch to gain insight into the relation between adjectives and nouns in natural language and the differences in their semantics, and to shed light on how reference to abstract objects in natural language works more generally. The analysis we develop is based on the following claims:

- The uninflected form (a) is nominal and denotes a description of the color/taste itself. The (neuter definite) article het has a standard interpretation (we assume the iota operator).

- The inflectional suffix $-e$ in (b) is a valence-changing operator that turns the adjective denotation into a relation between the object identified by the PP and its color/taste aspect. Het denotes Chierchia's [3] nominalizer $\cap$, creating the entity correlate of the color/taste aspect and embedding the AP directly under DP. 
- The derived noun in (c) describes realizations of the color/taste property associated with its adjective base. The (non-neuter definite) article $d e$ is interpreted as in (a).

In order to ground these semantic claims in the forms, we start with the morpho-syntactic analysis of uninflected, inflected and derived color and taste terms.

\section{Morpho-syntax}

\subsection{The Uninflected Form}

Uninflected color and taste forms are (neuter, mass) nouns. Three observations support this claim. (i) As nouns, they take adjectival (6a) but not adverbial modification (6a,b); note that there is no morphological distinction between uninflected adjectives and their adverbial counterparts:

(6) a Ook het mooie mA] $/ *$ mooi $_{[A d v]}$ geel van de akoniet wordt weer zichtbaar en
also the nice/ nice yellow of the aconite becomes again visible and also the nice/ nice yellow of the aconite becomes agair
daarna de crocussen, anemoontjes, narcissen en hyacinten. afterwards the crocuses, anemones, daffodils and hyacinths

'The nice yellow of the aconite is also visible again, and after that the crocuses, anemones, daffodils and hyacinths.'

b. *Het al te zuur van citrusvruchten is in deze confiture verdwenen.

the all too sour of citrus-fruits is in this jam disappeared 'The all too sour of citrus fruits is lacking in this jam.'

(ii) As regular nouns, they combine with a variety of determiners (7a-c):

(7) a. Meestal vertoonden deze noviteiten slechts bloemen in een bleek gele kleur, usually displayed these novelties only flowers in a pale yellow color terwijl dit geel ook nog spoedig naar rose verkleurde. while this yellow also again quickly to pink discolored

'Usually, these new varieties only show flowers in a pale yellow color, and moreover, this yellow soon changes into pink.'

b. Luister goed, wij willen ècht geen geel in onze tuin.

listen well we want really no yellow in our garden

'Listen to me, we really want no yellow in our garden.'

c. Het is een druif die weinig zuur, weinig zoet aan een wijn meegeeft.

it is a grape that little sour, little sweet to a wine adds

'It is a grape that adds little acid, little sweet to a wine.'

(iii) In their bare form, they have a generic use (8):

(8) a. Rood is een krachtige kleur. 'Red is a powerful color.' b. Zoet is één van de vier basissmaken. 'Sweet is one of the four basic tastes.'

\subsection{The Derived Form}

The derived color and taste terms built on -heid are clearly (non-neuter count or mass) nouns. Four observations support this claim. (i) In their count use, derived terms can be pluralized:

(9) De extra bleking van de houtpulp voor het bereiken van hogere witheden in het eindproduct is the extra bleaching of the wood-pulp for the reaching of higher whitenesses in the final-product is hier debet aan.

here indebted to

'The additional bleaching of the wood pulp to reach higher degrees of whiteness in the final product is responsible for this.' 


\section{General Program}

(ii) They take adjectival, not adverbial modification (10):

(10) Ik heb ook rosacea en gebruik altijd een licht dekkende foundation om de ergste/ *ergst I have also rosacea and use always a light covering foundation to the worst $_{[\mathrm{A}]} / \mathrm{worst}_{[\mathrm{Adv}]}$ roodheid te camoufleren.

redness to camouflage

'I have rosacea too, and I always use a light coverage foundation to camouflage the worst redness.'

(iii) As regular nouns, they combine with a variety of determiners (11a-b):

(11) a. Deze witheid kan verschillen per papiersoort. Bij kopieer/printpapier, enveloppen en this whiteness can vary per paper-type. With copy/ print-paper envelopes and offset wordt een witheid tussen de 160 en 170 cie verlangd.

offset is a whiteness between the 160 and 170 cie required.

'This whiteness varies according to paper type. Carbon and printing paper, envelopes and offset paper require a whiteness between 160 and 170 cie.'

b. Verwacht geen zoetheid, als je bitterheid schenkt.

expect no sweetness if you bitterness pour

'Expect no sweetness if you pour bitterness.'

(iv) In their bare form, derived terms have a generic use (12):

(12) Blijvende roodheid is het meest voorkomende kenmerk van rosacea. permanent redness is the most occurring characteristic of rosacea 'Permanent redness is the most common characteristic of rosacea.'

The syntactic features of derived color and taste terms are shared with other deadjectival nominalizations in -heid such as goedheid 'goodness', schoonheid 'beauty', dichtheid 'density', etc.

\subsection{The Inflected Form}

Somewhat surprisingly, in Dutch grammars there is no consensus as to whether the inflected forms in the (b) examples consist of an adjective with an elided nominal or a nominalization of the adjective (cf. [1], [6] for discussion). ${ }^{1}$ A closer investigation reveals that both analyses run into problems. Three facts argue against a nominalization analysis in which the inflected adjective changes category and becomes a noun. (i) Unlike regular nouns, the inflected color and taste term allows adverbial, but not adjectival modification (13):

(13) a.

Zonder licht, om het intens/ *intense rode van de ondergaande zon without light in-order the intense $e_{[\mathrm{Adv}]} /$ intense $_{[\mathrm{A}]} \operatorname{red}_{[+-\mathrm{e}]}$ of the setting niet te verstoren.

not to disturb

'Without light, so as not to disturb the intense red (aspect) of the sunset.'

b. Het wintert nu weldra en dan komt ook misschien het o zo witte van de sneeuw.

it winters now soon and then comes also maybe the oh so white $e_{[+\mathrm{e}]}$ of the snow

'It will soon be winter, and then perhaps will come the oh so white (aspect) of the snow.'

c. [het] al te zure van citrusvruchten is in deze confiture verdwenen.

the all too sour $_{[+-e]}$ of citrus-fruits is in this jam disappeared

'the all too sour (aspect) of citrus fruits is lacking from this jam.'

(ii) Unlike regular nouns, the inflected forms do not tolerate any other determiner besides het:

\footnotetext{
${ }^{1}$ Of course, it is possible to elide a nominal and leave behind an inflected adjective. Our point here is that this is not the analysis of examples like (3b, $4 \mathrm{~b}$ ), and our claims below do not apply to real elliptical uses, which permit the unambiguous identification of the missing nominal.
} 
(14) a. $\quad *$ een/dit/zijn/geen/veel rode $\quad$ b. $\quad *$ een/dit/zijn/geen/veel zoete

(iii) Inflected adjectives do not occur without het. They are not used generically, either with or without het.
(15) a
* Rode is...
b. *Zoete is...
${ }^{*} \operatorname{red}_{[+-e]}$ is...
$*_{\text {sweet }_{[+-\mathrm{e}]}}$ is...

The alternative maintains the adjectival status of the adjective, but posits an empty or elided noun (cf. [6]). Two arguments that argue against the ellipsis analysis. (i) It would be impossible to explain the determiner restrictions (14), and the lack of a generic reading (15). (ii) If ellipsis were involved, it is practically impossible to determine which noun is to be inserted in the structure. The general nouns kleur 'color' and smaak 'taste' do not fit, because they are non-neuter nouns and require the non-neuter $d e$ :

the.-neut $/ *$ the + neut red color of the strawberries

'the red color of the strawberries'

b. $\mathrm{de} / \quad$ het bittere smaak van witlof

the.-neut/*the.+neut bitter taste of Belgian-endive

'the bitter taste of Belgian endive'

In order to circumvent the problems raised by the nominalization analysis on the one hand and the empty nouon/ellipsis analysis on the other hand, we propose that the inflected word remains an adjective after affixation, but that this inflected adjective directly complements the head of a DP, taking the PP (if one is present) as its own complement:

(17) [DP het [AP rode [PP van de aardbeien]]]

The structure in (17) is the simplest surface analysis and immediately explains the use of an adverbial modifier with the inflected form. The neuter article het is expected, since het is the default article in nominalized constructions built from non-nominal categories such as infinitives (cf. het $/ *$ de zingen van Jan 'John's singing'). In the absence of an elided noun, other determiners besides het (including de) are not expected and the fact that a bare generic use is unavailable might very well be due to the special construction involved. Therefore, we take the structure in (17) as the starting point for the semantics of inflected color and taste terms.

\section{The Semantics of Uninflected, Derived, and Inflected Color and Taste Terms}

\subsection{Background Assumptions: Uncategorized Roots and Ontological Assumptions}

In the semantics we are setting up for reference to abstract objects, we assume a basic ontology that includes kinds and token individuals (cf. [2] a.o.). Token individuals can be either abstract or concrete (i.e. we can talk about a token of a color or taste, just as we can talk about a token person or bicycle). In order to capture what is common to corresponding nouns and adjectives (e.g. rood/rode), we posit that both are constructed from entitydenoting, uncategorized roots that refer to the kind of color or taste, as in (18).
(18) a.
$[[$ rood $]]=$ red
b. $\quad[[$ zuur $]]=$ acid

The uncategorized roots can lead to either nominal or adjectival denotations. Crucially, we take het to have a double denotation. In its regular use as a definite article with neuter nouns, het denotes the iota operator. In its use with inflected adjectives, het denotes Chierchia's [3] nominalizing (cap) operator $\cap$, directly embedding the adjective in a DP. These assumptions allow us to spell out the different semantic representations of uninflected, derived and inflected color and taste terms. 


\section{General Program}

\subsection{Uninflected Terms}

In section 2.1 we classified uninflected color and taste terms as regular (neuter) nouns. Direct kind reference based on the nominal version of (18) is found in the bare generic use in (8) for instance. Following [13] and others, we take a layered approach to the DP such that (e.g.) the noun $\operatorname{rood}_{\mathrm{N}}$ can denote the set of subkinds (shades) of the color via a relation of subkind instantiation, as in (19).

(19) $\left[\left[\operatorname{rood}_{N}\right]\right]=\lambda x_{k} \cdot \operatorname{subkind}\left(x_{k}\right.$, red $)$

The PP, if there is one (e.g. van de aardbeien 'of the strawberries'), functions as a modifier, introducing a contextually appropriate relation $R_{i}$ with the denotation of the DP complement to P (e.g. the strawberries, represented as s), as in (20).

(20) $[$ [van de aardbeien $]]=\lambda P \lambda x\left[P(x) \wedge R_{i}(x, \mathbf{s})\right]$

After the modifier is applied to the noun (see (21a)), the definite article het, interpreted as the iota operator, selects the unique subkind denoted by the modified nominal (see (21b)).

(21) a. $\quad[$ rood van de aardbeien $]]=\lambda x_{k}\left[\operatorname{subkind}\left(x_{k}\right.\right.$, red $\left.) \wedge R_{i}\left(x_{k}, \mathbf{s}\right)\right]$

b. $\quad[[$ het rood van de aardbeien $]]=1 x_{k}\left[\operatorname{subkind}\left(x_{k}\right.\right.$, red $\left.) \wedge R_{i}\left(x_{k}, \mathbf{s}\right)\right]$

This semantics captures the intuitive meaning of examples like (3a), in which the speaker refers to the particular shade of red found in the strawberries. Similarly, (4a) refers to the taste of olives, anchovies and cucumber as an abstract object (a kind), and qualifies it as sweet, salty or acidic respectively.

Uninflected color/taste terms can also refer to realizations of the color/taste, as in $(7 \mathrm{~b}, \mathrm{c})$. According to Zamparelli ([13]: 207ff.), quantifiers can range over subkinds or realizations of the kind. In line with this view, we posit, by way of example, the semantics of veel 'a lot of' in (22a), which leads to quantification over concrete instances of the color red in $(22 b)$ :

(22) a. $\quad[[$ veel $]]=\lambda P \lambda Q\left[\left(\operatorname{veel} y: \operatorname{Real}\left(y, x_{k}\left[P\left(x_{k}\right)\right]\right)[Q(y)]\right]\right.$

b. $\quad[[$ veel $\operatorname{rood}]]=\lambda Q\left[\left(\operatorname{veel} y: \operatorname{Real}\left(y, x_{k}\left[\operatorname{subkind}\left(x_{k}, \operatorname{red}\right)\right]\right)[Q(y)]\right]\right.$

Though color and taste terms denote abstract objects, the semantic representations in (19), (21), and (22) are not qualitatively different from what we would posit for examples involving concrete nominals.

\subsection{Derived Terms}

The suffix - heid creates nominalizations for a wide range of adjectives; it does not attach to nouns. Therefore, the morphology supports derivation of the nominal roodheid 'redness', bitterheid 'bitterness' from the adjective $\left(\operatorname{rood}_{\mathrm{A}}\right.$, bitter $\left._{\mathrm{A}}\right)$

For the semantics of these adjectives, follow [5] who argue that color adjectives (at least) are ambiguous. ${ }^{2}$ When referring to color (e.g. very red hair) the adjective embeds a measure function (mf) in its semantics and eventually denotes the set of entities whose value on that measure function exceeds the relevant standard degree for truthful application of the adjective; the uncategorized root supplies the identity of the measure function (23a). ${ }^{3}$ On the (frequently non-gradable) 'proxy' use (e.g. a (\#very) red traffic light), the adjective contributes an indexically identified property $P_{i}$ that is correlated (cor) with the color (23b) (e.g., the property of indicating that one must stop). For ease of readability, we abbreviate both of these denotations as in (23c).

(23) a. $\quad\left[\left[\operatorname{rood}_{\mathrm{A}[\mathrm{gr}]}\right]\right]=\lambda y\left[\mathbf{m f}_{\mathrm{red}}(y) \geq \operatorname{standard}\left(\mathbf{m f}_{\mathrm{red}}\right)\right]$ b. $\quad\left[\left[\operatorname{rood}_{\mathrm{A}[\text { nongr }]}\right]\right]=\lambda y\left[P_{i} \wedge \operatorname{cor}\left(y, P_{i}\right)\right]$

2 Whether taste adjectives are also ambiguous is something we haven't investigated. If they are not, only the sort of denotation in (23a) will be relevant.

3 (23a) simplifies Kennedy and McNally's analysis in leaving out the compositional details involving the introduction of the standard value, as the way in which that value is introduced into the semantics of the adjective is not crucial to our main point. 
c. $\quad\left[\left[\operatorname{rood}_{\mathrm{A}[\mathrm{grr}][\operatorname{nongr}]}\right]\right]=\lambda y[\operatorname{Red}(y)]$

Our semantics for the derivational suffix -heid builds on Chierchia's [3] proposal that properties such as that in (23c) have entity correlates that are formally assimilable to kinds: the suffix operates on such properties and returns the set of subkinds of their entity correlates, as in (24a). Thus, roodheid denotes the set of subkinds of redness (24b).

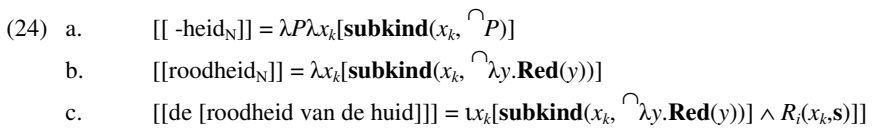

The derived form in (24b) can then undergo modification as in (24c), which specifies the meaning of (3c) as the unique subkind of the entity correlate of the property red that is associated with the skin (represented as $\mathbf{s}$ ). The derived term can combine with determiners in the same way as the uninflected form, leading to quantification over subkinds as well as realizations thereof, as in (11b). When the nominalization is used bare, without any modification whatsoever, as in (12), we assume that by default the most general subkind (i.e. the kind itself) is referred to, as must be the case for concrete bare plurals.

\subsection{Inflected Terms}

The morphosyntax and the semantics of the inflected color and taste terms are crucially different from those of their uninflected and derived counterparts. We take the inflection $-e$ to increase the valence of the adjective by one and to introduce a relation $\left(P_{\text {asp }}\right)$ indicating 'the $P$ aspect' of an argument $(25 \mathrm{a})$. The adjective provides a value for $P$, as in (25b):

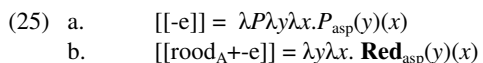

The internal argument is then saturated either by a PP (e.g. the one in (26a)) or, in the absence of the latter, contextually. The result in (26b) is the property of being the Adj aspect of the denotation of the DP complement to the P. We suggest that what allows embedding of this AP in a DP is crucially the article het, which contributes Chierchia's cap operator, reifying the property denoted by the inflected adjective in (26c).

(26) a. $\quad[[$ van de aardbeien $]]=\lambda R \lambda x \cdot R(\mathbf{s})(x)$

b. $\quad\left[\left[{ }_{\mathrm{AP}} \operatorname{rode}_{\mathrm{A}}\right.\right.$ van de aardbeien] ] ] $=\lambda x \cdot \operatorname{Red}_{\text {asp }}(\mathbf{s})(x)$

c. $\quad\left[\left[\left[_{\mathrm{DP}}\left[\mathrm{D}\right.\right.\right.\right.$ het $\left[\mathrm{AP}_{\mathrm{AP}} \operatorname{rode}_{\mathrm{A}}\right.$ van de aardbeien $\left.\left.\left.]\right]\right]\right]=\lambda \lambda x \cdot \operatorname{Red}_{\text {asp }}(\mathbf{s})(\mathrm{x})$

(26c) gives us the intuitive meaning of (3b), in which the color term indicates a particular color aspect of the strawberries (contrasted with those of the mascarpone, the cream and the mint leaves). Similarly, (4b) contrasts the sweet and bitter aspects of beer and malt. Because of the relational interpretation of the inflected adjectives, the meaning conveyed, the color/taste is not directly predicated of the object denoted by the PP complement. Abstract adjectives lend themselves better to this process than concrete adjectives, thus the frequent nominalized use of leuk 'nice', bijzonder 'special', vreemd 'strange', etc., but in fact the process generalizes to a wide range of adjectives, e.g. het gezonde van biologisch voedsel 'the healthy aspect of biological food', het grote van de $z e e$ 'the large aspect of the sea'. Note that the result of reification in (26) is not kind reference but rather the entity correlate of a particular property, i.e. what Moltmann [9-10] calls a trope, because that property is uniquely instantiated in exactly one particular individual. Thus whether kind reference results from nominalization depends on the nature of the property being reified and not the $\cap$ operator itself.

The inflected adjective is directly embedded under the DP, and as such it maintains it categorical status as an adjective. This explains why it tolerates modification by adverbials, but not by another adjective (cf. (13)). As far as the semantics is concerned, we propose that the adverbial operates on the adjective before it combines with the PP. This leads us to interpret het te zoute van de ansjovis 'the too salty $\mathrm{y}_{[+\mathrm{e}]}$ of the anchovies' as referring to the salt-aspect of the anchovies and saying by way of non-restrictive modification that it is excessive. This suggests that the modifier takes scope over the $-e$ inflection as in (27): 
(27) $\quad$ a. $\quad\left[\left[\right.\right.$ zout $\left.\left._{\mathrm{A}}+\mathrm{e}\right]\right]=\lambda y \lambda x$. $\operatorname{Salt}_{\text {asp }}(y)(x)$

d. $\quad[[$ te zoute $]]=\lambda y \lambda x$. $\left(\right.$ Too $\left(\right.$ Salt $\left.\left._{\text {asp }}\right)\right)(\mathrm{y})(\mathrm{x})$

e. $\quad[[$ pP van de ansjovis $]]=\lambda R \lambda y \cdot R(a)(y)$

e. $\quad[[$ te zoute van de ansjovis $\mathrm{A}]]=\lambda \times \mathbf{x} .\left(\mathbf{T o o}\left(\mathbf{S a l t}_{\text {asp }}\right)\right)(\mathbf{a})(\mathrm{x})$

f. $\quad\left[{ }_{D P}\left[{ }_{D}\right.\right.$ het $\left[{ }_{A P}\right.$ te zoute van de ansjovis $\left.]\right]=\lambda_{x}$. $\left(\mathbf{T o o}\left(\mathbf{S a l t}_{\text {asp }}\right)\right)(\mathrm{a})(\mathrm{x})$

The low interpretation of the adverbial modifier is in line with syntactic constraints as well as semantic intuitions.

\subsection{Parallels between Adjectives and Verbs}

Our analysis places the inflected adjective construction in direct parallel with Dutch het-nominalized infinitives and English imperfect -ing nominals in (28) (see [3] and [4]). ${ }^{4}$

(28) Het zingen van Jan stoorde mij.
the singing of Jan bothered me b. Zingen is leuk. 'John's singing bothered me.' singing is fun 'Singing is fun.'

If one might want to hypothesize that English -ing contributes the nominalization function, in Dutch this role is clearly assumed by het. We therefore take the neuter article het to directly embed the infinitive in a DP, and thus to denote the nominalization function for verbs and adjectives alike. Just like nominalized adjectives, nominalized infinitives do not occur with determiners other than het.

Interestingly, nominalized infinitives can occur bare in generic uses, as we see in (28b), but nominalized inflected adjectives strictly require the presence of the article het and never get a generic interpretation (cf. (15)). These properties correlate with the semantics proposed here. The inflection turns the adjective into a relational predicate, and the extra argument is always a specific object the $\mathrm{P}_{\mathrm{asp}}$ is predicated of; leaving out the argument contributed by the PP complement is simply not possible under the semantics posited in (25). The argument does not have to be realized overtly if it can be contextually retrieved, but it has to get a specific value. Similarly, when the infinitive is accompanied by overt arguments with specific reference, het must be used; (28a) is ungrammatical without the determiner. Bare generic uses of the infinitive normally do not have PP complements; the crucial difference between the infinitive and the inflected adjective is that the former allows binding of its external argument with a generic pro, whereas no such binding is possible for the internal argument of the latter.

\section{Comparison with Related Work}

We now turn to a brief comparison of our analysis with the most closely related work we know, namely Villalba's [12] analysis of Spanish lo-nominals (cf. (5b)) vs. deadjectival nominalizations (5c).

Villalba takes as his departure point Moltmann's [9-10] ontology, which includes both tropes and kinds of tropes. Tropes are particular instances of a property manifest in a particular individual in space and time (e.g. John's honesty). In contrast, kinds of tropes are not situated in space or time (e.g. honesty in general). Villalba observes that both lo-nominals and derived nominals pass Moltmann's tests for identifying tropes - e.g., they can appear as complements to perception predicates:

aprendió a ver la belleza de las cosas cotidianas.

learned to see the.fem beauty of the.fem.pl things daily

'(s)he learned to see the beauty of everyday things'

b. $\quad \mathrm{Al}$ ver lo imposible de su amor..

to-the see LO impossible of his/her love

'When (s)he saw the impossibility of his/her love...'

\footnotetext{
${ }^{4}$ Dutch infinitives also do some of the work of infinitives in English. We set aside these uses here as the correspondence we have observed is between the inflected adjective and -ing forms in English (and by extension het-marked infinitives in Dutch), and not between the inflected adjective and the English infinitive.
} 
However, he observes that lo-nominals with PP complements contrast with derived nominals with PP complements in not easily allowing generic uses. (lo-nominals without complements can easily appear in generic sentences; see (31)).
(30) a. En este país, la banalidad de la política es muy común. ([12]: (24a) in this country the.fem banality of the.fem politics is very common 'In this country, the banality of politics is very common.'
b. *En este país lo banal de la política es muy común.
in this country LO banal of the.fem politics is very common

(31) Lo cortés no quita lo valiente

LO polite not takes LO brave

'Being polite is compatible with being brave.'

Based on contrasts such that in (30), and other data as well, Villalba argues that Moltmann's ontology is insufficient to account for Spanish lo-nominals. Rather, an additional sort of abstract object must be introduced, namely qualities. The property/quality distinction is taken from [7-8]. For Levinson, a property is a condition or state that an object is in; as such, he argues that it cannot be quantified (e.g. ?? a bit of being happy). In contrast, a quality is an abstract substance that can be quantified (e.g. a bit of happiness). Taking advantage of this distinction, Villalba proposes that $l o$-nominals and derived nominals denote different subsorts of abstract objects: properties and qualities, respectively. The derived nominal in (30a) denotes a kind of quality trope. However, he does not have formalize the semantics or fully explain why lo-nominals only very selectively appear in generic sentences (where they would denote kinds of property tropes), while derived nominals are not restricted in this respect.

Our analysis and Villalba's are similar in positing a sortal difference between the denotation of Spanish $l o$ nominals/Dutch nominalized inflected adjectives vs. derived nominal DPs. However, our analysis does not make a linguistically interesting distinction between qualities and properties. As far as we can determine, what Villalba calls qualities behave for purposes of grammar just like concrete entities. In contrast, there is evidence that properties as abstract objects do have a special status in grammar. Thus, our analysis is ontologically somewhat more parsimonious than his. ${ }^{5}$

In addition, by making explicit the contrast in the internal semantics of nouns and inflected adjectives, our analysis allows us to predict the lack of generic interpretation for the latter. If we were to extend the analysis to Spanish, treating $l o$ as the $\cap$ operator and assigning the modified adjective a semantics like that of the inflected form, the contrast in (30) would follow directly. (31), which is not possible in Dutch, suggests that Spanish differs from Dutch in allowing for binding by a generic pro of the internal argument to the adjective. If the internal argument is generically interpreted, it is unsurprising that the entity correlate of the property can also be generically interpreted.

\section{Conclusions}

We conclude that Dutch inflected forms are adjectival, not nominal. In fact, the DPs containing them are an example of what used to be called an "exocentric" construction (see e.g. [11] on nominal gerunds). Nominalized adjectives are a previously unidentified sort of example of this construction, to our knowledge. The data indicate that het's interpretation as a nominalizing operator is shared with Spanish lo. English the apparently lacks this interpretation, which explains why there is no English counterpart of the Spanish and Dutch constructions. Crucially, het only embeds inflected adjectives in a DP. We take the $-e$ suffix to enrich the valence of the adjective by one, creating a relational semantics denoting the Adj-aspect of the object denoted by the PP complement. At a more general level, we conclude that natural languages exploit the distinction between (category preserving) inflection and (category changing) derivation to create subtle nuances of meaning that enrich the palette of reference to abstract objects. These nuances of meaning can be capture while maintaining a parsimonious ontology involving kind level and token level entities.

\footnotetext{
${ }^{5}$ In this respect, our analysis also contrasts with the approach to color terms presented in Moltmann (to appear, Chapter 6). Moltmann assigns the sort of abstract objects color terms denote a special status in her ontology.
} 


\section{Acknowledgements}

The research presented here has been supported by Spanish Ministry of Science and Innovation grant FFI201015006 and the Fundació ICREA, as well as by the Netherlands Organization for Scientific Research (grant 36070-340). We would like to thank Xavier Villalba, Angel Gallego, the audience at the UPF GLIF seminar (November 2011) and the Weak Referentiality group in Utrecht for helpful discussion.

\section{References}

1. Broekhuis, H., Keizer, M.E.: A modern Dutch grammar. Ms. (2011)

2. Carlson, G.: Reference to kinds in English. Ph.D. dissertation, U. Mass. Amherst (1977)

3. Chierchia, G.: Topics in the syntax and semantics of infinitives and gerunds. Ph.D. dissertation, U. Mass. Amherst. Published 1989 by Garland Press, New York (1984)

4. Hamm, F., van Lambalgen, M.: Formal foundations for semantic theories of nominalisation. E. Lang \& I. Zimmermann (eds.), ZAS Papers in Linguistics 27 (2002)

5. Kennedy, C., McNally, L.: Color, context and compositionality, Synthese 174, 79-98 (2010)

6. Kester, E.-P.: The nature of adjectival inflrection. PhD. Dissertation, U. of Utrecht (1996)

7. Levinson, J.: Properties and related entities. Philosophy and Phenomenological Research 39, 1-22 (1978)

8. Levinson, J.: The particularisation of attributes. Australasian Journal of Philosophy 58, 102-115 (1980)

9. Moltmann, F.: Properties and kinds of tropes: New linguistic facts and old philosophical insights. Mind $113,1-43(2004)$

10. Moltmann, F.: Abstract objects and the semantics of natural language, Oxford University Press, Oxford (to appear)

11. Pullum, G. K.: English nominal gerund phrases as noun phrases with verb phrase heads. Linguistics 29 , 763-799 (1991)

12. Villalba, X.: Definite adjective nominalizations in Spanish. M.T. Espinal, M. Leonetti \& L. McNally (eds.), Proceedings of the IV Nereus International Workshop "Definiteness and DP Structure in Romance Languages". Arbeitspapier 124, Fachbereich Sprachwissenschaft, Universität Konstanz, 139-153 (2009)

13. Zamparelli, R.: Layers in the Determiner Phrase. Ph.D. dissertation, U. Rochester (1995) 


\title{
Tropes, Intensional Relative Clauses, and the Notion of a Variable Object
}

\author{
Friederike Moltmann \\ IHPST (Paris 1/ENS/CNRS) \\ fmoltmann@univ-paris1.fr
}

\begin{abstract}
NPs with intensional relative clauses such as the impact of the book John needs to write pose a significant challenge for trope theory (the theory of particularize properties), since they seem to refer to tropes that lack an actual bearer. I will propose a novel semantic analysis of such NPs on the basis of the notion of a variable object. This analysis avoids a range of difficulties that an alternative analysis based on the notion of an individual concept would face.
\end{abstract}

keyword: tropes, intensional verbs, individual concepts, relative clauses, situations

\section{Introduction}

It is a common view, since Aristotle, that terms of the sort in (1) refer to tropes or particularized properties, that is, particular, non-sharable features of individuals (Williams 1953, Strawson 1959, Woltersdorff 1977, Campbell 1990, Lowe 2006, Mertz 1996):

(1) a. the wisdom of Socrates

b. the originality of the book

c. the simplicity of the dress

According to that view, (1a) refers to the particular manifestation of wisdom in Socrates, that is, a wisdom trope with Socrates as its bearer.

Given general diagnostics for trope reference, there are equally good reasons to take the terms below to be terms referring to tropes, namely quantitative tropes (Campbell 1990, Moltmann 2009, to appear a):
(2) a. the number of planets
b. the height of the building
c. the length of the vacation

According to that view (2a) refers to the instantiation of the property of being eight in the plurality of planets, a feature not shared by any equally numbered plurality.

There are closely related terms, however, that present a significant challenge to trope theory. These are NPs of the sort below with relative clauses containing an intensional verb: 


\section{General Program}

(3) a. the impact of the book John needs to write

b. the simplicity of the dress Mary needs for the occasion

c. the wisdom of the director that the institutes should hire

(4) a. the number of people that fit into the car

b. the height of the desk John needs

c. the length of the time John might be away

I will call apparent trope-referring NPs with intensional relative clauses of this kind $I R$-NPs.

Tropes as discussed in philosophy are meant to be real entities, involving a real object as bearers. In fact, tropes generally are taken to depend for their existence and their identity on their bearer. But the tropes the terms in (3) and (4) seem to refer to lack an actual bearer. In this paper, I will argue that nonetheless the terms in (3) and (4) refer to tropes, or rather, in most cases, what I will call variable tropes. Central on this account is the notion of a variable object, a particular case of the notion of a variable embodiment of Fine (1999). IR-NPs either refer to tropes with a variable object as bearer or else they themselves refer to variable tropes whose bearer is driven by the variability of the bearer. I will argue that making using variable objects avoids a range of serious difficulties for the more standard alternative account that would make use of individual concepts.

\section{Trope reference with NPs containing intensional relative clauses}

The NPs in (3) and (4) share a range of diagnostics for trope reference with the NPs in (1) and (2). Tropes, unlike properties, generally are taken to be perceivable and causally efficacious (Williams 1953, Lowe 2006). In fact both sorts of NPs allow for the application of perceptual predicates, as in (5), and predicates describing causal relations, as in (6):

(5) a. John observed Mary's politeness.

b. John noticed the small number of women that were present.

c. John noticed the number of screws that were missing.

(6) a. The weight of the lamp caused the table to break.

b. The great number of screws that were missing caused the table to fall apart.

Moreover both sorts of terms accept predicates of neutral evaluation such as exceed, great, high, or negligible, predicates which are not naturally applicable to the corresponding abstract objects (properties or numbers):

(7) a. The number of women exceeds the number of men.

b. The number of people that fit into the bus exceeds the number of people that fit into the car.

c. ?? Eight exceeds seven.

d. The impact of John's book is great / negligible.

e. The impact of the book John needs to write has to be great / negligible.

f. ?? The property of having an impact is great / negligible. 
In fact the exceed-relation appears to be inherited from the underlying pluralities in both cases in the very same way. Thus (7a) and (7b) are equivalent to (7a') and (7b') with the specifier of numerical respect in number:

(7) a'. The women exceed the men in number.

b'. The people that fit into the bus exceed the people that fit into the car in number.

Finally, trope reference is reflected in the application of the be of identity as opposed to the predicate is the same as. The observation is that whereas (8a) and (8c) can be true, (8b) and (8e) cannot:

(8) a. The number of men is the same as the number of women.

b. ?? The number of men is the number of women.

c. The number of people that fit into the bus is the same as the number of people that fit into the car.

d. ?? The number of people that fit into the bus is the number of people that fit into the car.

The same as in fact expresses close or exact similarity not numerical identity. Numerical identity is expressed only by identity be (Moltmann 2009, to appear). Tropes with different bearers that instantiate the same property are similar but not identical. Tropes that instantiate the same 'natural' property (for example the same number property) are exactly similar, and thus 'the same'.

\section{Approaches based on individual concepts}

Given standard semantics, an obvious approach to the terms in (3)-(4) would be to consider them terms referring to tropes with individual concepts as bearers, that is, (partial) functions from worlds and times ('circumstances' for short) to individuals (or collections of individuals) (Montague 1974). That individual concepts of some sort are the denotations of certain types of NPs with intensional relative clauses has in fact been argued by Moltmann (2008) for NPs as in (9a) and, for the closely related construction in (9b), by Grosu/Krifka (2007):

(9) a. The assistant John needs must speak French fluently.

b. The gifted mathematician that you claim to be could solve this problem in no time.

However, using reference to individual concepts and of individual concepts as bearers of tropes raises a range of problems, ontologically, conceptually, empirically, and regarding the compositional semantics of IR-NPs.

The ontological problem concerns the notion of a trope itself: tropes are entities in the world that are potentially causally efficacious and perceivable. This means that tropes have objects as bearers, not intensions or functions (unless of course the tropes are features of abstract objects, but this is not what is at stake).

The conceptual problem concerns substitution problems that reference to individual concepts in general give rise to: an abstract function has quite different properties (that is, is a bearer of quite different tropes) than 'the book that John needs to write'. Thus neither (5a') nor (6e') are valid inferences from (5b) and (6e):

(5) a'. John noticed the number of some function. 


\section{General Program}

(6) e'. The number of some function caused the table to fall apart.

The empirical problem concerns the particular behavior of NPs as in (3)-(4) with respect to the requirement that the predicate contain a modal. Sometimes IR-NPs are subject to the requirement, as in (10a), sometimes they are not, as in $(10 \mathrm{~b})$ :

(10) a. The impact of the paper John needs to write ?? exceeds /ok must exceed the impact of the papers he has so far written.

b. The number of people that fit into the bus exceeds the number of people that fit into the car

It is obscure how an account based on individual concepts could explain the difference.

Finally, there are problems regarding the compositional semantics of IR-NPs on the basis of individual concepts. There are two options of analyzing the book John needs to write as standing for an individual concept. For reasons of space, I can discuss those only briefly and only in their roughest outline.

The first option would be an extension of Grosu/Krifka's (2007) analysis of the gifted mathematician that John claims to be. Their analysis involves several assumptions. First, it involves type-lifting of all predicates to predicates of individual concepts and all singular terms (including proper names) to terms for individual concepts. Second, it requires treating all intensional verbs as operators quantifying over possible worlds. Finally, it interprets the head noun book in the upper position, rather than reconstructing it into the lower position inside the relative clause. Greatly simplifying, this analysis would yield the following as the denotation of the book John needs to write:

(11) $\min (\{\mathrm{f} \mid \operatorname{book}(\mathrm{f})\} \cap\{\mathrm{f} \mid$ John need to write (f) $\})$

(The second set would be the set of functions mapping a world w compatible with the satisfaction of John's needs to an object John writes in w.) This analysis raises a range of problems. First of all; it involves an excessive use of individual concepts as well as the assumption that all intensional verbs be analyzable as operators quantifying over words, an assumption that a great number of philosophers will find problematic. Furthermore, it poses a problem of uniqueness (a problem that did not arise for the construction for which Grosu and Krifka's analysis was originallydeveloped ${ }^{1}$ ). In a given word in which John's needs are satisfied, John may have written more than one book meeting his need. To account for uniqueness, not entire worlds should be taken into account in which John's needs are satisfied, but rather situations exactly satisfying the need. A given world in which John's needs are satisfied may then contain several situations satisfying his need. A given world may contain several such satisfaction situations. It is far from clear how the analysis in (11) would be able to accommodate the dependence on situations satisfying a need.

The second option of analysing the book John needs to write as standing for an individual concept would involve reconstructing the head noun into the lower position inside the relative clause yielding the analysis indicated below:

${ }^{1} \mathrm{Grosu} / \mathrm{Krifka}$ (2007) have no problem of uniqueness because they analyse the gifted mathematician John claims to be as involving identity be, which for them takes two individual concepts as arguments. 
This analysis raises the very same problem of uniqueness as the first analysis. Also, just like the first analysis, it is forced to treat all intensional verbs as modal operators quantifying over worlds. Moreover, in its attempt of avoiding type-shifting the analysis cannot go very far. Even though it is plausible that the head noun reconstructs into the lower position, reconstruction of the functional trope noun into a position inside the relative clause is in general impossible: there is no place inside the relative clause for a noun like impact in (3a), repeated below:

(3) a. the impact of the book John needs to write

Impact will have to be interpreted in the upper position. But this means that it will have to denote a function applying to individual concepts.

\section{The variable-objects approach}

The account I would like to propose is based both on the notion of a variable object and the notion of a variable trope. Variable objects are entities that fall under Fine's (1999) more general notion of a variable embodiment (see also Koslicki 2008). The notion of a variable embodiment for Fine is a central notion in metaphysics and accounts for a great variety of 'ordinary' objects. But Fine himself (p.c.) also meant to apply the notion of a variable embodiment to the semantic values of functional NPs as in (13) as well as NPs with intensional relative clauses such as the book John needs to write:

(13) a. John changed his trainer.

b. The temperature is rising.

c. The number of students has increased.

According to the standard Montagovian view, functional NPs such as (13a)-(13c) are of a different type than singular terms: they are of type $\langle e, t>$ rather than of type $\langle e>$. Functional NPs thus stand for individual concepts: functions from world-time pairs to objects. Some predicates such as change, rise, increase will apply to individual concepts directly. Other predicates will be type-shifted to predicates of individual concepts while imposing the following meaning postulate:

(14) For any predicate of individuals $P$ and any individual concept $f$, $\mathrm{P}^{, \mathrm{w}, \mathrm{t}}(\mathrm{f})=1$ iff $\mathrm{P}^{\mathrm{w}, \mathrm{t}}(\mathrm{f}(\mathrm{w}, \mathrm{t}))=1$.

There are various reasons to consider NPs of the sort in (13) as standing for objects (variable objects) rather than being of a different type than singular terms and as such denoting functions. For example, object-related predicates can apply to such NPs just as they apply to individuals (such as the predicates change, rise, and 


\section{General Program}

increase ). This also holds for NPs with intensional relative clauses. Most strikingly, the predicate count can apply with such NPs just as it applies with ordinary singular terms:

(15) a. John counted the books he needs to write.

b. John counted the screws that are missing.

c. John counted the things he needed to buy.

Moreover, functional NPs can naturally provide the bearers of tropes, as in the sentences below:

(16) a. The impact of the increasing number of students is noticeable.

b. The rise of the temperature caused the drought.

The notion of a variable embodiment allows an account of functional NPs that avoids type-shifting of predicates and also avoids treating their referents as abstract functions.

A variable embodiment, according to Fine, is an entity that allows for the replacement of constituting material or parts, and more generally that may have different manifestations in different circumstances. Organisms and artifacts are variable embodiments, but also entities like 'the water in the river'. 'The water in the river' is a variable embodiment that has different manifestations as different quantities of water at different times. Variable embodiments differ from 'rigid embodiments', entities which do not allow for a replacement of their immediate parts. An example is a token of the word $b e$, which has as its immediate parts a token of $b$ and a token of $e$, neither of which can be replaced.

Fine's theory of variable embodiments as formulated in Fine (1999) applies to variable embodiments that may have different manifestations at different times. But the theory is also meant to apply to entities that have different manifestations in different worlds and in fact may lack a manifestation in the actual world. 'The book John needs to write' is such an entity. It is an entity that has different manifestations as different objects in various counterfactual worlds. My term of a variable object is meant to apply to entities that have different manifestations as different objects at different times or in different worlds.

Let us then adopt the following conditions from Fine (1999) for variable objects:

(17) a. Existence: A variable object e exists in a circumstance i iff e has a manifestation in i.

b. Location: If a variable object e exists in a circumstance $i$, then e's location in is that of its manifestation in $\mathrm{i}$.

c. Property inheritance 1: A variable object e has a (world- or time-relative) property $\mathrm{P}$ in a circumstance $\mathrm{i}$ if e's manifestation in $\mathrm{i}$ has $\mathrm{P}$.

In addition to local properties, which they obtain in the way of (13b), variable objects may have global properties, properties that they may have on the basis of several of their manifestations at different times (for example properties of change, rise, or inccrease). Variable objects moreover may have properties that are not time- or world-relative (though may be attributed at a time or in a world). Thus we can add a second condition of property inheritance: 
(17) d. Property inheritance 2: A variable object has a (world- and time-independent) property P if all its manifestations in any circumstances have $P$.

These two conditions can be reformulated as follows when the property in consideration is understood as a particularized property (a trope):

(18) a. Trope 'inheritance' 1: A variable object e bears a trope $t$ relative to a circumstance i if e's manifestation in $\mathrm{i}$ bears a trope in $\mathrm{i}$ that is exactly similar to $\mathrm{t}^{2}$

b. Trope 'inheritance' 2: A variable object bears a trope t if for any circumstance i, e's manifestation in i bears a trope exactly similar to $t$ in $\mathrm{i}$.

Using variable objects in this sense has a significant advantage over the individual-concept approach to the compositional semantics of functional NPs and NPs with intensional relative clauses by avoiding a type ambiguity among predicates entirely. Let us first apply the account to (19a) and (19b):

(19) a. the impact of the number of students

b. the increase of the number of students

The functional trope noun in the upper position applies to a variable object and maps it onto a trope that is of either of two sorts: a local trope based on a single circumstance, as in (19a), or a global trope based on a series of circumstances, as in (19b). The two functional trope nouns in (19a) and (19b) denote different functions from variable objects to tropes, as indicated below, where $\mathrm{F}$ is the function mapping a variable object $\mathrm{e}$ and a circumstance $\mathrm{i}$ to the manifestation of $\mathrm{e}$ in $\mathrm{i}$ :

(20) a. For a variable object $\mathrm{e}$, impact $^{\mathrm{w}, \mathrm{t}}(\mathrm{e})=$ the trope that has e as its bearer and is exactly similar to $\operatorname{impact}^{\mathrm{w}, \mathrm{t}}(\mathrm{F}(\mathrm{e},(\mathrm{w}, \mathrm{t})))$.

b. For a variable object $\mathrm{e}$, increase $\mathrm{w}^{\mathrm{w}, \mathrm{t}}(\mathrm{e})=$ the trope that has $\mathrm{e}$ as its bearer and is the instantiation of the property $\lambda e^{\prime}\left[\left(F\left(e^{\prime}, w, t_{1}\right)\right.\right.$ less than $F\left(e^{\prime}, w, t_{2}\right)$ less than.... for subintervals $t_{1}, t_{2}$, and $t_{3}$ of $t$ and $\left.\mathrm{t}_{1}<\mathrm{t}_{2}<\mathrm{t}_{3} \ldots\right]$

A different case is that of the impact of the book John needs to write, which refers not to a single trope but rather to a variable trope. Let us first focus on what the variable object is that the book John needs to write stands for. Assuming that the head noun book is interpreted in the lower position inside the relative clause, the lower variable will stand for a variable object, an object to which the relative clause attributes certain properties in particular circumstances. But this variable object cannot be the variable object each of whose manifestations is a paper John writes in a world in which John's needs are satisfied. A world in which John's needs are satisfied may contain several papers that John writes in that world._Moreover, some of those papers may not qualify as

\footnotetext{
${ }^{2}$ In Moltmann (to appear b), I argue that in such cases the very same trope is inherited. This result is a trope with multiple bearers. This is of course a nonstandard view about tropes.
} 


\section{General Program}

'the paper John's needs to write': the complement of need gives only a partial characterization of the exact need. Rather to obtain uniqueness, use must be made of situations exactly satisfying John's needs. That is, 'the paper John needs to write' stands for the variable object each of whose manifestations is a paper John writes in a situation exactly satisfying John's needs. Uniqueness then holds relative to a situation of satisfaction of the need. The situation may impose various constraints on the paper John writes in it (constraints the speaker in fact need not know about). Given its dependence on satisfaction situations, the variable object that is 'the paper John needs to write' is an object that itself depends on a need.

But what is a need? A need is not a state of needing, and thus not a Davidsonian event argument. Only a need, but not a state of needing, can be 'satisfied' by a situation. How then do we obtain a 'need' in the interpretation of the sentence so that the variable object in question could depend on it? Without going into a greater discussion, I would simply like appeal to a particular syntactic proposal concerning the verb need by Harves/Kayne (to appear). According to their view, the verb need is the result of incorporating the copula have and the noun need. Given this proposal, an entity that is a need would be made available as part of the compositional semantics of the complex predicate have+need. Thus the analysis of the book John needs to write would be roughly as follows, where the variable object $d_{e}$ is dependent on a need e:

(21) the book [John needs to write e $]=$ the book [John has a need to write e $]=$ the $\mathrm{e}[\mathrm{John}$ has a need to write $[\mathrm{e}$ book $]]=\imath \mathrm{dd}\left[\exists \mathrm{e}\left(\right.\right.$ have $\left.(\mathrm{e}, \mathrm{John}) \& \operatorname{need}\left(\mathrm{e},{ }^{\wedge} \operatorname{write}\left(\mathrm{John}, \mathrm{d}_{\mathrm{e}}\right) \& \operatorname{book}\left(\mathrm{d}_{\mathrm{e}}\right)\right)\right]$

\section{The Modal Compatibility Requirement}

Let us then turn to the question of when a modal is required in the main clause of a sentence with an IR-NP. Following Grosu/Krifka (2007), who noticed the requirement for a related construction (see also Moltmann 2008), I will call this the Modal Compatibility Requirement:

(22) The Modal Compatbility Requirement (MCR)

IR-NPs require an appropriate modal in the main clause to 'access' the entities in the counterfactual circumstances.

The MCR does not hold for all sentences with IR-NPs. It does not hold in (22a) and (22b), though it does hold in (23a) and (23b):

(22) a.. The number of people that can fit into the bus exceeds the number of people that can fit into the car.

b. The length of the vacation John is allowed to take exceeds the length of the vacation Mary is allowed to take.

(23) a. The impact of the book John needs to write ?? exceeds / ok must exceed / ok might exceed the impact of the book he has already written.

b. The elegance of the dress the bridesmaid should wear ?? does not exceed / ok should not exceed the elegance of the dress that the bride will wear. 
This might suggest that IR-NPs referring to quantitative tropes are not subject of the MCR. But this is not right. The MCR is in place below:

(24) ?? The number of people John might invite exceeds the number of people Mary might invite.

Yet the distinction between quantitative and qualitative trope does matter. This can again be seen from the difference between (23a) and (23b) with a one-place evaluative predicate:

(25) a. The number of papers a student has to write during this program is too high.

b. The quality of the paper John must write ?? is very high / must be very high.

I propose an explanation of the MCR and exceptions to it based on general conditions on when a variable object can bear a trope on the basis of its instances. The cases in which the MCR is in place are cases in which the head noun applies to a variable object and maps it onto a variable trope. A variable trope driven by the variability of its bearer e has as its manifestation in a circumstance $i$ the trope $t$ that has as its bearer the manifestation of $\mathrm{e}$ in $\mathrm{i}$. A variable trope that has manifestations only in counterfactual circumstances requires a modal in the main clause in order to be attributed local properties in the first place. The noun impact in the impact of the book John needs to write thus denotes a function mapping a variable object onto a variable trope, as below:

(26) For a variable object e,

impact $^{\mathrm{w}, \mathrm{t}}(\mathrm{e})=$ the variable trope o such that for any circumstance $\mathrm{s}$ in which e has a manifestation $\mathrm{F}(\mathrm{e}, \mathrm{s})$, impact ${ }^{\mathrm{w}, \mathrm{t}}(\mathrm{F}(\mathrm{e}, \mathrm{s}))=$ the manifestation of $\mathrm{o}$ in $\mathrm{s}$.

Let us turn to the case of quantitative tropes not subject to the MCR. What is particular about such cases is that here the manifestations arguably are exactly similar: it is plausible to assume that the same number of people fit into the bus / the car in the various relevant circumstances, which means that the number tropes in the various circumstances are exactly similar. Recall that in such a case the variable object itself will bear an exactly similar number trope. That is, we won't have a variable trope, but an ordinary trope with a variable object as its bearer. Such cases are restricted to quantitative tropes because exact similarity among qualitative tropes is unlikely to obtain, given that natural language predicates in general do not express natural qualitative properties, but unspecific, determinable ones.

This account made use of general conditions on tropes to explain the exemption from the MCR. This in itself gives further support for the trope-based account.

There is another case of an exemption from the MCR:

(27) The originality of the paper John wants to write exceeds the originality of the papers he has so far written.

This case is an entirely different one. In (27), the trope 'the originality of the paper John wants to write' has as its bearer an intentional object, an 'object of thought', rather than a variable object (Moltmann 1997). An 


\section{General Program}

intentional object is fully present in the world in which the act generating it occurs. It is not an object that has different manifestations in worlds that may exclude the actual one. Clearly only psychological verbs allow for reference to intentional objects, modal verbs of absence like need do not:

(28) a. ?? The house John needs is very grand..

b. The house John imagines is very grand.

\section{Conclusion}

To summarize, the notion of a variable object allows an account of an otherwise very puzzling construction of apparent trope-referring terms. The notion of a variable object as such is not a peculiar notion, though, invoked only for the analysis of IR-NPs. Rather, it falls under the more general and ontologically central notion of a variable embodiment (in Fine's metaphysics). As subject, it is subject to the very same ontological conditions as drive variable embodiments in general.

\section{References}

1. Aristotle: The Categories.

2. Campbell, K. (1990): Abstract Particulars. Blackwell, Oxford (1990).

3. Fine, K. (1999): 'Things and Their Parts'. Midwest Studies of Philosophy 23, 61-74.

4. Grosu, A. / Krifka, M. (2007): 'The Gifted Mathematician that you Claim to be'. Linguistics and Philosophy 30, 445-485 (2007).

5. Harves, S. / R. Kayne: 'Having need and needing have'. Linguistic Inquiry (to appear).

6. Koslicki, K.: The Structure of Objects. Oxford UP (2008).

7. Lowe, J.: The Four-Category Ontology. A Metaphysics Foundation for Natural Science. Oxford UP (2006)

8. Mertz, D. W.: Moderate Realism and Its Logic. Yale UP, New Haven (1996).

9. Moltmann, F.: 'Intensional Verbs and Quantifiers'. Natural Language Semantics 5.1, 1-52 (1997).

10. Moltmann, F: 'Intensional Verbs and Their Intentional Objects'. Natural Language Semantics 16.3., 239-270 (2008)

11. Moltmann, F: 'Degree Structure as Trope Structure: A Trope-Based Analysis of Comparative and Positive Adjectives'. Linguistics and Philosophy 32, 51-94 (2009).

12. Moltmann, F.: 'Reference to Numbers in Natural Language'. Philosophical Studies(to appear a).

13. Moltmann, F.: 'Tropes, Bare Demonstratives, and Apparent Statements of Identity. Nous (to appear b).

14. Montague, R. 'The Proper Treatment of Quantification in Ordinary English'. In J. Hintikka et al.(eds.): Approaches to Natural Language. Reidel, Dordrecht (1973).

15. Strawson, P. Individuals. An Essay in Descriptive Metaphysics. Methuen, London (1959).

16. Williams, D. C.: 'On the Elements of Being'. Review of Metaphysics 7, 3-18 (1953).

17. Woltersdorff, N.: On Universals. Chicago UP, Chicago (1970). 
Tropes, Intensional Relative Clauses and the Notion of a Variable Object F. Moltmann 


\title{
A Theory of Names and True Intensionality
}

\author{
Reinhard Muskens \\ Tilburg Center for Logic and Philosophy of Science \\ r.a.muskens@uvt.nl \\ http://let.uvt.nl/general/people/rmuskens/
}

\begin{abstract}
Standard approaches to proper names, based on Kripke's views, hold that the semantic values of expressions are (set-theoretic) functions from possible worlds to extensions and that names are rigid designators, i.e. that their values are constant functions from worlds to entities. The difficulties with these approaches are well-known and in this paper we develop an alternative. Based on earlier work on a higher order logic that is truly intensional in the sense that it does not validate the axiom scheme of Extensionality, we develop a simple theory of names in which Kripke's intuitions concerning rigidity are accounted for, but the more unpalatable consequences of standard implementations of his theory are avoided. The logic uses Frege's distinction between sense and reference and while it accepts the rigidity of names it rejects the view that names have direct reference. Names have constant denotations across possible worlds, but the semantic value of a name is not determined by its denotation.
\end{abstract}

\section{Introduction}

Standard approaches to proper names, based on Kripke $(1971,1972)$, make the following three assumptions.

(a) The semantic values of expressions are (possibly partial) functions from possible worlds to extensions.

(b) These functions are identified with their graphs, as in set theory.

(c) Names are rigid designators, i.e. their extensions are world-independent.

In particular, the semantic values of names are taken to be constant functions from worlds to entities, possibly undefined for some worlds.

The difficulties resulting from these assumptions are well-known. On the one hand, there are general 'logical omniscience' problems with the possible worlds approach resulting from (a) + (b). Since functions, in the set-theoretic conception, are extensional entities, with their identity criteria given by input-output behaviour, the semantic values of far too many expressions will be identified. Implications and their contrapositives, for example, will be lumped together. That is incorrect since one may very well believe $p \rightarrow q$ but fail to believe $\neg q \rightarrow \neg p$, so that there is at least one property the semantic values of these expressions do not have in common. 
Adding (c) as a further restriction makes things worse, since if the semantic value of a name depends only on its bearer it is predicted that names with the same bearer can be substituted for one another in any context. This leads to philosophers claiming and dogmatically defending the position that the Ancients did know that Hesperus was Phosphorus before that identity was actually discovered, an armchair intuition that does not seem to be shared by many outside the profession. It also leads to the prediction that the following are equivalent.

(1) a. We do not know a priori that Hesperus is Phosphorus

b. We do not know a priori that Phosphorus is Phosphorus

(1a) is asserted in Kripke (1972, page 308); (1b) is obviously false. Traditional theorists are therefore confronted with the challenge to come up with a logic in which the values of (1a) and (1b) can be distinguished. No precise system seems to have been developed thus far.

The substitutivity problems that follow from the adoption of (a)-(c) show that this combination cannot stand, but this does not mean, of course, that (c), the idea that names denote rigidly, has to go. In this paper I will sketch a theory that does not suffer from the many problems that are connected with identifying intensions with certain functions in extension, but in which it is still possible to consistently formalize the intuition that names denote rigidly.

\section{A Truly Intensional Logic}

We move to a (higher order) logic that is truly intensional. By this we mean that the following axiom (schema) of extensionality fails. ${ }^{1}$

(2) $\forall X Y(\forall \vec{x}(X \vec{x} \leftrightarrow Y \vec{x}) \rightarrow \forall Z(Z X \rightarrow Z Y))$

There are now several approaches to type theory that manage to avoid making (2) valid. Fitting (2002) and Benzmüller et al. (2004) are two of them, but since both of these papers interpret the central machinery of type logic in some nonstandard way, ${ }^{2}$ the logic used here will be the ITL of Muskens (2007). In this logic all operators have standard interpretations and in fact the interpretation of the logic is a rather straightforward generalisation of that of Henkin (1950), making (2) invalid but retaining all classical rules for logical operators. The following somewhat impressionistic description mainly highlights ITL's minor differences with standard simple type theory. For precise definitions consult Muskens (2007).

\footnotetext{
${ }^{1}$ Our notion of true intensionality is just the notion of intensionality defined in Whitehead and Russell (1913), but using that term without modification may lead to confusion nowadays, as the word is now widely used for Carnap's imperfect approximation of the original concept. I will mostly, but not always, use true intensionality for intensionality in this paper. Hyperintensionality, another word for the same idea, is less than felicitous, as it suggests a property stronger than intensionality, while it is only stronger than Carnap's approximation.

${ }^{2}$ Fitting's (2002) interpretation of lambda abstraction is non-standard while Benzmüller et al.'s (2004) interpretation of application is.
} 


$$
\begin{array}{cc}
\frac{\Pi \Rightarrow \Sigma}{\Pi^{\prime} \Rightarrow \Sigma^{\prime}}[W], \quad \text { if } \Pi \subseteq \Pi^{\prime}, \Sigma \subseteq \Sigma^{\prime} \\
\frac{\Pi, \varphi \Rightarrow \Sigma, \varphi}{\Pi}[R] & \frac{\Pi, \perp \Rightarrow \Sigma}{\Pi, \mathrm{L}]} \\
\frac{\Pi, A\{x:=B\} \vec{C} \Rightarrow \Sigma}{\Pi,(\lambda x . A) B \vec{C} \Rightarrow \Sigma}[\lambda \mathrm{L}] & \frac{\Pi \Rightarrow \Sigma, A\{x:=B\} \vec{C}}{\Pi \Rightarrow \Sigma,(\lambda x . A) B \vec{C}}[\lambda \mathrm{R}] \\
\frac{\text { if } B \text { is free for } x \text { in } A}{\Pi, B \vec{C} \Rightarrow \Sigma} & \text { if } B \text { is free for } x \text { in } A \\
\hline \Pi, A \subset B \Rightarrow \Sigma & \frac{\Pi, A \vec{c} \Rightarrow \Sigma, B \vec{c}}{\Pi \Rightarrow \Sigma, A \subset B}[\subset \mathrm{R}] \\
\hline
\end{array}
$$

Table 1. Gentzen rules for ITL.

Type system ITL's type system is relational, rather than functional. Given some set of basic types, further types are formed by the rule that $\left\langle\alpha_{1} \ldots \alpha_{n}\right\rangle$ is a type if $\alpha_{1}, \ldots, \alpha_{n}$ are. Objects of type $\left\langle\alpha_{1} \ldots \alpha_{n}\right\rangle$ are n-ary relations in intension that take objects of type $\alpha_{k}$ in their $k$-th argument place. Readers familiar with functional type logics may identify $\left\langle\alpha_{1} \ldots \alpha_{n}\right\rangle$ with $\alpha_{1} \rightarrow \ldots \rightarrow \alpha_{n} \rightarrow t$, where $t$ is the type of propositions and truth values (and association is to the right). In linguistic semantics this would be written without the arrows: $\alpha_{1} \ldots \alpha_{n} t$. So $\langle e\rangle$ is the type of unary and $\langle e e e\rangle$ the type of ternary relations in intension of type $e$ objects, while $\langle\langle e\rangle\rangle$ is the type of properties of properties of individuals (quantifiers). The type \langle\rangle is a limiting case. It corresponds to $t$ in the functional set-up. Objects of this type are propositions, and their extensions are truth values.

Language Terms of the logic are built up in the usual way from variables and non-logical constants with the help of application, $\lambda$-abstraction and a few logical constants, here $\subset$ and $\perp$ ( $\subset$ is meant to denote inclusion of extensions and $\perp$ will be a proposition that is always false). Typing of terms is as expected, given the correlation between relational and functional types that was just described. For example, $(\lambda x . A)$ is of type $\left\langle\alpha_{1} \alpha_{2} \ldots \alpha_{n}\right\rangle$ if $A$ is of type $\left\langle\alpha_{2} \ldots \alpha_{n}\right\rangle$ and $x$ is of type $\alpha_{1}$, while $(A B)$ is of type $\left\langle\alpha_{2} \ldots \alpha_{n}\right\rangle$ if $A$ is of type $\left\langle\alpha_{1} \alpha_{2} \ldots \alpha_{n}\right\rangle$ and $B$ is of type $\alpha_{1}$. Successive applications can 'eat up' all the argument places of a relation until \langle\rangle is reached.

Intensional models Models for ITL distinguish between the intension of a term (given an assignment) and the extension associated with that intension. 


$$
\begin{aligned}
& \overline{\Pi \Rightarrow \Sigma, \top}[\top \mathrm{R}] \\
& \frac{\Pi, \psi \Rightarrow \Sigma \quad \Pi \Rightarrow \Sigma, \varphi}{\Pi, \varphi \rightarrow \psi \Rightarrow \Sigma}[\rightarrow \mathrm{L}] \quad \frac{\Pi, \varphi \Rightarrow \Sigma, \psi}{\Pi \Rightarrow \Sigma, \varphi \rightarrow \psi}[\rightarrow \mathrm{R}] \\
& \begin{array}{c}
\Pi, \varphi\{x:=A\} \Rightarrow \Sigma \\
\Pi, \forall x \varphi \Rightarrow \Sigma
\end{array}[\mathrm{L}] \quad \frac{\Pi \Rightarrow \Sigma, \varphi\{x:=c\}}{\Pi \Rightarrow \Sigma, \forall x \varphi}[\forall \mathrm{R}] \\
& \frac{\Pi, A \doteq B \Rightarrow \Sigma, \varphi\{x:=A\}}{\Pi, A \doteq B \Rightarrow \Sigma, \varphi\{x:=B\}}[=\mathrm{L}] \quad \frac{}{\Pi \Rightarrow \Sigma, A=A}[=\mathrm{R}] \\
& \text { where } A \doteq B \text { is } A=B \text { or } B=A
\end{aligned}
$$

Table 2. Some classical rules derivable in ITL.

Fitting (2002) uses a similar way of defining models and the set-up is strongly reminiscent of that of Frege (1892).

Collections of domains will be sets $\left\{D_{\alpha} \mid \alpha\right.$ is a type $\}$ of pairwise disjoint non-empty sets. There is no further restriction on collections of domains and in particular sets $D_{\left\langle\alpha_{1} \ldots \alpha_{n}\right\rangle}$ need not consist of relations over lower type domains, as is the case in the usual Henkin models. Assignments and notation for assignments are defined as usual. Intension functions are defined to be functions that send terms and assignments to elements of the $D_{\alpha}$ and respect the following constraints.

$-I(a, A) \in D_{\alpha}$, if $A$ is of type $\alpha$

$-I(a, x)=a(x)$, if $x$ is a variable

- $I(a, A)=I\left(a^{\prime}, A\right)$, if $a$ and $a^{\prime}$ agree on all variables free in $A$

- $I(a, A\{x:=B\})=I(a[I(a, B) / x], A)$, if $B$ is free for $x$ in $A$

These constraints are still very liberal and do not amount to the constraints imposed by the usual Tarski definition.

The next step associates extensions with intensions. For each $\alpha=\left\langle\alpha_{1} \ldots \alpha_{n}\right\rangle$, a function $E_{\alpha}: D_{\alpha} \rightarrow \mathcal{P}\left(D_{\alpha_{1}} \times \cdots \times D_{\alpha_{n}}\right)$ is called an extension function. A triple consisting of a collection of domains, an intension function, and a family of extension functions is called a generalised frame. (Note that in a generalised frame $E_{\langle\rangle}: D_{\langle\rangle} \rightarrow\{0,1\}$, if some standard identifications are made.) Generalised frames are intensional models if, for all $\alpha=\left\langle\alpha_{1} \ldots \alpha_{n}\right\rangle$, and for all terms $A$ of type $\alpha$, the extensions $E_{\alpha}(I(a, A))$, for which we write $V(a, A)$, satisfy the following constraints.

$$
\begin{aligned}
& -V(a, \perp)=0 \\
& -V(a, A B)=\{\langle\vec{d}\rangle \mid\langle I(a, B), \vec{d}\rangle \in V(a, A)\} \\
& -V\left(a, \lambda x_{\beta} . A\right)=\left\{\langle d, \vec{d}\rangle \mid d \in D_{\beta} \text { and }\langle\vec{d}\rangle \in V(a[d / x], A)\right\}
\end{aligned}
$$




$$
-V(a, A \subset B)=1 \Longleftrightarrow V(a, A) \subseteq V(a, B)
$$

These last clauses constrain extensions to behave as in the usual Tarski value definition. For the treatment of abstraction and application in a relational setting, see also Muskens (1995).

Entailment is defined in the usual way, with the help of intensional models. The rules for $\lambda$-conversion, $(\alpha),(\beta)$ and $(\eta)$, do not automatically hold (they preserve extension, but not necessarily intension), but it is possible to consistently add them to the logic. Extensionality is not universally valid. This is because the functions $E_{\alpha}: D_{\alpha} \rightarrow \mathcal{P}\left(D_{\alpha_{1}} \times \cdots \times D_{\alpha_{n}}\right)$ need not be injective. In fact, intensional models in which all extension functions are injective essentially are Henkin's general models, while a further requirement of surjectivity will give full models.

Proofs The Gentzen calculus in Table 1 is generalised complete for the semantic notion of entailment just defined (see Muskens (2007) for a proof). Table 2 gives derived rules for some operators defined from the two primitives $\subset$ and $\perp$. The identity here is Leibniz identity, having the same properties, i.e. $A=B$ is short for $\forall Z(Z A \rightarrow Z B)$.

\section{$3 \quad$ Names in a Truly Intensional Setting}

Given a truly intensional logic such as the one just defined, a theory of names can take the following form.

- Ordinary proper names are predicates.

- They are singular in the sense that their extensions are either empty or singletons.

- Meanings are represented by lambda terms and combine with the help of application and type shifters.

- Among the type shifters is Partee's type shifter A, i.e. $\lambda P^{\prime} P . \exists x\left(P^{\prime} x \wedge P x\right)$ (Partee, 1986). ${ }^{3}$

In defending a theory of names as predicates I side with Aristotle, I think, and the idea seems linguistically natural, as names accept modification, combine with determiners, etc., just like common nouns. Singularity can be enforced by adopting the following constraint, for all names $\mathrm{N}$.

(3) $\forall x y((\mathrm{~N} x \wedge \mathrm{N} y) \rightarrow x=y)$

The type shifter A provides the glue that is needed to get predication going. (4) provides a simple example. Let's say Zeus translates as the predicate Z (4a); then combining with Partee's type shifter $\lambda P^{\prime} P . \exists x\left(P^{\prime} x \wedge P x\right)$ leads to the translation

\footnotetext{
${ }^{3}$ We generally use $Q$ as a variable over type $\langle\langle e\rangle\rangle$ (quantifiers), $P$ as a variable of type $\langle e\rangle$ (properties of individuals), $R$ as a variable of type $\langle e e\rangle$ (binary relations in intension of individuals), and $x, y$ and $z$ as variables of type $e$ (individuals).
} 
in (4b) and a further combination with the translation of smiles, S say, to that in (4c) (here it is assumed that the rules of $\lambda$-conversion have indeed been added to the logic).

(4) a. Zeus $\sim \mathrm{Z}$

b. Zeus $\leadsto \lambda P \cdot \exists x(\mathrm{Z} x \wedge P x)$

c. Zeus smiles $\leadsto \exists x(\mathrm{Z} x \wedge \mathrm{S} x)$

(4c) also illustrates how non-referring names are dealt with. Atheists denying the existence of Zeus can consistently claim the statement $\exists x(\mathrm{Z} x \wedge \mathrm{S} x)$ to be false, a possibility that was also provided for in Russell (1905), but is not available in theories that translate names as individual constants.

Let us look at identity statements, such as the infamous Hesperus is Phosphorus case. In (5a) we translate Phosphorus as $\Phi$, a translation that, I take it, can be inherited by is Phosphorus. ${ }^{4}$ The translation in $(5 \mathrm{c})$ is then obtained in a way analogous to the one in (4).

(5) a. Phosphorus $\leadsto \Phi$

b. is Phosphorus $\leadsto \Phi$

c. Hesperus is Phosphorus $\sim \exists x\left(\mathrm{H} x \wedge \Phi_{x}\right)$

d. Hesperus is Hesperus $\leadsto \exists x(\mathrm{H} x \wedge \mathrm{H} x)$

Note that it is consistent to assume that the semantic value of Hesperus is Phosphorus, $\exists x(\mathrm{H} x \wedge \Phi x)$, and that of Hesperus is Hesperus, given in $(5 \mathrm{~d})$ as $\exists x(\mathrm{H} x \wedge \mathrm{H} x)$, are completely distinct propositions, even if the first is true. Also, while $\forall x(\mathrm{H} x \leftrightarrow \Phi x)$ follows from $\exists x(\mathrm{H} x \wedge \Phi x)$ and the singularity requirement for name denotations (from which we get $\forall x y((\mathrm{H} x \wedge \mathrm{H} y) \rightarrow x=y)$ and $\forall x y((\Phi x \wedge$ $\Phi y) \rightarrow x=y)$ ), it does not follow that $\mathrm{H}=\Phi$ and $\mathrm{H}$ may well have properties that $\Phi$ lacks or vice versa. Co-extensionality crucially does not entail identity, having the same properties, in our theory and the theory allows for the possibility that Phosphorus has, but Hesperus fails to have, the property ( $\lambda X$.we do not know a priori that Hesperus is $X$ ), as in (1).

\subsection{Worlds, Necessity, and Rigidity}

Possible worlds are not needed to obtain true intensionality, and in fact cannot provide it, but they are immensely useful for modeling all kinds of modal phenomena. Here we construct them as certain properties of propositions (see

\footnotetext{
${ }^{4}$ A traditional way to obtain the translation of is Phosphorus from that of Phosphorus is to start with (5a), to then observe that Partee's A shifter allows for an interpretation of Phosphorus as $\lambda P . \exists x(\Phi x \wedge P x)$, as in the Zeus case. To the latter we could apply the linear combinator $\lambda Q \lambda R \lambda x \cdot Q(\lambda y \cdot R y x)$, which is generally useful for combining transitive verb meanings with the meanings of their direct objects. This would result in a translation $\lambda R \lambda x \cdot \exists z(\Phi z \wedge R z x)$, which, combined with the translation of $i s, \lambda x y \cdot x=y$, would lead to $\lambda x \cdot \exists z(\Phi z \wedge z=x)$. The latter is extensionally, but not intensionally, equivalent to $\Phi$.
} 
Muskens (2007) for more details). Propositions have type \langle\rangle , so properties of propositions have type $\langle\langle\rangle\rangle$, and the property of being a world, a property of properties of propositions has type $\langle\langle\langle\rangle\rangle\rangle$. We will write $\Omega$ for this special property and stipulate the following.

$\mathrm{W} 1 \forall w(\Omega w \rightarrow \neg w \perp)$

$\mathrm{W} 2 \forall w(\Omega w \rightarrow(w(A \subset B) \leftrightarrow \forall \vec{x}(w(A \vec{x}) \rightarrow w(B \vec{x}))))$

W1 requires world extensions to be consistent while addition of W2 makes worlds 'distribute over logical operators'. Statements such as the following become derivable.
a. $\forall w(\Omega w \rightarrow(w(\neg \varphi) \leftrightarrow \neg(w \varphi)))$
b. $\forall w(\Omega w \rightarrow(w(\varphi \wedge \psi) \leftrightarrow((w \varphi) \wedge(w \psi))))$
c. $\forall w(\Omega w \rightarrow(w(\forall x \varphi) \leftrightarrow \forall x(w \varphi)))$
d. $\forall w(\Omega w \rightarrow(w(\exists x \varphi) \leftrightarrow \exists x(w \varphi)))$

The first of these statements says that worlds are complete, while the last two are 'Henkin properties' that enforce, for example, that if an existential proposition is an element of the extension of a given world some proposition witnessing the existential must also be an element. In general, given $\mathrm{W} 1$ and $\mathrm{W} 2$, worlds single out sets of propositions that could be simultaneously true. The term $\lambda p . p$ (with $p$ of type \langle\rangle$)$ will be a world if we assume $\Omega(\lambda p . p)$ and it will then have the function of the actual world, as, in any model, $\lambda p . p$ will hold of $\varphi$ iff $\varphi$ is indeed true. Let us make $\Omega(\lambda p . p)$ into an official postulate and let's consider two more.

$$
\begin{aligned}
& \text { W3 } \Omega(\lambda p . p) \\
& \text { W4 } \forall w w^{\prime}\left(\left(\Omega w \wedge \Omega w^{\prime}\right) \rightarrow\left(w\left(w^{\prime} \varphi\right) \leftrightarrow\left(w^{\prime} \varphi\right)\right)\right) \\
& \text { W5 } \forall w\left(\Omega w \rightarrow \forall w^{\prime}\left(\Omega w^{\prime} \leftrightarrow w\left(\Omega w^{\prime}\right)\right)\right)
\end{aligned}
$$

W4 says that whether a proposition holds in a world is a global property, and W5 says something similar about the question whether a property of propositions is a world.

Once worlds are introduced, it becomes useful to associate domains with them. Some objects may exist in some worlds but not in others. We introduce a constant $\mathrm{E}$ of type $\langle e\rangle$ that will function as an existence predicate. Quantification over existing objects can then be obtained by relativizing to $\mathrm{E}$. For example, the type shifter A may now be redefined as $\lambda P^{\prime} P \cdot \exists x\left(\mathrm{E} x \wedge P^{\prime} x \wedge P x\right)$. This will lead to slightly revised translations, e.g. Hesperus is Phosphorus will now go to $\exists x(\mathrm{E} x \wedge \mathrm{H} x \wedge \Phi x)$.

Having worlds at our disposal, we can now express that $\varphi$ is globally necessary by writing $\forall w(\Omega w \rightarrow w \varphi)$ and we may abbreviate this as $\square \varphi .^{5}$ The following scheme says that names have singleton extensions in all worlds.

(6) $\square \exists x \forall y(\mathrm{~N} y \leftrightarrow y=x)$

\footnotetext{
${ }^{5}$ Muskens (2007) discusses modalities based on accessibility relations, but here we can make do without these.
} 
This entails (3) but no longer leaves open the possibility of empty denotation that was useful for non-referring names. Since we now have an existence predicate at our disposal, that possibility is no longer needed.

We now come to rigidity. There are various ways to model variants of the notion. Here is a strong and straightforward one.

(7) $\exists x \square \forall y(\mathrm{~N} y \leftrightarrow y=x)$

The idea is that for all names there is a possible object $o$ such that the name's extension is $\{o\}$ across all possible worlds. Clearly, in the presence of this requirement $\exists x(\mathrm{E} x \wedge \mathrm{H} x \wedge \Phi x)$ will entail $\square \exists x(\mathrm{H} x \wedge \Phi x)$, so if Hesperus is Phosphorus, it is necessary that Hesperus is Phoshorus wherever it exists and the usual Kripkean intuitions are formalised.

On the other hand codesignating names cannot be replaced for one another in arbitrary contexts. While Hesperus and Phosphorus have the same extension in all possible worlds, they may still have distinct intensions, as intension is not determined by extension, not even by extension in all possible worlds. And since $\exists x(\mathrm{E} x \wedge \mathrm{H} x \wedge \Phi x)$, and $\exists x(\mathrm{E} x \wedge \mathrm{H} x \wedge \mathrm{H} x)$ are completely distinct propositions it is possible, for example, to bear the relation of belief to the second but not to the first.

\section{Conclusion}

In this paper I have shown that Kripke's intuitions with respect to the rigid designation of proper names can be formalised in a way that does not result in a theory predicting the intersubstitutivity of codesignating names in arbitrary contexts. This means that this intersubstitutivity does not follow from the intuitions. The theory I have developed accepts rigidity of names, but rejects the Millian idea of direct reference, the idea that the meaning of a name is its bearer or at least is determined by its bearer. In the present theory a person can have many names, all with different intensions. 


\section{Bibliography}

C. Benzmüller, C. E. Brown, and M. Kohlhase. Higher Order Semantics and Extensionality. Journal of Symbolic Logic, 69, 2004.

M. Fitting. Types, Tableaus, and Gödels God. Kluwer Academic Publishers, Dordrecht, 2002.

G. Frege. Über Sinn und Bedeutung. In G. Patzig, editor, Funktion, Begriff, Bedeutung. Fünf Logische Studien. Vanden Hoeck, Göttingen, 1892.

L. Henkin. Completeness in the Theory of Types. Journal of Symbolic Logic, 15:81-91, 1950 .

S. Kripke. Identity and Necessity. In M. Munitz, editor, Identity and Individuation, pages 135-164. New York University Press, 1971.

S. Kripke. Naming and Necessity. In D. Davidson and G. Harman, editors, Semantics of Natural Language, pages 253-355. Reidel, Dordrecht, 1972.

R.A. Muskens. Meaning and Partiality. CSLI, Stanford, 1995.

Reinhard Muskens. Intensional Models for the Theory of Types. Journal of Symbolic Logic, 72(1):98-118, 2007.

B. Partee. Noun Phrase Interpretation and Type Shifting Principles. In J. Groenendijk, D. de Jongh, and M. Stokhof, editors, Studies in Discourse Representation and the Theory of Generalized Quantifiers, pages 115-143. Foris, Dordrecht, 1986.

B. Russell. On Denoting. Mind, 14(56):479-493, 1905.

A. N. Whitehead and B. Russell. Principia Mathematica. Cambridge University Press, 1913. 


\title{
Multiple Foci in Japanese Clefts and the Growth of Semantic Representation
}

\author{
Tohru Seraku ${ }^{1}$ \\ ${ }^{1}$ St. Catherine's College, Manor Road, Oxford, UK. OX1 3UJ \\ tohru.seraku@stcatz.ox.ac.uk
}

\begin{abstract}
There are two types of Japanese clefts, depending on the presence of a case particle attached to a focus; multiple foci are possible only in clefts with particles. This paper proposes that this distinct behavior with respect to multiple foci emerges as a consequence of the incremental growth of semantic structure within Dynamic Syntax; multiple foci are licensed when an underspecification for each item is resolved by each case particle. From this analysis also follows the partially case-specified foci and the so-called clause-mate condition.
\end{abstract}

Keywords: incrementality, structural underspecification, Dynamic Syntax

\section{Introduction}

Japanese clefts are divided into two types, depending on whether a focus item has a case particle or not [5], as illustrated in (1). In this paper, clefts with a particle are called "clefts + ", and clefts without a particle are called "clefts_".

$\begin{array}{lllll}{\left[\begin{array}{l}\text { Tom-ga } \\ \text { [T.-NOM }\end{array} \quad \text { but-ta }\right.} & \text { no]-wa } & \text { ringo(-o) } & \text { da. } \\ \text { 'It is an apple that Tom bought.' } & & \text { NO]-TOP } & \text { apple(-ACC) } & \text { COP }\end{array}$

As observed in [12], multiple foci are possible only in clefts $+\mathrm{P}$.

\begin{tabular}{|c|c|c|c|}
\hline $\begin{array}{ll}{\left[e_{i}\right.} & e_{j} \\
{[} & \end{array}$ & $\begin{array}{l}\text { purezento-o } \\
\text { present-ACC }\end{array}$ & $\begin{array}{l}\text { age-ta } \\
\text { give-PAST }\end{array}$ & $\begin{array}{l}n o]-w a \\
\text { NOM]-TOP }\end{array}$ \\
\hline $\operatorname{Tom}_{\mathrm{i}}^{*}(-g a)$ & $\operatorname{Mary}_{\mathrm{j}}{ }^{*}(-n i)$ & $d a$ & \\
\hline T.-NOM & M.-DAT & COP & \\
\hline
\end{tabular}

Within Principles-and-Parameters theory, the distinct behavior of clefts -P $_{\mathrm{P}} / \mathrm{clefts}_{+\mathrm{P}}$ with respect to multiple foci has been a challenge to a uniform analysis $[3,4,11,12,13$, $16]$; the tendency is to assume that the particle no in clefts $_{+\mathrm{P}}$ is a complementizer, while no in clefts $-\mathrm{P}$ is a pronominal. Kizu [10] is exceptional in presenting a unitary account, but her account is not applicable to multiple foci, as admitted in [10, p.54]. 
There is also an empirical puzzle that has been overlooked in the literature. Though case particles have been presumed to be obligatory in multiple foci, a second focus, but not a first focus, may be case-less, as shown in the contrast between (3) and (4).

\begin{tabular}{|c|c|c|c|}
\hline $\begin{array}{ll}{\left[\mathrm{e}_{\mathrm{i}}\right.} & \mathrm{e}_{\mathrm{j}}\end{array}$ & $\begin{array}{l}\text { purezento-o } \\
\text { present-ACC }\end{array}$ & $\begin{array}{l}\text { age-ta } \\
\text { give-PAST }\end{array}$ & $\begin{array}{l}n o]-w a \\
\text { NO]-TOP }\end{array}$ \\
\hline Tom $_{\mathrm{i}}-g a$ & Mary $_{\mathrm{j}}$ & $d a$. & \\
\hline T.-NOM & M. & COP & \\
\hline
\end{tabular}

\begin{tabular}{|c|c|c|c|}
\hline$*\left[\mathrm{e}_{\mathrm{i}}\right.$ & $\begin{array}{l}\text { purezento-o } \\
\text { present-ACC }\end{array}$ & $\begin{array}{l}\text { age-ta } \\
\text { give-PAST }\end{array}$ & $\begin{array}{l}n o]-w a \\
\text { NO]-TOP }\end{array}$ \\
\hline Tom $_{\mathrm{i}}$ & Mary $_{\mathrm{j}}-n i$ & & \\
\hline
\end{tabular}

There is a passing remark on the partially case-specified foci data in [4], but no analysis is given.

This paper develops a uniform analysis of clefts in connection with multiple foci, from the perspective of the incremental growth of semantic structure, as modeled in Dynamic Syntax. After Section 2 introduces the framework, Section 3 articulates a unified analysis of clefts, and Section 4 examines the issues in multiple foci. Finally, the analysis is extended to long-distance clefts in Section 5, where it is argued that the clause-mate condition [11] naturally follows from the analysis presented.

\section{Framework: Dynamic Syntax}

Dynamic Syntax is a grammar formalism that models "Knowledge of Language", which is defined as a set of constraints on the building-up of semantic structure $[1,9]$. With such constraints, a parser processes a string of words in order, and builds up semantic structure gradually. In this view, a string is directly mapped onto semantic structure; thus, there is no syntactic structure.

The growth of semantic structure is driven by combination of (a) general action, (b) lexical action, and (c) pragmatic action. (Pragmatic action is not directly relevant in this paper, and thus disregarded.) For illustration, let's consider how the string (5) is progressively mapped onto its semantic structure word-by-word.

$$
\begin{array}{ll}
\text { Mary-ga } & \text { nai-ta. } \\
\text { M.-NOM } & \text { cry-PAST } \\
\text { 'Mary cried.' } &
\end{array}
$$

The first item to be parsed is Mary. Japanese allows the permutation of arguments, and thus a parser cannot see at this point whether Mary turns out to be a subject or an object, etc. So, the node for Mary is unfixed at this stage. Formally, the general action of LOCAL *ADJUNCTION induces structural underspecification, or an unfixed node, which must be fixed in a local proposition. The lexical action of Mary then decorates 
this unfixed node. These two actions give rise to the semantic structure (6). In (6), the structural underspecification is notated with the dashed line.

(6)

$$
\begin{aligned}
& \text { Parsing Mary } \\
& \left(\iota, \widehat{\mathrm{x}}, \widehat{\operatorname{Mry}^{\prime}}(\mathrm{x})\right): \mathrm{e}, \diamond
\end{aligned}
$$

In (6), the top node is decorated with ?t, a requirement that this node be decorated with a type-t content (i.e. proposition). The unfixed daughter node has three pieces of information. First, ( $\iota, \mathrm{x}$, Mary' $\left.^{\prime}(\mathrm{x})\right)$ is a content of Mary. ( $\iota$ is an iota operator that binds the variable $\mathrm{x}$, which is restricted by Mary'.) Second, e states that the content is of semantic type-e. Third, the pointer $\diamond$ indicates a node under current development.

The underspecification in (6) is fixed by the lexical action of the nominative case particle $g a$, which fixes the node for Mary as a subject node, as in (7), where the dashed line becomes solid.

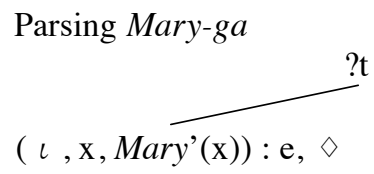

The next item is the predicate nai. Japanese being a pro-drop language, a predicate constructs an open proposition with argument slots. Thus, the lexical actions of nai updates (7) into (8), where a subject slot has been already identified.

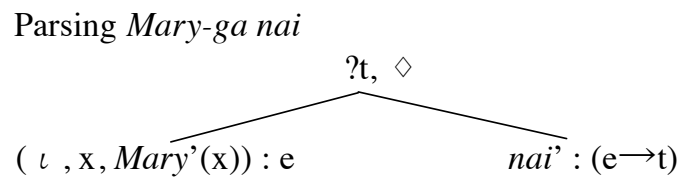

Finally, the general action of functional application composes the content at each daughter node, and the parse of $t a$ puts tense information PAST at the root node. ${ }^{1}$ The final state is given in (9); this structure is well-formed in the sense that no outstanding requirements? remain.

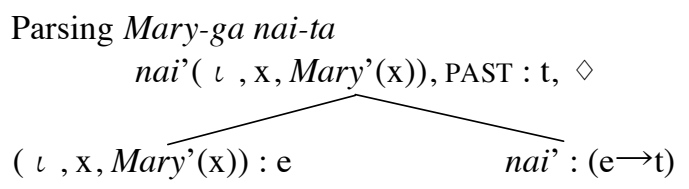

This section has introduced the framework of Dynamic Syntax: a parser constructs semantic structure incrementally on the basis of left-to-right word-by-word parsing.

${ }^{1}$ In a fully articulated structure, tense information is represented as an event term in Epsilon Calculus [2]. 


\section{Uniform Analysis of Clefts ${ }^{2}$}

\section{1 $\quad$ Clefts $_{+\mathbf{P}}$}

This sub-section provides a tree-transition for simple cases of clefts $_{+\mathrm{P}}$, such as (10). ${ }^{3}$

$\begin{array}{lllll}\text { [Nai-ta } & \text { no]-wa } & \text { gakusee-ga } & \text { san-nin } & \text { da. } \\ \text { [cry-PAST } & \text { NO]-TOP } & \text { student-NOM } & \text { 3-CL } & \text { COP } \\ \text { 'It is 3 students that cried.' } & & & \end{array}$

First, the parse of Nai-ta yields a proposition; the gap is notated as ( $\varepsilon, \mathrm{x}, \mathrm{P}(\mathrm{x})$ ), where $\varepsilon$ is an existential operator, and $P$ is an abstract restrictor [8, p.65]. Next, following [1, p.285], Seraku [15] regards no as a nominalizer that copies a type-e term within a proposition, and pastes it at a newly created node that is LINKed to the proposition. In (11), the LINK relation is notated by the curved arrow, and what is copied/pasted is the type-e term $(\varepsilon, x, P(x))$.

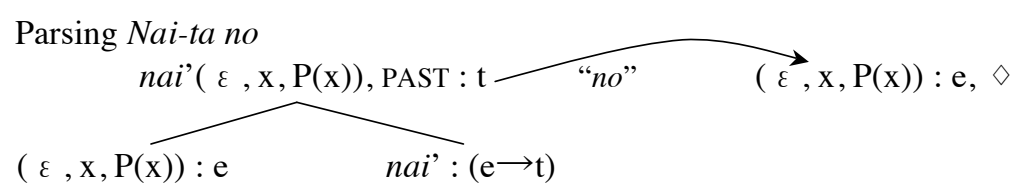

What comes next is the topic marker $w a$. The standard analysis of $w a[1, \mathrm{p} .268]$ is to put at a new type-t-requiring node the requirement $?<\mathrm{D}>(\varepsilon, \mathrm{x}, \mathrm{P}(\mathrm{x}))$, which requires that a node below this node should be decorated with $(\varepsilon, \mathrm{x}, \mathrm{P}(\mathrm{x})$ ). (This requirement will be met in (13) below, since the term $\left(\varepsilon, \mathrm{x}\right.$, gakusee' $\left.^{\prime}(\mathrm{x})\right)$ is stronger than the term $(\varepsilon, \mathrm{x}, \mathrm{P}(\mathrm{x}))$.)

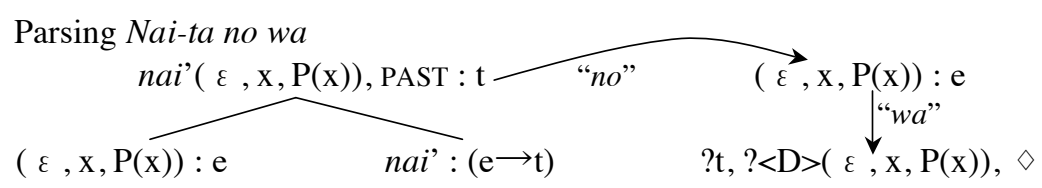

In order to parse the next item gakusee, structural underspecification is reuiqred. As mentioned in (6) in Section 2, the general action of LOCAL *ADJUNCTION induces an unfixed node (marked by the dashed line), which allows gakusee to be parsed.

2 The theoretical insights of this section are sketched in [15].

3 To many speakers, if a focus item has a nominative case particle $g a$, the string is not perfectly fine. As noted in [11], the string becomes acceptable if a numeral quantifier, such as san-nin in (10), is used. Also, a ga-marked focus is acceptable in multiple foci, as in (2) in Section 1. This tendency is stronger in Korean, where clefts with a nominative case-marked focus are completely unacceptable [6]. This paper sets aside the speaker variation concerning the acceptability judgments of case-marked foci in Japanese clefts. 


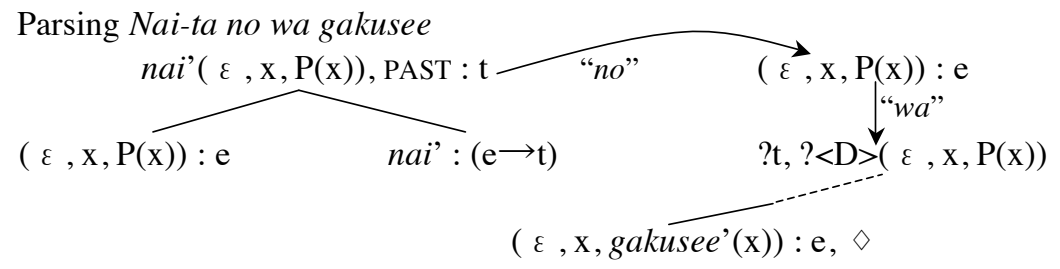

So far, the tree-transition is neutral between clefts $-\mathrm{P}$ and clefts $+\mathrm{P}$.

At this stage, the transition starts to differ between clefts $s_{-P}$ and clefts $+\mathrm{P}$, since only the latter has a case particle. In the cleft $_{+\mathrm{P}}(10)$, the case particle $g a$ is parsed, which fixes the unfixed node as a subject node, as indicated by the solid line in (14). (In (14), the quantifier san-nin has been parsed; note the strengthened operator $\varepsilon_{3}$.)

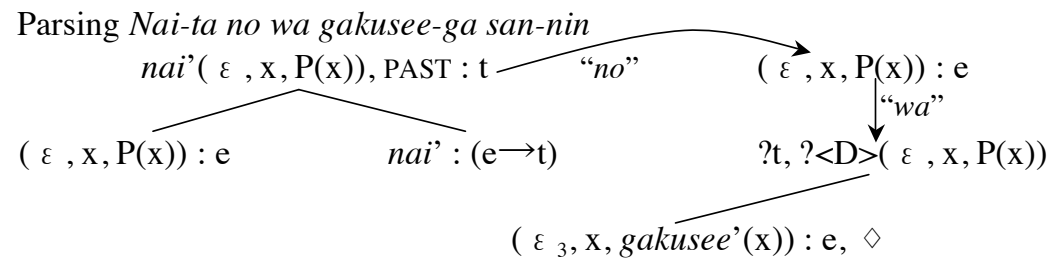

Finally, the copula $d a$ is parsed. Seraku [15] argues that $d a$ provides a type-t metavariable, which licenses the re-use of previous representation or actions. In the case of (15), the representation (i.e. the structure built up by the parse of Nai-ta) is re-used, where a subject node has been already present due to the parse of gakusee-ga.

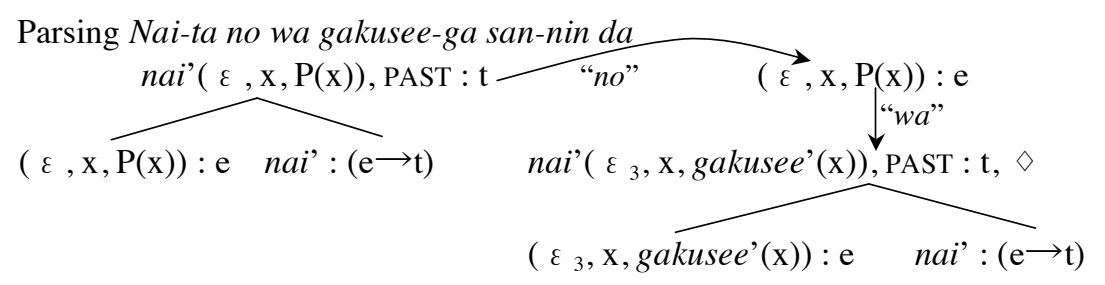

This semantic representation is the final state of the tree transition for the $\mathrm{cleft}_{+\mathrm{P}}(10)$.

\subsection{Clefts $_{-\mathrm{P}}$}

The analysis of clefts $_{+\mathrm{P}}$ is straightforwardly applicable to clefts_-P like (16).

$\begin{array}{lllll}\text { [Nai-ta } & \text { no]-wa } & \text { gakusee } & \text { san-nin } & \text { da. } \\ \text { [cry-PAST } & \text { NO]-TOP } & \text { student } & \text { 3-CL } & \text { COP } \\ \text { 'It is 3 students that cried.' } & & & \end{array}$

Given the transition above, the parse of Nai-ta no wa gakusee san-nin yields (17). 


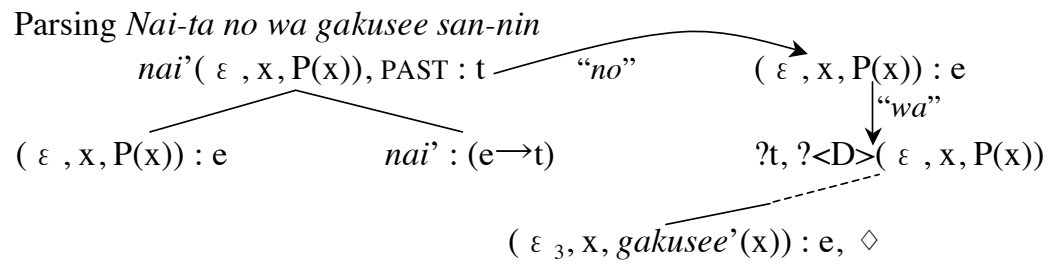

This time, the cleft $\mathrm{P}_{\mathrm{P}}(16)$ has no particle, and the transition proceeds without fixing the unfixed node. The parse of $d a$ then licenses the re-use of the previous structure.

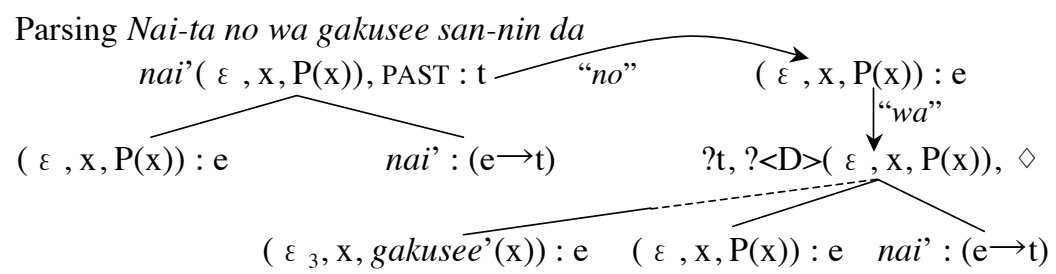

The subject node then unifies with the unfixed node by the general action of MERGE [1], engendering the final state (19). ${ }^{4}$

$$
\begin{aligned}
& \text { Parsing Nai-ta no wa gakusee san-nin da } \\
& n a i '(\varepsilon, \mathrm{x}, \mathrm{P}(\mathrm{x})) \text {, PAST : } \mathrm{t} \text { "no" }(\varepsilon, \mathrm{x}, \mathrm{P}(\mathrm{x})): \mathrm{e}
\end{aligned}
$$

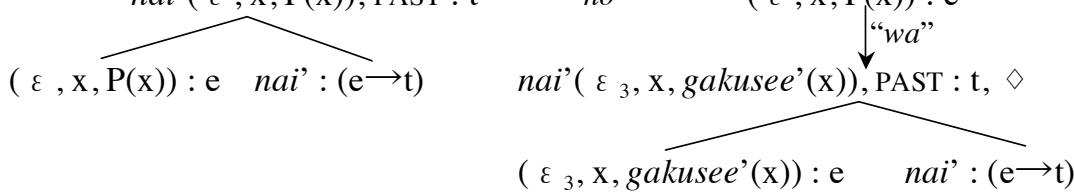

This structure of the cleft $_{-\mathrm{P}}(16)$ is identical to that of the cleft $_{+\mathrm{P}}(10)$. This ensures that the two types of clefts are truth-conditionally equivalent.

In sum, this section has sketched the unitary account of the two types of clefts in Japanese. The next section examines the issues in multiple foci.

\section{$4 \quad$ Multiple Foci}

In Section 3, when a focus item was parsed, an unfixed node was introduced as a result of the general action of LOCAL *ADJUNCTION. This general action may be conducted more than once from the same node only if an unfixed node has been fixed before another unfixed node is introduced [1, p.235]: this is because multiple nodes that are unfixed from the same node are indistinguishable, leading to inconsistency. This single-unfixed-node constraint accounts for the difference in the availability of

${ }^{4}$ Merge outputs $\left(\varepsilon_{3}, \mathrm{x}\right.$, gakusee $\left.^{\prime}(\mathrm{x}) \& \mathrm{P}(\mathrm{x})\right)$, but it is simply notated as $\left(\varepsilon_{3}, \mathrm{x}\right.$, gakusee $\left.^{\prime}(\mathrm{x})\right)$. The omission of the abstract restrictor $\mathrm{P}$ makes no difference if there is a concrete restrictor. 
multiple foci between the two types of clefts. First, in clefts $+\mathrm{P}$, multiple foci are licensed, since an unfixed node for each focus is fixed by each case particle. Second, in clefts $-\mathrm{P}$, the only means of fixing an unfixed node is a unification process. In order for unification to occur, however, a proposition must have been built up, and, as shown in Section 3, such a proposition is created only after the parse of $d a$. Thus, more than one item cannot be clefted in clefts - .

Within Dynamic Syntax, there are other two types of structural underspecification: *Adjunction and Generalized AdjunCtion [1]. A question, then, is: can these be utilized more than once from the same node? The answer is no, since an unfixed node induced by these actions is not fixed by the parse of a particle [1]. Thus, if these types of underspecification are induced, multiple foci are prohibited in clefts ${ }_{+\mathrm{P}}$ and clefts $\mathrm{P}$. One may object that this wrongly predicts that both clefts ${ }_{+P}$ and clefts $-P$ do not allow multiple foci. Nevertheless, the availability of multiple foci in clefts ${ }_{+P}$ is adequately modeled, as there is a successful tree transition based on LOCAL *ADJUNCTION.

This analysis also accounts for the partially case-specified foci in (3) in Section 1, repeated here as (20). In this case, the unfixed node for the second focus may be fixed by unification, and thus needs not to be fixed by the parse of a case particle.

$$
\begin{aligned}
& {\left[\mathrm{e}_{\mathrm{i}} \quad \mathrm{e}_{\mathrm{j}} \quad \text { purezento-o age-ta no }-w a\right.} \\
& \text { [ present-ACC give-PAST NO]-TOP } \\
& \text { Tom }_{\mathrm{i}^{-}} \text {ga } \text { Mary }_{\mathrm{j}} \quad \mathrm{da} \text {. } \\
& \text { T.-NOM M. COP } \\
& \text { Lit. 'It is } \mathrm{Tom}_{\mathrm{i}} \text { to } \mathrm{Mary}_{\mathrm{j}} \text { that } \mathrm{e}_{\mathrm{i}} \text { gave a present to } \mathrm{e}_{\mathrm{j}} \text {.' }
\end{aligned}
$$

\begin{tabular}{|c|c|c|c|}
\hline$*\left[\mathrm{e}_{\mathrm{i}}\right.$ & $\begin{array}{l}\text { purezento-o } \\
\text { present-ACC }\end{array}$ & $\begin{array}{l}\text { age-ta } \\
\text { give-PAST }\end{array}$ & $\begin{array}{l}n o]-w a \\
\text { NO]-TOP }\end{array}$ \\
\hline Tom $_{\mathrm{i}}$ & Mary $_{\mathrm{j}}-n i$ & & \\
\hline
\end{tabular}

To some speakers, (20) may not be completely fine, but it is much better than (21).

In (21), Tom has no particle, and the underspecification for Tom is not resolved. Thus, another structural underspecification cannot be induced for Mary.

As mentioned above, unification requires that a proposition be present. Given that a proposition is constructed by the parse of $d a$, unification is applicable only to a final focus. Thus, it is further predicted that a cleft is acceptable where there are three foci and only the final focus lacks a case particle. This prediction is borne out:

$$
\begin{aligned}
& {\left[\begin{array}{lllll}
\mathrm{e}_{\mathrm{i}} & \mathrm{e}_{\mathrm{j}} & \mathrm{e}_{\mathrm{k}} & \text { age-ta } & \text { no }-w a
\end{array}\right.} \\
& \text { [ give-PAST NO]-TOP } \\
& \text { Tom }_{\mathrm{i}} \text {-ga Mary }- \text { ni } \text { purezento }_{\mathrm{k}} d a \text {. } \\
& \text { T.-NOM M.-DAT present COP } \\
& \text { Lit. 'It is } \operatorname{Tom}_{\mathrm{i}} \text { to } \mathrm{Mary}_{\mathrm{j}} \text { a present }_{\mathrm{k}} \text { that } \mathrm{e}_{\mathrm{i}} \text { gave } \mathrm{e}_{\mathrm{k}} \text { to } \mathrm{e}_{\mathrm{j}} \text {.' }
\end{aligned}
$$

Again, (22) may not be perfectly fine, but it is much better than cases in which a case particle is dropped off foci other than the final focus (i.e. either the first or the second 
focus, or both). Such cases are all unacceptable, since any unfixed nodes for a nonfinal focus without a case particle cannot be fixed.

In a nutshell, multiple foci are constrained by the incremental updating of semantic structure that involves structural underspecification and subsequent resolution.

\section{Long-distance Clefts}

The analysis developed so far can be extended to long-distance clefts, like (23). Based on the treatment of long-distance scrambling proposed in [7], the transition for (23) is as in (24); after $w a$ is parsed, a type-t-requiring node is introduced by *ADJUNCTION, and the foci are parsed by LOCAL *ADJUNCTION below this node. ${ }^{5}$

$\begin{array}{lllll}\text { [Mary-ga } & {\left[\mathrm{e}_{\mathrm{i}} \quad \mathrm{e}_{\mathrm{j}}\right.} & \text { purezento-o } & \text { age-ta } & \text { to }] \\ \text { [M.-NOM } & {[} & \text { present-ACC } & \text { give-PAST } & \text { COMP] } \\ \text { it-ta } & n o]-w a & \text { Ken }_{\mathrm{i}} \text {-ga } & \text { Tom }_{\mathrm{j}}-n i & d a . \\ \text { say-PAST } & \mathrm{NO}]-\mathrm{TOP} & \text { K.-NOM } & \text { T.-DAT } & \text { COP }\end{array}$

Lit. 'It is $\mathrm{Ken}_{\mathrm{i}}$ to $\mathrm{Tom}_{\mathrm{j}}$ that Mary said that $\mathrm{e}_{\mathrm{i}}$ gave a present to $\mathrm{e}_{\mathrm{j}}$ ',

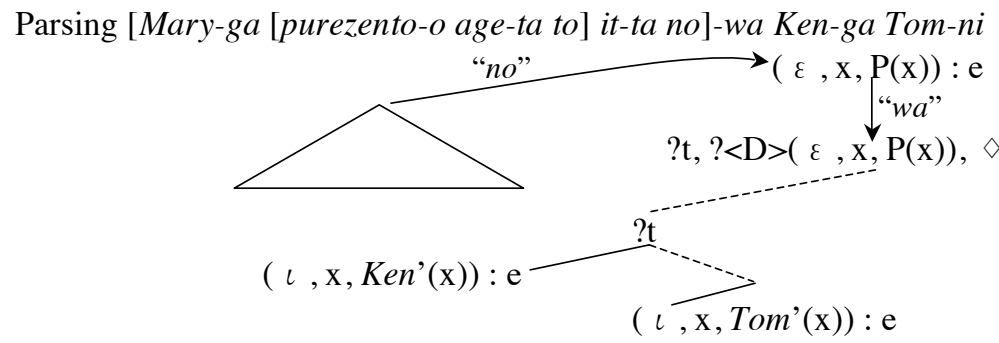

The rest of the transition is as usual; $d a$ licenses the re-use of the representation (i.e. the structure built up by the parse of Mary-ga purezento-o age-ta to it-ta), and after functional applications, the complete semantic structure emerges.

Interestingly, the string (23) is ambiguous; the following reading is also possible.

\begin{tabular}{|c|c|c|c|c|}
\hline$\left[\mathrm{e}_{\mathrm{i}}\right.$ & [Mary-ga & purezento-o & age-ta & to $]$ \\
\hline$\Gamma$ & [M.-NOM & present-ACC & give-PAST & COMP] \\
\hline it-ta & no]-wa & $K e n_{\mathrm{i}}-g a$ & $\operatorname{Tom}_{\mathrm{j}}-n i$ & $d a$. \\
\hline ay-PAST & NO]-TOP & K.-NOM & T.-DAT & $\mathrm{COP}$ \\
\hline
\end{tabular}

Lit. 'It is $\mathrm{Ken}_{\mathrm{i}}$ to $\mathrm{Tom}_{\mathrm{j}}$ that $\mathrm{e}_{\mathrm{i}}$ said to $\mathrm{e}_{\mathrm{i}}$ that Mary gave a present to someone.'

In this case, the foci are parsed without the use of *ADJUNCTION; that is, the foci are parsed by LOCAL *ADJUNCTION directly below the node decorated with ?t, ? $<\mathrm{D}>(\varepsilon$, $\mathrm{x}, \mathrm{P}(\mathrm{x}))$. The parse of $d a$ then licenses the re-use of the propositional structure, into which the content of each focus is reflected.

In this string, what is not possible is the reading (26).

4625 The node for Tom is not fixed at this point to move the pointer $\diamond$ back to the top node [7]. 


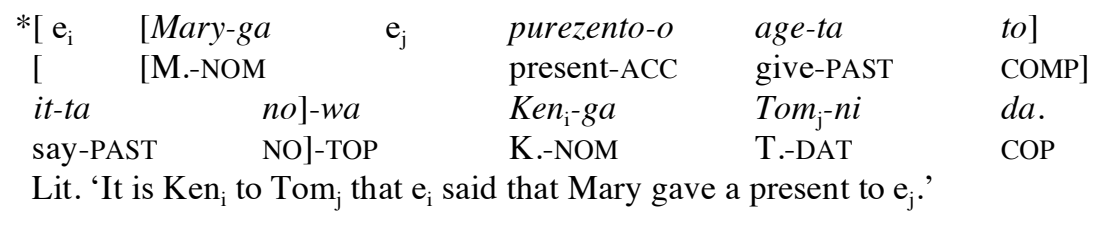

This indicates that foci must belong to the same local clause. This restriction has been already observed, and called the "clause-mate" condition [11]. There have been some attempts to account for this restriction, but they are not fully persuasive; Takano [16], one of the most advanced works on this matter, ends up positing the extra mechanism to create "surprising constituents".

In my analysis, however, the clause-mate condition emerges as a by-product of the gradual updating of semantic representation. First, in (23), the foci are parsed below the type-t-requiring node that is created by *ADJUNCTION. Second, in (25), the foci are parsed below the type-t-requiring node that is decorated by the parse of wa. Thus, the content of each focus is reflected into the same local proposition, and the reading in (26) is not possible. The upshot is that what is called the clause-mate condition is a result of the progressive development of semantic representation, which renders the condition merely an epiphenomenon.

As a further confirmation, if kinoo (= 'yesterday') is inserted between the foci Ken and Tom in (23)/(25), as in Ken-ga kinoo Tom-ni, the expected pattern occurs. In (23), kinoo can modify only the most embedded clause; in (25), kinoo can modify only the clause whose main predicate is it (= 'say'). This is because, in each case, kinoo is parsed below the same type-t-requiring node as the foci are parsed.

In closing, let me discuss a potential problem. To prevent a $g a$-marked item from being long-distance scrambled, Cann et al. [1, p.254] suggest that ga encodes a constraint that the node for a $g a$-marked item be fixed immediately. If this constraint is maintained, long-distance clefts with a $g a$-marked focus should not be possible; as shown in (23), however, such clefts are possible. Indeed, the long-distance scrambling of $g a$-marked item is acceptable if an embedded predicate is compatible only with the scrambled item [14, pp.176-7]. Thus, the immediate-closure constraint should be relaxed from the lexical entry of $g a$, and the acceptability of (23) is not unexpected. ${ }^{6}$

\section{Conclusion}

This paper has argued that Japanese clefts are uniformly characterized, and the issues in multiple foci are nicely handled, by the incremental growth of semantic structure that involves "structural underspecification and subsequent resolution". The analysis

${ }^{6}$ In Korean, the long-distance scrambling of a nominative-case-marked item is fine. Kempson and Kiaer [7, p.166] claim that in Korean, the immediate-closure constraint is not encoded, but a default option. My point is that in Japanese, too, the constraint is a default option, though the tendency for the default option is stronger in Japanese. In the case of clefts, the structure built up by the parse of a presupposition clause highlights the content of a gap ( $\varepsilon$, $x, P(x)$ ), and it signals a parser to override the default option. 
should be evaluated in four respects. First, the single entry of no as a nominalizer that copies a type-e term leads to the uniform treatment of clefts $_{-\mathrm{P}} / \mathrm{clefts}_{+\mathrm{P}}$. Second, the output of the tree growth captures the parallelism of the two types of clefts: truthconditional equivalence. Third, the process of tree growth captures the difference: availability of multiple foci. Fourth, restrictions on multiple foci (i.e. partially casespecified foci and the clause-mate condition) are explained as a by-product of the tree growth. These four achievements show clear advantages over previous studies within Principles-and-Parameters theory, where, as stated in Section 1, multiple foci have been an obstacle to a unified treatment, and the data such as partially case-specified foci and the clause-mate condition have posed empirical puzzles.

Finally, a general implication for linguistic theory. The issues in multiple foci are normally assumed to be "syntactic" in that they have been analyzed on the basis of syntactic operation/representation. In contrast, this paper has argued that they can be fruitfully dealt with semantically (i.e. in light of the incremental semantic-structure building). This reducibility of syntactic issues to semantic tree growth illustrates the significance of incrementality in linguistic theorizing.

Acknowledgments. I'd like to thank David Cram, Ruth Kempson, and Jieun Kiaer for their valuable comments on the draft of this paper.

\section{References}

1. Cann, R., Kempson, R., Marten, L.: The Dynamics of Language. Elsevier, Oxford (2005)

2. Gregoromichelaki, E.: Conditionals in Dynamic Syntax. In: Kempson, R., et al. (eds.) The Dynamics of Lexical Interfaces, pp. 237--278, CSLI, Stanford (2011)

3. Hasegawa, N.: A Copula-based Analysis of Japanese Clefts. In: Inoue, K. (ed.) Grand-inAid for COE Research Report (1), pp. 15--38, KUIS, Chiba (1997)

4. Hiraiwa, K., Ishihara, S.: Syntactic Metamorphosis. Syntax (to appear)

5. Hoji, H.: Theories of Anaphora and Aspects of Japanese Syntax. Ms., USC (1990)

6. Kang, B.: Some Peculiarities of Korean Kes Cleft Constructions. Studia Linguistica 60, 251-281 (2006)

7. Kempson, R., Kiaer, J.: Multiple Long-distance Scrambling. Journal of Linguistics 46, 127-$192(2010)$

8. Kempson, R., Kurosawa, A.: At the Syntax-Pragmatics Interface. In: Hoshi, H. (ed.) The Dynamics of Language Faculty. Kuroshio, Tokyo (2009)

9. Kempson, R., Meyer-Viol, W., Gabbay, D.: Dynamic Syntax. Blackwell, Oxford (2001)

10.Kizu, M.: Cleft Constructions in Japanese Syntax. Palgrave, New York (2005)

11.Koizumi, M.: String Vacuous Overt Verb Raising. Journal of East Asian Linguistics 9, 227-$285(2000)$

12.Kuroda, S.-Y.: Tokoro-Clause (Tokoro-Setsu). In: Kuroda, S.-Y. Generative Grammar from the Viewpoint of Japanese. (Nihongo kara Mita Seesee Bunpoo) Iwanami, Tokyo (2005)

13.Kuwabara, K.: Multiple Wh-Phrases in Elliptical Clauses and Some Aspects of Clefts with Multiple Foci. MITWPL 29, 97-116 (1996)

14.Mihara, K.: Tense Interpretation and Syntactic Phenomena. (Jisee Kaisyaku to Toogo Gensyoo) Kuroshio, Tokyo (1992)

15.Seraku, T.: On the Polyfunctionality of Copula Sentences in Japanese. Proceedings of the Sixth Cambridge Postgraduate Conference in Language Research (2011)

464 16.Takano, Y.: Surprising Constituents. Journal of East Asian Linguistics 11, 243--301 (2002) 


\title{
Nuu-chah-nulth Evidentials and the Origo
}

\author{
Ryan Waldie \\ University of British Columbia
}

\section{Incarnations of the Origo}

Evidentials are morphemes like apparently and reportedly which indicate the relation between a proposition and a particular person - the origo (Garrett 2001). In this paper I look at how the origo is determined in different clausal environments in a particular language, Nuu-chah-nulth. ${ }^{1}$ I propose to treat the origo as an argument of all propositions, and show how this can account for the assignments of origos in different kinds of clauses.

The origo as a concept is not new. Among its incarnations is the judge of Lasersohn (2005) and Stephenson $(2007,2008)$, which was proposed to account for the readings of embedded predicates of personal taste and epistemic modals. The centred worlds of Lewis (1979) and Chierchia (1989), which were proposed to account for the readings of embedded de se attitudes, are another incarnation of this idea.

Statements involving predicates of personal taste are only true relative to a particular individual (Kölbel 2002). In main clauses this individual, called the judge by Lasersohn (2005), is normally the speaker, while in embedded clauses this individual is the attitude holder specified in the main clause. Thus the sentence in (1a) is true if I brussels sprouts taste good to me, while that in (1b) is true if brussels sprouts taste good to Ken.

(1) a. The brussels sprouts taste good.

b. Ken thinks the brussels sprouts taste good.

Stephenson (2007) points out that epistemic modals are also interpreted relative to a judge. The judge is assigned the same way as in the cases of predicates of personal taste. In a main clause the judge is usually the speaker, while in embedded clauses it is the attitude holder. Thus in (2a) I am the one inferring that it rained, while in (2b) Kay is the one making the inference.

(2) a. It must have rained last night.

b. Kay thinks it must have rained last night.

Note that in both cases the problem to be solved is a reading of something embedded under a propositional attitude verb. Stephenson proposes that the embedded proposition has an individual argument in addition to a world and a time.

\footnotetext{
${ }^{1}$ Nuu-chah-nulth belongs to the Wakashan family, and is spoken on the west coast of Vancouver Island, British Columbia. The data presented here is from the Ahousaht dialect.
} 
Stephenson $(2007,71)$ also points out that in an interrogative, the judge of a predicate of personal taste is the addressee. As the speaker of (3) I am asking if the addressee thinks the brussels sprouts taste good, even though I do not like them myself.

(3) Do the brussels sprouts taste good?

The incarnation of this concept under the name "origo" is due to Garrett (2001), who used it in his account of evidentials in Tibetan. He identified the origo as "the person from whose perspective a given evidential is evaluated" (Garrett 2001, 4), and pointed out that, while the origo of an evidential is identified with the speaker in declarative sentences, in questions the origo is the addressee. This distinction is illustrated below in examples from Nuu-chah-nulth. In (4) the evidential -k'uk 'visual inference' occurs in a declarative, and the origo is the speaker - the speaker is the one making the inference from something she visually observed. Note also that the fact that this is a declarative is indicated by the indicative mood suffix -?irš.

(4) Scenario: Kay and her son Bill live in the same house as Kay's sister Ann. Ann went out of town for a few days, but hadn't been sure what day she would return. One day Kay and Bill came home and Kay saw Ann's shoes, and she said this to Bill.

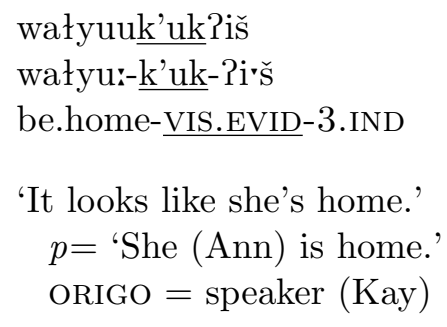

When the same evidential occurs in a question, as in (5), the origo is now the addressee. The addressee Bill is being asked by the speaker Kay whether his aunt is home, but the speaker assumes his answer will involved an inference from something he has visually observed. Note that the fact that this is an interrogative is indicated by the interrogative mood suffix -h.

(5) Scenario: Kay and her son Bill live in the same house as Kay's sister Ann. Ann went out of town for a few days and hadn't been sure what day she would return. One day Kay and Bill came home, and Bill went in first. Wondering if Bill could tell if his aunt was home, Kay asked him this.
wałyuuk'ukh
na?i
wałyu:-k'uk-h
na?i
be.home-VIS.EVID-3.INTER aunt/uncle 


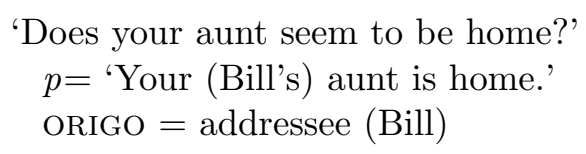

In Tibetan it is not possible for an evidential to be embedded under a propositional attitude verb, so the similarity between origos and judges and centred worlds is not so clear. In Nuu-chah-nulth, evidentials can be embedded under these verbs, and we can see that the origo patterns with judges in its behaviour. In (6) the origo of - $k$ 'uk 'visual inference' is the attitude holder, Linda, who is the subject of the main clause - she is the one who is making an inference from something she visually observed.

(6) Scenario: Linda saw lights on at Ken's place after he had been out of town for a few days, and she told Kay that it looked like Ken was home. Later, Kay said this to Bill.

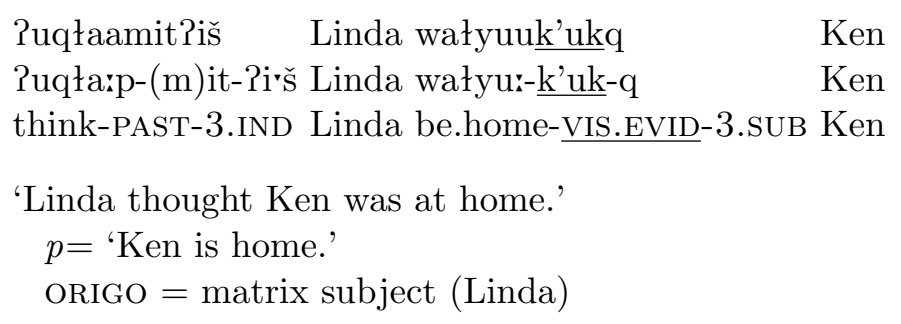

My main goal in this paper is to show that by making the origo an argument, we can account for the range of interpretations of evidentials in different clausal environments. I also discuss why the origo is distinct from the embedded contexts of Schlenker (2003): verbs of saying operate over contexts while propositional attitude verbs operate over origos, and

\section{Origo as a Semantic Argument}

An origo is equivalent to an ordered triplet $\langle j, w, t\rangle$ consisting of an sentient entity $j$, a world $w$ and a time $t$. It is possible to treat each element separately in the semantics, but for simplicity's sake I treat it as a single variable, $o$, of its own type.

The origo acts as an index of evaluation: every proposition can be thought of as the set of origos who believe it is true. In addition, situations (or events) are necessary for the semantics of evidentials, resulting in the basic schema for predicates, shown below:

(7) $\lambda s \lambda o[P(s)(o)]$

To give a concrete example of a predicate with an origo, a weather verb like miłaa 'be raining' will thus be translated as follows:

(8) $\quad$ mixaa $\Rightarrow \lambda s \lambda o[\operatorname{raining}(s)(o)]$ 
The approach outlined here is an extension of Stephenson's (2007) analysis of predicates of personal taste to all predicates. A predicate of personal taste, like taste good, treating it as an atomic predicate, will have the translation given in (9) below.

(9) $\lambda x \lambda s \lambda o[\operatorname{taste} \cdot \operatorname{good}(s)(o)(x)]$

\section{Origo Assigned by Mood}

Every clause in Nuu-chah-nulth contains a mood suffix which also expresses subject agreement. Several of these moods occur only in main clauses, not embedded clauses. Others occur in embedded clauses. I propose that main clause moods saturate the origo argument of the proposition of the clause, while the other moods do not. As I discuss in the next two sections, other mechanisms are at work in embedded clauses.

The indicative mood is used in making declarative statements; it assigns the speaker $s p$ as the origo. The speaker as origo corresponds to the triple $\langle s p *, w *, t *\rangle$, consisting of the speaker of the utterance, the utterance world and the utterance time.

(10) $\quad-\mathrm{i} \mathrm{i}\ulcorner\check{\mathrm{s}} \Rightarrow s p$

If there is an evidential in a clause with the indicative mood, as in (4) above, the evidential will describe the relation between the speaker and the prejacent proposition. Before showing how the derivation of such a sentence proceeds, I give the translation of the evidential $-k^{\prime} u k$ in (11) below. This translation is a much abbreviated form of the full meaning of $-k^{\prime} u k$. The relation vis.inf $(s)(o)\left(P\left(s^{\prime}\right)\right)$ indicates both that the origo is making an inference from some premise that holds at $s$ to the conclusion $P\left(s^{\prime}\right)$, and that the origo perceived $s$ visually. As well, $-k^{\prime} u k$ stipulates that the two situations $s$ and $s^{\prime}$ must overlap temporally. But for this paper it suffices treat this as a single relation. Also note that -k'uk introduces a context dependent situation variable, the situation in which the prejacent holds.

(11) $-k^{\prime} u k_{1} \Rightarrow \lambda P \lambda s \lambda o\left[\operatorname{vis.inf}(s)(o)\left(P\left(s_{1}\right)\right)\right]$

The structure shown in (12a) is for the sentence miłaak'uk?i'š '(It looks like) it's raining'. A null tense morpheme - $\varnothing$ will provide a contextually determined situation to saturate the situation argument of the predicate. The translation of this sentence is given in (12b); the speaker has visually perceived situation $s_{2}$, and from that infers the proposition that it is raining.

$$
\begin{aligned}
& \text { a. }\left[\left[\left[\min ^{\prime} \mathrm{ar}^{\prime}-\mathrm{k}^{\prime} \mathrm{uk}_{1}\right]-\emptyset_{2}\right]-\mathrm{Pi}\ulcorner\check{\mathrm{s}}]\right. \\
& \text { b. } \operatorname{vis.inf}\left(s_{2}\right)(s p)\left(\lambda o\left[\operatorname{raining}\left(s_{1}\right)(o)\right]\right)
\end{aligned}
$$

The interrogative mood assigns the addressee $a d$ as the origo. Again, the addressee is treated as an ordered triple, $\langle a d *, w *, t *\rangle$. This suffix also introduces interrogative semantics; in (13) I indicate this by the function Alt, which returns the set of alternatives of its argument. 
(13) $\quad-\mathrm{h} . \lambda p[\operatorname{Alt}(p(a d))]$

A question like miłaak'ukh 'Does it look like it's raining?' has a structure similar to that for the corresponding declarative sentence, and this structure is given in (14a) below, followed by its translation in (14b).

$$
\begin{aligned}
& \text { a. }\left[\left[\left[\operatorname{mì}^{\prime} \mathrm{ar}_{-}-\mathrm{k}^{\prime} \mathrm{uk}_{1}\right]-\varnothing_{2}\right]-\mathrm{h}\right] \\
& \text { b. } \operatorname{Alt}\left(\operatorname{vis} \cdot \inf \left(s_{2}\right)(a d)\left(\lambda o\left[\operatorname{raining}\left(s_{1}\right)(o)\right]\right)\right)
\end{aligned}
$$

\section{Origos Assigned by Propositional Attitude Verbs}

Clauses which are the complements of propositional attitude verbs do not have their origo argument saturated, but instead have it restricted by the propositional attitude verb to being its attitude holder, generally its subject.

$$
\text { Puqłaap } \Rightarrow \lambda p \lambda x \lambda s \lambda o\left[\operatorname{think}(s)(o)(x)\left(\lambda o^{\prime}\left[p\left(o^{\prime}\right)\right]\right)\right]
$$

A propositional attitude verb like this will be felicitous if the origo $o$ believes that $x$ is the origo for the content of the embedded clause. Thus, he sentence in (6) above, which contained -k'uk embedded under ?uqłaap 'think' will be felicitous if the speaker believes that Linda has inferred that Ken was home from something she has seen (as of some situation $s_{1}$ that precedes the utterance time).

(16) $\lambda o\left[\right.$ be.home $\left(s_{1}\right)(o)($ Ken $\left.)\right]$

\section{Context Dependent Origos in Adjunct and Relative Clauses}

Neither adjunct clauses nor relative clauses occur with main clause moods which assign an origo, nor do they occur as the complement of a propositional attitude verb which restricts the origo. Because these other mechanisms are not present, the origo is free to be context dependent.

In (17) below, the evidential -matak 'inference' occurs in a relative clause clause, and this sentence can be used in scenario 1 where the origo is the speaker, or in scenario 2 where the origo is the subject of the main clause.

a. Scenario 1 (origo = speaker):

Kay knows that some of Ken's money was stolen, and that John was staying with him. Linda doesn't know about the theft, but she recently saw John at the mall, and told the Kay this. Later Kay said this to Bill.

b. Secenario 2 (origo = main clause subject):

Kay knows that some of Ken's money was stolen, but not that anyone was staying with him. Linda knows that John was staying with him, and recently saw him at the mall. Linda told Kay that she saw the man who might have stole 
Ken's money. Later Kay said this to Bill. Kay still doesn't know that anyone was staying with Ken, only that Linda saw someone who she thinks might have stolen the money.

c. nhaatsiičiðitwa?iš Linda čakup yaqmatakitiič

na:tsi-ši $(\lambda)-(m)$ it-wa·?iš Linda čakup yaq ${ }^{\mathrm{w}}$-matak-(m)it-(y)irč

see-MOM-PAST-3.QUOT Linda man REL-IND.EVID-PAST-3.INDF.INF

kuuwiłčip Ken taana

kuwil-čip Ken ta:na

steal-BEN Ken money

'Linda saw the man who might have stole Ken's money.'

I have not found a suitable way to model the context dependent determination of the origo in adjunct and relative clauses. They will have the same structure as a complement clause, namely a proposition with an unsaturated origo argument. In order for its origo to be context dependent, it should be saturated with an indexed origo variable.

There is a resemblance between the bound and free origo readings and bound and free pronoun readings. A pronoun like he can be bound by an operator, or it can be context dependent. However, a pronoun can remain context-dependent in the scope of an quantifier, while an origo is always bound in the scope of a propositional attitude verb. Treating all origos as indexed variables will therefore not correctly capture their behaviour in complement clauses. The problem remains.

\section{Origos vs. Embedded Contexts}

The origo also shares some properties with embedded contexts, in the sense of Partee (1989), who invoked them to account for predicates with implicit context-dependent arguments, and Schlenker (2003), who invoked them for embedded shiftable indexicals. Here I argue that thy are distinct, and both are necessary for the correct interpretation of evidentials in Nuu-chah-nulth. One evidential in Nuu-chah-nulth, na?aat 'auditory evidence' has a restriction on it requiring its origo to be the speaker in a main clause, or the speaker of an embedded speech act. It thus behaves like a shifted indexical when it is embedded under a verb of saying.

I follow Schlenker (2003) in analyzing verbs of saying as introducing operators which bind a context variable in their complement clause, though I use a slightly different notation. The Nuu-chah-nulth verb wawaa 'say' will be translated as follows:

(18) wawaa $\Rightarrow \lambda P \lambda x \lambda s \lambda o[\operatorname{say}(s)(o)(x)(\lambda c[P(s p(c))])]$

The auditory evidential naPaat is felicitous in an embedded speech clause regardless of the identity of the origo. In (19) the verb wawaa 'say' introduces a complement clause which represents a speech act performed by the subject Linda. The speaker of the entire sentence is Kay, but she is not the one who has auditory perceptual grounding for the proposition that Ken is home. 
(19) Scenario: Linda lives next door to Ken, and was going to let Kay know when he got home. She heard noises from his apartment, and called Kay to tell her. Then Kay said this to Bill.

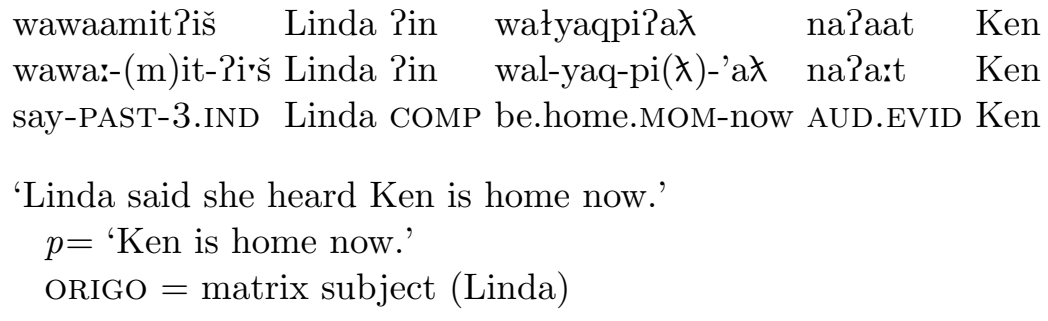

The auditory evidential na?aat cannot be embedded under a propositional attitude verb like Puqłaap 'think', as we see in (20), with one exception which I turn to shortly. Here the origo of the complement clause will be Linda, the attitude holder. Since she is not a speaker in any utterance context in the discourse, na?aat cannot be used to indicate that she has auditory evidence for Ken being home.

$$
\begin{aligned}
& \text { (20) *?uqłaamit?iš Linda wałyuuq Ken na?aat } \\
& \text { Puqła:p-(m)it-Pi'š Linda wałyus-q Ken na?a:t } \\
& \text { think-PAST-3.IND Linda be.home-3.suB Ken AUD.EvID }
\end{aligned}
$$

But what happens when the attitude holder happens to be the speaker? If na?aat only requires that its origo be the speaker of an utterance context, we would expect it to be felicitous in such a case. This prediction is borne out by the data. In (21) we have an example where the attitude holder is the speaker, indicated by first-person singular agreement. Because of this, the origo of the complement clause is also the speaker, and na?aat 'auditory evidence' is felicitous. The speaker Kay is saying that she herself thinks Ken is home, and na?aat is indicating that the speaker has auditory perceptual grounding for this.

(21) Scenario: Kay lives in the apartment next to Ken. He had been out of town for a while when she heard cupboard doors being closed in his apartment. Later she said this to Bill.

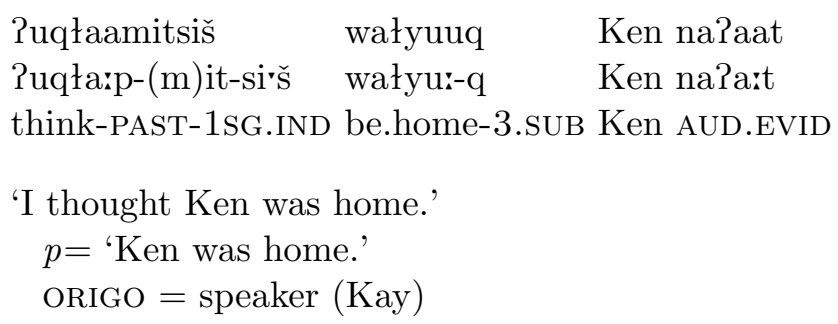


To summarize, the auditory evidential na?aat can only be used when the origo is a speaker. A verb of saying introduces embedded utterance context, thus allowing na?aat to occur in an embedded speech clause. This differs from propositional attitude verbs, where na?aat is only felicitous if the attitude holder is also the speaker. In order to capture the difference in behaviour between propositional attitude verbs and verbs of saying, we need to treat the former as introducing an operator over origo variables, and treat the latter as introducing an operator over context variables.

\section{The Origo and "Direct Evidence"}

The term "direct evidential" or "direct evidence" is vague (Matthewson 2010), and I need to be specific. By a direct evidential I mean an evidential which is used only when the origo is certain that the prejacent proposition is true. This contrasts with an indirect evidential, which is used only when the origo is uncertain about the truth of the prejacent proposition. This use of "direct evidential" is distinct from any sense in which the origo has observed the situation of the prejacent proposition itself, as opposed to inferring the prejacent proposition or hearing a report stating the prejacent proposition. My claim is that by treating the origo as an argument, direct evidence in the sense of origo certainty falls out from the pragmatics of assertion.

The indicative mood in Nuu-chah-nulth is used when the speaker is certain about a proposition, as in (22) below, yet this does not mean it is a direct evidential. In fact, it cannot be one, since it also occurs in clauses which also contain an indirect evidential, as we saw in (4) above.

(22) Scenario: Kay walks in from outside, shaking the rain off her jacket, and says this to Bill, who had the curtain closed.

$$
\begin{aligned}
& \text { miłaaa?iš } \\
& \text { miłar-Pi'řs } \\
& \text { rain.DuR-3.IND } \\
& \text { 'It's raining.' }
\end{aligned}
$$

The lack of a direct evidential in Nuu-chah-nulth follows from the fact that when there is no indirect evidential, the indicative mood suffix saturates the origo of the proposition directly, and identifies it with the speaker. Because the indicative mood also specifies that the proposition is asserted, and because an assertion is only felicitous if the speaker believes it to be true, it follows that the speaker is certain about the truth of the proposition. There is thus no need for a dedicated direct evidential.

\section{Conclusion}

In this paper I argued that the origo should be treated as an argument. By doing so we can account for the different assignments it gets in declaratives, interrogatives, and complement 
clauses. I also describe the assignments in adjunct and relative clauses, but as of yet I lack a way to model this assignment.

I also argued that propositional attitude verbs introduce an operator over origo variables, while verbs of saying introduce an operator over context variables. The behaviour of the auditory evidential na?aat supports this, as it is only felicitous when its origo is the speaker of some utterance context.

Finally, I discussed how direct evidentials, in the sense of indicating the origo's certainty, are unnecessary. The lack of an indirect evidential and the felicity conditions on assertion conspire to entail the origo's certainty in main clause declaratives. 


\section{Bibliography}

Chierchia, Gennaro (1989), Anaphora and attitudes De Se, in R. Bartsch, J. van Bentham, and P. van Emde Boas (eds.) Language in Context, Foris Publications, Dordrecht, $1-31$.

Garrett, Edward John (2001), Evidentiality and Assertion in Tibetan, Ph.D. thesis, University of California Los Angeles.

KÖLBEL, MAX (2002), Truth without Objectivity, Routledge, London.

Lasersohn, Peter (2005), Context dependence, disagreement, and predicates of personal taste, Linguistics and Philosophy 28: 643-686.

Lewis, David (1979), Attitudes de dicto and de se, The Philosophical Review 88: 513-543.

Matthewson, Lisa (2010), What are direct evidentials?, paper presented at Ohio State University, January 15, 2010.

Partee, Barbara H. (1989), Binding implicit variables in quantified contexts, in Chicago Linguistics Society (ed.) Proceedings of the 25th Meeting of the Chicago Linguistics Society, 342-365.

Schlenker, Phillippe (2003), A plea for monsters, Linguistics and Philosophy 26: 29120.

Stephenson, Tamina (2007), Towards a Theory of Subjective Meaning, Ph.D. thesis, University of Massachusetts at Amherst.

Stephenson, Tamina (2008), Judge dependence, epistemic modals, and predicates of personal taste, Linguistics and Philosophy 30: 487-525. 


\title{
Focus, Uniqueness and Soft Presupposition Triggers
}

\author{
Andreas Walker \\ University of Konstanz
}

\begin{abstract}
Von Heusinger (2007) shows that the definite article's uniqueness presupposition causes problems in a standard account of focus alternatives. He solves this problem by proposing a new lexical entry for the definite article, a solution adopted by Riester and Kamp (2010). This paper shows that the observed behavior is not limited to the definite article, making such a solution undesirable. Our argument is based on two observations: (1) The definite article is a soft presupposition trigger with respect to uniqueness and (2) soft presupposition triggers have a special behavior in focus alternatives.
\end{abstract}

\section{The Problem}

Von Heusinger (2007) observes that in a standard account of focus alternatives, the sentence in (1) receives the following analysis: the uttered sentence (excluding only) expresses the proposition in (2), and focus gives rise to the set of alternatives in (3).

(1) John only talked to the GERman ${ }_{F}$ professor.

(2) $\lambda w: \exists ! x[\operatorname{prof}(x, w) \wedge \operatorname{german}(x, w)] . \exists y[\mathrm{P}(y, w) \wedge \mathrm{G}(y, w) \wedge \mathrm{T}(j, y, w)]$

(3) $\{[\lambda w: \exists ! x[\mathrm{P}(x, w) \wedge \mathrm{G}(x, w)] \cdot \exists y[\mathrm{P}(y, w) \wedge \mathrm{G}(y, w) \wedge \mathrm{T}(j, y, w)]]$,

$$
\begin{aligned}
& {[\lambda w: \exists ! x[\mathrm{P}(x, w) \wedge \mathrm{F}(x, w)] \cdot \exists y[\mathrm{P}(y, w) \wedge \mathrm{F}(y, w) \wedge \mathrm{T}(j, y, w)]],} \\
& [\lambda w: \exists ! x[\mathrm{P}(x, w) \wedge \mathrm{D}(x, w)] \cdot \exists y[\mathrm{P}(y, w) \wedge \mathrm{D}(y, w) \wedge \mathrm{T}(j, y, w)]], \ldots\}
\end{aligned}
$$

That is, the uttered sentence roughly conveys (blending together presuppositions and assertions for the sake of readability) that there is a unique German professor such that John talked to him, and the alternatives are the propositions that there are unique professors of other nationalities (French, Dutch, ...), such that John talked to them. While this analysis might yield the correct readings in some contexts, von Heusinger (2007) notes that this analysis becomes problematic in the scenario described in (4).

(4) Scenario: At a party there are one German professor, one Dutch professor and two French professors.

The prediction would be that (1) suffers presupposition failure in (4), since there is no unique French professor. However, what we observe instead is that 
1. the sentence is felicitous and true in (4) if John talked to the German professor but none of the other professors.

2. the sentence is felicitous and false in (4) if John talked to any other professor, whether they are unique (i.e. Dutch) or non-unique (i.e. French).

Von Heusinger (2007) suggests solving this problem by assuming a different lexical entry for the definite article in the alternative semantics, namely $\llbracket$ the $\rrbracket^{f}=\cup$ (the generalized union). A computation in the alternative semantics of Rooth (1992) is given in (5). As the reader can see, the generalized union yields the set of all professors, which is passed up the tree in the alternative semantics computation without any uniqueness presupposition. The set of resulting propositional alternatives is then contextually restricted by Rooth's (1992) squiggle operator, yielding propositional alternatives concerning the contextually relevant professors, e.g. the ones at the party, regardless of their nationality or uniqueness. This way, the set of alternatives consists of all professors, rather than of all unique professors of a certain nationality. Von Heusinger's (2007) solution is adopted by Riester and Kamp (2010).

(5) $\llbracket \operatorname{GERman}_{F}$ professor $^{f}=\left\{\lambda x \cdot[\mathrm{Q}(x) \wedge \operatorname{PROF}(x)] \mid Q \in D_{\langle e, t\rangle}\right\}$

$\llbracket$ the $\rrbracket^{f}=[\lambda P . \cup P]_{\langle\langle\langle e, t\rangle, t\rangle,\langle e, t\rangle\rangle}$

$\llbracket$ the GERman Grofessor $^{f}=\cup\left\{\lambda x \cdot[\mathrm{Q}(x) \wedge \operatorname{PROF}(x)] \mid Q \in D_{\langle e, t\rangle}\right\}$

$=\{x \mid \exists Q[Q(x) \wedge P R O F(x)]\}$

$=\{x \mid \operatorname{PROF}(x)\}$

$\llbracket J o h n$ talked to the $\operatorname{GERman}_{F}$ professor $\rrbracket^{f}=\{\operatorname{TALK}(\mathrm{j}, \mathrm{x}) \mid \operatorname{PROF}(\mathrm{x})\}$

While this approach arrives at the correct reading, it misses an important generalization. The observed behavior is in fact not unique to the definite article, but can be shown to arise for the larger class of Soft Presupposition Triggers. In Sect. 2 we will discuss some of the data that has been presented on these Soft Presupposition Triggers in the literature, in order to provide the background for the observations presented in this paper. Two observations will be presented in Sect. 3: We will show that the definite article does in fact belong into the class of Soft Presupposition Triggers (Sect. 3.1), and that while Hard Presupposition Triggers must project their presuppositions in focus alternative sets, Soft Presupposition Triggers do no need to (Sect. 3.2). Further, we will point out that the non-projective behavior of Soft Presupposition Triggers in the alternative set does not amount to local accommodation, but to the eliminiation of the presupposed proposition as a whole. This is different from the behavior of Soft Presupposition Triggers in the environments investigated in the previous literature, where non-projection amounted to local accommodation (Sect. 3.3). Once the special behavior of Soft Presupposition Triggers is established, von Heusinger's (2007) example will be shown to be just one case of the general pattern (Sect. 3.4). Section 4 concludes the paper. 


\section{Background on Soft Presupposition Triggers}

The literature on presupposition triggers distinguishes between Soft and Hard Presupposition Triggers. Soft Presupposition Triggers (SPT) and Hard Presupposition Triggers (HPT) show different behavior in a number of contexts, a phenomenon that has been described by Simons (2001) and Abusch (2002) amongst others. In these contexts, the presuppositions of SPTs do not need to project, while projection is obligatory for HPTs. We are going to examine two such environments, disjunction and ignorance of the speaker with respect to the presupposition.

The first context, involving disjunction, is given in the following examples by Abusch (2002). Consider first a simple example of presupposition with a Soft Presupposition Trigger such as (6).

(6) John will continue missing meetings.

The presupposition that John has missed meetings before, triggered by continue, here projects to the whole sentence. However, in (7) the presuppositions of continue do not project to the whole sentence, but rather stay within the disjuncts, yielding a reading paraphrased in (8).

(7) After the first meeting, John will either continue missing meetings or continue attending meetings.

(8) "After the first meeting, John will either have missed the first meeting and continue missing meetings or have attended the first meeting and continue attending meetings."

The same projection behavior as in (6) can be shown for the HPT too in the simple case, where the presupposition that John missed the first meeting projects to the whole sentence.

(9) John missed the second meeting too.

However, HPTs do not show the non-projection behavior observed for SPTs under disjunction. In (10), the conflicting presuppositions of too must project, rendering the sentence pragmatically odd.

(10) \# After the first meeting, John will either miss the second meeting too or attend the second meeting too.

The second context, involving the speaker's explicite ignorance with respect to the presupposition, is given by Simons (2001). The presupposition of stop projects to the whole sentence as normal in (11), so that the sentence presupposes that John smoked before. The presupposition does not project in (12) where it instead becomes part of the question, as paraphrased in (13) 
(11) Did John stop smoking?

(12) I see that you keep chewing on your pencil. Have you recently stopped smoking?

(13) "Have you been a smoker and recently stopped smoking?

However, when a HPT is used, the same contrast as under disjunction arises. That is, the presupposition of again projects in the simple example (14) as well as in (15) where the speaker's ignorance is made explicit. Since the presupposition that Jane rented Manhattan before is incompatible with this context, (15) is rendered pragmatically odd.

(14) Jane is renting Manhattan again.

(15) \# I have no idea whether Jane ever rented "Manhattan", but perhaps she's renting it again.

Based on these contexts, several presupposition triggers have been classified as either hard or soft:

1. Soft Presupposition Triggers: e.g. factive verbs, change of state verbs

2. Hard Presupposition Triggers: e.g. again, even, too, also, negative polarity either, it-cleft

Missing from this list is the definite article. Its status is disputed in the literature, with no clear classification arising. Abusch (2002) describes it as "unclear". Abbott (2006) argues for treating the definite article as a HPT based on examples like (16). Here, the presupposition that the book has an owner, triggered by the must project. Since the speaker's ignorance with respect to the presupposition is explicitely stated, the sentence is pragmatically odd.

(16) \# Possibly no one owns this book, but if I find the owner I will return it.

However, these discussions are only concerned with the definite article's existential presupposition. The problematic presupposition in von Heusinger's (2007) puzzle is the uniqueness presupposition. The question arises whether the definite article is a soft or a hard presupposition trigger with respect to uniqueness.

\section{Observations}

The following sections present data on two observations: (a) The definite article is a SPT for uniqueness, and (b) SPTs show the same behavior as the definite article with respect to focus alternatives, but HPTs do not. Taken together, these two observations allow us to locate the original problem within a larger class of problems that need to be addressed together. 


\subsection{Observation A: The Definite Article is a Soft Presupposition Trigger for Uniqueness}

In the contexts described by Simons (2001) and Abusch (2002), the definite article behaves like a SPT, i.e. its uniqueness presupposition does not need to project in these contexts. Similar to the behavior of continue in (7), the definite article's presupposition does not project under disjunction in (18), given the scenario in (17). Instead it is locally accommodated within the disjuncts.

(17) Scenario: In a historical setting with a pope and a counterpope, a council is being held in order to settle the conflict by agreeing on one unique pope, but it is yet unclear whether this will succeed

(18) After the council, either the pope will unite Rome, or the popes will tear it apart.

This gives rise to the reading paraphrased in (19), i.e. the uniqueness is not a presupposition of the sentence but is only locally assumed in the disjunction.

(19) "After the council, either there will be a unique pope and this pope will unite Rome or there will be more than one pope and these popes will tear it apart."

In a scenario like (20) where we can assume the speaker's ignorance with respect to the presupposition, it is not projected either. Instead, it is locally accommodated, giving rise to the reading paraphrased in (22).

(20) Scenario: Pina needs a linear equation A to have exactly one solution. I do not know how many solutions A has. When I meet Pina she seems happy.

(21) Did you find the solution for A?

(22) "Is there a unique solution for A and did you find it?"

Since the definite article behaves as a Soft Presupposition Trigger in these contexts, we can assume that it is in fact a SPT with respect to uniqueness.

\subsection{Observation B: Soft Presupposition Triggers and Focus Alternatives}

We will now explore the behavior of Soft and Hard Presupposition Triggers with respect to focus alternatives. As expected, both SPTs and HPTs have the potential to project in focus alternatives. (24) demonstrates this for the SPT aware.

(23) Scenario: Three soccer players, A(lexandra), B(irgit) and C(elia), are pregnant. John believes that Birgit is pregnant.

(24) It's a good thing John is only aware that BIRgit $F$ is pregnant. 
Here, the presuppositions in the focus alternatives, i.e. that Alexandra is pregnant and that Celia is pregnant, do project as expected. A rough paraphrase (conflating assertions and presuppositions for the sake of readability) is given in (25).

(25) "B is pregnant and John believes that B is pregnant, A is pregnant and John doesn't believe that A is pregnant, and $\mathrm{C}$ is pregnant and John doesn't believe that $\mathrm{C}$ is pregnant."

The same can be shown for the HPT also in (27). Note that the relevant reading is the one in which only goes together with Birgit, and also with conjunctivitis.

(26) Scenario: John believes that all three players have a cold. On top, he thinks that Birgit has conjunctivitis.

(27) John only thinks that BIRgit also has conjunctivitis.

The presupposition - i.e. that John thinks that the players have a condition other than conjunctivitis - projects, and we obtain the rough paraphrase given in (28).

(28) "John thinks B has a condition other than conjunctivitis, and John thinks that B has conjunctivitis, and John thinks A has a condition other than conjunctivitis, and John doesn't think that A has conjunctivitis, and John thinks $\mathrm{C}$ has a condition other than conjunctivitis, and John doesn't think that C has conjunctivitis."

Interestingly, we note that a difference between the SPT and the HPT arises when the context does not support the presupposition. In the case of the SPT, the presupposition does not need to project, and the sentence is still acceptable.

(29) Scenario: There are rumours that the three players will not be able to play in the semi-final due to a pregnancy. It is not known whether any of the three actually is pregnant.

(30) The team's doctor is only aware that BIRgit $F$ is pregnant.

In (30) the sentence as a whole does not presuppose that Alexandra and Celia are pregnant. Instead we obtain the reading roughly paraphrased in $(31)^{1}$.

(31) " $\mathrm{B}$ is pregnant and the doctor believes that B is pregnant, the doctor does not believe that A is pregnant, and the team's doctor does not believe that $\mathrm{C}$ is pregnant."

\footnotetext{
${ }^{1}$ Note that the presupposition is not locally accommodated here, which we will discuss in more detail in Sect. 3.3.
} 
If we construct a similar example with the HPT also, we notice that the presupposition projection is obligatory here. We obtain the rough paraphrase in (34) which is at odds with the scenario in (32), rendering the sentence in (33) pragmatically odd.

(32) Scenario: It has been established that only Birgit has the flu and the doctor knows that.

(33) \# The team's doctor only thinks that BIRgit ${ }_{F}$ also has conjunctivitis.

(34) "The doctor thinks that B has a condition other than conjunctivitis and the doctor thinks that $\mathrm{B}$ has conjunctivitis, the doctor thinks that $\mathrm{A}$ has a condition other than conjunctivitis and the doctor does not think A has conjunctivitis, and the doctor thinks that $\mathrm{C}$ has a condition other than conjunctivitis and the doctor does not think that $\mathrm{C}$ has conjunctivitis."

In order to show that this observed difference is a general pattern for all SPTs and HPTs, let us briefly consider a different example that exhibits the same behavior, i.e. that the SPT does not need to project in whereas the HPT does. (35) provides a scenario in which the SPT stop in (36) does not project.

(35) Scenario: The Lamb \& Lion serves beer and wine, but the Rose \& Crown only serves beer. New legislation makes it illegal to serve either beer or wine but pubs will be financially compensated for this, based on what kind of alcohol they served before.

(36) The Lamb \& Lion stopped serving wine and beer. They will get $540 £$. The

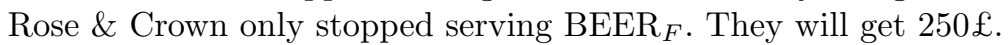

Here, the presupposition that the Rose \& Crown served wine before does not appear in the alternative set.

In contrast, in the case of a HPT, the presupposition must project. This is shown in (39).

(37) Scenario: Continuing the scenario in (35), the legislation is lifted.

(38) The Lamb \& Lion only serves BEER ${ }_{F}$ again.

(39) \# The Rose \& Crown only serves BEER $B_{F}$ again.

In both (38) and (39) the presupposition of again projects. That is, (38) and (39) presuppose that the Rose \& Crown and the Lamb \& Lion respectively served both wine and beer before. This is the case for the Lamb \& Lion, and thus (38) is acceptable in this scenario. But this presupposition is not satisfied for (39) in the present scenario, as the Rose \& Crown served beer but not wine before. This renders sentence (39) unacceptable in this scenario. 


\subsection{Local Accommodation or Cancellation?}

We haved observed that both in the contexts presented by Simons (2001) and Abusch (2002) and within focus alternatives, SPTs do not need to project. However, there is a difference between the exact ways in which this lack of projection comes about. The cases discussed in the literature assume that the presupposition is locally accommodated, i.e. instead of projecting it becomes part of the assertion. This can be seen both in the original examples such as (7) and (12), and in the examples we provided for the definite article, such as (18) and (21). In the case of focus alternatives however, the presuppositions of the Soft Presupposition Triggers simply do not appear in the alternative set. No local accommodation can be observed here. The following examples rule out local accommodation as the source of SPTs behavior with respect to focus alternatives. Instead, the empirical result is that the presuppositions are simply eliminated from the alternative set.

(40) Scenario: Only Birgit is pregnant. The pregnancy tests are faulty, leading the doctor to falsely believe that Alexandra and Celia are pregnant as well.

(41) I think there is something wrong with the pregnancy tests. After using them, the doctor believed that three players are pregnant. But he got it right for only one of them: \# The doctor is only aware that BIRgit $F$ is pregnant.

If local accommodation of the presupposition within each focus alternative was an option, (41) would have (42) as one of its possible readings, thus making the sentence felicitous in scenario (40). Since the sentence is infelicitous, this reading is not available for the sentence.

(42) " $\mathrm{B}$ is pregnant and the doctor believes that B is pregnant, and it is not the case that ( $A$ is pregnant and the doctor believes that $\mathrm{A}$ is pregnant), and it is not the case that ( $C$ is pregnant and the doctor believes that $\mathrm{C}$ is pregnant).

Instead of (42), the only reading available where presuppositions do not project is (43), where the presuposed proposition has been entirely eliminated from the focus alternatives.

(43) " $\mathrm{B}$ is pregnant and the doctor believes that B is pregnant, and the doctor does not believe that $\mathrm{A}$ is pregnant, and the doctor does not believe that $\mathrm{C}$ is pregnant."

It follows that the paraphrase in (43) should indeed be formalized as the set of alternatives in (44). 
(44) $\{\lambda w: \operatorname{PREGNANT}(a, w) \cdot \operatorname{believe}(d, \operatorname{PREGNANT}(a), w))$, $\lambda w: \operatorname{PREGNANT}(b, w) \cdot$ believe $(d, \operatorname{PREGNANT}(b), w))$,

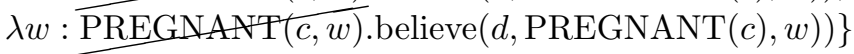

This provides a challenge to current accounts for SPTs, such as Romoli (2011) and Abrusán (2011). Since the literature usually assumes the local accommodation behavior this new observed behavior may proof difficult to integrate with these approaches ${ }^{2}$.

\subsection{Observation A+B and the Original Problem}

The observations made above now present us with a new perspective on the original problem presented in (1). The phenomenon there is not due to some peculiar property of the definite article's lexical entry in the alternative semantics, but rather due to its status as a SPT and the behavior of SPTs with respect to focus alternatives. The reading that we obtain for (45) (repeated from (1) above) is roughly paraphrased in (46).

(45) John only talked to the GERman ${ }_{F}$ professor.

(46) "There is a unique German professor and John spoke to the German professor, and John did not speak to a French professor and John did not speak to a Dutch professor."

As in the examples above, we obtain the correct formalization of this reading if we construct the alternative set as normal, but do not include the presuppositions in the alternatives.

$$
\begin{aligned}
& \{[\lambda w: \exists ! x[\mathrm{P}(x, w) \wedge \mathrm{G}(x, w)] \cdot \exists y[\mathrm{P}(y, w) \wedge \mathrm{G}(y, w) \wedge \mathrm{T}(j, y, w)]] \\
& {[\lambda w: \exists ! x[\mathrm{P}(x, w) \wedge \mathrm{F}(x, w)] \cdot \exists y[\mathrm{P}(y, w) \wedge \mathrm{F}(y, w) \wedge \mathrm{T}(j, y, w)]]} \\
& [\lambda w: \exists ! x[\mathrm{P}(x, w) \wedge \mathrm{D}(x, w)] \cdot \exists y[\mathrm{P}(y, w) \wedge \mathrm{D}(y, w) \wedge \mathrm{T}(j, y, w)]], \ldots\}
\end{aligned}
$$

We then arrive at the following truth conditions for (45):

(48a) Presupposition: $\lambda w \cdot \exists ! x[\mathrm{P}(x, w) \wedge \mathrm{G}(x, w)] \wedge \exists x[\mathrm{P}(x, w) \wedge \mathrm{G}(x, w) \wedge \mathrm{T}(j, x, w)]$ (48b) Assertion: $[\lambda w \cdot \neg[\exists y[\mathrm{P}(y, w) \wedge \mathrm{F}(y, w) \wedge \mathrm{T}(j, y, w)]] \wedge$ $\neg[\exists y[\mathrm{P}(y, w) \wedge \mathrm{D}(y, w) \wedge \mathrm{T}(j, y, w)]]$

Arriving at this reading by changing the lexical entry for the definite article overlooks the crucial fact that this behavior can be systematically observed for all Soft Presupposition Triggers when they appear together with focus alternatives.

\footnotetext{
${ }^{2}$ Note however that in some cases, e.g. (36), the local accommodation behavior seems to be available with focus alternatives. A possible explanation for this is that stop $P$, besides presupposing that $\mathrm{P}$ was true before, asserts that there was a change from $P$ to $\neg P$. If so the correct reading for the pub examples is still obtained by completely eliminating the presuppositions from the alternative set.
} 


\section{Conclusions}

Considering these observations, we arrive at the following conclusions:

1. The definite article is a Soft Presupposition Trigger for uniqueness.

2. Soft Presupposition Triggers and Hard Presupposition Triggers show different behavior not only in the contexts provided by Simons (2001) and Abusch (2002), but also within focus alternative sets: While Hard Triggers must obligatorily their presuppositions, Soft Triggers do not need to.

3. There is no need for a special lexical entry for the definite article in alternative semantics. Instead, a more general account for the interaction between Soft Presupposition Triggers and focus alternatives is necessary.

4. Any account of Soft Presupposition Triggers must be able to explain

(a) the behavior of Soft Presupposition Triggers (now including the definite article) within the previously known contexts, where the non-projection corresponds with readings that resemble a form of local accommodation,

(b) the behavior of Soft Presupposition Triggers within focus alternatives, where non-projection seems to amount to complete disappearance of the presupposition.

Further research will be needed to show how these conclusions interact with current accounts for Soft Presupposition Triggers, and whether these can be modified to account for the new behavior observed as well as the new Soft Trigger. The answers found there should then provide us with an answer to von Heusinger's (2007) puzzle as well.

\section{References}

Abbott, B.: Where Have Some of the Presuppositions Gone? In: Birner, B., Ward, G. (eds.) Drawing the Boundaries of Meaning. Neo-Gricean Studies in Pragmatics and Semantics in Honor of Laurence R. Horn. Benjamins, Philadelphia (2006)

Abrusán, M.: Triggering Verbal Presuppositions. In: Li, N., Lutz, D. (eds.), Semantics and Linguistic Theory (SALT) 20. Vancouver (2011)

Abusch, D.: Lexical Alternatives as a Source of Pragmatic Presuppositions. In: Jackson, B. (ed.) SALT XII. CLC, New York (2002)

Riester, A., Kamp, H.: Squiggly Issues. Alternative Sets, Complex DPs and Intensionality. In: Aloni, M. et al. (eds.) Logic, Language and Meaning. Revised Selected Papers from the 17th Amsterdam Colloquium. Springer, Berlin (2010)

Romoli, J.: The Presuppositions of Soft Triggers Are Not Presuppositions. In: Ashton, N., Chereches, A., Lutz, D. (eds.) Proceedings of SALT 21. Rutgers University (2011)

Rooth, M.: A Theory of Focus Interpretation. Natural Language Semantics 1 (1992) $75-116$

Simons, M.: On the Conversational Basis of Some Presuppositions. In: Hastings, R., Jackson, B., Zvolensky, Z. (eds.) Proceedings of Semantics and Linguistic Theory 11. CLC, New York (2001)

Von Heusinger, K.: Alternative Semantics for Definite NPs. In: Schwabe, K., Winkler, S. (eds.) On Information Structure, Meaning and Form. Generalizations Across Languages. Benjamins, Amsterdam (2007) 


\title{
You Again: How Is Its Ambiguity Derived?
}

\author{
Ting Xu \\ University of Connecticut, Storrs, USA \\ xuting.thu@gmail.com
}

\begin{abstract}
It is well-known that an English sentence with a complex predicate modified by again displays a repetitive vs. restitutive ambiguity. Like English again, Chinese you 'again' modifying a resultative verb compound also exhibits a repetitive vs. restitutive ambiguity. However, Chinese differs from English in that the position of you 'again' is relatively fixed: it can only occur preverbally but not postverbally. This study examines how the ambiguity of Chinese you is derived. Investigating the scope interaction between you 'again' and an indefinite object, I argue that the ambiguity of you 'again' is structural but not lexical. I further propose that you 'again' moves overtly as a last resort to satisfy a PF requirement specific to Chinese.
\end{abstract}

Keywords: ambiguity, restitutive again, scope, movement

\section{Introduction}

It is well-known that a sentence with a complex predicate modified by again (1) is ambiguous between a repetitive reading and a restitutive reading. The former presupposes that the subject has previously performed the action denoted by the VP (1a). The latter presupposes that the result state has held before. It either held from the very beginning (1b) or came into being as a result of someone else performing the action (1c).

(1) Sally painted the wall white again.

a. Sally had painted the wall white before.

b. The wall had been white before.

c. Someone else other than Sally painted the wall white before.

There exist different approaches to account for the ambiguity of English again: structural and lexical. The structural approach suggests that the ambiguity arises because (1) has different possible syntactic structures (see von Stechow, 1995, 1996; among others). The lexical approach instead argues that the ambiguity has nothing to do with the structure, but is a result of again having different meanings (e.g. Dowty, 1979; Fabricius-Hansen, 2001; Jäger \& Blutner, 2000).

Most of the previous studies focus on English again and German wieder, which can occur in different positions of a sentence. In this paper, I examine you 'again' in Mandarin Chinese. Like English, Chinese is also a head-initial language. Neverthe-

adfa, p. 1, 2011

(C) Springer-Verlag Berlin Heidelberg 2011 
less, Chinese differs from English in that the position of you 'again' is relatively fixed: it can only occur preverbally but not postverbally (2).

(2) ta you tu-hong le (*you) na-ge beike (*you). she again paint-red Asp (again) that-CL shell (again).

She painted two of the shells red again.

Like English again, however, Chinese you 'again' exhibits an ambiguity between a repetitive and a restitutive reading, which raises the following question: How do we account for the ambiguity? In this paper I argue that despite the preverbal position of you 'again', its ambiguity is structural rather than lexical. The crucial evidence comes from the scope interaction between you 'again' and an indefinite object.

\section{Previous Analyses of English again}

There are two main approaches to account for the ambiguity of English again in previous literature: structural analysis and lexical analyses. ${ }^{1}$

\subsection{The Structural Analysis}

Under the structural analysis (see von Stechow, 1995, 1996, among others), again always denotes repetition, as shown in (3). It introduces a presupposition that the proposition (a set of events) expressed by $\mathrm{P}$ was already true of another event $\mathrm{e}$ '.

(3) Let $\mathrm{P}$ be a property of eventualities and let e be an eventuality.

[[again]](P)(e) is defined only if $\exists \mathrm{e}^{\prime}\left[\mathrm{P}\left(\mathrm{e}^{\prime}\right)=1 \& \mathrm{e}^{\prime}<\mathrm{e}\right]$.

Where defined, [[again]](P)(e)=1 iff $\mathrm{P}(\mathrm{e})=1 . \quad($ adapted from von Stechow, 1996)

A complex predicate like painted the wall white in (4a) can be decomposed into an agentive event (wall painting) and a resultative state (the wall becoming white). The LF of (4a) is presented in (4b), in which the object NP the wall raises to semantically bind the empty pronominal (PRO) subject of AP.

(4) a. Sally painted the wall white.

b. [the wall 1 [vP Sally [v [ve $\mathrm{t}_{1}$ paint $\quad\left[{ }_{\mathrm{AP}} \mathrm{PRO}_{1}\right.$ white] $\left.\left.\left.]\right]\right]\right]$

1 There exists a naïve pragmatic analysis to analyze the ambiguity as well: We can treat the repetitive reading as a special case of the restitutive reading, as the former entails the latter. As far as I know, previous studies did not discuss the naïve pragmatic approach. However, Beck (2006) and Klein (2001) proposed a more complicated pragmatic account to explain the effect that focus has on the ambiguity of again. 
Sentence-final again can potentially adjoin to different positions in (4b), hence the ambiguity of Sally painted the wall white again. If again adjoins to vP or higher, we get the repetitive reading that Sally has painted the wall white before. If, on the other hand, again adjoins to the small clause AP, the restitutive reading is derived.

\subsection{The Lexical Analysis}

Contrary to the structural analysis, which argues that there is only one again, a lexical analysis claims that there is more than one again. The idea can be traced back to Dowty (1979), who account for the ambiguity by postulating a repetitive again, a restitutive again, and some type-shifting in between.

Different forms of lexical analysis have been proposed ever since. For example, Fabricius-Hansen (2001) proposes that in addition to expressing repetition, again can also express reversal of the direction (counterdirectional again), which leads to the restitutive reading.

\subsection{Two Kinds of Restitutive Readings}

So far we have been focusing on a two-way dichotomy of again: repetitive vs. restitutive. Notice that both scenarios in (1b) and (1c) are compatible with the restitutive reading of (1), with (1c) entailing (1b). Some researchers further argue that (1c) is a restitutive reading independent from (1b), and it is derived by adjunction of again to VP in (4) (Nissenbaum, 2006; Bale 2007; Dobler, 2008). (1b) and (1c) are called by Nissembaum (2006) the low restitutive reading and the high restitutive reading, respectively.

Here I summarize Nissembaum's (2006) argument in favor of two restitutive readings, which involves the interaction between again and an indefinite object. Assuming the LF in (5b), Nissembaum (2006) makes the following predictions about possible scope readings for (5a). First of all, for a low restitutive reading (with again adjoined to AP), the indefinite object can never be interpreted inside the scope of again, because it is base generated in Spec, VP. The prediction is borne out: It is felicitous to continue Scenario A with the sentence in (6a). The usage of pronoun in (6a) forces an interpretation in which the same tree was painted blue. As a contrast, (6b) does not serve as felicitous continuation of the scenario. This is expected assuming the structural analysis of again: The usage of an indefinite object in (6b) forces an interpretation in which a different tree was painted blue, which cannot be derived via the structure in (5b). Although for the low restitutive reading, wide scope reading of again with respect to the indefinite object is not available, the indefinite object can be interpreted outside the scope of again. This is illustrated in (7), in which the tree I painted blue has to be previously blue simply because of the universal quantifier in the scenario. Contrary to the low restitutive reading, for a high restitutive reading, the indefinite object can be interpreted inside the scope of again. (8) contains a scenario in which I painted a different tree blue from someone else. And it is felicitous to continue the scenario with a sentence having again and an indefinite object. 
(5) a. I painted one of my trees blue again.

b.

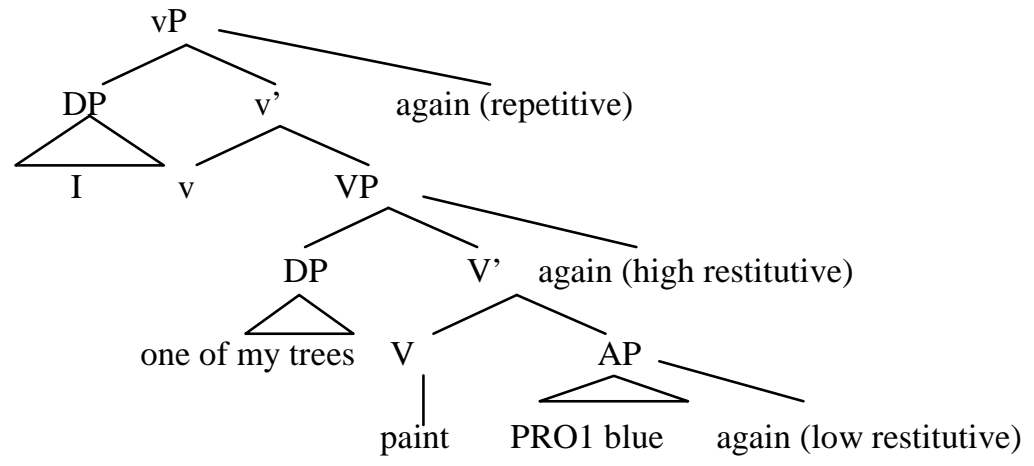

(6) Scenario A: One of my birch trees came up blue when it was a sapling; it later turned white like the rest. But I liked the idea of a blue birch tree so much that...

a. I painted it blue again.

b. \#I painted one of my trees blue again.

(7) Scenario B: All of my birch trees were blue when they were saplings; they later turned white like birch trees are supposed to be. But I liked the idea of a blue birch tree so much that...

I painted one of my trees blue again.

(8) Scenario C: One of my birch trees had been painted blue when I moved in. It later died and had to be cut down. But I liked the idea of a blue birch tree so much that...

I painted one of my trees blue again.

The scope facts is compatible with the structural analysis of again and challenge the lexical analyses. Under a lexicalist account, to the extent the facts may be described, there is no principled reason why only the low restitutive reading with wide scope of again is absent.

\section{The Puzzle: the Ambiguity of You in Mandarin Chinese}

Like English, a Chinese sentence with a complex predicate modified by you 'again' (2) is compatible with all three scenarios in (1). Different from English, you 'again' in Chinese has a restricted distribution: it can only occur preverbally (2).

The structural analysis cannot be extended directly to Chinese. On the assumption that the complex predicate ends up in $\mathrm{v}$, the preverbal you 'again' would appear to adjoin to $v \mathrm{P}$ or higher, thus only the repetitive reading is expected.

The Chinese $b a$-construction makes the ambiguity even more puzzling. A $b a$ sentence with you preceding ba also displays a three-way ambiguity (9). It is generally assumed in the literature that $b a$ is an overt realization of a recursive small $v$ (see Huang, Li \& Li, 2009). When you precedes $b a$, it must adjoin to vP or even higher, thus the restitutive reading becomes unexpected. 
(9) Zhangsan you ba men da-kai le.

Zhangsan again BA door hit-open Asp.

Zhangsan opened the door again. (repetitive, high restitutive, low restitutive)

Following Nissenbaum (2006), I examine how the ambiguity is derived by investigating the scope interaction between you 'again' and an indefinite object.

\section{Scope Interaction between You and a Quantifier}

Since you 'again' yields a three-way ambiguity, when it interacts with another scopebearing element, such as an indefinite object, there are altogether six logical possibilities. Native speakers were presented with scenarios for each of these possibilities and were asked to judge whether bare sentences and ba-constructions with you were felicitous. The judgment is summarized in Table 1: All readings are available except the $\underline{\text { low }}$ restitutive reading with you 'again' taking wide scope.

Table 1. Scope between you 'again' and an indefinite object in Chinese

\begin{tabular}{|l|l|l|}
\hline Low restitutive reading & $\exists>$ you 'again' & $\#$ you 'again' $>\exists$ \\
\hline High restitutive reading & $\exists>$ you 'again' & you 'again'> $>$ \\
\hline Repetitive reading & $\exists>$ you 'again' & you 'again' $>\exists$ \\
\hline
\end{tabular}

For expository convenience, I focus on the following three readings: a) low restitutive reading with you taking narrow scope; b) low restitutive reading with you taking wide scope; c) high restitutive reading with you taking wide scope.

First of all, when the low restitutive reading is intended, the indefinite object can be interpreted outside the presupposition of you 'again', i.e. the object in the presupposition and assertion can be the same (10)-(11). The ba-construction has the same interpretation as its corresponding bare sentence.

(10) Context: Lisa had a bunch of red shells. Unfortunately after a while they all got very dusty and the redness faded. In need of two red shells to decorate her Christmas tree, ...
a. ta you tu-hong she again paint-red
le
qizhong liang-ge
Asp among two-CL shell.
beike. $^{2}$

2 The test sentences in (10) and (11) involve a partitive marker qizhong 'among'. As a matter of fact, native speakers were asked to judge sentences either with or without a partitive marker for all the scenarios. It turned out when you 'again' takes wider scope than the indefinite object, speakers tended to prefer sentences without partitive markers. On the other hand, they preferred sentences with partitive markers when you 'again' takes narrow scope. For expository convenience, when concentrating on scenarios in which you takes narrow scope, I only present the judgment of sentences with partitive markers; when focusing on scenarios in which you takes wide scope, I only present the judgment of sentences without partitive markers. 
b. ta you ba qizhong liang-ge beike tu-hong le.

she again BA among two-CL shell paint-red Asp.

She painted two of the shells red again.

(11) Context: John ordered ten pocket watches. Unfortunately, all of them had always been open due to a manufacturing error. Yesterday he got all his watches fixed and closed them for the first time. Today, ...
a. ta you da-kai le
$\begin{array}{llll}\text { b. } & \text { you } & \text { ba } & \text { qizhong yi-kuai huaibiao } \\ \mathrm{He} & \text { again } & \text { BA } & \text { among one-CL pocket-w }\end{array}$
qizhong yi-kuai huaibiao.

He opened one of his pocket watches again.

For the low restitutive reading, although the indefinite object can take wide scope with respect to you 'again', but not vise versa. In other words, when a low restitutive reading is intended, the object cannot be interpreted within the presupposition of you 'again'. A number of my informants found the use of you 'again' odd in scenarios like (12) and (13), especially compared with (10) and (11).

(12) Context: Zhangsan went to the beach and collected a lot of white shells and two red shells. When his wife cleaned the house, she accidentally broke the two red shells. Worried that Zhangsan would notice the mishap, ... ${ }^{3}$
a. \#ta
she again paint-red
tu-hong
le liang-ge beike.
Asp
$\begin{array}{clll}\text { b. \#ta } & \text { you } & \text { ba } & \text { liang-ge } \\ \text { she } & \text { again } & \text { BA } & \text { two-CL }\end{array}$
she again BA two-CL
She painted two shells red again.
two-CL shell.
beike tu-hong
shell paint-red
le.
Asp.

(13) Context: John ordered many pocket watches. Unfortunately, two of them had always been open due to a manufacturing error. Yesterday he got them fixed and they closed for the first time. Today...
a. \#ta
you
again
da-kai le
yi-kuai huaibiao.
he again hit-open Asp one-CL pocket-watch.
b. \#ta you ba yi-kuai huaibiao da-kai
he again BA one-CL pocket-watch
He opened a pocket watch again.
Asp.

The reason why you 'again' sounds infelicitous in (12) and (13) is not because it cannot take wide scope. As shown in (14) and (15), when a high restitutive reading is intended, the indefinite object can be interpreted within the presupposition of you 'again'.

$\begin{array}{lll} & 3 & \text { The scenario is adapted from Dobler (2008) } \\ 490 & 4 & \text { The scenario is adapted from Bale (2007). }\end{array}$ 
(14) Context: John and Jane had some white shells. Since they needed two red shells to decorate their Christmas tree, John painted two shells red. Unfortunately, Jane accidentally broke the two red shells that John just painted. Therefore, ...
a. ta you tu-hong le liang-ge beike.

she again paint-red Asp two-CL shell.

b. ta you ba liang-ge beike tu-hong le.

She again BA two-CL shell paint-red Asp

She painted two shells red again.

(15)Context: Jane and John bought five pocket watches. Jane picked out one pocket watch, opened it and closed it. Later John needed to check the time. He wanted to open the watch that Jane opened just now, but he couldn't find it. Therefore,...
a. ta you da-kai le yi-kuai huaibiao.
He again hit-open Asp one-CL pocket-watch.
b. ta you ba yi-kuai huaibiao da-kai le.
$\mathrm{He}$ again BA one-CL pocket-watch hit-open Asp.

He opened a pocket watch again.

The scope facts suggest that syntactic structure is playing a role in the ambiguity of you. Let us take (16) as an example, whose LF is presented in (17). The scope facts would fall in place if you 'again' can adjoin to some lower projections such as VP or $\mathrm{XP}$ in (17). If you 'again' can indeed adjoin to XP, it will give rise to the low restitutive reading. As the indefinite object is base-generated in Spec, VP, it takes wider scope than you 'again', but not the other way around. On the other hand, if you 'again' can adjoin to VP, with the high restitutive reading, the indefinite object can be interpreted within the presupposition of you 'again'. Under a lexicalist account, to the extent the facts may be described, there is no principled reason why the low restitutive reading with the wide scope of you is absent.
(16) Lisi
tu-hong
le liang-ge beike.
Lisi
paint-red
Asp
two-CL shell.
Lisi painted two shells red.
(17) [IP... [vp Lisi v [vp two-shell 1 paint [FP F [xp $\mathrm{PRO}_{1}$ red]]]]

\section{$5 \quad$ Analysis}

Following Ernst (2004), who argues that adverbs are licensed in their base positions whenever the relevant semantic rule gives them their proper interpretation, I propose that you can adjoin to some lower projections in (17), such as a small clause XP or $\mathrm{VP}$, which gives rise to the low restitutive reading and the high restitutive reading, respectively.

First of all, you can modify a small clause. This is attested by Chinese deresultative constructions (18a). Following the analysis of Tang (1997), which is shown in (18b), particle de is base-generated in the head position of the functional projection, and incorporates to $\mathrm{V}$. Then $\mathrm{V}$-de as a whole undergoes $\mathrm{V}$-to-v movement. 
Since you 'again' can occur between the object Zhangsan and the secondary predicate $k u$ 'cry' (18a), it has to adjoin to XP in syntax to derive the correct linear order. ${ }^{5}$

$\begin{array}{rlllll}\text { (18) a. Lisi } & \text { da-de } & \text { Zhangsan } & \text { you } & \text { ku } & \text { le } \\ \text { Lisi } & \text { hit-de } & \text { Zhangsan } & \text { again } & \text { cry } & \text { Asp. }\end{array}$

Lisi hit Zhangsan to the extent that Zhangsan cried again.

b. [vP Lisi v $+\mathrm{da}_{\mathrm{k}}-\mathrm{de}_{\mathrm{j}}$ [vP $Z$ hangsan $\mathrm{t}_{\mathrm{k}}+\mathrm{t}_{\mathrm{j}}\left[\mathrm{FP}_{\mathrm{FP}} \mathrm{t}_{\mathrm{j}}\right.$ [you [xP $\mathrm{PRO}_{\mathrm{i}} \mathrm{ku}$ le]]

In addition to result-denoting XP, you can adjoin to VP in syntax. This is demonstrated by the Chinese $b a$-construction (19a). Here I follow Kuo (2009), who argues that $b a$ is the head of an Applicative phrase, as illustrated in (19b). ${ }^{6}$ Not only can you 'again' precede $b a$ (cf. (9)), it can also occur between the $b a$-NP (the NP immediately following $b a$ ) and the predicate (19). To derive the correct linear order, you 'again' has to adjoin to VP in the structure.

$\begin{array}{cllllll}\text { (19) a. wo } & \text { ba } & \text { Sara } & \text { you } & \text { da } & \text { shang } & \text { le } \\ \text { I } & \text { BA } & \text { Sara } & \text { again } & \text { hit } & \text { hurt } & \text { Asp. }\end{array}$

I hit Sara so that she got hurt again.

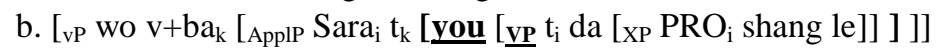

Furthermore, as Chinese adverbs in general occur preverbally in a bare sentence, I postulate here that there is a PF-requirement in Chinese, which requires adverbs like you to be preverbal. ${ }^{7}$

I propose a movement analysis of you 'again' to account for its ambiguity. In a bare sentence such as (16), when base-generated in a VP- or XP-attached position, you 'again' moves overtly as a last resort to satisfy this PF-requirement. When it gets interpreted, it undergoes LF reconstruction or semantic reconstruction (see Cresti, 1995; among others).

5 In fact, it is not clear whether you adjoins to FP or XP. To answer this question, we need to figure out the denotation of particle $d e$. The answer will not affect our analyses. For expository convenience, I assume in this paper that it adjoins to XP, which denotes the result state, and treat the functional head $d e$ as semantically vacuous. This is in line with the analysis which analyzes the functional head $d e$ as a complementizer (e.g. Wang, 2010).

6 Other analyses of $b a$-constructions (for example, treating $b a$ as an overt realization of a recursive $v$ ) are also compatible with my point here.

7 It has been widely observed that there exists a restriction in Mandarin that a transitive verb with an object cannot be modified by a post-verbal manner expression (i). The verb has to be doubled (i). The PF requirement proposed here may be part of a broader (but unexplained) generalization about modifiers in Chinese resisting VP-internal surface positions, even when they are interpreted there (Jonathan Bobaljik, pc).

(i) Wo pa shan *(pa) de kuai. I climb mountain climb DE fast. 


\section{Further Arguments and Consequences}

Under the movement analysis, several additional patterns follow directly. To begin with, only the surface scope reading is available when you precedes negation mei (20).

(20) a. Context: Zhangsan didn't clean the room last month. And...

Zhe-ge yue ta you mei dasao fangjian.
This-CLmonth he again Neg clean room.
Again, he didn't clean the room this month.
Context: Zhangsan cleaned the room last month. But...
\# the example in (20a)

Furthermore, neither high nor low restitutive reading is available when you 'again' immediately precedes negation (21a). In fact, to express the restitutive reading, a different lexical item zai, which is generally assumed to denote repetition in irrealis context, is used and it has to follow negation (21b).

(21) Scenario: The door was open before (either from the very beginning or got opened by someone else). Then somehow it got closed by someone other than John. John wants to open the door but he was too tired, so...

a. \#ta you mei ba men da-kai.

He you NEG BA door hit-open.

b. ta mei zai ba men da-kai.

He NEG again BA door hit-open.

He didn't open the door again.

There exist a tradition to treat the negative particle mei 'not' as a type of adverb (see Chao, 1968; Li \& Thompson 1981; Ernst, 1995). Assuming Tang's (1990, 2001) theory of licensing adverbials, negative particles may be licensed in an adjoined position on a par with other types of adverb in Mandarin. If this is on the right track, the above patterns follow directly from Relativized Minimality (Rizzi, 1990), a wellestablished syntactic constraint that bans movement of an adjunct like you across another adjunct.

\section{Conclusion}

In this study, I examine how the repetitive/restitutive ambiguity of you 'again' in Mandarin Chinese is derived. On the surface, Chinese poses a problem for the structural analysis because the adverb you can only be preverbal. However, the scope interaction between you 'again' and an indefinite object suggests that the ambiguity of you 'again' must be structural rather than lexical. The puzzle can be resolved if you 'again' moves overtly and gets reconstructed either at LF or in semantics. 


\section{References}

1. Bale, Alan. (2007). Quantifiers and verb phrases: An exploration of propositional complexity. Natural Language and Linguistic Theory, 25(3), 447-483.

2. Beck, Sigrid. (2006). Focus on again. Linguistics and Philosophy, 29(3), 277-314.

3. Chao, Yuen-Ren. (1968). A Grammar of Spoken Chinese. Berkeley, California: University of California Press.

4. Cresti, Diana. (1995). Extraction and Reconstruction. Natural Language Semantics 3, 79122.

5. Dobler, Eva. (2008). Again and the structure of result states. Proceedings of ConSOEL XV, 2008, 41-66.

6. Dowty, David R. (1979). Word Meaning and Montague Grammar. Dordrecht: Reidel.

7. Ernst, Thomas. (1995). Negation in Mandarin Chinese. Natural Language and Linguistic Theory 13.665-707.

8. Ernst, Thomas. (2004). Principles of adverbial distribution in the lower clause. Lingua 114: 755-777.

9. Fabricius-Hansen, Cathrine. (2001). 'Wi(e)der and Again(st)'. In C. Fery \& W. Sternefeld (eds). Audiatur Vox Sapientiae. A Festschrift for Arnim von Stechow. 101-130.

10. Huang, C.-T. James, Yen-hui Audrey Li, and Yafei Li. (2009). The syntax of Chinese. Cambridge: Cambridge University Press.

11. Jäger, Gerhard., \& Blutner, Reinhard. (2000). Against lexical decomposition in syntax. In A. Z. Wyner (ed.) Proceedings of the fifteenth annual conference of the Israeli Association for Theoretical Linguistics, 113-137. Reprinted in Reinhard Blutner and Gerhard Jäger (eds.), Studies in Optimality Theory, 5-29, University of Potsdam.

12. Klein, Wolfgang. (2001). Time and Again, in C. Fery and W. Sternefeld (eds.), Audiatur Vox Sapientiae. A Festschriftfor Arnim von Stechow, Akademie Verlag, Berlin, pp. 267286.

13. Kuo, Pei-Jung. (2009). IP internal movement and topicalization. PhD dissertation, University of Connecticut.

14. Li, Charles, and Sandra Thompson. (1981). Mandarin Chinese: A Functional Reference Grammar. Berkeley: University of California press.

15. Nissenbaum, Jon. (2006). Decomposing Resultatives: Two kinds of restitutive readings with Again, Poster presented at NELS37. University of Illinois, Urbana-Champain, Oct 1315 .

16. Rizzi, Luigi. (1990). Relativized Minimality. Cambridge: MIT Press.

17. Tang, Chih-Chen Jane. (1990). Chinese Phrase Structure and the Extended X'-Theory. Doctoral dissertation, Cornell University.

18. Tang, Chih-Chen Jane. (2001). Functional Projections and Adverbial Expressions in Chinese, Language and Linguistics 2.2: 203-241.

19. Tang, Sze-Wing. (1997). Parametrization of features in syntax. Ph.D. Dissertation, University of California, Irvine.

20. von Stechow, Arnim. (1995). Lexical Decomposition in Syntax, in U. Egli, et al., eds., Lexical Knowledge in the Organization of Language, Benjamins, Amsterdam, 81-118.

21. von Stechow, Arnim. (1996). The Different Readings of Wieder. Journal of Semantics 13, 87-138.

22. Wang, Chyan-an Arthur. (2010). The microparametric syntax of resultatives in chinese languages. $\mathrm{PhD}$ dissertation, New York University. 